The COMPASS Paradigm For The Systematic Evaluation Of U.S. ARMy COMMAND AND CONTROl Systems USING NeURAl NETWORK AND Discrete EVENT COMPUTER SimUlation

By

Sam E. Middlebrooks

Dissertation to be submitted to the faculty of the

Virginia Polytechnic Institute and State University

in partial fulfillment of the requirements for the degree of

Doctor of Philosophy

In

Industrial and Systems Engineering

Robert C. Williges, Ph.D., Chairman

C. Patrick Koelling, Ph.D.

Robert J. BeAton, Ph.D.

TONYA L. SMITH-JACKSON, PH.D.

EDWIN R. SMOOTZ, PH.D.

April 9, 2003

Blacksburg, Virginia

Keywords: human performance; task and workload modeling; command and control; command and control system; experimental design; polynomial regression; datamining; task network simulation; neural network simulation; discrete event simulation; naturalistic observation; ethnography 
This page left intentionally blank. 


\title{
The COMPASS Paradigm For The Systematic Evaluation Of U.S. ARMy Command AND CONTROL Systems Using NEURAL NETWORK AND DisCrete Event COMPUTER SimUlation
}

By

\author{
Sam E. Middlebrooks
}

\author{
Robert C. Williges, Ph.D., Chairman \\ Department of Industrial and Systems Engineering \\ Virginia Polytechnic Institute and State University
}

\section{(ABSTRACT)}

In today's technology based society the rapid proliferation of new machines and systems that would have been undreamed of only a few short years ago has become a way of life. Developments and advances especially in the areas of digital electronics and micro-circuitry have spawned subsequent technology based improvements in transportation, communications, entertainment, automation, the armed forces, and many other areas that would not have been possible otherwise. This rapid "explosion" of new capabilities and ways of performing tasks has been motivated as often as not by the philosophy that if it is possible to make something better or work faster or be more cost effective or operate over greater distances then it must inherently be good for the human operator. Taken further, these improvements typically are envisioned to consequently produce a more efficient operating system where the human operator is an integral component. The formal concept of human-system interface design has only emerged this century as a recognized academic discipline, however, the practice of developing ideas and concepts for systems containing human operators has been in existence since humans started experiencing cognitive thought.

An example of a human system interface technology for communication and dissemination of written information that has evolved over centuries of trial and error development, is the book. It is no accident that the form and shape of the book of today is as it is. This is because it is a shape and form readily usable by human physiology whose optimal configuration was determined by centuries of effort and revision. This slow evolution was mirrored by a rate of technical evolution in printing and elsewhere that allowed new advances to be experimented with as part of the overall use requirement and need for the existence of the printed word and some way to contain it. Today, however, technology is advancing at such a rapid rate that evolutionary use requirements have no chance to develop along side the fast pace of technical progress. One result of this recognition is the establishment of disciplines like human factors engineering that have stated purposes and goals of systematic determination of good and bad human system interface designs. However, other results of this phenomenon are systems that get developed and placed into public use simply because new technology allowed them to be made. This development can proceed without a full appreciation of how the system might be used and, perhaps even more significantly, what impact the use of this new system might have on the operator within it.

The U.S. Army has a term for this type of activity. It is called "stove-piped development." The implication of this term is that a system gets developed in isolation where the developers are only looking "up" and not "around." They are thus concerned only with how this system may work or be used for its own singular purposes as opposed to how it might be 
used in the larger community of existing systems and interfaces or, even more importantly, in the larger community of other new systems in concurrent development. Some of the impacts for the Army from this mode of system development are communication systems that work exactly as designed but are unable to interface to other communications systems in other domains for battlefield wide communications capabilities. Having communications systems that cannot communicate with each other is a distinct problem in its own right. However, when developments in one industry produce products that humans use or attempt to use with products from totally separate developments or industries, the Army concept of product development resulting from stove-piped design visions can have significant implication on the operation of each system and the human operator attempting to use it.

There are many examples that would illustrate the above concept, however, one that will be explored here is the Army effort to study, understand, and optimize its command and control (C2) operations. This effort is at the heart of a change in the operational paradigm in C2 Tactical Operations Centers (TOCs) that the Army is now undergoing. For the 50 years since World War II the nature, organization, and mode of the operation of command organizations within the Army has remained virtually unchanged. Staffs have been organized on a basic four section structure and TOCs generally only operate in a totally static mode with the amount of time required to move them to keep up with a mobile battlefield going up almost exponentially from lower to higher command levels. However, current initiatives are changing all that and while new vehicles and hardware systems address individual components of the command structures to improve their operations, these initiatives do not necessarily provide the environment in which the human operator component of the overall system can function in a more effective manner.

This dissertation examines $\mathrm{C} 2$ from a system level viewpoint using a new paradigm for systematically examining the way TOCs operate and then translating those observations into validated computer simulations using a methodological framework. This paradigm is called COmputer Modeling Paradigm And Simulation of Systems (COMPASS). COMPASS provides the ability to model TOC operations in a way that not only includes the individuals, work groups and teams in it, but also all of the other hardware and software systems and subsystems and human-system interfaces that comprise it as well as the facilities and environmental conditions that surround it.

Most of the current literature and research in this area focuses on the concept of $\mathrm{C} 2$ itself and its follow-on activities of command, control, communications (C3), command, control, communications, and computers (C4), and command, control, communications, computers and intelligence $(\mathrm{C} 4 \mathrm{I})$. This focus tends to address the activities involved with the human processes within the overall system such as individual and team performance and the commander's decision-making process. While the literature acknowledges the existence of the command and control system (C2S), little effort has been expended to quantify and analyze C2Ss from a systemic viewpoint. A C2S is defined as the facilities, equipment, communications, procedures, and personnel necessary to support the commander (i.e., the primary decision maker within the system) for conducting the activities of planning, directing, and controlling the battlefield within the sector of operations applicable to the system.

The research in this dissertation is in two phases. The overall project incorporates sequential experimentation procedures that build on successive TOC observation events to generate an evolving data store that supports the two phases of the project. Phase I consists of the observation of heavy maneuver battalion and brigade TOCs during peacetime exercises. The term "heavy maneuver" is used to connotate main battle forces such as armored and 
mechanized infantry units supported by artillery, air defense, close air, engineer, and other so called combat support elements. This type of unit comprises the main battle forces on the battlefield. It is used to refer to what is called the conventional force structure. These observations are conducted using naturalistic observation techniques of the visible functioning of activities within the TOC and are augmented by automatic data collection of such things as analog and digital message traffic, combat reports generated by the computer simulations supporting the wargame exercise, and video and audio recordings where appropriate and available. Visible activities within the TOC include primarily the human operator functions such as message handling activities, decision-making processes and timing, coordination activities, and span of control over the battlefield. They also include environmental conditions, functional status of computer and communications systems, and levels of message traffic flows. These observations are further augmented by observer estimations of such indicators as perceived level of stress, excitement, and level of attention to the mission of the TOC personnel. In other words, every visible and available component of the $\mathrm{C} 2 \mathrm{~S}$ within the $\mathrm{TOC}$ is recorded for analysis. No a priori attempt is made to evaluate the potential significance of each of the activities as their contribution may be so subtle as to only be ascertainable through statistical analysis. Each of these performance activities becomes an independent variable (IV) within the data that is compared against dependent variables (DV) identified according to the mission functions of the TOC. The DVs for the C2S are performance measures that are critical combat tasks performed by the system. Examples of critical combat tasks are "attacking to seize an objective", "seizure of key terrain", and "river crossings". A list of expected critical combat tasks has been prepared from the literature and subject matter expert (SME) input. After the exercise is over, the success of these critical tasks attempted by the $\mathrm{C} 2 \mathrm{~S}$ during the wargame are established through evaluator assessments, if available, and/or TOC staff self analysis and reporting as presented during after action reviews.

The second part of Phase I includes datamining procedures, including neural networks, used in a constrained format to analyze the data. The term constrained means that the identification of the outputs/DV is known. The process was to identify those IV that significantly contribute to the constrained DV. A neural network is then constructed where each IV forms an input node and each DV forms an output node. One layer of hidden nodes is used to complete the network. The number of hidden nodes and layers is determined through iterative analysis of the network. The completed network is then trained to replicate the output conditions through iterative epoch executions. The network is then pruned to remove input nodes that do not contribute significantly to the output condition. Once the neural network tree is pruned through iterative executions of the neural network, the resulting branches are used to develop algorithmic descriptors of the system in the form of regression like expressions.

For Phase II these algorithmic expressions are incorporated into the CoHOST discrete event computer simulation model of the $\mathrm{C} 2 \mathrm{~S}$. The programming environment is the commercial programming language Micro Saint ${ }^{\mathrm{TM}}$ running on a PC microcomputer. An interrogation approach was developed to query these algorithms within the computer simulation to determine if they allow the simulation to reflect the activities observed in the real TOC to within an acceptable degree of accuracy.

The purpose of this dissertation has been to introduce the COMPASS concept that is a paradigm for developing techniques and procedures to translate as much of the performance of the entire TOC system as possible to an existing computer simulation that would be suitable for analyses of future system configurations. 
The approach consists of the following steps:

- Naturalistic observation of the real system using ethnographic techniques.

- Data analysis using datamining techniques such as neural networks.

- Development of mathematical models of TOC performance activities.

- Integration of the mathematical into the CoHOST computer simulation.

- Interrogation of the computer simulation.

- Assessment of the level of accuracy of the computer simulation.

- Validation of the process as a viable system simulation approach. 


\section{DEDICATION}

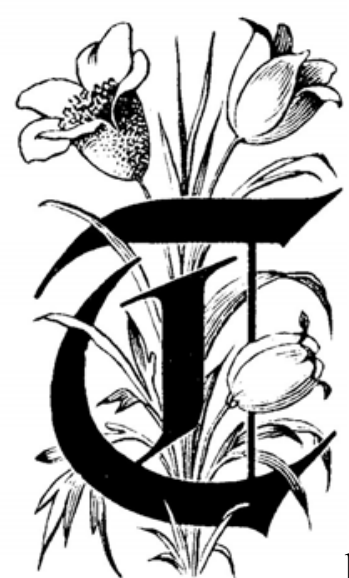

his dissertation is dedicated to Betty, my wife, mother of my

children, and my life partner. Her unfailing love, support and encouragement, through 35 plus years of marriage and a career spanning over 25 years across two continents through too many locations to remember, has made me the person I am today. This effort could not have been envisioned without her.

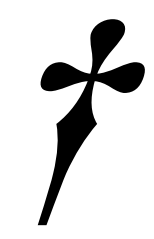


This page left intentionally blank. 


\section{ACKNOWLEDGEMENTS}

I want to acknowledge the support and assistance of the members of my dissertation committee, particularly the chairman, Dr. Robert C. Williges, for their professional leadership and ready expertise during this research effort. I consider it a distinct honor to have been Dr. Bob Williges' last Ph.D. student before his retirement and am humbled to see my name added to the end of a very long list of distinguished colleagues who have preceded me under his tutelage. Dr. Robert J. Beaton has added a wealth of knowledge in workload and system design. Dr. C. Patrick Koelling has opened my eyes to the real potential and capabilities of computer simulation analysis even though I thought I was a world class expert in that field when I came here. Dr. Tonya L. Smith-Jackson introduced me to the previously unknown area of ethnographic based research. Her depth of knowledge in this and the area of cognitive performance assessment has been of great help. Dr. Edwin R. Smootz, retired from my parent agency, the U.S. Army Research Laboratory's (ARL) Human Research and Engineering Directorate, along with Dr. Robin L. Keesee, my agency director, has been a constant source of encouragement in this educational pursuit at this stage of my life. Dr. Smootz has also applied his own psychologybased expertise to help guide my learning efforts. Finally, this effort would not have been possible without the research funding provided by ARL.

To all of you, Thankyou. 
This page left intentionally blank. 
(ABSTRACT) ..................................................................................................................................................III

DEDICATION..................................................................................................................... VII

ACKNOWLEDGEMENTS …......................................................................................................IX

TABLE OF CONTENTS _.........................................................................................................

LIST OF FIGURES .................................................................................................................................XV

LIST OF TABLES ........................................................................................................................ XVII

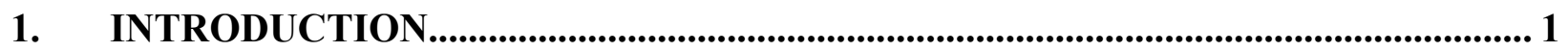

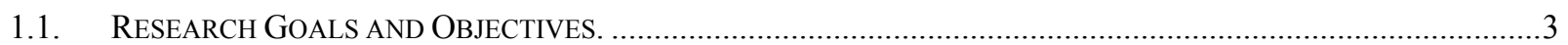

1.1.1. Research Objective ...........................................................................................................

1.1.2. Development of Procedures that will Allow the Screening and Identification of Significant IVs for

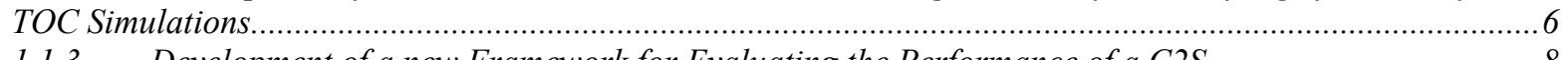

1.1.3. Development of a new Framework for Evaluating the Performance of a C2S...................................8

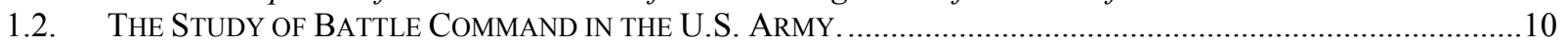

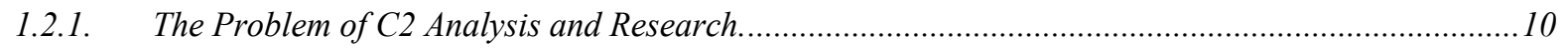

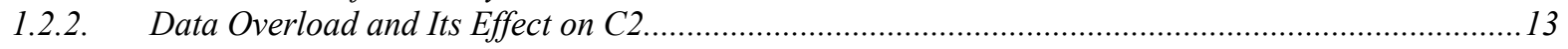

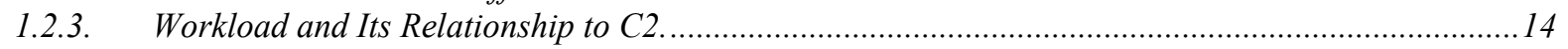

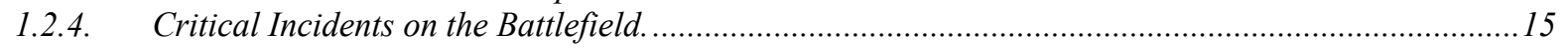

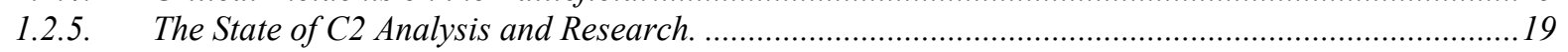

1.2.5.1. Operations Other Than War and a United Kingdom Land-Air Simulation. ...............................19

1.2.5.2. Army Command and Control Evaluation System...................................................................20

1.2.5.3. Using NNS to Evaluate Mental Workload in C2 Environments..............................................20

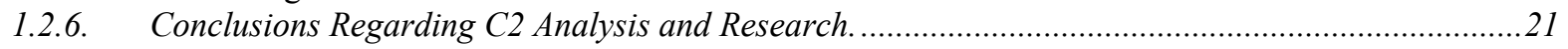

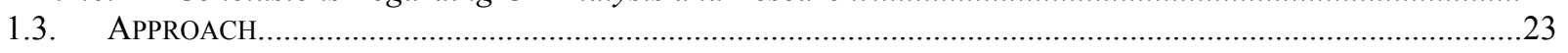

1.3.1. Phase I: Data Collection Through Naturalistic Observation Using Ethnographic Techniques and

Analysis Using Neural Network Simulation. ............................................................................................23

1.3.2. Phase II: C2 Analysis Using Discrete Event Simulation............................................................25

2. LITERATURE REVIEW............................................................................................... 27

2.1. Selected Military Techniques, ConcePtS, AND Procedures. .............................................................27

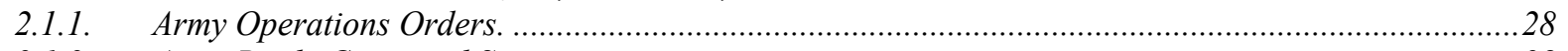

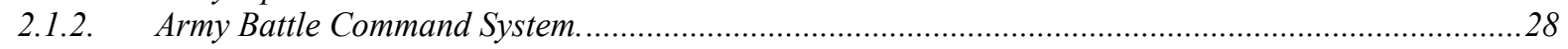

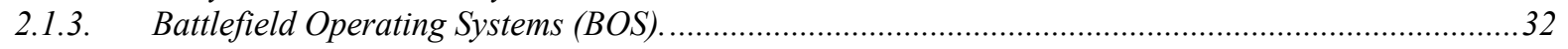

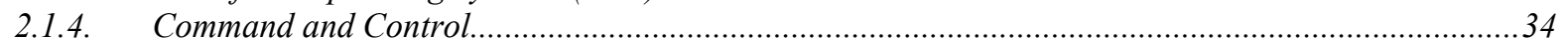

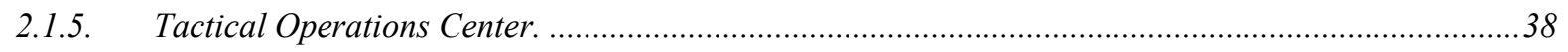

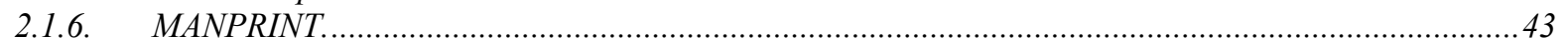

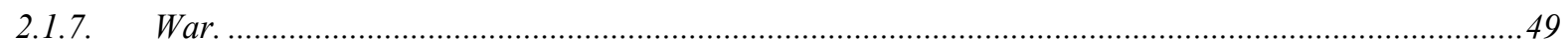

2.2. ISSUES IN HUMAN COGNITIVE PERformanCE. …..........................................................................51

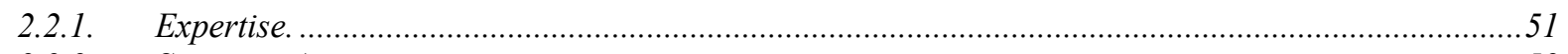

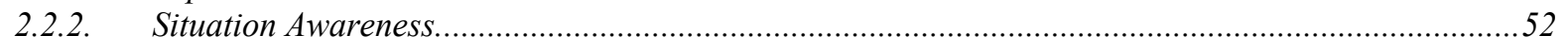

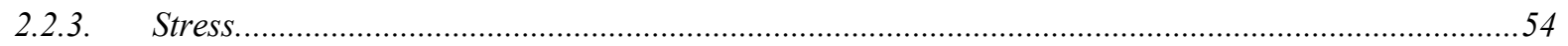

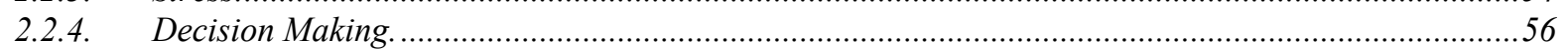

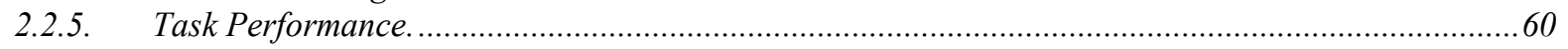

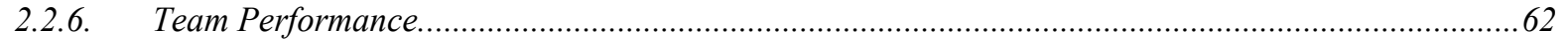




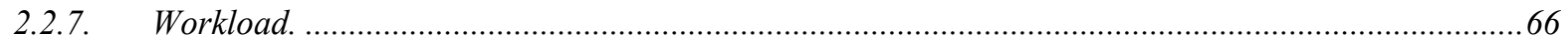

2.3. METHODS AND ProCEDURES FOR COGNITIVE PERFORMANCE ASSESSMENT................................................8

2.3.1. Evaluation of Human Cognitive Performance ....................................................................................8

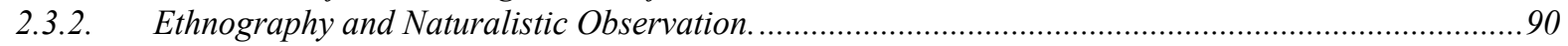

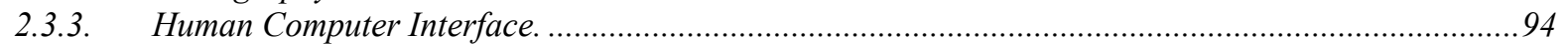

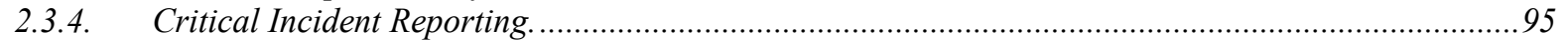

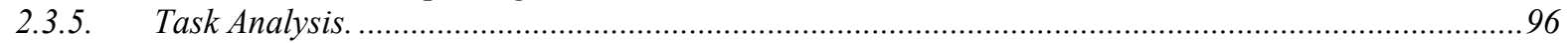

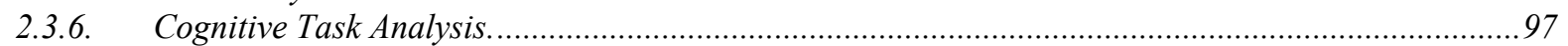

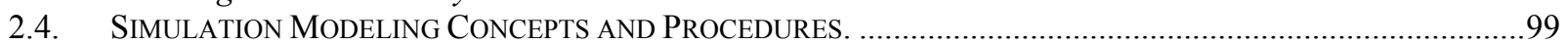

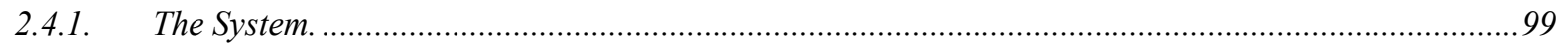

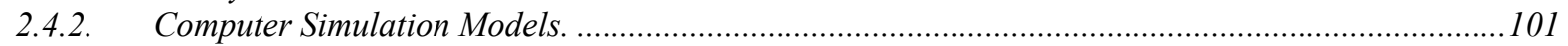

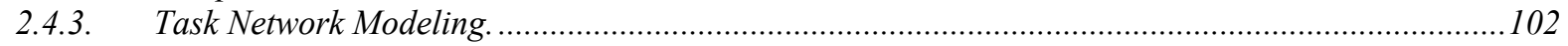

2.4.4. Optimization Techniques Using Computer Simulation Models....................................................106

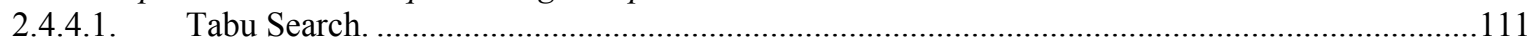

2.4.4.2. Goal Programming With Preemptive Priorities. .........................................................................112

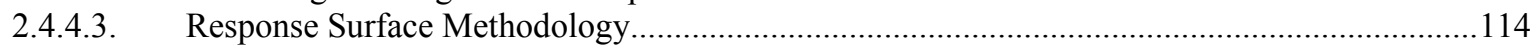

2.4.4.4. Box's Complex Method of Constrained Optimization. ..........................................................118

2.4.4.5. Gradient Based Optimization Approach..........................................................................118

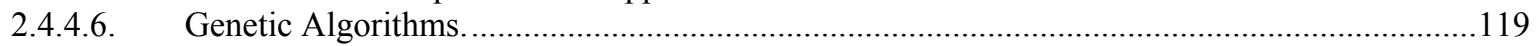

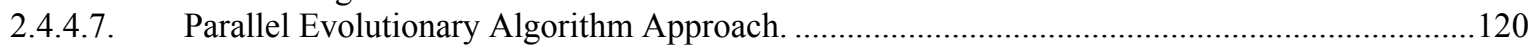

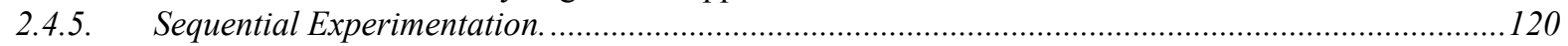

2.4.6. Simulations of Human System Performance ...................................................................................... 126

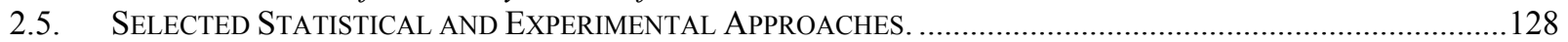

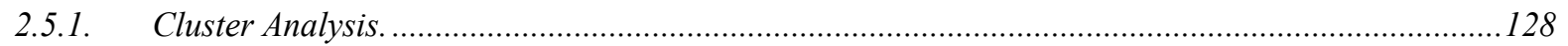

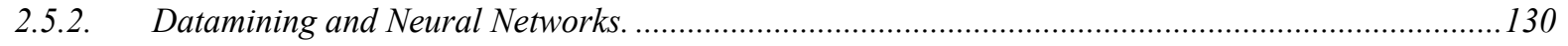

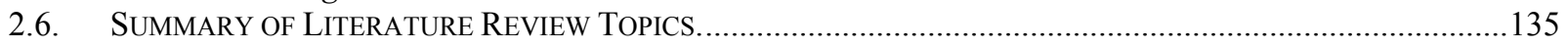

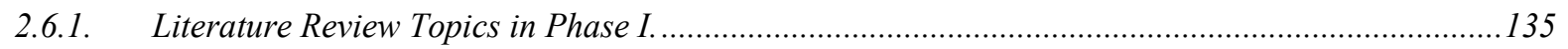

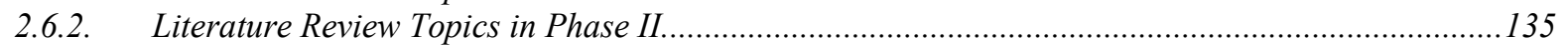

2.6.3. Literature Review Topics in Discussion Chapter................................................................. 136

3. PHASE I - ETHNOGRAPHIC BASED NATURALISTICALLY OBSERVED DATA COLLECTION AND NEURAL NETWORK ANALYSIS. ..................................................... 137

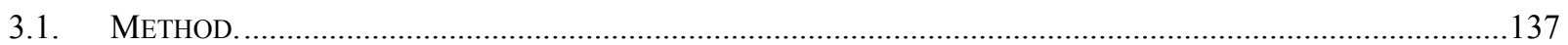

3.1.1. Ethnographic Based Naturalistic Observations of TOCs. .......................................................... 137

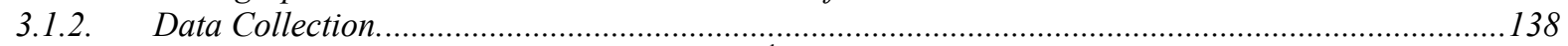

3.1.2.1. Exercise Raider Shadow, $1^{\text {st }}$ Brigade, $4^{\text {th }}$ Infantry Division, Fort Hood, Texas, April 2002 $\ldots . .141$

3.1.2.2. Exercise Ironhorse Bonecrusher, $1^{\text {st }}$ BCT, $1^{\text {st }}$ Cavalry Div., Fort Hood, Texas, Sept., $2002 \ldots . .146$

3.1.2.3. Exercise Warfighter, $1^{\text {st }}$ Brigade, $4^{\text {th }}$ Infantry Division, Fort Hood, Texas, November $2002 \ldots .147$

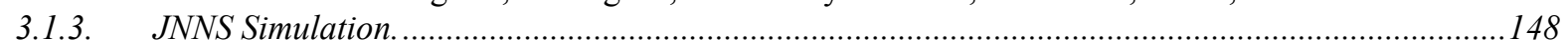

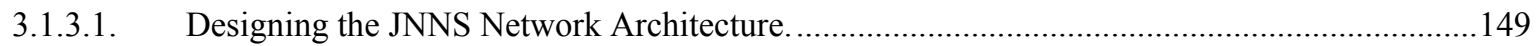

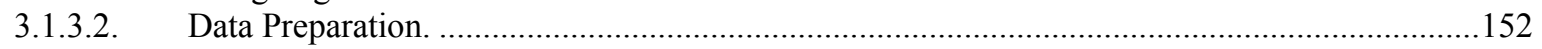

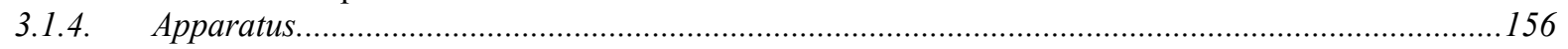

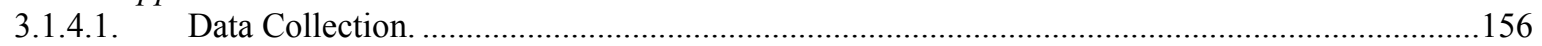

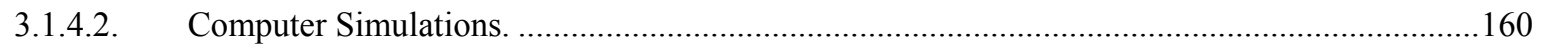

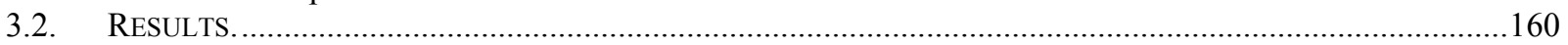

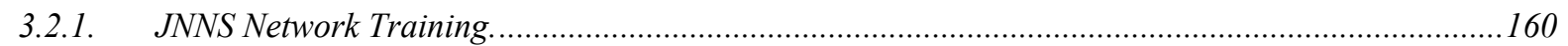

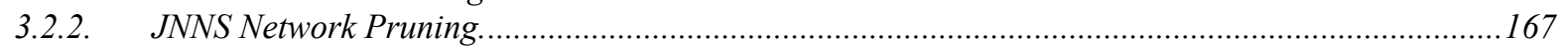

3.2.3. Development of Mathematical Models of DV Performance From JNNS.......................................172

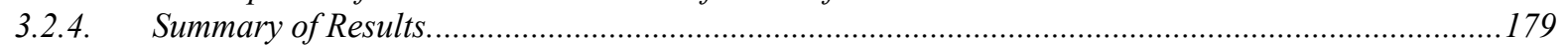

\section{PHASE II - DEVELOPMENT OF ALGORITHMIC CONTROL INTERFACES FOR TOC DISCRETE EVENT SIMULATIONS.................................................................. 181}

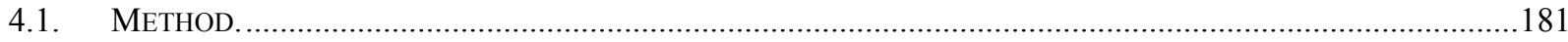

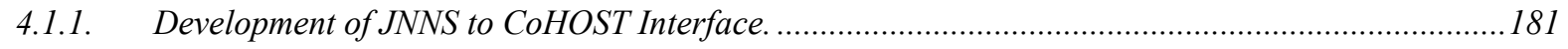

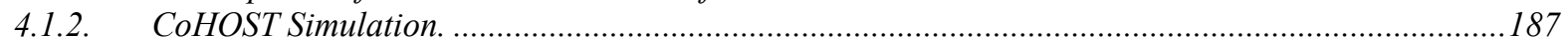




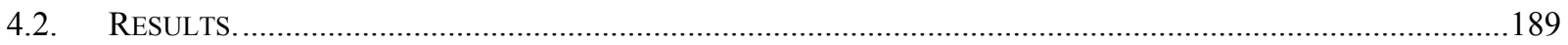

4.2.1. Evaluation Of the C2S With CoHOST Discrete Event Computer Simulation.....................................189

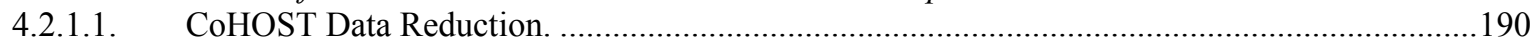

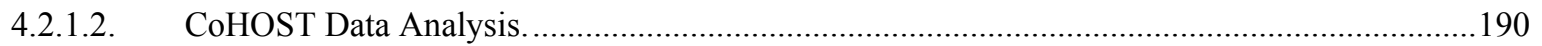

4.2.2. Predictions of TOC Activity From Mapping CoHOST Results to JNNS Predictors.........................199

4.2.2.1. Scenario Phase 1 - Preoperations Planning. ................................................................................20

4.2.2.2. Scenario Phase 2 - Movement To Contact (MTC).................................................................204

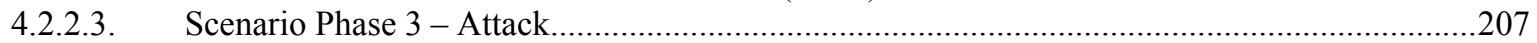

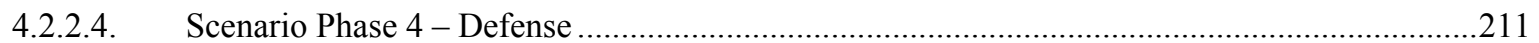

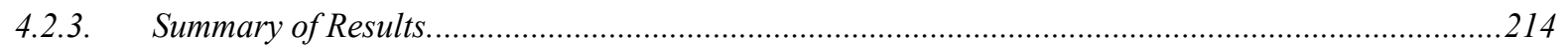

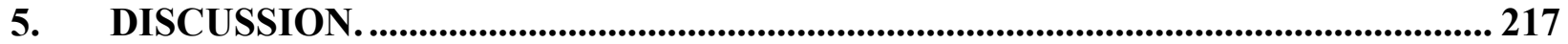

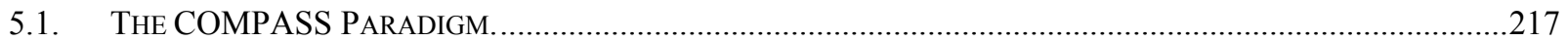

5.2. CONSIDERATIONS REGARDING THE COMPASS PARADIGM.............................................................222

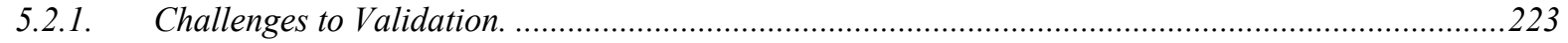

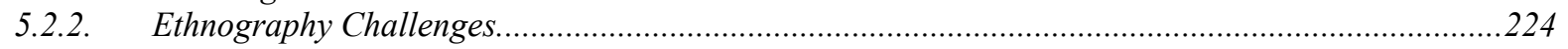

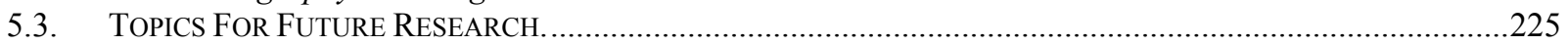

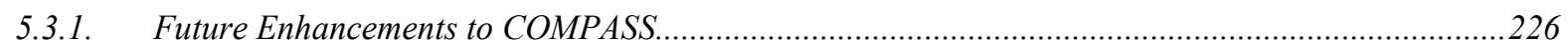

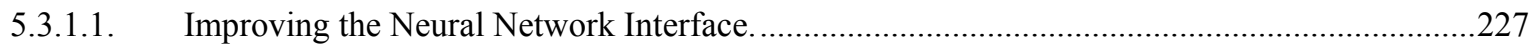

5.3.1.2. Improving the CoHOST Operator Interface-C3 TRACE. ........................................................231

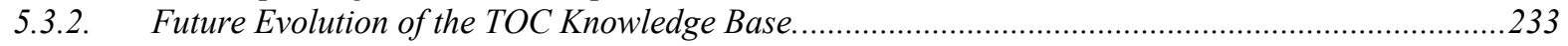

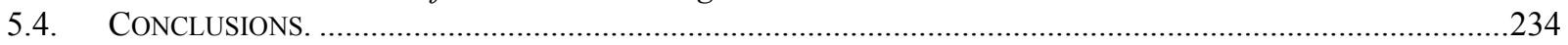

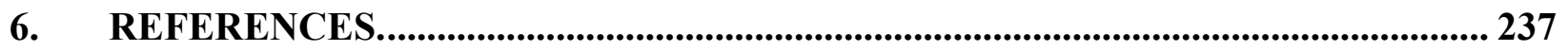

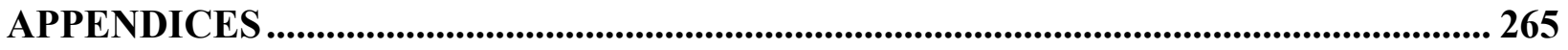

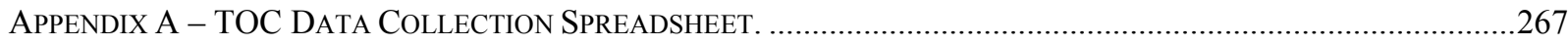

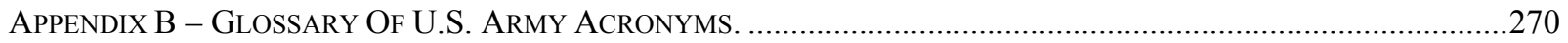

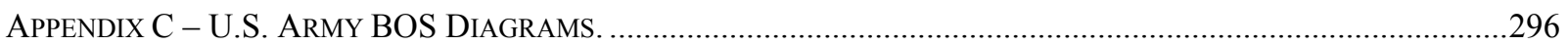

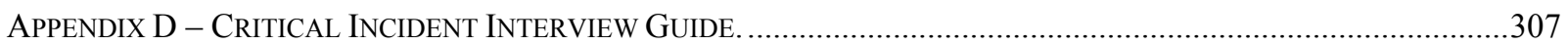

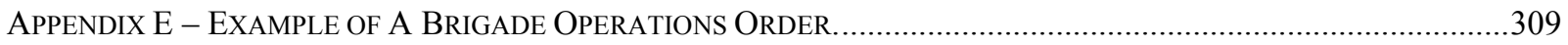

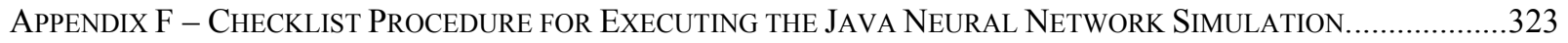

APPENDIX G - TRAINED JNNS OUTPUT NODE VALUES For TOC63 SiMULATION..............................................327

APPENDIX H - SAS PEARSON CORRELATION ANALYSIS OF JNNS OBSERVED TO TRAINED DV DATA....................329

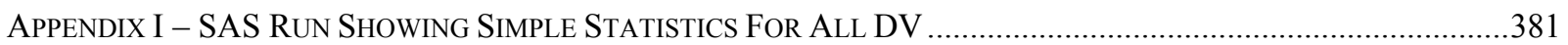

APPENDIX J - FIRST LINEAR REGRESSION RUN TO SELECT IV FOR INCLUSION IN DV MODELS..............................384

APPENDIX K - SECOND LINEAR REGRESSION RUN TO SELECT IV FoR INCLUSION IN DV MODELS .........................404

APPENDIX L - THIRD LINEAR REGRESSION RUN TO SELECT IV FOR INCLUSION IN DV MODELS..........................423

APPENDIX M - FOURTH LINEAR REGRESSION RUN TO SELECT IV FOR INCLUSION IN DV MODELS.......................435

APPENDIX N - LINEAR REGRESSION EXPRESSIONS FOR THE 17 DV MODELS....................................................447

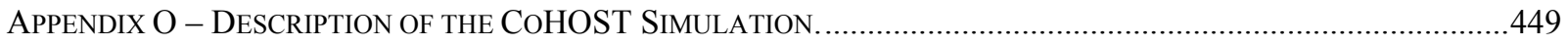

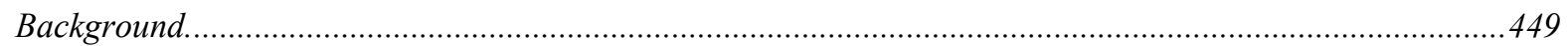

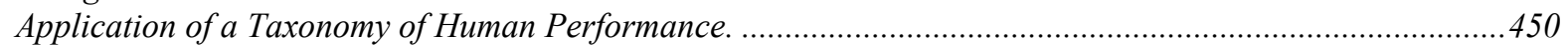

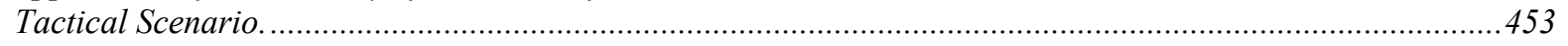

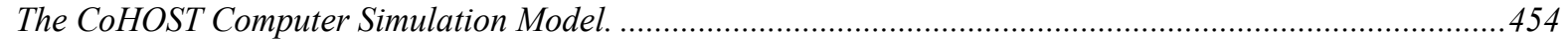

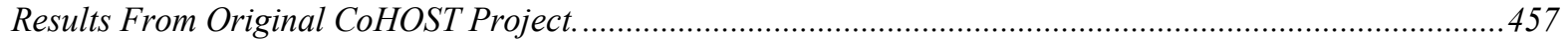

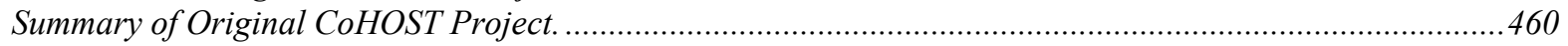

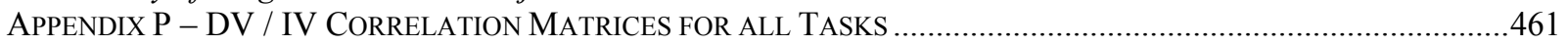

APPENDIX Q - DETERMINATION OF COHOST SiMULATION REPLICATION COUNT. ..............................................466

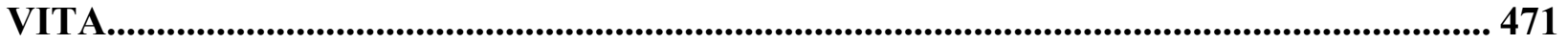


This page left intentionally blank. 


\section{LIST OF FIGURES}

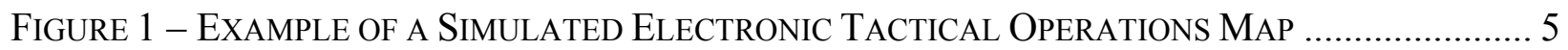

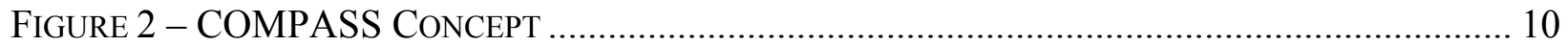

FIGURE 3 - EXERCISE OBSERVATIONS / DATA COLLECTION ..................................................... 24

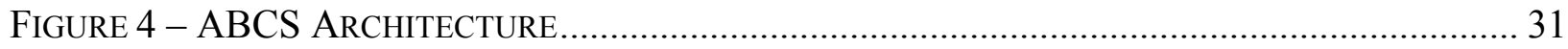

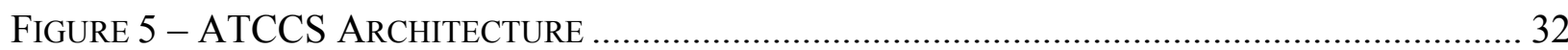

Figure 6 - SEVEn BATTLEFIELd OPERATING Systems (BOS) ............................................. 33

Figure 7 - MODEL OF COMMAND AND CONTROL PROCESS ......................................................... 37

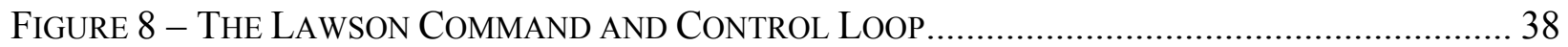

Figure 9 - TOC LAyOUT FOR $1^{\text {ST }}$ BDE, 1CD DuRING EXERCiSE PhANTOM THUNDER ................ 39

Figure 10 - The Military Decision MAKING ProceSS ........................................................ 59

FIGURE 11 - PAM TASK LEVEL ARCHITECTURE.................................................................... 80

FIGURE 12 - KNOWLEDGE, SKILLS, AND ABILITIES TAXONOMY ............................................ 83

FIGURE 13 - COHOST TASK FLOW SEQUENCE.................................................................... 84

FIGURE 14 - COHOST TASK DECOMPOSITION AND TRANSLATION FROM TAXONOMY ................ 85

FIGURE 15 - PERFORMANCE / WORKLOAD COMPARISON........................................................ 86

FIGURE 16 - A SIMPLE SYSTEM ............................................................................ 101

Figure 17 - Stages In SEQUENTIAL RESEARCH PARAdigM ............................................. 123

FigurE 18 - TACTICAL UNMANNED AERIAL VEHICLE (TUAV) ......................................... 141

FIGURE 19 - EXAMPLE VIDEO DISPLAY FROM TUAV …..................................................... 142

FIGURE 20 - EXCERPT OF RAW DATA COLLECTION SPREADSHEET ......................................... 146

FIGURE 21 - NNS NODE IDENTIFICATION AND NETWORK LAYOUT ......................................... 149

FIGURE 22 - COMPARISON OF BIOLOGICAL AND ARTIFICIAL NEURAL NETWORKS .................... 150

FigurE 23 - ARTIFICIAL NEURAL NETWORK ACTIVATION FUnCTIONS ................................. 151

FigURE 24 - JNNS TOC NETWORK AFTER LINK CREATION AND INITIALIZATION ..................... 152

FIGURE 25 - TRANSCRIBED PRELIMINARY DATA ............................................................ 154

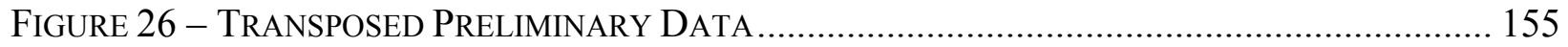

FIGURE 27 - JNNS PRELIMINARY PATTERN DATA FILE....................................................... 156

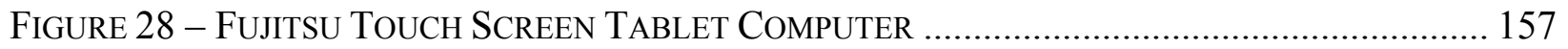

FIGURE 29 - SPECTRA MinI-SPOT LIGHTMETER................................................................ 158

Figure 30 - Quest Electronics Model 215 Sound LEVEL METER..................................... 159

FIGURE 31 - Vista SCIENTIFIC CORPORATION BATTERY OPERATED PSYCHROMETER ............... 159

FIGURE 32 - EXAMPLE ERROR GRAPH FOR A JNNS NEURAL NETWORK .................................. 161

Figure 33 - TOC JNNS Training NETWORK Diagram With ERror CURVE. ....................... 162

Figure 34 - JNNS TOC NEURAL Network DiAgram AfTER PRUNING ............................... 167

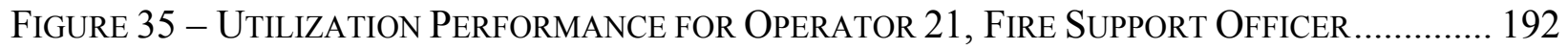

FIGURE 36 - WorklOAd PERFormancE FOR OPERATOR 21, FIRE SUPPORT OFFICER ............... 193

Figure 37 - TASK PERformance For Operator 12, BATtLE CAPTAin................................. 196

FIGURE 38 - TASK PERFORMANCE FOR OPERATOR 21, Fire SUPPORT OFFICER......................... 198

FIGURE 39 - BLOCK DiAgRAM OF THE COMPASS PARADIGM ............................................... 219

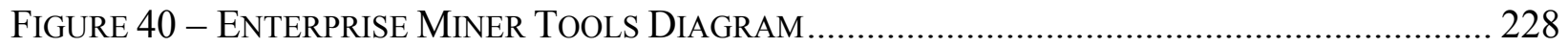

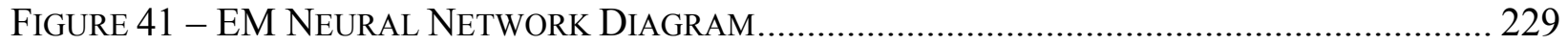

FIGURE 42 - EM ERROR GRAPH.................................................................................. 230

FIGURE 43 - C3 TRACE ORGANIZATION TREE WINDOW....................................................... 232

FIGURE 44 - C3 TRACE TASK DEFINITIONS .................................................................. 233 


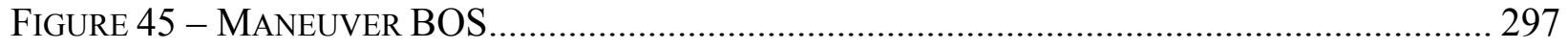

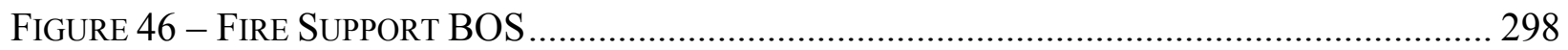

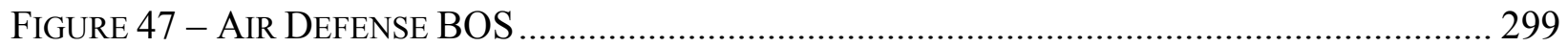

FIGURE 48 - COMMAND AND CONTROL BOS ................................................................... 300

FIGURE 49 - INTELLIGENCE BOS ............................................................................... 301

FIGURE 50 - MOBILITY AND SURVIVABILITY BOS - PART 1 ................................................. 302

FIGURE 51 - MOBILITY AND SURVIVABILITY BOS - PART 2 ….............................................. 303

FIGURE 52 - COMBAT SERVICE SUPPORT BOS - PART 1 ....................................................... 304

FIGURE 53 - COMBAT SERVICE SUPPORT BOS - PART 2 ................................................... 305

FIGURE 54 - COMBAT SERVICE SUPPORT BOS - PART 3 .................................................. 306

FIGURE 55 - TOC DIAGRAM ...................................................................................... 450

FiguRE 56 - Fleishman's KNOWLEDGE, SkILlS, AND ABILITIES TAXONOMY ....................... 452

FIGURE 57 - TACTICAL SCENARIO OVERLAY ..................................................................... 454

Figure 58 - CONCEPTUAL MOdEL OF TOC OPERATIONS..................................................... 455

FIGURE 59 - COHOST MODEL ACTION VIEW DiSPLAY ....................................................... 456

FIGURE 60 - COHOST MODEL NETWORK FlOW DIAGRAM ................................................. 457

Figure 61 - Percent of Time Spent In Each Performance Category of the TaXonomy 458 


\section{LIST OF TABLES}

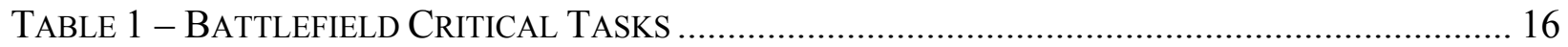

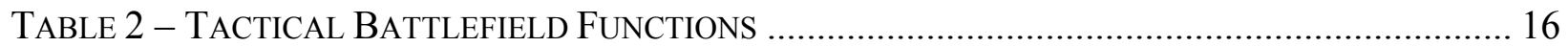

TABLE 3 - BATTALION AND BRIGADE BATTLEFIELD FunCTIONS GROUPED BY BOS ................... 18

TABLE 4 - TOC C2 FUNCTIONAL REQUIREMENTS ACCORDING TO BOS ................................... 40

TABLE 5 - CRITERIA For SELECTION OF WORKLOAD ASSESSMENT TECHNIQUES...................... 69

TABLE 6 - SubJECTIVE EVAluAtION METHOdS FOR WORKLOAD ASSESSMENT..........................8 88

TABLE 7 - SumMARY OF HuMAN SySTEM MODELING APPROACHES ....................................... 101

TABLE 8 - TOC OBSERVATION IVS BY BOS.................................................................... 143

TABLE 9 - TOC OBSERVATION IVS BY OTHER CATEGORIES .................................................. 144

TABLE 10 - TOC DEPENDENT MEASURES OF PERFORMANCE ................................................. 145

TABLE 11 - SELECTION VALUES For THE VARIABLE "Primary TASK" ................................... 153

TABle 12 - SAMPle OF OutPut DV VALues From the TOC OBSERVATION DATA................. 163

TABLE 13 - SAMPLE OF OUtPut DV VALUES From THE JNNS TRAined DATA....................... 164

TABLE 14 - PEARSON CORRELATION ANALYSIS OF OBSERVED TO TRAINED JNNS OUTPUTS.... 164

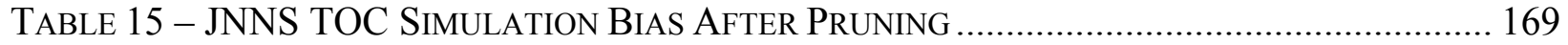

TABLE 16 - DV SELECTED For MODEL GENERATION ........................................................... 173

TABLE 17 - IV SELECTEd For INCLUSION IN DV MOdELS BASED ON JNNS PRUNING ............ 174

TABLE 18 - FIRST ITERATION TO SELECT IV FOR INCLUSION IN TP10 DV MODEL BASEd ON

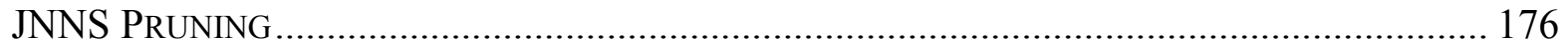

TABLE 19 - SECOND ITERATION TO SELECT IV For INCLUSION IN TP10 DV MODEL BASED ON

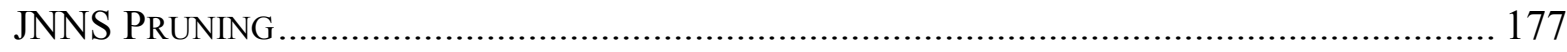

TABLE 20 - THIRD ITERATION TO SELECT IV FOR INCLUSION IN TP10 DV MODEL BASED ON

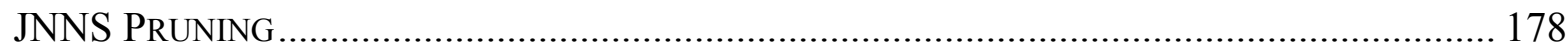

TABLE 21 - FoURTH ITERATION TO SELECT IV FOR INCLUSION IN TP10 DV MODEL BASED ON

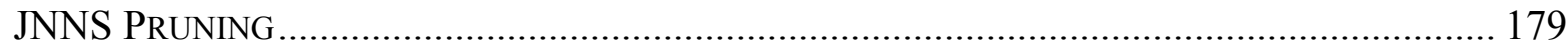

TABLE 22 - LINEAR REGRESSION EXPRESSION FOR DV TP10 ............................................ 179

TABLE 23 - JNNS REGRESSION MODELS / DVS .................................................................... 183

TABLE 24 - JNNS MODEL REGRESSORS / IVS ....................................................................... 183

TABLE 25 - DETERMINATION OF DV TARGET VALUES ........................................................... 186

TABLE 26 - SAMPLE DV / IV CORRELATION MATRIX FOR TASK TP10 ................................... 186

TABLE 27 - DV UtILIZATION / PSF SETTING CORRELATION.................................................... 188

TABLE 28 - DV / IV CORRELATION - PLANNING - PSF @ -10\% ............................................... 201

TABLE 29 - DV / IV CORRELATION - PLANNING - PSF @ -20\% ............................................... 202

TABLE 30 - DV / IV CORRELATION - PLANNING - PSF @ -30\% ................................................ 203

TABLE 31 - DV / IV CORRELATION - MTC - PSF @ -10\%...................................................... 204

TABLE 32 - DV / IV CORRELATION - MTC - PSF @ -20\%..................................................... 205

TABLE 33 - DV / IV CORRELATION - MTC - PSF @ -30\%...................................................... 206

TABLE 34 - DV / IV CORRELATION - ATTACK - PSF @ -10\% ................................................... 208

TABLE 35 - DV / IV CORRELATION - ATTACK - PSF @ -20\% ................................................ 209

TABLE 36 - DV / IV CORRELATION - ATTACK - PSF @ -30\% ................................................ 210

TABLE 37 - DV / IV CORRELATION - DEFENSE - PSF @ -10\% ................................................. 211

TABLE 38 - DV / IV CORRELATION - DEFENSE - PSF @ -20\% ….............................................. 212

TABLE 39 - DV / IV CORRELATION - DEFENSE - PSF @ -30\% ................................................. 213

TABLE 40 - GuIdelines For The Use Of THE COMPASS PARADIGM.................................... 220 
TABLE 41 - DV / IV CoRrelation MATRIX For EACH PERFORMANCE TASK.......................... 461

TABLE 42 - DATA From INITIAL 5 REPLiCATION SiMUlation Run...................................... 467

TABLE 43 - REPLICATION ANALYSIS FOR INITIAL 5 REPLICATION RUN .................................. 469 


\section{Introduction.}

Following the Persian Gulf War, when the U.S. Army determined that its current armored command and control vehicle was obsolete, the Human Research and Engineering Directorate of the U.S. Army Research Laboratory began a series of studies and projects focused on investigating the nature of military command and control (C2) operations. The questions seeking answers were whether these operations would be affected by the new operational paradigm mandated with the introduction of digitized battlefield systems in the area of command, control and communications (C3). These initiatives resulted in a number of projects, products, and studies aimed at addressing these issues. Among the modeling efforts were the IMPRINT modeling tool for developing models of individual and workstation soldier performance (Allender, Kelley, Archer, and Adkins, 1994), CrewCut (Dahl, Laughery, Hahler, Lockett, and Thein, 1991; Hahler, Dahl, Laughery, Lockett, and Thein, 1991) that provides an environment for the analysis of crew workload, WinCrew (Archer and Lockett, 1997) for modeling human performance and workload, the JACK anthropometric model (Kozycki, Faughn, Leiter, and Lockett, 1997), and the CoHOST task and workload models for battalion and brigade command and control teams (Middlebrooks et al., 1999b). A series of studies was also conducted to investigate the nature of the cognitive aspects of battlefield command and control and address how the operational shift to digitized operations would affect it (Adelman, Leedom, Murphy, and Killam, 1998; Cook, Leedom, Grynovicki, and Golden, 2000; Golden, Cook, Grynovicki, Kysor, and Leedom, 2000; Leedom, Adelman, and Murphy, 1998; Leedom and Fallesen, 1998; Leedom, Murphy, and Adelman, 1998a; Leedom, Murphy, and Adelman, 1998b; Murphy, Adelman, Leedom, Grynovicki, Golden, and Kysor, 1998).

These efforts have been in association with many other individuals, groups and agencies across the human factors domain (Bethmann, Malloy, and Hoever, 1989; Cooper, Shiflett, and Crotkin, 1984; D'Angelo, 1980; Levis and Athans, 1986; Maillefert, 1975; Monguillet and Levis, 1988; Perdu, 1988; Runals, 1985; Sutten and Hervey, 1986; Walker, Reimer, Brown, and Kloecker, 1984; Wildenberg, 1987; Wohl, Entin, and Eterno, 1983). These have all met with varying degrees of success and have typically addressed the issue from a specific point of reference. Examples of these points of reference include "command and control operations" (Ainslie, Leibrecht, and Atwood, 1991; Bolte, Black, and Mendel, 1991; Crumley and Sherman, 
1990; Fallesen, Lussier, and Michel, 1992a; McCann, 1990; Olsen, 1991; Swanson and Gibson, 1990; Zubal and Steinberg, 1989), “team performance” (Bowers, Jentsch, and Morgan, 2001; Campion, Brander, and Koritsas, 1998; Kay and Dolgin, 1998; Mathieu, Heffner, Goodwin, Salas, and Cannon-Bowers, 2000; Militello, Kyne, Klein, Getchell, and Thordsen, 1999; Peters, 1997; Sebok, 2000), "task analysis' to include cognitive task analysis (Ainsworth, 2001; Kieras and Meyer, 2000; Klein, 2000b; Luczak and Stahl, 2001; McNeese and Rentsch, 2001; Prietula, Feltovich, and Marchak, 2000; Schaafstal, Schraagen, and van Berlo, 2000; Schraagen, Chipman, and Shalin, 2000a; Schraagen, Chipman, and Shalin, 2000b; Schraagen, Chipman, and Shute, 2000; Seamster, Redding, and Kaempf, 2000; Vicente, 2000), and cognitive "task and workload" assessment (Adams, Tenney, and Pew, 1991; Hamilton, Bierbaum, and Fulford, 1991; Laughery, 1989a; Middlebrooks, 2001; Middlebrooks et al., 1999b; Wierwille, Rahimi, and Casali, 1986; Xie, 1997).

This work has predominately focused on the human component in the human systems interface with results that typically address issues that relate to the system operator(s). The goal of this research is to develop methodologies, modeling tools, and initial products that address the total system that encompasses a battalion or brigade command and control "operation." The term "operation" is used here to imply the entire C2 system. This includes the human team members, digital command and control systems, and external influences that affect the performance of this system. Because of the potentially huge numbers of variables and resultant data, this project started with the realization that the extent of the data may not be known until after it had been collected. At this point the final analysis plan could only then be finalized. It was presumed that traditional predictive techniques such as full and partial factorial experimentation and linear, multiple, and polynomial regression will be inefficient to attempt. In this research, these techniques are supplanted by datamining approaches that attempt to identify significant relationships in very large datasets.

This research had the potential to fall within one of two broad categories. A contentoriented research effort, in the context of tactical operations center (TOC) operations, would focus on the issue of how to design better TOCs and would have the primary goal of identifying key significant parameters that directly affect how a TOC system performs. The associated processes and procedures that dictate how these independent variable (IV) parameters would be used to evaluate existing TOC designs would be a byproduct of an effort focused on the 
development of a better understanding of how a TOC operates and what factors significantly affect that operation. Of follow-on importance would be the ability to predict the operation of future TOC designs and/or impacts of new TOC subsystem employments.

Alternatively, a methodology-based research effort would focus on the development of a framework to describe the processes and procedural tools that can be used to evaluate the performance of human-based systems such as military TOCs. These methodologies would have potential application to other similar systems where time pressure and high stress levels play critical roles in the performance of the overall system and its human component subsystem. This methodological framework would be developed using military TOCs as the exploratory medium and the process of developing the procedures would provide insight into critical elements of TOC operations. However, the primary goal of the research would be to develop techniques and procedures that would be applicable to a wide range of team-based, performance-oriented work groups and systems.

The distinction between using content versus methodological approaches for this research is subtle, but significant. For this research both approaches have similar end states as overall project objectives, however, the priorities to be followed during the course of the work are significantly different. Since this work is but the beginning of a long-term goal of establishing a new research program at ARL to investigate and improve TOC performance, the overall project is envisioned to have a content-oriented objective. However, since the purpose of this dissertation is to develop a framework of methodologies and procedures that will be used for the on-going effort, the research focus is methodological in nature.

\subsection{Research Goals and Objectives.}

The purpose of this dissertation is to take a systemic look at an activity the U.S. Army has been actively trying to understand for at least the last 50 years. Army command and control work teams have many similarities to counterparts in the civilian work sector where time pressure and high stress play critical roles. Counterpart examples from the civilian sector include hospital emergency room teams, nuclear power plant operator teams, and ship handling teams. What sets Army, and other military, teams apart from these counterparts are the dire consequences that could arise from ineffective performance. Instantaneous life and death, potentially across the entire battlefield that could encompass the team itself, are possible results of how well the team performs in its work domain. 
While much of the literature focuses on how well the human component of the overall system performs (i.e., the team), this research has a goal of examining the performance of the total command and control system. The focus is on Army battalion and brigade command and control centers as these two systems are very similar in organization and scope. In the U.S. Army the battalion, which consists of approximately 800 soldiers, is the lowest level where the formal command and control system is in existence. The brigade system, at the next higher level, directs the activities of three battalions. The complexity of the battlespace management tasks can be illustrated by looking at a tactical operations map used by a TOC during combat operations. Figure 1 is an example of this type of map where tactical control measure lines and symbols representing units on the battlefield is overlayed on top of a topographic map. The legend indicates the symbol color for different friendly units on the map while enemy units are shown in red. A thermometer like color filling of the unit symbols is used to represent approximate the strength of the unit. The image in this figure was produced from electronic imagery generated from a computer wargame simulation. While some units still use paper maps with manually posted unit symbol stickers during training exercises, there are digital battlefield systems in testing and initial trial use that provide this type of electronic imagery to the TOC from actual battlefield intelligence data. 


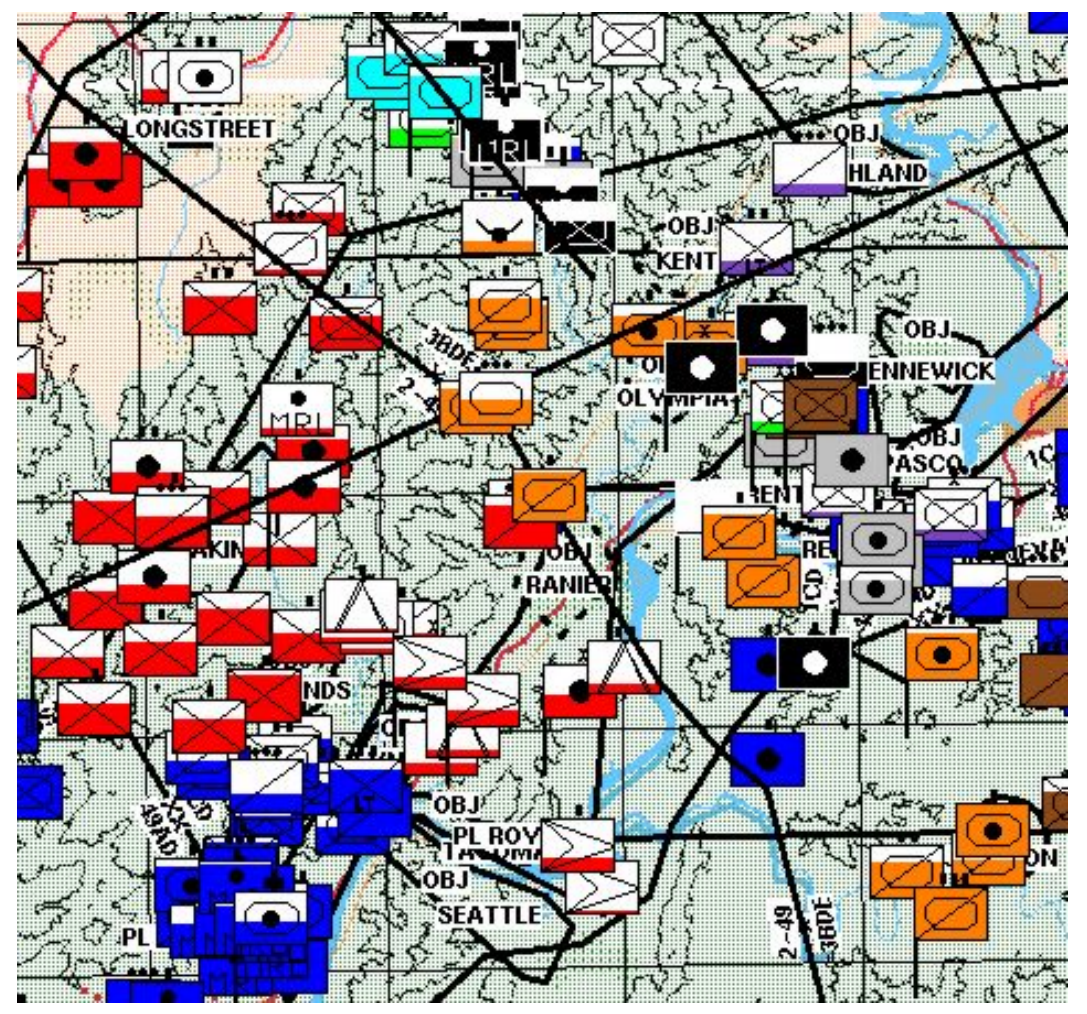

Figure 1 - Example of a Simulated Electronic Tactical Operations Map

\subsubsection{Research Objective.}

As the Army is struggling with the issues surrounding the change in the operational paradigm for how a TOC is to perform in a digitized and mobile environment, techniques and procedures need to be developed that will allow the identification of key parameters that should be included in TOC design. It is the intent of this dissertation to develop a framework of techniques and procedures that are based upon empirical methods but are usable in real world, uncontrolled environments which can only be approached using techniques such as naturalistic and ethnographic observation methods. Examples of some real world environments in addition to the military TOCs include hospital emergency rooms, shipboard bridges, and nuclear power plant control rooms. These are all examples of high stress, time critical work systems where the system performs in accordance to its input stressors in order to meet established performance guidelines. As such, they are totally intolerant to the application of any attempt at external control such as would be represented by experimental design treatment conditions. In order to achieve the desired fidelity in the analytical conclusions, the data must be rigorous enough to 
support empirical inquiry but must be recorded in an unconstrained, non-experimental environment where the process of gathering the data must not change the performance of the system being observed.

1.1.2. Development of Procedures that will Allow the Screening and Identification of Significant IVs for TOC Simulations.

The operation of military $\mathrm{C} 2$ is a complicated arena where many different factors can have an impact on the outcome of the performance of the system. While previous studies have identified $63 \mathrm{IV}$, the potential exists that this list could grow to 100 or more as more data are identified and gathered. In addition, while previous studies have considered 6 IV from a list of 22 possible performance measures, future studies could consider much larger performance specifications.

The consideration of data sets of this size requires techniques and procedures that transcend normal statistical inquiry. First, because different IV will be observed from different observation events, the use of sequential experimentation methods (Han, Williges, and Williges, 1997; Williges, Williges, and Han, 1992; Williges, Williges, and Han, 1993) is warranted as a systematic process to gather and organize the data. Once the data is gathered, however, some process is needed to identify meaningful relationships in the dataset and, further, to derive meaningful conclusions from that data. There is also the need to be able to identify the key parameters or IV that significantly describe the operation of the TOC so that these parameters can then be used in predictive computer simulations of the TOC. There are existing methods that can be applied to assist in this analysis effort, however, a framework that combines these methods into a new analytical paradigm is needed. Some of the techniques that are candidates to be incorporated into this paradigm include neural network simulation, regression analysis, and cluster analysis. Subject matter expert opinion, while not an analytical procedure, can also play a significant role in the determination of critical system components. These methods are summarized in the following paragraphs:

- Neural Network Simulation. Neural networks (Kwahk, 2002; Ntuen and Li, 2000; Reilly and Cooper, 1995) have the unique ability to investigate very large databases that contain many different factors. The drawback to their use is that the final results can be dependent on the technical ability and subjective opinion of the analyst as to when the network has achieved a state of being fully trained. 
Further subjective opinion is required to identify the level of pruning that is appropriate for the system being evaluated. As a result, the use of NNS can be considered an inexact science. However, this is probably not more so than many other multivariate techniques where the problem space must be probed with attempts to identify significant relationships between factors in the dataset.

- Regression Analysis. Before the advent of computer based statistical packages a regression analysis suffered the same problem of full factorial ANOVA experimentation. For small evaluations of up to 3 or 4 factors, regression analyses could be developed using manual means. Beyond this they became very large and inefficient to attempt. With computer based statistical analysis many of these limitations have been eliminated. However, early computer based packages that ran on mainframe computers in a batch oriented mode were difficult and cumbersome to use and the turnaround from submission of a statistical analysis run to the receipt of results could be days or even weeks. The advent of powerful desktop PC based computers has only recently afforded a true interactive interface with the ability to handle large regression situations that could not have been considered only a short time ago. If this approach can handle the large number of IV and dependent variables (DV) that are expected then the advantage would be a deterministic numerical solution that provides a very close mathematical description of the problem space.

- Cluster Analysis. Cluster Analysis (CA) is the generic name for mathematical models that can be used to find out which objects in a set are similar (Romesburg, 1984). It also allows the ability to find out which objects in a set are dissimilar. The most widely used form of CA is hierarchical CA (HCA). The steps in HCA are:

1. Collect a data matrix where the columns consist of the objects to be cluster analyzed and the rows are attributes that describe the objects.

2. Standardize the data matrix (optional).

3. Compute the values of a resemblance coefficient as a measure of the similarities among all the pairs of the data objects (from the standardized data matrix). 
4. Process the values of the resemblance coefficient using a clustering method which results in a tree diagram or dendrogram that graphically shows the relationship between the similarities among all the pairs of objects.

- Subject Matter Expert Opinion. This source of information can provide a rich store of data that is pertinent and current to the issues being investigated. Subject Matter Experts (SME) have a high degree of experience and knowledge that can be directly applied to a better understanding of the system. These individuals can, however, often be hard to find and they may not always be willing to participate in the study. The opinions from SME can also be highly subjective in nature and many different individuals may need to be approached before a consistent opinion can be realized.

This approach makes use of existing techniques and others that may be identified during the course of the study to develop a systematic framework that can be used to select key parameters that significantly describe the problem space. If a few number of IV can become known that address a large percentage of the response of the system then these IV are the logical candidates to be used to cause a simulation of that system to respond to its stimuli to within acceptable limits of precision. Assuming success in this effort, then, these few IV can be used for multiple purposes. First, they are the direct indicators of those factors that system designers should account for in the design of the system. Second, they provide the parameters that must be included in a computer simulation of that system in order to allow that simulation to be used for predictive purposes related to new system designs and proposed modifications. It is the merging and tailoring of all the original techniques and methods into a consistent methodological procedure or paradigm that will be the contribution of this research topic. This new paradigm would then be demonstrated using the case of the military TOC to illustrate how key parameters can be identified that describe the significant performance of the TOC.

\subsubsection{Development of a new Framework for Evaluating the Performance of a C2S.}

The development of an integrated framework to screen and identify significant IVs for TOC simulations is the focus of this research because it is considered to be a logical starting point for what is envisioned to be a continuing series of studies that will comprise a total project program that will continue past this dissertation. This integrated framework is a new paradigm 
for a direct path from the observation of unconstrained, real system performance and the ability to accurately model it in computer simulation. This new paradigm is called COmputer Modeling Paradigm And Simulation of Systems (COMPASS).

The COMPASS integrated framework, as shown in Figure 2, can be summarized as a systematic approach that provides an ability to look at a highly complex system that contains so many operational parameters as to make it virtually unapproachable using conventional experimental techniques such as factorial experimentation and regression analysis. This framework begins with system level observations of performance of the real system using naturalistic observations with an ethnographic approach. It derives pertinent information concerning key parameters that define the performance of that system to an identified level of confidence using exploratory approaches such as NNS. These parameters are then utilized in discrete event computer simulations of the system to provide the ability to model overall system performance and establish a platform for evaluating changes and modifications that may be projected for different portions of subsystems within the total system. The validation of the resulting simulation is then the comparison of its response back to the original observations of that system.

COMPASS consists of the specific observation process, the NNS exploratory process generation of descriptive and mathematical models, application of these models to a discrete event computer simulation, and the validation of the results of that simulation back to the original environmental conditions. The successful completion of the COMPASS procedures will produce a resultant computer simulation that not only is capable of performing evaluative studies on the original system, but will be flexible to updates from iterative uses of the methodology to keep pace with evolving changes in the system being evaluated.

The contribution of this research will be the development of the COMPASS paradigm for assessing the performance of complex systems that, in reality, are systems of systems that are reacting to the influence of hundreds of operational parameters and whose measure of performance can be spread across dozens of response characteristics. While COMPASS will make use of many techniques and procedures already in existence, it is the integration of these methods combined with novel use of exploratory capabilities contained in multivariate datamining techniques such as NNS that will make it a potentially new and powerful approach for investigating systems that have previously eluded systematic evaluation. 


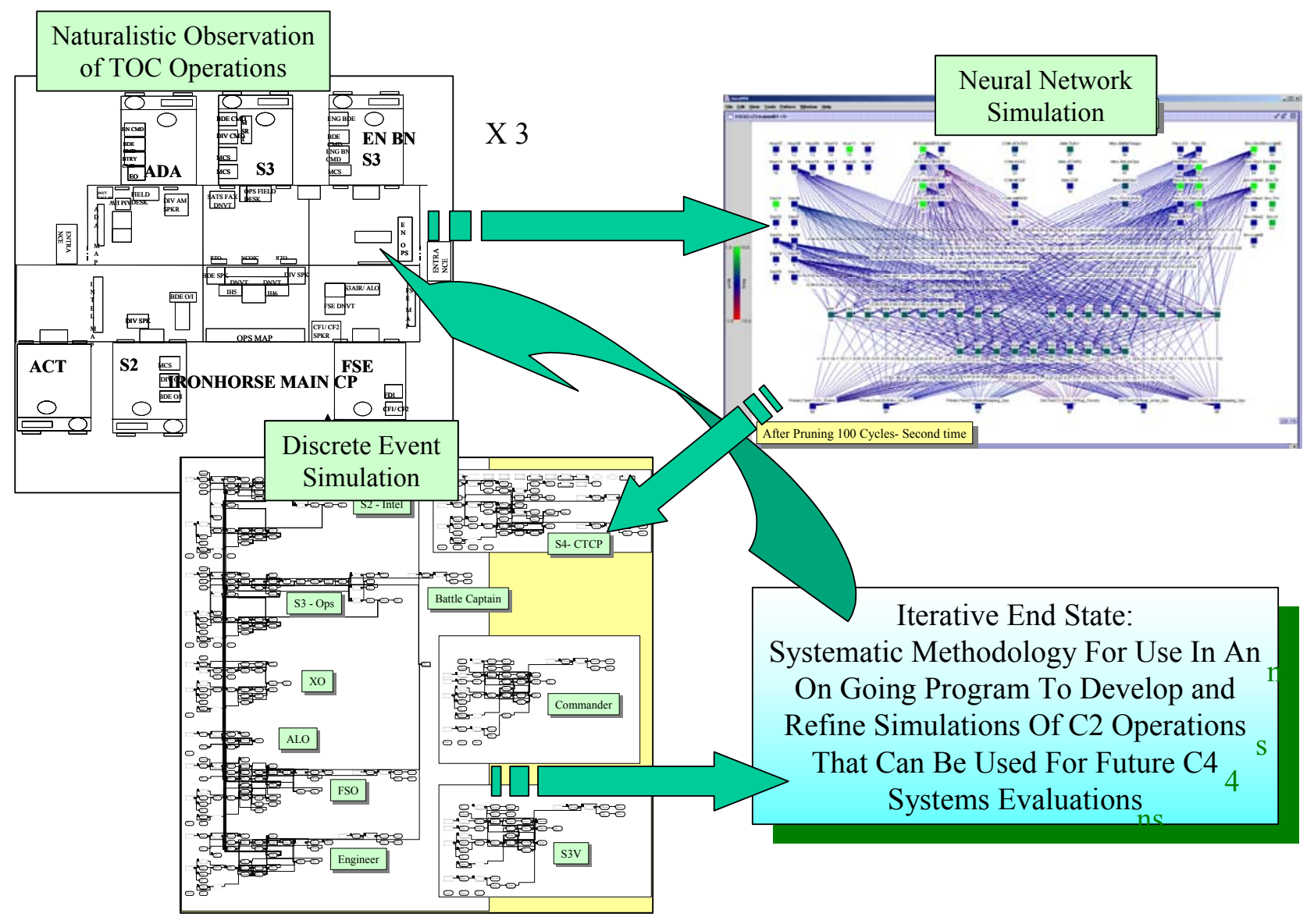

Figure 2 - COMPASS Concept

\subsection{The Study of Battle Command in the U.S. Army.}

An understanding of some of the concepts and techniques associated with U.S. Army doctrine for the control of land forces during periods of combat is essential to attempts to quantify, evaluate, and improve battle command procedures. Several topical areas are reviewed in preparation for the presentation of the COMPASS paradigm.

\subsubsection{The Problem of C2 Analysis and Research.}

Military $\mathrm{C} 2$ is described as the process by which commanders organize and employ forces to achieve military objectives (Mason and Moffat, 2000). Simulation and modeling of military $\mathrm{C} 2$ is recognized as one of the most challenging areas in defense analysis. Although NATO, for example, has been investigating military $\mathrm{C} 2$ for many years with great expenditure, little progress has been made. The vagaries of human decision making makes $\mathrm{C} 2$ extremely 
difficult to analyze. In fact, NATO's Research Study Group on Modeling of Command and Control has concluded that "no single measure exists that satisfactorily allows the assessment of either the overall effectiveness of $\mathrm{C} 2$ or the performance of C2 systems" (Jablunovsky, Dorman, and Yoworsky, 2000).

The modern problem of $\mathrm{C} 2$ is distinguished from its predecessors by the rate at which information must be handled and the resultant decisions that must be made utilizing that information (Adelson, 1961). Battlefield C2 is described as a constant balancing act where the need for detailed planning must be constantly weighed against the requirement for quick and decisive action (Fallesen et al., 1992a). The characteristics of modern C2 environments include time pressure, high risk, and ambiguous or missing information (Kaempf, Klein, Thordsen, and Wolf, 1996) while the goal of $\mathrm{C} 2$ organizations is to exercise decisive control of that hostile environment (Bent, 1983).

Timing has long been recognized as being of critical importance in combat with the associated implications for C2 (Cothier and Levis, 1986). Some definitions related to time and C2 are:

- System response time is the time delay between the moment when the $\mathrm{C} 3$ system receives a stimulus and the moment it can deliver a response.

- Tempo of operations is the number of actions per unit of time, which the system is executing and is indicative of how complex the environment is that the system can handle.

- Scenario describes those actions that are actually taking place.

- Timeliness is the systems ability to respond within an allotted time.

- Allotted time is the time interval over which the forces including the $\mathrm{C} 3$ system can affect the environment.

- The system consists of components, their interconnections, and a set of operating procedures.

- Boundaries are what defines what is included within the system whose effectiveness is to be assessed.

- The environment consists of our own forces and the adversary's forces upon which our forces can act and which can act upon ours.

- Parameters are the independent quantities used to specify the system and the mission requirements. 
- Measures of performance (MOP) are quantities that describe system properties or mission requirements. Example MOPs for a $\mathrm{C} 2$ system may include reliability, survivability, cost, and probability of kill.

- Measures of effectiveness are quantities that result from the comparison of the system MOPs to the mission requirements. MOEs reflect the extent to which the systems meets the requirements.

One critical aspect of time in the performance of $\mathrm{C} 2$ organizations is that it is likely that requests for services will exceed the capability of even the best C2 system (Witus and Blum, 1992). C2 is performed at all levels of military command, however, tactical C2 is described as a cyclical decision making and communication process performed under rigorous time constraints (Corker, Cramer, Henry, and Smoot, 1990). One view of tactical C2 describes it as having 4 steps:

1. Situation assessment.

2. Development of a course of action.

3. Execution of that course of action.

4. Feedback of the results of that execution.

Reflecting these steps, a realistic model of the C2 environment should include decision-making, taking account of the chain of command, course of action selection, and the communication process. The bottom line is that on the rapid paced battlefield of today, there is rarely enough time or resources to follow a systematic approach to achieve an optimum solution (Fallesen, Lussier, and Michel, 1992b).

$\mathrm{C} 2$ organizations are unique from other forces on the battlefield as their primary role is not to engage the enemy directly, but to direct the activities of those forces who are engaging the enemy. Some of the properties that tend to distinguish C2 organizations are (Sutherland, 1990):

- The units of the organization are both geographically dispersed and functionally specialized.

- Each of the individual units constituting a command and control organization will have critical missions they are expected to perform.

- The organization will have to contend with one or more ingenious, aggressive adversaries with few if any effective constraints on the nature of the competitive initiatives they might author.

- The key working challenge for the organization in aggregate and each of the units in particular is to be ready to deal with any threatening initiatives that might be mounted by their effective peers in the adversary organizations (enemy). 
Military forces throughout the world have developed an awareness of the systems approach to C2 (Harper, 1974), where a system is thought of as a collection of elements organized to perform a set of functions, during a specifiable era, in an environment known only uncertainly (Adelson, 1961).

\subsubsection{Data Overload and Its Effect on C2.}

One of the elements of interest in the simulation and modeling of any system where the human is a component is cognitive saturation. In other words, at what point is the human operator performing at the full potential level and is not capable of performing additional tasks or work without either suffering a degradation of total performance or by shedding some existing tasks. In command and control systems the summation effects of total task performance, which may or may not be combined with moderating effects of such elements as fatigue, noise, and vibration, can induce what has been called a "cognitive causality" where the individual is no longer capable of continued work or task performance (Middlebrooks, 2001). Stated in another way, data overload is a condition where an operator, supported by the physical components of the system and other operators, finds it extremely challenging to focus in on, assemble, and synthesize the significant portion of the data pertaining to the task or work being executed in a coherent manner. This generally is where the task being performed is a small portion of the requirements of the overall system (Patterson, Roth, and Woods, 2002). Some of the characteristics of data overload are (Woods, Patterson, and Roth, 2002):

- A clutter problem where there is too much data on the screen.

- A workload bottleneck where there is too much to do in the time available.

- A problem in finding the significance of data when it is not known a priori what data from a large data field will be informative.

Adding more technology, by itself, is usually not enough to solve generic and difficult problems like data overload that are problems that exist at the intersections of cognition, collaboration, and technology. Data overload is a condition where an operator, supported by artifacts and other operators, finds it extremely challenging to focus in on, assemble, and synthesize significant portions of a set of data into a valid situation assessment where the set of data being considered is part of a vast data field (Patterson et al., 2002). 
One reason that data overload causes problems in C2 organizations is the relationship of data to interests and expectations (Woods et al., 2002). While new technology often has the stated purpose of easing this condition, it often exacerbates it by impressing upon the user greater amounts of communications whose context must be analyzed before it becomes useful. It is noted that the human ability to assimilate information from artificial fields of data has expanded much more slowly, if at all, than the abilities of new technology to provide that data. The paradox of this situation is that while more and more data is available, in principle, to those who need it from increased technology, the human ability to interpret and understand what is available has not increased. Add to this the typical battlefield stressors of fatigue and stress and the results from cognitive overload often cause errors as human operators perform task operations (Braun et al., 1999).

\subsubsection{Workload and Its Relationship to C2.}

Although it is a subject of intense investigation, analysis, and debate, the simple fact of the matter is that nobody seems to know what workload is (Xie, 1997). While it can conceptually be defined as the amount of mental work or effort that an individual makes to perform tasks, and can be viewed as a consequence of human mental effort, one aspect seems to be recognized. This conclusion is that mental workload is significantly affected by time pressure. Hence, the effects of timing in $\mathrm{C} 2$, as just discussed, becomes a critical component of mental workload.

While workload may not be fully understood, there are significant and continuous efforts both in the human factors and psychology communities to establish its meaning and effects. Computer simulation plays a key role in this effort and in recognition of the fact that new system configurations may have the unintended effect of increasing operator workload and, therefore, contribute to accidents and errors, significant effort has been devoted to the development of workload prediction models (Dahl et al., 1991). These efforts, although to date are not conclusive on exactly what is mental workload, have nevertheless, developed significant levels of understanding on various aspects of workload and some insight on what it is not. For example, one author notes that it is widely accepted that the relationship between workload and individual performance is characterized by a curvilinear function where performance degrades at low and high levels of workload (Bowers, Braun, and Morgan, 1997). There are separate but 
parallel efforts that look at individual versus teamwork and workload. For example, one study found that the effect of communication on team coordination did facilitate team performance (Williges, Johnston, and Briggs, 1966).

\subsubsection{Critical Incidents on the Battlefield.}

As is the case with any team-oriented system that has human-in-the-loop types of activities, the identification of critical tasks that must be performed can form the basis for studying the performance of the team and system itself. Table 1 shows a list of these critical tasks. For battlefield command and control these critical tasks are the critical incidents that occur in battlefield operations and are identified as those major combat oriented tasks on the battlefield that must be directed and coordinated by the $\mathrm{C} 2$ system. A partial list includes those things that the decision maker or commander may wish to analyze (Fallesen et al., 1992a): 
Table 1 - Battlefield Critical Tasks

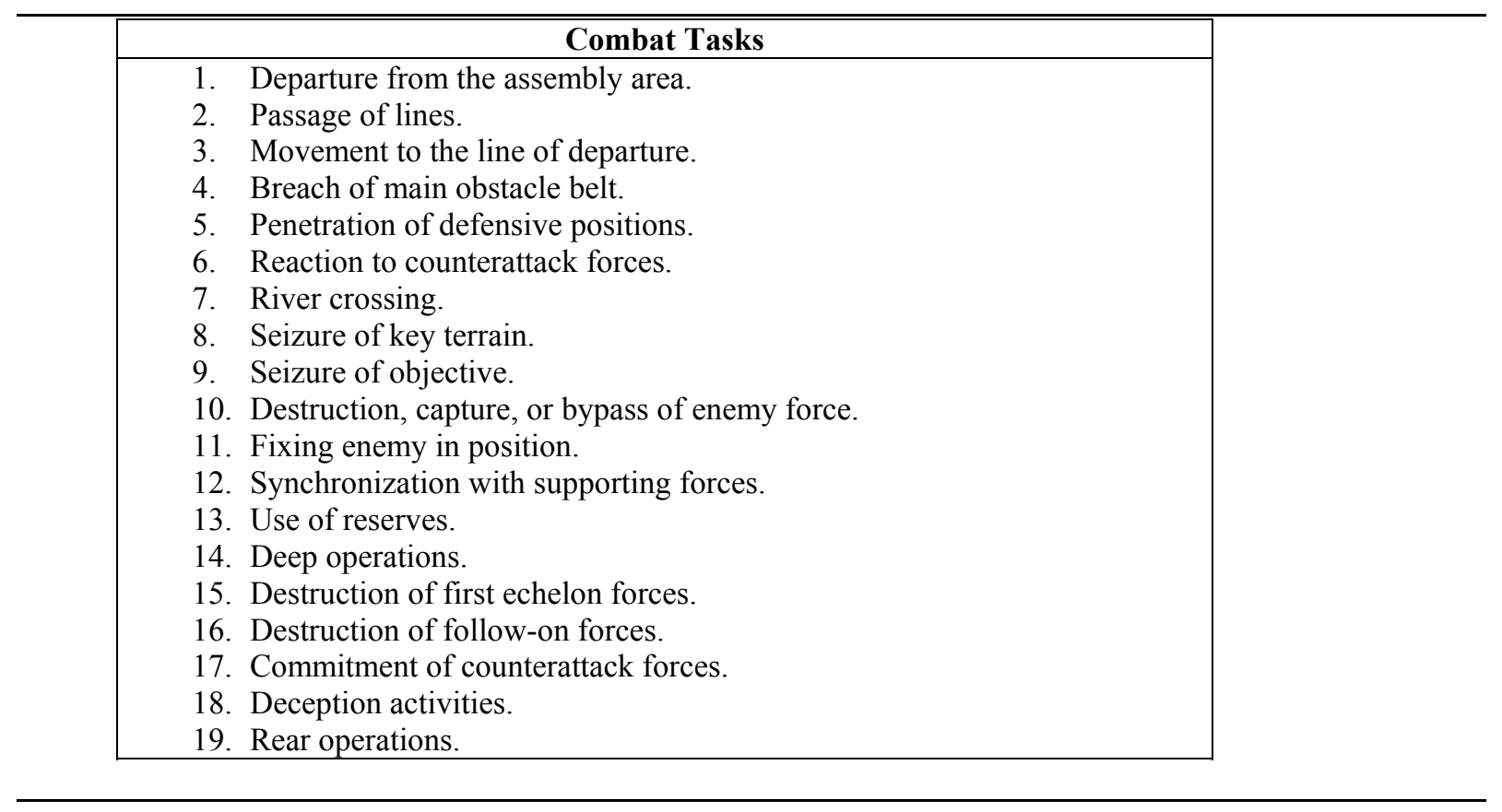

The Army categorizes these battlefield functions into four types of operations that include offensive, defensive, stability, and support. These are outlined in Table 2.

Table 2 - Tactical Battlefield Functions

(Army, 2001c)

\begin{tabular}{|l|l|l|}
\hline \multicolumn{1}{|c|}{ Category } & \multicolumn{1}{|c|}{ Forms } & \multicolumn{1}{c|}{ Function } \\
\hline Offensive & Maneuver & Envelopment \\
Operations & & Turning Movement \\
& & Infiltration \\
& & Penetration \\
& & Frontal Attack \\
& & \\
& Types & Movement To Contact \\
& & Attack \\
& & -Hasty \\
& & -Deliberate \\
& & -Special Purpose \\
& & + Spoiling \\
& & + Counterattack \\
& & + Raid \\
& & + Ambush \\
& & + Feint \\
& & + Demonstration \\
& & Exploitation \\
& & Pursuit \\
\hline
\end{tabular}




\begin{tabular}{|l|l|l|}
\hline \multicolumn{1}{|c|}{ Category } & \multicolumn{1}{|c|}{ Forms } & \multicolumn{1}{c|}{ Function } \\
\hline Defensive & Types & Mobile Defense \\
Operations & & Area Defense \\
& & Retrograde Defense \\
& & - Withdrawal \\
& & -Delay \\
& - Retirement \\
\hline Stability & Types & Peacekeeping \\
Operations & & Foreign Internal Defense \\
& & Security Assistance \\
& & Humanitarian and Civic Assistance \\
& & Support to Insurgencies \\
& & Support to Counter drug Operations \\
& & Combating Terrorism \\
& & Noncombatant Evacuation Operations \\
& & Arms Control \\
& & Show of Force \\
\hline Support & Types & Domestic Support Operations \\
Operations & & Foreign Humanitarian Assistance \\
& & \\
& Forms & Relief Operations \\
& & Support to Domestic Consequence \\
& & Management \\
& & Support to Civil Law Enforcement \\
& & Community Assistance \\
\hline
\end{tabular}

Several approaches have been used for attempting to quantify the operations of battlefield command and control systems. One approach is the function analysis methodology (Ford, Mullen, and Keesling, 1997) (from a previous report by R.J. Mullen in 1996). This approach has been used to identify systematic structures and organization of the tasks critical to battlefield success. Critical command and control tasks were identified as battlefield functions (BFs) and were organized according to battlefield operating systems (BOS). Table 3 shows these task functions and their applicability to battalion and brigade TOCs. The task functions in this table are another view of the task list presented above. 
Table 3 - Battalion and Brigade Battlefield Functions Grouped by BOS

(Ford et al., 1997)

\begin{tabular}{|c|c|c|c|}
\hline BOS & Battlefield Function (BF) & Applies & To: \\
\hline & & Battalion & Brigade \\
\hline Intelligence & $\begin{array}{ll}\text { 1. } & \text { Conduct Intelligence Planning } \\
\text { 2. } & \text { Collect Information } \\
\text { 3. } & \text { Process Information } \\
\text { 4. } & \text { Disseminate Intelligence } \\
\end{array}$ & $\begin{array}{l}X \\
X \\
X \\
X\end{array}$ & $\begin{array}{l}X \\
X \\
X \\
X\end{array}$ \\
\hline Maneuver & $\begin{array}{l}\text { 5. Conduct Tactical Movement } \\
\text { 6. Engage Enemy with Direct Fire and Maneuver }\end{array}$ & $\begin{array}{l}X \\
X\end{array}$ & $\mathrm{X}$ \\
\hline Fire Support & $\begin{array}{ll}\text { 7. } & \text { Employ Mortars } \\
\text { 8. } & \text { Employ Field Artillery } \\
\text { 9. } & \text { Employ Close Air Support } \\
\text { 10. } & \text { Conduct Electronic Collection and Attack } \\
\text { 11. } & \text { Conduct PSYOP } \\
\text { 12. Employ Chemical Weapons } \\
\text { 13. Conduct Counter Target Acquisition Operations } \\
\text { 14. Employ Naval Surface Fires } \\
\text { 15. Coordinate, Synchronize, and Integrate Fire } \\
\text { Support }\end{array}$ & $\begin{array}{l}X \\
X \\
X \\
X\end{array}$ & $\begin{array}{l}X \\
X\end{array}$ \\
\hline Air Defense & $\begin{array}{l}\text { 16. Take Active Air Defense Measures } \\
\text { 17. Take Passive Air Defense Measures }\end{array}$ & $\begin{array}{l}X \\
X\end{array}$ & $\mathrm{X}$ \\
\hline $\begin{array}{l}\text { Command } \\
\text { and Control }\end{array}$ & $\begin{array}{l}\text { 18. Plan for Combat Operations } \\
\text { 19. Direct and Lead Unit During the Preparation Phase } \\
\text { of the Battle } \\
\text { 20. Direct and Lead Unit in Execution of Battle }\end{array}$ & $\begin{array}{l}\mathrm{X} \\
\mathrm{X} \\
\mathrm{X}\end{array}$ & $\begin{array}{l}X \\
X \\
X\end{array}$ \\
\hline $\begin{array}{l}\text { Mobility and } \\
\text { Survivability }\end{array}$ & $\begin{array}{l}\text { 21. Overcome Obstacles } \\
\text { 22. Enhance Movement } \\
\text { 23. Provide Countermobility } \\
\text { 24. Enhance Physical Protection } \\
\text { 25. Provide Operations Security } \\
\text { 26. Conduct Deception } \\
\text { 27. Conduct NBC Defense }\end{array}$ & $\begin{array}{l}X \\
X \\
X \\
X\end{array}$ & $\begin{array}{l}X \\
X \\
X \\
X\end{array}$ \\
\hline $\begin{array}{l}\text { Combat } \\
\text { Service } \\
\text { Support }\end{array}$ & $\begin{array}{l}\text { 28. Provide Transport Services } \\
\text { 29. Conduct Supply Operations } \\
\text { 30. Provide Personnel Services } \\
\text { 31. Maintain Weapons Systems and Equipment } \\
\text { 32. Provide Health Services } \\
\text { 33. Treat and Evacuate Battlefield Casualties } \\
\text { 34. Conduct Enemy Prisoners of War Operations } \\
\text { 35. Conduct Law and Order Operations } \\
\text { 36. Conduct Civil Affairs Operations } \\
\text { 37. Provide Sustainment Engineering } \\
\text { 38. Evacuate Non-combatants from Area of Operations } \\
\text { 39. Provide Field Services }\end{array}$ & $\begin{array}{l}\mathrm{X} \\
\mathrm{X} \\
\mathrm{X} \\
\mathrm{X} \\
\mathrm{X}\end{array}$ & $\begin{array}{l}X \\
X \\
X \\
X \\
X \\
X \\
X\end{array}$ \\
\hline
\end{tabular}

The effective performance of critical tasks by the TOC can result in decisive points in the battle. Battlefield decisive points are defined as (Army and Marines, 1997; BCTP, 2002): 
- A point, if retained, that provides a commander with a marked advantage over his opponent. Decisive points are usually geographic in nature, but could include other physical elements such as enemy formations.

- A time or location where enemy weakness is positioned that allows overwhelming combat power to be generated against it.

- Conveys to a subordinate a potential point of decision that a commander has identified through his estimate process to apply overwhelming combat power.

\subsubsection{The State of C2 Analysis and Research.}

In recent years there has been an increasing volume of work in the area of $\mathrm{C} 2$ as its importance continues to be emphasized but its meaning and ways of optimization remain vague. This work predominately focuses on the areas of individual and team performance, mental workload, decision making (including the Military Decision Making Process, MDMP), knowledge elicitation, to name just a few. These are all very significant topics and are certainly worthy of continued and in depth research, however, while the literature acknowledges the existence of the $\mathrm{C} 2$ system there is very little on going effort on trying to describe it and how to address C2 from the system level. The reasons are obvious as while human performance and workload are extremely complicated and diverse topics, the performance of a military $\mathrm{C} 2$ center is even more so by at least an order of magnitude. This is why it is felt that an approach like the COMPASS paradigm has such a great potential to add to the knowledge base. However, there has been some work identified that addresses various components of the $\mathrm{C} 2$ work system and a few of them are summarized here.

\subsubsection{Operations Other Than War and a United Kingdom Land-Air Simulation.}

One approach for representing the whole $\mathrm{C} 2$ process in simulation was attempted for an Operations Other Than War (OOTW) simulation and a United Kingdom (UK) land-air combat simulation (Mason and Moffat, 2000). The simulation represented a range of C2 operations from top down rapid planning activities to bottom up deliberate planning activities. It attempted to generically describe the activities the $\mathrm{C} 2$ process from a component level viewpoint. Modeled in the simulation were the commander, communications officer, intelligence officer, force planner, and a promulgator that disseminated the plan to subordinates. The model used the recognitionprimed decision (RPD) model (Klein, 1998) of the decision making process and was a 
constructive simulation of $\mathrm{C} 2$ in combat based on an intelligent agent framework of $\mathrm{C} 2$ processes that operated in accordance with simple rules.

\subsubsection{Army Command and Control Evaluation System.}

The U.S. Army Command and Control Evaluation System (ACCES) is a measurement system developed to evaluate the effectiveness of $\mathrm{C} 2$ at various levels (Halpin, 1992). The premise of ACCES is that $\mathrm{C} 2$ effectiveness is defined as the effectiveness of the headquarters staff (synonymous with TOCs at battalion and brigade levels in the field). This program was a very intensive effort to evaluate overall performance of actual TOCs in the field. It employed a team of data collectors to collect all relevant information exchange in a TOC over a 12-hour shift. ACCES is a bottom up manually intensive data collection technique that relies on both qualitative and quantitative observation in the TOCs and battle simulation center (BSC) for ground truth. Data requirements are rigorous and the data collectors were required to complete a 14 lesson-training program before being sent to collect data. This was a system level approach before the days of sophisticated computer simulation that employed a large team to collect data for analysis through traditional statistical evaluation techniques.

\subsubsection{Using NNS to Evaluate Mental Workload in C2 Environments.}

One study was conducted to test the predictions of a mental workload model (Hancock and Caird, 1993). As it was noted that the basic premise of human factors is a primary concern for the human in system design and operation, an evaluation of a mental workload model was conducted that predicted that mental workload grew as perceived distance from the task goal increases and the effective time for action decreases. This study encountered the same problem found in many other studies. This problem is understanding how to transform the composition of performance tasks from physical to cognitive demands. Here, mental workload was conceptualizes in the three dimensions of effective time for action, perceived distance from the desired goal state, and the level of effort required to achieve the desired goal.

Another study utilized neural network simulation models to predict subjective workload measures using condition and performance measures as input variables. The goal was in attempting to learn how condition and performance factors relate to differences in subjective workload measures in TOC structures (Schvaneveldt, Reid, Gomez, and Rice, 1997). Data was collected using the Subjective Workload Assessment Technique (SWAT) (Reid, Potter, and 
Bressler, 1986) during pursuit tracking, sequential reaction time, and tone counting tasks to provide workload training data. The NNS models were trained using the performance data, the condition information, and the subjective judgments of workload from the SWAT results. The NNS model had 3 input variables, 12 hidden nodes, and one output node representing workload redline condition. Epoch tests went from less than 100 to over 100,000 with training achieved for the 3-12-1 model at 3020 epochs. Study results indicated that linear regression models perform as well as nonlinear neural network models in predicting "redline" workload level in training data.

A recent Ph.D. dissertation (Cioppa, 2002) used the agent-based simulation MANA to analyze 22 variables in a complex military peace enforcement operation. Here a set of experimental designs were developed that combined orthogonal Latin Hypercubes and uniform designs to create designs having near orthogonality. These designs were used to develop a capability to search an intricate simulation model with a large number of inputs and a resulting complex response surface. This study provided an interesting approach for investigating command system performance while trying to account for as many of the independent measures as possible. The paper presented a solution using the Latin Hypercubes for models of up to 22 IV.

Another recent study (Jablunovsky et al., 2000) was conducted that looked at approximating C2 network behavior using a neural network trained on the relationships between the network state and performance. Training data for the NNS was obtained by sampling the surface defined by network latency performance as a function of the $\mathrm{C} 2$ network state to provide the known input and output training pairs of the NNS training set. The training algorithm employed was the standard iteratively updated, feed-forward, error backpropagation algorithm. The NNS contained $41 \mathrm{C} 2$ input nodes, 30 hidden nodes in one layer, and 5 output nodes with 1250 training pairs and 125 validation pairs. The intent of the study was to integrate the NNS into a two sided theater level simulation called THUNDER with the objective to show the effects of interdiction and degradation of the $\mathrm{C} 2$ network state on campaign model measures.

\subsubsection{Conclusions Regarding C2 Analysis and Research.}

While there is a great amount of research and investigative work into C2 operations, there is little aimed at the overall battlefield management system. This is understandable due to the complexities of trying to quantify and understand individual mental workload, let alone overall 
system performance. The application of the COMPASS paradigm does not event attempt to address internal relationships in the $\mathrm{C} 2 \mathrm{~S}$, it simply tries to quantify it through the identification of significant relationships in an ever-growing knowledge base that drives a simulation of the TOC processes. One of the big challenges in this performance domain is the transformation the U.S. Army is undergoing with the conversion to digital communication processes. It is not enough to model the current state of the system, the analysis must be able to address perceived future systems. This is one reason that the quick turnaround capabilities of modeling from preexisting knowledge bases has merit. There are many obstacles to achieving this capability that include developing an understanding of the true nature of the system along with the ability to overcome false impressions or myths about it. Some of the current myths of digital technology are (Lynch, 2001):

1. TOCs will get smaller using information technology.

2. Training will take less time.

3. We need "contractor battalions" to support us.

4. Digitization will show us an immediate impact on battlefield operations.

While the effectiveness of military systems will continue to be inextricably linked to the performance capabilities of human operators (Bowers et al., 1997), it is the overall performance of the system that is important. A common characteristic of sub-system components in current use is that they often provide vast quantities of partially relevant data, while failing to identify the information which the decision maker actually needs to solve his problem (Cohen and Freeling, 1981). Some of the issues that have appeared with the introduction of automated systems into C2 environments are (Corker et al., 1990):

1. Will human workload saturate a particular system and will procedural bottlenecks be revealed?

2. What will the duty cycle or workload of an operator be in an automated system?

3. What is the impact of automation initiatives on manpower and training for new systems?

4. What will be the effect of automation on the information and data requirements for system operation?

5. How can automation be effectively transferred into the existing environment?

6 . What are the procedures associated with system verification and validation? 
Finally, it is noted that in the continuous effort to improve overall $\mathrm{C} 2$ system performance it often the case that those engaged in improving human performance seem to arrive on the scene a little too late (Harper, 1974). Analytical tools, like COMPASS, that are intended to decrease the analytical response time, is one approach to addressing some of the issues presented here.

\subsection{Approach.}

The research in this dissertation was conducted in two phases. Phase I was be the collection of data from TOC operations and the evaluation of it with datamining techniques such as neural networks and cluster analysis. The neural network models were utilized to identify and select those IV that are significant for use in Phase II. Phase II took the descriptive and numerical models developed in the first phase and applied them into an appropriate pre-existing computer simulation of TOC operations.

\subsubsection{Phase I: Data Collection Through Naturalistic Observation Using Ethnographic}

Techniques and Analysis Using Neural Network Simulation.

The three TOC deployments observed for this research, which are depicted in Figure 3, provided a total of 164 hourly observations. The raw data from the exercises were standardized and converted, as appropriate, to binary for evaluation with neural network simulation. These data consists of $159 \mathrm{IV}$, or input nodes and $75 \mathrm{DV}$ or target nodes. 


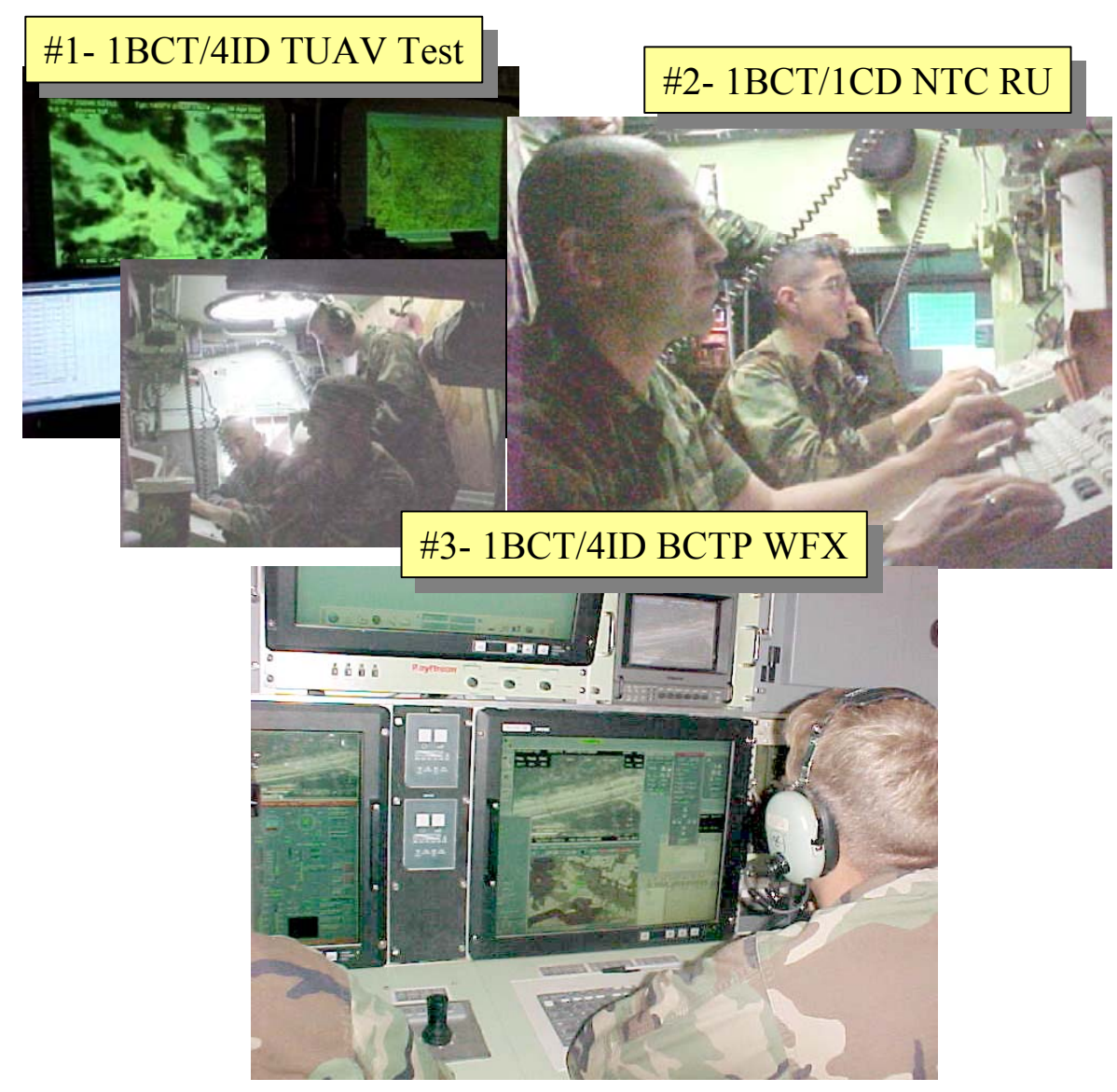

Figure 3 - Exercise Observations / Data Collection

These three TOC observation events represent only the start of what is envisioned to be a very large dataset containing data from 30, 40 or more TOC deployments over the next few years. Data from the existing observations is deemed satisfactory for the exploratory development of the COMPASS paradigm, however, it falls short of providing satisfactory depth for the purpose of substantiated conclusions regarding TOC performance in general. It has been said that all TOCs are different while all TOCs are the same. This statement alludes to the fact that each TOC deployment configuration and operational configuration is dependent upon the personal preferences of the TOC members who establish its layout and procedural actions. It also means that all similar type and size units have, in essence, the same basic building blocks of hardware, software, and tactical equipment with which to set up the TOC.

As the TOC observation dataset grows to include information from a dozen and more deployments then conclusions can start to be generated that transcend the interpersonal differences of individual opinion for setup and operation, and allow the analysis to concentrate 
on those activities and constructs that are germain to the overall configuration and operation of TOCs that allows for the most efficient and successful operation.

The exercise data was evaluated using a neural network simulation called Java Neural Network Simulation (JNNS) (Fischer, Hennecke, Bannes, and Zell, 2001) developed at the University of Tübingen, Wilhelm-Schickard Institute in Tübingen, Germany. JNNS runs in a Microsoft Windows environment and is a derivative and subset of a more complete neural network simulation called Stuttgart Neural Network Simulation (SNNS) that executes on Unix based computers. This simulation allows the development of neural networks that can be trained and pruned to reveal relationships to be identified as to the significance of input nodes (corresponding to IV) and output nodes (corresponding to DV). The results of the NNS analysis are used to provide mathematical models of the relationships of the IVs to the DVs for use as algorithmic drivers for discrete event simulations of TOC performance in Phase II of this research.

\subsubsection{Phase II: C2 Analysis Using Discrete Event Simulation.}

The mathematical models developed in Phase I are applied to discrete event simulations of TOC performance developed in the late 1990s by the U.S. Army to study C2 organizations based on Army needs stemming from the Persian Gulf war. The resulting CoHOST simulations examined human mental and physical performance capabilities resulting from proposed new digital communications systems. The CoHOST model architecture was developed based on a taxonomy of human performance that included 52 knowledge, skills, and abilities taxons organized into eight categories ranging from highly physical to completely cognitive in nature. A CoHOST design was implemented with these taxonomic based descriptors of human performance in the military command and control domain using the commercial programming language Micro Saint ${ }^{\mathrm{TM}}$. For this research the results of the JNNS analysis, in the form of mathematical models, are used to control the operation of the CoHOST model to optimize its performance according to the observed processes in real TOCs.

The process of collecting naturalistically observed data, its analysis in neural network simulations with resulting generation of mathematical models of performance, and the use of these models to tailor the performance of discrete event simulations of the original work domain is what is termed the COMPASS paradigm framework. The development of COMPASS by this 
dissertation is intended to provide a research basis for future investigative work in $\mathrm{C} 2$ work environments. 


\section{Literature Review.}

There are a number of concepts and subject areas whose understanding and current meanings are critical to the development of the research presented in this dissertation. First, a review of some of the U.S. Army techniques, concepts, and procedures (TCP), that have applicability in the $\mathrm{C} 2$ domain, are presented to provide a conceptual application basis for this research. Then, there are a number of research areas in human cognitive performance that have significance in the understanding of how and why human operators tend to perform the way they do in the $\mathrm{C} 2$ arena. Cognitive task analysis procedures and concepts form the basis for making observations and data collection to support the research. This is followed by a review of some simulation concepts and developmental techniques followed by a review of some analytical techniques used to draw conclusions from the results of the computer simulations.

\subsection{Selected Military Techniques, Concepts, and Procedures.}

While military based C2 can be considered to have many areas of commonality with civilian sector counterparts, it is unique in many respects. First, approaches to military $\mathrm{C} 2$ tend to be highly procedural concepts that are well documented in voluminous staff, technical, and field manuals. Equipment and systems that support military C2 are highly specialized that are conceived of and built according to rigid specification designed not only to meet performance needs, but also to have an ability to operate in widely varying location and climatic conditions. Finally, the military focus on mission accomplishment, especially in combat conditions, far exceeds any possible counterpart in the civilian work ethic.

It is noted that the U.S. Army, very much like other Federal agencies and services, is famous or infamous for its development and use of acronyms in its publications and normal operations. To the uninitiated this can become daunting while trying to read and understand publications containing this verbiage. While significant effort has been made to immediately identify all acronyms used in this dissertation, there may be some omissions as well as references in appendices and elsewhere containing terminology that seems perfectly normal to the military reader but may be meaningless to others. For this reason a glossary of terminology is included that has been compiled from official Army sources (Army, 1993; Army, 1998) augmented with terms from various Army development programs such as the Command and Control Vehicle 
(C2V), the Tactical Unmanned Aerial Vehicle (TUAV), the Force XXI Battle Command Brigade and Below system (FBCB2), and the Future Combat System (FCS). This glossary of acronyms is at Appendix B.

The TCPs are presented in a logical sequence to allow an understanding of concepts followed by techniques and procedures (it is just a coincidence that they are in alphabetical order.) These TCPs are:

\subsubsection{Army Operations Orders.}

A major element in military operations orders (OPORD) is the commander's intent statement. The Commander's Intent statement helps the soldiers read the commander's mind if they run into uncertainty about how to carry out the orders under field conditions (Klein, 1998). One description of the commander's intent statement has the following elements (Weick, 1983):

- Here is what I think we face.

- Here's what I think we should do.

- Here is why.

- Here is what we should keep our eye on.

- Now, talk to me.

Some of the considerations that should be included in communicating the commander's intent are (Klein, 1998):

- The purpose of the task.

- The objective of the task.

- The sequence of steps in the plan.

- The rationale for the plan.

- The key decisions that may have to be made.

- Anti-goals or unwanted outcomes.

- Constraints and other considerations.

\subsubsection{Army Battle Command System.}

On the modern battlefield there frequently occurs information overload conditions where the amount of information may reach greater quantities than can be assimilated even though the number of communications channels employed may be reduced. The realization that 
information about one's enemy is a key to success on the battlefield is not a new one. In 1832 the Prussian General Carl von Clausewitz (Clausewitz, 1832) wrote in his classic treatise concerning the nature of war that "By the word "information" we denote all the knowledge which we have of the enemy and his country; therefore, in fact, the foundation of all our ideas and actions." Concerning the nature of information he stated that the "Great part of the information obtained in War is contradictory, a still greater part is false, and by far the greatest part is of a doubtful character." Some technology based assistance can be given to information acquisition and $\mathrm{C} 2$ operations, however, the key to effective $\mathrm{C} 2$ performance rests on the judgments and actions of key personnel, both individually and collectively (Olmstead, Christensen, and Lackey, 1973). In order to provide the best technology based assistance to these key decision makers in the field the Army has developed and is in the process of fielding a suite of digital systems to provide much more efficient and powerful communications systems that greatly increase the amount of information provided to military decision makers without overloading their ability to assimilate it.

The Army Battle Command System (ABCS) was designed to be an interoperable system of systems using a client-server architecture on a local area network (LAN) to achieve communications interoperability for $\mathrm{C} 2$ systems. The ABCS client-server architecture provides ability for any ABCS operator to access the data and view the displays from any other ABCS workstation on the same tactical LAN. The ABCS components are designed to answer the following six questions for the commander:

- Where am I?

- What is my status?

- Where are the other friendly units?

- What is their status?

- Where is the enemy?

- What is the enemy's status?

ABCS provides a common operational picture on an electronic map of the battlefield that can display various types of information such as friendly unit locations, enemy unit locations, fire support control measures, air and tactical ballistic missile tracks, and logistics information (Army, 2002a). 
Many of these systems and their interconnectivity are shown in Figure 4. The ABCS systems include:

- GCCS-A (Global Command and Control System). This is a corps level system that provides joint information on force tracking, host nation, civil affairs, theater air defense, targeting, psychological operations, C2, logistics, medical, and personnel status.

- MCS (Maneuver Control System). This system is found at the battalion through corps levels and provides the operations staff the ability to monitor the current battle and to plan the future battle.

- MCS-L (Maneuver Control System - Light). This system is a reduced version of MCS that operates on a Windows NT 4.0 desktop or laptop computer system.

- ASAS (All Source Analysis System). This system is found at the battalion through corps levels and receives and processes enemy information from national, theater, and tactical echelons to include FBCB2.

- ASAS-L (All Source Analysis System - Light). This system is a reduced version of ASAS that operates on a Windows NT 4.0 desktop or laptop computer system.

- AFATDS (Advanced Field Artillery Tactical Data System). This system is positioned at the firing battery through echelon above corps (EAC) levels and provides the artillery operations staff with automated fire support planning, coordination, and control of close support, counterfire, interdiction, suppression of enemy air defenses, and deep operations.

- AMDPCS (Air and Missile Defense Planning and Control System). AMDPCS is located at the air defense artillery (ADA) battery through EAC levels and provides the commander with the ability to monitor current air operations while planning for future events.

- $\quad$ FAADC ${ }^{3}$ I (Forward Area Air Defense Command, Control, Communications, and Intelligence). FAADC $^{3}$ I provides automated interfaces between the forward area air defense nodes and weapon systems

- CSSCS (Combat Service Support Control System). CSSCS is deployed at the battalion through corps levels and provides the logistical staff with the ability for planning and controlling the logistics support of combat operations.

- $\quad$ FBCB2 (Force XXI Battle Command - Brigade and Below, also known as $\mathrm{AB}^{2}$ - Army Brigade and Below Command And Control System). FBCB2 is deployed throughout the battlefield from commander to platform and even soldier level. It provides $\mathrm{C} 2$ and situational understanding (SU) to the lowest tactical echelons with the transmission and receipt of orders, reports, and data via combat messages.

- TAIS (Tactical Airspace Integration System). TAIS is found at the division through EAC levels and is a system that provides the ground commander the ability to take part in the 
management of the air battle. It is the interface to the Army Airspace Command and Control (A2C2) system for the planning and operations of air traffic services.

- DTSS (Digital Topographic Support System). DTSS is found at the brigade through corps levels and enables topographic support personnel to receive, format, store, retrieve, create, update, and manipulate digital topographic data.

- IMETS (Integrated Meteorological System). IMETS is found at the aviation brigade and the division and corps levels providing commanders and staffs an automated, highresolution weather system to receive, process, and disseminate current weather observations, forecasts, and environmental effects decision aids.

Five of these systems (MCS, ASAS, AFATDS, AMDPCS, CSSCS) comprise a subset of the overall ABCS called the Army Tactical Command and Control System (ATCCS).

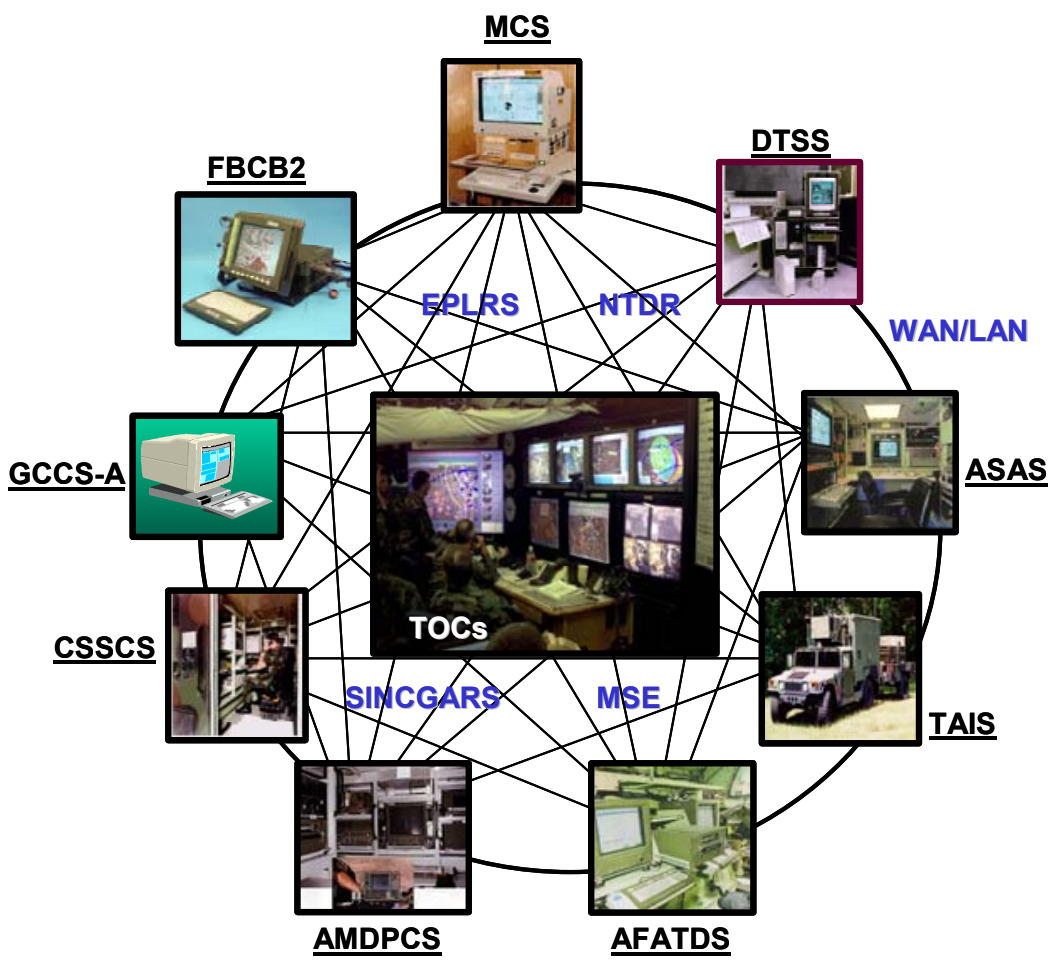

Figure 4 - ABCS Architecture

(Adapted From: (Brown, 2002))

The ABCS systems are interconnected by a tactical communications system that includes the following components:

- Mobile Subscriber Equipment (MSE).

- Near Term Digital Radio (NTDR). 
- Single Channel Ground and Airborne Radio System (SINCGARS).

- Enhanced Position Location and Reporting System (EPLARS).

- Wide Area Network (WAN) / Local Area Network (LAN) switch router architecture.

ABCS provides the linkages to individual soldiers and vehicles to the overall Army Tactical Command and Control System (ATCCS). ATCCS links together all of the functional area control systems such as MCS, FAADC ${ }^{3}$ I, CSSCS, AFATDS, and FBCB2/AB ${ }^{2}$. ATCCS connectivity is provided by the Area Common User System (ACUS), the combat net radio (CNR) systems, and the Army Data Distribution System (ADDS) (Army, 1995). The overall ATTCS architecture is shown in Figure 5.

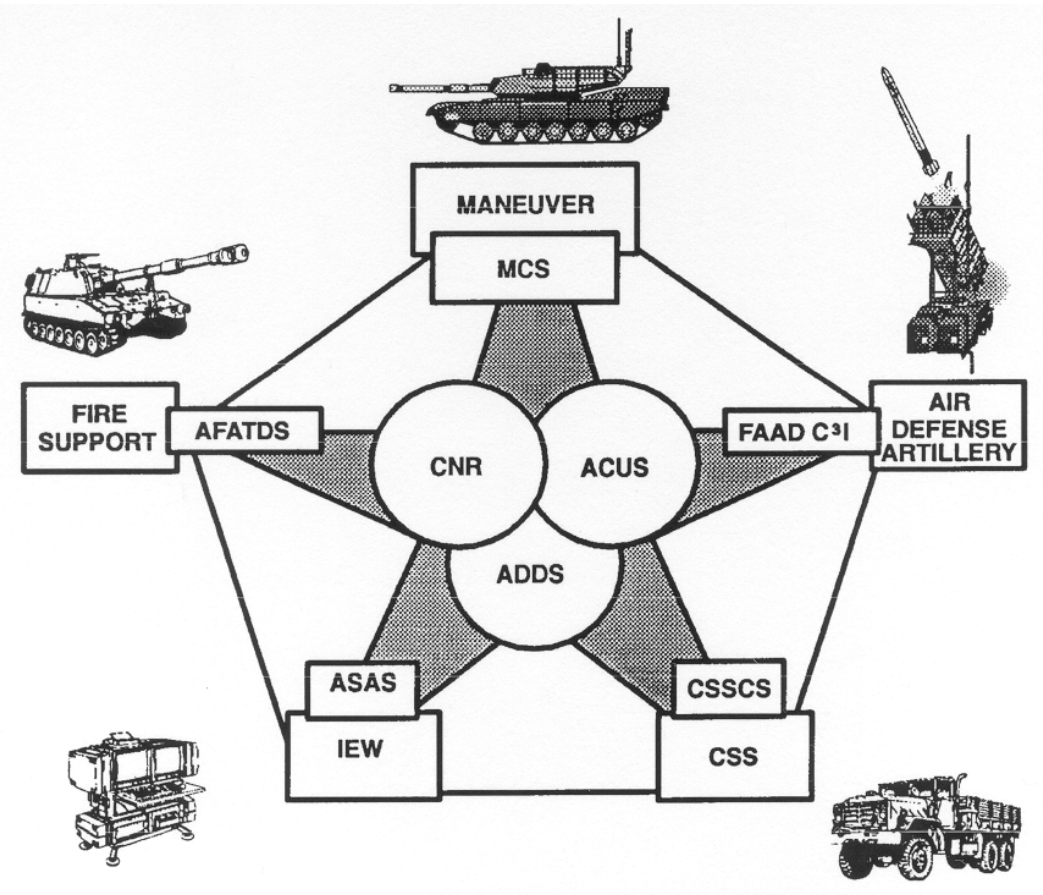

Figure 5 - ATCCS Architecture

(Army, 1995)

\subsubsection{Battlefield Operating Systems (BOS).}

Battlefield Operating Systems are groups of hardware and performance tasks that comprise critical tactical activities on the ground battlefield. The BOS provide a means of reviewing preparations or execution in discrete subsets. Army BOSs include intelligence, maneuver, fire support, mobility and survivability, air defense, combat service support, and 
command and control. These are illustrated in Figure 6. Specifically not included as identified BOSs are intangible activities such as timing, tempo, reconnaissance, information operations, and tactics (Army and Marines, 1997). BOS are defined as the major functions that occur on the battlefield. These are performed by the ground forces to successfully execute battles and engagements to accomplish the military objectives that have been directed by the ground force commander. The seven BOSs are described below (Gibbings, 1991). Appendix C contains detailed diagrams showing the functions for each BOS.

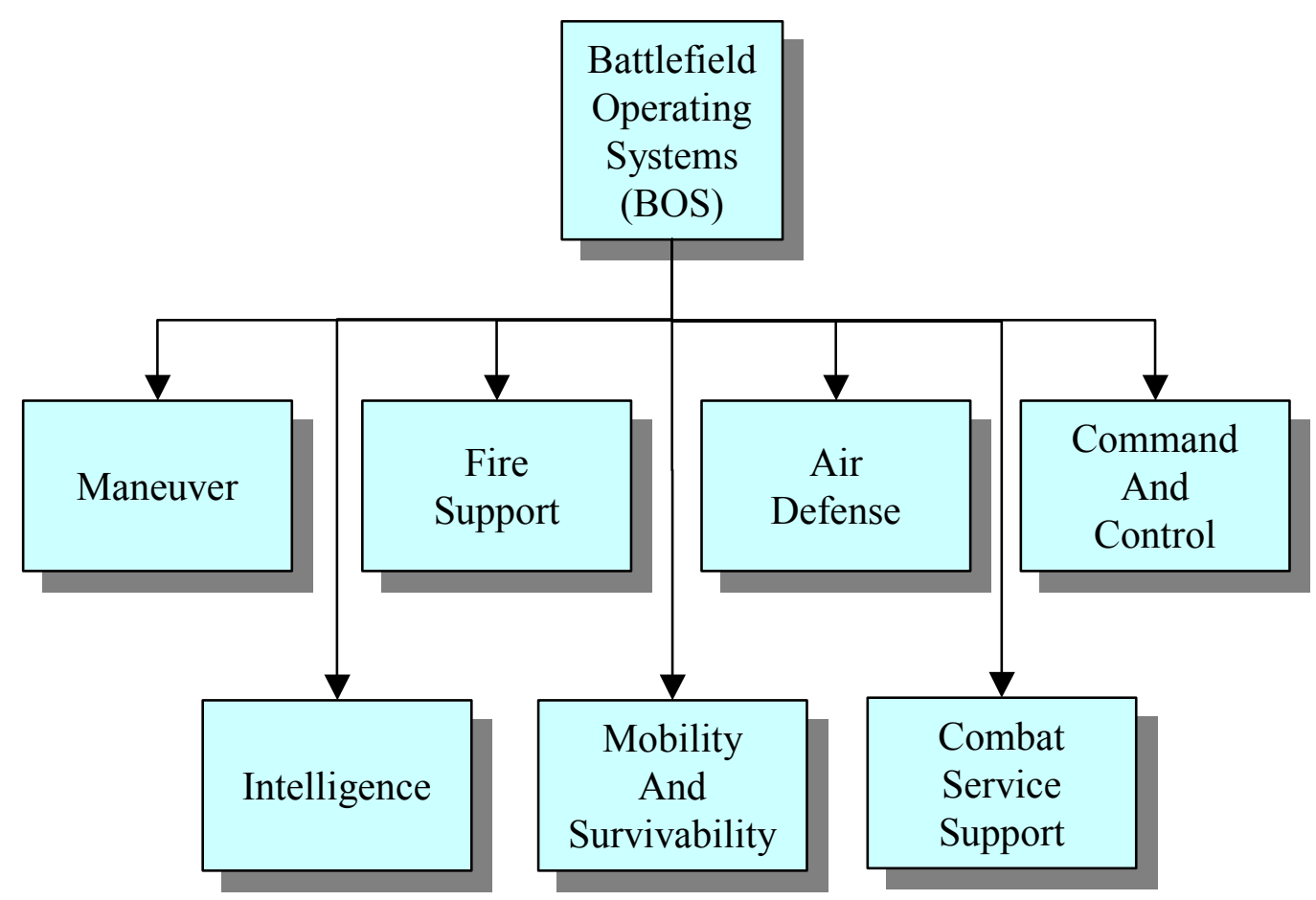

Figure 6 - Seven Battlefield Operating Systems (BOS)

(Gibbings, 1991)

- The Maneuver BOS is the employment of forces on the battlefield through movement and direct fires. It is supported by fire support artillery and has the goal of achieving a position of advantage over the enemy to accomplish the mission.

- The Fire Support BOS is the collective and coordinated use of target acquisition data by indirect fire weapons, armed aircraft (excluding armed helicopters), and other lethal and nonlethal means against ground targets. This BOS is always employed in support of maneuver force operations. Indirect fire weapons includes tube artillery, rocket and missile artillery, mortars, naval gunfire, close air support, electronic countermeasures, and all other non-line-of-sight fires. 
- The Air Defense BOS includes all measures designed to nullify or reduce the effectiveness of attack by hostile aircraft or missiles after they are airborne. This BOS includes all weapon systems with the potential to engage aerial targets.

- The Command and Control BOS is the exercise of authority and direction by a commander over subordinate forces to achieve mission accomplishment. C2 functions are performed through an arrangement of personnel, equipment, facilities, and procedures employed by the commander and the staff in the planning, directing, coordinating, and controlling of forces and operations to achieve mission accomplishment.

- The Intelligence BOS is the collection of activities that generate knowledge of the enemy, weather, and geographical features required by the commander for planning and conducting combat operations. This BOS is derived by analyzing information on the enemy's capabilities, intentions, vulnerabilities, and the environment.

- The Mobility and Survivability BOS describes the functions of the ground force that permits freedom of movement relative to the enemy while retaining the ability to fulfill the primary mission. It also includes those measures taken to remain viable and functional by achieving protection from the effects of enemy weapons as well as natural occurrences.

- The Combat Service Support BOS is the support and assistance provided to the ground force to sustain it in the fields of logistics, personnel services, and health services. This BOS describes activities that may be provided by the U.S. Army, the host nation, or by contracted support to man, arm, fuel, fix, and move the Army ground forces during times of combat operations. It also includes the functions to build and maintain facilities and provide military police support.

Military tactical units are excellent examples of organizations that must adapt readily to fast changing environmental conditions (Olmstead et al., 1973). The ABCS system is an integral component of the Army's Objective Force (Army, 2002b) program development strategy.

\subsubsection{Command and Control.}

It has been stated that there is nothing more important to success on the battlefield than effective C2 (Goedkoop, 1988). The United States military in general and the U.S. Army in particular have an enormous investment in the expenditure to study, analyze, quantify, and understand the concept of $\mathrm{C} 2$. Even today, after more than 50 years of intense effort in the post World War II era this topic and process remains an enigma that is the subject of constant study, analysis, and debate. The new operational paradigm that is promulgating throughout the Army as a result of the decision to digitize the force has only served to heighten the intensity with which this subject is addressed. Military tactical environments, that are both turbulent and 
unpredictable, are characteristic of the present and anticipated future environments that do and will require $\mathrm{C} 2$ functions to respond flexibly to a more or less constant flow of situations characterized by uncertainty (Olmstead et al., 1973). The advent of digitization of C2 has caused a change in the way that operators in these environments operate as they are mainly faced with performing tasks related to information processing (Schaafstal and Schraagen, 1992). The proliferation of this information technology has spawned a dire need for the ability to measure and evaluate system performance in a comprehensive manner (Kleiner, 1997).

The U.S. defense establishment has manuals, regulations, and publications on every imaginable topic and sources for formal definitions of command and control are no exception. Some of these authoritative definitions are (Huron, 1997; JCS, 1994):

- Command - The authority that a commander in the Armed Forces lawfully exercises over subordinates by virtue of rank or assignment. Battle command is the application of leadership as an element of combat power. It involves four functions: visualizing, describing, directing, and leading. It is the exercise of command in operations against a hostile, thinking enemy (Army, 2001c). It is also described as the art of battle decision making and the leading and motivating of soldiers and their organizations into battle. It is the natural expansion of $\mathrm{C} 2$ as a result of changes in the scope and intensity of current operations and the prospect of future operations (Army, 1996).

- Control - Authority which may be less than full command exercised by a commander over part of the activities of subordinate or other organizations.

- Command and Control System (C2S) - The facilities, equipment, communications, procedures, and personnel essential to a commander for planning, directing, and controlling operations of assigned forces pursuant to the missions assigned. However, it is not just the arrangement of equipment such as a communications system. The concept of a $\mathrm{C} 2 \mathrm{~S}$ is that it is the personnel, equipment, communications, facilities and procedures that facilitate the commander's ability to command and control. The C2S is the organization of resources available to the commander to help plan, direct, coordinate, and control military operations within the unit. The goal is the assurance of mission accomplishment (Army, 1997). It is also the brain of a tactical unit, collating all 
information and sending appropriate instructions to personnel who are in contact with the enemy. The extent to which this system functions flexibly, efficiently, and effectively determines the ability of the unit to succeed on the battlefield (Olmstead et al., 1973).

- Command and Control - The exercise of authority and direction by a properly designated commander over assigned and attached forces in the accomplishment of the mission (Army, 1997).

Another definition of the C2S is that it is comprised of: (Pendergrass and Hughes, 1993):

- Physical elements - transmitters, signal lights, computers, codebooks, tapes, deciphering equipment, etc.

- Human elements - the commander, staff, military analysts in the chain of command, etc.

- Procedural elements - used to conduct the process including training manuals, equipment manuals, organization charts, and command relationships.

Command and control is described as having two components. These are the commander and the C2S. Subsystems to the C2S include communications, intelligence, and computer networks, which provide the backbone for the overall C2S. The effective use of these systems and subsystems is what allows the commander the ability to lead from any point on the battlefield (Army, 2001c).

The U.S. Army and U.S. Marine Corps formally define C2 as (Army and Marines, 1997):

"The exercise of authority and direction by a properly designated commander over assigned and attached forces in the accomplishment of the mission. Command and control functions are performed through an arrangement of personnel, equipment, communications facilities, and procedures employed by a commander in planning, directing, coordinating, and controlling forces and operations in the accomplishment of the mission."

Procedures for effective $\mathrm{C} 2$ can be highly individualistic and interactive and are therefore "not cut and dried". Battlefield C2 is described as a constant balancing act where the need for detailed planning must be balanced against the need for quick and decisive action (Fallesen et al., 1992a). A symbolic model of these activities is at Figure 7. Tactical command and control is made up of three primary activities: 
- Planning -A continuous activity conducted to prepare for assigned or assumed tasks.

- Directing-The commander decides on a particular action and then directs its execution.

- Monitoring-The process of discerning what is happening and what it means.

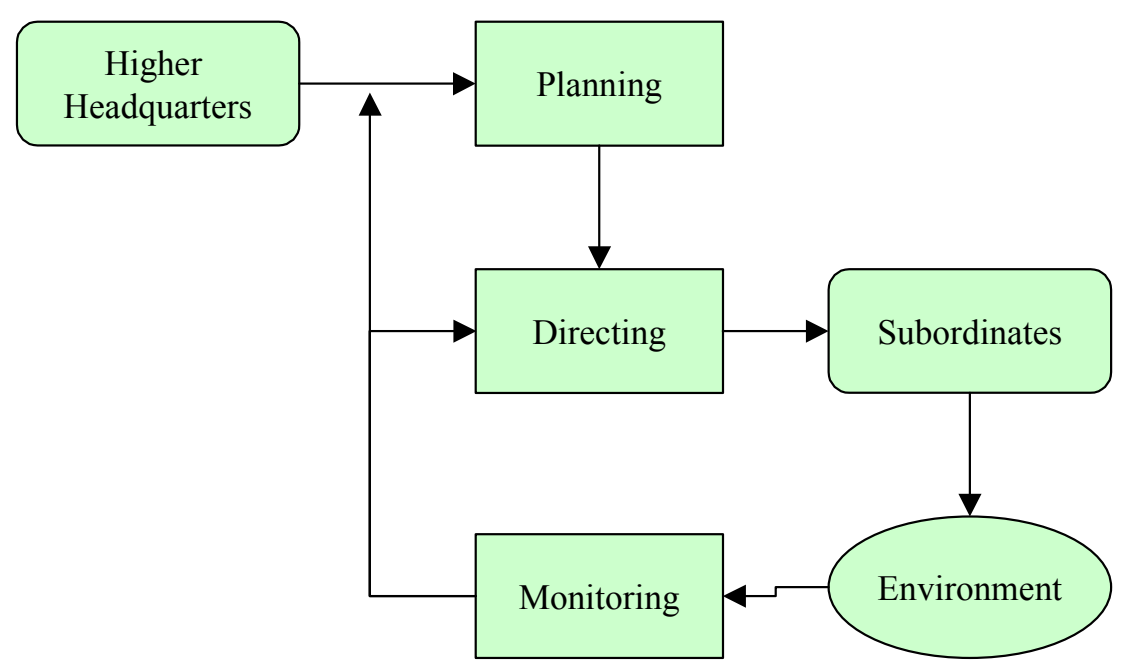

Figure 7 - Model of Command and Control Process

(Fallesen et al., 1992a)

Another accepted model of command and control process is the Lawson Loop. This model was developed by Joel S. Lawson and is considered applicable to $\mathrm{C} 2$ processes ranging from the Napoleonic era to the 1990s. This model consists of five functions (Huron, 1997):

- Sense - the collection of data from the environment.

- Process - pulls together and correlates the data gathered from the sense function.

- Compare - comparison of the information just processed in the process function to the current state of the environment to the desired state of the environment.

- Decide - decisions, performed normally by the commander, from the courses of action presented by the commander's staff.

- Act - the manifestation of the commander's decision.

This model is illustrated in Figure 8. 


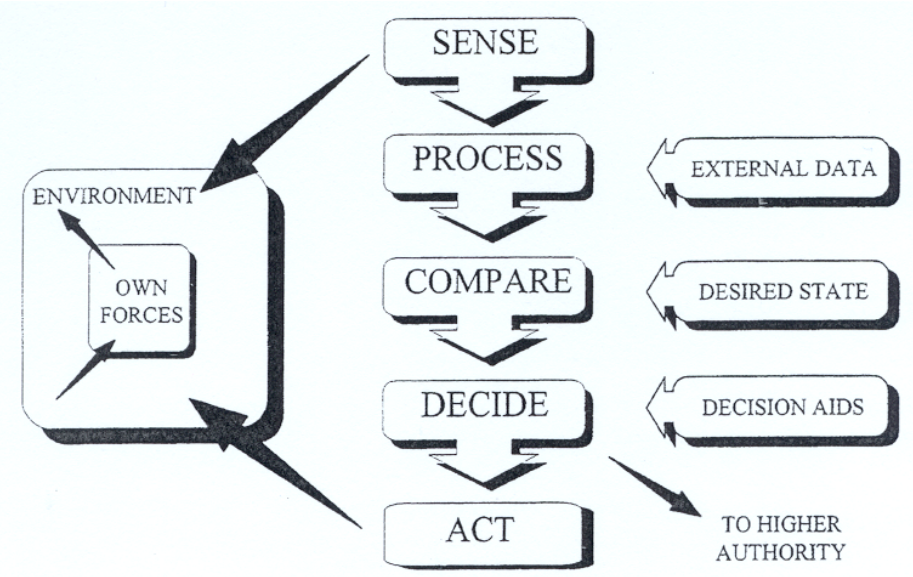

Figure 8 - The Lawson Command and Control Loop (Huron, 1997)

Evaluations of $\mathrm{C} 2, \mathrm{C} 3$, and $\mathrm{C} 4$ systems all need to be performed with the aim to establish the extent to which the system will support the effective prosecution of combat operations. It should also be to answer the question of whether an investment in a more capable system would result in more combat effectiveness than an equal investment in alternatives, including the fighting forces themselves (Snyder, 1993).

\subsubsection{Tactical Operations Center.}

A TOC is that group of personnel and equipment used by combat unit headquarters to control operations on a battlefield. TOCs for battalion and brigade level units tend to be similar as they use the same type of equipment and general organization. TOCs for division and corps level units are also similar in nature but are significantly bigger and more complex as these levels of headquarters exercise authority over a much larger sector of the battlefield. An example of a brigade TOC layout that was actually used during the Battle Command Training Program (BCTP) Warfighter computer simulation wargame exercise Phantom Thunder conducted at Fort Hood, Texas in February of 2002 is shown in Figure 9. BCTP is a training program based in Fort Leavenworth, Kansas that, along with the National Training Center (NTC) at Fort Irwin, California, evaluates units on their warfighting capabilities. BCTP performs their evaluations through the use of computer wargame simulations and the NTC performs its evaluations through live field exercises conducted against live opposing force (OPFOR) units. 
This TOC layout was used by the $1^{\text {st }}$ Brigade of the $1^{\text {st }}$ Cavalry Division during this exercise (BCTP, 2002). The individual sections such as the S3, ADA, and S2 are formed around M1068 tracked command post vehicles. The area in the center between the M1068s is formed using tactical tents interconnected together and to the vehicles.

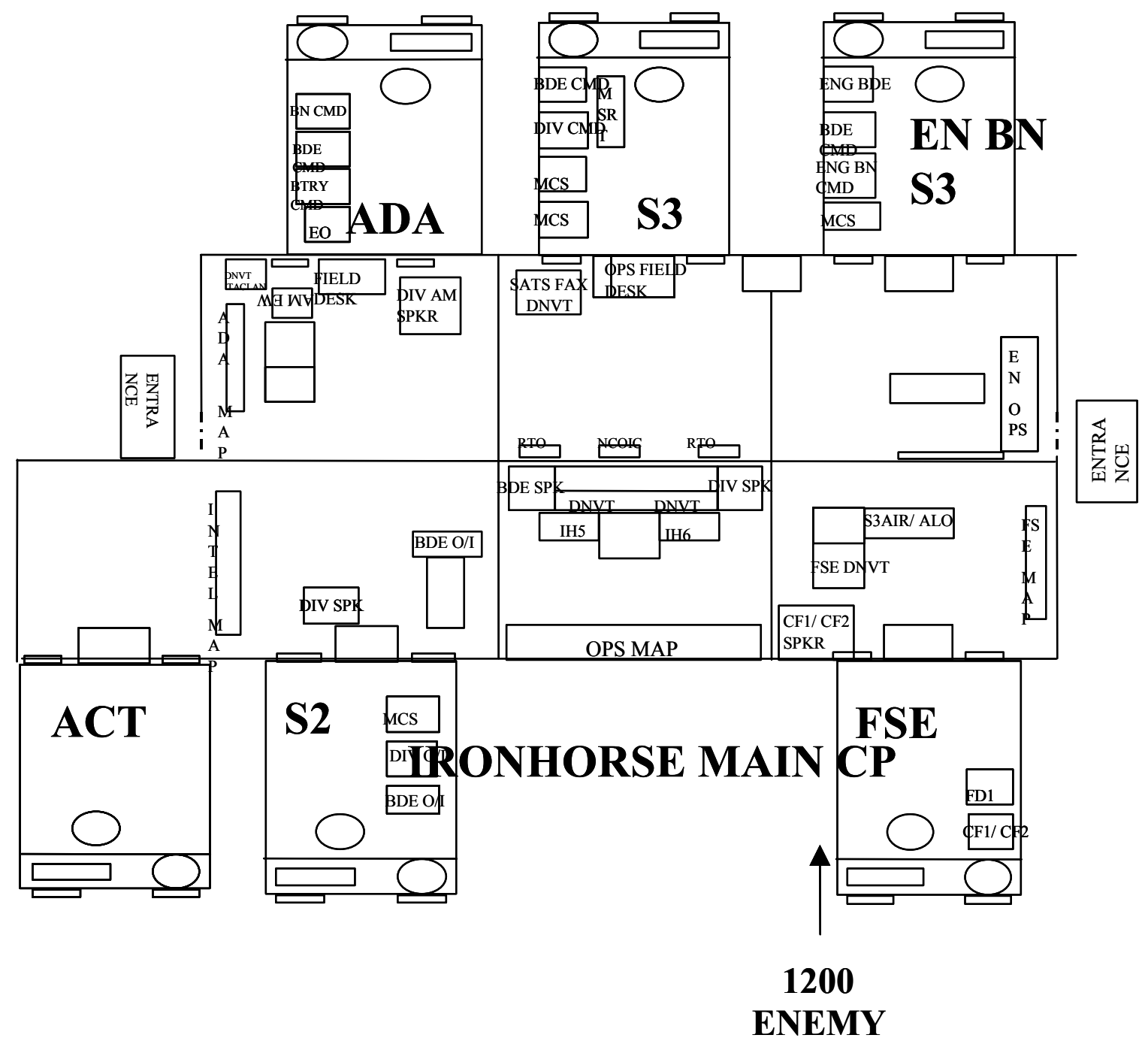

Figure 9-TOC Layout for $1^{\text {st }}$ Bde, 1 CD During Exercise Phantom Thunder

The officers assigned to the TOC and their enlisted assistants frequently suffer from viscerogenic deficiencies, especially the gross lack of rest, incomplete diet, and often overwhelming psychological influences resulting from the very nature of combat operations. In addition to functioning as a control center, the TOC frequently is placed under bombardment or ground attack by the enemy forces which requires that the personnel assigned to the command 
center be able to render judgments and decisions while engaged in close combat, repelling the enemy, and often while attempting to destroy classified documents and equipment. Military personnel employed within the TOC are required to accumulate a sizeable number of facts, relationships, and hypotheses while being constantly conversant about the friendly and enemy force situations and intentions. It is within the TOC that the unit commander, operations officer, intelligence officer, and supply officer direct and support the operations of the command. The essence of success in this role is to remain in control of one's emotions and faculties while responding to one's training (Gordon, 1973).

Effective operation of the TOC is tied to its ability to ensure effective $\mathrm{C} 2$ within the battalion/task force and brigade/brigade combat team organizations (Goedkoop, 1988). The key to doing this is the successful integration of the commander's tactical intent throughout all phases of the TOCs tactical operations. These tactical operational requirements can be tied to the seven BOS systems. TOC C2 requirements organized according to BOS requirements are shown in Table 4.

Table 4 - TOC C2 Functional Requirements According to BOS (Goedkoop, 1988)

\begin{tabular}{|c|c|}
\hline BOS & TOC Performance Tasks \\
\hline $\begin{array}{l}\text { 1. Maneuver of combat } \\
\text { elements of the unit. }\end{array}$ & $\begin{array}{l}\text { - Recommends new maneuver courses of action to the commander when } \\
\text { the situation dictates. } \\
\text { - Tracks movement of maneuver elements in accordance with the tactical } \\
\text { plan. } \\
\text { - Analyzes unit reporting to ensure execution in accordance with the } \\
\text { tactical plan; adjusts as necessary. } \\
\text { - Tracks movement of adjacent, higher, and rear units. } \\
\text { - Integrates attack helicopters into maneuver scheme. }\end{array}$ \\
\hline $\begin{array}{l}\text { 2. Intelligence that } \\
\text { provides the ability to } \\
\text { "see" the battlefield. }\end{array}$ & 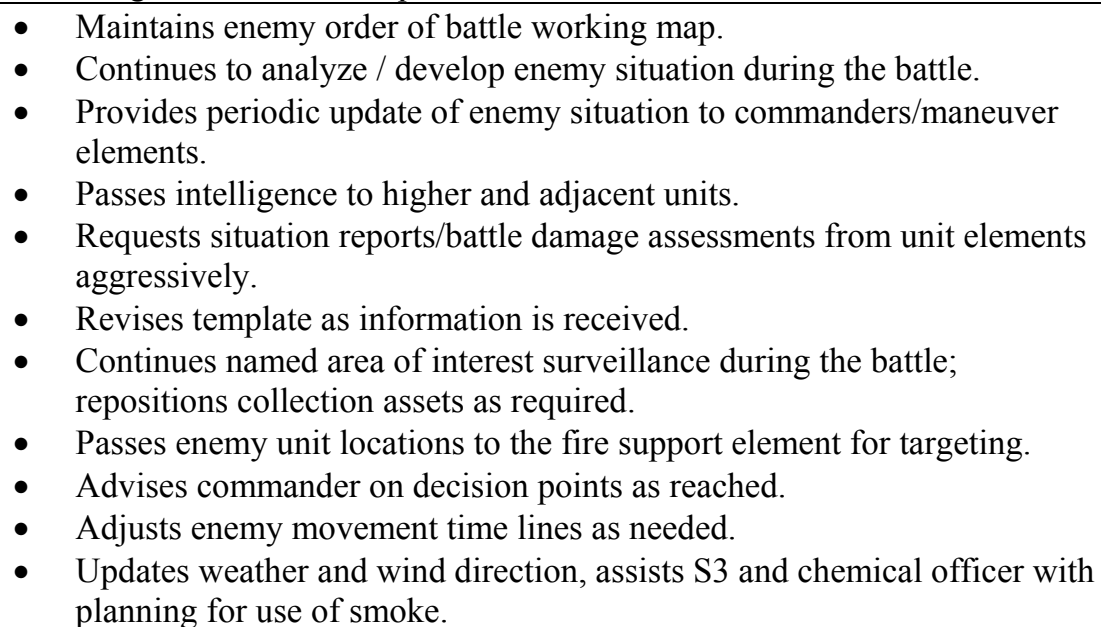 \\
\hline
\end{tabular}




\begin{tabular}{|c|c|}
\hline$B O S$ & TOC Performance Tasks \\
\hline & $\begin{array}{l}\text { - Advises on possibility of enemy nuclear, biological, or chemical weapon } \\
\text { usage. } \\
\text { - } \text { Maintains record of enemy losses by type of vehicle to assist in } \\
\text { - Maintaing status of task force collection assets. } \\
\text { - Maintains ground surveillance radar and remote sensor locations and } \\
\text { - } \text { adjusts as necessary. } \\
\text { - Renitors counter-reconnaissance battle. } \\
\text { - Procests and integrates information from higher/adjacent units. } \\
\text { - Opomb, and mortar reports. } \\
\text { - Screens information from enemy prisoners of war. }\end{array}$ \\
\hline $\begin{array}{l}\text { 3. Fire Support that } \\
\text { masses firepower to } \\
\text { delay, disrupt, or } \\
\text { destroy enemy forces } \\
\text { in support of the } \\
\text { scheme of maneuver. }\end{array}$ & $\begin{array}{l}\text { - Maintains maneuver unit front-line trace; provides to the direct support } \\
\text { artillery unit and the higher fire support element. } \\
\text { - } \quad \text { Clears indirect fires within the unit's sector/zone of operations. } \\
\text { - } \text { artillery support of priorities of fire/final protective fires to the field } \\
\text { - } \text { Coordinates for placement of field artillery supporting units in } \\
\text { sector/zone of maneuver unit. } \\
\text { - } \text { Ensures that the fire support plan is executed per the fire support matrix. } \\
\text { - } \text { locations. } \\
\text { - } \text { Adjusts fire coordination measures as required. } \\
\text { Engages targets of opportunity as directed by the Executive Officer/S3 } \\
\text { Air. } \\
\text { Maintains communications with the mortar platoon, field artillery fire } \\
\text { direction center, and fire support teams (FISTs). } \\
\text { - Updates FISTs on the status of fire support availability. } \\
\text { Relays FIST calls for fires to direct support battalion when FIST cannot } \\
\text { do so. } \\
\text { Ensures mortars are integrated into the fire support plan; Recommends } \\
\text { mortar repositioning as necessary. } \\
\text { - Provides current artillery ammunition status to maneuver unit. } \\
\text { Plans with the engineer/ executes artillery delivered family of scatterable } \\
\text { mines (FASCAM). } \\
\text { - Coordinates close air support missions. } \\
\text { Ensures airspace coordination areas are put into effect and cancelled as } \\
\text { needed. } \\
\text { Plans and requests suppression of enemy air defense missions to support } \\
\text { close air support / joint air attack team strikes. } \\
\text { Coordinates requests for additional fire support. }\end{array}$ \\
\hline $\begin{array}{l}\text { 4. Combat Service } \\
\text { Support actions taken } \\
\text { to sustain the unit's } \\
\text { ability to fight. }\end{array}$ & $\begin{array}{l}\text { - } \text { Ensures combat trains command post tracks friendly situation/status. } \\
\text { - } \text { Ensures units are reporting losses via Administrative/Logistics radio } \\
\text { nets. } \\
\text { - } \quad \text { Maintains status of critical supply/ammunition items. } \\
\text { - } \quad \text { Informs S4 when to displace combat trains. } \\
\text { - Maintains location of key logistical facilities; Field trains, combat trains, } \\
\text { unit maintenance collection point. } \\
\text { - } \quad \text { Coordinates time/location of unit logistical package (LOGPAC) arrival. } \\
\text { Directs priorities of movement on task force supply routes. }\end{array}$ \\
\hline 5. Air Defense system & - Updates air defense warnings/weapons control status. \\
\hline
\end{tabular}




\begin{tabular}{|c|c|}
\hline$B O S$ & TOC Performance Tasks \\
\hline $\begin{array}{l}\text { providing security } \\
\text { from enemy close air } \\
\text { support aircraft and } \\
\text { attack helicopters. }\end{array}$ & $\begin{array}{l}\text { - } \quad \text { Disseminates "Red Air" early warning over command radio net. } \\
\text { - } \quad \text { Announces friendly aircraft arrival on station. } \\
\text { - Employs passive and active air defense measures as required. } \\
\text { - } \quad \text { Disseminates "Red" air assault operations in sector. } \\
\text { - Monitors status of air defense weapons/units; adjusts coverage as } \\
\text { necessary based on combat losses or new contingencies. } \\
\text { - Ensures air defense elements maintain location/situation of task force } \\
\text { lead elements. } \\
\text { - Assists communications between Vulcan/Stinger assets and parent } \\
\text { organizations. }\end{array}$ \\
\hline $\begin{array}{l}\text { 6. Mobility, } \\
\text { Countermobility, } \\
\text { Survivability that: } \\
\text { - Preserves freedom of } \\
\text { maneuver of friendly } \\
\text { forces; } \\
\text { - Obstructs maneuver of } \\
\text { the enemy; } \\
\text { - Enhances survivability of } \\
\text { friendly forces. }\end{array}$ & 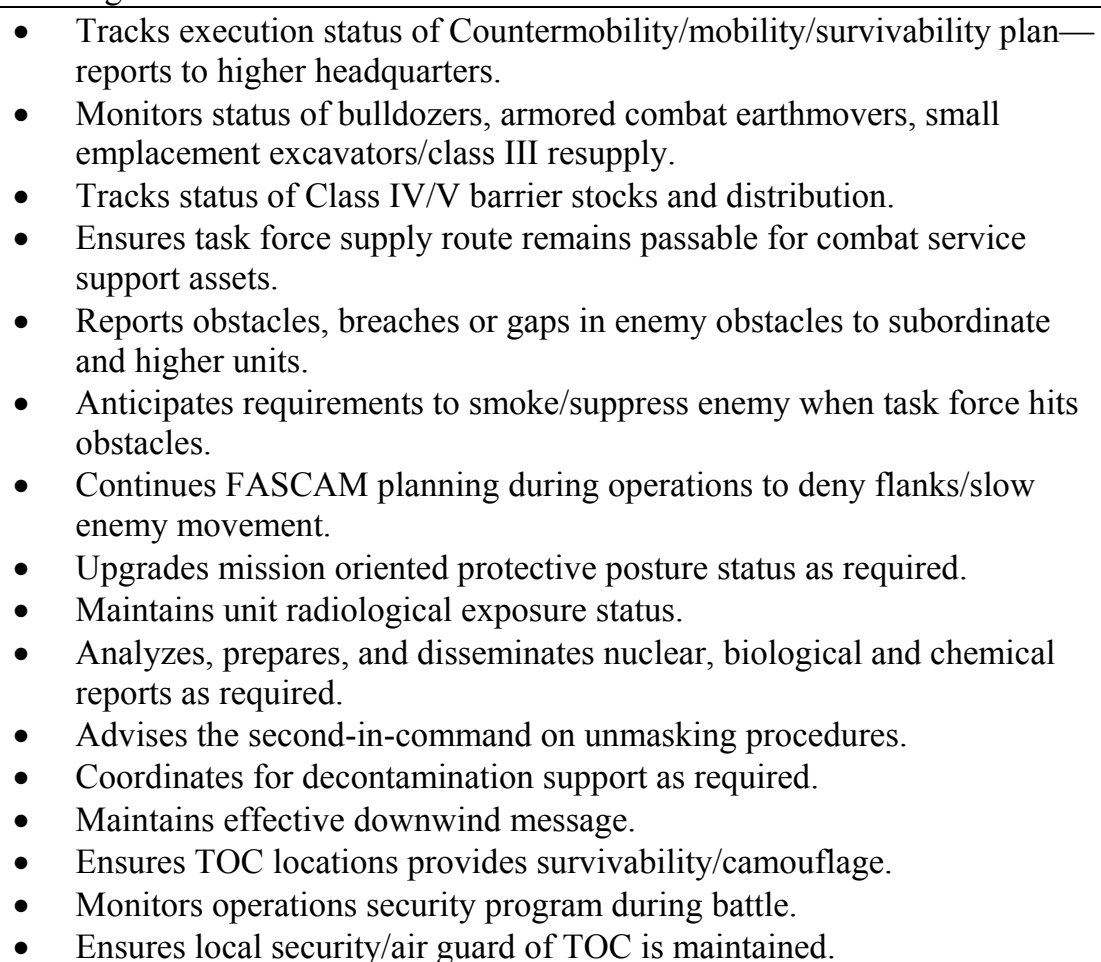 \\
\hline $\begin{array}{l}\text { 7. Command and Control } \\
\text { system that plans, } \\
\text { coordinates, and } \\
\text { executes combat } \\
\text { operations. }\end{array}$ & $\begin{array}{l}\text { - Issues fragmentary orders. } \\
\text { - Maintains radio communications with higher, adjacent, and subordinate } \\
\text { headquarters. } \\
\text { - } \text { Maintains net discipline as command net control station. } \\
\text { - } \text { relays as necessary. } \\
\text { - } \text { Monitors critical subordinate command nets during contact. } \\
\text { necessary. } \\
\text { - } \text { Activates "Battle Net" if key elements within the task force lose secure } \\
\text { capabilities. } \\
\text { - Maintains accurate status charts for critical information items. } \\
\text { - } \text { Manages synchronization matrix in accordance with tactical plan. } \\
\text { - Disseminates tactical information to subordinate units. } \\
\text { - Ensures subordinates continue to report during the conduct of operations. } \\
\text { - Second-in-Command responds for commander on higher command net } \\
\text { - to allow the commander to fight the battle. } \\
\text { Requests and coordinates additional combat/combat support assets from }\end{array}$ \\
\hline
\end{tabular}




\begin{tabular}{|l|ll|}
\hline BOS & \multicolumn{1}{c|}{ TOC Performance Tasks } \\
\hline & brigade. \\
& - Provides routine or requested situation updates/reports to higher \\
& - headquarters. \\
& - Lays land line to combat trains command post when possible. \\
& - Initiates planning for future operations. \\
\hline
\end{tabular}

As a result of hundreds of battalion and brigade TOCs that have trained at the National Training Center (NTC) at Fort Irwin, California, there have been countless insights on how TOCs function during combat operations. These experiences provide insights into problem areas that are systemic in nature to TOC operations (at least in the U.S. Army) and transcend individual problems and deployments. Some of these noted weaknesses are (Goedkoop, 1988):

- Inability of the TOC to track the flow of the battle, and synchronize the actions of the task force.

- The performance of the TOC is generally unsatisfactory when the executive officer does not operate from it during combat operations.

- A displacement plan is seldom produced to guide the movement of the TOC in consonance with the tactical plan with the result that the TOC often is left behind and loses communication with the lead elements.

- Local security and small arms air defense protection of the TOC are often neglected during combat operations.

- If the TOC is destroyed the alternate TOC (normally the combat trains command post (CTCP)) must cease its normal duties and assume the duties, albeit in a degraded mode, of the TOC with the result that a rippling effect goes throughout the entire organization causing the effectiveness of the whole organization to suffer.

- TOCs have an obvious visual signature due to the large number of vehicles, which normally compose it. This often causes it to be targeted and subsequently destroyed by high performance aircraft.

- Most TOCs do not practice adequate communications security. The large number of radio nets normally operated at the TOC produce an electronic signature that is subject to exploitation by electronic means such as direction finding equipment and jammers.

\subsubsection{MANPRINT.}

It was not until World War II that the U.S. Department of War recognized the importance of designing systems that meet human performance requirements (O'Brien, 1985). Beginning in 
the early 1980's with a realization of this requirement, the U.S. Army acknowledged that developing and fielding the right kind of force in the face of continued technological advancement combined with evolving changes in the strategic environment is a big challenge (Army, 2001b). A predominate issue is the integration of human performance abilities into the system design and acquisition process. The Army program to incorporate human factors issues into its materiel acquisition and development process is called Manpower and Personnel Integration (MANPRINT).

MANPRINT is mandated by Army Regulation (Army, 1991) and is a management and technical effort to integrate soldier performance and reliability issues into the acquisition process and is a comprehensive technical effort to promote system effectiveness by integrating into the materiel development and acquisition process all relevant information concerning human factors engineering, system safety, and health hazards (Barber, Ching, Jones, and Miles, 1990; Guerrier, Lowry, Jones, Guthrie, Barber, and Miles, 1991). MANPRINT is the Army's execution of DoD's Human systems Integration (HSI). D0D 5000.2-R, Part Four, Paragraph 4.3.8, states, “A comprehensive management and technical strategy shall be initiated early ... to ensure that human performance ... is considered throughout the system design and development process (Walker, 1997)." It is an Army program developed to optimize the human dimensions of system design through early involvement in systems design / systems engineering (Thurmond and Collins, 1988). It is a comprehensive management and technical program to enhance human performance and reliability in the operation, maintenance and use of weapon systems and equipment.

The total MANPRINT system includes all of the people, equipment, doctrine, training, etc., necessary to field and sustain the system in peacetime and combat. The goal of MANPRINT is to optimize total system performance by influencing the design and fielding of new weapon and other systems to improve battlefield effectiveness and reduce operations and support costs through the continuous integration of human factors engineering, manpower, personnel, training, system safety and health hazard considerations throughout the materiel development and acquisition process(Blackwood and Dice, 1988; Bogner, 1989; Lickteig, 1987). Some of the so-called "MANPRINT Rules of Thumb" are:

1. Soldier performance affects system performance.

2. Aptitude plus training $=$ skill. 
3. Measure soldier performance by time and accuracy.

4. Equipment design determines soldier tasks.

5. Make the designer responsible for soldier performance.

MANPRINT, in practice, is the recognition that the capabilities and limitations of the soldiers who operate, maintain, and support Army equipment must be an important consideration when designing or selecting hardware. The MANPRINT process refers to those specific actions that must be accomplished to ensure that soldier performance issues are identified, addressed, and managed throughout the design, development, and acquisition of a new materiel system (Johnson and Wright, 1990). MANPRINT is a comprehensive management and technical program that focuses attention on human capabilities and limitations throughout the systems life cycle including concept development, documentation, design, development, fielding, operation and modernization of systems (Walker, 1997). The MANPRINT program is a comprehensive management and technical program to assure total system effectiveness by continuous integration into materiel development and acquisition of all relevant information concerning Manpower, Personnel, Training, System Safety, Health Hazards, and Human Factors engineering (Hiemstra, Korzym, Barila, and Imbs, 1987; Johnson, Rossmeissl, Kracov, and Shields, 1988).

The search for decisive weapons has led to a continued high level of investment in advanced technologies. The implementation of well conceived, integrated HFE programs is intended to mitigate or resolve many of the identified human performance deficiencies in the design of military systems (Price, Sawyer, and Kidd, 1983). During the years of the draft, military personnel were viewed as essentially cost-free (which was, of course, an illusion) which resulted in little attention being paid to personnel issues by the military. However, the rapidly accelerating shift toward technological sophistication in the weapon and support systems utilized by the services has led to significant labor demand shifts for specific types of labor. It has also led to more complex career behaviors of soldiers themselves (BDM, 1985). The MANPRINT program itself focuses on the needs and capabilities of the soldier (Williges, 1990).

MANPRINT database and information sources include (Barila, 1987):

- Army Modernization Information Memorandum (AMIM).

- Army Training Requirements and Resources System (ATRRS).

- Basis of Issue Plan (BOIP) / Tables of Organization and Equipment (TO\&E).

- Defense Technical Information Center (DTIC).

- Manpower Requirements Criteria (MARC) Maintenance Data Base. 
- Personnel Structure and Composition system (PERSACS).

- Qualitative and Quantitative Personnel Requirements Information (QQPRI).

- Standard Study Number (SSN) / Line Item Number (LIN).

- Center for Army Lessons Learned (CALL).

- Directorate of Soldier Advocacy (DSA).

- Force Structure and Programs Directorate (FSPD).

$\circ$ Manning Integration directorate (MID).

- Military Occupational Development Directorate (MODD).

○ Personnel Proponent Coordination directorate (PPCD).

Chapter 2 of Army Regulation 602-1 (Army, 1991), states that Human Factors Engineering (HFE) is defined as a "comprehensive technical effort to integrate into Army doctrine, materiel development, and materiel acquisition (to insure operational effectiveness) all relevant information concerning" (Weisz, 1989):

○ Human characteristics.

- Skill capabilities.

○ Performance.

- Anthropometric data.

- Biomedical factors.

- Safety factors.

○ Training.

○ Manning implications.

The MANPRINT program encourages the use of predecessor or reference systems in the analysis and development of new systems (Lysaght, Hill, Dick, Plamondon, Linton, Wierwille, Zaklad, Bittner, and Wherry, 1989). Army Regulation 602-2 (Army, 2001a) identifies the critical MANPRINT domains as:

- Manpower. The number of personnel, both military and civilian, required, authorized and potentially available to train, operate, maintain, and support each system acquisition.

- Personnel. The human aptitudes, skills, and capabilities required to operate, maintain, and support a system in peacetime and war.

- Training. The instruction and resources required to provide Army personnel with requisite knowledge, skills, and abilities to properly operate, maintain, and support Army systems.

- Human Factors Engineering. The comprehensive integration of human capabilities and limitations into system definition, design, development, and 
evaluation to promote effective soldier-machine integration for optimal total system performance.

- System Safety. The design and operational characteristics of a system that minimize the possibilities for accidents or mishaps caused by human error or system failure.

- Health Hazards. The systematic application of biomedical knowledge, early in the acquisition process, to identify, assess, and minimize health hazards associated with the systems operation, maintenance, repair or storage, such as: Acoustic energy, toxic substances (biological and chemical), oxygen deficiency, radiation energy, shock, temperature extremes, trauma and vibration.

- Soldier Survivability. The characteristics of a system that reduce fratricide as well as reduce detectability of the soldier, prevent attack if detected, prevent damage if attacked, minimize medical injury if wounded or otherwise injured, and minimize physical and mental fatigue.

Some of the human engineering analysis methods used to conduct MANPRINT assessments include (DOD, 1999):

1- Mission Analysis is used to define what tasks the total system (hardware, software, and liveware) must perform.

2- Task description / analysis is a method designed to record and analyze how the human is involved in a system and provides an organized listing of how the human interacts with the system.

3- Predetermined time standards (PTSs), which are internationally recognized time standards used for work measurement. They are employed to estimate performance times for tasks that can be decomposed into smaller units for which execution times can be determined or estimated. Examples are Methods Time Measurement (MTM) and Modular Arrangement of Predetermined Time Standards (MODAPTS).

4- Cognitive Task Analysis (CTA) is a task analysis method that focuses on describing the cognitive skills and abilities needed to perform a task proficiently and is used to analyze and understand task performance in complex real world situations, especially those involving change, uncertainty, and time pressure. Example is the Critical Decision Method (CDM), which emphasizes the elicitation of knowledge from individuals skilled at performing a given task.

5- Functional flow diagrams that provide a detailed outline of all system requirements.

6- Operational Sequence Diagrams (OSD) that is a graphic presentation of operator tasks as they relate sequentially to both equipment and other operators.

7- Flow process charts (FPCs), which are plots of the sequence of operator activities or information, transfer as a part of a system.

8- Decision / action diagrams which show the flow of required system data in terms of operations and decisions. 
9- Action / information requirements which define those specific actions necessary to perform a function and, in turn, those specific information elements that must be provided to perform the action.

10- Timeline, which is a plot of task, flow as a function of time.

11- Integrated Computer Aided Manufacturing Definition (IDEF) is a method of system modeling that enables understanding of system functions and their relationships. The IDEF methodology defines a system in terms of its functions and its inputs, outputs, controls, and mechanisms (ICOMS).

+ IDEF0 models the functions and resources of a system rather than the process flow of the system.

+ IDEF1X is a method for developing logical data models that describe the structure of information within a system.

12- Function allocation trades which perform preliminary trade off studies of human machine allocations for each of the functions being considered.

13- Workload analysis that provides an appraisal of the extent of operator or crew task loading, based on the sequential accumulation of task times.

14- Situation Awareness (SA) analysis where SA is the experience of fully understanding what is going on in a given situation, of seeing each element within the context of the overall mission or goal, and of having all the pieces fit together into a coherent picture.

15- Link Analysis that describe the interactions between components in a system (human or machine).

16- Human performance reliability analysis (HPRA), which is an analysis of the factors that determine how reliably, a person will perform within a system or process.

Test and analysis methods used to conduct MANPRINT assessments include (DOD, 1999):

1- Continuous direct observation.

2- Sampled direct observation.

3- Specification compliance summary sheet.

4- Technical manual functional evaluation.

5- Human Factors Engineering Data Guide for Evaluation (HEDGE).

6- Use of Environment and engineering measurement equipment.

7- System records review.

8- Test participant history record.

9- Interview.

10- Questionnaire. 
11- Motion pictures.

12- Sound recording.

13- Video recording.

14- Still photography.

15- Event recording.

16- Secondary task monitoring.

17- Physiological Instrumentation.

18- Physical measurement.

19- Online interactive simulation.

20- Statistical analysis.

Unfortunately, human factors continues to be rediscovered nearly every time there is a well publicized disaster in which "human error" is involved (Booher, 1990). It is noted that it is not a trivial or simple matter to engineer down complexity at the soldier-machine interface (DePuy and Bonder, 1982). The MANPRINT program is designed to enable the Army to overcome many of these oversights. In 1985, when the program was just beginning, it was noted that many times a system program manager will choose not to implement human engineering changes during development because of the potential for cost overruns and procurement delays (O'Brien, 1985). This is sometimes still true today, however, the MANPRINT program provides the framework for the development of systems optimized for human performance especially if the human performance and MANPRINT assessments are made early enough in the program development cycle.

\subsubsection{War.}

Some thoughts on the concept of war itself is considered necessary as a basis for the study of techniques to deal with it such as command and control. A recent white paper published by the U.S. Army stated that, at its most fundamental level, war is a brutal contest of wills (Army, 2002b). At the strategic level this is characterized as a contest of national will and interests. At the tactical level this is characterized as life and death struggles between units and individuals. The white paper goes on to say that leaders must know how to conduct rapid tactical decision making where information superiority via a web- enhanced, knowledge- based common operating picture is the key to force effectiveness. At its most abstract war is an uncertain, mentally complex, physically demanding, and intensely emotional experience. Combat, by its 
very nature, is a hostile environment intended to diminish human performance. In this context, it is noted that the sum of the factors which degrade human performance may be much greater than the sum of their parts (Parry, Collins, and VanNostrand, 1990). The central fact of combat in war is danger to life as armies exist to fight and fighting means casualties (Williams, 1984).

The U.S. Army has defined war to exist at three levels. These are strategic, operational, and tactical. These are described as (Army, 2001c; Gibbings, 1991):

- The strategic level of war is described as the level of war "at which a nation or group of nations determines national or alliance security objectives and develops and uses national resources to accomplish those objectives."

- The operational level of war is described as "the level of war at which campaigns and major operations are planned, conducted, and sustained to accomplish strategic objectives within theaters or areas of operations."

- The tactical level of war is described as "the level of war at which battles and engagements are planned and executed to accomplish military objectives assigned to tactical units or task forces."

General Frederick M. Franks, Jr., the VII Corps Commander during Operation Desert Storm in 1991, is quoted as saying that "modern land warfare is tough, uncompromising, and highly lethal" where casualties are sudden, and the combat results are final and frozen for a lifetime (Army, 2001c). This is why combat leadership is so demanding. Future military environments will be evolutionary and chaotic, often presenting ill-defined, high-bandwidth information dilemmas that proliferate across traditional geopolitical boundaries under highly stressful conditions. This situation is termed "cognition in the wild" (McNeese and Rentsch, 2001).

A war using modern weapons systems is likely to be both intense and short. There are three types of stressors that come into play during combat. The first are the physical stressors of heat, cold, humidity, noise, overpressure, toxic substances and fatigue. Then there are psychological stressors of confinement and isolation, crowding, and psychological warfare.

Finally, there are NBC stressors from the effects of weapons of mass destruction such as nuclear, biological and chemical weapons (Kubala and Warnick, 1979).

Few activities of mankind are more complex than combat operations, and few have been studied as assiduously. Notwithstanding this, man's understanding of the process of warfare is 
incomplete and inadequate. In many respects certain elements known to have an important bearing on the outcome of battle can neither be predetermined nor measured. War is an example of the complexity, the uncontrolled variability, and the impossibility of obtaining and recording desired data through manipulation and observation (Hausrath, 1971).

\subsection{Issues in Human Cognitive Performance.}

While this dissertation is focused on an analysis of the total $\mathrm{C} 2 \mathrm{~S}$, it is realized that the human operator is the key component in this system. For this reason, an understanding of selected concepts of human performance, especially human cognitive performance, is of paramount importance in understanding how the role of the operator affects the performance of the overall system. It is noted that each topic is worthy of and the subject of much research in its own right, and are presented briefly here as a review of the topic. These are explored briefly here in order to gain some understanding of how each might be considered in computer simulations of the overall system.

\subsubsection{Expertise.}

Experts see the world differently. They see things the rest of us cannot. One view of experts is that they have accumulated lots of knowledge. Often experts do not realize that others are unable to detect what seems obvious to them (Klein, 1998). Some of the many things experts can see that are invisible to everyone else:

- Patterns.

- Anomalies.

- The big picture (situation awareness).

- The way things work.

- Past and / or future events that might or might not happen.

- Opportunities and improvisations.

- Differences that are too small for novices to detect.

- Their own limitations.

People with greater expertise have a larger resource of procedures to apply. They notice problems more quickly and have a have richer mental simulation to use in diagnosing problems and in evaluating courses of action. In other words, they have more analogies to draw upon. On the negative side, expertise can cause problems by causing individuals to view problems in 
stereotyped ways. Expert systems are computer programs that embody domain-specific knowledge and that perform at levels typical of human experts, but not necessarily in exactly the same manner as human experts (Cooke, 1999).

\subsubsection{Situation Awareness.}

Situation awareness (SA) is defined as the perception of the elements in the environment within a volume of time and space, the comprehension of their meaning, and the projection of their status in the near future (Endsley, 1988). It is a state of knowledge from the processes used to achieve that state (Endsley, 1995b). SA refers to the up-to-the-minute cognizance required to operate or maintain a system (Adams, Tenney, and Pew, 1995). One view of SA is that it is an adaptive, externally directed consciousness (Smith and Hancock, 1995). It can be described simply as "knowing what is going on so that one can figure out what to do" (AGARD, 1998) or simply knowing what is going on around you (Endsley, 2000) or the up to the minute cognizance or awareness required to move about, operate equipment, or maintain a system (Pew and Mavor, 1998h). Another definition states that SA is the perception of reactions to a set of changing events (Klein, 2000a). The definition of SA has evolved to a description of an operator's comprehension of a complex system in which the environment is dynamically changing and in which the operator is responsible for maintaining or achieving particular states or goals (Durso and Gronlund, 1999). Operator SA is comprised of the total understanding about the physical environment, system states, one's own status, etc. This awareness, or knowledge, forms the basis for making critical decisions. A more formal definition of SA is that it is "the perception of the elements in the environment within a volume of time and space, the comprehension of their meaning and the projection of their status in the near future". SA can be assessed with objective or subjective ratings and may be inferred from other measures of performance (AGARD, 1998).

Historical evidence has shown that in periods of rapid movement and intensive fighting the "fog of battle" becomes unforgettably evident with a lack of information not only in the wider context but also of what was happening in one's own immediate situation. A very striking feature of combat is the prevalence of extreme restriction, ignorance, and uncertainty. The chaos of battle obscures nearly everything (Williams, 1984).

The enhancement of SA has become a major design goal in the development of system interfaces between the operator and the hardware and software of the system. One of the chief 
reasons to measure SA is for the purpose of evaluating new system and interface designs. Three levels of SA are defined as (Endsley, 2000):

- Level $1 \mathrm{SA}$ - Perception of important information.

- Level $2 \mathrm{SA}$ - Comprehension of how people combine, interpret, store, and retain information.

- Level $3 \mathrm{SA}$ - Projection or the ability to forecast future situation events and dynamics. This marks operators who have the highest level of understanding of the situation or highest SA.

One way to study SA is through critical incidents, which can be presented in the form of simulations, workplace observations, or can be elicited and / or probed through interviews that generate narratives from memory. The acquisition and development of SA by a system operator is an active process of guided information seeking rather than passively receiving and storing details (Klein, 2000a).

Individual situation models and shared mental models can be considered precursor products of team SA in that they are cognitive structures that are brought to the task of interpreting the situation by team members. The shared mental model is a precursor to team SA where each team member brings to the task not only momentary or transient understandings of the current situation, i.e., individual situation models, but also long term, relevant individual knowledge pertinent to the environment, task, or team members. The information that is critical for SA is not always obvious, even to an expert (Cooke, Stout, and Salas, 2001).

It is hypothesized that working memory constitutes the main bottleneck for SA. SA is a complex process of perception and pattern matching greatly limited by both working memory and attention capacities. Several mechanisms, attention sharing and automated processing, may serve to alleviate these limitations to some degree. Attention can also be seen as an important constraint on SA. Direct attention is needed for not only perception and working memory processing, but also for decision-making and response executions (Endsley, 1988).

Some general principles for improving SA are (Endsley, 1988):

- Information presentation should feature the grouping of information in terms of spatial proximity and the use of multiple information imbedded within objects.

- The use of information grouping should tie multiple attributes to each object while minimizing the number of objects presented.

- Information presentation should allow rapid access to long-term memory storage. 
- Holistic processing strategies should be employed.

- Information filtering by the system should be employed to reduce information processing filtering requirements on the operator.

- Information that allows for the determination of trend/rate of change of components should be available to the operators.

- The most important information should be the most salient perceptually to insure focused attention.

- Peripheral vision can be employed for the non-attentive monitoring of qualitative state or changes in simple secondary information.

- Verbal information requirements on short-term memory should be minimized, particularly for spatial information.

- Additional types of information input, such as auditory or tactile, can be utilized to provide information simultaneously to the visual channel.

- Methods of providing simultaneous access to secondary information should be employed which will not induce decrements on the primary task.

- Spatial information should be rapidly relatable to the operator's cognitive map and their orientation in it.

- Designs/technologies should be employed which will reduce pilot workload and improve upon the quality of information needed.

The impact of a person achieving or not achieving good SA can be significant. In general, it can be expected that poor performance will occur when SA is incomplete or inaccurate (Endsley, 1995b). Current military thinking insists that SA provides an information advantage that translates to success to the military decision maker who can achieve and maintain battlefield SA (Lee, 1999). Judging the level of SA in an operator when faced with multiple, competing demands on their attention during system operations is important in evaluating the effects of attempts to improve SA. Global measures of SA across many elements of interest is desirable if designers are to be able to evaluate the impact of projected design concepts on operator SA (Endsley, 1995a). These concepts are not new. Over 2400 years ago the Chinese military genius Sun Tzu wrote: "If you know the enemy and know yourself, you need not fear the result of a hundred battles (Tzu, 1910).”

\subsubsection{Stress.}

Stress is an important concept in the context of this research according to its potential to affect individual and group performance and decision-making. The issue of stress and performance is of special importance on the high tech battlefield because of the possibility that 
equipment may not be optimally operated by soldiers whose cognitive and sensory-motor skills are degraded by stress (Buckalew, 1990). While the evidence that shows that stress results in decision making errors is not convincing, stress does effect the way decisions are made, however, it does not cause bad decisions to be made that are based on available information. Stress, in itself, does not result in faulty decision-making but it may limit the information that is considered while making the decisions. Stressors such as time pressure, noise, and ambiguity can result in effects such as less information being gathered to support decision making, a disruption of working memory to sort out details, and a distraction to the task at hand. The evidence that shows that stress results in decision errors is not convincing nor does stress effect the way decisions are made as it does not cause bad decisions based on the available information. Stressors may result in the following effects (Klein, 1998):

- Not as much information can be gathered.

- The ability to use working memory to sort things out is disrupted.

- The attention to the task at hand is distracted.

Some key points in decision making are (Klein, 1998):

- Decision biases do not seem to explain poor decisions.

- Stress does not result in faulty decision making strategies but may limit the information to be considered in making the decisions.

- Most poor decisions may result from having inadequate knowledge and expertise.

- Experience does not translate directly into expertise if the domain is dynamic, feedback is inadequate, and the number and variety of experiences is too small.

The types of stressors include extreme temperature, noise, sleep deprivation, time constraints, frustration, and performance pressure, etc. Stress affects the speed/accuracy tradeoff and the effect of stress on decision making can largely be explained in the terms of less time being allotted to sub tasks such as gathering information. Each stressor requires attention for adjusting to and managing the stress itself and this may be seen as constituting a secondary task, so that degraded performance under stress may also be a function of fewer cognitive resources available (Klein and Crandall, 1996). Stress casualties can be expected to be as high as 1:4 to 1:3 in comparison to casualties such as wounded in action (WIA). Temperature extremes, rough terrain, and high altitudes cause fatigue that degrades performance (Vandivier, 1990).

In the case of sleep deprivation, soldiers become militarily ineffective after only 48 to 72 hours with no sleep and suffer a degradation of 75 percent in performance on most tasks after 72 
hours of work with no sleep. In addition, cognitive abilities begin to degrade as soon as 18 hours into sustained operations. For physical performance and routine motor tasks, the degradation is less rapid than cognitive tasks. High levels of cohesiveness, morale, and motivation can reduce stress casualties. Assuming all other factors are equal, leadership, training, and high mental aptitude can result in better performance on the battlefield (Vandivier, 1990).

\subsubsection{Decision Making.}

A decision-making episode occurs whenever the flow of action is interrupted by a choice between conflicting alternatives. A decision is made when one of the competing alternatives is executed, producing a change in the environment and yielding consequences relevant to the decision maker (Pew and Mavor, 1998b). The crucial activities for decision making are actions whose controlled execution consolidates fragments of policy that are lying around, gives them direction, and closes off other possible arrangements. Decisions that are tied more closely to action are more likely to contain improvisation (Weick, 1983). Also, planning, or the generation of a plan, is critical to successful operations where it plays a key role in the tactical decisionmaking process in the military throughout all echelons (Pew and Mavor, 1998g).

Some comments (Klein, 1998) on inaccurate decision making include the statement that decisions can be considered to be poor if the knowledge gained would lead to a different decision if a similar situation arose. Poor decisions can also be caused by factors such as lack of experience according to naturalistic decision-making theory. Some of the causes of poor decision-making are:

- Lack of experience.

- Lack of information.

- Mental simulation, the de minimus error where the signs of the problem were noticed but were explained away.

In complex and dynamic environments, attention demands resulting from information overload, complex decision making, and multiple tasks can quickly exceed a person's attention capacity (Endsley, 1995b).

The evidence that shows that stress results in decision errors is not convincing nor does stress effect the way decisions are made but it does not cause bad decisions based on the available information. Stressors such as time pressure, noise, and ambiguity result in the following effects (Klein, 1998): 
- Not as much information can be gathered.

- The ability to use working memory to sort things out is disrupted.

- The attention to the task at hand is distracted.

Some key points in decision making are (Klein, 1998):

- Decision biases do not seem to explain poor decisions.

- Stress does not result in faulty decision making strategies but may limit the information to be considered in making the decisions.

- Most poor decisions may result from having inadequate knowledge and expertise.

- Experience does not translate directly into expertise if the domain is dynamic, feedback is inadequate, and the number and variety of experiences is too small.

The field of naturalistic decision making (NDM) is a recent approach to describing how system operators actually make judgments and decisions, during emergencies as well as routine conditions (Klein, 1993). NDM is concerned with poorly defined procedures as opposed to laboratory studies where decision-making is distinct from problem solving. Skilled military commanders, for example, will evaluate a plan of action by mental simulation and will apply a sense of predictability to notice that their adversary can anticipate their moves and will take the necessary precautions to prevent it (Klein, 1998).

Recognition primed decision (RPD) making is the process of making decisions based on experiential knowledge. For example, an experienced commander's secret was that their experience let them see a situation, even a nonroutine one, as an example of a prototype, so they knew the typical course of action right away even though it was not easy to classify the decision points. Experts are able to see the world differently and have the ability to recognize things that others cannot. One view of experts is that they have accumulated lots of knowledge (Klein, 1998).

The RPD model of decision-making describes a decision strategy commonly employed by proficient personnel called upon to make decisions in operational settings by high risk, time constraints, and ambiguous or incomplete information. It is a decision strategy that appears well suited for operational settings marked by time pressure, ambiguity, incomplete information, and ill defined and shifting goals. A key question from the NDM perspective is how people are able to use experience to handle difficult conditions. The RPD model asserts that experience enables decision makers to recognize the essential characteristics of a situation, and thereby to identify feasible goals and plausible courses of action through a fusing of two processes, situation 
assessment and mental simulation. The hypothesis for the RPD model claims that decision makers quickly recognize a favorite option, and a next best option, and that the decision making consists of trying to show that the favorite option dominates the next best option on all evaluation dimensions (Klein and Crandall, 1996).

Findings and conclusions of the RPD model:

- Experienced decision makers rely more on situation assessment while novices rely more on option evaluation strategies.

- Situation assessment seems to involve schematic or prototypical knowledge of cues, goals, and expectancies that apply to a given class of events.

- Whereas experts and novices notice the same cues in a situation, novices draw fewer inferences based on these cues and tend to miss the tactical implications of the situational cues.

- In the command and control domain decisions are most likely to be made without any conscious deliberation between option alternatives.

- When deliberation does occur, decision makers are more likely to use serial evaluation strategies than concurrent evaluation of options. Serial strategies appear to offer a means of minimizing the calculation burden as well as maximizing the speed with which a decision may be implemented.

- Serial evaluation is associated with satisficing rather than optimizing strategies, and is preferred under time-pressured conditions.

- Options are frequently evaluated through the use of images or a "mental model" that operates as a simulation for judging whether an option will be successful in a specific case.

- Expert decision makers rely on a process of "progressive deepening" or reasoning into the future.

- Analogical reasoning is infrequently reported, suggesting that the processes involved in selecting and using analogues are relatively automatic and unconscious.

- When analogues are used (often to address non-routine aspects of a problem), they are critical to option selection. Thus, inappropriate analogues are a primary cause of errors.

- Time pressure does not affect the quality of decisions made by experts as much as novices, due to experts greater reliance on rapid recognition processes.

The RPD model shows how the proficient decision maker becomes aware of events that have occurred, and relies on experience to recognize the events as largely typical (Klein and Crandall, 1996). 
The U.S. Army approach to decision making is called the Military Decision Making Process (MDMP). This process was first described in the 1932 version of FM 101-5, Staff Officers Field Manual, Part I, as the "Estimate of the Situation" (Army, 1997). The steps in that process included a statement of the mission, the disposition of the enemy forces, an analysis of the enemy situation, an analysis of the friendly force situation, and the decision to be made regarding the next action (Charlton, 1997). Today's MDMP is diagrammed in Figure 10 and consists of six steps which are receipt of mission, mission analysis, course of action (COA) development, COA analysis, COA comparison, and COA approval and orders production.

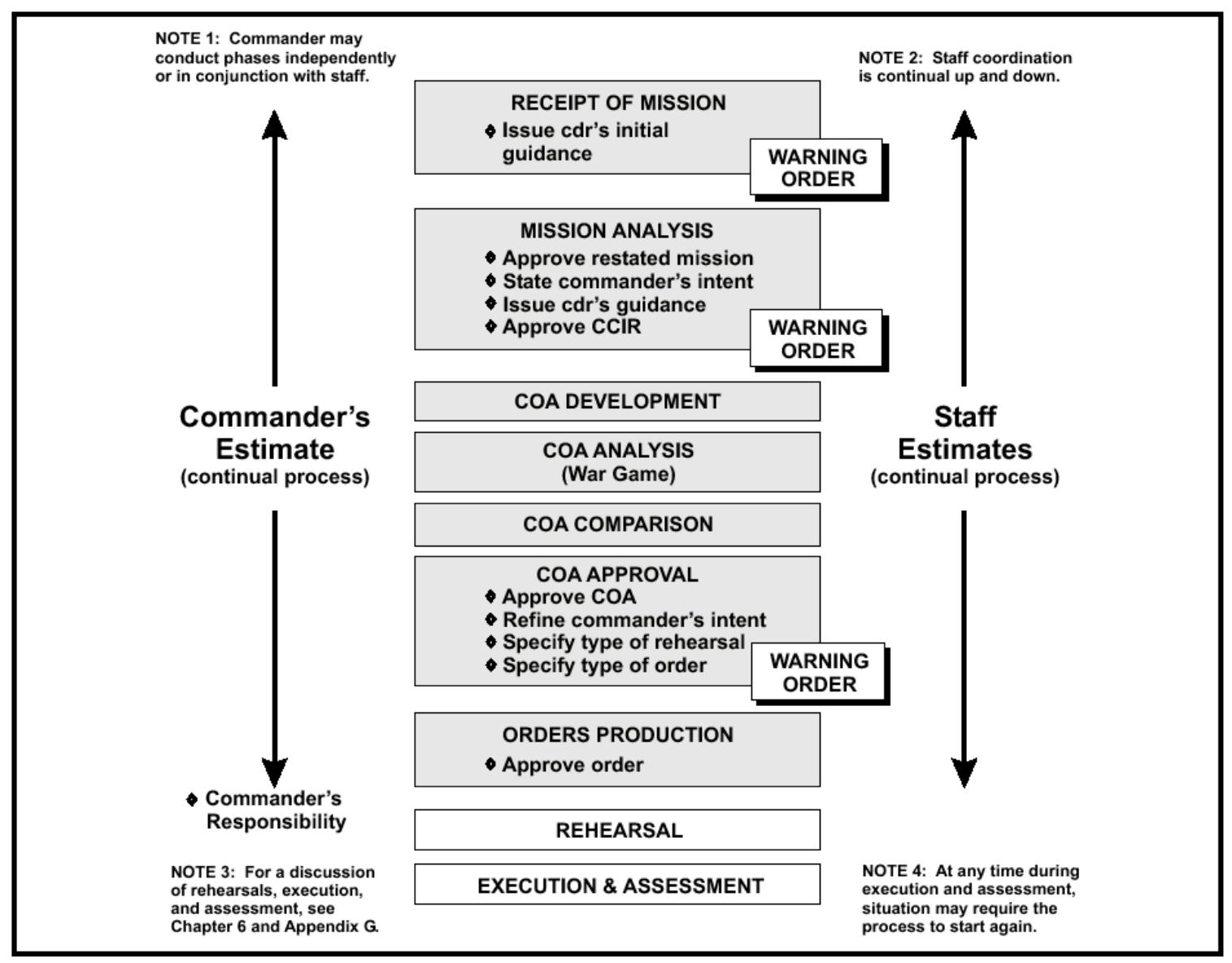

Figure 10 - The Military Decision Making Process

(Army, 1997)

However, the fundamental dilemmas faced by the Army using the MDMP are fivefold (Centric and Salter, 1999): 
- While the Army has become increasingly involved since the demise of the cold war in the operational level of war (for example, peacekeeping and direct action as opposed to full scale conventional warfare) there is currently little experience at this level.

- While the MDMP describes a tactical process, it is also being used at the joint planning level because there is no alternative.

- The MDMP was designed for the Army's mission during the Cold War as opposed to current missions that are focused on the operational level.

- While the MDMP works well in potentially time consuming and complex and uncertain environments typical of full-scale land warfare, it has deficiencies in missions that can call for time compressed decision cycles.

- The MDMP does not accurately model the way experienced decision makers naturally make decisions (reference, for example, Klein's RPD model (Klein, 1998)).

There is a considerable discussion in the literature concerning ways to optimize, enhance, and/or generally improve the ways that decisions are made especially in the military under combat conditions. It is noted that properly designed decision support systems should include provisions for the heuristics that are likely to be employed by a decision maker when faced with rapidly changing and information intensive situations along with incomplete or questionable data (Colton and Ganze, 1993).

\subsubsection{Task Performance.}

The ability of individuals and teams to perform tasks as part of the overall function of battlespace management is very important to the successful operation of a C2S. Human task performance has been described in a variety of models and descriptions and a widely accepted description is in the multiple resources model. The multiple resources model presents a formal framework for describing the extent to which two human information channels, each described by one of four formats of perception, two codes of central processing and response, and three stages of processing, will compete or interfere with each other.

Anatomical research, looking at the different human cognitive processing abilities, has established that visual information presented to the right visual field is relayed directly to the left 
cerebral hemisphere, and left visual field information directly accesses the right hemisphere. Since the left and right hemispheres are generally associated with verbal and spatial processing respectively, the direct access principle asserts that information of a given code (verbal or spatial) will be best processed if it is presented to the fovea and contra-lateral visual field (i.e., verbal, right field; spatial, left field). One of the strong intuitive appeals of the use of voice and auditory displays is that information thus displayed may be perceived without disrupting visual processing.

The multiple resources model predicts that as tasks occupy different "cells" of processing resources, interference between them will be reduced. In contrast to the weak effects observed when factors such as compatibility and competition are in opposition, the magnitude of effects when the two factors work together can be quite pronounced. Changing a task from an incompatible resource competitive configuration to a compatible configuration with separate resources has three positive influences. First, dual task performance decrement is reduced when separate resources are employed. Second, compatibility produces a higher baseline of performance. Third, high compatibility also reduces the resource demands of a task, and so further reduces the level of dual task interference.

For example, if the choice is to be made as to whether to use auditory displays for presenting information for a spatial or verbal task, the greatest gains by far will result from allocating the display to the verbal task. Any advantages of using an auditory display for the spatial task will be offset and nullified by the low level of compatibility. The greatest gains to auditory displays will be realized by the verbal tasks. Examples of visual and auditory verbal and spatial displays are:

- Visual spatial format can appear as an arrow emanating from the symbol and pointing up or down.

- Visual verbal format can use the word "up" or "down" that appears beside the corresponding symbol.

- Auditory verbal format can consist of the symbol flashing concurrently with the spoken word "up" or "down".

- Auditory spatial format can consist of a high or low tone presented in conjunction with the flashing symbol. 
Other considerations include the observation that the auditory modality may present problems in noisy environments, while the visual modality is more sensitive to disruptive effects of anoxia, high $\mathrm{G}$ forces, and vibration (Wickens, 1984).

\subsubsection{Team Performance.}

A team is defined as a distinguishable set of two or more people who interact, dynamically, interdependently, and adaptively toward a common and valued goal / objective / mission, who have each been assigned specific roles or functions to perform, and who have a limited life-span of membership (Mathieu et al., 2000; Salas, Dickinson, Converse, and Tannenbaum, 1992). A similar definition states that a team is defined as two or more interdependent individuals performing coordinated tasks toward the achievement of specific task goals who interact, dynamically, interdependently, and adaptively toward a common and valued goal/objective/mission. They have each been assigned specific roles or functions to perform, and have a limited life span of membership (Salas et al., 1992).

Team performance has two primary components. These are individual task behaviors and coordinated task related processes/functions/behaviors (Fleishman and Zaccaro, 1992). Work teams of individuals form a subconscious identity that can be described as the team mind. Some of the functions of the team mind include the ability to hold information for brief periods of time (working memory), the ability to store information permanently (long term memory), the ability to focus on only one thing at a time (limited attention), sensory mechanisms that convert mechanical energy into patterns of neural activity (perceptual filters), and the ability to acquire new procedures while discarding inefficient behaviors and determining how to become more effective (Klein, 1998). The nature and type of problems experienced by teams in operational settings are naturalistic and involve many elements that may emerge in situations (McNeese and Rentsch, 2001). Most decision-making activities in large scale systems and organizations are performed by a team of people who are interconnected by communication networks (Kleinman, Luh, Pattipati, and Serfaty, 1992).

Key points in describing team performance include the recognition that a team is an intelligent entity. Its cognition can be inferred from such things as the team behaviour, the contents of the team's collective consciousness, and the team's preconscious. The collective team mind has the ability to develop basic competencies and routines. It forms a clear identity 
while learning to manage the flow of ideas and learning to monitor itself to adjust its thinking when necessary (Klein, 1998).

The ability of the individuals to effectively function together as a team is dependent on many factors and is vitally important to the success of many organizations and work groups. One of the factors that has been studied is the differential effects from human interaction face to face versus through remote communications systems. Systems in which visual cues such as selective gaze are absent produce no differences in turn taking or in any other aspect of the structure of conversation. In fact, (in this experiment) turn taking was unaffected even when visual information was completely absent. However, in videoconferencing, failure to make eye contact tends to be a problem because of the separation of the camera and monitor. One implication of multiparty videoconferences involving several sites using the Picture In Picture (PIP or Hollywood Squares) approach is that it fails to support selective gaze and selective listening (Sellen, 1995).

Some of the most profound changes in social and organizational behavior in this century can be traced to tools that support remote individual and team performance cooperation. While collaborative work at a distance will be difficult to do for a long time, if not forever, effective communication between people requires that the communicative exchange take place with respect to some level of common ground. Common ground refers to that knowledge that the participants have in common, and they are aware that they have it in common (Olson and Olson, 2000). Common ground means two or more individuals working together and articulating perspectives on problems to jointly arrive at a shared meaning that references goals, terms, content, context, and process of a situation (McNeese and Rentsch, 2001). For example, people describe the same event or idea quite differently talking to a spouse, a coworker, a distant relative, a neighbor, a stranger from across the country and a stranger from overseas (Olson and Olson, 2000). In 1898 Arthur Mee stated:

"If, as it is said to be not unlikely in the near future, the principle of sight is applied to the telephone as well as that of sound, earth will be in truth a paradise, and distance will lose its enchantment by being abolished altogether (Mee, 2000)"

The shared mental model theory offers an explanation of what the mechanisms of adaptability might be, that is, how teams can quickly and efficiently adjust their strategy "on the fly." The function of shared mental models is to allow team members to draw on their own well- 
structured knowledge as a basis for selecting actions that are consistent and coordinated with those of their teammates. A person's mental model of the domain is described as the structure in which the declarative knowledge framework is organized. The shared mental models approach proposes that the overlap of individuals' mental models leads to greater shared expectations and explanations within a team which leads to improved coordination, communication and other team behaviors which in turn leads to superior team performance (Banks and Millward, 2000; Zachary, Ryder, and Hicinbothom, 1998). Military organizations use teams to plan, initiate, and coordinate battles (Streufert and Nogami, 1992). These teams that are operating complex command and control networks with life and death literally hanging in the balance, are among the most highly skilled and trained teams in existence. Nevertheless, history has shown that breakdowns do occur and even these teams make mistakes.

All models of team functioning share an input-process-outcome (I-P-O) framework according to the general systems theory. Inputs to such models are conditions that exist prior to a performance episode and may include member, team, and organizational characteristics. Processes describe how team inputs are transformed into outputs. Outcomes are results and by products of team activity that are valued by one or more constituencies. Types of outcomes include performance, i.e., quality and quantity, team longevity and team members's affective reactions (Mathieu et al., 2000). Examples of models of team performance are the normative model where the basic assumption is that organizational context and group design (i.e., input variables) affect the member interaction process, and that, in turn, affects the quality of team performance (i.e., the output variables). The time and transition model (TTM) where the team is assumed to start out with a method of performing at the beginning of the project only to pursue a different strategy halfway through the task. The task group effectiveness model (TGFM) where group process and group effectiveness are central to the model with group task demands serving as a moderating factor. The team evolution and maturation model (TEAM) is a model of team performance that predicts the stages that teams go through before, during, and after performance of a task. The team performance model (TEM) is where the team has the two major components of task behaviors by individuals, and task functions at the team level. Finally, the task oriented model (TOM) emphasizes that team performance is a function of the subtasks that members must perform effectively for the accomplishment of team goals (Salas et al., 1992).

A taxonomy of team performance is presented as (Fleishman and Zaccaro, 1992): 
I. Orientation Functions.

A. Information Exchange Regarding Member Resources and Constraints.

B. Information exchange Regarding Team Task and Goals / Mission.

C. Information Exchange Regarding Environmental Characteristics and Constraints.

D. Priority Assignment Among Tasks.

II. Resource Distribution functions.

A. Matching Member Resources to Task Requirements.

B. Load Balancing.

III. Timing Functions (Activity Pacing).

A. General Activity Pacing.

B. Individually Oriented Activity Pacing.

IV. Response Coordination functions.

A. Response Sequencing.

B. Time and Position Coordination of Responses.

V. Motivational Functions.

A. Development of Team Performance Norms.

B. Generating Acceptance of Team Performance Norms.

C. Establishing Team Level Performance Rewards Linkages.

D. Reinforcement of task Orientation.

E. Balancing Team Orientation with Individual Competition.

F. Resolution of Performance-Relevant Conflicts.

VI. Systems Monitoring Functions.
A. General Activity Monitoring.
B. Individual Activity Monitoring.
C. Adjustment of Team and Member Activities in Response to Errors and Omissions.

VII. Procedure Maintenance.
A. Monitoring of General Procedural Based Activities.
B. Monitoring of Individual Procedural Based Activities.
C. Adjustments of Nonstandard Activities. 
Another view of a team performance taxonomy is presented as (Nieva, Fleishman, and Rieck, 1985):

I. Team Orientation Functions
A. Elicitation and distribution of information about team goals.
B. Elicitation and distribution of information about team tasks.
C. Elicitation and distribution of information about member resources and constraints.

II. Team Organizational Functions.
A. Matching member resources to task requirements.
B. Response coordination and sequencing of activities.
C. Activity pacing.
D. Priority assignment among tasks.
E. Load balancing of tasks by members.

III. Team Adaptation Functions.
A. Mutual critical evaluation and correction of error.
B. Mutual compensatory performance.
C. Mutual compensatory timing.

IV. Team Motivational functions.
A. Development of team performance norms.
B. Generating acceptance of team performance norms.
C. Establishing team level performance - rewards linkages.
D. Reinforcement of task orientation.
E. Balancing team orientation with individual competition.
F. Resolution of performance - relevant conflicts.

\subsubsection{Workload.}

It was stated 20 years ago that there is no consistent definition of mental workload, no agreement on how to measure it, and no single universal metric describing it (Lysaght et al., 1989; Moray, 1982; Williges and Wierwille, 1979). This statement is apparently still true today. The problem is that it is not known for certain what it is that a human senses when making judgments of difficulty as there is no mental force that has been operationally defined with consistent internal dimensions. Human task based activities during system performance have 
been described to exist in the four categories of perceptual, cognitive, communications, and motor (Wierwille and Casali, 1983). Mental workload clearly relates to such factors as operator stress and effort, however, these factors are as lacking in operational definitions as mental workload. The fact that mental workload is multidimensional in nature presupposes that any one measure can describe its effect. Therefore, attempts to measure mental workload should include multiple measures such as subjective opinion, spare mental capacity, and primary task measures along with physiological indices (Williges and Wierwille, 1979). In comparison to other scientific inquiry, there has not been a lot of research into what makes a human experience excessive mental workload (Moray, 1982).

There are many different definitions of workload that arise from individual situations and contexts. The dictionary (Webster, 1979) defines it as: " the amount of work or of working time expected from or assigned to an employee." However, in terms of human cognitive performance it is much more than this and much less capable of being precisely defined. One definition is the capacity to perform (Lysaght et al., 1989). It can also be described as the demand placed upon humans during task performance (Rouse, Edwards, and Hammer, 1993). It is the total attention demand placed on the operators as they perform the mission tasks (Hamilton et al., 1991). A more formal definition is related to subjective opinion of the operator on how much work is being performed. Thus, subjective workload is that load that is perceived by the operator and may fluctuate as a function of experience, sensory acuity, cognitive flexibility, affective condition, and state of fatigue of the operator (Warren, Stern, Eddy, Horst, Kramer, Parasuraman, Sanquist, and Wilson, 1985).

The Army defines workload as the amount of work, stated in predetermined work units, that organizations or individuals perform or are responsible for performing (Army, 1983). Attention, with its components of cognitive, psychomotor, and sensory, plays a major role in invoking workload. Workload can be characterized as the demand on each of these components imposed by all the tasks an operator is currently performing. When these demands exceed the capacity of the operator then the condition of operator overload can be experienced. Operator overload is defined as the level of workload at which operator performance begins to degrade (Hamilton et al., 1991). However, it is noted that there is no fully accepted formal model that describes the factors comprising the concept of workload nor is there a model that describes the 
contribution of individual factors to the overall workload concept. Three broad categories of workload definitions have been presented as (Lysaght et al., 1989):

- The amount of work and number of tasks to be performed.

- The aspect of time that the operator is concerned with.

- The subjective psychological experiences of the operator.

An assessment of potential operator workload is one of the major considerations in any human based system (Williges and Williges, 1981). However, the concept of mental workload has yet to achieve a consistent definition in the research community. Part of the problem is that workload is both task specific and individual specific (Rouse et al., 1993). A start is the definition is that workload is the capacity to perform (Fallesen and Quinkert, 1990). Another definition is the cost incurred by the human operator in accomplishing the imposed task requirements and involves both physical and mental activities (AGARD, 1998). This workload cost is indicative of the combined effects of the demands imposed by the tasks themselves, the information and equipment used, the task environment, operator skills and experience, operator strategies, the effort expended in performance of the work, and the emotional response to the situation. There are many accepted methods for measuring human physical and mental workload, (Hamilton et al., 1991; Hendy, Hamilton, and Landry, 1993; Kumashiro, 1995; Reid et al., 1986; Tijerina, Kiger, Rockwell, and Tornow, 1995; Vidulich, Ward, and Schueren, 1991; Wilson and Eggemier, 2001; Young and Stanton, 2001) to quote only a small sampling from the literature.

However, methods for predicting mental workload is less precise, owing, in part to the absence of a consistent definition of what constitutes mental workload. The general aim of workload prediction techniques is to predict accurately the relationship between operator task demands and the capacity of the operator (AGARD, 1998). The issue is whether changes in performance can be predicted given the characteristics of an individual task or the relationships between multiple tasks. This form of workload prediction is generally performed using a task analysis technique usually implemented in a discrete event computer simulation environment such as Micro Saint ${ }^{\mathrm{TM}}(\mathrm{MA} \& D, 1996)$. Another form of workload analysis is mission timeline analysis, which computes the ratio between time available and time required to perform the task. A ratio greater than 1.0 implies that the work tasks cannot be performed. Values of between 0.85 and 1.0 are considered to be indicative of workload problems (AGARD, 1998). 
Workload assessment is the topic of extensive discussion in the literature, (reference such examples as (Gopher and Donchin, 1986a; Hancock and Caird, 1993; Hancock and Meshkati, 1988; Hart and Wickens, 1993; Hendy et al., 1993; Rouse, Edwards, and Hammer, 1992; Rouse et al., 1993; Whitaker, Oatman, and Shank, 1987; Wickens, 1995; Wierwille et al., 1986; Xie, 1997)) but even today there is no generally accepted paradigm for representing it in human performance models. In the paragraphs that follow, I will examine the concept as to its meaning and use for the general application of predictive modeling of human performance systems that is typically carried out on the computer using discrete event simulation techniques. All of the example applications that will be presented are implemented in the commercial programming language Micro Saint ${ }^{\mathrm{TM}}$, developed by Micro Analysis and Design Corporation in Boulder, Colorado. It appears that Micro Saint ${ }^{\mathrm{TM}}$ has become the de facto programming environment of choice for human factors research. In my opinion this is primarily due to the human factors background of its developers (Laughery and Corker, 1997) as there are many other competing products in the discrete event programming world. Some of the better known "general purpose" languages include Arena, AweSim, GPSS, MODSIM III, Simple++, and ProModel, in addition to Micro Saint ${ }^{\mathrm{TM}}$ (Law, 1997). The primary application area of the majority of these and other languages like them is in the area of manufacturing and process control. While Micro Saint ${ }^{\mathrm{TM}}$ is also touted as a general purpose discrete event language, and has been used for other applications such as process manufacturing simulation (Tan, 1991), its primary focus is in the area of human performance modeling.

One set of criteria for selection of workload assessment techniques (O'Donnell and Eggemeier, 1994) includes the measures of sensitivity, diagnosticity, intrusiveness, implementation requirements, and operator acceptance. These factors are summarized in Table 5.

Table 5 - Criteria For Selection Of Workload Assessment Techniques

(from: (O'Donnell and Eggemeier, 1994))

\begin{tabular}{|l|l|}
\hline \multicolumn{1}{|c|}{ Criterion } & \multicolumn{1}{c|}{ Explanation } \\
\hline Sensitivity & $\begin{array}{c}\text { Capability of a technique to discriminate significant variations in the workload imposed } \\
\text { by a task or group of tasks }\end{array}$ \\
Diagnosticity & $\begin{array}{c}\text { Capability of a technique to discriminate the amount of workload imposed on different } \\
\text { operator capacities or resources (e.g., perceptual versus central processing versus }\end{array}$ \\
\hline
\end{tabular}




\begin{tabular}{|l|c|}
\hline Intrusiveness & \multicolumn{1}{|c|}{ motor resources) } \\
The Tendency for a technique to cause degradations in ongoing primary task performance \\
Requirements
\end{tabular}$\quad \begin{gathered}\text { Factors related to the ease of implementing a particular technique. Examples include } \\
\text { instrumentation requirements and any operator training that might be required. } \\
\text { Operator Acceptance } \\
\begin{array}{c}\text { Degree of willingness on the part of operators to follow instructions and actually utilize a } \\
\text { particular technique }\end{array}\end{gathered}$

In 1992 a dissertation was presented (Moscovic, 1992) that attempted the development and validation of a methodology to incorporate a predetermined time system (PTS) and the cognitive workload metric SWAT (Tsang and Wilson, 1997) into a model of human performance for making decisions while using interactive display consoles. This model was implemented in Micro Saint ${ }^{\mathrm{TM}}$ and was a display console interaction task network simulation using a work measurement technique called MODular Arrangement of Predetermined time Standards (MODAPS). MODAPS is based upon the PTS methodology and is a work measurement technique that embodies 44 elements of human body movements in addition to numerous activities and consists of a set of databases containing standard time performance values for human body movements. There are other PTS systems such as the Methods Time Measurement (MTM) system based on film time studies of various industrial jobs and Computerized Maynard Operation Sequence Technique (CMOST) where work is measured by the movement of objects. However, the conclusion was that MODAPS was the most effective of these approaches for assigning task completion times for use in the Micro Saint ${ }^{\mathrm{TM}}$ model. As Moscovic did not actually provide a name for her model, here it will be referred to as the Moscovic Display Model (MDM) for ease of reference.

Moscovic's aim was to develop the MDM methodology incorporating the MODAPS PTS along with the SWAT workload metric into a model capable of predicting human performance in this work setting. Findings from the MDM effort indicated that it was a valid way to predict time performance and provided a strong indicator of workload, however, statistically, it did not provide an exact prediction of workload scores compared to SWAT observations of actual operators. Complicating the issue was the observation that regression analyses of SWAT scores from two test groups indicated that they were not homogeneous with respect to the workload ratings. It is hard to validate a computer model when its baseline is, itself, not conclusive. 
An evaluation of some of the issues surrounding the difficulty in workload estimation starts with an examination of what workload is and how it can be measured in real world situations. One definition of workload is that it is an expression of the degree of qualitative and quantitative load induced by work and is closely related to stress and industrial fatigue (Kumashiro, 1995). Mental workload is that load placed on the cognitive capabilities such that it taxes the information processing capabilities of human operators. Another definition of mental workload states that it is an intervening variable that may be viewed as the difference between the capacities of the information processing system that are required for task performance to satisfy performance expectations and the capacity available at any given time (Gopher and Donchin, 1986b). The definition of an intervening variable, as opposed to a hypothetical construct, as provided by MacCorquadale and Meehl in 1948, discussed it as a theoretical concept as:

... simply a quantity obtained by a specified manipulation of the values of empirical variables; it will involve no hypothesis as to the existence of unobserved entities or the occurrence of unobserved processes; it will contain, in its complete statement for all purposes of theory and prediction no words which are not defined either explicitly or by reduction sentences in terms of the empirical variables.

A workload measurement procedure is one in which an attempt is made to characterize the conditions under which task demands can or cannot be met by the operator. A workload measure is one by which the latter differences are expressed in relation to the overall ability of the human processing system to process information and generate responses. Three categories of workload measures are presented as (Gopher and Braune, 1984):

- Where demands are expressed in terms of the objective parameters of tasks.

- Measures of response (behavioral or physiological).

- Subjective appraisal given by the performer to the load he or she experiences during task performance.

The key appears to be its effect on the information processing abilities of the human mind. Moscovic's three categories of mental workload measures, which have some similarities to the three above are:

- Psychophysiological measures are based on the premise that states of cognitive workload can be inferred from physiological conditions such as sinus arrhythmia 
(SA) or heart rate where the heart rate variability decreases as the cognitive workload increases. Its primary advantage is objectivity.

- Performance measures assess impairments to performance levels of such things as primary and secondary task performance and provide immediate and direct responses that are objective.

- Subjective measures assess the conscious experience of the operator through self report estimates from the operator and can provide the most valid and sensitive way to tap cognitive workload. Techniques include: 1) unidimensional numerical ratings; 2) multidimensional evaluations; 3) rank ordering of tasks; 4) task specific protocols and checklists; 5) stereo tape recorder monitoring. Examples include:

- Cooper-Harper Scales.

- NASA-TLX.

- Subjective Workload Assessment Technique (SWAT).

It is important to select a categorization of workload measures which groups the various workload techniques in a logical way so that conflicts and discrepancies on workload concepts is minimized (Williges and Wierwille, 1979).

Fourteen behavioral workload measures have been identified (Williges and Wierwille, 1979) and are summarized as:

1. Rating scales:

- Advantages - is a sensitive measure of workload and results in little intrusion on the primary task.

- Disadvantages -

- Some approaches fail to follow rigorous psychometric procedures in the development of the workload scale.

- Confusion over the distinction over physical and mental workload.

- Respondent ratings can vary according to mental state, experience, learning and natural abilities.

- Respondent may simply not be aware of the degree of mental loading of a given task.

2. Interviews / questionnaires:

- Advantages - Used as a supplemental measure in workload assessment. 
- Disadvantages - Results cannot be given a high priority because the data can only be used in a supportive way in workload assessment.

3. Task Component / Time Summation:

- Advantages - Can be used in an activity analysis format.

- Disadvantages:

- The basic assumption of a constant workload capacity could cause a bias in the results.

- The more remote the actual application is from the laboratory where the results are measured the more inaccurate the workload assessment is likely to be.

4. Information / Theoretic:

- Advantages - Used for:

○ Applications for visual monitoring.

- Applications for continuous tracking.

- Applications for complex information processing activities.

- Disadvantages (at least as of 1979) -

○ The theoretical formulations underlying these procedures need further development.

- Only a limited amount of validation data are available to support these procedures.

5. Nonadaptive, Arithmetic / Logic:

- Advantages - Useful for monitoring, shadowing, mental math, memory, choice reaction time, auditory detection, simple reaction time, problem solving, random sequence generation, and classification tasks.

- Disadvantages -

○ The most difficult aspect of the secondary task methodology for assessing workload is intrusion.

- Another problem in workload estimation by the secondary task methodology is the underlying assumption of task regularity or stationary, which assumes that the primary task is uniform during the analysis period.

6. Nonadaptive, Tracking:

- Advantages -

- Allows the validation and development of pilot models of workload.

○ Allows the analysis of concurrent workload involving monitoring functions.

- Disadvantages - 
○ Data does not exist (as of 1979...) for real world tasks, thus this method valid for laboratory use only.

- Extra tracking tasks in performance studies of actual task performance can lead to safety issues for operator in the study.

7. Time Estimation:

- Advantages - Can be used to imply the attention demands required for task performance.

- Disadvantages -

- The display of time information can have a pronounced effect on primary task performance.

○ Only relative, not absolute, workload assessment evaluations can be made.

- Post hoc interpretations can vary considerably depending on the mode of production assumed.

8. Adaptive, Arithmetic / Logic:

- Advantages -

- Tests show that differences in the adaptive tasks are sufficiently large to demonstrate changes in primary task workloads.

- Cross - adaptive procedures eliminate the intrusion of the secondary task on primary task performance.

- Disadvantages - Technique probably limited to laboratory and flight simulator situations

9. Adaptive / Tracking:

- Advantages - Can be used as a critical tracking task as a secondary task measure that is useful as a sensitive measure of varying levels of primary task workload.

- Disadvantages - Useful in laboratory environments but have limited capability in actual performance.

10. Occlusion: This is a time-sharing technique that is similar to the secondary task method.

- Advantages -

- Useful for studying attention demand.

- Quite sensitive to control task difficulty and operator skill.

- Primarily applicable to simulation research

- Disadvantages -

- Safety in actual task performance situations. 
○ Method not particularly sensitive and intruded more when compared to other techniques.

- Substantial lengths of time may be necessary for operators to learn to use the occlusion apparatus.

11. Handwriting Analysis:

- Advantages -

- Used as a secondary task.

- Reductions possible in handwriting legibility and sentence structure as a function of "distraction stress".

- Disadvantages -

○ Requirement for a dedicated writing hand.

- Environment must be nearly vibration and acceleration free.

○ Operator's hand must be available and ungloved.

12. Single Measures (Primary Task):

- Advantages -

○ Often used as a means of validating other workload measures.

- Performance on primary task as a means of examining the effect of the secondary task to assess workload.

○ Takes advantages of implications regarding workload and its relationship to primary task measures.

- Disadvantages -

○ While high workload situations (near operator overload) are discernable by primary task measures, low workload situations may not be.

○ Measurement of primary task variables tends to be complicated.

13. Multiple Measures (Primary Task):

- Advantages - Measurement of workload in multiple task environments.

- Disadvantages - The more variables that are measured means that some will not change reliably as a function of workload.

14. Mathematical Modeling:

- Advantages - Useful for areas of human operator decision processes, supervisory processes, team interactions, and operator workload.

- Disadvantages -

- Usually confined to specific, well constrained, and perhaps repetitive, human operator tasks. 
- As higher mental processes become more involved, modeling techniques tend to be less applicable.

This is but one example of attempts to categorization of workload measures. There are others. While there is mutual agreement over the importance of mental workload, there is substantial controversy over the best type of workload measurement. It is argued that all three forms of measures are needed in any workload measurement attempt, however, practical limitations make this unfeasible. Further, the controversy over the best type of workload measure for actual evaluation of real human performance complicates the issue of how to attempt to predict it in a computer simulation.

Attempting to put some form to this effort, Moscovic turned to task network modeling and Laughery's Micro Saint ${ }^{\mathrm{TM}}$ simulation tool (Laughery, 1989a). Regardless of the ongoing theoretical debate on how to measure it and what it actually is, researchers have and are continuing to build on the knowledge of what both physical and mental workload constitute. Laughery proposed that the theoretical aspects of the operator approach to workload analysis consist of a combination of task network modeling and the multiple resource theory (Wickens, Sandry, and Vidulich, 1983).

Task network modeling is a technique where human performance is decomposed into a series of sub functions, which are then subsequently decomposed into tasks, which are represented in a task network. It is noted that task network modeling, in and of itself, is not inherently a model of human workload but provides output that is the time required to perform a set of tasks and the sequence in which the tasks are performed. However, a promising theory of operator workload which is consistent with task network modeling is Wicken's multiple resource theory (Laughery, 1989a). The multiple resource theory (Wickens et al., 1983) suggests that humans have more than just one information processing source that can be accessed singly. Rather, they have several resources, which can be accessed simultaneously. Laughery summarizes his comments on human workload by listing his set of issues associated with the evaluation of workload:

- What are the channels of workload?

- How should attention demands within a workload channel be combined across tasks?

- How should attention demands be combined across workload channels? 
- What values for workload represent "excessive" workload?

- What about task dumping?

Thus, parlaying on Laughery's comment that excessive human workload is not usually caused by one particular task required of the operator, but rather is a result of the human having to perform several tasks simultaneously that leads to overload, Moscovic used the multiple resource theory as the theoretical approach to evaluating workload and the visual, auditory, cognitive and psychomotor (VACP) (McCracken and Aldrich, 1984) technique for characterization of workload demand as a representation of human information processing. This technique states that each operator's activity in a task network is characterized by the workload demand required in each of four channels. These channels are the auditory channel, the visual channel, the cognitive processing channel, and the psychomotor output channel.

Unfortunately, Moscovic was not able to claim complete success with her MDM methodology. While the PTS / MODAPS approach did provide a successful means to predict task time performance, the effort to generate a regression equation based predictive model of human workload based on an analysis of SWAT data was not. She states that the findings indicate that either the methodology of determining workload was not adequate or that the groups were heterogeneous in terms of workload ratings. She noted that the small number of participants in each empirical group could have had an impact on the results.

This finding, however, did not dampen the enthusiasm for the approach, which is an enthusiasm that I endorse and also have as a personal research goal. The utility of using regression equation techniques in predictive models that use beta weights for variables as a means of workload projection and prediction is just too powerful to ignore. A technique known as the Subjective Workload Dominance (SWORD) (Vidulich et al., 1991) method has been used in studies to project workload. The SWORD technique has human operators assign retrospective ratings from an abstract workload scale to a task without comparison to other tasks. The three steps in the SWORD technique are collecting the raw judgment data, constructing the judgment matrices, and calculating the SWORD ratings. As the SWORD technique is an expert opinion extraction technique it is hypothesized that it could be useful as a projective workload tool. Tests have shown that it can be used as a predictive tool provided SME opinion is available to establish the required workload parameters. 
In 1995 another dissertation was presented (Green, 1995) that introduced a variant on traditional task analysis called the Performance Assessment Methodology (PAM). This methodology was developed that utilized observable events and actions along with inferred decisions to describe, assess, and predict performance of operators in a complex human - system - task performance situation. PAM consisted of a new task analysis methodology that was developed to generate objective information from human performance involving complex tasks. SME opinion was used to break down each complex task into observable elements along with unobservable elements that were inferred to have occurred in support of the observable events. Guidelines were developed to specify the level of detail for the task analysis breakdown. From this information a simulation model was constructed using Micro Saint ${ }^{\mathrm{TM}}$ that represented the task elements. Possibly concurrent with the development of the computer simulation was the observation of operators trained in the task(s) being investigated, which resulted in objective data on their actions that could be observed. Data from these observations then was used as numeric input to the computer simulation. The simulation was then run and the results compared to the real events that were observed. Following these initial runs the model was altered to produce results similar to what was observed during the data collection phase. This process is stated to have produced a model capable of accurately reflecting human performance on the task.

The key to the PAM methodology, and the thing that sets it apart from other similar techniques, appears to be the breakdown of the complex tasks into first, observable elements, and second, unobservable elements that were predicted to have taken place in support of the observed task elements. Critical to this analysis is the participation of proficient SMEs capable of making these assessments. Once the PAM model was validated against the observable performance parameters then it could be used for purposes that are ascribed to computer simulations in general which is to investigate performance conditions under different task environments that may or may not be observable in the real world. In other words, if a computer model is accepted for its ability to produce a realistic simulation under one given set of circumstances then it has a probable ability to produce realistic results for other performance circumstances. Unfortunately, the farther the investigative domain drifts from the validated situation, the less likely the simulation's results can be accepted. However, risk assessment and acceptance is another topic completely and deviates from the topic here. 
Although workload is not directly addressed in this report, it is implied through the task analysis and resulting human performance measures. However, the Micro Saint ${ }^{\mathrm{TM}}$ model used in this study was only capable of predicting task performance time thereby providing a "taskload" prediction or component of workload. The taskload times in this case were the overall functional recognition time values observed in the model. These results mirror the Moscovic tests where the modeling accurately reflected the temporal component of workload as it simulated the operator performance during functional tests. The functional recognition task that Green chose to evaluate the operator performance where the operators were required to determine the purpose of the information on the display required operators to process both auditory and visual information. His finding validated the task time predictive nature of the simulation where there was a high correlation between the Micro Saint ${ }^{\mathrm{TM}}$ predictions and observed operator performance of the functional recognition task.

Two years later an updated PAM methodology was applied to the relationships describing visual events, decisions, and actions while operating a visual display system (Orrell, 1997). In this case PAM provides a quantitative basis for evaluating display image quality based on the visual events that occur in a task and is defined as a framework that shows the relationships among operator tasks, display system parameters, and performance measures for these parameters. With the PAM methodology, operator tasks are decomposed into events, decisions, and actions. When a triggering event occurs, the operator makes a decision and then responds with an action. Thus, observable perceptions or events lead to decisions and actions. A task might have many events, decisions, and actions associated with it.

In this application PAM is based on a network analysis model of time sequential events and is an aggregate measure of performance (e.g., time, errors, and workload) for individual task parameters. It allows consideration of any human performance measure including accepted measures such as speed (time) and accuracy (errors). Stress or workload provides an additional measure of performance and is defined as the difference between the perceived demands of the task and a person's perceived capacity to cope when coping is important.

Orrell refines the previous definition of PAM as a framework that shows the relationships among operator tasks, system parameters, and performance measures for those parameters. PAM decomposes the operator tasks into events, decisions and actions (EDA) and provides a 
framework for describing the relationships between them. When events occur, the operator makes a decision and then responds with an action. A task might have many events, decisions, and actions associated with it. In the taxonomy that is developed for PAM, parameters may be associated with many events, and individual decisions and actions can be isolated for further evaluation. Thus, a parameter may be related to any level of an EDA so that multiple decisions may use the same parameter. The evaluation may start with actions and then determine how events and decisions influence these actions. For operator tasks PAM shows the interrelationships between events, how system parameters influence individual events, and how these parameters are related to performance measures. This sequence is diagrammed in Figure 11 .

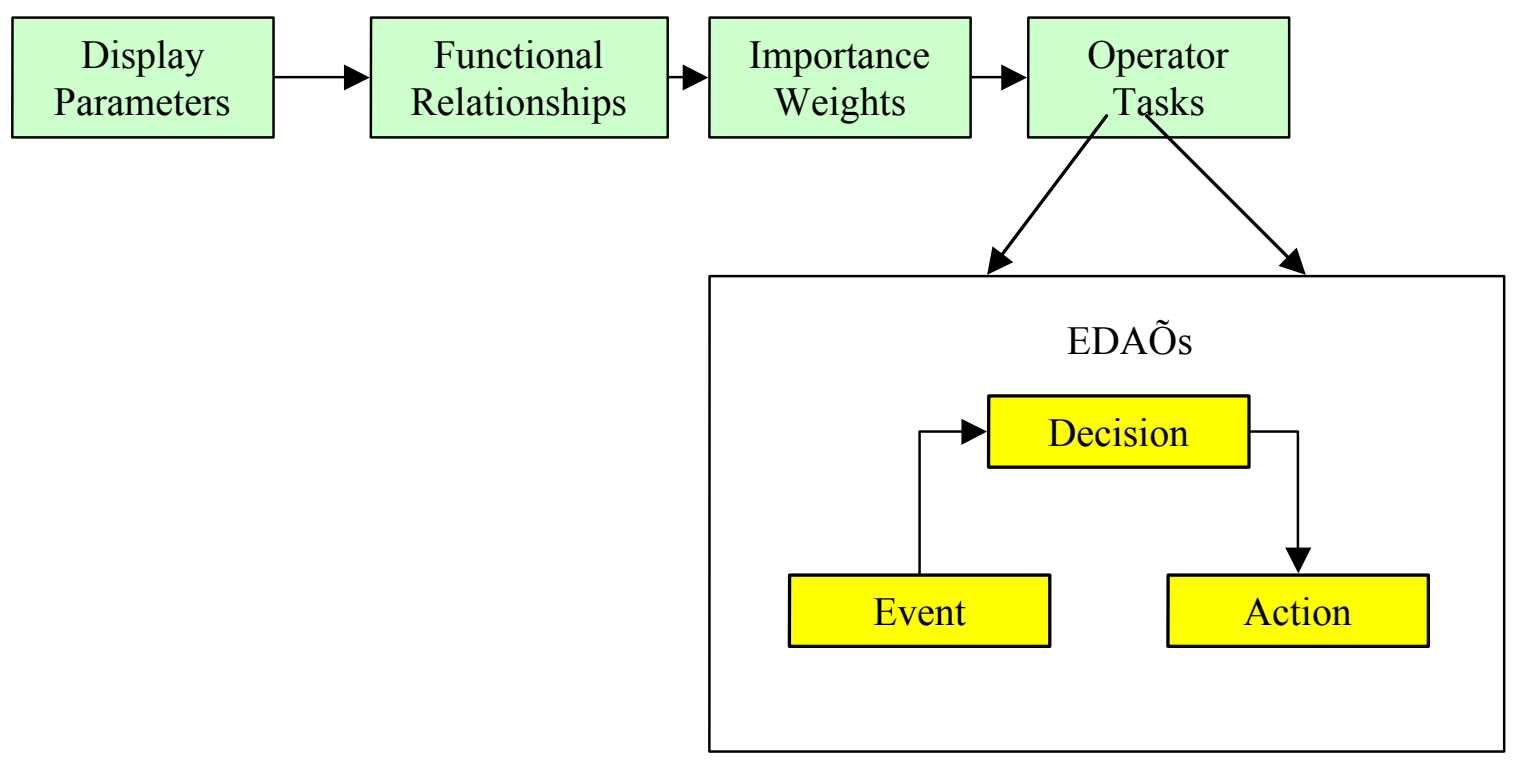

Figure 11 - PAM Task Level Architecture

(Adapted From: (Orrell, 1997))

The influence of system parameters is determined for each task relevant event. Functional relationships between performance measures and parameters are then determined. A PAM efficiency measure is created by combining and weighting performance scores across like parameters. Thus, efficiency is an aggregate measure of performance according to time, errors and workload for individual system parameters. Any human performance measure can be used with PAM including such measures as speed (time) and accuracy (errors). Workload is 
measured according to stress and is defined as the difference between the perceived demands of the task and a person's perceived capacity to cope when coping is important.

When setting up the database there are two elements that require definition SMEs. These are task relevant events and system parameters. A ranking and rating methodology is used to determine the important events and parameters. With this procedure each factor is ranked in order of importance within its category on a scale of 0 to 1 with ties permitted. The factors with the highest ratings are then selected for inclusion in the appropriate task where the SME determine the cutoff scores.

A visual theoretical model called the Model of Visual Events (MOVE) was used to describe the relationships between visual events, decisions, and resulting actions and implemented the PAM methodology for this visual display application. MOVE was developed to describe categories of perceptual decisions that are associated with visual events. These categories included the ability to detect, identify, discriminate, and evaluate visual objects or targets. The purpose of the investigation was to develop a quantitative basis for image quality evaluation. The PAM top-level architecture previously shown in Figure 11 is applied to the visual display application to create the MOVE model. In this application, MOVE expands on the EDA model by incorporating the components of perception, decision-making, and response / action sequence. In MOVE the flow of the model is dependent on the nature of the task. For simple tasks the image would be immediately detected and evaluated. However, for more complex tasks the actions first detects, identifies or recognizes, discriminates and then interprets or evaluates.

Summarizing the use of PAM, the preparation stages for this methodology include a general task analysis and an event analysis to be conducted with SME assistance. Using the PAM based model includes the steps of calculating the event efficiency across all the individual parameters, calculating the task efficiency for each task completion pathway, and calculating the network efficiency.

While Orrell claims workload, and synonymously, stress as a measure of performance there is no direct mention of how he measures or attempts to predict it. While he defines it as the difference between the perceived demands of the task and a 
person's perceived capacity to cope with it, it is only assumed that it must be a part of the task and network efficiency calculations.

A multi-year effort conducted by the Human Research and Engineering Directorate of the U.S. Army Research Laboratory was the Computer Modeling of Human Operator System Tasks (CoHOST) project (Middlebrooks et al., 1999a; Middlebrooks et al., 1999b). This project also provided the computer simulation model for a followon master's thesis (Middlebrooks, 2001). This project was undertaken with the objective to investigate potential effects on human mental and physical performance capabilities during combat operations from the introduction of a new command and control vehicle equipped with modernized digital communications systems. The objective of the project was to produce a task performance and workload model for a maneuver battalion task force using this mobile computer communications system using the discrete event programming environment in Micro Saint ${ }^{\mathrm{TM}}$. The intent of the model was to investigate the efficiency of information flow and task loading during the conduct of an extended mission and to compare soldier task and workload predictions in order to answer the following questions:

- Is one configuration of personnel and communications equipment better or worse than another?

- Can the human operator continue to function effectively during extended periods of on-the-move operations in the vehicle?

A taxonomy of human performance (Fleishman and Quaintance, 1984) was chosen to provide a qualitative basis for workload and task performance evaluation in the model. This taxonomy consists of 52 human physical and mental knowledge, skill, and ability (KSA) taxons that are designed to be able to describe human performance in any generalized work setting. Fifty of the 52 KSAs were subsequently chosen for use with the CoHOST model as two of the auditory KSAs were deemed to be repetitive of others for the work setting being simulated. The remaining $50 \mathrm{KSAs}$ were grouped into eight cognitive and physical performance clusters so that selected performance demand weightings could be applied to selected clusters to account for the specifics of this work situation. These $50 \mathrm{KSAs}$ in their respective cluster assignments are shown in Figure 12.

A numerical database was established that contained ratings on a scale of from 1 to 7 of how much each of the taxons applied to work performance for each job category in the work group the simulation was representing. SME opinion was used to develop the ratings for this 
database according to a computer based questionnaire using a 7 point Likert like behaviorally anchored rating scale.

Cognitive Skill and Experience Clusters

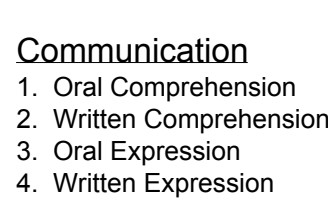
Vision
24. Near Vision
25. Far Vision
26. Night Vision
27. Visual Color
Discrimination
28. Peripheral Vision
29. Depth Perception
30. Glare Sensitivity

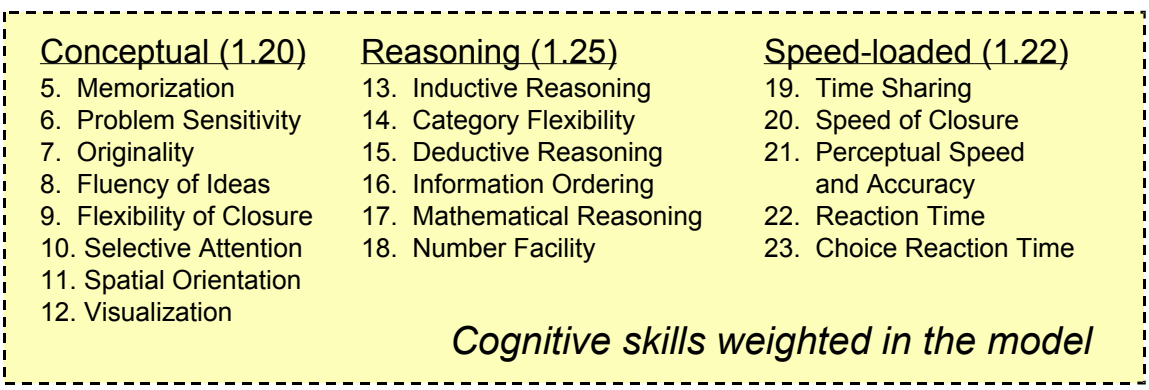

Perceptual-Motor Ability Clusters

$\begin{array}{lll}\text { Audition } & \text { Psychomotor } & \text { Gross Motor } \\ \text { 31. General Hearing } & \text { 34. Control Precision } & \text { 41. Extent Flexibility } \\ \text { 32. Auditory Attention } & \text { 35. Rate Control } & \text { 42. Dynamic Flexibility } \\ \text { 33. Sound Localization } & \text { 36. Wrist-Finger Speed } & \text { 43. Speed of Limb Movement } \\ & \text { 44. Gross Body Equilibrium } \\ & \text { 37. Finger Dexterity } & \text { 45. Gross Body Coordination } \\ & \text { 38. Manual Dexterity } & \text { 46. Static Strength } \\ \text { 39. Arm-hand Steadiness } & \text { 47. Explosive Strength } \\ \text { 40. Multi-Limb Coordination } & \text { 48. Dynamic Strength } \\ & \text { 49. Trunk Strength }\end{array}$

Fleishman, E. A. and Quaintance, M. K. (1984) Taxonomies of Human Performance: The Description of Human Tasks., Orlando: Academic Press.

Figure 12 - Knowledge, Skills, and Abilities Taxonomy

(Middlebrooks et al., 1999b)

The CoHOST computer simulation used these ratings for instantaneous (performed every 100 seconds in simulation time) calculations of task loading by summing up all the KSA values that were being applied in the execution of tasks that were being performed at the time of the calculation by the simulated operator. The further summing of the 100 second interval taskload calculations over the course of selected time intervals and the total scenario of the simulation run produced what was called a "workload" value for the operator for the performance of the tasks that were conducted during the course of the time interval. A sample of the types of tasks being performed by the operators in the workgroup is shown in Figure 13 and the decomposition of these performance tasks into taxons is illustrated in Figure 14. This decomposition follows procedures previously described for how task based performance indices are established for inclusion in a task network simulation. 
Thus, what was called workload in the original CoHOST simulation was a distinct departure from workload assessment methodologies in the literature that use such techniques as SWAT and NASA-TLX. In fact, it caused enough of a controversy that the term was changed to taskload in a master's thesis on this subject (Middlebrooks, 2001).

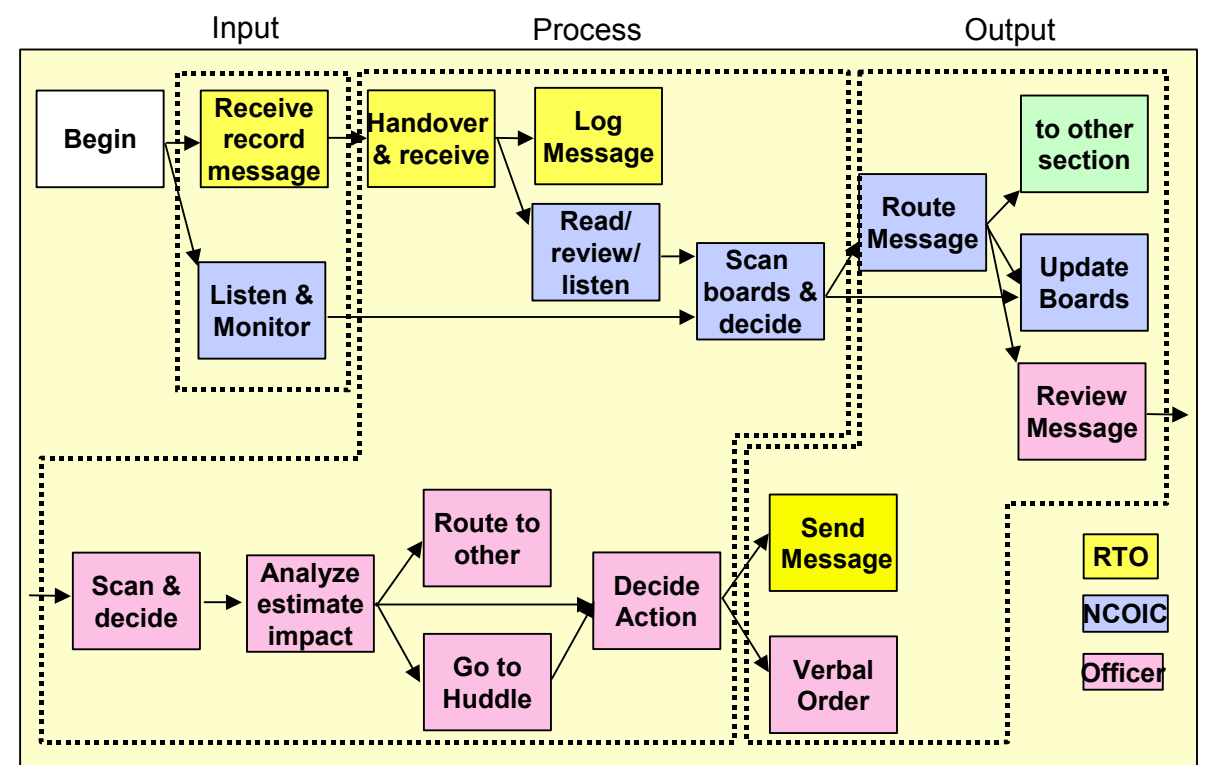

Figure 13 - CoHOST Task Flow Sequence

(Middlebrooks et al., 1999b)

The issue, then, is how can a computer simulation be used to predict human performance "workload"? The CoHOST definition as the "performance of tasks over time", in hindsight, is not acceptable. Moscovic made what appears to be a creditable attempt to incorporate workload predictions into her simulation using SWAT techniques but, by her own admission, came up short. Even though her experiments were inconclusive, Moscovic stated "there is merit to using the regression equation technique in a predictive model by identifying beta weights for variables using projective workload techniques.

Figure 15 illustrates Moscovic's experimental findings that indicate that workload is a separate measure from performance. Her comment is that performance is a metric of the number of errors committed over time while the definition of SWAT scores is that they are relative ratings based on the rank ordering of workload dimensions. If performance does not correlate to workload, then what does? If workload is an independent metric of human performance, then, the question is "how can it be quantitatively represented?" Can algorithmic predictors of 
workload be developed based on regression or other techniques? This is a topic area of interest for future research.

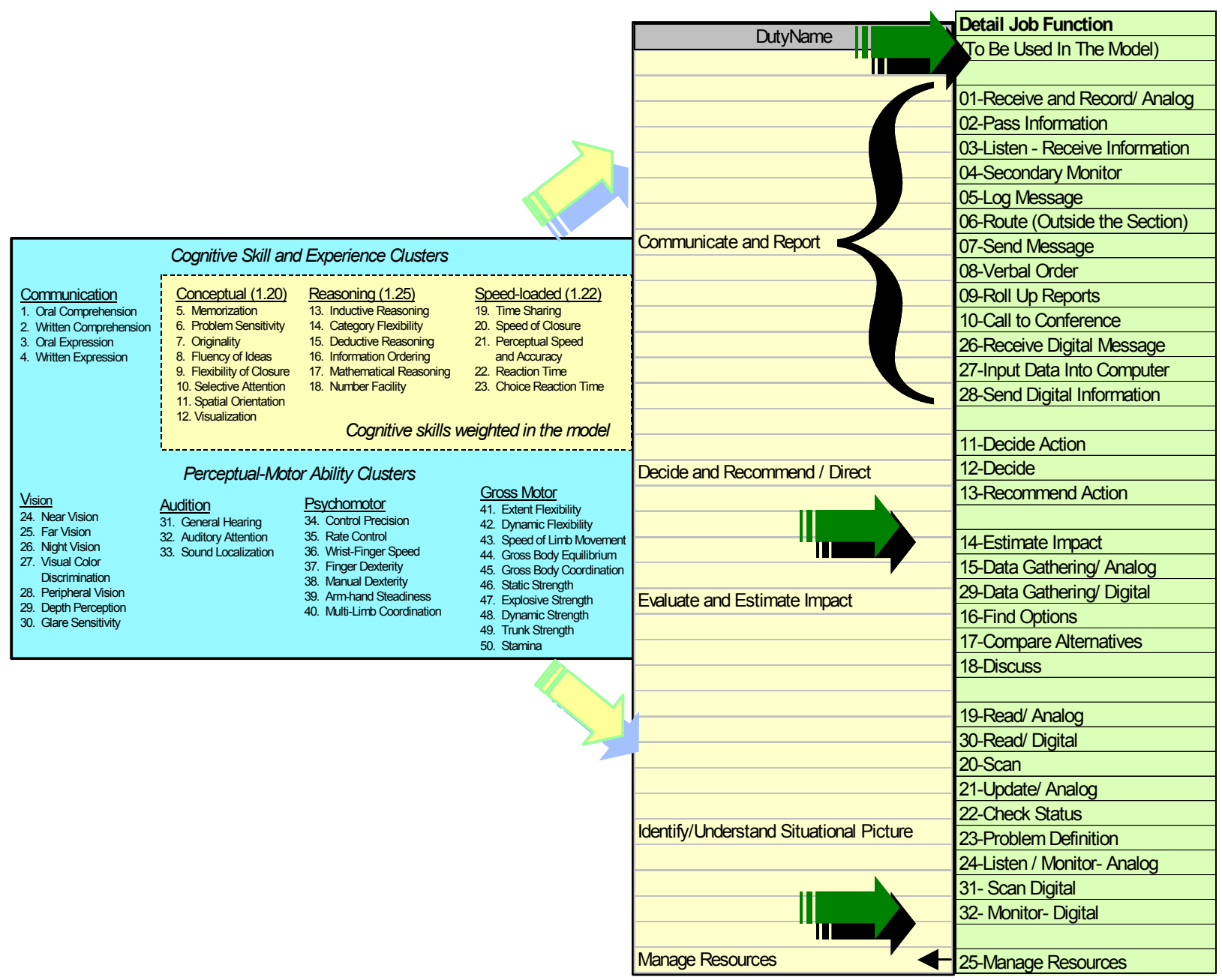

Figure 14 - CoHOST Task Decomposition and Translation From Taxonomy (Middlebrooks et al., 1999b) 


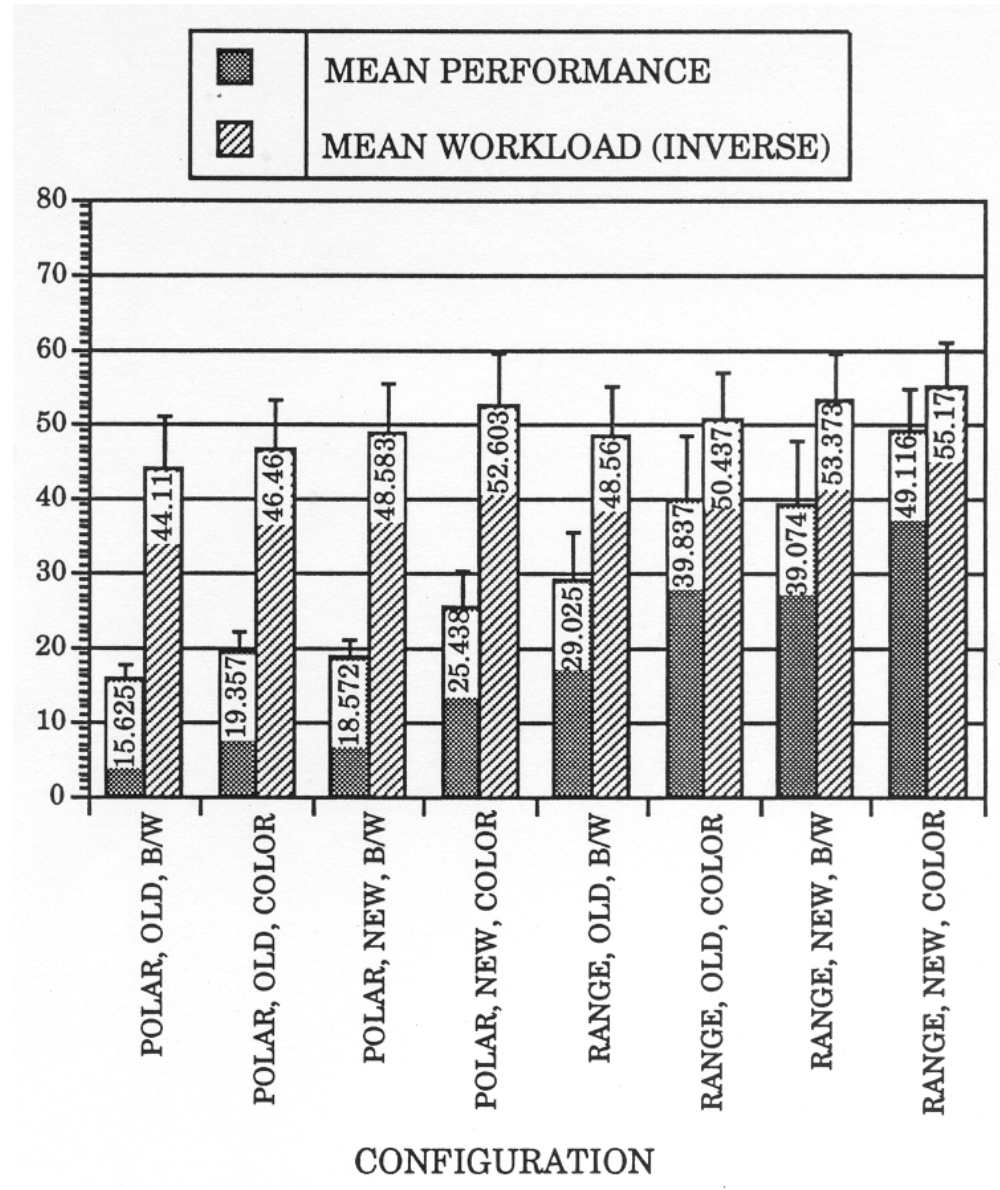

Figure 15 - Performance / Workload Comparison

(Moscovic, 1992)

There are five interrelated issues that are associated with attempts to extend the scope and applicability of human performance models (HPM) to more complex problems and designs (Baron, Kruser, and Huey, 1990):

1. Complex / comprehensive models: Most existing HPMs have been developed only for relatively simple situations.

2. Model parameterization: As models become more complex, the number of parameters related to human performance in the model is likely to increase.

3. Model validation: As models become more complex, they also become more difficult and costly to validate.

4. Underutilization / inaccessibility of HPMs: Most complex HPMs have not been used widely or subjected to independent evaluation. 
5. Potential for misuse / misunderstanding: As models become more complex, they also become significantly more difficult to use.

Three additional issues related to modeling the future role of operators in and of complex systems are (Baron et al., 1990):

1. Accounting for mental aspects of tasks: In an attempt to deal with cognitive aspects of thee operator's tasks, there has been increasing interest in incorporating aspects of the operator's tasks, there has been increasing interest in incorporating mental models into HPMs.

2. Developing and using knowledge based models: Along with the increased interest and popularity of artificial intelligence (AI), there has been a rush toward the development, integration, and use of intelligent or knowledgebased models (or sub models).

3. Accounting for individual differences: The effects of individual differences have been largely ignored in HPMs (as of 1990), in favor of using average indices of human characteristics representing the ideal, fully trained operator. A conclusion reached in this dissertation is that this is still the case today.

An early modeling effort can provide quantitative and qualitative analyses that allow design trade-off studies to include a variety of human performance factors along with other system variables (Baron et al., 1990) (p.86). Amen.

The literature is rich with narrative comment on the subject of the evaluation and estimation of mental workload, however, it remains a topic that is open to interpretation and subjective opinion. Table 6 provides a summary of some of the more common subjective techniques along with the performance measure of each. 
Table 6 - Subjective Evaluation Methods for Workload Assessment

(Braun et al., 1999)

\begin{tabular}{|l|c|l|}
\hline \multicolumn{1}{|c|}{ Method } & Technique & \multicolumn{1}{c|}{ Outcome Measures } \\
\hline $\begin{array}{l}\text { NASA Bipolar } \\
\text { (Vidulich and Tsang, 1985) }\end{array}$ & Rating & $\begin{array}{l}\text { Overall workload, performance, } \\
\text { frustration, task difficulty, fatigue }\end{array}$ \\
\hline $\begin{array}{l}\text { Multidimensional bipolar rating scale } \\
\text { (Braun et al., 1999) }\end{array}$ & Rating & $\begin{array}{l}\text { Overall workload, frustration level, } \\
\text { stress, performance, fatigue }\end{array}$ \\
\hline $\begin{array}{l}\text { NASA-TLX (NASA-Task Load Index) } \\
\text { (Braun et al., 1999) }\end{array}$ & Rating & $\begin{array}{l}\text { Overall index of mental demand, } \\
\text { physical demand, temporal demand, } \\
\text { performance, effort, frustration }\end{array}$ \\
\hline $\begin{array}{l}\text { SWAT (Subjective Workload } \\
\text { Assessment Technique) } \\
\text { (Vidulich and Tsang, 1985) }\end{array}$ & Rating & Time load, mental load, stress load \\
\hline $\begin{array}{l}\text { MCH (Modified Cooper Harper) } \\
\text { (Wierwille and Casali, 1983) }\end{array}$ & Rating & Task difficulty \\
\hline $\begin{array}{l}\text { McDonnell Rating Scale } \\
\text { (Braun et al., 1999) }\end{array}$ & Rating & $\begin{array}{l}\text { Control difficulty, attentional } \\
\text { demand }\end{array}$ \\
\hline $\begin{array}{l}\text { Bedford Workload Scale } \\
\text { (Braun et al., 1999) }\end{array}$ & Rating \\
\hline $\begin{array}{l}\text { Borg Scale } \\
\text { (Braun et al., 1999) }\end{array}$ & Rating & Perceived exertion \\
\hline
\end{tabular}

\subsection{Methods and Procedures for Cognitive Performance Assessment.}

A key factor in trying to determine cognition requirements for system analyses is the ability to estimate the cognitive performance responses of real human operators working in real work domains. There are several approaches and methodologies that have been explored in previous research and proven to be effective for certain applications. A set of these approaches is reviewed here as a description of those approaches that are considered to be useful in formulating the COMPASS paradigm.

\subsubsection{Evaluation of Human Cognitive Performance.}

Understanding and evaluating the various aspects of human mental performance will often begin with field observations of individual and group activities as they occur within the system domain being studied. Some of the performance parameters and their definitions that can be used in observations to gather these types of human performance data include (Gawron, Travale, and Neal, 1989):

1) Decision making-the ability to choose between two or more alternatives. 
2) Detection-the ability to discover or become aware of a visual, auditory, tactile, olfactory, proprioceptive, or kinesthetic stimulus.

3) Fine manipulation-the ability to manipulate controls through a physical effort, which requires sensitive movement, and touch rather than physical strength.

4) Gross manipulation-the ability to manipulate controls that require significant physical strength.

5) Numeric manipulation-the ability to estimate and perform mathematical calculations.

6) Probability estimation-the ability to predict the chance of an event occurring.

7) Recognition-the ability to identify a detected stimulus.

8) Team coordination-the ability to organize and implement a team effort.

9) Time estimation-the ability to predict how long it will take a moving body to travel a fixed distance.

10) Tracking-the ability to follow a moving target with a control, e.g., joystick.

Once these data are collected, a traditional approach to providing input to a quantitative analysis of this performance is to use curve-fitting techniques to develop regression equations representative of the performance data. Types of regression equations that can result and their forms are (Gawron et al., 1989):

- Simple regression with exponents:

- Simple regression with inverse exponents:

- Simple regression with squared exponents:

- Simple regression with inverse squared exponents:

- Multiple regression:

- Polynomial regression:
- Simple regression:

$$
\begin{aligned}
& y=m x+b \\
& y=x^{m}+b \\
& y=x^{(-m)}+b \\
& y=x^{\left(m^{* * 2}\right)}+b \\
& y=x^{\left(-m^{* * 2}\right)}+b \\
& y=m_{1} x_{1}+m_{2} x_{2}+\ldots+b \\
& y=m x^{2}+m x+b
\end{aligned}
$$

In very large data sets containing variables numbering in the dozens, hundreds, or higher, these techniques have been cumbersome to attempt. This has helped to spawn alternative, though less precise, techniques such as datamining that are supported by cluster analysis and neural network evaluations. However, the development of high speed desktop computers and statistical software that support immediate interactive analysis by the researcher now allow regression approaches for these larger problems to be considered. It is the use of all these techniques that provide the best opportunity for success in exploratory evaluations of variable 
interactions in large sets of data such as can be produced by observation of human cognitive performance.

\subsubsection{Ethnography and Naturalistic Observation.}

Ethnographic research is concerned with revealing the routine and the "paramount reality" of the everyday world of individuals and groups. Ethnography is defined as the analytic descriptions or reconstructions of intact cultural scenes and groups. Ethnographies recreate the shared beliefs, practices, artifacts, folk knowledge, and behaviors of some group of people. What distinguishes ethnographic research is its purpose-which is cultural description. Ethnographic research seeks to build a systematic understanding of all human cultures from the perspective of those who have learned. It stresses things like the actor's understanding and theorizing about their actions as opposed to traditional social science research where the researcher attempts to explain human action in terms of psychological theories such as attribution theory. One aim of ethnographic research is to record processes of change rather than stability (Uzzell, 2000). It involves the creation and ongoing renegotiations of relationships between researchers and informants (Lawlor and Mattingly, 2001). Ethnographies are analytic descriptions or reconstructions of intact cultural scenes and groups which delineate the shared beliefs, practices, artifacts, folk knowledge, and behaviors of some group of people (LeCompte and Goetz, 1982). One of the most salient characteristics of qualitative research, especially ethnography, is that the researcher is preeminently the research tool (Borman, LeCompte, and Goetz, 1986).

Some aspects of this type of technique are:

- It demonstrates that the social situations or context in which action takes place is fundamental to the analysis of the behaviour.

- Ethnographic techniques are empirical and are almost without exception employed in naturalistic settings. The researcher is interested in how individuals and groups behave in their own real world setting unmanipulated by the researcher.

- Ethnographic research attempts to present the totality of the phenomenon under investigation.

Ethnographic methods are used to study people "in the wild," as they go about their everyday activities in offices, homes, schools, etc. The point of ethnography is not to find out how people respond to a constructed situation in which narrowly pinpointed variables are 
studied, as in experimental psychology, but to learn how people actually work and play. The chief ethnographic methods are interviews, observations, and participant-observation. One of the greatest strengths of ethnography is its flexible research design. The study takes shape as the work proceeds (Nardi, 1997b).

Ethnography makes extensive use of qualitative data and one aim of collecting ethnographic data is to assist in the development and verification of theory in order to account for human behavior. One of the most salient characteristics of qualitative research, especially ethnography, is that the researcher is preeminently the research tool (Borman et al., 1986). Ethnographers begin their documentation by accepting the fact that what they record in their field notes is already an interpretation of an event (Segall, 1991). Ethnographic research involves the creation and ongoing renegotiations of relationships between researchers and informants (Lawlor and Mattingly, 2001).

Ethnographies are analytic descriptions or reconstructions of intact cultural scenes and groups, which delineate the shared beliefs, practices, artifacts, folk knowledge, and behaviors of some group of people. Ethnographic research involves the acquisition of first hand, sensory accounts of phenomena as they occur in real world settings. Ethnographers begin their documentation by accepting the fact that what they record in their field notes is already an interpretation of an event (Segall, 1991). There are three types of data that is provided by ethnographic research (LeCompte and Goetz, 1982):

- Baseline data-information about the human and technological context of the research population and program setting.

- Process data-information determining what happened in the course of a curricular program or innovation.

- Values data-information about the values of the participants, the program administrators, and the policymakers who financed the program.

Types of ethnographic data collection include:

- Interactive methods:

○ Participant observation.

○ Key informant interviewing.

- Career histories.

○ Surveys.

- Noninteractive methods: 
○ Nonparticipant observation.

- Archival and demographic collection.

- Physical trace collection.

The point of ethnography is to find out not how people respond to a constructed situation in which narrowly pinpointed variables are studied, but to learn how people actually work and play (Nardi, 1997b). Many products are designed and brought to market with very little idea of how people will use them or whether they will use them at all. Ethnography provides a basis on which to judge a product's potential impact and can be a fertile source of design ideas. The leading theoretical perspectives for ethnographically oriented HCI studies are activity theory, distributed cognition, and situated action. The role of the ethnographer in design and evaluation is:

- Conducting specific studies for a given project or product.

- Project management.

- Acting as the "first user" of a prototype.

- Informing usability studies.

- Keeping up with the literature.

- Injecting the users perspective throughout the project.

The object of activity theory is to understand the unity of consciousness and activity. It incorporates strong notions of intentionality, history, mediation, collaboration and development in constructing consciousness. Mediation is where all human experience is shaped by the tools and sign systems we use. Ethnographic and participatory design methods have the problem that every account is an ad hoc description cast in specific terms according to the situation. Activity theory proposes that activity cannot be understood without understanding the role of artifacts in everyday existence, especially the way artifacts are integrated into social practice. Activity theory is concerned with practice, that is doing and activity, which significantly involves the mastery of external devices and tools of labor activity (Nardi, 1997a).

Activity theory is both culturally given and socially formed which makes it not easy to grasp and handle in empirical research. Activity theory postulates that activity is not simply a prism, but rather it is a prism that moves and changes all the time as a consequence of the process of learning. It is not to be mistaken as development. Development is the result of the 
learning that has taken place because parts of the world moved into the scope of the prism and were reflected in and by it (Christiansen, 1997).

Two methodologies used in this kind of psychological research include momothetic methodology where the data is collected from a large number of people (for example, through questionnaire surveys) and by some process of averaging, purport to generalize with some degree of confidence to a larger population and thereby imply a wider validity. Idiographic methodology (for example, in depth interviews) captures the richness and complexity of the phenomenon under investigation but at the risk of basing conclusions on a small number of potentially atypical cases.

What distinguishes ethnographic research is its purpose, which is cultural description. Ethnographic research seeks to build a systematic understanding of all human cultures from the perspective of those who have learned. This approach stresses things like the actor's understanding and theorizing about their actions as opposed to traditional social science research where the researcher attempts to explain human action in terms of psychological theories such as attribution theory. Ethnography is defined as the analytic descriptions or reconstructions of intact cultural scenes and groups. Ethnographies recreate the shared beliefs, practices, artifacts, folk knowledge, and behaviors of some group of people. Aspects of ethnographic techniques include the social situations or context in which action takes place, which is fundamental to the analysis of the behaviour.

Ethnographic techniques are empirical and are almost without exception employed in naturalistic settings. The researcher is interested in how individuals and groups behave in their own real world setting unmanipulated by the researcher. This type of research attempts to present the totality of the phenomenon under investigation. Ethnography makes extensive use of qualitative data and has an air of assisting in the development and verification of theory in order to account for human behaviour. It is concerned with revealing the routine and the "paramount reality" of the everyday world of individuals and groups.

One touchstone of scientific endeavor is described as the replicability of the investigation. In ethnographic research it is impossible to duplicate naturally occurring events in all their complexity and their history because the situation is constantly changing, but this does not necessarily invalidate the findings. One must separate statistical or scientific significance from behavioral significance. The significance of an event is independent of its probability of 
occurrence. One aim of ethnographic research is to record processes of change as opposed to the stability in the environment (Uzzell, 2000).

\subsubsection{Human Computer Interface.}

Human computer interface (HCI) is a discipline concerned with the study and design of interactive computing systems used by people towards satisfying their goals (Pirolli, 1999). It consists of all the objects and actions presented to the user during the process of communicating with computer based programs and applications. HCI includes but is not limited to (Keane, 1992):

- The look and feel, or style, of the communications devices. This guides the appearance and behavior of the interface.

- All of the physical interaction devices including displays, keyboards, and pointer devices.

- Graphical interaction objects present on the communication display(s) such as windows, icons, buttons, and scroll bars.

- Other means of interaction such as touch screen or voice.

- Environmental factors such as illumination, seating, work place management, keyboard layout, display contrast, and symbol size.

- Data handling procedures, data storage method, and data processing logic.

- Supporting hardware such as workstations and printers.

- The techniques employed by the user to enter and retrieve information.

Many important elements of human to human communication such as age, sex, and race, are totally absent from human to computer interaction (Williges, Ehrich, Williges, Hartson, and Greenstein, 1984). The design of any system typically has a goal of minimizing both equipment and personnel costs. Typically, however, these two goals are mutually exclusive. In the past, and in many cases today, design tradeoffs favoring the hardware, primarily the computers, are made at the expense of the human operator. One study suggests that high visual display terminal (VDT) work is prone to the development, for example, of stress related disorders in the operator (Boucsein, 2000). In terms of human performance, however, the system must attempt to achieve a low error rate along with an acceptable cost from the human operator (Williges and Williges, 1981). 
One of humankind's oldest and most persistent dreams is to build reasoning devices of which the computer can be viewed as a modern implementation. However, in order to be effective, system designers must have zero tolerance for user-hostile systems (Hoffman, Hayes, and Ford, 2001; Hoffman, Klein, and Laughery, 2002). This philosophy and requirement is at the heart of the requirement for effective HCI design.

Almost 20 years ago, as the U.S. Army was seriously starting the development and fielding of computer based weapon and communications systems, it was recognized that inadequate design of displays and input/output devices can degrade overall system performance below the required levels for functional effectiveness. The problem is that, with the advent of computer based control and communications devices, the human operator has become the focal point of a potentially inundating volume of information. This is because the computer can acquire, correlate, and present data at a rate that far exceeds the information processing capabilities of the human operator (Benel and Avery, 1985). While one of the fundamental reasons for introducing automation into complex system designs is to reduce the probability of human error by reducing the anticipated operator workload, in practice this reduction does not always occur (Parasuraman and Riley, 1997).

While these comments were made at a time when many of the $\mathrm{C} 2$ and $\mathrm{C} 3$ systems in final development and fielding today were just in the visionary stage of evolution, many of the sentiments expressed here and in numerous similar reports and journals, have yet to find an adequate solution. Benel goes on to state that the $\mathrm{C} 2$ operators must rely on the system designer's ability to anticipate their needs and provide the information that is needed when it is needed in order to be able to extract specifically desired information from the vast store of information contained within the computer system. It is observed that this is a need that is still very much in demand today and an understanding of that need and a solution for it seems to still elude $\mathrm{C} 2$ system designers more often than not.

\subsubsection{Critical Incident Reporting.}

Critical incident reporting is one way to study situational awareness where the critical incidents can be presented in the form of simulations, workplace observations, or can be elicited and / or probed through interviews that generate narratives from memory (Klein, 2000a). Critical incident reports can be a good source of information about how people make use of teamwork schemas, especially in unusual situations where emerging conditions increase risk and 
uncertainty. Low schema similarity among team members may be one reason teams have difficulty addressing decision making requirements (McNeese and Rentsch, 2001).

The critical incident technique consists of a set of procedures for collecting direct observations of human behavior in such a way as to facilitate their potential usefulness in solving practical problems and developing broad psychological principles. An incident is defined as any observable human activity that is sufficiently complete in itself to permit inferences and predictions to be made about the person performing the act. A critical incident is an incident that must occur in a situation where the purpose or intent of the act seems fairly clear to the observer and where its consequences are sufficiently definite to leave little doubt concerning its effects. Critical incidents obtained from interviews can be relied on to provide a relatively accurate account of job performance if suitable precautions are taken to prevent systematic bias. The essence of the critical incident technique is that only simple types of judgments are required of the observer, reports from only qualified observers are included, and all observations are evaluated by the observer in terms of an agreed upon statement of the purpose of the activity.

The critical incident technique is essentially a procedure for gathering certain important facts concerning behavior in defined situations. Steps in the critical incident technique include classification of the critical incidents and making inferences regarding practical procedures for improving performance based on the observed incidents. A summary of the steps in using the critical incident activity (Flanagan, 1954):

- Define the general aims of the activity where the job is being performed.

- Precise instructions must be given to the observers.

- Data for behaviors or results observed should be evaluated, classified, and recorded while the facts are still fresh in the mind of the observer.

- Analyze the data.

- Interpreting and Reporting.

Knowledge concerning operator performance during critical incidents is often obtained immediately following the critical incident through personal interviews (Randel, Pugh, and Wyman, 1996). A suggested guide for conducting critical incident interviews is at Appendix D.

\subsubsection{Task Analysis.}

Task Analysis (TA) is a time oriented description of personnel, equipment, and software interactions brought about by an operator, controller, or maintainer in accomplishing a unit of 
work with an item of equipment or within a system. Critical tasks are those tasks involving human performance that, if not accomplished in accordance with system requirements, will most likely have adverse effects on mission effectiveness, cost, system reliability, efficiency, or safety (Myers, Tijerina, and Geddie, 1987). TA mainly focuses on an analysis of users' knowledge, preferences, perceptions, and actions, with respect to the goal and environment (Pirolli, 1999).

TA is described as a top down decomposition of the overall operation of the system. The steps in a task analysis include developing a composite mission scenario, dividing the composite mission scenario into phases, identifying the segments in each phase, identifying the functions in each segment and identifying the tasks in each function (Hamilton et al., 1991).

A task inventory taxonomy is presented as (Myers et al., 1987):

- Mission-what the system is supposed to accomplish.

- Scenario/conditions-categories of factors for constraints under which the system will be expected to operate and be maintained.

- Function-categories of activity performed by a system.

- Job-the combination of all human performance required for operation and maintenance of one personnel position in a system.

- Duty-a set of operationally related tasks within a job.

- Task-A composite of related activities performed for an immediate purpose.

- Subtask-Activities (perceptions, decisions, and responses) that fulfill a portion of the immediate purpose within a task.

- Task element-The smallest logically and reasonably definable unit of behavior required in completing a task or subtask.

\subsubsection{Cognitive Task Analysis.}

Individual cognition is influenced by cognitive styles among individuals. Cognitive styles are differences that describe individuals' preferred information gathering and decisionmaking abilities. Personality research has indicated the presence of five recurring personality dimensions (Costa and McCrae, 1995; Thompson, 1998):

- Extroversion-interpersonally based traits such as sociability, assertiveness, dominance, and the tendency to be outgoing versus reserved, aloof, shy, and solemn.

- Agreeableness-interpersonal in nature and includes tendencies to be tolerant, cooperative, and warm versus malicious, harsh, irritable, and insincere. 
- Conscientiousness: includes thoroughness, persistence, predictability, rigidity and dependability versus carelessness, absent-mindedness, forgetful and erratic.

○ Neuroticism: one's emotional resilience, calmness, stability, confidence, and independence versus a tendency to be anxious, fearful, sensitive, and self-critical.

- Openness to Experience-includes tendencies to be intellectually complex, insightful, original, curious, and studious versus dull, illogical and narrow minded.

Much of modern cognitive science has been devoted to providing detailed models of cognitive operations and developing experimental methods and paradigms to infer these processes (Williges, 1987). However, cognitive processes such as decision time, accuracy of answers, good vs. bad decisions, etc., cannot be casually observed (Braun et al., 1999).

Methodologies that can be tools for identification of cognitive requirements early in the design cycle include Cognitive Task Analysis (CTA), Scenarios and Team Integrated Design Environment (TIDE). CTA comprises both knowledge elicitation (interviews and observations with SMEs) and knowledge representation (analysis and meaningful representation of the data) (McDermott, Klein, Thordsen, Ransom, and Paley, 2000). CTA is the description of the expertise needed to perform complex tasks (Klein, 1998). The difference in task analysis (TA) and CTA is that TA focuses only on observable behavior with the result that there is no information gained about the overall organization of knowledge. CTA focuses on the psychological processes underlying the behavior and concentrates on the critical decisions and cognitive processes that separate the expert from the novice (Brenner, Sheehan, Arthur, and Bennett, 1999). In essence, it analyzes the thought processes of performers while they complete a task (Randel et al., 1996). CTA is a method for capturing expertise and making it accessible for training and system design (Klein, 1998). CTA goes beyond traditional task analysis that have concentrated on the procedures to be followed and have had relatively little to say about perception, judgment, and decision making skills. The steps in the process are:

- Locate sources of expertise (and acquire background knowledge in the process).

- Evaluate the quality of the expertise.

- Perform knowledge elicitation to get inside the head of the skilled decision makers.

- Process the findings so they can be interpreted to others. 
- Apply the findings.

Key points of CTA are identified as:

- Experts can perceive things that are invisible to novices.

- Skilled chess players show high quality moves, even under extreme time pressure, and high quality moves as the first ones they consider.

- Training to high skill levels should emphasize perceptual skills, along with mastery of procedures.

CTA can be a tool for identification of cognitive requirements early in the design cycle. It comprises both knowledge elicitation (interviews and observations with SMEs) and knowledge representation (analysis and meaningful representation of the data) (McDermott et al., 2000). The purpose of CTA techniques is to analyze and model the cognitive processes that give rise to human task performance in specific domains, as the basis for design and evaluation of computerbased systems and their user interfaces (Zachary et al., 1998).

\subsection{Simulation Modeling Concepts and Procedures.}

Modern computer simulation software architectures and hardware platforms have made possible a level of modeling sophistication that simply did not exist as recently as 20 years ago. Conventional linear programming techniques supplied by languages such as Fortran and $\mathrm{C}$ have been supplanted by discrete event language capabilities that allow model developers and computer programmers to focus more on the design of the software rather than the details of the coding language. This has greatly enhanced the ability to translate conceptual models into working computer simulations that are not only easier to code into computer programs, but also provide a more accurate and realistic representation of the environment being simulated.

This research uses two of these modern simulation approaches in the development of the COMPASS paradigm. These are neural network simulations and discrete event simulations. Some of the underlying principles behind these modeling approaches along with certain concepts pertinent to an understanding of computer simulation based investigative approaches are presented here to facilitate an understanding of this process.

\subsubsection{The System.}

A system is an assemblage or combination of things or parts forming a complex or unitary whole (Hiemstra et al., 1987). Systems typically include various kinds of real-world 
facilities or processes. At its top level it is an entity that exists to carry out some purpose and is typically composed of some combination of humans, machines, and other things such as environmental factors. It exists to carry out some goal that cannot be accomplished by the individual components working independently. A "human-machine" system is a combination of one or more humans and one or more physical components interacting to bring about some desired output resulting from given inputs (Sanders and McCormick, 1993). It is defined in more detail as a group of objects that are joined together with some form of interaction or interdependence for the purpose of accomplishing some goal or goals (Banks, Carson, and Nelson, 1996). It is a collection of entities such as people and/or machines that act and interact together to accomplish a goal (Law and Kelton, 2000). In more general terms, a system is an aggregation of elements according to some structure to accomplish system goals and objectives. All systems include the following characteristics:

- Interaction of elements.

- Structure.

- Purpose and goals.

- Inputs.

- Outputs.

Systems are usually composed of some combination of humans and machines. They have a defined structure and organizations. Systems are further characterized as having external boundaries that separate them from elements outside of the system (Czaja, 1997). The systems approach considers total system performance rather than concentrating on individual parts. This concept is based on the understanding that even if the various subparts of a system are optimized for performance, the performance of the overall system may be sub optimal (Pegden, Shannon, and Sadowski, 1995). The field of macroergonomics provides the concept of "work system" which involves two or more people interacting with the environment. This environment may include hardware and/or software, external environment, internal environment, or an organizational design (Hendrick and Kleiner, 2001). For the purposes of this research the term system is defined as including the weapon systems and all of the people and equipment necessary to field and sustain these weapon systems in peacetime and combat. Figure 16 shows the components of a basic system. 


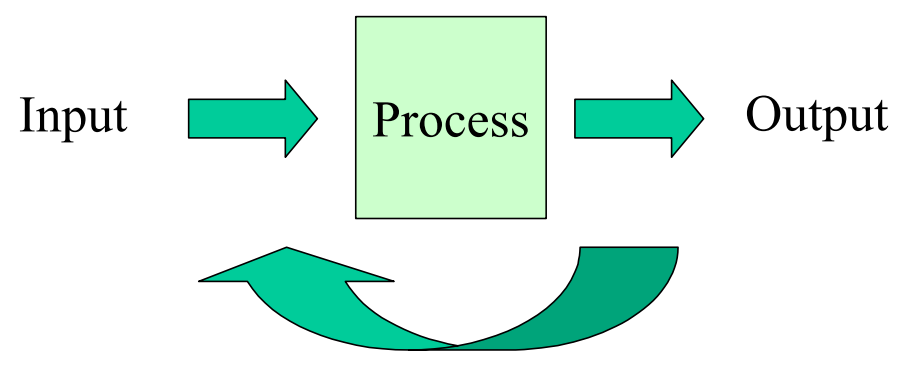

\section{Feedback}

Figure 16 - A Simple System

(Littlefield, 1998)

\subsubsection{Computer Simulation Models.}

Most computer simulations used in human computer interface design are task network models which structure the interface around the task, subtasks, interconnections of subtasks, rules for connecting subtasks and time to complete the subtasks (Williges, 1987). One simulation language in particular, Micro Saint ${ }^{\mathrm{TM}}$ has been specifically designed for modeling systems where the human is a part of the system (Laughery, 1989b; Laughery, 1999; Laughery and Corker, 1997; Williges, 1987). Williges goes on to point out that no single complete model of the human computer interface exists. This statement seems just as valid today as it was then and will likely remain so for the indefinite future. He goes on to point out that the challenge for user modeling is to develop models that can be readily used by designers. Table 7 shows a summary of Williges recommended modeling approaches to human-computer systems.

Table 7 - Summary of Human System Modeling Approaches

(Williges, 1987)

\begin{tabular}{|c|l|l|l|}
\hline \multicolumn{1}{|c|}{ Type of Model } & \multicolumn{1}{c|}{ Emphasis of Model } & \multicolumn{1}{c|}{ Design Tools } & \multicolumn{1}{c|}{ Design Stage } \\
\hline 1. Conceptual & & & Initial design \\
Cognitive processes & Procedural representation & $\begin{array}{l}\text { User actions } \\
\text { Error analysis } \\
\text { Verbal protocols }\end{array}$ & Formative evaluation \\
\hline Cognitive structure & $\begin{array}{l}\text { Task representation } \\
\text { Device representation }\end{array}$ & $\begin{array}{l}\text { Production rules } \\
\text { Transition networks }\end{array}$ & Initial design \\
\hline Cognitive strategy & $\begin{array}{l}\text { Task representation } \\
\text { Goal representation }\end{array}$ & $\begin{array}{l}\text { Task analysis } \\
\text { Goal analysis }\end{array}$ & Initial design \\
\hline 2. Quantitative & Keystroke analysis & $\begin{array}{l}\text { Initial design } \\
\text { Formative evaluation }\end{array}$ \\
\hline Performance & $\begin{array}{l}\text { User performance } \\
\text { prediction }\end{array}$ & &
\end{tabular}




\begin{tabular}{|c|l|l|l|}
\hline Type of Model & \multicolumn{1}{|c|}{ Emphasis of Model } & \multicolumn{1}{c|}{ Design Tools } & \multicolumn{1}{c|}{ Design Stage } \\
\hline Ergonomic & Procedural representation & $\begin{array}{l}\text { Computer aided design } \\
\text { Anthropometric } \\
\text { representation } \\
\text { Bechanical } \\
\text { representation }\end{array}$ & Initial design \\
\hline Computer simulation & $\begin{array}{l}\text { Task sequence } \\
\text { representation }\end{array}$ & Simulation languages & Initial design \\
\hline Statistical & $\begin{array}{l}\text { Task representation } \\
\text { User performance } \\
\text { prediction }\end{array}$ & $\begin{array}{l}\text { Clustering algorithm } \\
\text { Polynomial regression }\end{array}$ & $\begin{array}{l}\text { Initial design } \\
\text { Formative evaluation }\end{array}$ \\
\hline
\end{tabular}

\subsubsection{Task Network Modeling.}

Network models in general evolved from the difficulty to develop formal mathematical models of crew performance. This occurred because theoretical constructs were not available to develop equations that could describe the performance of multi-operator systems. While network models are often criticized as to their validity and because they are often viewed as being too general, there is an opinion that many times they are the only alternative for systematically evaluating alternative system designs (McMillan et al., 1991). Network models based on task performance, i.e., task network modeling, can provide a sound method for modeling human behaviors or performance in systems (Laughery, 1989b). Human behavior is defined as any human action generally defined by a stimulus or cue and a response. A basic stimulus-organism-response constituent of behavior is comprised of the smallest logically definable set of perceptions, decisions, and responses required to complete a task (Godowski, King, Ronco, and Askren, 1978).

Approaches in task network model are based on the concepts of task allocation and workload prediction. These are tools that address the problem of allocating functions to the human or to the machine. Some of the approaches to task allocation and workload prediction include time line analysis which compares the time required to complete assigned tasks to the time available and multiple resource or attention demand which recognizes the fact that some tasks can be accomplished essentially in parallel (low attention demand), while other tasks must be done serially (high attention demand).

The steps that are followed in task network modeling start with the identification of the predecessor tasks that must be completed before the task in question can begin. Then the 
statistical characteristics of the task in question are developed. Finally, the branching to other tasks to be performed upon task completion is identified (McMillan and Martin, 1989).

Current thinking classifies the world of human - system performance models into two categories. The first is termed reductionist models. These use human system task sequences as the primary organizing structure of the model. The term reductionist is used to describe the process of taking larger aspects of human system behavior and successively reducing it to smaller elements of behavior until reasonable estimates of human performance for the task elements can be made. Task networking models are an example of reductionist models where the basis of a representation of human performance is the task analysis (Laughery and Corker, 1997).

In task network models human performance is decomposed into a series of sub functions that are further decomposed into tasks. The sequence of tasks to be performed to replicate the system according to a task analysis is what constitutes the task network. The term task is defined as a composite of related activities (behaviors) performed by an individual and directed toward accomplishing a specific amount of work within a specific work context (Godowski et al., 1978). Each task has a goal. Another definition is the simplest level of behavior that describes the performance of a meaningful function in a job under consideration. Task analysis is defined as a process of reviewing actual job content and context in order to classify information into units of work within a job (Hiemstra et al., 1987). Another definition is that is an analytic process employed to determine the specific behaviors required of a human component in a man-machine system (Godowski et al., 1978). This form of modeling has become popular for several reasons. First, it is a direct means of applying conventional task analysis procedures into a simulation-modeling environment. This modeling system also typically provides the ability to establish sub-task networks of detailed systems and established closed loop approximations of the system performance. Next, recent advances in technology have made task network modeling relatively easy to understand and to implement and has made it accessible to a wide audience. Finally, task network modeling can address many different types of issues and allow many different types of systems to be described and evaluated (Laughery and Corker, 1997). An example of a programming environment that 
implements this type of modeling tool is the Micro Saint ${ }^{\mathrm{TM}}$ discrete event programming language.

A second major category of models of human behavior is termed the First Principle models, which are structured around an organizing framework that represents the goals and constructs of the human performance system being modeled. The term, first principle, is based on the fundamental principles of humans and their interactions with the system and the environment. These models have structures embedded into them that directly represent elemental components of human performance. Examples of these behavioral structures might include goal seeking, sensation, perception, cognition, and motor output. First Principle models require descriptions of how the system and environment interacts with the human process being modeled. An example of this type of modeling is the Man Machine Integrated Design and Analysis System (MIDAS) (Laughery and Corker, 1997).

When using task network modeling to describe human system performance where basic human behavior characteristics are lacking, one approach to overcome the limitations of the task network model is to augment it with elements of First Principle models to represent behavioral phenomena accurately (Laughery and Corker, 1997). The advantages of this approach would be to capitalize on the strengths of each modeling type to provide a strong representation of human system performance that is moderated with human behavior traits. The disadvantage of this approach, as evidenced that there is no example in the literature reflecting it, is that integrating two totally different modeling structures, each with their own database structures and simulation mechanisms, is apparently much more difficult to perform than it is to state.

Other approaches to modeling human performance, especially in military command and control as well as conventional managerial situations, includes classical linear programming and descriptive programming techniques that are provided by conventional programming languages such as Fortran and $\mathrm{C}++$. It also includes the whole general category of mathematical modeling.

Mathematical modeling has strong application in the physical sciences arena because of its ability to provide a continuous description of the physical system over 
time. The "only" requirement is that the system under investigation must be able to be described in terms of mathematical functions and equations. Chemical reactions, nuclear explosions, even weather patterns are examples of physical phenomena that have been modeled in this way. However, the inexact science of trying to estimate human performance characteristics has, so far, eluded acceptable mathematical models that describe it. There are attempts to do so where the model is augmented by Monte Carlo randomness to account for human variability.

These types of models generally follow the predictive equations to predict force on force attrition (Lanchester, 1916) developed early in the $20^{\text {th }}$ century. Because of the random nature of human variability these models typically have found application in the military training arena as command and control training platforms (Middlebrooks, 1991). They are typically implemented in a conventional linear programming environment with languages such as Fortran and $\mathrm{C}++$ and require the model developer to account for all aspects of the system being modeled as the environment only provides technical tools to support the programming constructs. Thus, all algorithms, interactions, interaction techniques, and model constructs must be provided by the model developer in order to describe the system being modeled.

There are other methods for modeling decision making in command and control that include human participation, game theory, optimization, mechanical statistical techniques, controlled experimentation and expert systems (Farrell, Bonder, Proegler, Miller, and Thompson, 1986). However, most of these predate modern computer simulation capabilities. Human participation translates to full-scale field exercises, which almost always had to be scripted with predetermined outcomes because of peacetime maneuver limitations. Game theory models usually were pre-computer board games with outcomes determined many times by actual rolls of the dice. Optimization modeling techniques using mathematical equation descriptors still find favor for force on force evaluations but lack the component of the human in the system as already described. Mechanical statistical techniques follow the limitations of other statistical models already discussed. Controlled experimentation would be a logical first choice for command and control evaluations except for the fact that qualified test participants are almost nonexistent for command and control evaluations. To run a controlled experiment evaluating 
battalion level command and control requires the availability of at least one, and probably several, battalion command and control team(s) of approximately 23 people for the duration of the experiment. However, there are instances where this has been attempted such as a study conducted at Fort Benning, Georgia in the early 1970s where 10 - 12 man teams of combat experienced officers were brought together in an ad hoc fashion over a three week period to role play TOC operations under experimentally controlled scenarios (Olmstead et al., 1973). The drawback to this type of study is that there is no team cohesion or familiarity among the team members because they were nominally brought together just for the study and had no part in the preparation phase of the tactical operation as would have been the case in a real battalion operation.

The only place to obtain qualified C2 teams is from actual military battalions, which is generally precluded by their own requirements for training and mission support. Expert systems, after a brief promise of great expectations in the middle 1980s, have generally failed to live up to their billing primarily because of the same problems with the mathematical models, which is the unavailability of suitable and accurate algorithmic predictors of human performance under varying conditions.

\subsubsection{Optimization Techniques Using Computer Simulation Models.}

Computer simulation is defined as the establishment of a mathematical-logical model of a system and the experimental manipulation of that model on a digital computer (Biles and Ozmen, 1987; Biles and Swain, 1979). Four major classes of approaches to simulation optimization are described as (Pierreval, Tautou, and Bzeznik, 1995):

-1. Gradient based search methods.

-2. Stochastic approximation methods.

-3. Response surface methodology.

-4. Heuristic methods.

The aim of each of these approaches is to propose a strategy to explore the solution space with a limited number of simulation experiments. From these approaches there are three principal experimental design techniques for statistical analysis and optimization using computer simulation, especially in a multi-computing environment. These are factor 
screening experiments, experiments of comparison, and response surface methodology (Biles and Ozmen, 1987). Another view of optimizing the output proposes the steps (Clayton, Weber, and Taylor, 1982):

- Enumerate all possible combinations of inputs in the model and evaluate the output for each combination. This is feasible only for models with a few inputs and short computer simulation time.

- Run various combinations of input variable values picked from an experimental design analysis. Then, using regression analysis, estimate the equations that produce the output values.

- Use a direct search procedure that considers multi-objectives and does not require knowledge of the exact model equations. The simulation model is treated as a "black box" in which the inputs are varied according to the search procedure. This makes decisions on the next set of inputs to try based on the observed model outputs from the current inputs.

Another categorization states that optimization procedures fall into the three categories of (Biles and Swain, 1979):

- Direct search techniques.

- First order response surface methods.

- Second order response surface procedures.

The term design of experiments (DOE), which is related to the term experimental design, is a subdiscipline within mathematical statistics. While classical experimental design techniques can allow simulation outputs to be approximated by polynomials that are functions of input parameters, DOE techniques can be used to conduct, for example, $2^{8}-2$ level full factorial experiments. This design would involve 256 treatment combinations, or more, which is not normally associated as being possible in conventional experimental design (Pucik, Curry, Dziuban, and Senseny, 1999). DOE includes designs such as $2^{\mathrm{k}-\mathrm{p}}$ (fractional factorial experiments) as they are applied to computer simulation. DOE gives good estimators of the main effects, interactions, and quadratic effects through regression metamodels. Thus, DOE improves the effectiveness 
of simulation experimentation. DOE requires relatively few simulation runs resulting in improved efficiency of both the experimental design and the use of computer resources. Once the factor effects have been quantified through regression estimates they can be used for optimization through response surface modeling which augments regression analysis and DOE through the use of techniques such as the steepest ascent hill climbing technique (Kleijnen, 1998).

Some of the peculiarities of computer simulation include, first, the fact that there can be a great many factors in practical simulation models. As many as 281 factors would not be unusual while standard experimental design would typically not have more than 15 , if that many. Stochastic simulation models use pseudo random numbers, which gives the analysts more control over the noise in their experiments than investigators in standard statistical experiments. Both common and antithetic seeds may be used. (Anththetic random numbers are used to induce a negative covariance between paired replications. If a random number in a replication is $r_{j}$ derived as a uniform random number in the range from 0 to 1 , i.e., $r_{j} \sim \operatorname{UNIF}(0,1)$, then the corresponding antithetic random number is $1-r_{j}$. Antithetic random number seeds are determined using the same inverse relationship (Pegden et al., 1995) (Law and Kelton, 2000) ).

In non-simulation experiments randomization is a major concern where the experimental units are assigned in a random nonsystematic way in order to avoid bias. For simulation based DOE this concern disappears as pseudorandom number streams take over. Finally, blocking can be an important technique in conventional experimentation to reduce systematic differences among experimental units. DOE simulations exercise complete blocking control over the experiment which eliminates the need for blocking although it may be used to assign common and antithetic pseudo random numbers (Kleijnen, 1998).

Terminology associated with DOE includes (Kleijnen, 1998):

- Factor - a parameter, input variable, or a module of a simulation model or simulation computer program. The factor may be qualitative.

- Number of combinations of factor levels $-2^{281}$, this would not be unusual in computer simulations. 
- What if analysis - DOE analyzes the input / output (I/O) data of the experiment or simulation to derive conclusions about the importance of the factors.

- Sensitivity analysis - the systematic investigation of the reaction of the simulation responses to extreme values of the model's input or to drastic changes in the model's structure.

- Regression analysis - (also known as analysis of variance - ANOVA) is based on a metamodel, which is defined as a model of the underlying simulation model. A metamodel is an approximation of the simulation program's I/O transformation and is also called a response surface.

- Responses - simulation outputs. Most simulations have multiple responses.

DOE Advantages:

- DOE can be applied to all simulation models, either deterministic or stochastic.

- DOE provides better estimates of the factor effects than does the one factor at a time approach.

- DOE may be used not only for sensitivity analysis and optimization, but also for validation.

DOE Disadvantages:

- DOE cannot take advantage of the specific structure of a given simulation which requires more simulation runs for the analysis than do perturbation analysis and modern importance sampling which is also known as likelihood ratios or score functions.

One purpose of DOE is the optimization of the simulated system. Some examples of optimization techniques include sequential simplex search, simulated annealing that is a network flow method based on a physical process in metallurgy, so called genetic methods that seek to imitate the biological phenomenon of evolutionary reproduction and 
tabu search that may be regarded as a technique based of selected concepts from artificial intelligence that is a general heuristic procedure for guiding search to obtain good solutions in complex solution spaces (Glover, Taillard, and de Werra, 1993). Commercial software is available for simulation modeling using some of these techniques. Some of these packages include ProModel's SimRunner, Witness' Optimizer, and Micro Saint ${ }^{\mathrm{TM}}$ 's OptQuest. All of these programs can handle single response univariate cases, however, as of 1996 there was no known software for optimization of multiple responses in the multivariate case (Kleijnen, 1998).

The use of parallel-distributed processors (PDP) and/or multi-computers is often employed in computer simulation because of the complex and extensive computational loading that these simulations can place on the computer. A multi-computer is defined as a set of tightly coupled but autonomous computers, capable of synchronizing and communicating in parallel while operating independently. They often employ shared resources such as memory and input-output devices. PDP networks, on the other hand involve groupings of completely independent computer systems that may or may not be collocated that work together to solve complex problems and simulations and typically are coordinated in their efforts through a central executive computer system that provides directives and receives responses back from the individual processors on the network. A distributed simulation is defined as the process by which large, complex simulation models are decomposed onto a set of processors. Two steps involved in this are event decomposition in which each of the several events making up a model is assigned to a specific processor and task decomposition in which the various simulation functions such as $\mathrm{I} / \mathrm{O}$, event processing, random number and random variates generation, statistics collection, and report generation are allocated to different processors (Biles and Ozmen, 1987).

One multi-computer approach uses a JAVA based system for allocating simulation trials to a set of $P$ parallel processors for carrying out simulations involving direct search optimization or response surface methodology procedures. As opposed to a PDP based simulation where the simulation model is decomposed and its parts run in a parallel environment, the parallel replications approach allows a simulation model to run 
to completion with a unique set of input conditions. Since a simulation study typically involves executing $R$ replications of the model at each of $S$ sets of input conditions, the server's task in managing a parallel replications approach is to allocate the $R S \mathrm{x}$ simulation trials to $P$ client processors in a manner that balances the workload on each processor. The objective is to complete the simulation study in a time interval approaching $1 / P$ of that which would be required of a single processor operating in a purely sequential mode (Biles and Kleijnen, 1999).

A sampling follows of some of these techniques from the literature for optimizing the results of computer simulation models.

\subsubsection{Tabu Search.}

TABU Search (TS) is a heuristic for providing solutions to combinatorial problems by moving from one solution to another in a way that avoids becoming trapped in local optimal solutions. TS records the best solutions discovered during the search and, often, these solutions are optimal. TS, however, does not guarantee finding an optimal solution, nor will it recognize an optimal solution if it encounters one (Ryan, Bailey, Moore, and Carlton, 1998). One of the main components of TS is its use of flexible (adaptive) memory, which plays an essential role in the search process. The method behind TS can be viewed as an iterative technique which explores a set of problem solutions, denoted by $X$, by repeatedly making moves from one solution $s$ to another solution $s$ ' located in the neighborhood $N(s)$ of $s$. These moves are performed with the aim of efficiently reaching a solution that qualifies as "good" (optimal or near optimal) by the evaluation of some objective function to be minimized. The goal is to make improving moves to the fullest extent allowed while balancing trade-offs between solution quality and computational effort in examining larger samples. TS may be viewed as a variable neighborhood method where each step redefines the neighborhood from which the next solution will be drawn, based on the conditions that classify certain moves as tabu. Experience has shown that TS is able to obtain results that match or surpass the best known outcomes from other techniques in a variety of optimization settings. One advantage of TS is its ability to adapt a rudimentary prototype implementation to encompass additional model elements such as new types of constraints and objective functions (Glover et al., 1993). 


\subsubsection{Goal Programming With Preemptive Priorities.}

Many times the simulation analyst is not interested in the detailed specifications of the model, they only want to know what combination of input variables will provide the optimal output. Some of the possible approaches to optimizing the output include the enumeration of all possible combinations of inputs in the model and evaluate the output for each combination. However, this is feasible only for models with a few inputs and short computer simulation execution times. A second approach is to run various combinations of input variable values picked from an experimental design analysis. Then, using regression analysis, estimate the equations that produce the output values and apply math programming optimization techniques to the estimated model. This, however, may result in an estimated model that is nonlinear and requires multiple objectives. A third approach is to use a direct search procedure that considers multiple objectives and does not require knowledge of the exact model equations. The simulation model is treated as a "black box" in which the inputs are varied according to the search procedure. This makes decisions on the next set of inputs to try based on the observed model outputs from the current inputs. This third technique is a method for optimizing multiple response simulation models that applies modified pattern search and gradient search techniques to the simulation model responses that may be linear or nonlinear. For this procedure the model equations generating the responses may be known or unknown, however, the simulation must not contain Monte-Carlo random effects (Clayton et al., 1982).

This technique is a procedure for solving integer nonlinear optimization problems. The process consists of a ten-step procedure, which is summarized as:

-Step 1: Choose an initial starting point and evaluate the goal values associated with it.

-Step 2: Attempt to find a direction vector using the pattern search exploratory move method.

-Step 3: Check to determine whether or not the direction vector from step 2 is equal to zero and then send the program in the proper direction.

-Step 4: Make a pattern move.

-Step 5: Ascertains that the first goal level has been achieved.

-Step 6: Perform the gradient move. 
-Step 7: Test the gradient move for improvement.

-Step 8: If no improvement found in step 7, determine whether or not it is possible to find other rays.

-Step 9: Reached only if no optimum is found and no exploratory move or ray move has found a direction in which the IVs can be moved to improve the selected goal and allows the user to restart the search at a new initial point a specified distance away from the original start point.

-Step 10: Reached only if no optimum is found and no exploratory move or ray move has found a direction in which the IVs can be moved to improve the selected goal then the maximum value for the goal has been determined and the desired goal level cannot be met. Decision logic either terminates the search or returns to step two with a new desired goal level.

The advantages and disadvantages of this algorithm are:

$>$ Advantages:

-The technique is efficient because of its systematic search procedures and because it exploits a direction of change in the IVs until it no longer leads to an improvement in the goal levels.

-The technique is very efficient allowing an optimum solution to be reached in only a fraction of the computer time required to reach a non-linear solution when solved by enumeration.

-Like all direct search optimization methods, this technique does not necessarily require knowledge of the functional forms of the goal equations although it does require that goal values be obtained that are dependent upon given vectors of IVs.

-The method does not require linear or polynomial approximation of the fundamental forms of the goal equations if they are unknown, as would be the case of multiple response surface methods are being used. Thus the method can be tied directly to the output of a nonMonte-Carlo simulation model, and the solution found by this technique will be a solution to the simulation model, not a solution to an approximation of it.

$>$ Disadvantages:

-The algorithm does not guarantee that the global optimal solution will be found. 
-There is no guarantee that the method is the most efficient in the sense of requiring the fewest number of point evaluations of simulations in order to achieve an optimum.

-The technique ignores the stochastic behavior of the simulation response on the performance of the algorithm.

-The model algorithm has a restriction of dealing only with integer problems even though there are large numbers of realistic problems, which are non-integer.

\subsubsection{Response Surface Methodology.}

Response Surface Methodology (RSM), originally developed by Box and Wilson in 1951, has emerged as one of the primary tools for determining optimum values of the experimental space and is the topic of a wide variety of discussion in the literature. RSM was specifically developed to provide data for satisfying the needs to collect data in a more efficient manner than conventional factorial experiments. Its purpose is to collect a "foundation" of information that can be useful for predicting performance when system equipment parameters are not currently known and to determine what combination of these parameters can optimize overall system performance where system performance must be expressed as a function of equipment parameters, where the independent factors are measurable on a quantitative, continuous scale, and interaction and higher order terms may be included (Simon, 1968). As opposed to traditional ANOVA factorial and fractional factorial designs, RSM focuses on determining the functional relationship that exists between the response and specified continuous, quantitative factors, rather than merely determining the significance of the various individual factors (Clark and Williges, 1972b; Clark and Williges, 1973).

As a tool for gradient estimation and sensitivity analysis in discrete simulation, RSM possesses noteworthy advantages in comparison to other techniques. RSM is based on wellknown principles of regression analysis and analysis of variance and is more completely developed than recent extensions of techniques such as perturbation analysis and likelihood ratio methods. The main disadvantage of RSM is the cost of making all of the simulation runs required by the experimental design. This cost can be prohibitive when the response of interest has a large variance so that excessive run lengths are required. This naturally invokes the interest for searches for effective variance reduction techniques that can be incorporated into the overall experimental design. One distinct advantage of RSM is that the Monte Carlo method of 
control variates can be effectively used in simulation based RSM studies with a negligible computational overhead (Wilson, 1987).

The RSM methodology involves the classical application of experimental design based on standard least squares theory. Generally in simulation-based designs, it is assumed that the response surface resulting from the continuous parameters of the simulation model is smooth enough to be described by either a first or second-degree polynomial over the region of interest. The methodology is sequential in nature with each successive experiment building on the results and insights of earlier experiments. It is, therefore, ideally suited to simulation because of the relative ease with which data can be obtained in the simulation context (Hood and Welch, 1993).

Often it is desired to utilize the simulation model to attempt to find the optimum conditions for operating the system. RSM represents a body of techniques by which the optimum set of system conditions is determined. A procedure for employing a first order response surface approach is (Biles and Ozmen, 1987):

-1. Identify the known experimental region.

-2. Perform simulation trials at each of the experimental design points and record the responses.

-3. Apply the appropriate mathematical programming technique to locate the next center point in the search.

-4. Repeat steps 1-3 until an "optimum" solution is located. It may be necessary to add design points to complete a second order response surface design to test the optimum solution.

Some assumptions and characteristics of RSM are (Kleijnen, 1998):

- $\mathrm{RSM}$ assumes that the decision variables are quantitative.

- RSM assumes a single type of response.

- RSM relies on first and second order polynomial regression meta models or response surfaces. The responses are assumed to have white noise.

- RSM uses classical designs.

- $\quad$ RSM adds to regression models and DOE the mathematical (not statistical) technique of steepest ascent; that is, the estimated gradient determines in which direction the decision variables are changed. 
- RSM uses the mathematical technique of canonical analysis to analyze the shape of the optimal region: Does that region have a unique maximum, a saddle point, or a ridge (stationary points)?

In a parallel processing system RSM approach, the desire is to determine the $2^{\mathrm{n}-\mathrm{p}}+2 \mathrm{~N}+$ $\mathrm{P}$ design points in a central composite design and to systematically allocate the determination of these points to $\mathrm{P}$ client processors (Biles and Kleijnen, 1999).

A generalized outline for applying the RSM methodology to optimization problems is (Hood and Welch, 1993):

- Select an initial experimental region.

- Fit a first order model to the region.

- Check to see if the first order model is a reasonable fit and that a minimum does not fall within the region.

- If the first order model is valid, follow the path of steepest descent to an estimated minimum of the function along the path.

- Repeat this procedure for a region about this minimum point.

- If it is determined that it is likely that a minimum exists within the region then, augment the design and fit a second order model.

- Analyze the model to determine whether or not a minimum exists and continue.

The first order response surface methods attempt to accomplish experimentally what the "method of steepest ascent" accomplishes computationally. From a selected point in the experimental space a designed experiment is conducted with a simulation trial at each design point to estimate the gradient direction. Simulation trials are then conducted at points along this direction to a new point, which represents the best solution obtained along the gradient direction. The gradient direction is then estimated by placing an appropriate first order experimental design, such as a $2^{\mathrm{n}}$ factorial, $2^{\mathrm{n}-\mathrm{p}}$ fractional factorial, or $n$ - dimensional simplex design around the current point of analysis. A simulation trial is performed at each point in the selected experimental design. From these observations a multiple linear regression model can be estimated. A simulation trial is conducted as each design point in the selected first order design and observations are recorded at each design point. Multiple linear regression is applied to each observation (assuming independence among the responses) producing a model for each response (Biles and Swain, 1979). 
These estimates are then employed in an optimization scheme to produce an improved solution. Simulation optimization procedures that can be employed for this purpose include Box's Complex Search, Rosen's gradient projection method to evaluate a first order response surface procedure, a central composite design coupled with a computational version of Box's complex search for a second order response surface. One point that this author notes, which may seem minor but is the type of thing that often consumes an inordinate amount of time and energy, is that computer programs for the optimization procedures and the computer programs from the simulation must often be "custom fitted" together in order to perform this analysis (Biles and Swain, 1979).

Another statement of the generalized procedure to be followed for the case of employing a first order response surface approach to the multiple-response simulation problem is (Biles and Swain, 1979):

1. Identify the known experimental region. Select a starting point within the region. With the initial point as its center, array an orthogonal first order response surface design within a selected design radius.

2. Perform simulation trials at each of the $N$ experimental design points and record the responses. Using multiple linear regression, fit linear models to the responses.

3. Apply the appropriate mathematical programming technique to locate the next center point in the search.

4. Repeat steps $1-3$ until an "optimum" solution is located.

A summary of advantages of the use of RSM for computer simulation experimentation is (Hood and Welch, 1993):

- It is sequential so it matches well the ready availability of data in the simulation context.

- It is sequential both in the model fitting and estimation at each experimental stage and in the generation of a sequence of experimental stages.

- It produces confidence intervals on the estimates of interest and powerful diagnostics for the model fitting.

- It provides a solid theoretical and intuitive base in classical regression theory for the experimental process. 


\subsubsection{Box’s Complex Method of Constrained Optimization.}

This is a method for finding the maximum of a general non-linear function of several variables within a constrained region that is described to be efficient when compared with existing methods when the required optimum lies on one or more constraints. The definition of a constrained optimum is meant one for which the solution corresponds to certain variables lying at the edges of their permissible ranges. If this is not the case a method with no provision for bounding the variables will produce the same result. The constrained complex method searches for the maximum value of a function subject to $M$ constraints where the lower and upper constraints are either constants or functions.

This approach, using gradient based optimization, utilizes successive, alternative phases, with the first phase consisting of a first order experimental design which estimates an "optimal" improving direction and a second phase determining the "optimal" step along this direction. (Box, 1965)

+ Advantage: Each phase lends itself nicely to the assignment of a set of S simulation trials to $\mathrm{P}$ parallel processors.

+ Disadvantages:

- Requires a parallel computer to execute.

- The lack of the ability to perform efficient unconstrained optimization.

- Continuation of the search when one or more constraints become effective.

- Some of the randomly generated points will be relatively remote from the initial point and may be in the vicinity of a higher peak.

\subsubsection{Gradient Based Optimization Approach.}

This is an optimization approach that takes advantages of the simultaneous execution of $P$ simulations trials on parallel processors. Gradient - based optimization utilizes successive, alternating phases. The first phase consists of a first order experimental design that estimates an "optimal" improving direction and a second phase that determines the "optimal" step in the direction of the first phase. An advantage of this technique is that each phase can be tailored to 
the assignment of a set of $S$ simulation trials to $P$ parallel processors (Biles and Kleijnen, 1999). However, experimental studies comparing actual human performance to a gradient algorithm based on a two variable optimization task found the algorithm to always be inferior to the human operator (Laughery and Drury, 1979).

\subsubsection{Genetic Algorithms.}

The Genetic Algorithms (GA) approach is where each client processor is assigned its own initial random number seed and a GA search is undertaken completely independently on each processor. GA concepts, developed by John Holland, are based on natural evolution phenomena where only the strongest individuals, i.e., the best adapted to the environment, survive. GA algorithms begin their search of the optimal solution from a set of potential solutions (i.e., individuals represented by their chromosomes), which is called the population. An initial population is made that evolves towards a population, which contains the best solution. From generation to generation, the population contains better and better solutions of the problem and converges to an optimum (Pierreval et al., 1995).

The GA approach greatly reduces the interaction between the Simulation Manager and the clients. Under this approach each client processor is assigned its own initial random number seed and an independent GA search is undertaken on each processor. Each of the $P$ processors acts, analogically, as an island continent for the purposes of evolution, where the evolutionary process takes place as if it were completely unaware of the existence of its "neighbor" continents. After a complete GA search has taken place on each of the $P$ client processors, the Simulation Manager receives the collective statistical results and determines the best solution (Biles and Kleijnen, 1999).

In a formal sense, data analysis is not the main field of application of GA. They should be viewed rather as a powerful technique for solution of various combinatorial or optimization problems. Nevertheless, GA are applicable for datamining. The name of the method derives from its similarity to the process of natural selection. Let the problem be to find a solution to a question that would be the most optimal from the point of view of a certain criterion. Assume that each solution can be exhaustively described by some set of numerical or non-numerical parameters. For example, if the task is to select a fixed number of performance parameters influencing the $\mathrm{C} 2$ performance the most, then the names of these parameters comprise such a 
descriptive set. One can think of this set as of a set of chromosomes determining qualities of an "organism"-a solution of the problem. Following this analogy, values of parameters determining a solution correspond to genes. A search for the optimal solution is similar then to the process of evolution of a population of organisms, where each organism is represented by a set of its chromosomes (Megaputer, 2002). The four main components of GA are chromosome representation, evaluation function, genetic operators and initialization of the population. When compared to other rule discovery methods such as artificial neural networks and statistical models, the advantage of techniques such as genetic algorithms is that the rules evolved are selfexplanatory (Lopes, Pacheco, Vellasco, and Passos, 1999).

\subsubsection{Parallel Evolutionary Algorithm Approach.}

Evolutionary algorithms (EA) is an extension of GA that concern the coding of chromosomes, the genetic operators or the selection techniques. Parallel Evolutionary Algorithms (PEA) is EA implemented on a parallel processing computer. In PEA the selection is replaced by a local search, generally considered as a "local hill-climbing," where each chromosome tries to improve its fitness by itself in a neighborhood. An example of a fine grained PEA sequence is (Pierreval et al., 1995):

- Step 0: Define a genetic representation of the problem.

- Step 1: Create an initial population.

- Step 2: Each chromosome does local hill climbing.

- Step 3: Each chromosome selects a neighbor for mating.

- Step 4: An offspring is created with genetic operators working on the genotypes of its parents.

- Step 5: The offspring does local hill climbing. It replaces the parent, if it is better than some criterion.

- Step 6: If not finished, return to Step 3.

\subsubsection{Sequential Experimentation.}

Experimental designs based on full factorial evaluations very quickly become inefficient to the point of being impractical to conduct as the number of factors goes up. Considering experiments with only two treatment levels, for example, five factors would generate $2^{5}=32$ treatment combinations. However, many experiments in human factors can involve 10, or 20 or 
more IV factors. A ten factor - two level full factorial experiment involves $2^{10}=1024$ treatments to be conducted. While certain techniques such as fractional factorial replicates can reduce these numbers, the situations that occur when different factors have different numbers of treatment levels greater than two and when all the treatments cannot be conducted at the same time, place, or by the same experimenter, many times result in the fact that no explicit data reduction techniques are available that can gather the required data. When there are large numbers of factors to be evaluated over extended periods of time among different locations and by multiple experimenters then the technique of sequential experimentation becomes viable.

In the mid 1970s investigations were undertaken to examine traditional experimental design methods as they applied to human factors engineering (then referred to as engineering psychology) where the two distinct approaches of experimental and correlational empirical investigation were compared. Arguments were made for merging the more productive features of each into a new research paradigm for experimental psychology called the sequential process. This proposed systematic multi-factor experiments that could be performed economically. With this paradigm the total data collection process for deriving equations for all critical variables affecting the system under investigation would be less than that used for a 4 or 5 factor experiment using traditional means. This paradigm was described in five phases (Simon, 1977b):

- Define the systems research problem.

- Identify the critical variables.

- Develop a response surface.

- Refine the regression equation.

- Verify the experimental results.

Further proposals were made that described methods for constructing Resolution IV screening designs ascribed as being robust to linear, quadratic, and cubic trend effects. Complete designs of up to 32 variables were presented using this technique. These screening designs, in some ways a precursor and in some ways a component of sequential experimentation, was described as a class of fractional factorials with systematic data collection plans that enabled the effects of large numbers of factors to be estimated economically. The purpose of screening 
designs was to identify the most important factors not to obtain an accurate representation of the full experimental space. However, the full benefit of screening designs was that, once the most important factors were identified the same data can be further utilized by supplementing the original experiments by a relatively few additional observations with new experimental conditions that would have the ability to describe the response surface that would be capable of representing the full experimental space of the original large number of IVs (Simon, 1977a).

As computer simulations began providing an ever more capable tool for complex human performance investigation, it was noted that the primary difficulty of using simulation for experimental design could be summarized as too many factors and too few runs. Factor screening methods were acknowledged as being able to reduce the number of factors in large experiments where the basic aim was to efficiently and effectively classify as active or as inactive the $\mathrm{K}$ factors under investigation. Two approaches for this were described as the use of expert judgment where the analyst decides which factors are important and which are not, and group screening where "group factors" are created by partitioning the individual factors into a number of groups (Smith and Mauro, 1981).

Building on the work of Simon and others, efforts were undertaken to describe systematic methods that could be applied to human performance system studies that could only be realistically described by large numbers of factors that could number 100 or more (Han et al., 1997; Williges, 1981; Williges and Williges, 1989; Williges et al., 1993). The goal of sequential experimentation is to develop integrated empirical models describing user performance as a function of a large number of IVs investigated across a series of small studies (Williges et al., 1993). Three general approaches for conducting empirical research with systems that deal with complex relationships among many IVs include the use of direct observations of the operational system or prototype if possible, the use of analytic methods of modeling and computer simulation, and real time (human - in - the - loop) simulation with manipulation of various system parameters according to experimental designs (Williges, 1981). Some of the experimental design methods that can be used to provide for efficient data collection and reduction in complex system design and experimentation include:

- Single observation factorials where only one observation is made in each treatment combination. 
- Hierarchical designs where factors other than subjects are nested causing the level of one factor to appear at only one level of another factor which results in a hierarchical, or pyramidal, shape of the treatment conditions.

- Blocking designs where the data is collected in stages or blocks over time or between locations.

- Fractional factorial designs where the nature and extent of confounding is determined by specifying the subset of treatment combinations to be observed from the full factorial design.

- Empirical model building where the researcher's primary aim is the determination of a quantitative relationship between human performance and quantitative system parameters.

- Central-Composite Designs where the aim is to determine the optimal combination of various factors from the total system design.

Some alternative sequential design procedures include the use of random and independent selections of experimental data points, a series of single factor experiments, and / or the use of the method of steepest ascent in response surface exploration (Williges, 1981).

The stages involved in the use of sequential research are shown in Figure 17 and include selecting, describing, and optimizing IVs. The goal of this research strategy is to obtain estimates of the functional relationships of a large number of IVs in a realistic, efficient, and systematic manner.

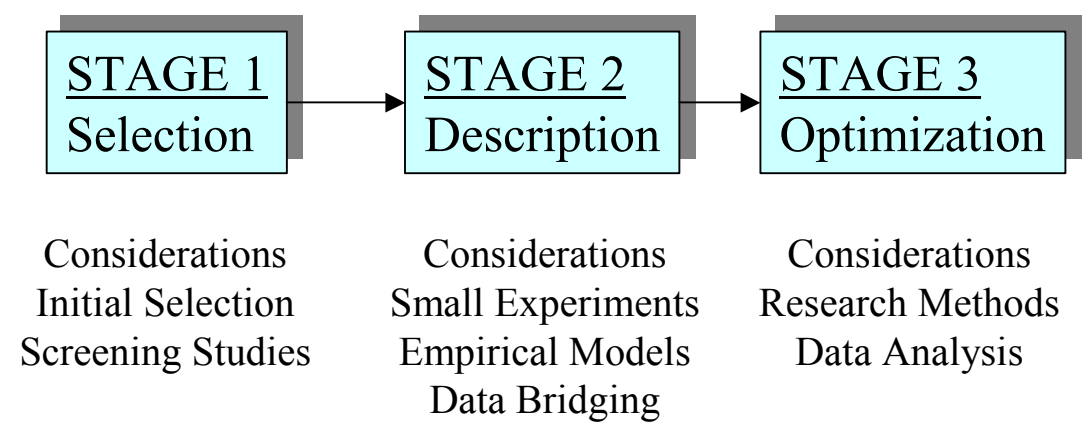

Figure 17 - Stages In Sequential Research Paradigm

(Williges, 1981; Williges, 2001) (Used With Permission of the Author)

Figure 17 shows the stages involved in the preparation of IVs for sequential research. The methods involved for each stage include (Williges, 2001):

- Stage 1: Brainstorming, prototyping, subjective ratings, literature review, and screening studies. 
- Stage 2: $2^{\mathrm{k}}$ factorials, $1 / 2$ replicates, central composite design, polynomial regression, integrated database.

- Stage 3: Steepest ascent, random selection, partial derivatives.

The first stage of this methodology involves the identification of all possible variables that could affect human performance in the system being evaluated. The second stage is the conduct of several small experiments to gather the necessary data to evaluate the variables identified in stage 1 (Diamond, 1981). The experimental designs that are used in this stage generally only focus on main effects and two-way interactions. This is because, in human factors research, it generally is not feasible to interpret human performance interactions above second order. While interactions of variables such as "talking on a cell phone" and "driving an automobile" can generally be evaluated, the interpretation of a third variable such as "reading an information display" generally is not possible in human factors research. For this reason Resolution $\mathrm{V}$ experiments that examine the main effects of the individual variables and two-way interactions are the highest level experiments that are generally employed. The key to these experiments is efficient experimental designs that allow for data collection economy. The third stage of sequential design is to determine the optimum combination of levels of the important IVs. From this an empirical model is developed that integrates the relationships from the most important IVs. Optimization procedures can include response surface methodology, ridge analysis, and mixed integer programming (Williges, 1981; Williges et al., 1993).

Before beginning a program of sequential research a plan must be developed that considers the constraints and region of interest for the project. The selection of IVs for a sequential research plan involves both the initial design factors and screening studies to reduce the number of factors to a reasonable size for the experimentation and model building. Once the set of IVs to be used in the experiment has been identified from the larger set, a series of small experiments is conducted to gather data to describe the effects of these variables. The results from each of these small experiments is then combined together by a data bridge so that the functional relationships pertaining to all the IVs can be identified and analyzed. From this single data set an integrated empirical model can be generated. Finally, the last stage of the sequential design procedure is to determine the optimum combination of important design variables (Han et al., 1997; Williges et al., 1993). 
When embarking on studies involving sequential experimentation, distinct consideration must be given on what types of experimental designs might be appropriate for the individual data collection runs. First, discussions of two types of IVs that are associated with sequential experimentation are presented (Williges et al., 1993):

- Dichotomous variables are discontinuous factors encountered in human factors research. Examples are gender (male or female) or availability of a specific feature. This class of variables is evaluated with two level, $2^{\mathrm{k}}$, factorial designs. When the amount of data precludes its full collection because of such things as time or budget restraints and the researcher can be satisfied with a Resolution $\mathrm{V}$ experiment where only the main effects and two way interactions are of interest, then fractional factorial, $2^{\mathrm{k}-\mathrm{p}}$, designs can provide an efficient alternative for simultaneous consideration of multiple factors.

- Continuous variables can be evaluated using central composite designs, which provide an efficient means for handling this class of variable. Second order models, including main effects, pure quadratics, and two way interactions, can be estimated with the CCD design. A central composite design consists of three portions: 1) A factorial portion, $2^{\mathrm{k}}$ or $2^{\mathrm{k}-\mathrm{p}}$, 2) An axial portion, and 3) A center point.

If the researcher can assume that the main effects and two way interactions are of the primary importance for human factors research, and that higher order effects are not of interest, then useful designs for sequential experimentation are (Williges and Williges, 1989; Williges et al., 1993):

1. Two-level full- or fractional-factorial designs to be used in the evaluation of dichotomous variables.

2. Central-composite designs to be used to evaluate continuous variables.

3. Data bridging designs to add required evaluations for the investigation of interactions not investigated in the set of sequential experiments. These data points are generated with the view to minimize multicollinearity effects that include large variances of coefficients, poor power of partial - F tests, incorrect signs on coefficients, and poor prediction performance. Controls on serious multicollinearity in a regression model are implemented by insuring that the variance inflation factor (VIF) should be smaller than 10 and the eigenvalue ratios should be smaller than 100 in order to test model items for statistical significance. 


\subsubsection{Simulations of Human System Performance.}

Modeling the cognitive processes that are involved in information processing is central to understanding human capabilities and limitations in computer tasks (Williges, 1987). Simulations can permit a much more precise level of control of many variables than could ever be realized in physiological or behavioral experimental paradigms (Grossberg and Mingolla, 1986). The significant dimensions of the human system include the task dimension, the performance-learning-development dimension and the individual difference dimension. The human information processing system is a serial system consisting of an active processor, input (sensory) and output (motor) systems, an internal long-term and short-term memory and an external memory. There is general agreement that human memory is best modeled in terms of a basic division between active short term memory and a passive storehouse called long term memory (Pew and Mavor, 1998e). For modeling purposes memory can be broken down into three broad categories:

- Episodic, generic, and implicit memory.

- Short term and working memory.

- Long-term memory and retrieval.

Some of the sources of information providing input to the human system include (Newell and Simon, 1972):

- The task instructions themselves.

- Previous experience with the same task.

- Previous experience with tasks that are recognized as somehow analogous to the given task.

- Stored programs in long-term memory of substantial generality that can be applied to a range of tasks.

- Stored programs in long term memory for constructing problem spaces.

- The course of problem solving itself.

Human behavior is a complex result of many factors that include environmental moderators and interpersonal differences. Human behavior moderators include (Pew and Mavor, 1998a) external moderators such as physiological stressors that include environmental conditions such as temperature, toxic substances, noise, vibration, etc, and other conditions such as physical work and fatigue-effects of continuous performance. They may also include cognitive workload stressors, which are described as a consistent human adaptation to overwhelming task loads in 
information processing tasks (such as command and control). The reaction to this is to exclude some portion of the signal flow or postpone the processing response until the peak period has passed. Some responses to cognitive overload are dysfunctional. In this case, sequence sense is often lost. For example, where a tank battalion commander receives messages regarding unit losses in a high signal flow load, the initial signals may become overlooked with the commander not being aware of the true extent of the overall losses.

Internal moderators of human behavior include intelligence such as ASVAB scores and level and type of expertise and cognitive abilities. If a task is within the physical capability of the soldier, the cognitive aspects of the task will display greater variation in performance than will physical aspects that are frequently practiced. Personality traits frequently judged by observers of military affairs to have a high likelihood of salience for the military character include need achievement, risk taking and innovativeness and a general alertness and vigilance. Likewise, emotions such as anxiety, obsessiveness, and depression are an internal moderator as well as attitudes and expectancies where expectancies are a product of the interaction between beliefs and attitudes. Finally, cultural values can induce a profound effect on the behavior of the individual warrior.

Another view to human performance modeling is called the normative-descriptive approach where motivated expert human decision makers strive for optimality but are constrained from achieving it by their inherent perceptual limitations and cognitive biases. This approach includes information processing models such as the distributed information processing (DIP) model that predicts how team members weight and combine sequential information from distributed sources. The hierarchical information-processing (HIP) Model predicts how a leader in a hierarchical team combines the opinions and confidences of subordinates to solve an event identification (or hypothesis-testing) problem. It also includes resource allocation models such as the distributed resource allocation and management model (DREAM) that represents the time evolution of task appearance and movement, as well as resource flow, within a dynamic programming framework and then obtains a sub optimal solution to the formulated optimization problem and the team distributed scheduling (TDS) Model that examines how team decision making and coordination strategies adapt to increased task load and resource scarcity under different responsibility structures (Kleinman et al., 1992). 
There are three major categories of simulations in use in the military. These are training simulations for individual combatants or leaders and teams. The second type is analytical simulations for analyses of systems, doctrine, and tactics for purposes of acquisition and advanced development. Then there are those simulations that address questions associated with improving command and control and the interoperability of joint forces. While a model is defined as a physical, mathematical, or otherwise logical representation of a system, entity, phenomenon, or process, a simulation is defined as a method for implementing a model over time. Types of simulations in use in the military include (Pew and Mavor, 1998c):

- Live simulations, which are live, field exercises.

- Virtual simulations where real humans operate simulated equipment in simulated environments.

- Constructive simulations where simulated people operate simulated equipment and may or may not be in real time.

Three common approaches used to represent the C3 architecture in models that examine the behavior and decision making of organizational units are the rule based approach the network approach and the Petri Net approach (Pew and Mavor, 1998f).

\subsection{Selected Statistical and Experimental Approaches.}

Investigations of the order of magnitude envisioned for the COMPASS paradigm, where a hundred or more IV may be evaluated for dozens of DV, require analytical approaches that transcend normal statistical and experimental designs. Multivariate techniques that are augmented by datamining approaches are seen as an appropriate venue to investigate the amount and type of data that this study will produce. This section presents a review of some of these techniques.

\subsubsection{Cluster Analysis.}

Cluster Analysis (CA) is the generic name for mathematical models that can be used to find out which objects in a set are similar (Romesburg, 1984). It also allows the ability to find out which objects in a set are dissimilar. There are a number of methods of CA but the most widely used is hierarchical CA (HCA). Other CA methods are the single linkage, the complete linkage, Ward's method, and the centroid clustering method. The steps in HCA are: 
1. Collect a data matrix where the columns consist of the objects to be cluster analyzed and the rows are attributes that describe the objects.

2. Standardize the data matrix (optional).

3. Compute the values of a resemblance coefficient as a measure of the similarities among all the pairs of the data objects (from the standardized data matrix).

4. Process the values of the resemblance coefficient using a clustering method which results in a tree diagram or dendrogram that graphically shows the relationship between the similarities among all the pairs of objects.

Clustering can be generally defined as a problem where " $\mathrm{N}$ " points are given in a dimensional feature space, with the requirement to find " $\mathrm{d}$ " interesting groups of points. There is no definitive way to quantify what "interesting" means in this context, however, many algorithms assume that there are a certain number of expected clusters, "k," with the requirement to find these clusters so as to minimize some error metric. Examples of applications where clustering is applicable include classification problems in machine learning, information retrieval to identify concepts, to improve the presentation of web search results, and by physicists to find the spatial grouping of stars into galaxies. In general the desire is to find relationships in the data and to succinctly model the data distribution (Palmer and Faloutsos, 1999).

Applications include the commercial sector where data from billions of credit card transactions and cash register receipts are processed annually in order to analyze purchasing patterns and uncover evidence of fraud. In the national security arena, vast amounts of electronic and image data are processed in near real time with the requirement to search for features of special interest (Joyce, Abarbanel, Callan, Dally, and Dyson, 2000).

Cluster analysis is not a typical statistical test but is a collection of different algorithms that "put objects into clusters." Statistical significance testing is not appropriate with cluster analysis even in cases when p-levels are reported (as in k-means clustering). The purpose of cluster analysis is to join together objects into successively larger clusters, using some measure of similarity or distance. Typically this clustering is shown as the hierarchical tree. The tree clustering method uses the dissimilarities or distances between objects in forming the clusters. Types of distances that can be used with cluster analysis:

- Euclidean distance-the most commonly used type of distance. It simply is the geometric distance in the multimensional space. 
- Squared Euclidean distance-places progressively greater weight on objects that are further apart.

- City-block (Manhattan) distance-the average difference across dimensions.

- Chebychev distance-appropriate when the desire is to define two objects as "different" if they are different on any one of the dimensions.

- Power distance-where the desire is to increase or decrease the progressive weight that is placed on dimensions on which the respective objects are very different.

- Percent disagreement-used for data for the dimensions included in the analysis are categorical in nature.

One form of cluster analysis uses $k$ means clustering where the expected number of clusters is predetermined by a hypothesis (StatSoft, 2002).

\subsubsection{Datamining and Neural Networks.}

The merging of statistics, machine learning and database management has resulted in the emerging technology area called datamining (Clifton and Thuraisingham, 2001). Datamining is the search for and extraction of hidden and useful patterns of information and structures in large multidimensional datasets that were originally collected for another purpose. It is the search of observational data for the relationships between parameters in that data rather than the measurement of experimental data. A major limitation of datamining is that the search algorithms chosen for the search may miss an important and interesting pattern or even a class of patterns. There is no systematic method currently known to preclude this (Ceruti and McCarthy, 2000). Datamining techniques are used to extract previously unknown information from a large database that typically can house millions of pieces of information about customers and customer relationships. It is the process of posing queries and extracting information often previously unknown from large quantities of data. Datamining outcomes include forming clusters as well as making associations and correlations (Thruraisingham, Clifton, Maurer, and Ceruti, 2001). Part of the problem is that there is simply not enough knowledge about the many factors that govern human behavior to accurately predict results of such actions as decision-making. While datamining is no panacea to overcome this deficiency, it can provide correlations according to behavior patterns and can help guide the search for types and timing of decisions that are made 
but cannot accurately predict what the outcomes of the decisions will be. Behavioral processes are next to impossible to predict with a high degree of accuracy. Emotions make it possible for a person to make a decision for no obvious reason and then to change his mind seconds later. This is a domain where a mere matter of seconds can make the difference between one response or another (Wisner, 1999).

Datamining is an emerging research area with a focus to extract significant patterns or interesting rules from large databases (Zaki, Parthasarathy, Li, and Ogihara, 1996). The technique relies on mathematical algorithms and neural networks to reach into relational data bases and uncover new patterns among the data (Asbrand, 1997). It is the process of posing queries and extracting information often previously unknown from large quantities of data where the outcomes include forming clusters as well as making associations and correlations (Thruraisingham et al., 2001). At present Evolutionary Programming is the youngest and evidently the most promising branch of datamining. The underlying idea of the method is that the system automatically formulates hypotheses about the dependence of the target variable on other variables (Megaputer, 2002). Datamining is the process of extracting patterns as well as predicting (previously unknown) trends from large quantities of data by posing (automatically) repeated queries.

One classification of datamining models is (Clifton and Thuraisingham, 2001):

- Tree Classification.

- Polynomial Regression.

- General Regression.

- Association rules.

- Neural Networks.

- Clustering.

Nonlinear Regression Methods are based on searching for a dependence of the target variable on other variables in the form of function of some predetermined form. For example, in one of the most successful implementation of algorithms of this type, group attribute accounting method, a dependence is sought in the form of polynomials. Such methods must provide solutions with a larger statistical significance than neural networks do. An obtained formula, a polynomial, is more suitable for analysis and interpreting in principle (in reality it is usually still 
too complex for that). Thus this method is useful in providing reliable solutions in such involved applications as financial markets or medical diagnostics (Megaputer, 2002).

Of these datamining approaches, neural networks and clustering are among the more widely used. Neural networks are computer programs whose architecture is patterned after the neurons in the human brain. It's made up of a web of electronic neurons that send signals to each other through thousands of connections, which are adjusted up or down as the program learns a particular application (Anonymous, 1997). It can be defined as a distributed computational system composed of individual processing nodes that operate in parallel and are interconnected according to a specific architecture. Learning, in the context of neural networks, is the capacity to self-modify connection strengths and processing element parameters (Reilly and Cooper, 1995). Neural networks have a long and interesting history dating back to the 1950s (Darling, 1997). Neural networks are approximation tools that learn the relationships between IVs and DVs, much like regression or other more traditional approaches. The principal difference between neural networks and statistical approaches is that neural networks make no assumptions about the statistical distribution of properties of the data and therefore tend to be more useful in practical situations (Smith and Gupta, 2000).

Types of Neural Network Models include:

- Multilayered feedforward neural networks (MFNN)-appropriate for solving problems that involve learning the relationships between a set of inputs and known outputs. They are a supervised learning technique in the sense that they require a set of training data in order to learn the relationships. The neurons are connected in layers, and the weights are modified throughout the algorithm to reflect the leaning process.

- Hopfield neural networks (HNN)-a fully interconnected system of N neurons where the weights of the network are fixed and symmetric and store information about the memories or stable states of the network. HNNs are principally used to solve optimization problems.

- Self-organizing neural networks (SOFM)-similar to MFNNs but are used as a clustering technique when no training data are available.

Neural networks (MFNNs and SOFMs) are used by most commercial datamining packages such as the SAS Enterprise Miner and the IBM Intelligent Miner. 
The functioning of a biological brain depends on networks of nerve cells, called neurons that are connected with each other by links called synapses. Some of the functions of a biological brain can now be mimicked with computer-based models of neural networks. However, models of neural networks that are simulated in computers are far simpler than the highly complex and often messy neural systems that have been devised by nature (Sejnowski, 1998). Artificial Neural Networks are relatively crude electronic models based on the neural structure of the brain that follows the realization from current research that shows that brains store information as patterns. The term "crude" is utilized with the knowledge that biological human brains have about 100 billion neurons. Each neuron can connect with up to 200,000 other neurons with a typical connection pattern of around 1,000 to 2,000. Biological neurons receive inputs from other sources, combines them in some way, performs a generally nonlinear operation on the result, and then outputs the final result (Anderson and McNeill, 1992). Neural Networks is a large class of diverse systems whose architecture to some extent imitates structure of live neural tissue built from separate neurons. One of the most widespread architectures, multilayered perceptron with back propagation of errors, emulates the work of neurons incorporated in a hierarchical network, where the input of each neuron of the next layer (narrower) is connected with the outputs of all neurons of the previous (wider) layer. Analyzed data are treated as neuron excitation parameters and are fed to inputs of the first layer. These excitations of a lower layer neurons are propagated to the next layer neurons, being amplified or weakened according to weights (numerical coefficients) ascribed to corresponding intraneural connections. As a final result of this process, the single neuron, comprising the topmost neuron layer, acquires some value (excitation strength) which is considered to be a prediction-the reaction of the whole network to the processed data (Megaputer, 2002).

Artificial neurons are called "processing elements" and neural networks are the simple clustering of the primitive artificial neurons. There are two primary ways that neural networks are employed. These are called supervised and unsupervised "training" (Sejnowski, 1998). In supervised training both the inputs and outputs are provided and the network processes the inputs and compares its resulting outputs against the desired outputs. Errors are then propagated back through the system, causing the system to adjust the weights, which control the network. The process is repeated over and over until a threshold is reached. Unsupervised or adaptive training is where the network is provided with inputs but not with desired outputs. The system itself must 
then decide what features it will use to group the input data. This is referred to as selforganization. Another classification of types of neural networks lists the following types (Anderson and McNeill, 1992):

- Networks for prediction-networks that attempt to make projections of the future.

○ Feedforward, Back propagation.

- Delta Bar Delta.

- Extended Delta Bar Delta.

○ Directed Random Search.

○ Functional Link Network.

○ Self-organizing map into back propagation.

- Networks for classification-

○ Learning Vector Quantization.

○ Counter Propagation Network.

- Probabilistic Neural Network.

- Networks for data association-recognition of occurrences of bad data that can span all classifications.

○ Hopfield Network.

○ Boltzmann Machine.

○ Hamming Network.

○ Bi-directional Associative Memory.

- Spatio Temporal Pattern Recognition (Avalanche).

- Networks for Data Conceptualization-provide the ability to group data into classifications.

- Adaptive Resonance Network.

○ Self-organizing Map.

- Networks for data filtering-

- Recirculation.

NNS models may not have a way to determine the output of the model when given a certain input short of "running" the model in a numerical computer simulation. Thus, "experiments" can be run on a model, in ways that are similar in some respects to experiments run on human subjects. 


\subsection{Summary of Literature Review Topics.}

The topics in this literature review are intended to provide a knowledge understanding of many of the concepts that apply to the COMPASS paradigm throughout this dissertation to those not versed in U.S. Army operational concepts, concepts in human factors engineering and research psychology, computer simulation, and certain multivariate statistical approaches. The following paragraphs summarize which topics apply to various sections of this dissertation.

\subsubsection{Literature Review Topics in Phase I.}

The following topics from the literature review that apply to discussions in Phase I include:

- Army Operations Orders.

- Army Battle Command System.

- Battlefield Operating Systems.

- Command and Control.

- TOC.

- MANPRINT.

- War.

- Evaluation of Human Cognitive Performance.

- Ethnography and Naturalistic Observation.

- Human Computer Interface.

- Critical Incident Reporting.

- Task Analysis.

- Cognitive Task Analysis.

- Cluster Analysis.

- Datamining and Neural Networks.

- Sequential Experimentation.

2.6.2. Literature Review Topics in Phase II.

The following topics from the literature review that apply to discussions in Phase II include: 
- Expertise.

- Situation Awareness.

- Stress.

- Decision Making.

- Task Performance.

- Team Performance.

- Workload.

- The System

- Concept of the Model.

- Computer Simulation Models.

- Task Network Modeling.

- Optimization Techniques Using Computer Simulation Models.

- Simulations of Human System Performance.

2.6.3. Literature Review Topics in Discussion Chapter.

The following topics from the literature review that apply to discussions in the Discussion chapter include:

- Command and Control.

- TOC.

- Decision Making.

- Task Performance.

- Team Performance.

- Characteristics of Models. 


\section{Phase I - Ethnographic Based Naturalistically Observed Data Collection And Neural Network Analysis.}

The overall goal of this research is to develop a paradigm of experimental tools and methodologies that can be utilized to provide an evaluation test bed for the examination of future systems that are envisioned to be deployed into a TOC environment. Currently this is not known to exist. While the literature reflects an extensive amount of work that is focused on the description and evaluation of the military $\mathrm{C} 2$ environment, all of the literature surveyed focuses on the human and team performance aspect of this work space, (Cowings, Toscano, DeRoshia, and Tauson, 1999; Dryer, 1998; Ford et al., 1997; Huron, 1997; McGlynn and Pierce, 1997; Rasker, Post, and Schraagen, 2000; Reynolds, 1997), to name a small representative of recent work. This study goes beyond the human performance aspect of the $\mathrm{C} 2 \mathrm{~S}$ and is intended to consider all aspects that make up the total system surrounding the $\mathrm{C} 2$ effort.

\subsection{Method.}

This data collection effort is expected to generate large amounts of raw data with a hundred or more IV and a dozen or more DV through hundreds of observation cycles. First, neural network and datamining procedures are used to identify those IV that are suitable candidates for further experimental evaluation. The experimental environment that will be utilized to evaluate these IV will be similar to previous work (Middlebrooks, 2001) where an existing computer simulation of the TOC system provided the controlled environment whereby these IV can be evaluated.

\subsubsection{Ethnographic Based Naturalistic Observations of TOCs.}

This phase consisted of naturalistic observations and data collection from the operation of brigade TOCs during simulated combat operations. Direct observation of activities during critical incidents that occur during the combat scenario, augmented by impromptu overheard self reporting during critical incident interactions, forms a basis for the collection of performance data. This is especially true when the observer is accepted and, even better, ignored while in the midst of the group being observed as a result of ethnographic and other operator interaction techniques. Because of the size and complexity of the data, procedures were developed to evaluate it using datamining techniques such as neural networks and cluster analysis. The data from the study provided information on 159 IVs and 75 DVs with 164 hourly observations. In 
the future this dataset will grow with each successive TOC observation. A benefit of the datamining approach is that the data can grow longitudinally with the observations from each successive exercise adding to a growing data store.

\subsubsection{Data Collection.}

In order to evolve the ability to determine those things that are of significance to TOCs in general and are not specific to any one TOC, many observations over time are required. Some of these will involve longitudinal observations of the same unit through successive deployments of their TOC. It is noted that Army units conduct training exercises generally for one of three reasons. First, an exercise might be conducted as a pure training event where the unit commander is working to develop and maintain the warfighting skills of the unit. Second, the exercise can be conducted where the unit is being evaluated. Typically, this is the case where an external cadre of evaluators will deploy to the exercise with the unit and observe and evaluate their performance. Third, an exercise might be conducted to test some new weapon system, communications system, or tactical procedure being considered for fielding to the general Army population. This type of exercise is conducted in conjunction with a test and evaluation organization, and/or the new system's developer program manager to determine if the new system performs as designed and required, and if it actually improves the battlefield performance capability of the unit.

The data collection for this effort was able to observe one of each type of these exercises. Exercise Raider Shadow, conducted in April 2002, was a test exercise evaluating the new Tactical Unmanned Aerial Vehicle (TUAV) that was being prepared for fielding to brigade TOCs. Exercise Ironhorse Bonecrusher, conducted in September 2002, was a unit conducted training exercise where the commander conducted the exercise according to his own agenda and training objectives. Exercise Warfighter, conducted in November 2002, was an evaluated exercise where a team from Operations Group B of the Battle Command Training Program (BCTP) from Fort Leavenworth, Kansas deployed to the exercise to evaluate the battlefield proficiency of the unit. All three exercises were conducted at Fort Hood, Texas. While the research in this dissertation observed three TOC deployments, the intent is to develop techniques and procedures that will form the basis for an ongoing effort. As the data store grows through successive TOC observations, the possibility will exist that the simulations will be able to 
relegate to the noise level the interpersonal differences between deployments of individual TOCs and will be able to focus on those core issues that are relevant to TOC operations in general.

Each TOC observation begins weeks before the start of the exercise with the acquisition and assimilation of information that describes how the exercise will be conducted and what its tactical objectives will be. These materials include OPORDs, fragmentary operations orders (FRAGO) as they are developed that modify the original OPORD, tactical map overlays, and intelligence collection plans, for example. In other words, all of the tactical materials that the exercise unit prepares that describe how it intends to conduct combat operations in this simulated training scenario. An example of a brigade OPORD is at Appendix $\mathrm{E}$ for an exercise that was conducted in April 2002 by the $1^{\text {st }}$ Brigade, $4^{\text {th }}$ Infantry Division at Fort Hood, Texas. These OPORDs are supported by numerous annexes and attachments, however, the basic document provides the reader with a feel for the level of detail that goes into the planning for these exercise events which mirror what is anticipated to be required for a real combat operation.

Once the exercise began the TOC was observed in operation by a data gathering effort designed to assimilate as much information as is possible from a totally non-interference effort. This included observations of the activities of the personnel in the TOC along with their interactions with various TOC systems such as the ABCS. It also incorporated aspects of cognitive task analysis techniques to correlate mental processes being employed to the tasks and activities being attempted. One intent during this process was not take any action or make any inquiry of TOC personnel that could potentially cause a change in their mode of operation or what their span of focus and attention is at any one time.

The ability to perform naturalistic observations within the limited space available in a TOC requires equipment that not only is capable of performing the observation but also must be highly portable with self contained power sources. One of the requirements for a non-obtrusive presence in the midst of the human operators within the TOC is that the observer must make little or no demands for resources in terms of power, workspace, furniture, or any other asset that has been placed within the TOC for use in conducting the mission. To be sure, it takes a considerable effort on the part of the $\mathrm{C} 2$ team to set up the workspace and populate it with the tools and equipment required for $\mathrm{C} 2$ operations. Even minor considerations such as sitting down in an empty chair must be avoided as that chair was carried in and placed there for the use of some soldier who has a role to perform in the functioning of the TOC. Along with 
considerations for ethnographic role associations that are established with members of the TOC team, the naturalistic observer team member must be totally self sufficient within the workspace confines in order to blend into the background and achieve the state of having one's presence essentially ignored.

One of the only exceptions to this philosophy is when a member of the $\mathrm{C} 2$ team approaches the observer with inquiries such as what they are doing there. Following ethnographic considerations the observer should treat all direct contact with team members with equal priority regardless of whether they are the highest-ranking commanding officer or the lowest-ranking radio operator. Once the individual has approached the observer on their own volition then the rules of non-interference are superseded by considerations for establishing rapport with the team member. In these cases if a chair or cup of coffee is offered and it would be impolite to refuse, then it should be accepted. These conversations and encounters often provide a rich opportunity to fill in gaps in the knowledge of how the TOC operation is organized and what the current problems and priorities for the $\mathrm{C} 2$ team are. However, once the encounter has run its logical course and the team member returns to his or her duties then the observer should return to the previous non-interference state of presence.

Once the exercise begins observations are made and the data previously described are recorded at selected intervals, usually hourly, in order to develop a longitudinal database. The philosophy is to make observations that can be recorded in a quantitative rather than qualitative manner. The further desire is to record these observations using automated rather than manual techniques. This eliminates any requirement for manual transcription of the data with the resultant time delays, probability for transcription error, and requirement for labor to perform the transcription. It also provides data that is immediately ready for reduction from the raw observed form into data tables and analytical evaluation with statistical and simulation software packages. To accomplish this with totally portable equipment while retaining the ability for the observer to be able to move around in the TOC requires the selection of pseudo-specialized equipment that meets these objectives.

The three exercises observed during this study were: 
3.1.2.1. Exercise Raider Shadow, $1^{\text {st }}$ Brigade, $4^{\text {th }}$ Infantry Division, Fort Hood, Texas, April 2002.

This exercise was conducted by a brigade combat team (BCT) and was performed in conjunction with the Operational Test Command (OTC) at Fort Hood to test the unmanned aerial drone called the Tactical Unmanned Aerial Vehicle (TUAV) prior to its initial deployment to brigades across the Army. The TUAV is designed to provide live video imagery of enemy forces behind the line of contact and to provide the ability to direct artillery fire against them. A picture of the TUAV is at Figure 18 and a sample of its video display is at Figure 19. The exercise was conducted using the wargame simulation JANUS (not an acronym) where live friendly and enemy forces engage each other on an electronic battlefield. The algorithms in JANUS provide the ability to adjudicate all battlefield losses and results. Normally this simulation is used for training purposes in battlefield command and control, however, this exercise also provided the ability to evaluate the new TUAV battlefield asset envisioned to be assigned to the brigade headquarters.

During the course of the two-week exercise there were 74 hourly observations made in the TOC 63 independent measures and 6 dependent measures. Naturalistic observation procedures were followed that employed ethnographic interaction techniques to blend into the TOC environment so that observations of activity occurring within the TOC could be conducted without interfering with the activity being observed.

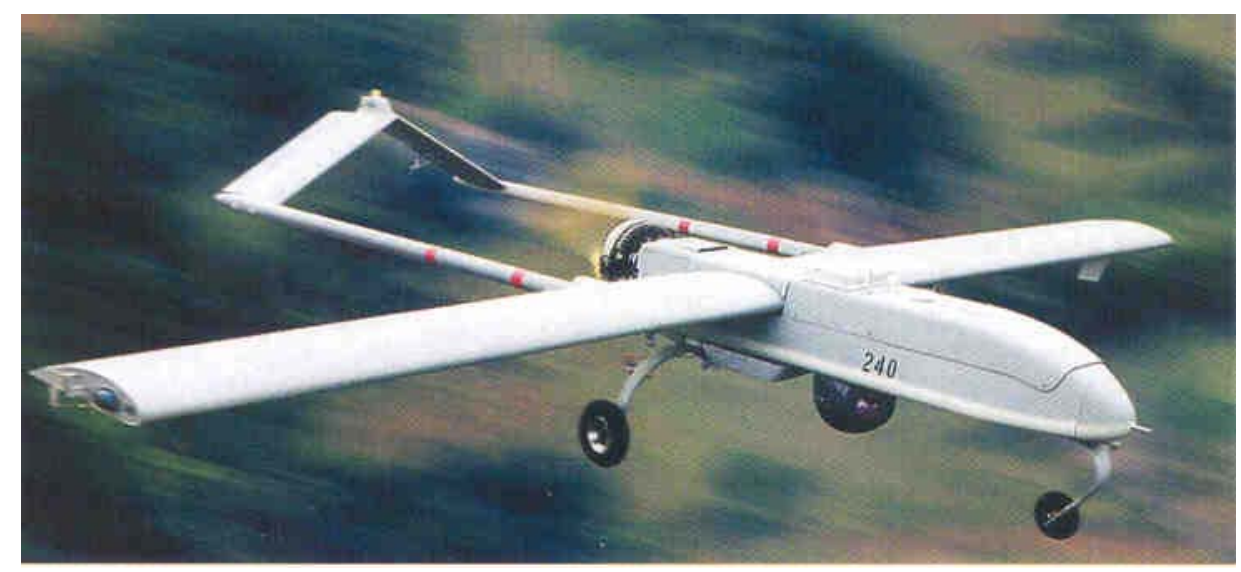

Figure 18 - Tactical Unmanned Aerial Vehicle (TUAV) 


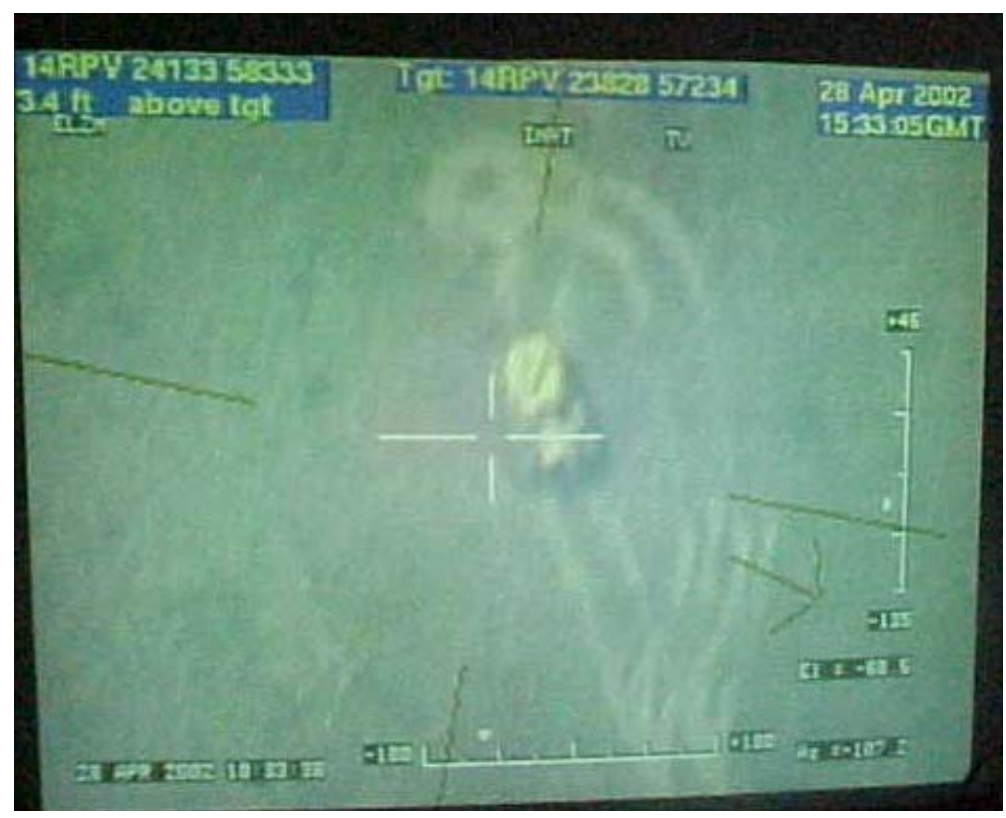

Figure 19 - Example Video Display From TUAV

Before the start of the exercise, OPORDs and other tactical documents was collected that provided an insight as to how the unit intended to react to the tactical scenario. While this exercise was conducted on a 24 hour basis only about eight hours in the day could be observed because of the size of the observation team, which was one. Other demands included the upload of the observed data at the end of the day to the main simulation computer and time required to recharge batteries and care for observation equipment. During the course of the exercise careful attention was paid to ethnographic considerations for interaction with the members of the TOC and after about the $3^{\text {rd }}$ day of the exercise the COMPASS observer's presence was generally ignored except when a TOC member became bored and looked around for someone to talk to.

The independent measures observed fell into several categories. These independent measures provided the set of IVs used in subsequent analyses. The first set was oriented around the first six of seven categories of Army BOSs. These IV and the BOS they relate to are shown in Table 8. 
Table 8 - TOC Observation IVs by BOS

(Ford et al., 1997)

\begin{tabular}{|c|c|}
\hline BOS & Performance Measure \\
\hline Intelligence & $\begin{array}{l}1 \text { Conduct Intelligence Planning } \\
2 \text { Collect Information } \\
3 \text { Process Information } \\
4 \text { Disseminate Intelligence } \\
\end{array}$ \\
\hline Maneuver & $\begin{array}{l}1 \text { Conduct Tactical Movement } \\
2 \text { Engage Enemy with Direct Fire and Maneuver }\end{array}$ \\
\hline Mobility \& Survivability & $\begin{array}{l}1 \text { Overcome Obstacles } \\
2 \text { Enhance Movement } \\
3 \text { Provide Countermobility } \\
4 \text { Enhance Physical Protection } \\
5 \text { Provide Operations Security } \\
6 \text { Conduct Deception } \\
7 \text { Conduct NBC Defense }\end{array}$ \\
\hline Fire Support & $\begin{array}{l}1 \text { Employ Mortars } \\
2 \text { Employ Field Artillery } \\
3 \text { Employ Close Air Support } \\
4 \text { Conduct Electronic Collection and Attack } \\
5 \text { Conduct PSYOP } \\
6 \text { Employ Chemical Weapons } \\
7 \text { Conduct Counter Target Acquisition Operations } \\
8 \text { Employ Naval Surface Fires } \\
9 \text { Coordinate, Synchronize, and Integrate Fire Support }\end{array}$ \\
\hline Air Defense & $\begin{array}{l}1 \text { Take Active Air Defense Measures } \\
2 \text { Take Passive Air Defense Measures }\end{array}$ \\
\hline Command \& Control & $\begin{array}{l}\text { 1 Plan for Combat Operations } \\
2 \text { Direct \& Lead Unit During the Preparation Phase of the Battle } \\
3 \text { Direct \& Lead Unit in Execution of Battle }\end{array}$ \\
\hline
\end{tabular}

The second set of observation IVs evaluated the use of the various factors including communications systems, intelligence collection assets, the presence or absence of key personnel, environmental conditions, and certain miscellaneous factors concerning the operation of the TOC. Table 9 lists these systems. 
Table 9 - TOC Observation IVs by Other Categories

\begin{tabular}{|c|c|}
\hline Category & Performance Measure \\
\hline Communications Usage & $\begin{array}{l}\text { - AFATDS - Advanced Field Artillery Tactical Data System } \\
\text { - AMPS - Aviation Mission Planning System } \\
\text { - Appliqué } \\
\text { - ASAS - All Source Analysis System } \\
\text { - CTIS - The Combat Terrain Information System } \\
\text { - CSSCS - The Combat Service Support and Control System } \\
\text { - FAADC2 - Forward Area Air Defense Command and Control } \\
\text { - FBCB2 - Force XXI Battle Command Brigade and Below } \\
\text { - IMETS - Integrated Meteorological System } \\
\text { - MCS/P - Maneuver Control Systems/Phoenix } \\
\text { - AMDWS. ADA } \\
\text { - CGS. JSTARS }\end{array}$ \\
\hline Intelligence Collection Usage & $\begin{array}{l}\text { - TUAV - Tactical Unmanned Aerial Vehicle } \\
\text { - JSTARS - Joint \& Strategic Tactical Airborne Radar System } \\
\text { - GSR - Ground Surveillance Radar }\end{array}$ \\
\hline Key Personnel Present or Absent & $\begin{array}{l}\text { - Commanding Officer } \\
\text { - Executive Officer } \\
\text { - Battle Captain } \\
\text { - S3 } \\
\text { - S3 RTO } \\
\text { - S2 } \\
\text { - FSO } \\
\text { - Engineer } \\
\text { - ALO }\end{array}$ \\
\hline Environmental Conditions & $\begin{array}{l}\text { - Sky Condition: Clear-1; Overcast-2; Rain-3 } \\
\text { - Wind Condition: 0- none; 1- low; 2- moderate; 3- high } \\
\text { - Light Level, Foot Lamberts - Background at } 20 \mathrm{ft} . \\
\text { - Light Level, Foot Lamberts - Map Display (Rear Projection) at } 20 \mathrm{ft} \text {. } \\
\text { - Noise, dbA } \\
\text { - Dry Bulb Temperature, degrees F } \\
\text { - Wet Bulb Temperature, degrees F } \\
\text { - Relative Humidity, \% } \\
\text { - Hour of the Day } \\
\text { - Day of the Exercise }\end{array}$ \\
\hline Miscellaneous Factors & $\begin{array}{l}\text { - Battle Timing } \\
\text { - Battle Tempo } \\
\text { - Reconnaissance Operations } \\
\text { - Information Operations } \\
\text { - Tactics } \\
\text { - Observed Activity / Stress Level in TOC } \\
\end{array}$ \\
\hline
\end{tabular}

The dependent measures that were observed are those activities that the TOC is trying to perform at the system level. Generally, these measures are the mission oriented tactical activities or their components whose activity the TOC is trying to direct on the battlefield. These observations were made first simply as what activity was being attempted when. They were 
recorded in the three categories of primary task performance, secondary task performance, and tiertiary task performance as being representative of the fact that the TOC work group will usually be performing more than one activity at a time. The first, second, and third performance tasks are observed as those goal oriented activities in progress and their relative priority. These dependent measures become the DVs in the subsequent analyses. Table 10 lists these dependent measures. At the end of the exercise when the TOC performance is rated by either the training group themselves or by external evaluators in the After Action Review (AAR) meeting, the ratings are correlated back to the observations made during the exercise to indicate a measure of success for the TOC system in the performance of its mission activities as indicated by a percentile rating for the appropriate DV.

Table 10 - TOC Dependent Measures of Performance

\begin{tabular}{|l|l|}
\hline \multicolumn{1}{|c|}{ Category } & \multicolumn{1}{c|}{ Performance Measure } \\
\hline & \\
\hline & -1 Departure from the assembly area. \\
& -2 Passage of lines. \\
& -3 Movement to the line of departure. \\
& -4 Breach of main obstacle belt. \\
& -5 Penetration of defensive positions. \\
& -6 Reaction to counterattack forces. \\
& -7 River crossing. \\
& -8 Seizure of key terrain. \\
& -9 Seizure of objective. \\
& -10 Destruction, capture, or bypass of enemy force. \\
& -11 Fixing enemy in position. \\
& -12 Synchronization with supporting forces. \\
& -13 Use of reserves. \\
& -14 Deep operations. \\
& -15 Destruction of first echelon forces. \\
& -16 Destruction of follow-on forces. \\
& -17 Commitment of counterattack forces. \\
& -18 Deception activities. \\
& -19 Rear operations. \\
& -20 Entry into area of operations. \\
& -21 Peacekeeping operations. \\
& -22 Transfer of mission. \\
\hline
\end{tabular}

These preliminary data contained elements that were categorical, binary, and interval in nature and were coded into a spreadsheet for field observation. An excerpt of the final data collection spreadsheet is shown in Figure 20. 


\begin{tabular}{|c|c|c|c|c|c|c|}
\hline & $\mathbf{A}$ & $\mathrm{B}$ & $\mathrm{C}$ & ET & EU & $E V$ \\
\hline 2 & & & Central Standard Time (CST) - & 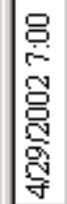 & 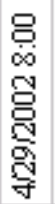 & 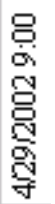 \\
\hline 3 & & & Hour Df The Day: & 7 & 8 & 9 \\
\hline 4 & & & Day Df The Exercise: & 7 & 7 & 7 \\
\hline 12 & Primary Task & & Select Task Number From List:|1 Departure from the assembly area.|2 F & 11 & 11 & 11 \\
\hline 13 & Secondary Task & & Select Task Number From List:|1 Departure from the assembly area.|2 F & 12 & 12 & 12 \\
\hline 14 & Ancillary Task & & Select Task Number From List:|1 Departure from the assembly area.|2 F. & \multirow{2}{*}{\multicolumn{3}{|c|}{${ }^{21} \quad 21 \quad 21$}} \\
\hline 16 & \multicolumn{3}{|c|}{ Battlefield Operating Systems (BDS) } & & & \\
\hline 17 & Intelligence & & Battlefield Function (BF) -|lndicate Which BF Is Being Performed|1 Cor & 2 & 2 & 2 \\
\hline 18 & Maneuver & & Battlefield Function (BF) - |lndicate Which BF Is Being Performed|1Co: & & & \\
\hline 19 & Fire Support & & Battlefield Function (BF) - Indicate W'hich BF Is Being Performed|1Eme & 9 & 9 & 9 \\
\hline 20 & Mobility and Survivability & & Battlefield Function (BF) - Indicate W'Wich BF Is Being Performed|10ut & 5 & 5 & 5 \\
\hline 21 & Air Defense & & Battlefield Function (BF) - |ndicate W'Wich BF Is Being Performed|1 Tal & 2 & 2 & 2 \\
\hline 22 & \multirow{3}{*}{\multicolumn{2}{|c|}{$\begin{array}{l}\text { Command and Control } \\
\text { Communications }\end{array}$}} & Battlefield Function (BF) - Indicate Which BF Is Being Performed|1Pla & \multirow{6}{*}{$\begin{array}{c}3 \\
3 \\
3\end{array}$} & \multirow{6}{*}{3} & \multirow{3}{*}{3} \\
\hline 23 & & & & & & \\
\hline 24 & & & Rate usage of Each System: $1-10,10$ Highest & & & \\
\hline 25 & & 1 & AFATDS - Advanced Field Artillery Tactical Data System & & & 3 \\
\hline 26 & Rate each system & 2 & AMPS - Aviation Mission Planning System & & & \\
\hline 27 & on a scale of 1 (low) & 3 & Appliqué & & & \\
\hline 28 & to 10 (high) as to its & 4 & ASAS - All Source Analysis System & \multirow[t]{4}{*}{3} & \multirow[t]{4}{*}{3} & 3 \\
\hline 29 & level of observed usage & 5 & CTIS - The Combat Terrain Information System & & & \\
\hline 30 & & 6 & CSSCS - The Combat Service Support and Control System & & & \\
\hline 31 & & 7 & FA.ACD2 - Forward Area Air Defense Command and Control & & & \\
\hline 32 & & 8 & FBCB2 - Force XXI Battle Command Brigade and Below & \multirow[t]{2}{*}{1} & \multirow[t]{2}{*}{1} & \multirow[t]{2}{*}{1} \\
\hline 33 & & 9 & IMETS - Integrated Meteorological System & & & \\
\hline 34 & & 10 & MCSIP - Maneuver Control SystemstPhoenix & 2 & 2 & 2 \\
\hline 35 & & 11 & AMDW'ADA & 2 & 2 & 2 \\
\hline 36 & & 12 & CGS. JSTARS & 2 & 2 & 2 \\
\hline 37 & Intelligence Collection & & & & & \\
\hline 38 & & & Rate usage of Each System: 1-10, 10 Highest: & & & \\
\hline 39 & & 1 & TUAV - Tactical Unmanned Aerial Vehicle & 6 & 6 & 6 \\
\hline 40 & & 2 & JSTARS - Joint \& Strategic Tactical Airborne Radar System & 3 & 3 & 3 \\
\hline 41 & & 3 & GSR - Ground Surveillance Radar & 3 & 3 & 3 \\
\hline
\end{tabular}

Figure 20 - Excerpt of Raw Data Collection Spreadsheet

\subsubsection{Exercise Ironhorse Bonecrusher, $1^{\text {st }} \mathrm{BCT}, 1^{\text {st }}$ Cavalry Div., Fort Hood, Texas, Sept., 2002.}

This exercise was an internal training event that was conducted as the unit prepared itself for deployment to the National Training Center (NTC) at Fort Irwin, California. An NTC deployment is the only time a brigade sized unit undergoes full scale field maneuvers against a live opposing force playing the enemy. The NTC exercise rotation provides a full up evaluation of the unit's warfighting capability and will only occur once during the tour of duty for a commander and his staff. This is the most intensive tactical field event that a unit will undergo in peacetime and, as a result, an intensive amount of preparation and training is conducted to prepare for it. Exercise Ironhorse Bonecrusher was just such a preparatory training event. It 
represents one of the few times at Fort Hood that a brigade sized unit will conduct live field training of the entire brigade as opposed to simulations based training.

During the course of the two-week exercise there were 44 hourly observations made in the TOC for 159 independent measures and 75 dependent measures. Naturalistic observation procedures were again followed that employed ethnographic interaction techniques to blend into the TOC environment so that observations of activity occurring within the TOC could be conducted without interfering with the activity being observed. Data was collected and merged into the previous dataset.

\subsubsection{Exercise Warfighter, $1^{\text {st }}$ Brigade, $4^{\text {th }}$ Infantry Division, Fort Hood, Texas, November} 2002.

The division level equivalent to the brigade's NTC rotation is called the Battle Command Training Program (BCTP). Instead of the unit deploying to the exercise location as with the NTC rotation, a BCTP team from Fort Leavenworth, Kansas deploys to the division's location and conducts an evaluation exercise in computer simulation. While the division commander and staff are conducting the exercise on the simulation's electronic battlefield, all three brigade headquarters TOCs from the division deploy to the field to support the exercise. The organization of the exercise is that a cadre from each battalion role plays the activities of their battalion on the computer simulation terminals and report activities and receive command guidance from the brigade TOC who further coordinates with the division TOC. Both the division and all three brigade TOCs are fully deployed to the field and if they did not know that the battle was actually being played out in simulation they might think the battle was in actual progress. This is because at the TOCs in the field the commanders and staffs are receiving communications from role-playing subordinates and actual adjacent and higher headquarters command centers.

This observation was with the first brigade of the division while it was deployed during one of these exercises. During the course of the two-week exercise there were 46 hourly observations made in the TOC for 159 independent measures and 75 dependent measures. Naturalistic observation procedures were again followed that employed ethnographic interaction techniques to blend into the TOC environment so that observations of activity occurring within the TOC could be conducted without interfering with the activity being observed. Data was collected and merged into the previous dataset. 


\subsubsection{JNNS Simulation.}

The neural network simulation used in this study is called Java Neural Network Simulation (JNNS) developed at the University of Tübingen, Wilhelm-Schickard Institute in Tübingen, Germany (Fischer et al., 2001). JNNS runs in a Microsoft Windows environment and is a derivative and subset of a more complete neural network simulation called Stuttgart Neural Network Simulation (SNNS) that executes on Unix based computers. This simulation allows the development of neural networks that can be trained and pruned to reveal relationships to be identified as to the significance of input nodes (corresponding to IV) and output nodes (corresponding to DV). It supports many different learning algorithms and architectures including the feed-forward, back-propagation learning algorithm and multi-layer perceptron architecture used in this dissertation.

For this study the JNNS was configured in a multi-layer perceptron (MLP) architecture with a feed-forward, back-propagation learning algorithm. There where three layers in the network. Input and output layers represented the independent (input) variables, dependent (output layer) variables along with one hidden layer of 30 nodes. Utilizing the information collected from the exercise observations, the independent measures provided data for 159 nodes in the input layer and the dependent measures provided data for 75 nodes in the output layer. The number of hidden layers and the number of nodes in each hidden layer is determined by subjective opinion based upon the following general guidelines:

- There should be as few hidden layers as possible, however, for MLP architectures there must be at least one. If successful training of the network cannot be achieved with one hidden layer then increase the number of layers one at a time until full training can be accomplished.

- The number of nodes in each hidden layer should be as small as possible to create an efficient network. The general guideline is to start with approximately half the number of input nodes. If successful training of the network cannot be achieved with this number then make successive runs with ever-greater numbers of hidden nodes until training can be achieved.

In this case the number of hidden layers was selected as one, and the number of nodes in this layer as 90. Successful training was achieved at approximately 350 epochs with no over training. Successful pruning was achieved at 100 cycles and the pruned results were used to factor screen the input parameters to set up post processing of the results. The remaining IV after pruning were used to generate mathematical models using traditional linear regression 
analysis. The DV to be modeled were selected as those dependent measures that had at least 10 observations in the dataset.

The checklist procedure used to configure and run JNNS for this dataset is at Appendix F. A general description of the process and the results is in the following paragraphs:

\subsubsection{Designing the JNNS Network Architecture.}

JNNS uses a graphical user interface (GUI) to lay out and design neural networks for analysis. The first step is to place all of the input, hidden, and output nodes into a design pallet as shown in Figure 21. There is one input node for each independent measure in either binary or standardized $(0,1)$ form, a set number of hidden nodes according to the network design guidelines previously discussed, and one output node for each dependent measure in either binary or standardized $(0,1)$ form.

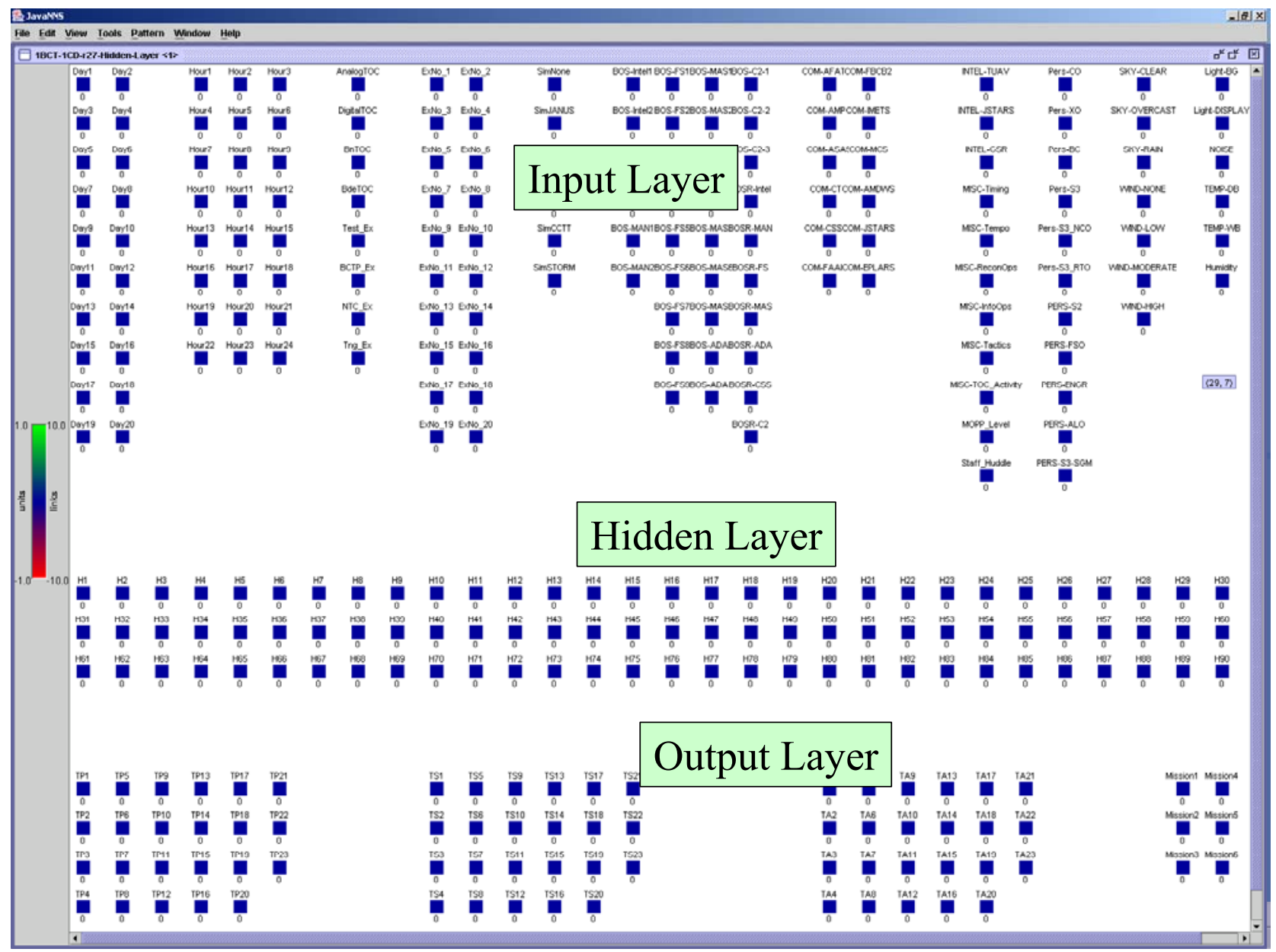

Figure 21 - NNS Node Identification and Network Layout 
The next step is to establish the linkages between the nodes in each layer. While in a biological neural network many nodes or neurons are connected to many other neurons, in an artificial neural network each input node is connected to each hidden layer node that is then forward connected to each output node. The similarity between biological and artificial neural networks and nodes is illustrated in Figure 22.

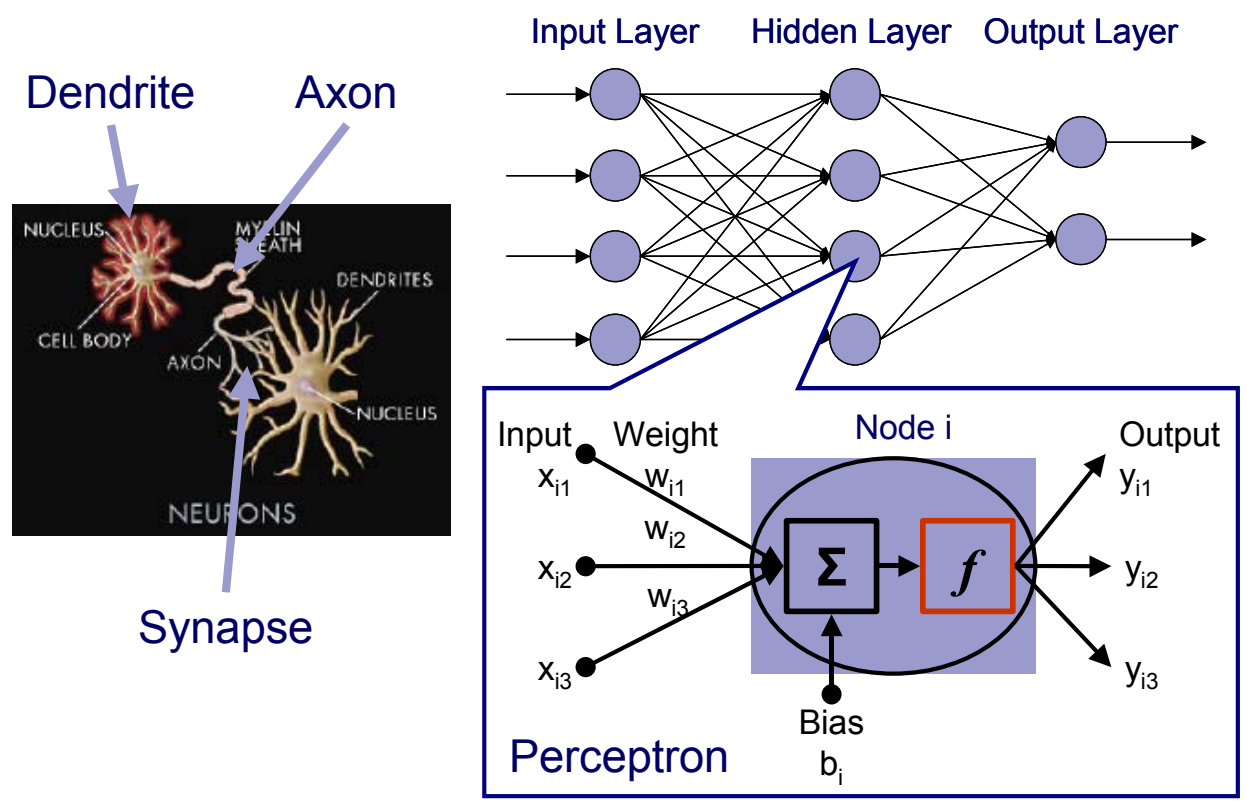

Figure 22 - Comparison of Biological and Artificial Neural Networks

(Kwahk, 2002)

(Used With Permission of the Author)

While each neuron performs a component of the thought process, each artificial node translates the input value weight from the link connecting it to another node according to a predetermined activation or transfer function. Possible activation functions for artificial neural networks is shown in Figure 23. 

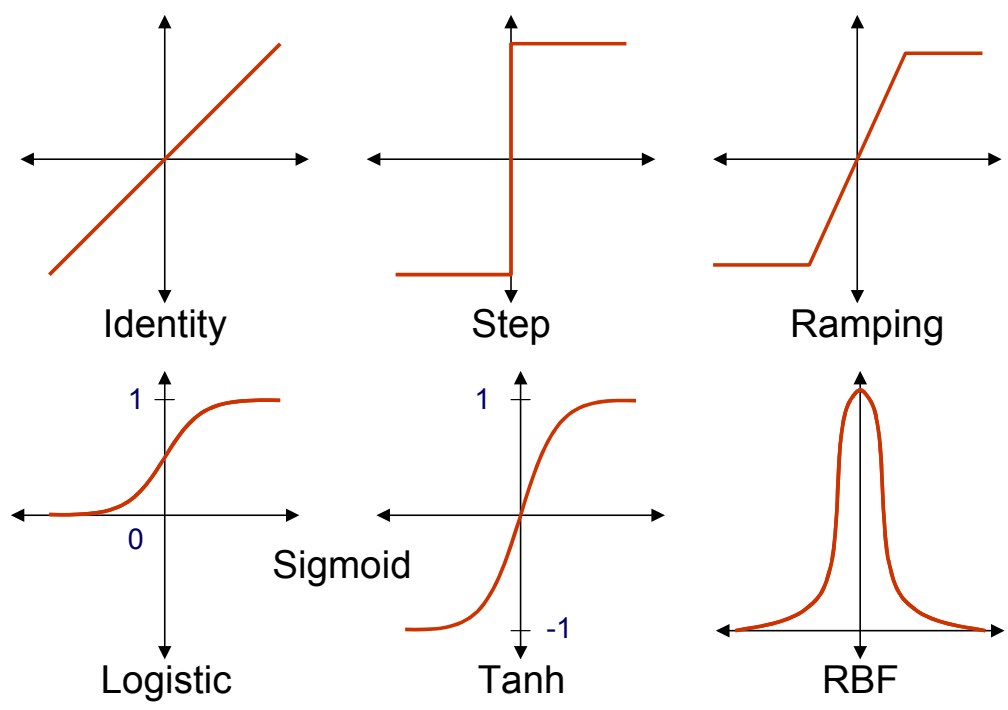

Figure 23 - Artificial Neural Network Activation Functions

(Kwahk, 2002)

(Used With Permission of the Author)

When the command to establish all the interconnecting links is submitted to JNNS it connects each input node to each hidden node and forward connects each hidden node to each output node. Each link value is then initialized with a random number as a starting value to begin the process of training where each training cycle recalculates each link value according to the activation function being performed by the nodes. The initialized preliminary data neural network that is ready for training with JNNS is shown at Figure 24. This and the following neural network diagram figures presented later are acknowledged to be shown at a scale that makes them unreadable, however, they are shown only to provide a graphical view of the complexity of the networks used in this research. 


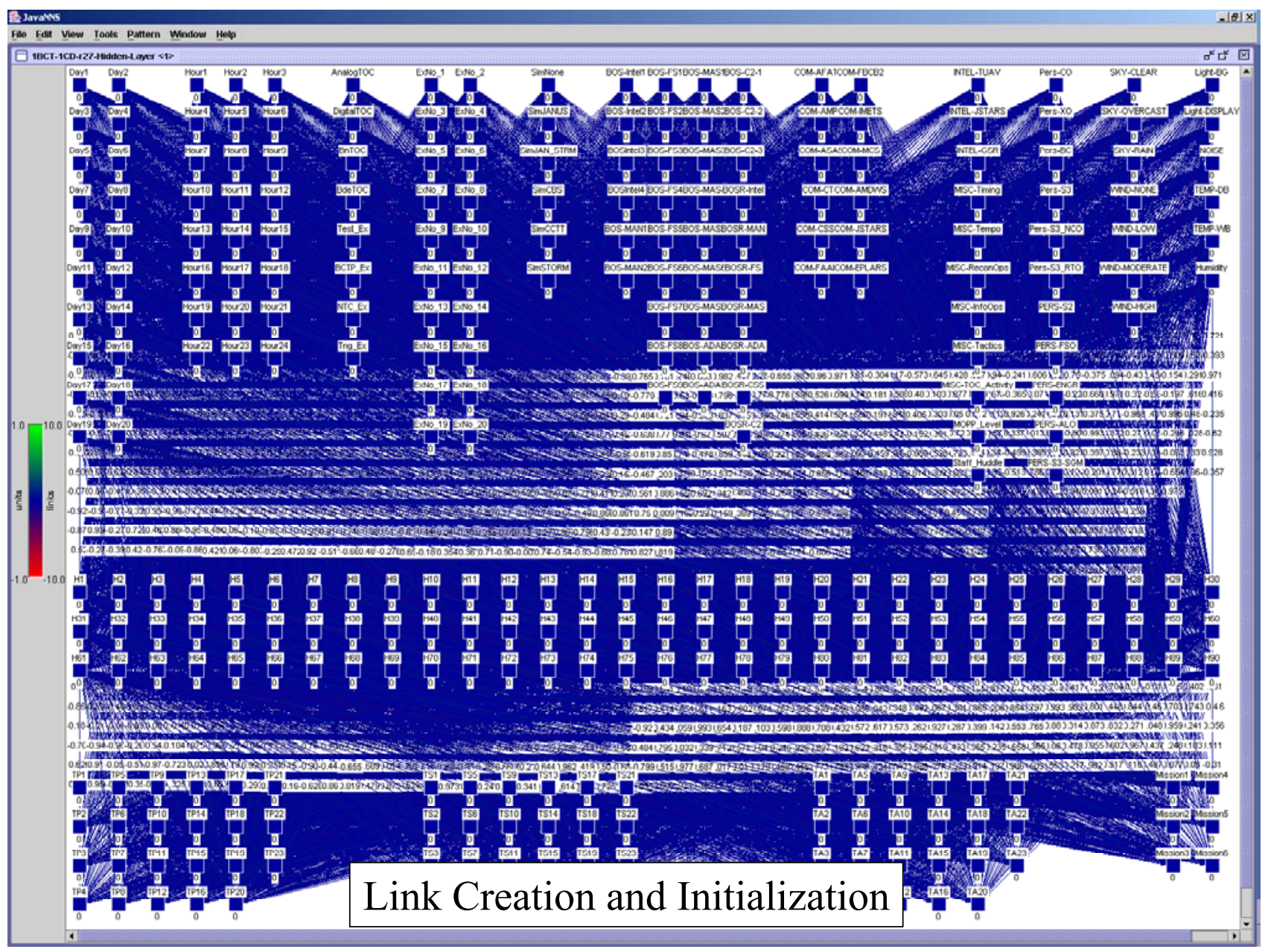

Figure 24 - JNNS TOC Network After Link Creation and Initialization

\subsubsection{Data Preparation.}

Preparation of the exercise data for analysis follows the JNNS requirement that the input data streams be organized into patterns of binary or standardized information. Allowable data types are binary $(0,1)$ and standardized in the range of -1 to +1 or 0 to +1 . The binary data could be transcribed and used in its original form. The interval data such as \% humidity was standardized into a range of 0 to +1 . The categorical data was separated into a subset of binary variables. For example, the variable "Primary Task", had categorical values as shown in Table 11. 
Table 11 - Selection Values For The Variable "Primary Task"

Select Task Number From List:

1 Departure from the assembly area.

2 Passage of lines.

3 Movement to the line of departure.

4 Breach of main obstacle belt.

5 Penetration of defensive positions.

6 Reaction to counterattack forces.

7 River crossing.

8 Seizure of key terrain.

9 Seizure of objective.

10 Destruction, capture, or bypass of enemy force.

11 Fixing enemy in position.

12 Synchronization with supporting forces.

13 Use of reserves.

14 Deep operations.

15 Destruction of first echelon forces.

16 Destruction of follow-on forces.

17 Commitment of counterattack forces.

18 Deception activities.

19 Rear operations.

20 Entry into area of operations.

21 Peacekeeping operations.

22 Transfer of mission.

Figure 20, shown previously, shows that selections of 11, 12, and 21 were made from this selection list during collection of the data. Therefore, three new binary variables were created called Primary_Task_11, Primary_Task_12, and Primary_Task_21 were created and assigned values of 0 or 1 depending on whether or not they were selected during the observation period. Likewise, new binary variables Day1 to Day10 and Hour1 to Hour24 were created to represent 
the day of the exercise and hour of the day that the observation was made. In JNNS terminology each hourly data collection became a pattern of data for each observation. An excerpt of this transcribed preliminary data is shown in Figure 25.

\begin{tabular}{|c|c|c|c|c|c|c|c|c|c|c|c|c|}
\hline & A & $\mathrm{B}$ & $\mathrm{CD}$ & \begin{tabular}{|l|l|}
$D$ & $E$ \\
\end{tabular} & $\mathrm{~F}$ & $\mathbf{G}$ & $\mathrm{H}$ & 1 & 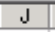 & K & $\mathrm{L}$ & $M$ \\
\hline 1 & & & & 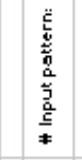 & 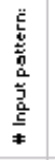 & 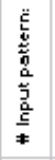 & 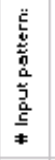 & 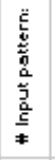 & 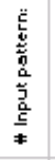 & 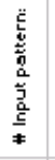 & 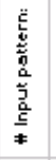 & 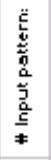 \\
\hline 2 & & \multicolumn{2}{|c|}{ Pattern count-> } & - & $N$ & $m$ & + & $\omega$ & $\omega$ & $m$ & $\infty$ & $\sigma$ \\
\hline 3 & BOS- Intel- BF 2 & Input Node & 1 & 1 & 1 & 1 & 1 & 1 & 1 & 1 & 1 & 1 \\
\hline 4 & BOS-Intel- BF 3 & Input Node & 2 & 0 & 0 & 0 & 0 & 0 & 0 & 0 & 0 & 0 \\
\hline 5 & BOS-FS-BF 2 & Input Node & 3 & 0 & 0 & 0 & 0 & 0 & 0 & 0 & 0 & 0 \\
\hline 6 & BOS-FS-BF 9 & Input Node & 4 & 0 & 0 & 0 & 0 & 0 & 0 & 0 & 0 & 0 \\
\hline 7 & BOS-MAS-BF 5 & Input Node & 5 & 1 & 1 & 1 & 1 & 1 & 1 & 1 & 1 & 0 \\
\hline 8 & BOS-C2-BF 1 & Input Node & 6 & 0 & 0 & 1 & 0 & 0 & 0 & 0 & 0 & 0 \\
\hline 9 & $\mathrm{BOS}-\mathrm{C} 2-\mathrm{BF} 2$ & Input Node & 7 & 1 & 1 & 0 & 1 & 1 & 1 & 1 & 1 & 1 \\
\hline 10 & $\mathrm{BOS}-\mathrm{C} 2 \cdot \mathrm{BF} 3$ & Input Node & 8 & 0 & 0 & 0 & 0 & 0 & 0 & 0 & 0 & 0 \\
\hline 11 & INTTEL-TUAV & Input Node & 9 & 0.3 & 0.3 & 0.3 & 0.4 & 0.4 & 0.0 & 0.0 & 0.0 & 0.4 \\
\hline 12 & INTEL- JSTARS & Input Node & 10 & 0.1 & 0.1 & 0.1 & 0.1 & 0.1 & 0.0 & 0.1 & 0.0 & 0.0 \\
\hline 13 & INTEL-GSR & Input Node & 11 & 0.0 & 0.0 & 0.0 & 0.0 & 0.0 & 0.0 & 0.0 & 0.0 & 0.0 \\
\hline 14 & PERS-CMDR & Input Node & 12 & 0 & 0 & 0 & 0 & 0 & 0 & 0 & 0 & 0 \\
\hline 15 & PERS-XO & Input Node & 13 & 0 & 0 & 0 & 0 & 0 & 0 & 0 & 0 & 0 \\
\hline 16 & PERS-BC & Input Node & 14 & 1 & 1 & 1 & 1 & 1 & 1 & 1 & 1 & 1 \\
\hline 17 & PERS- S3RTO & Input Node & 15 & 0 & 0 & 0 & 0 & 0 & 0 & 0 & 0 & 0 \\
\hline 18 & PERS-S3RTO & Input Node & 16 & 0 & 0 & 0 & 0 & 0 & 0 & 0 & 0 & 0 \\
\hline 19 & PERS- S2 & Input Node & 17 & 1 & 1 & 0 & 1 & 0 & 1 & 1 & 1 & 0 \\
\hline 20 & PERS-FSO & Input Node & 18 & 1 & 1 & 1 & 1 & 1 & 1 & 1 & 1 & 0 \\
\hline 21 & PERS-ENGR & Input Node & 19 & 1 & 1 & 1 & 1 & 1 & 1 & 1 & 1 & 0 \\
\hline 22 & PERS-ALO & Input Node & 20 & 1 & 1 & 1 & 1 & 1 & 0 & 1 & 1 & 0 \\
\hline 23 & SKY-CLEAR & Input Node & 21 & 1 & 1 & 1 & 1 & 1 & 1 & 1 & 1 & 1 \\
\hline 24 & SKY-OVERCAST & Input Node & 22 & 0 & 0 & 0 & 0 & 0 & 0 & 0 & 0 & 0 \\
\hline 25 & WIND- NONE & Input Node & 23 & 1 & 1 & 1 & 0 & 1 & 0 & 0 & 0 & 1 \\
\hline 26 & WIND-LOW & Input Node & 24 & 0 & 0 & 0 & 0 & 0 & 0 & 0 & 1 & 0 \\
\hline 27 & W'NDD-MODERATE & Input Node & 25 & 0 & 0 & 0 & 1 & 0 & 1 & 1 & 0 & 0 \\
\hline 28 & Noise & Input Node & 26 & 0.66 & 0.66 & 0.66 & 0.70 & 0.71 & 0.72 & 0.65 & 0.70 & 0.68 \\
\hline 29 & TEMP.DB & Input Node & 27 & 0.74 & 0.74 & 0.77 & 0.78 & 0.79 & 0.88 & 0.87 & 0.88 & 0.72 \\
\hline 30 & TEMP. WB & Input Node & 28 & 0.70 & 0.70 & 0.71 & 0.71 & 0.71 & 0.73 & 0.73 & 0.73 & 0.68 \\
\hline 31 & Humidity & Input Node & 29 & 0.82 & 0.82 & 0.75 & 0.72 & 0.71 & 0.54 & 0.52 & 0.50 & 0.82 \\
\hline 32 & & & & 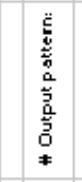 & 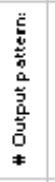 & 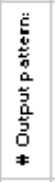 & 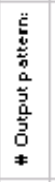 & 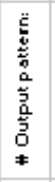 & 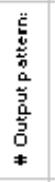 & 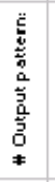 & 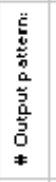 & 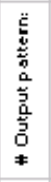 \\
\hline 33 & & \multicolumn{2}{|c|}{ Pattern count-> } & - & $N$ & $m$ & + & $\omega$ & $\omega$ & $m$ & $\infty$ & $\sigma$ \\
\hline 34 & Task Primary 11 & Output Node & 1 & 0 & 0 & 0 & 0 & 0 & 0 & 0 & 0 & 0 \\
\hline 35 & Task Primary 20 & Output Node & 2 & 1 & 1 & 1 & 1 & 1 & 1 & 0 & 0 & 0 \\
\hline 36 & Task Primary 21 & Output Node & 3 & 0 & 0 & 0 & 0 & 0 & 0 & 1 & 1 & 1 \\
\hline 37 & Task Secondary 12 & Output Node & 4 & 0 & 0 & 0 & 0 & 0 & 0 & 0 & 0 & 0 \\
\hline 38 & Task Secondary 19 & Output Node & 5 & 0 & 0 & 0 & 0 & 0 & 0 & 0 & 0 & 0 \\
\hline 39 & Task Ancillary 21 & Output Node & 6 & 0 & 0 & 0 & 0 & 0 & 0 & 0 & 0 & 0 \\
\hline
\end{tabular}

Figure 25 - Transcribed Preliminary Data

Text strings were then added to the spreadsheet to set up the required data text formats for JNNS and the data was transposed to make the hourly collection patterns appear horizontally. A sample of this transposed data is at Figure 26. 


\begin{tabular}{|c|c|c|c|c|c|c|c|c|c|c|c|c|c|c|c|c|c|c|c|c|c|c|c|c|c|}
\hline & $\mathrm{A}$ & $\mathrm{B}$ & C & $\mathrm{D}$ & $E$ & $F$ & $\mathbf{G}$ & $\mathrm{H}$ & 1 & J & $\mathrm{K}$ & $\mathbf{L}$ & 1 & $\mathrm{AD}$ & $\mathrm{AE}$ & AF & $A G$ & $\mathrm{AH}$ & Al & \multicolumn{6}{|c|}{ A. Af Al Al Al AO } \\
\hline 1 & \# Input pattern & 1 & : & 1 & 0 & 0 & 0 & 1 & 0 & 1 & 0 & 0.3 & ¿ & 0.74 & 0.70 & 0.82 & \# Qutput pattern & 1 & : & 0 & 1 & 0 & 0 & 0 & 0 \\
\hline 2 & \# Input pattern & 2 & : & 1 & 0 & 0 & 0 & 1 & 0 & 1 & 0 & 0.3 & c & 0.74 & 0.70 & 0.82 & \# Dutput pattern & 2 & : & 0 & 1 & 0 & 0 & 0 & 0 \\
\hline 3 & \# Input pattern & 3 & $:$ & 1 & 0 & 0 & 0 & 1 & 1 & 0 & 0 & 0.3 & $\mathrm{c}$ & 0.77 & 0.71 & 0.75 & \# Output pattern & 3 & : & 0 & 1 & 0 & 0 & 0 & 0 \\
\hline 4 & \# Input pattern & 4 & . & 1 & 0 & 0 & 0 & 1 & 0 & 1 & 0 & 0.4 & C & 0.78 & 0.71 & 0.72 & \# Dutput pattern & 4 & : & 0 & 1 & 0 & 0 & 0 & 0 \\
\hline 5 & \# Input pattern & 5 & : & 1 & 0 & 0 & 0 & 1 & 0 & 1 & 0 & 0.4 & c & 0.79 & 0.71 & 0.71 & \# Dutput pattern & 5 & : & 0 & 1 & 0 & 0 & 0 & 0 \\
\hline 6 & \# Input pattern & 6 & : & 1 & 0 & 0 & 0 & 1 & 0 & 1 & 0 & 0.0 & 0 & 0.88 & 0.73 & 0.54 & \# Dutput pattern & 6 & : & 0 & 1 & 0 & 0 & 0 & 0 \\
\hline 7 & t pattern & 7 & : & 1 & 0 & 0 & 0 & 1 & 0 & 1 & 0 & 0.0 & ¿ & 0.87 & 0.73 & 0.52 & $\# 0$ & 7 & : & 0 & 0 & 1 & 0 & 0 & 0 \\
\hline 8 & \# Input pattern & 8 & . & 1 & 0 & 0 & 0 & 1 & 0 & 1 & 0 & 0.0 & 0 & 0.88 & 0.73 & 0.50 & ut pattern & 8 & : & 0 & 0 & 1 & 0 & 0 & 0 \\
\hline 9 & \# Input pattern & 9 & : & 1 & 0 & 0 & 0 & 0 & 0 & 1 & 0 & 0.4 & 0 & 0.72 & 0.68 & 0.82 & \# Du & 9 & : & 0 & 0 & 1 & 0 & 0 & 0 \\
\hline 10 & \# Input pattern & 10 & : & 1 & 0 & 0 & 0 & 0 & 0 & 1 & 0 & 0.4 & 0 & 0.73 & 0.68 & 0.78 & ut pattern & 10 & : & 0 & 0 & 1 & 0 & 0 & 0 \\
\hline 11 & $\# \ln$ & 11 & . & 1 & 0 & 0 & 0 & 0 & 0 & 1 & 0 & 0.5 & ¿ & 0.77 & 0.70 & 0.72 & $\# 0$ & 11 & 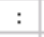 & 0 & 0 & 1 & 0 & 0 & 0 \\
\hline 12 & \# Input pattern & 12 & $:$ & 1 & 0 & 0 & 0 & 0 & 0 & 1 & 0 & 0.5 & c & 0.80 & 0.70 & 0.62 & ut pattern & 12 & . & 0 & 0 & 1 & 0 & 0 & 0 \\
\hline 13 & \# Input pattern & 13 & : & 1 & 0 & 0 & 0 & 0 & 0 & 1 & 0 & 0.5 & c & 0.82 & 0.70 & 0.56 & \# & 13 & : & 0 & 0 & 1 & 0 & 0 & 0 \\
\hline 14 & tern & 14 & $:$ & 1 & 0 & 0 & 0 & 0 & 0 & 1 & 0 & 0.5 & 0 & 0.90 & 0.73 & 0.45 & & 14 & : & 0 & 0 & 1 & 0 & 0 & 0 \\
\hline 15 & \# In & 15 & $:$ & 1 & 0 & 0 & 0 & 0 & 0 & 1 & 0 & 0.5 & C & 0.92 & 0.74 & 0.43 & & 15 & - & 0 & 0 & 1 & 0 & 0 & 0 \\
\hline 16 & \# Input pattern & 16 & $:$ & 1 & 0 & 0 & 0 & 0 & 0 & 1 & 0 & 0.5 & c & 0.92 & 0.74 & 0.43 & ut pattern & 16 & : & 0 & 0 & 1 & 0 & 0 & 0 \\
\hline 17 & ern & 17 & . & 1 & 0 & 0 & 0 & 0 & 0 & 1 & 0 & 0.5 & C & 0.93 & 0.75 & 0.44 & $\#$ & 17 & . & 0 & 0 & 1 & 0 & 0 & 0 \\
\hline 58 & & 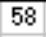 & 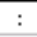 & 0 & 1 & 0 & 1 & 1 & 0 & 0 & 1 & 0.1 & ᄃ & & & & & , & 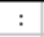 & 1 & 0 & 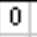 & 1 & 0 & 1 \\
\hline 59 & attern & 59 &. & 1 & 0 & 0 & 1 & 0 & 0 & 0 & 1 & 0.4 & C & 0.76 & 0.70 & 0.75 & ttern & 59 & - & 1 & 0 & 0 & 1 & 0 & 1 \\
\hline 60 & \# Input pattern & 60 & . & 1 & 0 & 0 & 1 & 0 & 0 & 0 & 1 & 0.4 & c & 0.76 & 0.70 & 0.75 & ttern & 60 & : & 1 & 0 & 0 & 1 & 0 & 1 \\
\hline 61 & \# Input pattern & 61 & . & 1 & 0 & 0 & 1 & 0 & 0 & 0 & 1 & 0.5 & C & 0.80 & 0.70 & 0.62 & attern & 61 & : & 1 & 0 & 0 & 1 & 0 & 1 \\
\hline 62 & $\# \ln$ & 62 & $:$ & 1 & 0 & 0 & 1 & 0 & 0 & 0 & 1 & 0.5 & ¿ & 0.80 & 0 & 2 & & 62 & ${ }^{\circ}$ & 1 & 0 & 0 & 1 & 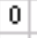 & 1 \\
\hline 63 & $\# \ln$ & 63 & $:$ & 1 & 0 & 0 & 1 & 0 & 0 & 0 & 1 & 0.5 & 0 & 0.85 & 0.72 & 0.54 & & 63 & ${ }^{\circ}$ & 1 & 0 & 0 & 1 & 0 & 1 \\
\hline 64 & \# Input pattern & 64 & : & 1 & 0 & 0 & 1 & 0 & 0 & 0 & 1 & 0.1 & c & 0.92 & 0.75 & 0.46 & \# Dutput & 64 & : & 1 & 0 & 0 & 1 & 0 & 1 \\
\hline 65 & \# Input pattern & 65 &. & 1 & 0 & 0 & 1 & 0 & 0 & 0 & 1 & 0.1 & c & 0.92 & 0.75 & 0.46 & \# Dutput pattern & 65 & : & 1 & 0 & 0 & 1 & 0 & 1 \\
\hline 66 & \# Input pattern & 66 & $:$ & 1 & 0 & 0 & 1 & 0 & 0 & 0 & 1 & 0.5 & 0 & 0.91 & 0.73 & 0.43 & \# & 66 & : & 1 & 0 & 0 & 1 & 0 & 1 \\
\hline 67 & ttern & 67 & $:$ & 1 & 0 & 0 & 1 & 0 & 0 & 0 & 1 & 0.6 & C & 0.90 & 0.76 & 0.54 & \# & 67 & ? & 1 & 0 & 0 & 1 & 0 & 1 \\
\hline 68 & \# Input pattern & 68 & : & 1 & 0 & 0 & 1 & 0 & 0 & 0 & 1 & 0.6 & 0 & 0.77 & 0.71 & 0.75 & \# Dutput & 68 & : & 1 & 0 & 0 & 1 & 0 & 1 \\
\hline 69 & \# Input pattern & 69 & : & 1 & 0 & 0 & 1 & 0 & 0 & 0 & 1 & 0.6 & 0 & 0.83 & 0.73 & 0.63 & \# Dutput pattern & 69 & : & 1 & 0 & 0 & 1 & 0 & 1 \\
\hline 70 & \# Input pattern & 70 & $:$ & 1 & 0 & 0 & 1 & 0 & 0 & 0 & 1 & 0.6 & 0 & 0.83 & 0.73 & 0.63 & \# Dutput pattern & 70 & : & 1 & 0 & 0 & 1 & 0 & 1 \\
\hline 71 & \# Input pattern & 71 & $:$ & 0 & 1 & 0 & 1 & 0 & 0 & 0 & 1 & 0.6 & 0 & 0.86 & 0.75 & 0.61 & \# Dutput pattern & 71 & : & 1 & 0 & 0 & 0 & 1 & 1 \\
\hline 72 & \# Input pattern & 72 & : & 0 & 1 & 0 & 1 & 0 & 0 & 0 & 1 & 0.6 & 0 & 0.79 & 0.67 & 0.55 & \# Dutput pattern & 72 & : & 1 & 0 & 0 & 0 & 1 & 1 \\
\hline 73 & \# Input pattern & 73 & : & 1 & 0 & 0 & 1 & 0 & 0 & 0 & 1 & 0.6 & 0 & 0.77 & 0.66 & 0.57 & \# Dutput pattern & 73 & : & 1 & 0 & 0 & 0 & 1 & 1 \\
\hline 74 & \# Input pattern & 74 & : & 1 & 0 & 0 & 1 & 0 & 0 & 0 & 1 & 0.3 & 0 & 0.77 & 0.66 & 0.57 & \# Dutput pattern & 74 & : & 1 & 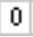 & 0 & 0 & 1 & 1 \\
\hline
\end{tabular}

Figure 26 - Transposed Preliminary Data

The data were then saved as a pure text file with space delimiters between each cell from the spreadsheet. Appropriate JNNS header markings were added, the file extension was changed to .PAT and the file was ready to be read by the JNNS simulation. An excerpt of the final pattern file is at Figure 27. 


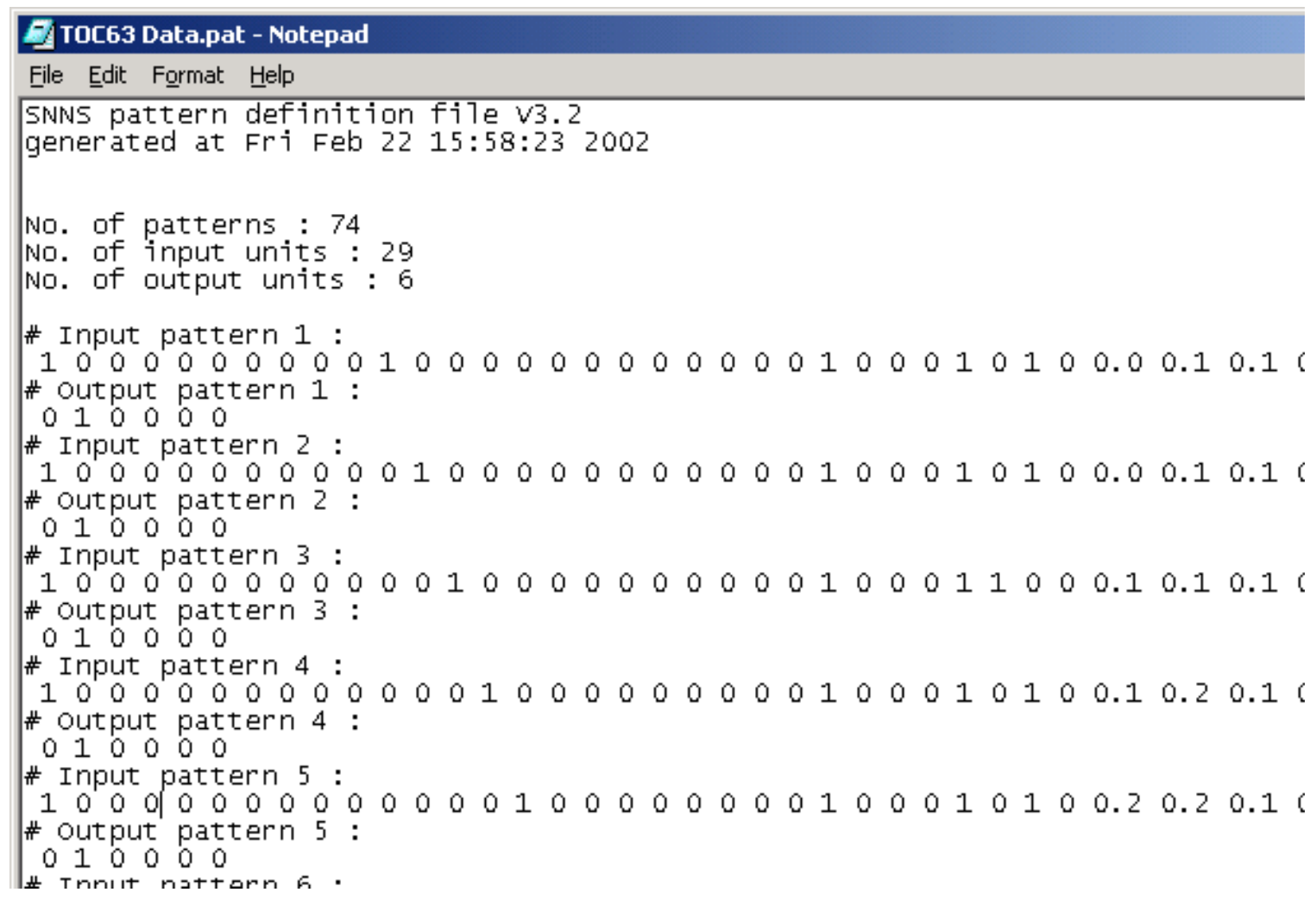

Figure 27 - JNNS Preliminary Pattern Data File

\subsubsection{Apparatus.}

The equipment employed for this research, although highly specialized, consists entirely of off-the-shelf instrumentation, computer hardware, and software programs. Field equipment, including the tablet computer, was procured from commercial sources and each unit met the requirement to operate with self contained power and be completely portable. The analysis computer was custom built using commercially available components with the design goal to configure a hardware and software system with then state of the art capabilities in terms of processor speed, data throughput, and storage capability. The JNNS simulation was available as freeware from its developers. The Micro Saint ${ }^{\mathrm{TM}}$ discrete event simulation system, the CoHOST TOC simulation, and all of the equipment used was provided by funding made available by the U.S. Army Research Laboratory.

\subsubsection{Data Collection.}

The naturalistic observations were made at hourly time intervals and included all activities that could be observed and recorded in the TOC regardless of how seemingly 
insignificant the observation might be. These recorded data included information regarding the current tactical mission, the battlefield operating systems (BOS) in current employment, communications systems usage, intelligence collection efforts in progress, types of activity and relative stress levels in the work group, which key team members are present at the time of the observation, and observable environmental factors. The equipment to perform these observations must have self-contained power and be highly portable. This equipment included:

- A tablet computer running a standard Microsoft Windows operating system and Excel spreadsheet that was used to record the observations which were made on quantitative scales set up for that purpose. The computer was a Fujitsu model Point 510 running Windows 95 with Microsoft Excel installed and is illustrated in Figure 28. It is a compact unit with a touch screen display integrated into the body of the unit. A stylus is used to enter data on the touch screen and handwriting recognition software translates the handwritten entry into computer text for entry into the data file.

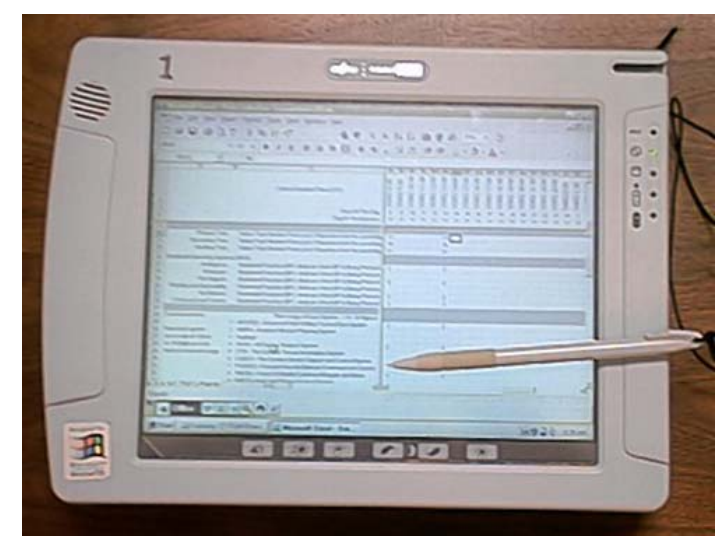

Figure 28 - Fujitsu Touch Screen Tablet Computer

The data file was set up in an Excel spreadsheet. A full listing of the spreadsheet for the first few hours of data collection is at Appendix A.

- A light meter for recording ambient and display light levels in the TOC. Figure 29 shows a Spectra Mini-Spot Silicon Cell Spotmeter by Photo Research / Kollmorgen Corporation. This meter provides a spot reading in foot-lamberts of the targeted surface. 


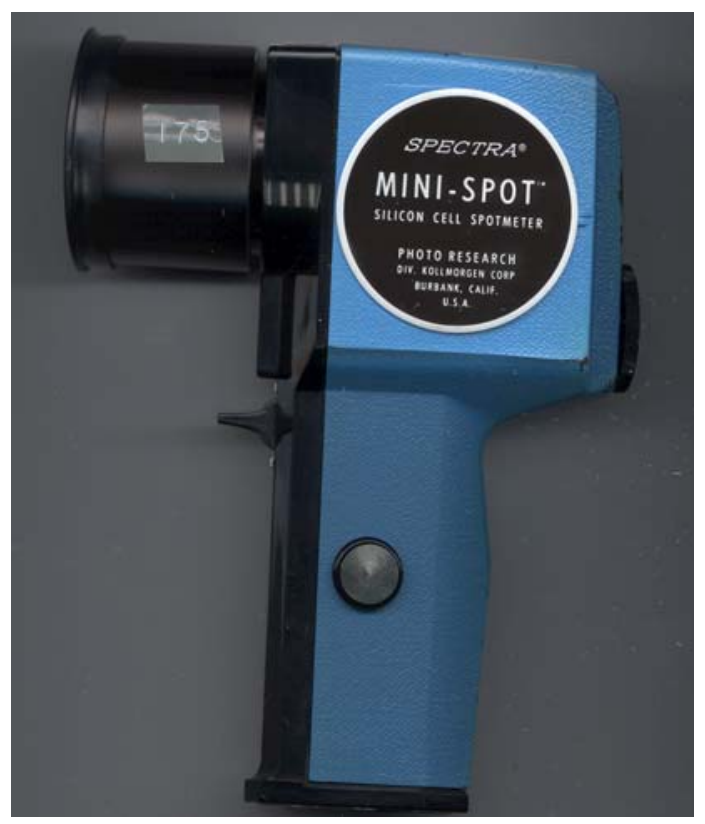

Figure 29 - Spectra Mini-Spot Lightmeter

- A sound meter for recording the ambient noise level within the TOC. Figure 30 shows a Quest Electronics model 215 Sound Level Meter capable of recording noise levels in decibels in either the $\mathrm{A}, \mathrm{B}$, or $\mathrm{C}$ weighted scales in $\mathrm{Db}$ ranges of 30 to +140 . The A weighted scale will be utilized as it most closely matches the frequency response pattern of the human ear (Casali, 2001). 


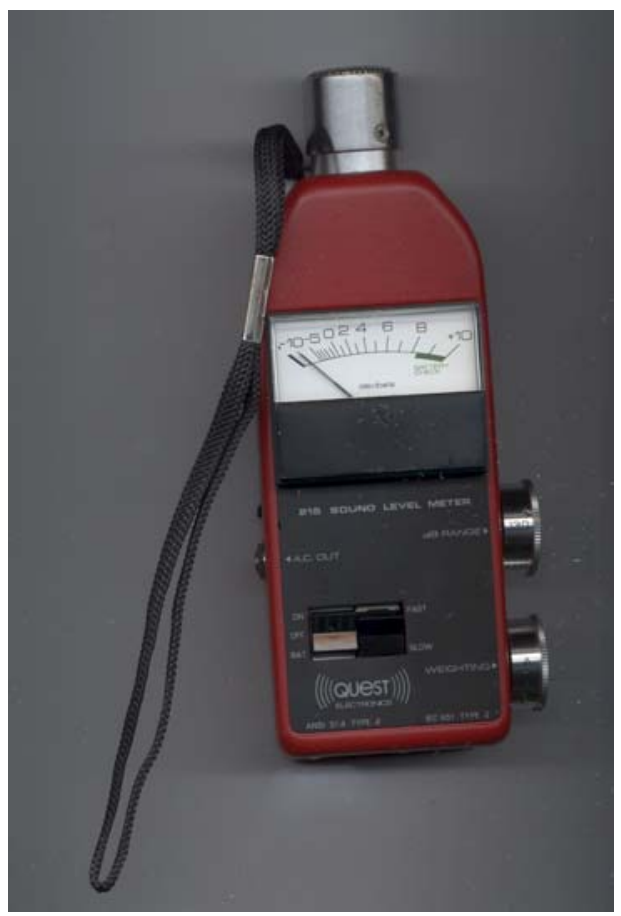

Figure 30 - Quest Electronics Model 215 Sound Level Meter

- A Psychrometer for measuring wet and dry bulb temperature from which relative humidity can be determined. Figure 31 shows a Vista Scientific Corporation psychrometer with identical mercury thermometers. A fabric sock covers one of the thermometer bulbs. The unit has a built in battery operated fan that cools the sock covered bulb when it is moistened to provide the wet bulb reading. A slide rule scale on the side of the unit allows the determination of the relative humidity from the recorded wet and dry bulb readings.

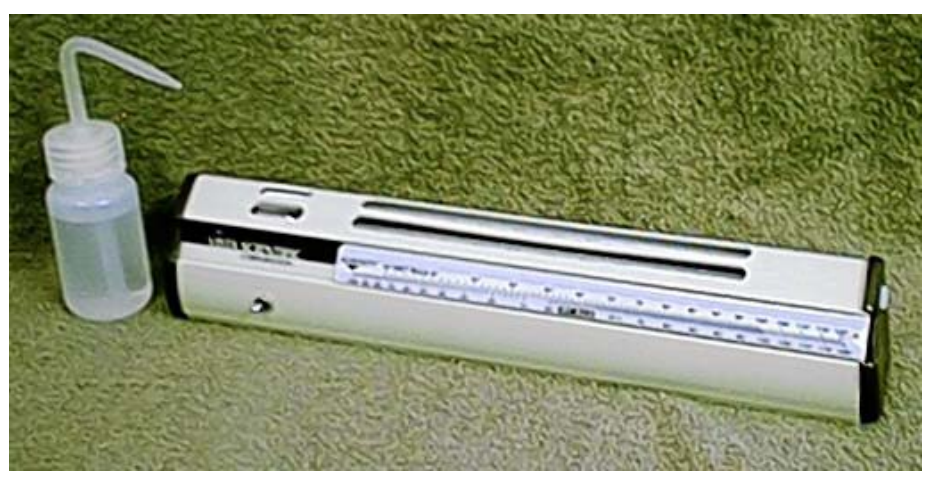

Figure 31 - Vista Scientific Corporation Battery Operated Psychrometer 


\subsubsection{Computer Simulations.}

Because of the potentially large databases and number of required simulation iterations anticipated for the analysis, a state of the art Microsoft Windows ${ }^{\mathrm{TM}}$ based dual processor computer was configured to support the effort. The specifications for this computer system are:

- Dual Pentium IV Xeon processors each with a clock speed of 1.8 GigaHertz (GHZ).

- Bus Rate: 400 MegaHertz (MHZ).

- Memory: 1 GigaBytes (GB) of DDR high speed memory.

- Disk storage: $180 \mathrm{~GB}$ in a striped RAID array.

- Operating System - Windows 2000 Professional $^{\mathrm{TM}}$.

- Dual 21" Monitors each with a screen area of $1600 \times 1200$ pixels at a 16 bit color depth.

\subsection{Results.}

The results of this Phase I study are mathematical models that quantitatively describe the relationship of observed independent performance measures in the TOC against the mission based dependent performance measures of what activities the TOC was trying to accomplish or control on the battlefield. Deviation of these models includes the training and pruning of the neural network followed by generation of the models through traditional linear regression procedures.

\subsubsection{JNNS Network Training.}

In neural network terminology, the term "training" is used to describe the NNS state where it has programmed itself to replicate the set of output conditions that were observed for each input condition in each of the observations in the dataset. Each observation is called a "pattern" of data. Thus, the 164 hourly observations from the 3 exercises that were observed become 164 patterns of input and output data.

The determination of when a network has achieved full training is a subjective one. If the training process is stopped before a 100\% training state has been achieved then the network outputs do not completely represent the observed output states for each input state. If the training process is allowed to continue beyond a 100\% training state then the possibility exists 
that the network will over train itself and start representing network noise. The actual identification of a fully trained network is when the mean square of the error of the outputs is at a minimum.

JNNS provides an error curve display to assist the analyst in identifying the number of epochs to allow the network to run to achieve full training. To effectively utilize the error curve, a portion of the dataset is separated from the rest and is used as a validation dataset. Approximately $25 \%$ of the original data is typically used for this purpose. For this study, as a result, $25 \%$ of the data was randomly selected from the original training dataset and was separated into a validation dataset. During training JNNS displays the error curves from both the training and validation datasets for analysis. If the validation curve flattens to a horizontal line across successive epochs then it is interpreted to mean that the network does not go into an over training state regardless of the number of epochs. If the validation curve starts to increase after an initial decrease, then the point of the increase indicates the number of epochs required for full training and that should not be exceeded to prevent over training. An example of this relationship is shown in an error curve display in Figure 32.

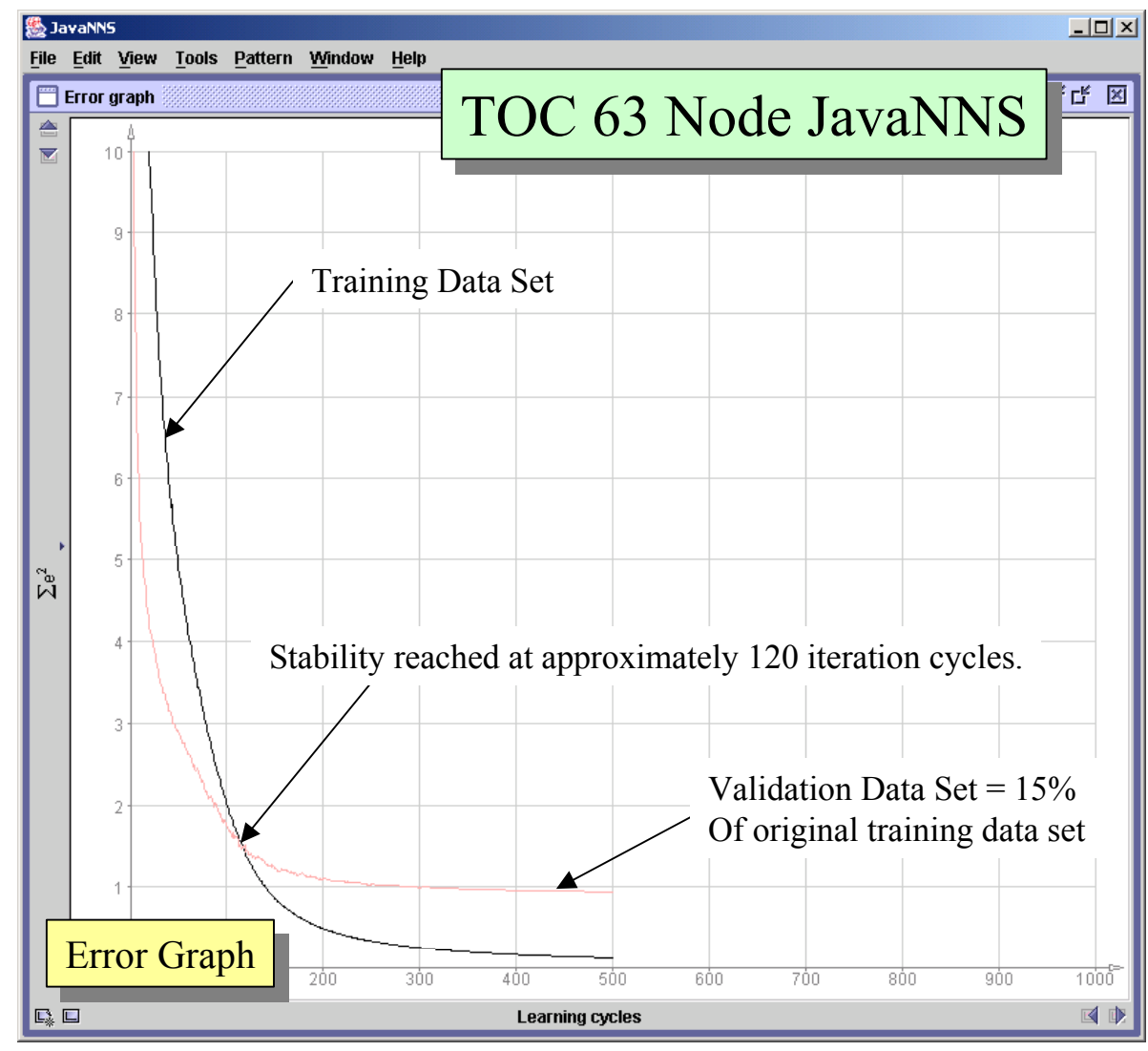

Figure 32 - Example Error Graph for a JNNS Neural Network 
For this network both curves, as shown in Figure 33, approach a flat line over the number of epochs reflecting that the network is stable and will not attempt to over train itself. The dark curve is the error curve from the training dataset and the light line is from the validation dataset. These curves indicate that full training was achieved at approximately 550 epochs for this test and that additional epochs will not cause over training.

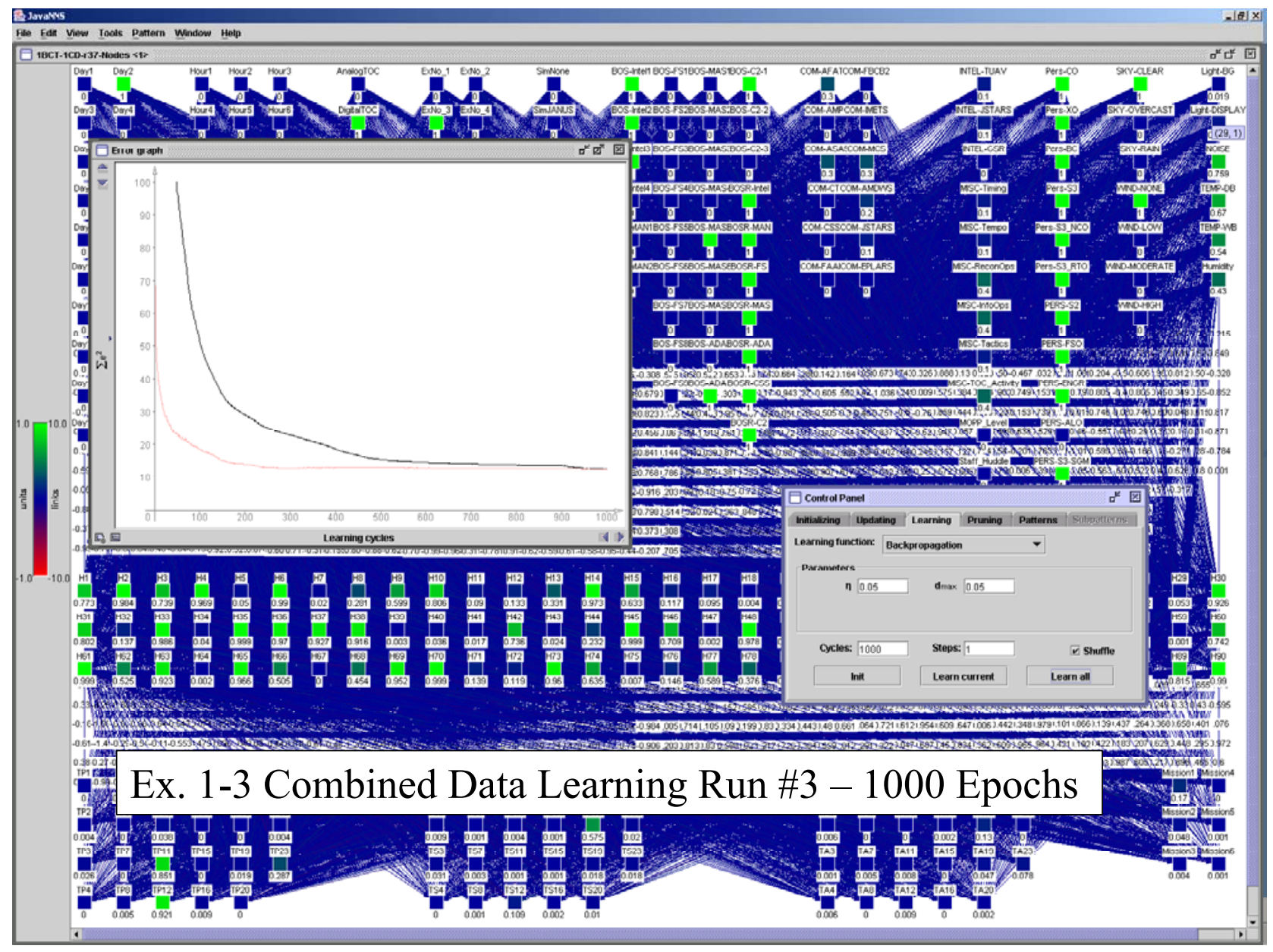

Figure 33 - TOC JNNS Training Network Diagram With Error Curve.

Following completion of the training cycle the network is declared to be fully trained and ready for further analysis. However, to validate the results of the network training run, a correlation analysis can be performed comparing the output node values of the trained network to the values in the original dataset that show the field observations. A sample of the output DV values as 
they were observed in the TOCs is at Table 12, and a sample of the output DV values from the trained JNNS TOC simulation is at Table 13. For reference, the input and trained output DV values from a small study are presented at Appendix G. Casual inspection of Tables 12 and 13 show what appears to be closely matching data where the trained values approach either " 0 " or " 1 " in patterns that match the binary values in the observation data. However, trying to manually compare two tables of 75 columns representing the DVs, and 139 rows representing the 139 observation patterns is not possible. A simple correlation analysis will verify if this relationship is consistent for all the trained patterns thereby validating the accuracy of the trained status of the JNNS simulation.

Table 12 - Sample of Output DV Values From the TOC Observation Data.

\begin{tabular}{|l|c|c|c|c|c|c|c|c|c|c|}
\hline \multicolumn{10}{|c|}{ Observed DV Values } \\
\hline & ITP1 & ITP2 & ITP3 & ITP4 & ITP5 & ITP6 & ITP7 & ITP8 & ITP9 & ITP10 \\
\hline \# Output Pattern 1: & 0 & 0 & 0 & 0 & 0 & 0 & 0 & 0 & 0 & 1 \\
\hline \# Output Pattern 2: & 0 & 0 & 0 & 0 & 0 & 0 & 0 & 0 & 0 & 1 \\
\hline \# Output Pattern 3: & 0 & 0 & 0 & 0 & 0 & 0 & 0 & 0 & 0 & 0 \\
\hline \# Output Pattern 4: & 0 & 0 & 0 & 0 & 0 & 0 & 0 & 0 & 0 & 0 \\
\hline \# Output Pattern 5: & 0 & 0 & 0 & 0 & 0 & 0 & 0 & 0 & 0 & 0 \\
\hline \# Output Pattern 6: & 0 & 0 & 0 & 0 & 0 & 0 & 0 & 0 & 0 & 0 \\
\hline \# Output Pattern 7: & 0 & 0 & 0 & 0 & 0 & 0 & 0 & 0 & 0 & 0 \\
\hline \# Output Pattern 8: & 0 & 0 & 0 & 0 & 0 & 0 & 0 & 0 & 0 & 0 \\
\hline \# Output Pattern 9: & 0 & 0 & 0 & 0 & 0 & 0 & 0 & 0 & 0 & 0 \\
\hline \# Output Pattern 10: & 0 & 0 & 1 & 0 & 1 & 0 & 0 & 0 & 0 & 0 \\
\hline \# Output Pattern 11: & 0 & 0 & 0 & 0 & 0 & 0 & 0 & 0 & 0 & 0 \\
\hline \# Output Pattern 12: & 0 & 0 & 1 & 0 & 0 & 0 & 0 & 0 & 0 & 0 \\
\hline \# Output Pattern 13: & 0 & 0 & 0 & 0 & 0 & 0 & 0 & 0 & 0 & 0 \\
\hline \# Output Pattern 14: & 0 & 0 & 0 & 0 & 0 & 0 & 0 & 0 & 0 & 0 \\
\hline \# Output Pattern 15: & 0 & 0 & 0 & 0 & 0 & 0 & 0 & 0 & 0 & 0 \\
\hline
\end{tabular}


Table 13 - Sample of Output DV Values From the JNNS Trained Data.

\begin{tabular}{|c|c|c|c|c|c|c|c|c|c|c|}
\hline \multicolumn{11}{|c|}{ Trained JNNS DV Values } \\
\hline & OTP1 & OTP2 & OTP3 & OTP4 & OTP5 & OTP6 & OTP7 & OTP8 & OTP9 & OTP10 \\
\hline \#1.1 & 0.008 & 0.014 & 0.025 & 0.012 & 0.036 & 0.000 & 0.031 & 0.020 & 0.013 & 0.984 \\
\hline$\# 2.1$ & 0.005 & 0.040 & 0.029 & 0.002 & 0.027 & 0.002 & 0.016 & 0.013 & 0.002 & 0.989 \\
\hline$\# 3.1$ & 0.002 & 0.003 & 0.010 & 0.002 & 0.012 & 0.001 & 0.019 & 0.004 & 0.017 & 0.004 \\
\hline$\# 4.1$ & 0.004 & 0.000 & 0.006 & 0.000 & 0.000 & 0.002 & 0.002 & 0.011 & 0.003 & 0.000 \\
\hline$\# 5.1$ & 0.006 & 0.000 & 0.004 & 0.000 & 0.000 & 0.008 & 0.001 & 0.008 & 0.003 & 0.000 \\
\hline \#6.1 & 0.004 & 0.001 & 0.009 & 0.000 & 0.000 & 0.013 & 0.000 & 0.001 & 0.002 & 0.042 \\
\hline$\# 7.1$ & 0.003 & 0.002 & 0.011 & 0.002 & 0.012 & 0.001 & 0.022 & 0.003 & 0.022 & 0.004 \\
\hline$\# 8.1$ & 0.001 & 0.001 & 0.050 & 0.001 & 0.008 & 0.006 & 0.001 & 0.010 & 0.001 & 0.000 \\
\hline \#9.1 & 0.004 & 0.000 & 0.013 & 0.002 & 0.000 & 0.004 & 0.005 & 0.050 & 0.003 & 0.000 \\
\hline$\# 10.1$ & 0.001 & 0.020 & 0.951 & 0.005 & 0.962 & 0.012 & 0.006 & 0.002 & 0.007 & 0.049 \\
\hline \#11.1 & 0.002 & 0.000 & 0.011 & 0.002 & 0.000 & 0.002 & 0.013 & 0.012 & 0.016 & 0.000 \\
\hline \#12.1 & 0.003 & 0.001 & 0.950 & 0.003 & 0.013 & 0.007 & 0.000 & 0.003 & 0.005 & 0.034 \\
\hline \#13.1 & 0.004 & 0.008 & 0.000 & 0.027 & 0.026 & 0.002 & 0.017 & 0.003 & 0.005 & 0.026 \\
\hline$\# 14.1$ & 0.006 & 0.001 & 0.003 & 0.001 & 0.000 & 0.005 & 0.002 & 0.007 & 0.008 & 0.000 \\
\hline \#15.1 & 0.004 & 0.000 & 0.022 & 0.001 & 0.001 & 0.012 & 0.001 & 0.010 & 0.001 & 0.000 \\
\hline
\end{tabular}

To perform this correlation analysis the observed data and trained data were concatenated into a single dataset with the observed data variables given a prefix of "I" or input to the analysis and the trained data variables given a prefix of "O" or output to the analysis. This dataset is loaded into SAS and a correlation table generated comparing each input and output variable to each other using a standard Pearson Correlation Coefficient. The SAS run is shown in Appendix H. From this data a t-test is performed between each input-output variable pair to determine if there is a significant correlation at the $\alpha=0.05$ level. A standard decision rule hypothesis is established for the test where the " $t$ " observed value is from the SAS run and the " $t$ " tabled value from a reference source. Table 14 shows the results of the significance determinations for all of the DV Observed-Trained variable pairs.

Table 14 - Pearson Correlation Analysis of Observed To Trained JNNS Outputs

\begin{tabular}{|c|c|c|c|c|l|l|l|l|l|}
\hline & $\begin{array}{c}\text { Input } \\
\text { Variable } \\
\text { Label }\end{array}$ & $\begin{array}{c}\text { Output } \\
\text { Variable } \\
\text { Label }\end{array}$ & $\begin{array}{c}\text { Pearson } \\
\mathbf{r} \\
\text { (From } \\
\text { SAS } \\
\text { Run) }\end{array}$ & $\begin{array}{c}\mathbf{P} \\
\text { value }\end{array}$ & Variable ID & $\mathbf{n}$ & $\begin{array}{c}\boldsymbol{t} \\
\text { Observed }\end{array}$ & $\begin{array}{c}\boldsymbol{t} \\
\text { tabled }\end{array}$ & $\begin{array}{c}\text { Test } \\
\text { Result, } \\
\text { Signif- } \\
\text { icant? }\end{array}$ \\
\hline $\mathbf{1}$ & ITP2 & OTP2 & -0.0515 & 0.5471 & $\begin{array}{l}\text { 2 Passage of } \\
\text { Lines }\end{array}$ & 139 & 0.6036 & 1.97 & No \\
\hline $\mathbf{2}$ & ITP3 & OTP3 & 0.99832 & $<.0001$ & 3 Movement to & 139 & 201.6707 & 1.97 & Yes \\
\hline
\end{tabular}




\begin{tabular}{|c|c|c|c|c|c|c|c|c|c|}
\hline & $\begin{array}{c}\text { Input } \\
\text { Variable } \\
\text { Label }\end{array}$ & $\begin{array}{c}\text { Output } \\
\text { Variable } \\
\text { Label }\end{array}$ & $\begin{array}{c}\text { Pearson } \\
\text { r } \\
\text { (From } \\
\text { SAS } \\
\text { Run) }\end{array}$ & $\begin{array}{c}\mathbf{P} \\
\text { value }\end{array}$ & Variable ID & $\mathbf{n}$ & $\begin{array}{c}t \\
\text { Observed }\end{array}$ & $\begin{array}{c}t \\
\text { tabled }\end{array}$ & $\begin{array}{c}\text { Test } \\
\text { Result, } \\
\text { Signif- } \\
\text { icant? }\end{array}$ \\
\hline & & & & & $\begin{array}{l}\text { the line of } \\
\text { departure. }\end{array}$ & & & & \\
\hline 3 & ITP4 & OTP4 & 0.9857 & $<.0001$ & $\begin{array}{l}4 \text { Breach of main } \\
\text { obstacle belt. }\end{array}$ & 139 & 68.4668 & 1.97 & Yes \\
\hline 4 & ITP5 & OTP5 & 0.99784 & $<.0001$ & $\begin{array}{l}5 \text { Penetration of } \\
\text { defensive } \\
\text { positions. }\end{array}$ & 139 & 177.7927 & 1.97 & Yes \\
\hline 5 & ITP7 & OTP7 & 0.99782 & $<.0001$ & 7 River crossing. & 139 & 176.9726 & 1.97 & Yes \\
\hline 6 & ITP8 & OTP8 & 0.99383 & $<.0001$ & $\begin{array}{l}8 \text { Seizure of key } \\
\text { terrain. }\end{array}$ & 139 & 104.8783 & 1.97 & Yes \\
\hline 7 & ITP10 & OTP10 & 0.99906 & $<.0001$ & $\begin{array}{l}10 \text { Destruction, } \\
\text { capture, or } \\
\text { bypass of enemy } \\
\text { force. }\end{array}$ & 139 & 269.7584 & 1.97 & Yes \\
\hline 8 & ITP11 & OTP11 & 0.99938 & $<.0001$ & $\begin{array}{l}11 \text { Fixing enemy } \\
\text { in position. }\end{array}$ & 139 & 332.2366 & 1.97 & Yes \\
\hline 9 & ITP12 & OTP12 & 0.99855 & $<.0001$ & $\begin{array}{l}12 \\
\text { Synchronization } \\
\text { with supporting } \\
\text { forces. }\end{array}$ & 139 & 217.1144 & 1.97 & Yes \\
\hline 10 & ITP15 & OTP15 & 0.99877 & $<.0001$ & $\begin{array}{l}15 \text { Destruction of } \\
\text { first echelon } \\
\text { forces. }\end{array}$ & 139 & 235.7718 & 1.97 & Yes \\
\hline 11 & ITP22 & OTP22 & 0.99806 & $<.0001$ & $\begin{array}{l}22 \text { Transfer of } \\
\text { mission. }\end{array}$ & 139 & 187.6341 & 1.97 & Yes \\
\hline 12 & ITP23 & OTP23 & 0.99886 & $<.0001$ & 23. Planning & 139 & 244.9183 & 1.97 & Yes \\
\hline 13 & ITS1 & OTS1 & 0.99189 & $<.0001$ & $\begin{array}{l}1 \text { Departure from } \\
\text { the assembly } \\
\text { area. }\end{array}$ & 139 & 91.3441 & 1.97 & Yes \\
\hline 14 & ITS2 & OTS2 & 0.99872 & $<.0001$ & $\begin{array}{l}2 \text { Passage of } \\
\text { lines. }\end{array}$ & 139 & 231.1123 & 1.97 & Yes \\
\hline 15 & ITS5 & OTS5 & 0.99818 & $<.0001$ & $\begin{array}{l}5 \text { Penetration of } \\
\text { defensive } \\
\text { positions. }\end{array}$ & 139 & 193.7386 & 1.97 & Yes \\
\hline 16 & ITS8 & OTS8 & -0.04592 & 0.5914 & $\begin{array}{l}8 \text { Seizure of Key } \\
\text { Terrain }\end{array}$ & 139 & 0.5380 & 1.97 & No \\
\hline 17 & ITS11 & OTS11 & 0.99624 & $<.0001$ & $\begin{array}{l}11 \text { Fixing enemy } \\
\text { in position. }\end{array}$ & 139 & 134.5935 & 1.97 & Yes \\
\hline 18 & ITS12 & OTS12 & 0.99904 & $<.0001$ & $\begin{array}{l}12 \\
\text { Synchronization } \\
\text { with supporting } \\
\text { forces. } \\
\end{array}$ & 139 & 266.9296 & 1.97 & Yes \\
\hline 19 & ITS15 & OTS15 & 0.99642 & $<.0001$ & $\begin{array}{l}15 \text { Destruction of } \\
\text { first echelon } \\
\text { forces. }\end{array}$ & 139 & 137.9543 & 1.97 & Yes \\
\hline 20 & ITS18 & OTS18 & 0.99919 & $<.0001$ & $\begin{array}{l}18 \text { Deception } \\
\text { activities. }\end{array}$ & 139 & 290.6289 & 1.97 & Yes \\
\hline 21 & ITS19 & OTS19 & 0.99836 & $<.0001$ & $\begin{array}{l}19 \text { Rear } \\
\text { operations. }\end{array}$ & 139 & 204.1215 & 1.97 & Yes \\
\hline 22 & ITS20 & OTS20 & 0.98841 & $<.0001$ & 20 Entry into area & 139 & 76.2084 & 1.97 & Yes \\
\hline
\end{tabular}




\begin{tabular}{|c|c|c|c|c|c|c|c|c|c|}
\hline & $\begin{array}{c}\text { Input } \\
\text { Variable } \\
\text { Label }\end{array}$ & $\begin{array}{c}\text { Output } \\
\text { Variable } \\
\text { Label }\end{array}$ & $\begin{array}{c}\text { Pearson } \\
\text { r } \\
\text { (From } \\
\text { SAS } \\
\text { Run) } \\
\end{array}$ & $\begin{array}{c}P \\
\text { value }\end{array}$ & Variable ID & $\mathbf{n}$ & $\begin{array}{c}t \\
\text { Observed }\end{array}$ & $\begin{array}{c}t \\
\text { tabled }\end{array}$ & $\begin{array}{c}\text { Test } \\
\text { Result, } \\
\text { Signif- } \\
\text { icant? }\end{array}$ \\
\hline & & & & & of operations. & & & & \\
\hline 23 & ITS22 & OTS22 & 0.99121 & $<.0001$ & $\begin{array}{l}22 \text { Transfer of } \\
\text { mission. }\end{array}$ & 139 & 87.6946 & 1.97 & Yes \\
\hline 24 & ITS23 & OTS23 & 0.99863 & $<.0001$ & 23. Planning & 139 & 223.3770 & 1.97 & Yes \\
\hline 25 & ITA2 & OTA2 & 0.98799 & $<.0001$ & $\begin{array}{l}2 \text { Passage of } \\
\text { lines. }\end{array}$ & 139 & 74.8401 & 1.97 & Yes \\
\hline 26 & ITA3 & OTA3 & 0.99872 & $<.0001$ & $\begin{array}{l}3 \text { Movement to } \\
\text { the line of } \\
\text { departure. }\end{array}$ & 139 & 231.1123 & 1.97 & Yes \\
\hline 27 & ITA7 & OTA7 & 0.99755 & $<.0001$ & 7 River crossing. & 139 & 166.9026 & 1.97 & Yes \\
\hline 28 & ITA10 & OTA10 & 0.99663 & $<.0001$ & $\begin{array}{l}10 \text { Destruction, } \\
\text { capture, or } \\
\text { bypass of enemy } \\
\text { force. }\end{array}$ & 139 & 142.2101 & 1.97 & Yes \\
\hline 29 & ITA11 & OTA11 & 0.9975 & $<.0001$ & $\begin{array}{l}11 \text { Fixing enemy } \\
\text { in position. }\end{array}$ & 139 & 165.2189 & 1.97 & Yes \\
\hline 30 & ITA12 & OTA12 & 0.99632 & $<.0001$ & $\begin{array}{l}12 \\
\text { Synchronization } \\
\text { with supporting } \\
\text { forces. }\end{array}$ & 139 & 136.0568 & 1.97 & Yes \\
\hline 31 & ITA18 & OTA18 & 0.99792 & $<.0001$ & $\begin{array}{l}18 \text { Deception } \\
\text { activities. }\end{array}$ & 139 & 181.1904 & 1.97 & Yes \\
\hline 32 & ITA19 & OTA19 & 0.99483 & $<.0001$ & $\begin{array}{l}19 \text { Rear } \\
\text { operations. }\end{array}$ & 139 & 114.6597 & 1.97 & Yes \\
\hline 33 & ITA21 & OTA21 & 0.99931 & $<.0001$ & $\begin{array}{l}21 \text { Peacekeeping } \\
\text { operations. }\end{array}$ & 139 & 314.9169 & 1.97 & Yes \\
\hline 34 & ITA23 & OTA23 & 0.99915 & $<.0001$ & 23. Planning & 139 & 283.6997 & 1.97 & Yes \\
\hline 35 & IM1 & OM1 & 0.99813 & $<.0001$ & $\begin{array}{l}\text { 1- Pre Operations } \\
\text { Planning }\end{array}$ & 139 & 191.1238 & 1.97 & Yes \\
\hline 36 & IM2 & OM2 & 0.99073 & $<.0001$ & $\begin{array}{l}\text { 2- Movement to } \\
\text { Contact }\end{array}$ & 139 & 85.3630 & 1.97 & Yes \\
\hline 37 & IM3 & OM3 & 0.99664 & $<.0001$ & 3-Attack & 139 & 142.4227 & 1.97 & Yes \\
\hline 38 & IM4 & OM4 & 0.96609 & $<.0001$ & 4- Defense & 139 & 43.7938 & 1.97 & Yes \\
\hline 39 & IM5 & OM5 & 0.99683 & $<.0001$ & 5- River Crossing & 139 & 146.6497 & 1.97 & Yes \\
\hline
\end{tabular}

Table 14 shows a high correlation for all of the DV input-output pairs except ITP2-OTP2 Passage of Lines, and ITS8-OTS8, Seizure of Key Terrain. An examination of the original observation data for these two variable pairs provides the explanation of their non-correlation. During the original observation of the TOC exercises these two variables were only observed to occur two or three times with the result that there was insufficient data for the JNNS training algorithm to achieve significance.

This correlation analysis provides a high degree confidence in the accuracy of the state of training in the JNNS simulation as the analysis proceeds to the pruning stage. 


\subsubsection{JNNS Network Pruning.}

Pruning, in neural network terminology, is the process of identifying those input nodes that are predicted to be less significant than others in contributing to the performance of the network in the replication of the trained network to the observed data. The training process previously performed has tuned the NNS simulation model to represent the interactions between the input nodes and the output nodes to a certain acceptance level. Pruning is where nodes are eliminated according to a performance threshold. Through multiple pruning iterations nodes are identified that are potential candidates to be declared less important than other nodes. Figure 34 shows an example of the JNNS TOC network after a successful pruning operation.

Superimposed on the chart are the threshold parameters set into JNNS for the run. Those nodes that have been eliminated are shown with their links removed.

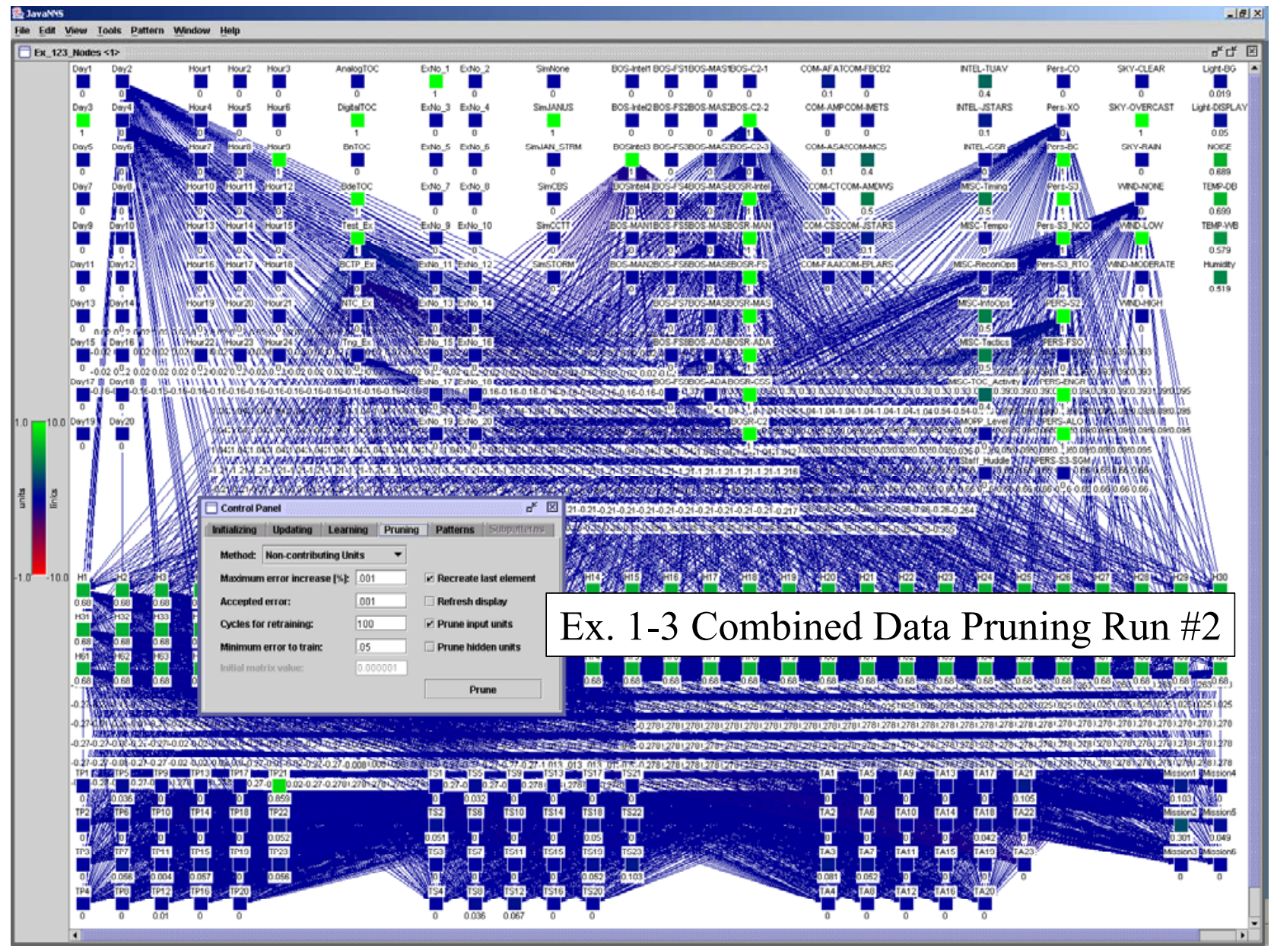

Figure 34 - JNNS TOC Neural Network Diagram After Pruning 
While this display is visually informative, the exact state of the node relationships is indicated by text results files. During execution JNNS saves its network design into ASCII text files that includes a bias parameter containing the calculated link coefficient determined during training. During the pruning stage if the node is eliminated then this bias coefficient is set to zero. Table 15 shows the bias information for this network before and after pruning. 
Table 15 - JNNS TOC Simulation Bias After Pruning

\begin{tabular}{|c|c|c|c|}
\hline Node no. & Unit Name & Trained bias & Pruned bias \\
\hline 1 & Day1 & -0.98737 & -0.9975 \\
\hline 2 & Day2 & -0.92248 & 0.12717 \\
\hline 3 & Day3 & 0.41398 & -0.61339 \\
\hline 4 & Day4 & -0.56047 & 0.61748 \\
\hline 5 & Day5 & -0.62572 & 0.17002 \\
\hline 6 & Day6 & -0.81347 & -0.04025 \\
\hline 7 & Day7 & -0.05356 & -0.29942 \\
\hline 8 & Day8 & 0.93939 & 0.79192 \\
\hline 9 & Day9 & -0.44438 & 0.64568 \\
\hline 10 & Day10 & -0.31748 & 0.49321 \\
\hline 11 & Day11 & 0.09232 & 0 \\
\hline 12 & Day12 & -0.66192 & 0 \\
\hline 13 & Day13 & 0.63536 & 0 \\
\hline 14 & Day14 & -0.73986 & 0 \\
\hline 15 & Day15 & -0.02396 & 0 \\
\hline 16 & Day16 & 0.63854 & 0 \\
\hline 17 & Day17 & -0.52257 & 0 \\
\hline 18 & Day18 & -0.28916 & 0 \\
\hline 19 & Day19 & 0.07138 & 0 \\
\hline 20 & Day20 & 0.87304 & 0 \\
\hline 21 & Hour1 & 0.62236 & 0 \\
\hline 22 & Hour2 & 0.50078 & 0 \\
\hline 23 & Hour3 & -0.28043 & 0 \\
\hline 24 & Hour4 & -0.19962 & 0 \\
\hline 25 & Hour5 & 0.44682 & 0 \\
\hline 26 & Hour6 & 0.53917 & 0 \\
\hline 27 & Hour7 & -0.92743 & 0.06333 \\
\hline 28 & Hour8 & -0.67498 & 0.14237 \\
\hline 29 & Hour9 & -0.84307 & 0.20353 \\
\hline 30 & Hour10 & 0.45128 & 0.21433 \\
\hline 31 & Hour11 & -0.47478 & -0.66753 \\
\hline 32 & Hour12 & 0.45109 & 0.32609 \\
\hline 33 & Hour13 & -0.82531 & -0.09842 \\
\hline 34 & Hour14 & -0.33256 & -0.29575 \\
\hline 35 & Hour15 & -0.30235 & -0.88592 \\
\hline 36 & Hour16 & -0.41539 & 0.21537 \\
\hline 37 & Hour17 & -0.51799 & 0.56664 \\
\hline 38 & Hour18 & -0.35899 & 0.60521 \\
\hline 39 & Hour19 & -0.96869 & 0 \\
\hline 40 & Hour20 & -0.00424 & 0 \\
\hline 41 & Hour21 & 0.48454 & 0 \\
\hline 42 & Hour22 & -0.49205 & 0 \\
\hline 43 & Hour23 & 0.06101 & 0 \\
\hline 44 & Hour24 & -0.74664 & 0 \\
\hline 45 & AnalogTOC & 0.95685 & 0 \\
\hline 46 & DigitalTOC & -0.49998 & 0 \\
\hline 47 & BnTOC & -0.57561 & 0 \\
\hline 48 & BdeTOC & 0.4453 & 0 \\
\hline
\end{tabular}




\begin{tabular}{|c|c|c|c|}
\hline Node no. & Unit Name & Trained bias & Pruned bias \\
\hline 49 & Test Ex & -0.36729 & 0.72448 \\
\hline 50 & ВСТP Ex & 0.4246 & -0.5808 \\
\hline 51 & NTC Ex & 0.67315 & 0 \\
\hline 52 & Tng Ex & -0.00436 & 0.68731 \\
\hline 53 & ExNo 1 & -0.99768 & 0 \\
\hline 54 & ExNo 2 & 0.52312 & 0 \\
\hline 55 & ExNo 3 & 0.94678 & 0 \\
\hline 56 & ExNo 4 & -0.10709 & 0 \\
\hline 57 & ExNo 5 & -0.12125 & 0 \\
\hline 58 & ExNo 6 & 0.02145 & 0 \\
\hline 59 & ExNo 7 & 0.5728 & 0 \\
\hline 60 & ExNo 8 & -0.85998 & 0 \\
\hline 61 & ExNo 9 & 0.29673 & 0 \\
\hline 62 & ExNo 10 & 0.68365 & 0 \\
\hline 63 & ExNo 11 & -0.3455 & 0 \\
\hline 64 & ExNo 12 & 0.24058 & 0 \\
\hline 65 & ExNo 13 & -0.69433 & 0 \\
\hline 66 & ExNo 14 & 0.68236 & 0 \\
\hline 67 & ExNo 15 & 0.71026 & 0 \\
\hline 68 & ExNo 16 & -0.46886 & 0 \\
\hline 69 & ExNo_17 & -0.7796 & 0 \\
\hline 70 & ExNo_18 & 0.09799 & 0 \\
\hline 71 & ExNo_19 & -0.57469 & 0 \\
\hline 72 & ExNo_20 & -0.82434 & 0 \\
\hline 73 & SimNone & 0.89038 & 0 \\
\hline 74 & SimJANUS & -0.64043 & 0 \\
\hline 75 & SimJAN_STRM & 0.58007 & 0 \\
\hline 76 & SimCBS & -0.36912 & 0 \\
\hline 77 & SimCCTT & -0.44829 & 0 \\
\hline 78 & SimSTORM & -0.0607 & 0 \\
\hline 79 & BOS-Intel1 & -0.78387 & 0.48888 \\
\hline 80 & BOS-Intel2 & 0.39775 & -0.78344 \\
\hline 81 & BOSIntel3 & -0.17637 & 0.1981 \\
\hline 82 & BOSIntel4 & -0.3867 & -0.22953 \\
\hline 83 & BOS-MAN1 & 0.10361 & 0.47002 \\
\hline 84 & BOS-MAN2 & -0.1298 & 0.21793 \\
\hline 85 & BOS-FS1 & -0.58824 & 0 \\
\hline 86 & BOS-FS2 & -0.39818 & -0.27732 \\
\hline 87 & BOS-FS3 & -0.42888 & 0 \\
\hline 88 & BOS-FS4 & 0.09452 & 0 \\
\hline 89 & BOS-FS5 & 0.0809 & 0 \\
\hline 90 & BOS-FS6 & -0.09977 & 0 \\
\hline 91 & BOS-FS7 & 0.7622 & 0.03421 \\
\hline 92 & BOS-FS8 & -0.74938 & 0 \\
\hline 93 & BOS-FS9 & -0.5399 & 0.5031 \\
\hline 94 & BOS-MAS1 & 0.1146 & -0.30888 \\
\hline 95 & BOS-MAS2 & 0.37938 & -0.66204 \\
\hline 96 & BOS-MAS3 & -0.33091 & 0 \\
\hline 97 & BOS-MAS4 & 0.24223 & -0.01621 \\
\hline 98 & BOS-MAS5 & 0.11295 & -0.87292 \\
\hline 99 & BOS-MAS6 & -0.02036 & 0.39952 \\
\hline 100 & BOS-MAS7 & 0.19572 & 0.00961 \\
\hline
\end{tabular}




\begin{tabular}{|c|c|c|c|}
\hline Node no. & Unit Name & Trained bias & Pruned bias \\
\hline 101 & BOS-ADA1 & 0.34001 & -0.70501 \\
\hline 102 & BOS-ADA2 & -0.19413 & 0.89917 \\
\hline 103 & BOS-C2-1 & -0.11728 & -0.71685 \\
\hline 104 & BOS-C2-2 & 0.71606 & 0.81024 \\
\hline 105 & BOS-C2-3 & 0.56273 & 0.38578 \\
\hline 106 & BOSR-Intel & 0.36876 & -0.3939 \\
\hline 107 & BOSR-MAN & 0.75542 & 0 \\
\hline 108 & BOSR-FS & -0.34721 & 0 \\
\hline 109 & BOSR-MAS & -0.62645 & 0.93323 \\
\hline 110 & BOSR-ADA & 0.21708 & 0 \\
\hline 111 & BOSR-CSS & 0.93377 & 0 \\
\hline 112 & BOSR-C2 & 0.24827 & 0 \\
\hline 113 & COM-AFATDS & -0.70714 & 0.64336 \\
\hline 114 & COM-AMPS & 0.0491 & 0 \\
\hline 115 & COM-ASAS & 0.54698 & -0.6173 \\
\hline 116 & COM-CTIS & 0.4445 & 0 \\
\hline 117 & COM-CSSCS & 0.50725 & 0 \\
\hline 118 & COM-FAADC2 & 0.2667 & 0 \\
\hline 119 & COM-FBCB2 & 0.50334 & -0.68889 \\
\hline 120 & COM-IMETS & 0.17344 & 0 \\
\hline 121 & COM-MCS & 0.24894 & 0.46403 \\
\hline 122 & COM-AMDWS & -0.9118 & -0.18882 \\
\hline 123 & COM-JSTARS & -0.59697 & 0 \\
\hline 124 & COM-EPLARS & 0.87005 & 0 \\
\hline 125 & INTEL-TUAV & -0.49297 & 0.36448 \\
\hline 126 & INTEL-JSTARS & 0.6646 & 0 \\
\hline 127 & INTEL-GSR & 0.43852 & 0 \\
\hline 128 & MISC-Timing & -0.97967 & -0.04941 \\
\hline 129 & MISC-Tempo & -0.36699 & -0.75396 \\
\hline 130 & MISC-ReconOps & -0.48753 & -0.26438 \\
\hline 131 & MISC-InfoOps & -0.65392 & 0.66936 \\
\hline 132 & MISC-Tactics & 0.55724 & 0 \\
\hline 133 & MISC-TOC_Activity & 0.40379 & 0.03403 \\
\hline 134 & MOPP_Level & 0.57585 & 0.32597 \\
\hline 135 & Staff_Huddle & -0.52947 & -0.14756 \\
\hline 136 & Pers-CO & -0.0839 & -0.79064 \\
\hline 137 & Pers-XO & 0.10361 & 0.89868 \\
\hline 138 & Pers-BC & -0.77074 & 0.84277 \\
\hline 139 & Pers-S3 & -0.99457 & 0.09909 \\
\hline 140 & Pers-S3_NCO & 0.56377 & -0.30802 \\
\hline 141 & Pers-S3_RTO & -0.8114 & -0.05655 \\
\hline 142 & PERS-S2 & 0.53502 & -0.25004 \\
\hline 143 & PERS-FSO & 0.47233 & 0.69396 \\
\hline 144 & PERS-ENGR & 0.81188 & -0.36625 \\
\hline 145 & PERS-ALO & 0.09568 & -0.0878 \\
\hline 146 & PERS-S3-SGM & 0.06632 & -0.45622 \\
\hline 147 & SKY-CLEAR & -0.50023 & 0.96594 \\
\hline 148 & SKY-OVERCAST & -0.27134 & -0.4044 \\
\hline 149 & SKY-RAIN & 0.75817 & 0 \\
\hline 150 & WIND-NONE & -0.38993 & 0.13456 \\
\hline 152 & WIND-LOW & 0.96655 & -0.60802 \\
\hline & & & 0.52263 \\
\hline
\end{tabular}




\begin{tabular}{|c|c|c|c|}
\hline Node no. & Unit Name & Trained bias & Pruned bias \\
\hline 153 & WIND-HIGH & 0.39128 & 0 \\
\hline 154 & Light-BG & 0.0604 & 0 \\
\hline 155 & Light-DISPLAY & -0.42998 & 0 \\
\hline 156 & NOISE & -0.72582 & 0 \\
\hline 157 & TEMP-DB & -0.89026 & 0 \\
\hline 158 & TEMP-WB & 0.81652 & 0 \\
\hline 159 & Humidity & 0.88336 & 0.14518 \\
\hline
\end{tabular}

Table 15 provides the set of those IV to be carried forward in the analysis as those IV with a pruned bias greater than zero. Some of the eliminated IV nodes are intuitively obvious. For example, the IV for exercise day was eliminated after day 11. This reflects the fact that no exercise had longer than a 10 day recording period. Circadian rhythm information variables eliminated all hours of the day except for hours 7:00 a.m. through 6:00 p.m. Because only one person was collecting data there was no data collected at hours other than these. However, many other performance parameters that were not immediately obvious were eliminated by the JNNS model. Many of the artillery battlefield operating systems (BOS-FS) IV were shown to be less significant. Many of the communications systems (COM-xxx) were identified as less significant than others. This was partly due to the fact that observation abilities limited the scope of data that could be collected, but also it was due to the observations that were observed of several of the more significant systems. All of the personnel IV remained after pruning. Most of the environmental variables, with the exception of the wind and humidity factors, were removed by the pruning calculations.

\subsubsection{Development of Mathematical Models of DV Performance From JNNS.}

As the JNNS simulation is not capable of generating descriptive expressions of how the DV respond to the various IV, an alternative procedure was established to develop these mathematical relationships. The first step was to identify those DV for which sufficient data had been collected to allow an attempt to develop a model of their performance. A subjective judgment was made that there must be at least 10 observations that were made against a DV to allow sufficient data for it to be modeled. This examination of the data was performed with a SAS correlation run of each DV to each of the other DV that generated simple descriptive statistics for all the DV. One of the parameters that this run produced was the sum of the data values for each DV. For the binary DV this sum provided a count of the number of observations 
in the dataset for that DV. For the standardized $(0,1) \mathrm{DV}$, this sum provided a value that approached the number of observations for that DV in the dataset. Table 16 shows the list of the 17 DV that were selected for modeling by selecting those DV with a sum of 10 or greater. Appendix I contains the output from the SAS run that was used to determine this list of DV.

Table 16 - DV Selected For Model Generation

\begin{tabular}{|c|l|l|c|}
\hline Model & \multicolumn{1}{|c|}{ Category } & \multicolumn{1}{|c|}{ Name } & Label \\
\hline 1 & Primary Task & 10 Destruction, capture, or bypass of enemy force. & TP10 \\
\hline 2 & Primary Task & 11 Fixing enemy in position. & TP11 \\
\hline 3 & Primary Task & 12 Synchronization with supporting forces. & TP12 \\
\hline 4 & Primary Task & 15 Destruction of first echelon forces. & TP15 \\
\hline 5 & Primary Task & 21 Peacekeeping operations. & TP21 \\
\hline 6 & Primary Task & 23. Planning & TP23 \\
\hline 7 & Secondary Task & 12 Synchronization with supporting forces. & TS18 \\
\hline 8 & Secondary Task & 18 Deception activities. & TS19 \\
\hline 9 & Secondary Task & 19 Rear operations. & TS23 \\
\hline 10 & Secondary Task & 23. Planning & TA3 \\
\hline 11 & Tertiary Task & 3 Movement to the line of departure. & TA21 \\
\hline 12 & Tertiary Task & 21 Peacekeeping operations. & TA23 \\
\hline 13 & Tertiary Task & 23. Planning & Mission1 \\
\hline 14 & Mission & 1- Pre Operations Planning & Mission2 \\
\hline 15 & Mission & 2- Movement to Contact & Mission3 \\
\hline 16 & Mission & 3- Attack & Mission5 \\
\hline 17 & Mission & 5- River Crossing &
\end{tabular}

For each DV to be modeled, the next step was to identify those IV that are predicted to significantly contribute to a model of the DV. This was performed through simple linear regression analysis. Using SAS, an initial model of each of the DV was constructed using IV remaining from the JNNS pruning stage. Those IV that are predicted to have significance for model generation are shown in Table 17. In addition, administrative variables such as day of the exercise, hour of the day, type of TOC, exercise number, and type of simulation were also eliminated as they were recorded in the observation data for possible future analysis. 
Table 17 - IV Selected For Inclusion in DV Models Based On JNNS Pruning

\begin{tabular}{|c|c|c|c|}
\hline Node no. & Variable Name & Trained bias & Pruned bias \\
\hline 79 & BOS-Intel1 & -0.78387 & 0.48888 \\
\hline 80 & BOS-Intel2 & 0.39775 & -0.78344 \\
\hline 81 & BOSIntel3 & -0.17637 & 0.1981 \\
\hline 82 & BOSIntel4 & -0.3867 & -0.22953 \\
\hline 83 & BOS-MAN1 & 0.10361 & 0.47002 \\
\hline 84 & BOS-MAN2 & -0.1298 & 0.21793 \\
\hline 86 & BOS-FS2 & -0.39818 & -0.27732 \\
\hline 91 & BOS-FS7 & 0.7622 & 0.03421 \\
\hline 93 & BOS-FS9 & -0.5399 & 0.5031 \\
\hline 94 & BOS-MAS1 & 0.1146 & -0.30888 \\
\hline 95 & BOS-MAS2 & 0.37938 & -0.66204 \\
\hline 97 & BOS-MAS4 & 0.24223 & -0.01621 \\
\hline 98 & BOS-MAS5 & 0.11295 & -0.87292 \\
\hline 99 & BOS-MAS6 & -0.02036 & 0.39952 \\
\hline 100 & BOS-MAS7 & 0.19572 & 0.00961 \\
\hline 101 & BOS-ADA1 & 0.34001 & -0.70501 \\
\hline 102 & BOS-ADA2 & -0.19413 & 0.89917 \\
\hline 103 & BOS-C2-1 & -0.11728 & -0.71685 \\
\hline 104 & BOS-C2-2 & 0.71606 & 0.81024 \\
\hline 105 & BOS-C2-3 & 0.56273 & 0.38578 \\
\hline 106 & BOSR-Intel & 0.36876 & -0.3939 \\
\hline 109 & BOSR-MAS & -0.62645 & 0.93323 \\
\hline 113 & COM-AFATDS & -0.70714 & 0.64336 \\
\hline 115 & COM-ASAS & 0.54698 & -0.6173 \\
\hline 119 & COM-FBCB2 & 0.50334 & -0.68889 \\
\hline 121 & COM-MCS & 0.24894 & 0.46403 \\
\hline 122 & COM-AMDWS & -0.9118 & -0.18882 \\
\hline 125 & INTEL-TUAV & -0.49297 & 0.36448 \\
\hline 128 & MISC-Timing & -0.97967 & -0.04941 \\
\hline 129 & MISC-Tempo & -0.36699 & -0.75396 \\
\hline 130 & MISC-ReconOps & -0.48753 & -0.26438 \\
\hline 131 & MISC-InfoOps & -0.65392 & 0.66936 \\
\hline 133 & MISC-TOC_Activity & 0.40379 & 0.03403 \\
\hline 134 & MOPP_Level & 0.57585 & 0.32597 \\
\hline 135 & Staff_Huddle & -0.52947 & -0.14756 \\
\hline 136 & Pers-CO & -0.0839 & -0.79064 \\
\hline 137 & Pers-XO & 0.10361 & 0.89868 \\
\hline 138 & Pers-BC & -0.77074 & 0.84277 \\
\hline 139 & Pers-S3 & -0.99457 & 0.09909 \\
\hline 140 & Pers-S3_NCO & 0.56377 & -0.30802 \\
\hline 141 & Pers-S3 RTO & -0.8114 & -0.05655 \\
\hline 142 & PERS-S2 & 0.53502 & -0.25004 \\
\hline 143 & PERS-FSO & 0.47233 & 0.69396 \\
\hline 144 & PERS-ENGR & 0.81188 & -0.36625 \\
\hline 145 & PERS-ALO & 0.09568 & -0.0878 \\
\hline 146 & PERS-S3-SGM & 0.06632 & -0.45622 \\
\hline 147 & SKY-CLEAR & -0.50023 & 0.96594 \\
\hline 148 & SKY-OVERCAST & -0.27134 & -0.4044 \\
\hline
\end{tabular}




\begin{tabular}{|c|c|c|c|}
\hline Node no. & Variable Name & Trained bias & Pruned bias \\
\hline 150 & WIND-NONE & -0.38993 & 0.13456 \\
\hline 151 & WIND-LOW & 0.96655 & -0.60802 \\
\hline 152 & WIND-MODERATE & 0.96008 & 0.52263 \\
\hline 159 & Humidity & 0.88336 & 0.14518 \\
\hline
\end{tabular}

An initial SAS model was constructed for each of the DV containing each of the IV and a simple linear regression was executed. The results of the first regression run are shown in Table 18 using Primary Task 10 (TP10-Destruction, capture, or bypass of enemy force) as an example. The results for all $17 \mathrm{DV}$ is at Appendix J. 
Table 18 - First Iteration To Select IV For Inclusion in TP10 DV Model Based On JNNS Pruning

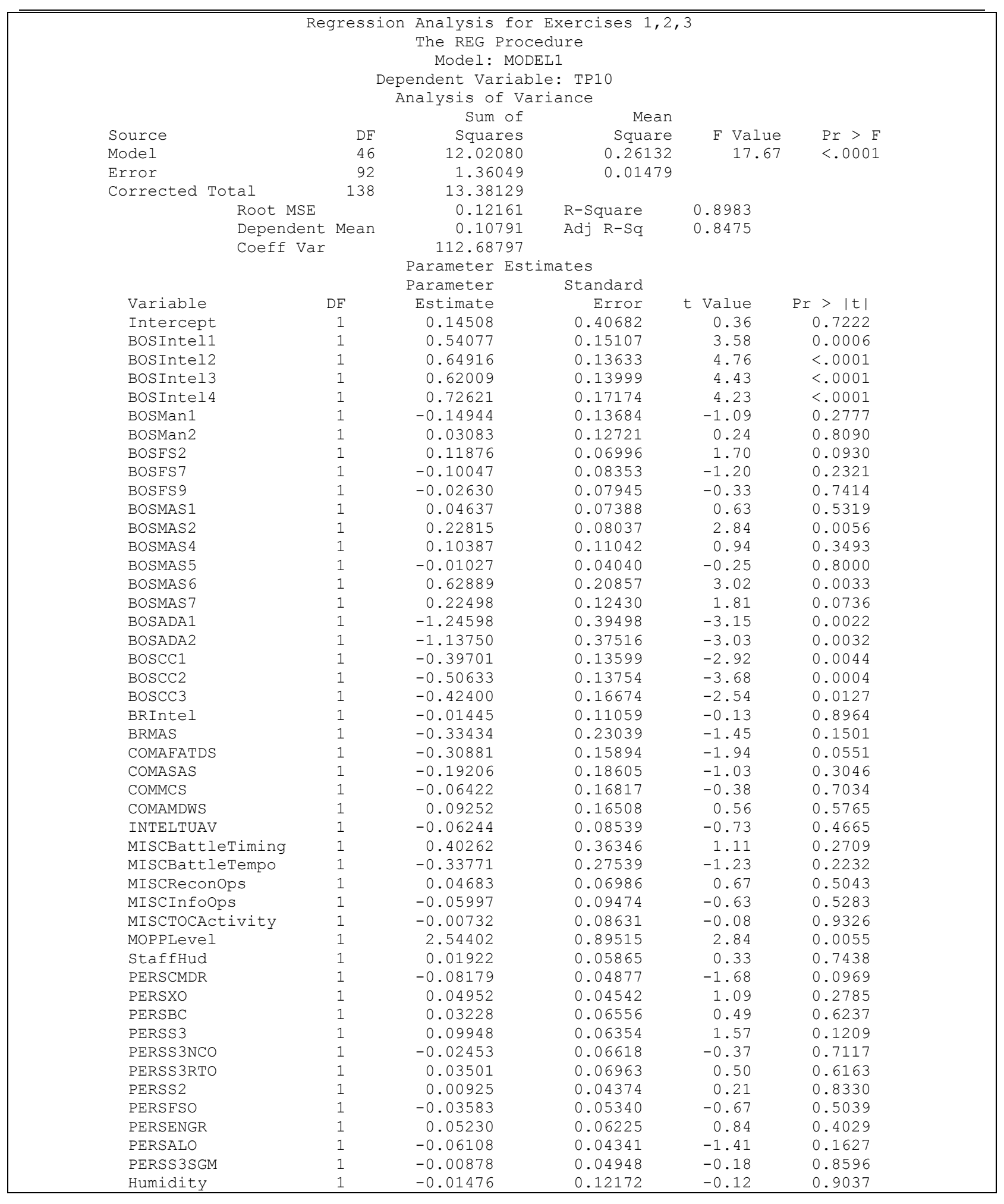


The results of the second iterative regression run are shown in Table 19 using Primary Task 10 (TP10- Destruction, capture, or bypass of enemy force) as an example. The results for all $17 \mathrm{DV}$ is at Appendix K.

Table 19 - Second Iteration To Select IV For Inclusion in TP10 DV Model Based On JNNS Pruning

\begin{tabular}{|c|c|c|c|c|c|c|c|c|}
\hline \multicolumn{9}{|c|}{ Second level Regression Analysis for Exercises 1,2,3 } \\
\hline \multicolumn{9}{|c|}{$\begin{array}{l}\text { The REG Procedure } \\
\text { Model: MODEL1 }\end{array}$} \\
\hline \multicolumn{9}{|c|}{$\begin{array}{c}\text { Dependent Variable: TP10 } \\
\text { Analysis of Variance }\end{array}$} \\
\hline & & & Sum of & & Mean & & & \\
\hline Source & & DF & Squares & & Square & & F Value & $\mathrm{Pr}>\mathrm{F}$ \\
\hline Model & & 10 & 7.97293 & & 0.79729 & & 18.87 & $<.0001$ \\
\hline Error & & 128 & 5.40837 & & 0.04225 & & & \\
\hline \multirow[t]{6}{*}{ Corrected Tot } & Total & 138 & 13.38129 & & & & & \\
\hline & Root MSE & & 0.20556 & $\mathrm{R}-\mathrm{S}$ & quare & & 0.5958 & \\
\hline & Dependent & Mean & 0.10791 & Adj & $\mathrm{R}-\mathrm{Sq}$ & & 0.5643 & \\
\hline & Coeff Var & & 190.48100 & & & & & \\
\hline & & & Parameter Est & ates & & & & \\
\hline & & & Parameter & Star & ndard & & & \\
\hline Variable & & DF & Estimate & & Error & $\mathrm{t}$ & Value & $\operatorname{Pr}>|t|$ \\
\hline Intercept & & 1 & 0.44886 & & 17174 & & 2.61 & 0.0100 \\
\hline BoSIntel3 & & 1 & 0.00466 & & 05049 & & 0.09 & 0.9266 \\
\hline BosIntel4 & & 1 & 0.27463 & & 08769 & & 3.13 & 0.0022 \\
\hline BOSMAS2 & & 1 & 0.25440 & & 06685 & & 3.81 & 0.0002 \\
\hline BOSMAS6 & & 1 & -0.21173 & & 14555 & & -1.45 & 0.1482 \\
\hline BOSADA1 & & 1 & 0.22378 & & 16293 & & 1.37 & 0.1720 \\
\hline BOSADA2 & & 1 & -0.01263 & & 12326 & & -0.10 & 0.9186 \\
\hline Boscc1 & & 1 & -0.22450 & & 14833 & & -1.51 & 0.1326 \\
\hline Boscc2 & & 1 & -0.53501 & & 16576 & & -3.23 & 0.0016 \\
\hline Boscc3 & & 1 & -0.34403 & & 17206 & & -2.00 & 0.0477 \\
\hline MOPPLevel & & 1 & -0.68254 & & 25038 & & -2.73 & 0.0073 \\
\hline
\end{tabular}

The results of the third iterative regression run are shown in Table 20 using Primary Task 10 (TP10- Destruction, capture, or bypass of enemy force) as an example. The results for all 17 DV is at Appendix L. 
Table 20 - Third Iteration To Select IV For Inclusion in TP10 DV Model Based On JNNS Pruning

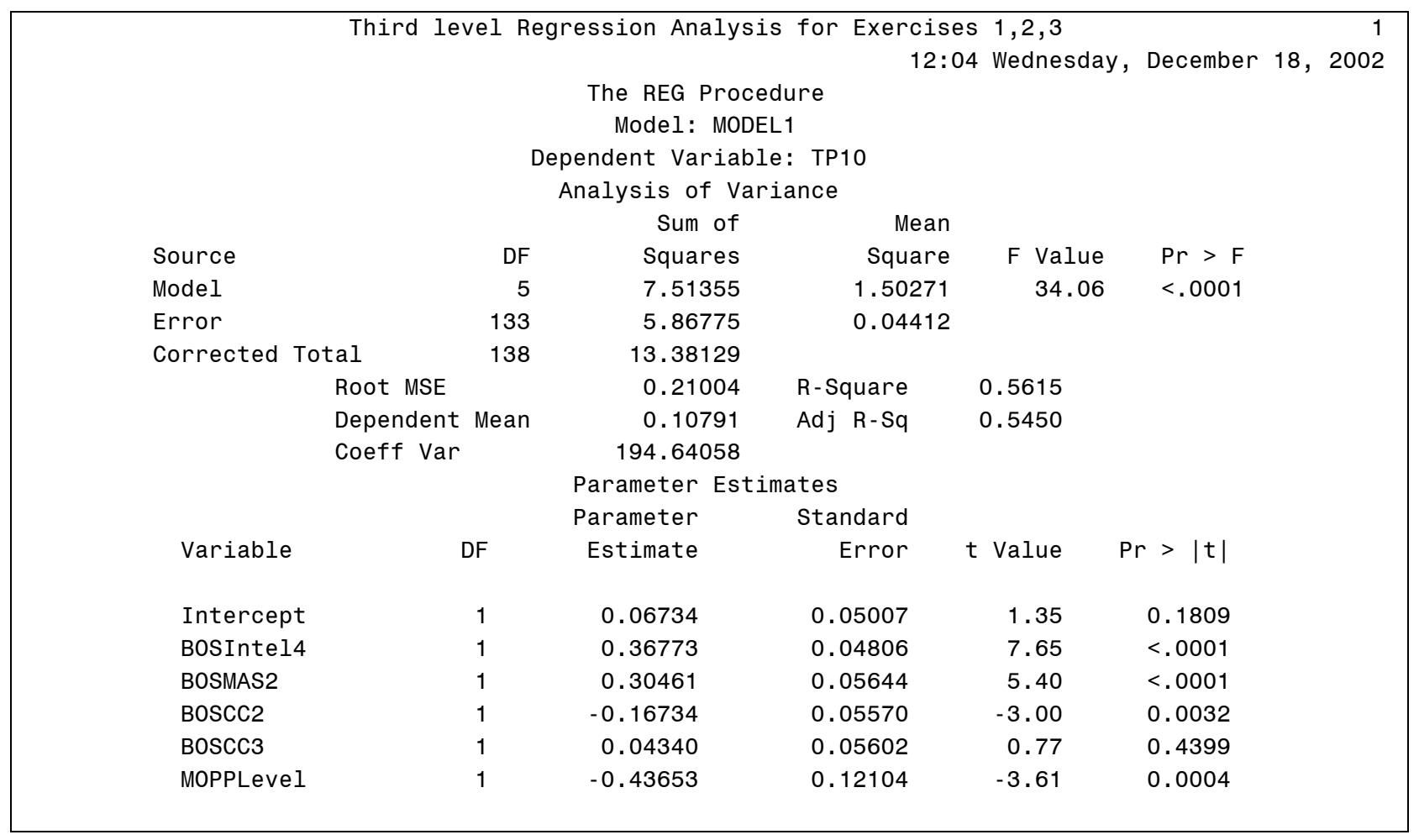

The results of the fourth iterative regression run are shown in Table 21 using Primary Task 10 (TP10- Destruction, capture, or bypass of enemy force) as an example. The results for all $17 \mathrm{DV}$ is at Appendix M. The results from this regression run show that all the remaining IV for each of the DV models have achieved significance at the $\alpha=.05$ level. Thus, the parameter estimates, or Beta Weights, along with the intercept in the SAS output for each DV form the linear regression equation, or mathematical model, that describe the input from each significant IV to each of the DV models. 
Table 21 - Fourth Iteration To Select IV For Inclusion in TP10 DV Model Based On JNNS Pruning

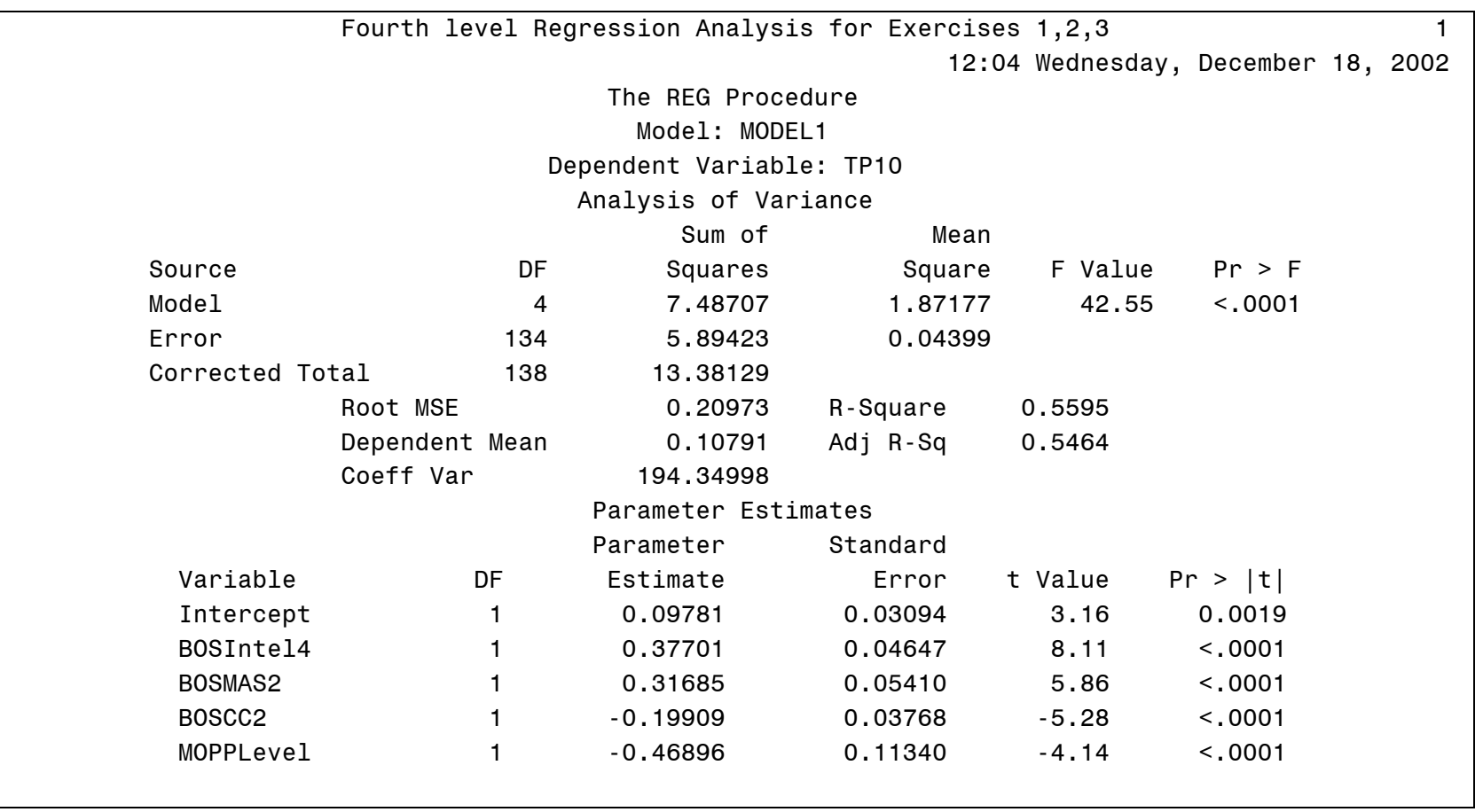

From these results the linear regression equation for the TP10 variable is represented in Table 22. The similar derivation of linear regression expressions for all the DV models is at Appendix N.

Table 22 - Linear Regression Expression For DV TP10

\begin{tabular}{|c|c|c|c|c|c|c|}
\hline \multicolumn{7}{|c|}{ \# 1 - Primary Task10. Destruction, capture, or bypass of enemy force. } \\
\hline & Variable & Intercept & BOSIntel4 & BOSMAS2 & BOSCC2 & MOPPLevel \\
\hline TP10 & Beta Weight & 0.09781 & 0.37701 & 0.31685 & -0.19909 & -0.46896 \\
\hline
\end{tabular}

\subsubsection{Summary of Results.}

The use of neural networks, as implemented by JNNS, along with post processing of its output with traditional linear regression modeling, provides a straightforward approach to analyzing the data. The intent is to, first, identify those IV that are predicted to significantly affect the outcomes of the system. JNNS provides this ability through its pruning capability after successful training of the network. In fact, JNNS is being used here as a factor screening method 
to identify those relationships contained in the dataset that potentially affect the performance of the system. After those IV that are predicted to be less important were rejected during the pruning process, the dataset became more manageable with traditional modeling approaches such as stepwise linear regression. The results of the regression analysis provided the algorithmic models for use in the remainder of the COMPASS framework to provide control inputs for the CoHOST discrete event simulation of the $\mathrm{C} 2 \mathrm{~S}$. 


\section{Phase II - Development of Algorithmic Control Interfaces for TOC Discrete Event Simulations.}

Phase II of this research consisted of applying the mathematical control models developed in Phase I into a suitable simulation of the performance of the overall system. This assumes that a simulation of this type already exists which is the case here. This discrete event simulation is the CoHOST model previously developed by the U.S. Army (Middlebrooks, 2001; Middlebrooks et al., 1999a; Middlebrooks et al., 1999b). CoHOST provides simulated human test subjects who act as avatars to evaluate the effects of the different IV through experimental evaluations of the data. Appendix O provides an overall description of the CoHOST simulation for readers unfamiliar with it along with a brief description of the results from the project that developed it.

The interface of the mission based mathematical performance models from Phase I to the operator task and workload performance structure of the CoHOST model is a critical component of the overall COMPASS framework. In the following sections procedures are developed that enable CoHOST to respond to mission based stressors so that the use of the simulation can be extended to include predictions of the effectiveness of the different subsystems and associated performance measures that exist within the $\mathrm{C} 2 \mathrm{~S}$.

\subsection{Method.}

The methodology for this research is the implementation of the mathematical models developed by the JNNS simulation into a form usable by the CoHOST simulation. The interface points were first developed in the last part of Phase I. These interfaces take observational data of mission based overall system level performance of the TOC and correlates it to task and workload performance characteristics for the human operators in the TOC. CoHOST simulation runs are made according to the interface parameters and the results of the runs are analyzed to determine the predicted effects from changes in mission characteristics.

\subsubsection{Development of JNNS to CoHOST Interface.}

The process to translate the results of the JNNS (Fischer et al., 2001) into performance parameters that determine how a discrete event simulation of the $\mathrm{C} 2$ system performs is critical to the establishment of the COMPASS paradigm. While the $\mathrm{C} 2$ system field observations that are evaluated by the neural network are characterized as mission based performance parameters 
of the overall system, the CoHOST discrete event simulation models the C2 system in terms of human performance, workload, and utilization of the human operators in the system. The overall intent of the COMPASS approach is to systematically evaluate the C2 system so that human performance can be quantitatively described within that system. Once this goal is achieved the COMPASS approach can be used to evaluate modifications of that system in terms of the human ability to perform where the proposed changes to the system are compared to the baseline as characterized by the current existing system.

The correlation of the mission based JNNS data structures to the human operator task and workload based CoHOST data structures requires a procedural approach where JNNS mission performance is cross tabulated with CoHOST performance shaping factors (PSF) that modify the amount of time required to perform a task. The resulting operator workload is determinant upon the instantaneous workload as determined by a knowledge, skills, and abilities assessment using the operator performance categories of gross motor, psychomotor, auditory, visual, communications, conceptual, and speed loaded activity (Fleishman and Quaintance, 1984) multiplied by the amount of time that the tasks are being performed. By establishing PSF definitions for modifying the task time performance in CoHOST a set of user defined PSF factors can be defined that will cause the amount of time required to perform tasks across the CoHOST model to change accordingly. The CoHOST simulation work and task load performance then becomes responsive to $\mathrm{C} 2$ system mission task demands as they result in subsequent operator task time performance.

Logically, the IVs in the JNNS regression equations are independent measures whose activity level was determined from the field observations of TOCs. Some of the IVs, such as specific events that were either occurring or not occurring at the time of the observation, were recorded as binary. Other variables were observed on an interval scale of 1-10 indicating level of usage, such as level of usage of a communications system or intelligence collection asset. Environmental variables were recorded directly in their scale of observation such as temperature in degrees $\mathrm{F}$ and percent relative humidity. All of the non-binary variables were standardized into a range of 0 to 1 for the analysis. The presumption is that the IVs represent subsystems whose efficient use are designed to enhance the operation of the TOC. The efficient use of these subsystems do this by allowing the operator to perform more tasks over time. The continuous work and task load remain fairly constant over time but the task based parameters such as the 
number of tasks queued, dropped, interrupted, and suspended by individual operators goes down. As a result, the operator continues to work at a steady rate but is able to accomplish more.

The process is to determine regressor values from the JNNS results that will provide DV values at selected points in the response range of each regression equation. The DV product points were selected as $0 \%, 25 \%, 50 \%$, and $75 \%$ as providing representative points for the response of the DV. The previous JNNS output analysis provided 17 mathematical performance models for the 17 DV mission tasks as shown again in Table 23. The IVs used across all 17 models are listed in Table 24 .

Table 23 - JNNS Regression Models / DVs

\begin{tabular}{|c|l|}
\hline DV Label & \multicolumn{1}{|c|}{ Dependent Variable Description } \\
\hline & \\
\hline TP10 & $\# 1$ - Primary Task10. Destruction, capture, or bypass of enemy force. \\
\hline TP11 & $\#$ 2 - Primary Task11. Fixing enemy in position. \\
\hline TP12 & $\#$ 3 - Primary Task12. Synchronization with supporting forces. \\
\hline TP15 & $\# 4$ - Primary Task15. Destruction of first echelon forces. \\
\hline TP21 & $\#$ 5 - Primary Task21. Peacekeeping operations. \\
\hline TP30 & $\# 6$ - Primary Task23. Planning. \\
\hline TS12 & $\# 7$ - Secondary Task12. Synchronization with supporting forces. \\
\hline TS18 & $\# 8$ - Secondary Task18. Deception activities. \\
\hline TS19 & $\# 9$ - Secondary Task19. Rear operations. \\
\hline TS30 & $\# 10$ - Secondary Task23. Planning. \\
\hline TA3 & $\# 11$ - Tertiary Task3. Movement to the line of departure. \\
\hline TA21 & $\# 12$ - Tertiary Task21. Peacekeeping operations. \\
\hline TA30 & $\# 13$ - Tertiary Task23. Planning. \\
\hline Mission1 & $\# 14$ - Mission1. Pre Operations Planning. \\
\hline Mission2 & $\# 15$ - Mission2. Movement to Contact. \\
\hline Mission3 & $\# 16$ - Mission3. Attack. \\
\hline Mission5 & $\# 17$ - Mission5. River Crossing. \\
\hline &
\end{tabular}

Table 24 - JNNS Model Regressors / IVs

\begin{tabular}{|c|l|}
\hline \hline IV Label & \\
\hline & \\
\hline BOSIntel1 & $\# 1$ - Intel BOS- 1 Conduct Intelligence Planning \\
\hline BOSIntel2 & $\# 2$ - Intel BOS- 2 Collect Information \\
\hline BOSIntel3 & $\# 3$ - Intel BOS- 3 Process Information \\
\hline BOSIntel4 & $\# 4$ - Intel BOS- 4 Disseminate Intelligence \\
\hline BOSMan1 & $\# 5$ - Maneuver BOS- 1 Conduct Tactical Movement \\
\hline BOSMan2 & $\# 6$ - Maneuver BOS- 2 Engage Enemy with Direct Fire and Maneuver \\
\hline BOSFS2 & $\# 8$ - Fire Support BOS- 2 Employ Field Artillery \\
\hline BOSFS7 & $\# 13$ - Fire Support BOS- 7 Conduct Counter Target Acquisition Operations \\
\hline
\end{tabular}




\begin{tabular}{|c|c|}
\hline IV Label & IV Description \\
\hline BOSFS9 & \#15 - Fire Support BOS- 9 Coordinate, Synchronize, and Integrate Fire Support \\
\hline BOSMAS1 & \#16 - Mobility \& Survivability BOS- 1 Overcome Obstacles \\
\hline BOSMAS2 & \#17 - Mobility \& Survivability BOS- 2 Enhance Movement \\
\hline BOSMAS4 & \#19 - Mobility \& Survivability BOS- 4 Enhance Physical Protection \\
\hline BOSMAS5 & \#20 - Mobility \& Survivability BOS- 5 Provide Operations Security \\
\hline BOSMAS6 & \#21 - Mobility \& Survivability BOS- 6 Conduct Deception \\
\hline BOSMAS7 & \#22 - Mobility \& Survivability BOS- 7 Conduct NBC Defense \\
\hline BOSADA1 & \#23 - Air Defense BOS- 1 Take Active Air Defense Measures \\
\hline BOSADA2 & \#24 - Air Defense BOS- 2 Take Passive Air Defense Measures \\
\hline BOSCC1 & \#25 - Command and Control BOS- 1 Plan for Combat Operations \\
\hline BOSCC2 & $\begin{array}{l}\text { \#26 - Command and Control BOS- } 2 \text { Direct and Lead Unit During the Preparation } \\
\text { Phase of the Battle }\end{array}$ \\
\hline BOSCC3 & \#27 - Command and Control BOS- 3 Direct and Lead Unit in Execution of Battle \\
\hline BRIntel & \#28 - BOS Ratings- Intelligence \\
\hline BRMAS & \#31 - BOS Ratings- Mobility and Survivability \\
\hline COMAFATDS & \#35 - Communications- AFATDS - Advanced Field Artillery Tactical Data System \\
\hline COMASAS & \#37 - Communications- ASAS - All Source Analysis System \\
\hline COMMCS & \#43 - Communications- MCS - Maneuver Control System (Heavy \& Light) \\
\hline COMAMDWS & \#44 - Communications- AMDWS. ADA \\
\hline INTELTUAV & \#47 - Intel Collection- TUAV - Tactical Unmanned Aerial Vehicle \\
\hline MISCBattleTiming & \#50 - Misc Factors- Battle Timing \\
\hline MISCBattleTempo & \#51 - Misc Factors- Battle Tempo \\
\hline MISCReconOps & \#52 - Misc Factors- Reconnaissance Operations \\
\hline MISCInfoOps & \#53 - Misc Factors- Information Operations \\
\hline MISCTOCActivity & \#55 - Misc Factors- Observed Activity / Stress Level in TOC \\
\hline MOPPLevel & \#56 - Misc Factors- MOPP Level \\
\hline StaffHud & \#57 - Misc Factors- Staff Huddle \\
\hline PERSCMDR & \#58 - Personnel- Commanding Officer present in TOC \\
\hline PERSXO & \#59 - Personnel- Executive Officer present in TOC \\
\hline PERSBC & \#60 - Personnel- Battle Captain present in TOC \\
\hline PERSS3 & \#61 - Personnel- S3 present in TOC \\
\hline PERSS3NCO & \#62 - Personnel- S3 NCO present in TOC \\
\hline PERSS3RTO & \#63 - Personnel- S3 RTO present in TOC \\
\hline PERSS2 & \#64 - Personnel- S2 present in TOC \\
\hline PERSFSO & \#65 - Personnel- FSO present in TOC \\
\hline PERSENGR & \#66 - Personnel- Engineer present in TOC \\
\hline PERSALO & \#67 - Personnel- ALO present in TOC \\
\hline PERSS3SGM & \#68 - Personnel- S3 SGM present in TOC \\
\hline Humidity & \#69 - Environmental Factor- Relative Humidity, \% \\
\hline
\end{tabular}

The steps to determine the regressor values required to produce the desired DV responses for each of the 17 performance models are:

- Set all binary IVs (regressors) to 1, indicating that they are in play or in use, etc, since they were observed as binary active or not active variables.

- Set all real IVs (regressors) also to 1 indicating that they are at the full range of their performance. 
- Calculate DV from this setting, which becomes the MAX range value.

- Recognizing that the intercept is the value when all IVs are set to zero, set the MIN range value to the value of the intercept.

- Calculate the RANGE of the DV response $=$ MAX - MIN .

- Calculate the MEDIAN of the range as the mid point in the RANGE.

- Determine the DV Levels in the range of $25 \%, 50 \%, 75 \%$ :

- Calculate the quartile of the range, QTR $=$ RANGE $/ 4$.

- Calculate the $25 \%$ point in the range $=$ MEDIAN - QTR .

- Calculate the $50 \%$ point in the range $=$ MEDIAN .

- Calculate the $75 \%$ point in the range $=$ MEDIAN + QTR .

- Calculate the DV Target Value for each of the 3 DV Levels of $25 \%, 50 \%$, and $75 \%$ by:

- Iteratively adjust the IV values in each DV regression equation to one of their two possible binary values, and the real values from 0 to 1 until a combination of the IVs is reached where the regression equation result provides a value approximating as closely as possible the $25 \%, 50 \%$, and $75 \%$ levels determined from the RANGE of the DV.

- The resulting set of IVs identified as TRUE, or with a value greater than zero indicate those IVs that are predicted to be significant in the performance of that DV at each of the respective treatment levels.

The calculations to determine the DV Target Values for the $25 \%, 50 \%$, and $75 \%$ utilization levels is at Table 25 and a sample of the corresponding IV matrix for the DV model TP10 is at Table 26. A complete table of all the DV / IV correlation matrices for each of the 17 DV models is at Appendix P. Listed beside the IV matrix in Table 25 are those regressors that were required to make the regression equation provide the required DV value to correspond with each of the 3 utilization levels. These regressors then become the factors that are identified as those performance tasks that are predicted to significantly impact the mission task at that utilization level. 
Table 25 - Determination of DV Target Values

\begin{tabular}{|l|l|l|l|l|l|l|l|l|l|l|l|}
\hline & DV & Target & Value & & & \multicolumn{1}{|l|}{ DV } & \multicolumn{1}{|c|}{ Stats } & & DV & Levels \\
\hline $\begin{array}{c}\text { DV } \\
\text { Label }\end{array}$ & $\mathbf{0 . 2 5}$ & $\mathbf{0 . 5}$ & $\mathbf{0 . 7 5}$ & MAX & MIN & Range & $\begin{array}{c}\text { Qtr= } \\
\text { Range } \\
/ \mathbf{4}\end{array}$ & Median & $\mathbf{0 . 2 5}$ & $\mathbf{0 . 5}$ & $\mathbf{0 . 7 5}$ \\
\hline TP10 & 0.0978 & 0.1236 & 0.1236 & 0.1236 & 0.0978 & 0.0258 & 0.0065 & 0.1107 & 0.1043 & 0.1107 & 0.1172 \\
\hline TP11 & 0.2042 & 0.7804 & 1.2037 & 1.7186 & -0.2610 & 1.9796 & 0.4949 & 0.7288 & 0.2339 & 0.7288 & 1.2237 \\
\hline TP12 & -0.4774 & -0.0857 & 0.5515 & 0.9788 & -0.8666 & 1.8454 & 0.4614 & 0.0561 & -0.4053 & 0.0561 & 0.5175 \\
\hline TP15 & -0.3028 & 0.0022 & 0.3139 & 0.6282 & -0.6194 & 1.2476 & 0.3119 & 0.0044 & -0.3075 & 0.0044 & 0.3163 \\
\hline TP21 & -0.5036 & -0.0440 & 0.4460 & 0.4755 & -0.5332 & 1.0087 & 0.2522 & -0.0288 & -0.2810 & -0.0288 & 0.2234 \\
\hline TP23 & 0.9153 & 1.6095 & 1.9265 & 2.6450 & 0.3903 & 2.2547 & 0.5637 & 1.5177 & 0.9540 & 1.5177 & 2.0813 \\
\hline TS12 & -0.0436 & -0.0436 & -0.0436 & -0.0436 & 0.0412 & -0.0848 & -0.0212 & -0.0012 & 0.0200 & -0.0012 & -0.0224 \\
\hline TS18 & 0.1338 & 0.2935 & 0.4047 & 0.5595 & -0.0209 & 0.5804 & 0.1451 & 0.2693 & 0.1242 & 0.2693 & 0.4144 \\
\hline TS19 & 0.9781 & 0.9781 & 0.9781 & 0.9781 & 0.0989 & 0.8791 & 0.2198 & 0.5385 & 0.3187 & 0.5385 & 0.7583 \\
\hline TS23 & -0.3058 & 0.0886 & 0.2260 & 0.4519 & -0.5006 & 0.9525 & 0.2381 & -0.0243 & -0.2624 & -0.0243 & 0.2138 \\
\hline TA3 & 0.3078 & 0.4981 & 0.5768 & 0.8565 & 0.0898 & 0.7667 & 0.1917 & 0.4731 & 0.2815 & 0.4731 & 0.6648 \\
\hline TA21 & -0.2860 & -0.2163 & -0.1712 & -0.1015 & -0.3323 & 0.2308 & 0.0577 & -0.2169 & -0.2746 & -0.2169 & -0.1592 \\
\hline TA23 & 0.8937 & 1.1810 & 2.4907 & 3.3385 & -0.0603 & 3.3989 & 0.8497 & 1.6391 & 0.7894 & 1.6391 & 2.4888 \\
\hline Mission1 & -0.0886 & -0.0886 & 0.1815 & 0.3222 & -0.2187 & 0.5409 & 0.1352 & 0.0517 & -0.0835 & 0.0517 & 0.1869 \\
\hline Mission2 & 0.2245 & 0.2256 & 0.3112 & 0.3112 & 0.2142 & 0.0970 & 0.0243 & 0.2627 & 0.2384 & 0.2627 & 0.2869 \\
\hline Mission3 & 0.0426 & 0.3974 & 0.4620 & 0.8169 & -0.1561 & 0.9730 & 0.2432 & 0.3304 & 0.0871 & 0.3304 & 0.5736 \\
\hline Mission5 & -0.1868 & 0.1075 & 0.4407 & 0.9907 & -0.6554 & 1.6461 & 0.4115 & 0.1676 & -0.2439 & 0.1676 & 0.5792 \\
\hline
\end{tabular}

Table 26 - Sample DV / IV Correlation Matrix For Task TP10

Model \# 1 - Primary Task10. Destruction, capture, or bypass of enemy force.

\begin{tabular}{|c|c|c|c|c|c|l|}
\hline Variable & $\begin{array}{c}\text { Inter } \\
\text { cept }\end{array}$ & $\begin{array}{c}\text { BOS } \\
\text { Intel4 }\end{array}$ & $\begin{array}{c}\text { BOS } \\
\text { MAS2 }\end{array}$ & $\begin{array}{c}\text { BOS } \\
\text { CC2 }\end{array}$ & $\begin{array}{c}\text { MOPP } \\
\text { Level }\end{array}$ & \\
\hline $\begin{array}{c}\text { All IVs } \\
1.0\end{array}$ & 1 & 1 & 1 & 1 & \\
\hline $\begin{array}{c}\text { All IVs } \\
0.0\end{array}$ & 0 & 0 & 0 & 0 & \\
\hline DV .25 & & 0 & 0 & 0 & 0 & No IVs \\
\hline DV 0.5 & 1 & 1 & 1 & 1 & $\begin{array}{l}\text { BOSIntel4, BOSMAS2, BOSCC2, } \\
\text { MOPPLevel }\end{array}$ \\
\hline DV .75 & 1 & 1 & 1 & 1 & $\begin{array}{l}\text { BOSIntel4, BOSMAS2, BOSCC2, } \\
\text { MOPPLevel }\end{array}$ \\
\hline $\begin{array}{c}\text { Beta } \\
\text { Weight }\end{array}$ & 0.09781 & 0.37701 & 0.31685 & -0.19909 & -0.46896 & \\
\hline
\end{tabular}




\subsubsection{CoHOST Simulation.}

CoHOST is a discrete event computer simulation of TOC operations developed by ARLHRED in support of various Army studies evaluating current and proposed TOC configurations. It models 23 members of the battalion or brigade command staff and tracks workload / taskload and utilization over time for each of the 23 operators. It also tracks task activity for each operator that includes the number of tasks queued, dropped, interrupted, and suspended for each of the 23 operators. The original project developed two scenarios for use with this simulation. The first was a four phase combat scenario consisting of planning, movement to contact, attack, and defense phases occurring over an approximate 8 hour period. The second scenario was a long movement Desert Storm like scenario where the friendly forces conducted a movement to contact tactical movement across approximately 180 kilometers spanning 24 hours before making contact with the enemy.

The CoHOST simulation was executed in the Microsoft Windows ${ }^{\mathrm{TM}}$ environment running the Micro Saint ${ }^{\mathrm{TM}}$ programming language. Micro Saint ${ }^{\mathrm{TM}}$ is a discrete event simulation language that is designed to support human performance studies and is applicable for a wide range of human performance domains (Laughery and Corker, 1997). The version used here was Release 3.1 Build A with ActionView and OptQuest, Standard Version, that was released on October 27, 1999 and operates in a Windows 98 / NT / 2000 / XP environment.

The CoHOST simulation model was configured to execute with the PSF set to represent the effects of each of the 4 values of the DV response of $0 \%, 25 \%, 50 \%$, and $75 \%$. As higher DV response utilizations represent more efficient operation while higher PSF settings represent longer task times (i.e., less efficient operation), an inverse relationship exists between the DV utilization percentiles and the PSF settings. For the purposes of these developmental runs a SME estimation was made that presumed a $75 \%$ DV utilization equated to a $30 \%$ decrease in task times resulting from the PSF setting (The value of 30\% was subjectively chosen based upon individual observation of field TOC performance. For the purposes of this exploratory process development this is deemed a satisfactory reference point upon which to build the analytical technique. Future research will validate and / or update this value with further SME interrogation). Thus, each of the DV utilization levels resulted in PSF settings as shown in Table 27. 
Table 27 - DV Utilization / PSF Setting Correlation

- $0 \% \mathrm{DV}$ utilization $==\quad \mathrm{PSF}=0$ (This is the condition where all the IVs are set to zero, i.e., turned off or not in play, and the numerical value of the DV becomes the regression intercept.)

- $25 \%$ DV utilization $==\quad$ PSF $=-10$.

- $50 \%$ DV utilization $==\quad \mathrm{PSF}=-20$.

- $75 \%$ DV utilization $==\quad \mathrm{PSF}=-30$.

With stochastic simulations such as CoHOST, multiple replications of the simulation must be performed to control the effects of random variability in the model. In this study the desire was to have the output data exhibit a $95 \%$ probability of falling within the confidence limit which gives a specified error level, $\varepsilon$, equal to \pm .05 of the mean for each data element. Using Banks' procedures (Banks et al., 1996), the number of replications required for each simulation run to achieve this desired error level was determined to be 15 (see Appendix Q). Each of the simulation conditions was run for this number of replications.

Prior to running the simulation, the scenario start and stop times for each of the combat phases was determined in order to apply the appropriate DV model to the combat phase for which it applies. The four combat phases in the scenario are (1) Pre-Operations Planning, (2) Movement to Contact, (3) Attack, and (4) Defense. The number of simulation runs was therefore one combined run for each PSF setting where each combined run contained the four scenario phases for a total of four simulation runs with each run consisting of 15 replications.

The PSF functions are established and the CoHOST simulations executed by:

- Use Banks' replication analysis (Banks et al., 1996) to determine the number of replications to run the CoHOST simulation for each treatment condition, $\mathrm{R}$, to account for random variability in the simulation. In this case, this is established as 15 (See Appendix Q).

- Modify the CoHOST task parameter definition arrays in the model to reflect the desired PSF settings of 0 (default), $-10,-20$, and -30 , meaning to reduce the task times by $0 \%, 10 \%, 20 \%$, and $30 \%$ as the different treatment conditions to be evaluated.

- Execute each of the resulting four simulation models with all output turned on and $\mathrm{R}$ set equal to 15 . Also, set the initial random number seed equal to one, "1," at the beginning of each treatment run for consistency. 


\subsection{Results.}

The mathematical models developed in Phase I were applied as algorithmic predictors used during the execution of the CoHOST TOC simulation. Interface characteristics to translate the mission based models from the JNNS to the task and workload constructs existent in the CoHOST model were developed that allow the translation of the mission based parameters that were recorded during the naturalistic observations in the TOC to the human operator task and workload characteristics that are modeled in CoHOST. Using these interfaces, CoHOST was executed to produce results that provided the basis for discussion and analysis.

\subsubsection{Evaluation Of the C2S With CoHOST Discrete Event Computer Simulation.}

The CoHOST run results are analyzed to make predictions as to which IV performance elements make contributions during various types of combat actions to improve overall operator performance. This is one of many possible approaches for trying to optimize the performance of a system through computer simulation. The presumption is that a decrease in task performance time will result in more efficient operation of the TOC along with the ability to achieve better situational awareness of the battlefield and allow the commander an enhanced ability to make better decisions. This is achieved by allowing the operator to be more effectively utilized with a greater workload throughput performance as a result of less time being required to perform individual tasks. The application of the COMPASS paradigm allows hypotheses to be generated regarding the overall performance of the $\mathrm{C} 2 \mathrm{~S}$. One such hypothesis regarding the overall performance of the system is:

Hypothesis: Decreasing the amount of time required to perform individual tasks in a TOC based work environment results in more effective operator performance through better utilization of the operator's time by providing greater workload throughput performance because of the ability to perform more tasks over the same amount of time.

The COMPASS paradigm allows a relationship of overall task based operator workload to be tested against those mission, hardware, and software task components that can affect operator performance during combat activities in the TOC. 


\subsubsection{CoHOST Data Reduction.}

Each CoHOST simulation run produces a number of ASCII text results files with an extension of .RES. There is one file containing workload and utilization data for each operator, one file containing task queue, dropped, and interrupted data for all operators, and one file containing summarized utilization information for all operators. The general procedure to first reduce the operator utilization and workload data for analysis is:

- Load the individual data for each operator into a separate spreadsheet and then combine all the operator spreadsheets into one.

- Sort the combined spreadsheet by replication number, clock time, and operator id.

- Extract the individual components for operator id, clock time, utilization, and overall workload into separate spreadsheets for each replication.

- Determine the mean for the clock time, utilization, and overall workload for the 15 replication runs.

- As the operator data is categorical it cannot be averaged. Determine a representative replication from the 15 for the operator id and use it with the subsequent analysis along with the rest of the data.

- Paste the data for the selected operator ID replication, and the mean values for the clock time, utilization, and workload into a new spreadsheet.

- Sort the new worksheet by the operator ID to block the data into performance blocks for each operator.

- For each operator to be analyzed, plot the utilization and workload data by time for each of the four simulation runs for a total of 8 plots for each operator. Add a linear regression trendline onto each plot along with the equation of the line for analysis and to more effectively represent the difference between the plot for each treatment. A sample of these plots for operator 21, Fire Support Officer, is at Figures 35 for utilization, and 36 for workload.

\subsubsection{CoHOST Data Analysis.}

Figures 35 and 36 show the workload and utilization profiles over time for the Fire Support Officer. The four phases in the simulation scenario, preoperations planning, movement to contact (MTC), hasty attack, and defense in sector, are delineated on each of the plots. A linear regression trendline is also shown on each plot along with its equation under the legend. The first point to note in examining these plots is that overall utilization remains roughly constant, although with a slight increase, as the task times are reduced from $0 \%$ to $10 \%$, to $20 \%$, and $30 \%$. Workload has a slight increase initially at $10 \%$ and then stabilizes becoming almost 
identical at $20 \%$ and $30 \%$. The initial conclusion, from only regarding these graphs, is that decreasing the overall times to perform tasks has little effect and might actually increase workload and utilization slightly. 

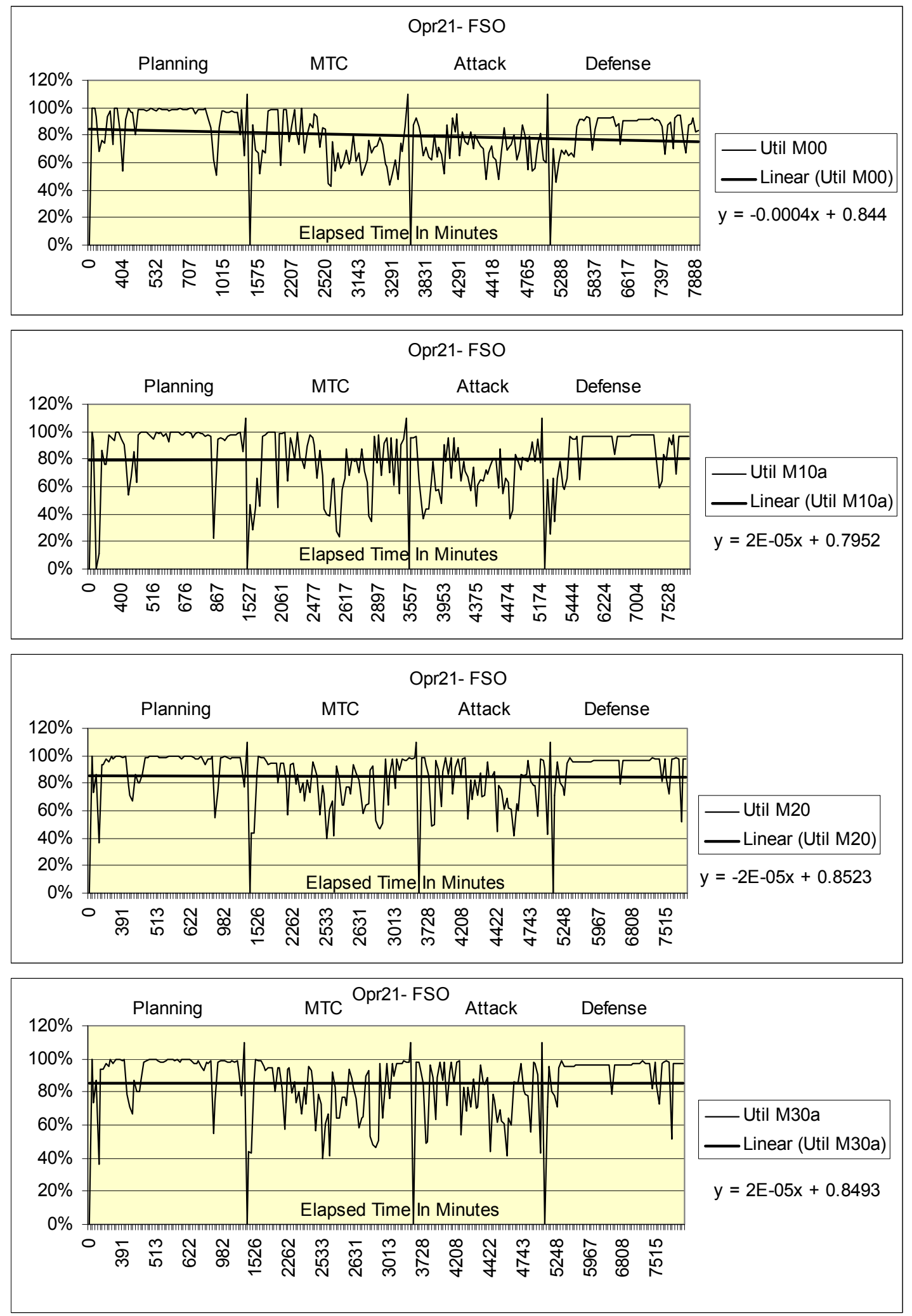

Figure 35 - Utilization Performance for Operator 21, Fire Support Officer 

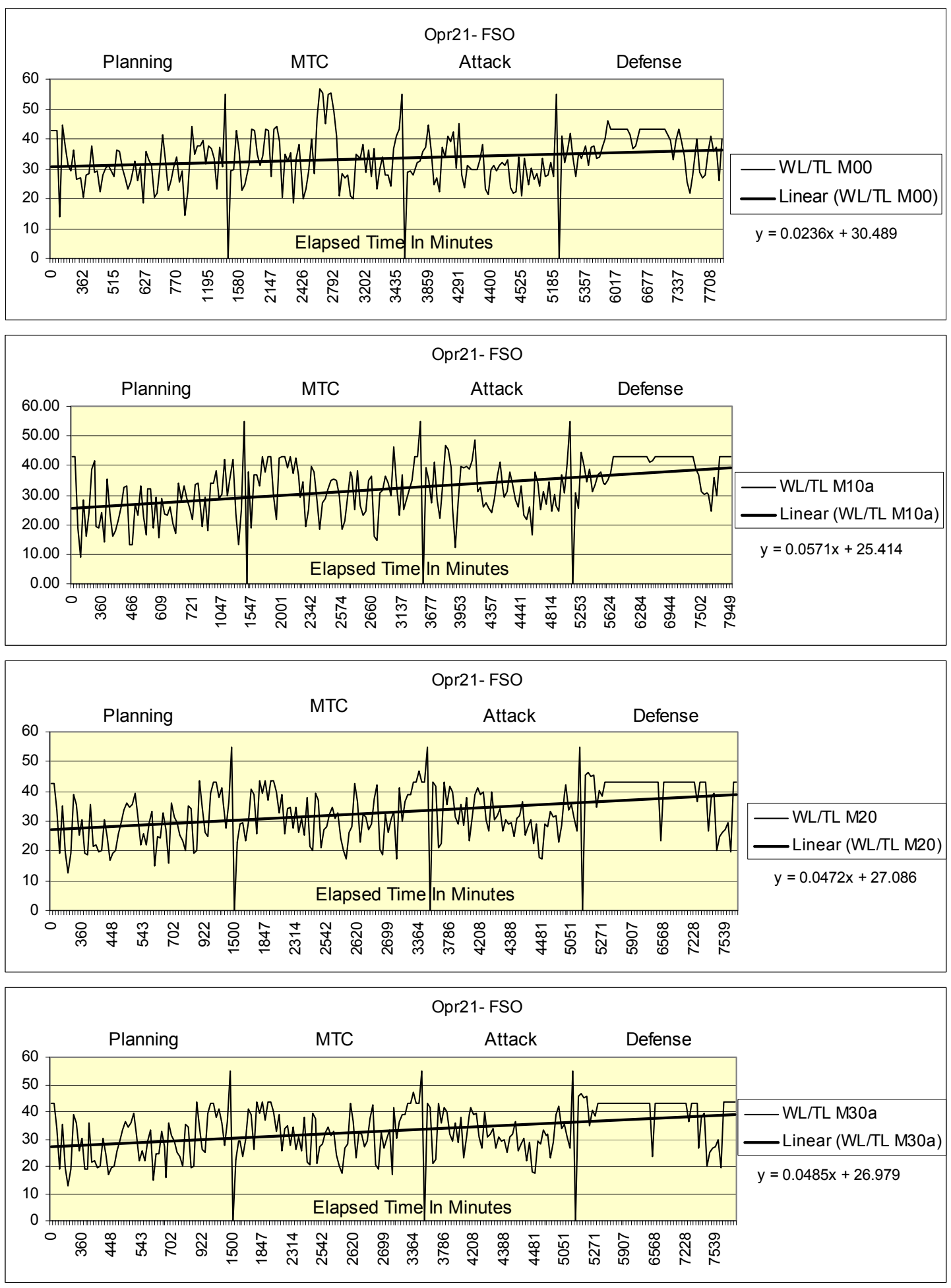

Figure 36 - Workload Performance for Operator 21, Fire Support Officer 
- Operator task performance data:

- Load the task data results file from each of the four simulation runs into a spreadsheet.

- Tabulate the data for number of task queues, number of task interrupts, number of task suspensions, and number of task drops against the four simulation runs for each operator.

- Plot the four task conditions over the four simulation runs for each operator to provide a task performance profile for each operator. A sample plot of these task conditions for operator 12, Battle Captain, is at Figure 37 and for operator 21, Fire Support Officer at Figure 38. The horizontal axis indicates the results from the four simulation runs where the M00 point indicates the baseline run with " 0 " reduction in task time performance, the M10 point represents the run with a $10 \%$ reduction in task time performance, the M20 point represents the run with a $20 \%$ reduction and the $\mathrm{M} 30$ point represents the run with a $30 \%$ reduction.

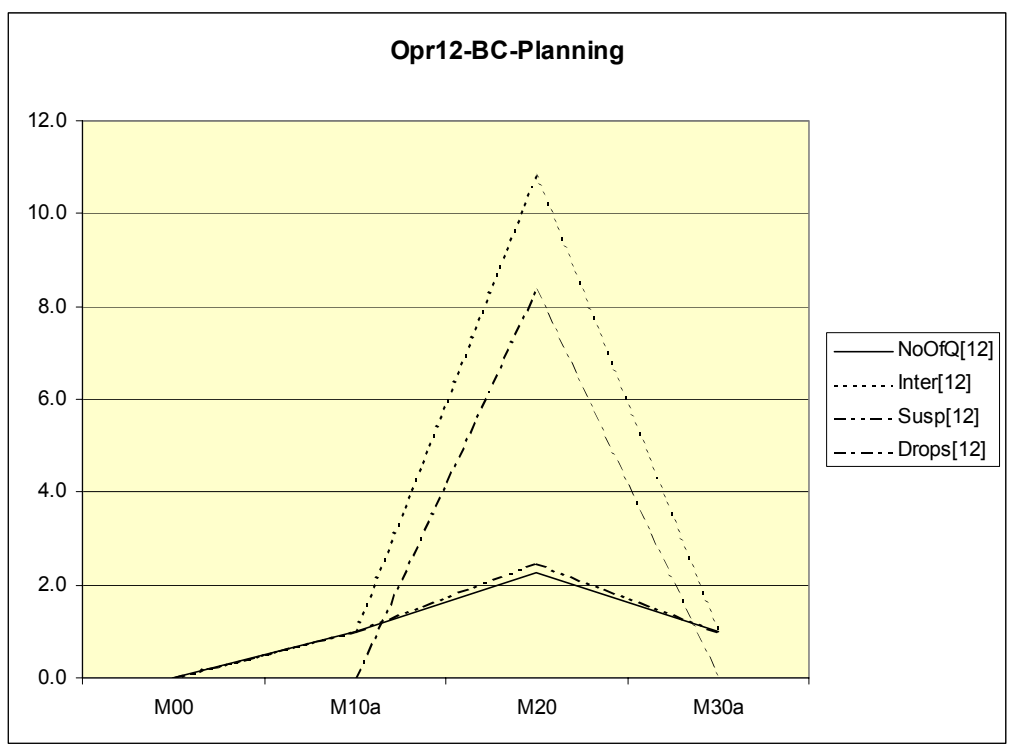



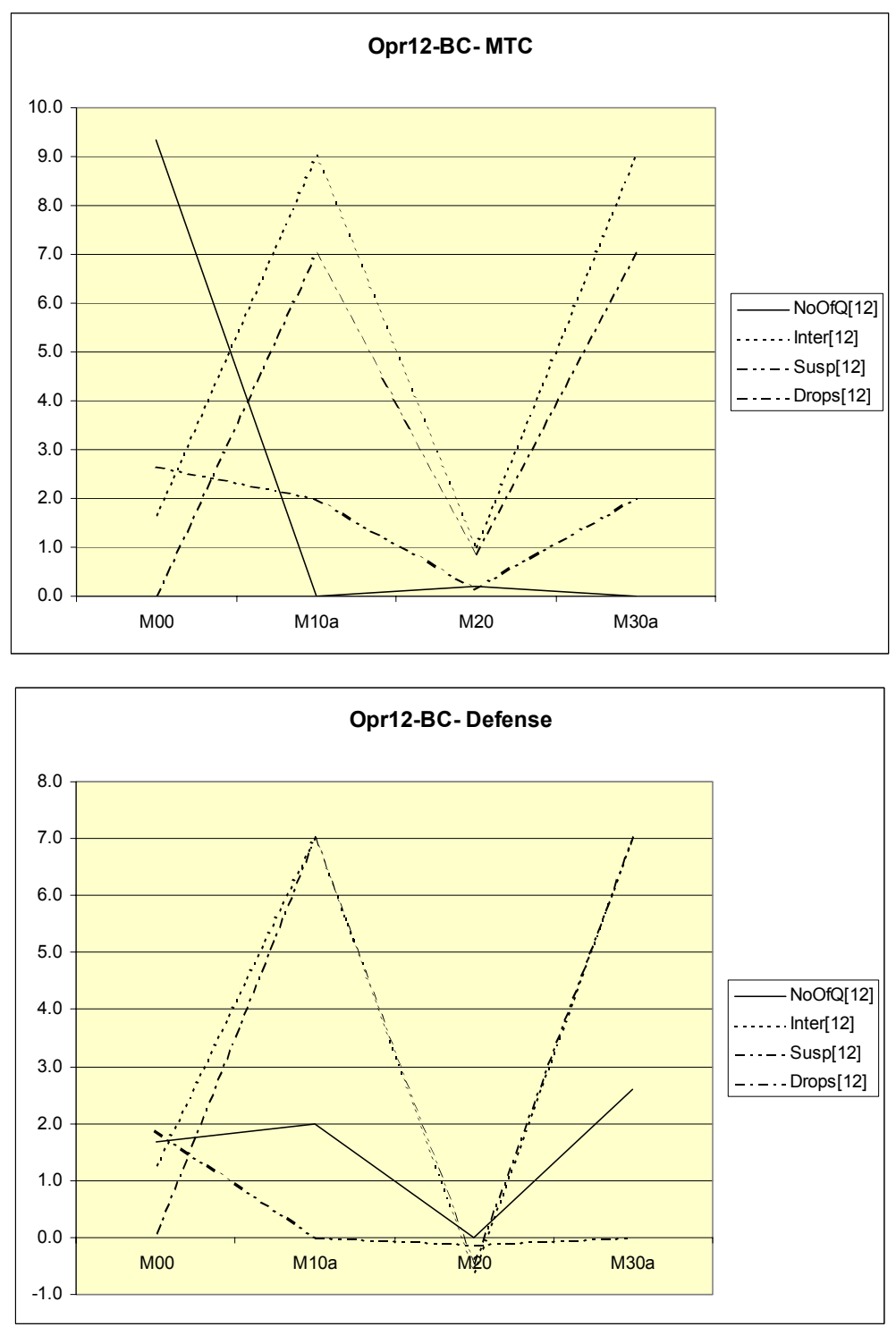


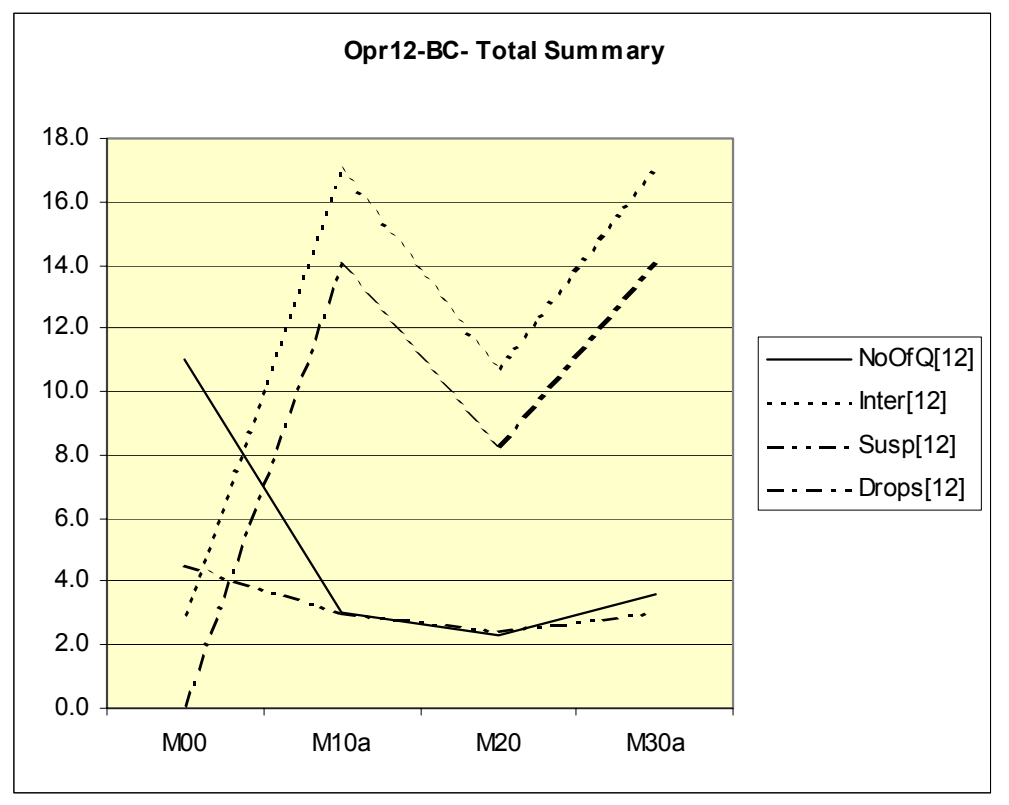

Figure 37 - Task Performance for Operator 12, Battle Captain

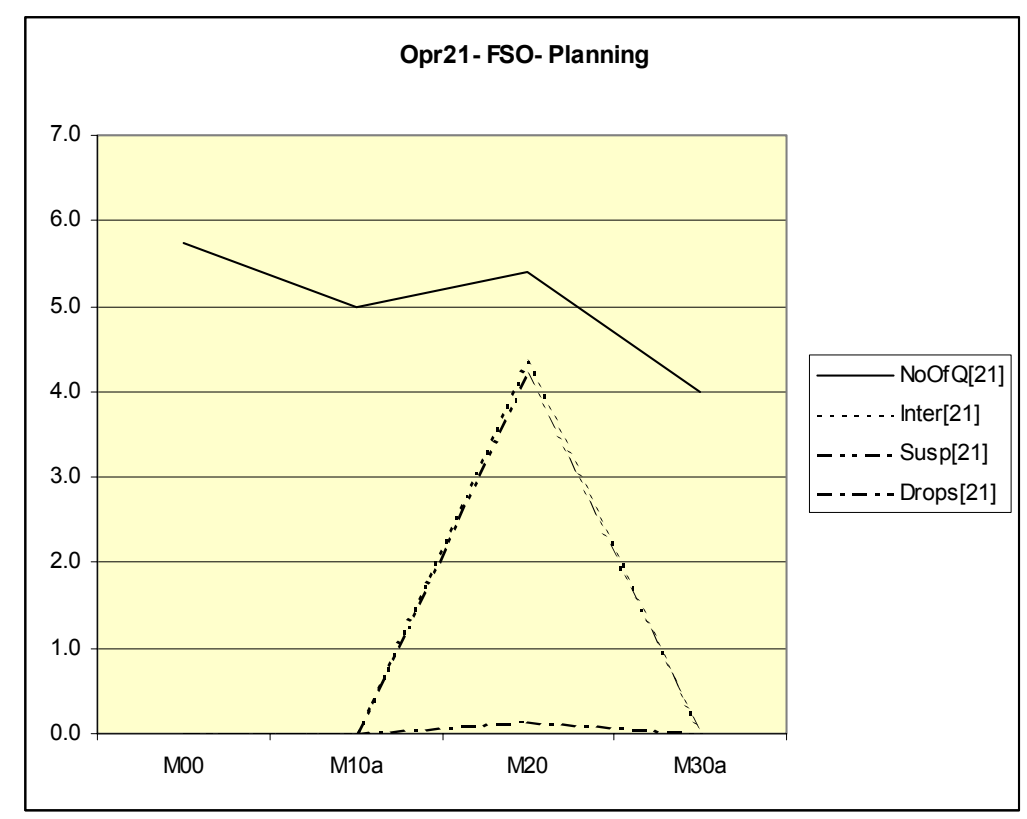



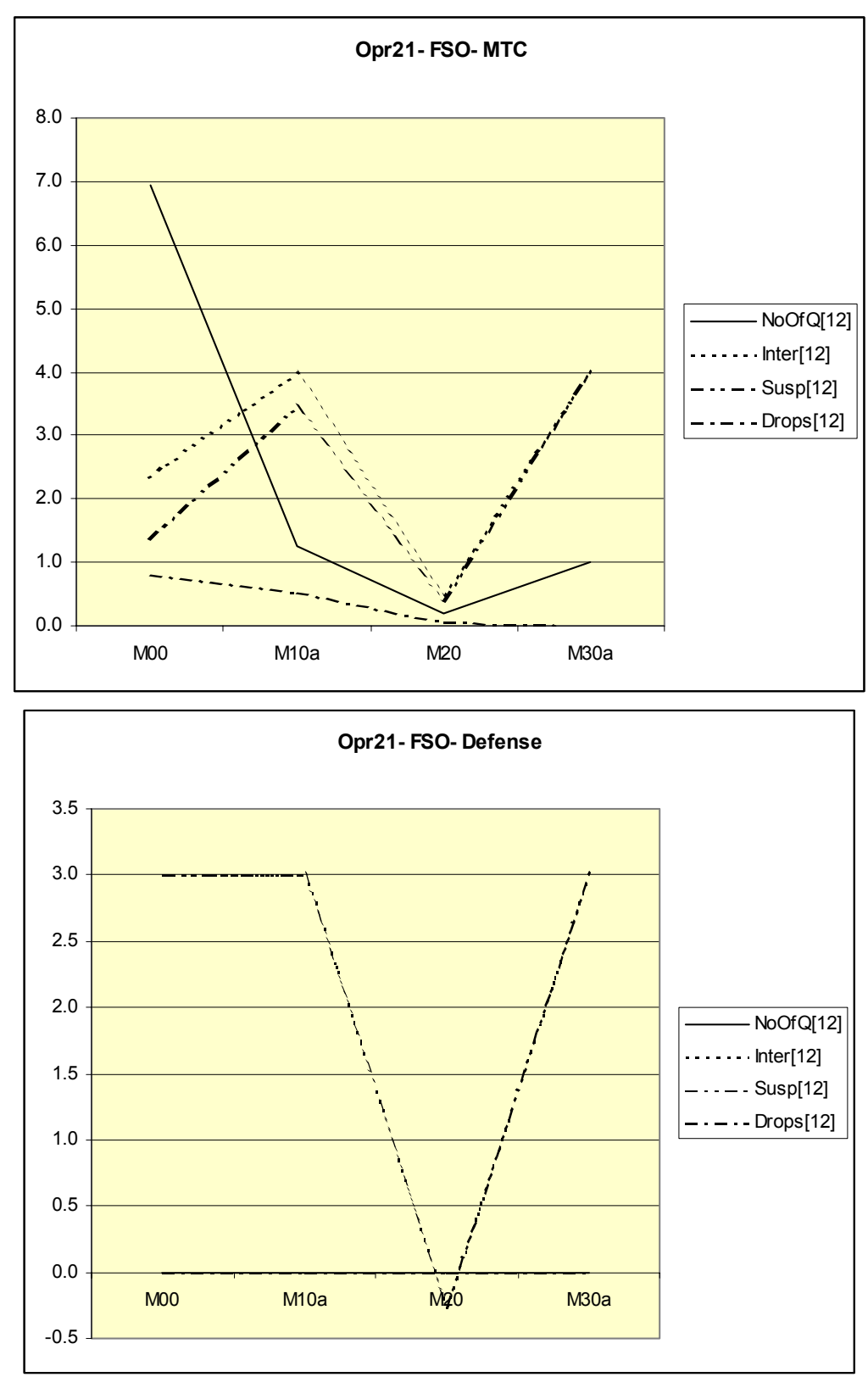


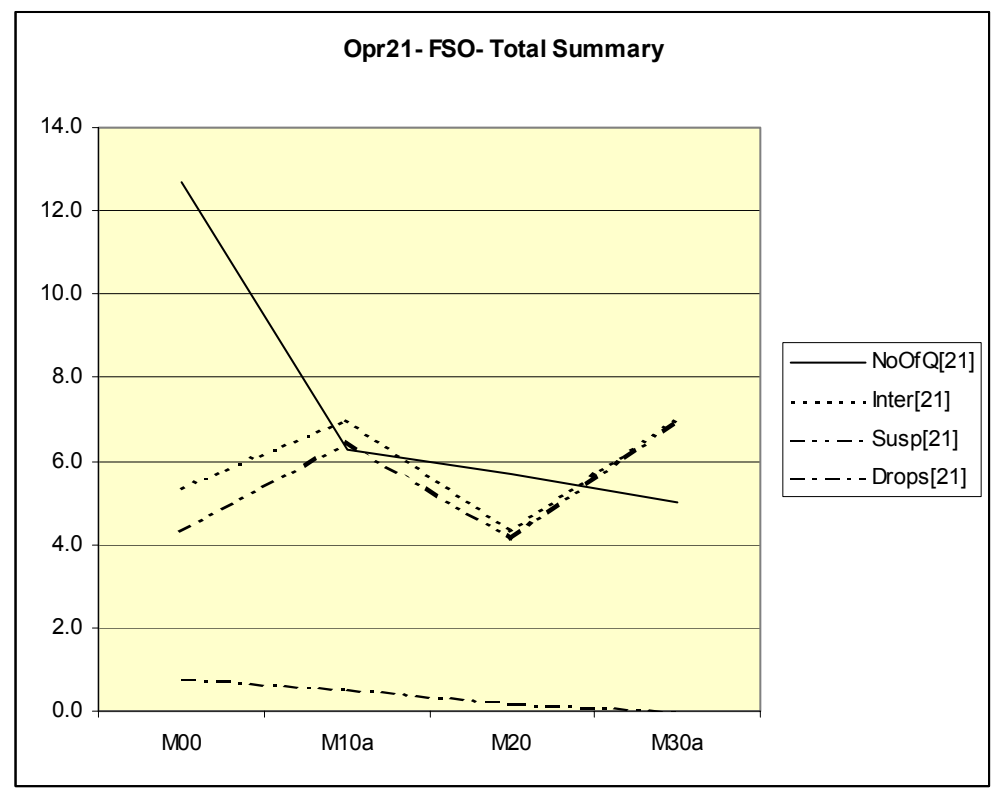

Figure 38 - Task Performance for Operator 21, Fire Support Officer

However, when these observations are combined with the task performance results shown in Figure 35, a more complete picture starts to emerge. With shorter task performance times more tasks can be performed over the same period of time. Similar results are shown in Figure 36 for the Battle Captain. If an operator finishes a task early or more quickly, that operator is not going to just wait idle until the next task arrives thereby achieving a lower workload. Considering the mission-oriented focus of the TOC work group, operators will immediately proceed on to the next task when the current performance effort is completed. This is reflected in the utilization and workload performance profiles in Figures 37 and 38.

On the other hand, this trend can only continue up to a point. This condition is where the operator becomes physically and / or cognitively saturated and can no longer keep pace with increasing performance demands resulting from quicker task execution. This condition is reflected in the charts in Figures 37 and 38 in the Total Summary chart for the Battle Captain and Fire Support Officer, which shows the number of tasks that were queued, interrupted, suspended, and dropped during the overall scenario. When task performance times are reduced from $10 \%$ to $20 \%$, while the utilization and workload profiles remain fairly constant, the task performance increases with fewer numbers of tasks that are queued up, interrupted, suspended and dropped. This predicts increased operator effectiveness because more tasks can be performed because they take less time to execute as long as the operator has the psychophysiological ability to keep up 
with the increased task demand. But, with further reductions in task time requirements that limit can be exceeded. Between a task time reduction of $20 \%$ to $30 \%$ the Total Summary chart in Figure 38 shows that the operator is apparently no longer able to keep up with increased task demands with the result that task performance begins to degrade with increases in the number of queues, interrupts, suspended tasks, and dropped tasks. The conclusion is that the tasks performance profiles between $10 \%$ to $30 \%$ reflect that an operator overload condition has occurred somewhere along this performance period causing a transition from peak to degraded operator performance.

\subsubsection{Predictions of TOC Activity From Mapping CoHOST Results to JNNS Predictors.}

The final stage of this analysis is to relate the predictors from the JNNS neural network simulation to performance parameters in the CoHOST simulation. Estimates can then be made as to the causal relationships between the factors influencing TOC performance at the system level as represented by JNNS and human performance in response to task stimuli as represented in CoHOST. These associations can then be interpreted as to the resulting impact on human performance that might result from proposed changes to TOC systems.

The relationship between task time decrease in CoHOST and the DV response by the regression models from JNNS, as stated previously, was determined from applying the subjective experience of one observer from working in and observing TOC operations. While the level of accuracy of the numbers may be in need of continued refinement, the relationship that they represent is not. The COMPASS paradigm states that there is a relationship between DV utilization levels in the TOC and overall resulting task performance time that this imposes on the operators. The exact level of the PSF that corresponds to utilization levels of the various DVs is a subject for continued field observation and simulation testing and evaluation. However, the use of initial subjective opinion for test parameters provides the basis for the examination of the causal relationships that exist here and allows the development of procedures to systematically examine them.

The DV / IV correlation matrices at Table 26 and Appendix P show IV from the JNNS simulation that have been determined to have some significance at various stages of the scenario as overall task time decreases. Comparing these predictors to the CoHOST simulation results such as those presented in Figures 35-38 allows this analysis to occur. 
This analysis will focus on operator \#21, the Fire Support Officer (FSO), and operator \#12, the Battle Captain (BC). Both of these operators play primary roles in the TOC and both are critical to the overall success of the TOC operation. Their taskload and utilization profiles are representative of the type of activity expected by operators whose job is to work at high levels of coordination and interaction with other operators. It is the quality of efforts by individuals such as these that will determine how good the conditions are upon which the actual decision makers base their judgments.

The role of the FSO is to act as the TOC single point of contact for directing and coordinating supporting cannon and missile artillery during combat. This operator has dedicated voice and digital communications links with fire direction control centers and firing batteries and is the one who initiates calls for fire missions based on the decisions made in the TOC. The FSO is responsible for carrying out the priority allocation of artillery missions as directed by the commander and for seeing that all requests for fire support are directed to the appropriate receiver.

The role of the $\mathrm{BC}$ can be compared to that of an office manager in the civilian world. The $\mathrm{BC}$ coordinates the minute-to-minute activities of the various sections in the TOC and insures that all the commander's policies, procedures, guidelines, and directives are implemented as ordered when they are required. When neither the commander, executive officer, or S3 operations officer are present, the $\mathrm{BC}$ is in command of the TOC.

\subsubsection{Scenario Phase 1 - Preoperations Planning.}

This phase of the scenario consists of moving combat forces forward to the line of departure (LD) and final preparation planning of combat activities immediately prior to the commencement of actual combat. During this phase the CoHOST simulation represents the FSO as being fairly busy with a constant high utilization rate and constant workload (previously shown in Figures 35 and 36). As task times are decreased from $0 \%$ to $30 \%$ below the default, the task performance of the FSO drops across all categories of task performance (number of task queues, number of interrupted tasks, number of suspended tasks, and number of dropped tasks as shown in Figure 38).

- Table 28 shows those tasks, predicted by JNNS at the $-10 \%$ PSF level, during this tactical phase whose increased utilization can cause these task time reduction effects. At the $-10 \%$ PSF level this includes such things as the requirement to 
coordinate and integrate fire support. A way to do this is by more effective utilization of the artillery digital fire communications system AFATDS or by more effective design of this system. The presence of the ALO in the TOC during this time can also improve overall performance as the ALO is also responsible for air supported fire missions and works closely with the FSO.

Table 28 - DV / IV Correlation - Planning - PSF @-10\%

\begin{tabular}{|c|c|c|}
\hline \multicolumn{3}{|c|}{ Phase 1- Planning (DV@0.25,-10\% PSF) } \\
\hline Dependent Variable / Model & Independent Variable & $\begin{array}{l}\text { Multi- } \\
\text { plication } \\
\text { Factor }\end{array}$ \\
\hline $\begin{array}{c}\text { \# } 05 \text { - Primary Task21. } \\
\text { Peacekeeping } \\
\text { operations. }\end{array}$ & \#51 - Misc Factors- Battle Tempo & 1 \\
\hline $\begin{array}{c}\text { \# } 06 \text { - Primary Task23. } \\
\text { Planning }\end{array}$ & \#28 - BOS Ratings- Intelligence & 2 \\
\hline $\begin{array}{c}\text { \# } 06 \text { - Primary Task23. } \\
\text { Planning }\end{array}$ & \#57 - Misc Factors- Staff Huddle & 2 \\
\hline $\begin{array}{l}\text { \# } 08 \text { - Secondary Task18. } \\
\text { Deception activities. }\end{array}$ & $\begin{array}{l}\text { \#25 - Command and Control BOS-1 Plan } \\
\text { for Combat Operations }\end{array}$ & 1 \\
\hline $\begin{array}{r}\text { \# } 09 \text { - Secondary Task19. } \\
\text { Rear operations. }\end{array}$ & $\begin{array}{l}\text { \#21 - Mobility \& Survivability BOS- } 6 \\
\text { Conduct Deception }\end{array}$ & 1 \\
\hline $\begin{array}{r}\text { \# } 09 \text { - Secondary Task19. } \\
\text { Rear operations. }\end{array}$ & \#64 - Personnel- S2 present in TOC & 1 \\
\hline $\begin{array}{l}\text { \# } 10 \text { - Secondary Task23. } \\
\text { Planning } \\
\end{array}$ & \#02 - Intel BOS- 2 Collect Information & 1 \\
\hline $\begin{array}{c}\text { \# } 10-\text { Secondary Task23. } \\
\text { Planning } \\
\end{array}$ & \#03 - Intel BOS- 3 Process Information & 1 \\
\hline $\begin{array}{c}\text { \# } 10 \text { - Secondary Task23. } \\
\text { Planning }\end{array}$ & $\begin{array}{l}\text { \#15 - Fire Support BOS-9 Coordinate, } \\
\text { Synchronize, and Integrate Fire } \\
\text { Support }\end{array}$ & 2 \\
\hline $\begin{array}{c}\text { \# } 12 \text { - Tertiary Task21. } \\
\text { Peacekeeping } \\
\text { operations. }\end{array}$ & $\begin{array}{c}\text { \#55 - Misc Factors- Observed Activity / } \\
\text { Stress Level in TOC }\end{array}$ & 1 \\
\hline $\begin{array}{c}\text { \# } 12 \text { - Tertiary Task21. } \\
\text { Peacekeeping } \\
\text { operations. }\end{array}$ & \#67 - Personnel- ALO present in TOC & 1 \\
\hline $\begin{array}{c}\text { \# } 13-\text { Tertiary Task23. } \\
\text { Planning } \\
\end{array}$ & $\begin{array}{l}\text { \#01 - Intel BOS- } 1 \text { Conduct Intelligence } \\
\text { Planning }\end{array}$ & 1 \\
\hline $\begin{array}{c}\text { \# } 13 \text { - Tertiary Task23. } \\
\text { Planning }\end{array}$ & \#50 - Misc Factors- Battle Timing & 1 \\
\hline $\begin{array}{l}\text { \# } 14 \text { - Mission1. Pre } \\
\text { Operations Planning }\end{array}$ & $\begin{array}{l}\# 16 \text { - Mobility \& Survivability BOS- } 1 \\
\text { Overcome Obstacles }\end{array}$ & 1 \\
\hline
\end{tabular}

- Table 29 shows those tasks, predicted by JNNS at the $-20 \%$ PSF level, during this tactical phase whose increased utilization can cause these task time reduction effects. Decreasing the amount of time to perform tasks $20 \%$ below the default 
focuses on tasks that improve the collection of intelligence such as the conduct of information operations, the presence of the S2 intelligence officer in the TOC and the planning and collecting of intelligence information. Systems critical to this activity includes the all source analysis system (ASAS), which is the digital communications system for this purpose.

Table 29 - DV / IV Correlation - Planning - PSF @ -20\%

\begin{tabular}{|c|c|c|}
\hline \multicolumn{3}{|c|}{ Phase 1- Planning (DV@0.5,-20\% PSF) } \\
\hline Dependent Variable / Model & Independent Variable & $\begin{array}{l}\text { Multi- } \\
\text { plication } \\
\text { Factor }\end{array}$ \\
\hline $\begin{array}{r}\text { \# } 05 \text { - Primary Task21. } \\
\text { Peacekeeping } \\
\text { operations. }\end{array}$ & \#51 - Misc Factors- Battle Tempo & 1 \\
\hline $\begin{array}{l}\text { \# } 05 \text { - Primary Task21. } \\
\text { Peacekeeping } \\
\text { operations. }\end{array}$ & \#53 - Misc Factors- Information Operations & 1 \\
\hline $\begin{array}{c}\text { \# } 06 \text { - Primary Task23. } \\
\text { Planning }\end{array}$ & $\begin{array}{l}\text { \#21 - Mobility \& Survivability BOS- } 6 \\
\text { Conduct Deception }\end{array}$ & 2 \\
\hline $\begin{array}{c}\text { \# } 06 \text { - Primary Task23. } \\
\text { Planning }\end{array}$ & \#57 - Misc Factors- Staff Huddle & 1 \\
\hline $\begin{array}{l}\text { \# } 08 \text { - Secondary Task18. } \\
\text { Deception activities. }\end{array}$ & $\begin{array}{l}\text { \#08 - Fire Support BOS- } 2 \text { Employ Field } \\
\text { Artillery }\end{array}$ & 1 \\
\hline $\begin{array}{r}\text { \# } 08 \text { - Secondary Task18. } \\
\text { Deception activities. }\end{array}$ & $\begin{array}{l}\text { \#25 - Command and Control BOS-1 Plan } \\
\text { for Combat Operations }\end{array}$ & 1 \\
\hline $\begin{array}{c}\text { \# } 09 \text { - Secondary Task19. Rear } \\
\text { operations. }\end{array}$ & \#64 - Personnel- S2 present in TOC & 1 \\
\hline $\begin{array}{l}\text { \# } 10 \text { - Secondary Task23. } \\
\text { Planning } \\
\end{array}$ & $\begin{array}{c}\text { \#19 - Mobility \& Survivability BOS- } 4 \\
\text { Enhance Physical Protection }\end{array}$ & 1 \\
\hline $\begin{array}{c}\text { \# } 12 \text { - Tertiary Task21. } \\
\text { Peacekeeping } \\
\text { operations. }\end{array}$ & $\begin{array}{c}\text { \#55 - Misc Factors- Observed Activity / } \\
\text { Stress Level in TOC }\end{array}$ & 1 \\
\hline $\begin{array}{c}\text { \# } 13 \text { - Tertiary Task23. } \\
\text { Planning }\end{array}$ & $\begin{array}{l}\text { \#01 - Intel BOS- } 1 \text { Conduct Intelligence } \\
\text { Planning }\end{array}$ & 1 \\
\hline $\begin{array}{c}\text { \# } 13 \text { - Tertiary Task23. } \\
\text { Planning }\end{array}$ & \#02 - Intel BOS- 2 Collect Information & 1 \\
\hline $\begin{array}{l}\text { \# } 14 \text { - Mission1. Pre } \\
\text { Operations Planning }\end{array}$ & $\begin{array}{l}\# 16 \text { - Mobility \& Survivability BOS- } 1 \\
\text { Overcome Obstacles }\end{array}$ & 1 \\
\hline
\end{tabular}

- Table 30 shows those tasks, predicted by JNNS at the $-30 \%$ PSF level, during this tactical phase whose increased utilization can cause these task time reduction effects. Further decreasing the overall task performance time $30 \%$ below the default can be propagated by even more effective intelligence operations such as more effective planning, collecting, processing, and disseminating of intelligence information. Activities associated with the coordination and synchronization of 
fire support assets also is critical. Increased message traffic from both ASAS and AFATDS can contribute to the more effective implementation of these activities.

Table 30 - DV / IV Correlation - Planning - PSF @ -30\%

\begin{tabular}{|c|c|c|}
\hline \multicolumn{3}{|c|}{ Phase 1- Planning (DV @ 0.75, -30\% PSF) } \\
\hline Dependent Variable / Model & Independent Variable & $\begin{array}{l}\text { Multi- } \\
\text { plication } \\
\text { Factor }\end{array}$ \\
\hline $\begin{array}{c}\text { \# } 05 \text { - Primary Task } 21 . \\
\text { Peacekeeping } \\
\text { operations. }\end{array}$ & $\begin{array}{l}\text { \#15 - Fire Support BOS- } 9 \text { Coordinate, } \\
\text { Synchronize, and Integrate Fire } \\
\text { Support }\end{array}$ & 2 \\
\hline $\begin{array}{l}\text { \# } 05 \text { - Primary Task } 21 . \\
\text { Peacekeeping ops. }\end{array}$ & $\begin{array}{c}\text { \#20 - Mobility \& Survivability BOS- } 5 \\
\text { Provide Operations Security }\end{array}$ & 1 \\
\hline $\begin{array}{c}\text { \# } 05 \text { - Primary Task } 21 . \\
\text { Peacekeeping } \\
\text { operations. }\end{array}$ & \#53 - Misc Factors- Information Operations & 2 \\
\hline $\begin{array}{c}\text { \# } 06 \text { - Primary Task } 23 . \\
\text { Planning }\end{array}$ & $\begin{array}{l}\text { \#21 - Mobility \& Survivability BOS- } 6 \\
\text { Conduct Deception }\end{array}$ & 3 \\
\hline $\begin{array}{c}\text { \# } 06 \text { - Primary Task } 23 . \\
\text { Planning }\end{array}$ & \#28 - BOS Ratings- Intelligence & 2 \\
\hline $\begin{array}{l}\text { \# } 06 \text { - Primary Task } 23 . \\
\text { Planning }\end{array}$ & \#57 - Misc Factors- Staff Huddle & 2 \\
\hline $\begin{array}{l}\text { \# } 08 \text { - Secondary Task18. } \\
\text { Deception activities. }\end{array}$ & $\begin{array}{l}\text { \#08 - Fire Support BOS- } 2 \text { Employ Field } \\
\text { Artillery }\end{array}$ & 1 \\
\hline $\begin{array}{l}\text { \# } 08 \text { - Secondary Task18. } \\
\text { Deception activities. }\end{array}$ & $\begin{array}{l}\# 59 \text { - Personnel- Executive Officer present } \\
\text { in TOC }\end{array}$ & 1 \\
\hline $\begin{array}{l}\text { \# } 09 \text { - Secondary Task19. Rear } \\
\text { operations. }\end{array}$ & \#64 - Personnel- S2 present in TOC & 1 \\
\hline $\begin{array}{l}\text { \# } 10 \text { - Secondary Task23. } \\
\text { Planning }\end{array}$ & $\begin{array}{c}\text { \#19 - Mobility \& Survivability BOS- } 4 \\
\text { Enhance Physical Protection }\end{array}$ & 1 \\
\hline $\begin{array}{c}\text { \# } 12 \text { - Tertiary Task } 21 . \\
\text { Peacekeeping } \\
\text { operations. }\end{array}$ & \#67 - Personnel- ALO present in TOC & 1 \\
\hline $\begin{array}{c}\text { \# } 13 \text { - Tertiary Task } 23 . \\
\text { Planning }\end{array}$ & $\begin{array}{l}\# 01 \text { - Intel BOS- } 1 \text { Conduct Intelligence } \\
\text { Planning }\end{array}$ & 1 \\
\hline $\begin{array}{c}\text { \# } 13 \text { - Tertiary Task } 23 . \\
\text { Planning }\end{array}$ & \#02 - Intel BOS- 2 Collect Information & 1 \\
\hline $\begin{array}{c}\text { \# } 13 \text { - Tertiary Task } 23 . \\
\text { Planning }\end{array}$ & \#03 - Intel BOS- 3 Process Information & 1 \\
\hline $\begin{array}{c}\text { \# } 13 \text { - Tertiary Task } 23 . \\
\text { Planning }\end{array}$ & $\begin{array}{l}\text { \#04 - Intel BOS- } 4 \text { Disseminate } \\
\text { Intelligence }\end{array}$ & 1 \\
\hline $\begin{array}{c}\text { \# } 13 \text { - Tertiary Task } 23 . \\
\text { Planning }\end{array}$ & \#50 - Misc Factors- Battle Timing & 1 \\
\hline $\begin{array}{l}\text { \#13 - Tertiary Task } 23 . \\
\text { Planning }\end{array}$ & \#63 - Personnel- S3 RTO present in TOC & 1 \\
\hline $\begin{array}{l}\text { \# } 14 \text { - Mission1. Pre } \\
\text { Operations Planning }\end{array}$ & $\begin{array}{l}\text { \#16 - Mobility \& Survivability BOS- } 1 \\
\text { Overcome Obstacles }\end{array}$ & 2 \\
\hline $\begin{array}{l}\text { \# } 14 \text { - Mission1. Pre } \\
\text { Operations Planning }\end{array}$ & $\begin{array}{c}\text { \#17 - Mobility \& Survivability BOS- } 2 \\
\text { Enhance Movement }\end{array}$ & 1 \\
\hline $\begin{array}{l}\text { \# } 14 \text { - Mission1. Pre } \\
\text { Operations Planning }\end{array}$ & $\begin{array}{c}\text { \#43 - Communications- MCS - Maneuver } \\
\text { Control System (Heavy \& Light) }\end{array}$ & 1 \\
\hline
\end{tabular}




\subsubsection{Scenario Phase 2 - Movement To Contact (MTC).}

This phase of the scenario involves the initiation of movement toward the supposed location of the enemy forces. The line of departure (LD) is crossed according to a time movement table and forces are deployed and prepared for combat but no shots are being fired, as there is no contact with the enemy. Once the enemy has been sighted and is within weapons range this phase of the scenario ends. During this phase the activities of the FSO become more diverse according to the CoHOST simulation with workload and utilization rates constantly changing as the FSO works to meet constantly changing requirements for possible artillery support as the forces move forward.

- Table 31 shows those tasks, predicted by JNNS at the $-10 \%$ PSF level, during this tactical phase whose increased utilization can cause these task time reduction effects. The activities that can contribute to an overall $10 \%$ reduction in task time requirements according to the JNNS simulation include the presence of the executive officer $(\mathrm{XO})$ and the $\mathrm{S} 3$ non-commissioned officer (S3NCO) in the TOC, the coordination and employment of integrated fire support and active use of systems that improve command and control or those things that improve continuous planning and directing of tactical operations. Systems that can affect these activities include the AFATDS, the maneuver control system (MCS) which is the digital system used to communicate with friendly forces, and the effective employment of reconnaissance operations.

Table 31 - DV / IV Correlation - MTC - PSF @-10\%

\begin{tabular}{|c|c|c|}
\hline \multicolumn{2}{|c|}{ Phase 2- MTC (DV @ 0.25,-10\% PSF) } & $\begin{array}{c}\text { Multi- } \\
\text { plication } \\
\text { Factor }\end{array}$ \\
\hline $\begin{array}{c}\text { Dependent Variable / Model } \\
\text { - Primary Task11. Fixing } \\
\text { enemy in position. }\end{array}$ & $\# 03$ - Intel BOS- 3 Process Information & 1 \\
\hline $\begin{array}{c}\# 02 \text { - Primary Task11. Fixing } \\
\text { enemy in position. }\end{array}$ & $\# 57$ - Misc Factors- Staff Huddle & 1 \\
\hline $\begin{array}{c}\# 02 \text { - Primary Task11. Fixing } \\
\text { enemy in position. }\end{array}$ & $\# 63$ - Personnel- S3 RTO present in TOC \\
\hline $\begin{array}{c}\# 07 \text { - Secondary Task12. } \\
\text { Synchronization with }\end{array}$ & $\# 05$ - Maneuver BOS- 1 Conduct Tactical \\
Movement & 2 \\
\hline
\end{tabular}




\begin{tabular}{|c|c|c|}
\hline Dependent Variable / Model & Independent Variable & $\begin{array}{c}\text { Multi- } \\
\text { plication } \\
\text { Factor }\end{array}$ \\
\hline \multicolumn{3}{|l|}{ supporting forces. } \\
\hline $\begin{array}{c}\text { \# } 07 \text { - Secondary Task12. } \\
\text { Synchronization with } \\
\text { supporting forces. }\end{array}$ & $\begin{array}{l}\text { \#15 - Fire Support BOS- } 9 \text { Coordinate, } \\
\text { Synchronize, and Integrate Fire } \\
\text { Support }\end{array}$ & 2 \\
\hline $\begin{array}{c}\text { \# } 07 \text { - Secondary Task12. } \\
\text { Synchronization with } \\
\text { supporting forces. }\end{array}$ & $\begin{array}{l}\text { \#16 - Mobility \& Survivability BOS- } 1 \\
\text { Overcome Obstacles }\end{array}$ & 2 \\
\hline $\begin{array}{l}\text { \# } 08 \text { - Secondary Task18. } \\
\text { Deception activities. }\end{array}$ & $\begin{array}{l}\text { \#25 - Command and Control BOS- 1 Plan } \\
\text { for Combat Operations }\end{array}$ & 2 \\
\hline $\begin{array}{l}\text { \# } 15 \text { - Mission2. Movement to } \\
\text { Contact }\end{array}$ & $\begin{array}{l}\# 08 \text { - Fire Support BOS- } 2 \text { Employ Field } \\
\text { Artillery }\end{array}$ & 1 \\
\hline $\begin{array}{l}\text { \# } 15 \text { - Mission2. Movement to } \\
\text { Contact }\end{array}$ & $\begin{array}{l}\text { \#27 - Command and Control BOS- } 3 \text { Direct } \\
\text { and Lead Unit in Execution of } \\
\text { Battle }\end{array}$ & 1 \\
\hline $\begin{array}{l}\text { \# } 15 \text { - Mission2. Movement to } \\
\text { Contact }\end{array}$ & \#62 - Personnel- S3 NCO present in TOC & 1 \\
\hline
\end{tabular}

- Table 32 shows those tasks, predicted by JNNS at the $-20 \%$ PSF level, during this tactical phase whose increased utilization can cause these task time reduction effects. Decreasing the task performance time during this MTC phase to the $20 \%$ level below default is predicted as being possible by the same activities that predict a $10 \%$ reduction but with utilization rates.

Table 32 - DV / IV Correlation - MTC - PSF @-20\%

\begin{tabular}{|c|c|c|}
\hline \multicolumn{3}{|c|}{ Phase 2- MTC (DV@0.5,-20\% PSF) } \\
\hline Dependent Variable / Model & Independent Variable & $\begin{array}{l}\text { Multi- } \\
\text { plication } \\
\text { Factor }\end{array}$ \\
\hline $\begin{array}{c}\text { \# } 02 \text { - Primary Task11. Fixing } \\
\text { enemy in position. }\end{array}$ & \#03 - Intel BOS- 3 Process Information & 1 \\
\hline $\begin{array}{c}\text { \# } 02 \text { - Primary Task11. Fixing } \\
\text { enemy in position. }\end{array}$ & $\begin{array}{l}\text { \#26 - Command and Control BOS- } 2 \text { Direct } \\
\text { and Lead Unit During the } \\
\text { Preparation Phase of the Battle }\end{array}$ & 2 \\
\hline $\begin{array}{c}\text { \# } 02 \text { - Primary Task11. Fixing } \\
\text { enemy in position. }\end{array}$ & \#57 - Misc Factors- Staff Huddle & 1 \\
\hline $\begin{array}{c}\text { \# } 02 \text { - Primary Task11. Fixing } \\
\text { enemy in position. }\end{array}$ & $\begin{array}{l}\text { \#59 - Personnel- Executive Officer present } \\
\text { in TOC }\end{array}$ & 1 \\
\hline $\begin{array}{l}\text { \# } 07 \text { - Secondary Task12. } \\
\text { Synchronization with } \\
\text { supporting forces. } \\
\end{array}$ & $\begin{array}{l}\text { \#05 - Maneuver BOS- } 1 \text { Conduct Tactical } \\
\text { Movement }\end{array}$ & 2 \\
\hline $\begin{array}{l}\text { \# } 07 \text { - Secondary Task12. } \\
\text { Synchronization with } \\
\text { supporting forces. }\end{array}$ & $\begin{array}{l}\text { \#15 - Fire Support BOS- } 9 \text { Coordinate, } \\
\text { Synchronize, and Integrate Fire } \\
\text { Support }\end{array}$ & 1 \\
\hline $\begin{array}{l}\text { \# } 07 \text { - Secondary Task12. } \\
\text { Synchronization with }\end{array}$ & $\begin{array}{l}\text { \#16 - Mobility \& Survivability BOS- } 1 \\
\text { Overcome Obstacles }\end{array}$ & 1 \\
\hline
\end{tabular}




\begin{tabular}{|c|c|c|}
\hline Dependent Variable / Model & Independent Variable & $\begin{array}{c}\text { Multi- } \\
\text { plication } \\
\text { Factor }\end{array}$ \\
\hline \multicolumn{3}{|l|}{ supporting forces. } \\
\hline $\begin{array}{r}\text { \# } 08 \text { - Secondary Task18. } \\
\text { Deception activities. }\end{array}$ & $\begin{array}{l}\text { \#08 - Fire Support BOS- } 2 \text { Employ Field } \\
\text { Artillery }\end{array}$ & 2 \\
\hline $\begin{array}{l}\text { \# } 08 \text { - Secondary Task18. } \\
\text { Deception activities. }\end{array}$ & $\begin{array}{l}\text { \#25 - Command and Control BOS- } 1 \text { Plan } \\
\text { for Combat Operations }\end{array}$ & 1 \\
\hline $\begin{array}{l}\text { \# } 15 \text { - Mission2. Movement to } \\
\text { Contact }\end{array}$ & $\begin{array}{l}\text { \#27 - Command and Control BOS- } 3 \text { Direct } \\
\text { and Lead Unit in Execution of } \\
\text { Battle }\end{array}$ & 1 \\
\hline $\begin{array}{l}\text { \# } 15 \text { - Mission2. Movement to } \\
\text { Contact }\end{array}$ & $\begin{array}{c}\# 52 \text { - Misc Factors- Reconnaissance } \\
\text { Operations }\end{array}$ & 1 \\
\hline $\begin{array}{l}\text { \# } 15 \text { - Mission2. Movement to } \\
\text { Contact }\end{array}$ & \#62 - Personnel- S3 NCO present in TOC & 1 \\
\hline
\end{tabular}

- Table 33 shows those tasks, predicted by JNNS at the $-30 \%$ PSF level, during this tactical phase whose increased utilization can cause these task time reduction effects. Reducing the task performance times $30 \%$ below default is predicted as being possible through a continuation of existing activities along with higher activities with fire support battlefield operating systems (BOS) and more effective mobility operations.

Table 33 - DV / IV Correlation - MTC - PSF @-30\%

\begin{tabular}{|c|c|c|}
\hline \multicolumn{3}{|c|}{ Phase 2-MTC (DV@0.75,-30\% PSF) } \\
\hline Dependent Variable / Model & Independent Variable & $\begin{array}{l}\text { Multi- } \\
\text { plication } \\
\text { Factor }\end{array}$ \\
\hline $\begin{array}{c}\text { \# } 07 \text { - Secondary Task12. } \\
\text { Synchronization with } \\
\text { supporting forces. }\end{array}$ & $\begin{array}{l}\text { \#05 - Maneuver BOS- } 1 \text { Conduct Tactical } \\
\text { Movement }\end{array}$ & 2 \\
\hline $\begin{array}{c}\text { \# } 07 \text { - Secondary Task12. } \\
\text { Synchronization with } \\
\text { supporting forces. }\end{array}$ & $\begin{array}{l}\text { \#15 - Fire Support BOS- } 9 \text { Coordinate, } \\
\text { Synchronize, and Integrate Fire } \\
\text { Support }\end{array}$ & 2 \\
\hline $\begin{array}{c}\text { \# } 07 \text { - Secondary Task12. } \\
\text { Synchronization with } \\
\text { supporting forces. }\end{array}$ & $\begin{array}{c}\text { \#16 - Mobility \& Survivability BOS- } 1 \\
\text { Overcome Obstacles }\end{array}$ & 3 \\
\hline $\begin{array}{r}\text { \# } 08 \text { - Secondary Task18. } \\
\text { Deception activities. }\end{array}$ & $\begin{array}{l}\# 08 \text { - Fire Support BOS- } 2 \text { Employ Field } \\
\text { Artillery }\end{array}$ & 3 \\
\hline $\begin{array}{r}\text { \# } 08 \text { - Secondary Task18. } \\
\text { Deception activities. }\end{array}$ & $\begin{array}{l}\text { \#21 - Mobility \& Survivability BOS- } 6 \\
\text { Conduct Deception }\end{array}$ & 1 \\
\hline $\begin{array}{r}\text { \# } 08 \text { - Secondary Task18. } \\
\text { Deception activities. }\end{array}$ & $\begin{array}{l}\# 59 \text { - Personnel- Executive Officer present } \\
\text { in TOC }\end{array}$ & 1 \\
\hline $\begin{array}{l}\text { \# } 11 \text { - Tertiary Task3. } \\
\text { Movement to the line } \\
\text { of departure. }\end{array}$ & $\begin{array}{l}\text { \#25 - Command and Control BOS-1 Plan } \\
\text { for Combat Operations }\end{array}$ & 1 \\
\hline $\begin{array}{l}\text { \# } 11 \text { - Tertiary Task3. } \\
\text { Movement to the line }\end{array}$ & \#63 - Personnel- S3 RTO present in TOC & 1 \\
\hline
\end{tabular}




\begin{tabular}{|c|c|c|}
\hline Dependent Variable / Model & Independent Variable & $\begin{array}{c}\text { Multi- } \\
\text { plication } \\
\text { Factor }\end{array}$ \\
\hline \multicolumn{3}{|l|}{ of departure. } \\
\hline $\begin{array}{l}\text { \# } 15 \text { - Mission2. Movement to } \\
\text { Contact }\end{array}$ & $\begin{array}{l}\text { \#26 - Command and Control BOS- } 2 \text { Direct } \\
\text { and Lead Unit During the } \\
\text { Preparation Phase of the Battle }\end{array}$ & 1 \\
\hline $\begin{array}{l}\text { \# } 15 \text { - Mission2. Movement to } \\
\text { Contact }\end{array}$ & $\begin{array}{l}\text { \#27 - Command and Control BOS- } 3 \text { Direct } \\
\text { and Lead Unit in Execution of } \\
\text { Battle }\end{array}$ & 1 \\
\hline $\begin{array}{l}\text { \# } 15 \text { - Mission2. Movement to } \\
\text { Contact }\end{array}$ & $\begin{array}{l}\text { \#52 - Misc Factors- Reconnaissance } \\
\text { Operations }\end{array}$ & 1 \\
\hline $\begin{array}{l}\text { \# } 15 \text { - Mission2. Movement to } \\
\text { Contact }\end{array}$ & \#62 - Personnel- S3 NCO present in TOC & 1 \\
\hline
\end{tabular}

\subsubsection{Scenario Phase 3 - Attack.}

This phase of the scenario is characterized by the fact that the friendly forces have encountered the enemy and are attacking them. The enemy may also be moving thus causing a meeting engagement, or they may be in a stationary defense where the action becomes an attack against fixed fortifications. In this situation the FSO is fairly constantly utilized with a workload that includes the actual directing and coordinating of artillery and airstrike missions against the enemy.

- Table 34 shows those tasks, predicted by JNNS at the $-10 \%$ PSF level, during this tactical phase whose increased utilization can cause these task time reduction effects. $10 \%$ reductions in task time can be influenced by the presence of the $\mathrm{XO}$, negatively by TOC personnel being in chemical protective clothing (MOPP) and negatively by abnormally high or low humidity. The key activity, however, is the actual employment of field artillery assets as indicated by a higher multiplication factor in the table. For the FSO, the key to success in this effort is effective use of communications systems both to know and understand the enemy situation as well as the ability to direct the artillery assets supporting the battle. The AFATDS and ASAS digital communications systems provide this communications abilities and an increased efficiency to this performance level could be the key to these improvement effects. 
Table 34 - DV / IV Correlation - Attack - PSF @ -10\%

\begin{tabular}{|c|c|c|}
\hline Dependent Variable / Model & Independent Variable & $\begin{array}{l}\text { Multi- } \\
\text { plication } \\
\text { Factor }\end{array}$ \\
\hline $\begin{array}{l}\text { \# } 03 \text { - Primary Task12. } \\
\text { Synchronization with } \\
\text { supporting forces. }\end{array}$ & $\begin{array}{l}\text { \#08 - Fire Support BOS- } 2 \text { Employ Field } \\
\text { Artillery }\end{array}$ & 2 \\
\hline $\begin{array}{l}\text { \# } 03 \text { - Primary Task12. } \\
\text { Synchronization with } \\
\text { supporting forces. }\end{array}$ & $\begin{array}{l}\text { \#21 - Mobility \& Survivability BOS- } 6 \\
\text { Conduct Deception }\end{array}$ & 1 \\
\hline $\begin{array}{l}\text { \# } 03 \text { - Primary Task12. } \\
\text { Synchronization with } \\
\text { supporting forces. }\end{array}$ & $\begin{array}{l}\text { \#25 - Command and Control BOS- } 1 \text { Plan } \\
\text { for Combat Operations }\end{array}$ & 1 \\
\hline $\begin{array}{l}\text { \# } 03 \text { - Primary Task12. } \\
\text { Synchronization with } \\
\text { supporting forces. }\end{array}$ & $\begin{array}{c}\text { \#69 - Environmental Factor- Relative } \\
\text { Humidity, } \%\end{array}$ & 1 \\
\hline $\begin{array}{c}\text { \# } 04 \text { - Primary Task15. } \\
\text { Destruction of first } \\
\text { echelon forces. }\end{array}$ & \#50 - Misc Factors- Battle Timing & 1 \\
\hline $\begin{array}{l}\text { \# } 04 \text { - Primary Task15. } \\
\text { Destruction of first } \\
\text { echelon forces. }\end{array}$ & $\begin{array}{l}\text { \#59 - Personnel- Executive Officer present } \\
\text { in TOC }\end{array}$ & 1 \\
\hline \# 16 - Mission3. Attack & \#56 - Misc Factors- MOPP Level & 1 \\
\hline $\begin{array}{c}\text { \# } 17 \text { - Mission5. River } \\
\text { Crossing }\end{array}$ & $\begin{array}{l}\text { \#24 - Air Defense BOS- } 2 \text { Take Passive Air } \\
\text { Defense Measures }\end{array}$ & 1 \\
\hline
\end{tabular}

- Table 35 shows those tasks, predicted by JNNS at the $-20 \%$ PSF level, during this tactical phase whose increased utilization can cause these task time reduction effects. The measures that are predicted to cause additional efficiencies to this level include the intelligence BOS of intelligence dissemination, and battle timing or the ability to synchronize forces on the battlefield to meet enemy threats. Providing a strong negative correlation is the performance degradation while in MOPP status (when the TOC personnel are wearing chemical protective clothing and equipment). The presence of the $\mathrm{XO}$ and $\mathrm{S} 3 \mathrm{NCO}$ in the TOC is also predicted to contribute to this increased performance level. 
Table 35 - DV / IV Correlation - Attack - PSF @ -20\%

\begin{tabular}{|c|c|c|}
\hline \multicolumn{3}{|c|}{ Phase 3- Attack (DV@0.5,-20\% PSF) } \\
\hline Dependent Variable / Model & Independent Variable & $\begin{array}{l}\text { Multi- } \\
\text { plication } \\
\text { Factor }\end{array}$ \\
\hline $\begin{array}{l}\text { \# } 01 \text { - Primary Task10. } \\
\text { Destruction, capture, } \\
\text { or bypass of enemy } \\
\text { force. }\end{array}$ & $\begin{array}{l}\text { \#04 - Intel BOS- } 4 \text { Disseminate } \\
\text { Intelligence }\end{array}$ & 4 \\
\hline $\begin{array}{l}\text { \# } 01 \text { - Primary Task10. } \\
\text { Destruction, capture, } \\
\text { or bypass of enemy } \\
\text { force. }\end{array}$ & $\begin{array}{c}\text { \#17 - Mobility \& Survivability BOS- } 2 \\
\text { Enhance Movement }\end{array}$ & 1 \\
\hline $\begin{array}{l}\text { \# } 01 \text { - Primary Task10. } \\
\text { Destruction, capture, } \\
\text { or bypass of enemy } \\
\text { force. }\end{array}$ & $\begin{array}{l}\text { \#26 - Command and Control BOS- } 2 \text { Direct } \\
\text { and Lead Unit During the } \\
\text { Preparation Phase of the Battle }\end{array}$ & 1 \\
\hline $\begin{array}{l}\text { \# } 01 \text { - Primary Task10. } \\
\text { Destruction, capture, } \\
\text { or bypass of enemy } \\
\text { force. }\end{array}$ & \#56 - Misc Factors- MOPP Level & 3 \\
\hline $\begin{array}{l}\text { \# } 03 \text { - Primary Task12. } \\
\text { Synchronization with } \\
\text { supporting forces. }\end{array}$ & $\begin{array}{l}\text { \#21 - Mobility \& Survivability BOS- } 6 \\
\text { Conduct Deception }\end{array}$ & 1 \\
\hline $\begin{array}{l}\text { \# } 03 \text { - Primary Task12. } \\
\text { Synchronization with } \\
\text { supporting forces. }\end{array}$ & $\begin{array}{l}\text { \#22 - Mobility \& Survivability BOS- } 7 \\
\text { Conduct NBC Defense }\end{array}$ & 1 \\
\hline $\begin{array}{l}\text { \# } 03 \text { - Primary Task12. } \\
\text { Synchronization with } \\
\text { supporting forces. }\end{array}$ & \#50 - Misc Factors- Battle Timing & 3 \\
\hline $\begin{array}{l}\text { \# } 04 \text { - Primary Task15. } \\
\text { Destruction of first } \\
\text { echelon forces. }\end{array}$ & $\begin{array}{l}\text { \#59 - Personnel- Executive Officer present } \\
\text { in TOC }\end{array}$ & 1 \\
\hline $\begin{array}{l}\text { \# } 17 \text { - Mission5. River } \\
\text { Crossing }\end{array}$ & \#62 - Personnel- S3 NCO present in TOC & 1 \\
\hline
\end{tabular}

- Table 36 shows those tasks, predicted by JNNS at the -30\% PSF level, during this tactical phase whose increased utilization can cause these task time reduction effects. Adding to many of the same factors present at the $-20 \%$ PSF level is success at the employment of field artillery assets and the effective use of the field artillery digital communications system AFATDS. 
Table 36 - DV / IV Correlation - Attack - PSF @ -30\%

\begin{tabular}{|c|c|c|}
\hline Dependent Variable / Model & Independent Variable & $\begin{array}{l}\text { Multi- } \\
\text { plication } \\
\text { Factor }\end{array}$ \\
\hline $\begin{array}{l}\text { \# } 01 \text { - Primary Task10. } \\
\text { Destruction, capture, } \\
\text { or bypass of enemy } \\
\text { force. }\end{array}$ & $\begin{array}{l}\text { \#04 - Intel BOS- } 4 \text { Disseminate } \\
\text { Intelligence }\end{array}$ & 3 \\
\hline $\begin{array}{l}\text { \# } 01 \text { - Primary Task10. } \\
\text { Destruction, capture, } \\
\text { or bypass of enemy } \\
\text { force. }\end{array}$ & $\begin{array}{c}\text { \#17 - Mobility \& Survivability BOS- } 2 \\
\text { Enhance Movement }\end{array}$ & 1 \\
\hline $\begin{array}{l}\text { \# } 01 \text { - Primary Task10. } \\
\text { Destruction, capture, } \\
\text { or bypass of enemy } \\
\text { force. }\end{array}$ & $\begin{array}{l}\text { \#26 - Command and Control BOS- } 2 \text { Direct } \\
\text { and Lead Unit During the } \\
\text { Preparation Phase of the Battle }\end{array}$ & 1 \\
\hline $\begin{array}{l}\text { \# } 01 \text { - Primary Task10. } \\
\text { Destruction, capture, } \\
\text { or bypass of enemy } \\
\text { force. }\end{array}$ & \#56 - Misc Factors- MOPP Level & 2 \\
\hline $\begin{array}{l}\text { \# } 03 \text { - Primary Task12. } \\
\text { Synchronization with } \\
\text { supporting forces. }\end{array}$ & $\begin{array}{l}\text { \#08 - Fire Support BOS- } 2 \text { Employ Field } \\
\text { Artillery }\end{array}$ & 2 \\
\hline $\begin{array}{l}\text { \# } 03 \text { - Primary Task12. } \\
\text { Synchronization with } \\
\text { supporting forces. }\end{array}$ & $\begin{array}{l}\text { \#15 - Fire Support BOS- } 9 \text { Coordinate, } \\
\text { Synchronize, and Integrate Fire } \\
\text { Support }\end{array}$ & 1 \\
\hline $\begin{array}{l}\text { \# } 03 \text { - Primary Task12. } \\
\text { Synchronization with } \\
\text { supporting forces. }\end{array}$ & $\begin{array}{l}\text { \#35 - Communications- AFATDS - } \\
\text { Advanced Field Artillery Tactical } \\
\text { Data System }\end{array}$ & 1 \\
\hline $\begin{array}{l}\text { \# } 03 \text { - Primary Task12. } \\
\text { Synchronization with } \\
\text { supporting forces. }\end{array}$ & \#67 - Personnel- ALO present in TOC & 1 \\
\hline $\begin{array}{l}\text { \# } 03 \text { - Primary Task12. } \\
\text { Synchronization with } \\
\text { supporting forces. } \\
\end{array}$ & $\begin{array}{c}\text { \#69 - Environmental Factor- Relative } \\
\text { Humidity, \% }\end{array}$ & 1 \\
\hline $\begin{array}{l}\text { \# } 04 \text { - Primary Task15. } \\
\text { Destruction of first } \\
\text { echelon forces. }\end{array}$ & \#50 - Misc Factors- Battle Timing & 2 \\
\hline $\begin{array}{l}\text { \# } 04 \text { - Primary Task15. } \\
\text { Destruction of first } \\
\text { echelon forces. }\end{array}$ & $\begin{array}{l}\text { \#59 - Personnel- Executive Officer present } \\
\text { in TOC }\end{array}$ & 1 \\
\hline $\begin{array}{c}\text { \# } 17 \text { - Mission5. River } \\
\text { Crossing }\end{array}$ & $\begin{array}{l}\text { \#05 - Maneuver BOS- } 1 \text { Conduct Tactical } \\
\text { Movement }\end{array}$ & 1 \\
\hline $\begin{array}{c}\text { \# } 17 \text { - Mission5. River } \\
\text { Crossing }\end{array}$ & $\begin{array}{l}\text { \#24 - Air Defense BOS- } 2 \text { Take Passive Air } \\
\text { Defense Measures }\end{array}$ & 1 \\
\hline $\begin{array}{c}\text { \# } 17 \text { - Mission5. River } \\
\text { Crossing }\end{array}$ & \#62 - Personnel- S3 NCO present in TOC & 1 \\
\hline
\end{tabular}




\subsubsection{Scenario Phase 4 - Defense}

This phase of the scenario is characterized by the fact that the friendly forces have seized or otherwise occupied a battle position and they conduct a defense against enemy forces who try to take the position away from them through hostile attack. In this situation, as in the attack phase of the scenario, the FSO is fairly constantly utilized with a workload that includes the actual directing and coordinating of artillery and airstrike missions against the enemy.

- Table 37 shows those tasks, predicted by JNNS at the $-10 \%$ PSF level, during this tactical phase whose increased utilization can cause these task time reduction effects. $10 \%$ reductions are predicted to be achieved most importantly through effective employment of field artillery assets. Also contributing are the effective coordination of field artillery assets, an effective plan for the conduct of the defense, and good battle rhythm or battle timing in the TOC operation. The presence of the $\mathrm{XO}$ is also predicted to contribute to TOC effectiveness improvements to this level.

Table 37 - DV / IV Correlation - Defense - PSF @-10\%

\begin{tabular}{|c|c|c|}
\hline Dependent Variable / Model & Independent Variable & $\begin{array}{l}\text { Multi- } \\
\text { plication } \\
\text { Factor }\end{array}$ \\
\hline $\begin{array}{l}\text { \# } 03 \text { - Primary Task12. } \\
\text { Synchronization with } \\
\text { supporting forces. }\end{array}$ & $\begin{array}{l}\text { \#08 - Fire Support BOS- } 2 \text { Employ Field } \\
\text { Artillery }\end{array}$ & 2 \\
\hline $\begin{array}{l}\text { \# } 03 \text { - Primary Task12. } \\
\text { Synchronization with } \\
\text { supporting forces. }\end{array}$ & $\begin{array}{l}\text { \#21 - Mobility \& Survivability BOS- } 6 \\
\text { Conduct Deception }\end{array}$ & 1 \\
\hline $\begin{array}{l}\text { \# } 03 \text { - Primary Task12. } \\
\text { Synchronization with } \\
\text { supporting forces. }\end{array}$ & $\begin{array}{l}\text { \#25 - Command and Control BOS-1 Plan } \\
\text { for Combat Operations }\end{array}$ & 1 \\
\hline $\begin{array}{l}\text { \# } 03 \text { - Primary Task12. } \\
\text { Synchronization with } \\
\text { supporting forces. }\end{array}$ & $\begin{array}{c}\text { \#69 - Environmental Factor- Relative } \\
\text { Humidity, } \%\end{array}$ & 1 \\
\hline $\begin{array}{c}\text { \# } 04 \text { - Primary Task15. } \\
\text { Destruction of first } \\
\text { echelon forces. }\end{array}$ & \#50 - Misc Factors- Battle Timing & 1 \\
\hline $\begin{array}{c}\text { \# } 04 \text { - Primary Task15. } \\
\text { Destruction of first } \\
\text { echelon forces. }\end{array}$ & $\begin{array}{l}\text { \#59 - Personnel- Executive Officer present } \\
\text { in TOC }\end{array}$ & 1 \\
\hline $\begin{array}{l}\text { \# } 07 \text { - Secondary Task12. } \\
\text { Synchronization with } \\
\text { supporting forces. }\end{array}$ & $\begin{array}{l}\text { \#05 - Maneuver BOS- } 1 \text { Conduct Tactical } \\
\text { Movement }\end{array}$ & 1 \\
\hline
\end{tabular}




\begin{tabular}{|c|c|c|}
\hline Dependent Variable / Model & Independent Variable & $\begin{array}{c}\text { Multi- } \\
\text { plication } \\
\text { Factor }\end{array}$ \\
\hline$\# 07$ - Secondary Task12. \\
$\begin{array}{c}\text { Synchronization with } \\
\text { supporting forces. }\end{array}$ & $\begin{array}{c}\text { \#15 - Fire Support BOS- 9 Coordinate, } \\
\text { Synchronize, and Integrate Fire } \\
\text { Support }\end{array}$ & 1 \\
\hline $\begin{array}{c}\text { \#07 - Secondary Task12. } \\
\text { Synchronization with } \\
\text { supporting forces. }\end{array}$ & $\begin{array}{c}\# 16 \text { - Mobility \& Survivability BOS- 1 } \\
\text { Overcome Obstacles }\end{array}$ & 1 \\
\hline
\end{tabular}

- Table 38 shows those tasks, predicted by JNNS at the $-20 \%$ PSF level, during this tactical phase whose increased utilization can cause these task time reduction effects. In addition to factors at the $10 \%$ level such as battle timing and the presence of the $\mathrm{XO}, 20 \%$ reductions are predicted to be achieved through the effective dissemination of intelligence and effective counter battery target operations where enemy artillery is identified, located, and fired upon with friendly artillery and airstrikes. Effective use of the ASAS and AFATDS digital communications systems along with effective liaison with the ALO can contribute to these effects.

Table 38 - DV / IV Correlation - Defense - PSF @-20\%

\begin{tabular}{|c|c|c|}
\hline \multicolumn{3}{|c|}{ Phase 4- Defense (DV@ @ 0.5, -20\% PSF) } \\
\hline Dependent Variable / Model & Independent Variable & $\begin{array}{l}\text { Multi- } \\
\text { plication } \\
\text { Factor }\end{array}$ \\
\hline $\begin{array}{l}\text { \# } 01 \text { - Primary Task10. } \\
\text { Destruction, capture, } \\
\text { or bypass of enemy } \\
\text { force. }\end{array}$ & $\begin{array}{l}\text { \#04 - Intel BOS- } 4 \text { Disseminate } \\
\text { Intelligence }\end{array}$ & 2 \\
\hline $\begin{array}{l}\text { \# } 01 \text { - Primary Task10. } \\
\text { Destruction, capture, } \\
\text { or bypass of enemy } \\
\text { force. }\end{array}$ & $\begin{array}{c}\text { \#17 - Mobility \& Survivability BOS- } 2 \\
\text { Enhance Movement }\end{array}$ & 1 \\
\hline $\begin{array}{l}\text { \# } 01 \text { - Primary Task10. } \\
\text { Destruction, capture, } \\
\text { or bypass of enemy } \\
\text { force. }\end{array}$ & $\begin{array}{l}\text { \#26 - Command and Control BOS- } 2 \text { Direct } \\
\text { and Lead Unit During the } \\
\text { Preparation Phase of the Battle }\end{array}$ & 1 \\
\hline $\begin{array}{l}\text { \# } 01 \text { - Primary Task10. } \\
\text { Destruction, capture, } \\
\text { or bypass of enemy } \\
\text { force. }\end{array}$ & \#56 - Misc Factors- MOPP Level & 1 \\
\hline $\begin{array}{l}\text { \# } 03 \text { - Primary Task12. } \\
\text { Synchronization with } \\
\text { supporting forces. }\end{array}$ & $\begin{array}{l}\text { \#13 - Fire Support BOS- } 7 \text { Conduct } \\
\text { Counter Target Acquisition } \\
\text { Operations }\end{array}$ & 1 \\
\hline \# 03 - Primary Task12. & \#21 - Mobility \& Survivability BOS- 6 & 1 \\
\hline
\end{tabular}




\begin{tabular}{|c|c|c|}
\hline Dependent Variable / Model & Independent Variable & $\begin{array}{l}\text { Multi- } \\
\text { plication } \\
\text { Factor }\end{array}$ \\
\hline $\begin{array}{l}\text { Synchronization with } \\
\text { supporting forces. }\end{array}$ & Conduct Deception & \\
\hline $\begin{array}{l}\text { \# } 03 \text { - Primary Task12. } \\
\text { Synchronization with } \\
\text { supporting forces. }\end{array}$ & \#50 - Misc Factors- Battle Timing & 2 \\
\hline $\begin{array}{l}\text { \# } 04 \text { - Primary Task15. } \\
\text { Destruction of first } \\
\text { echelon forces. }\end{array}$ & $\begin{array}{l}\text { \#59 - Personnel- Executive Officer present } \\
\text { in TOC }\end{array}$ & 1 \\
\hline $\begin{array}{l}\text { \# } 07 \text { - Secondary Task12. } \\
\text { Synchronization with } \\
\text { supporting forces. }\end{array}$ & $\begin{array}{l}\text { \#05 - Maneuver BOS- } 1 \text { Conduct Tactical } \\
\text { Movement }\end{array}$ & 1 \\
\hline $\begin{array}{l}\text { \# } 07 \text { - Secondary Task12. } \\
\text { Synchronization with } \\
\text { supporting forces. }\end{array}$ & $\begin{array}{l}\text { \#15 - Fire Support BOS- } 9 \text { Coordinate, } \\
\text { Synchronize, and Integrate Fire } \\
\text { Support }\end{array}$ & 1 \\
\hline $\begin{array}{l}\text { \# } 07 \text { - Secondary Task12. } \\
\text { Synchronization with } \\
\text { supporting forces. }\end{array}$ & $\begin{array}{c}\# 16 \text { - Mobility \& Survivability BOS- } 1 \\
\text { Overcome Obstacles }\end{array}$ & 1 \\
\hline
\end{tabular}

- Table 39 shows those tasks, predicted by JNNS at the $-30 \%$ PSF level, during this tactical phase whose increased utilization can cause these task time reduction effects. $30 \%$ reductions are predicted to be achieved through most of the same effects as existed at the $20 \%$ level with increased emphasis on the use of AFATDS and the more effective use of the field artillery BOSs.

Table 39 - DV / IV Correlation - Defense - PSF @-30\%

\begin{tabular}{|c|c|c|}
\hline \multicolumn{2}{|c|}{ Phase 4- Defense (DV @ 0.75, -30\% PSF) } & $\begin{array}{c}\text { Multi- } \\
\text { plication } \\
\text { Factor }\end{array}$ \\
\hline $\begin{array}{c}\text { Dependent Variable / Model } \\
\text { - Primary Task10. } \\
\begin{array}{c}\text { Destruction, capture, } \\
\text { or bypass of enemy } \\
\text { force. }\end{array}\end{array}$ & Independent Variable & 2 \\
\hline $\begin{array}{c}\text { \# 01 - Primary Task10. } \\
\text { Destruction, capture, } \\
\text { or bypass of enemy } \\
\text { force. }\end{array}$ & $\begin{array}{c}\text { Intelligence } \\
\text { Enhance Movement }\end{array}$ & 1 \\
\hline $\begin{array}{c}\text { Destruction, capture, } \\
\text { or bypass of enemy } \\
\text { force. }\end{array}$ & $\begin{array}{c}\text { \#26 - Command and Control BOS- 2 Direct } \\
\text { and Lead Unit During the } \\
\text { Preparation Phase of the Battle }\end{array}$ & 1 \\
\hline $\begin{array}{c}\text { Destruction, capture, } \\
\text { or bypass of enemy } \\
\text { force. }\end{array}$ & $\begin{array}{c}\text { \#56 - Misc Factors- MOPP Level } \\
\text { \# 01 - Primary Task10. }\end{array}$ & 1 \\
\hline
\end{tabular}




\begin{tabular}{|c|c|c|}
\hline Dependent Variable / Model & Independent Variable & $\begin{array}{l}\text { Multi- } \\
\text { plication } \\
\text { Factor }\end{array}$ \\
\hline $\begin{array}{l}\text { \# } 03 \text { - Primary Task12. } \\
\text { Synchronization with } \\
\text { supporting forces. }\end{array}$ & $\begin{array}{l}\text { \#08 - Fire Support BOS- } 2 \text { Employ Field } \\
\text { Artillery }\end{array}$ & 2 \\
\hline $\begin{array}{l}\text { \# } 03 \text { - Primary Task12. } \\
\text { Synchronization with } \\
\text { supporting forces. }\end{array}$ & $\begin{array}{l}\text { \#15 - Fire Support BOS- } 9 \text { Coordinate, } \\
\text { Synchronize, and Integrate Fire } \\
\text { Support }\end{array}$ & 2 \\
\hline $\begin{array}{l}\text { \# } 03 \text { - Primary Task12. } \\
\text { Synchronization with } \\
\text { supporting forces. }\end{array}$ & $\begin{array}{l}\text { \#35 - Communications- AFATDS - } \\
\text { Advanced Field Artillery Tactical } \\
\text { Data System }\end{array}$ & 1 \\
\hline $\begin{array}{l}\text { \# } 03 \text { - Primary Task12. } \\
\text { Synchronization with } \\
\text { supporting forces. }\end{array}$ & \#67 - Personnel- ALO present in TOC & 1 \\
\hline $\begin{array}{l}\text { \# } 03 \text { - Primary Task12. } \\
\text { Synchronization with } \\
\text { supporting forces. }\end{array}$ & $\begin{array}{c}\text { \#69 - Environmental Factor- Relative } \\
\text { Humidity, \% }\end{array}$ & 1 \\
\hline $\begin{array}{l}\text { \# } 04 \text { - Primary Task15. } \\
\text { Destruction of first } \\
\text { echelon forces. }\end{array}$ & \#50 - Misc Factors- Battle Timing & 1 \\
\hline $\begin{array}{l}\text { \# } 04 \text { - Primary Task15. } \\
\text { Destruction of first } \\
\text { echelon forces. }\end{array}$ & $\begin{array}{l}\text { \#59 - Personnel- Executive Officer present } \\
\text { in TOC }\end{array}$ & 1 \\
\hline $\begin{array}{c}\text { \# } 07 \text { - Secondary Task12. } \\
\text { Synchronization with } \\
\text { supporting forces. }\end{array}$ & $\begin{array}{l}\text { \#05 - Maneuver BOS- } 1 \text { Conduct Tactical } \\
\text { Movement }\end{array}$ & 1 \\
\hline $\begin{array}{c}\text { \# } 07 \text { - Secondary Task12. } \\
\text { Synchronization with } \\
\text { supporting forces. }\end{array}$ & $\begin{array}{l}\# 16 \text { - Mobility \& Survivability BOS- } 1 \\
\text { Overcome Obstacles }\end{array}$ & 1 \\
\hline
\end{tabular}

\subsubsection{Summary of Results.}

The analyses presented in this section illustrate the successful use of the COMPASS framework. A systematic step by step approach has been shown that allows a cross-walk identification of the mission performance effects as compared to human performance characteristics. These impacts on human performance capabilities provide the means to identify C2S subsystems whose performance either successfully or unsuccessfully contributes to overall changes in the way the TOC functions. An unsuccessful contribution of the performance of a particular subsystem is considered to be just as important in the overall analysis as a successful one. If the efficient performance of a $\mathrm{C} 2 \mathrm{~S}$ subsystem, such as an individual intelligence collection asset, actually causes an overall degradation in the performance capability of the C2S, then that is an important piece of information for $\mathrm{C} 2 \mathrm{~S}$ designers. The conclusion might then be 
to eliminate or alter the characteristics of that subsystem rather than continuing to try and improve it. 
This page left intentionally blank. 


\section{Discussion.}

The processes, discussions, and analyses in Phase I and Phase II have provided a proof of concept that an approach such as provided by the COMPASS paradigm can be used to look at complex work domains. Certainly, there has been much highly creditable work performed over the last 50 years in the area of military $\mathrm{C} 2$ in general and various aspects of it in specific. Topics such as decision making (including MDMP), individual and team performance, cognition, task analysis, knowledge elicitation, workload, situation awareness, and span of control are but a few of the highly relevant areas of investigation that have been explored in this domain. However, the complexity of each of these research areas and the open ended nature of the research in them has apparently precluded substantive efforts at looking at the overall work system in military C2.

Underlying the development of the COMPASS paradigm is an acknowledgement that all of these areas are critical and highly significant in their own right, however, an understanding of what makes the overall system perform more or less effectively is the key to understanding how to improve it. The use of COMPASS to investigate the overall $\mathrm{C} 2 \mathrm{~S}$ as a system with its own performance parameters is the goal here. Some of these performance parameters are human based, some are hardware and software based, some are environmentally based, and some are based upon the interaction from and to other like systems. Implicit in this definition is an acknowledgement of the role that each of the subsystem areas play in the performance of the system while attempting to account for the effects of these internal systems to the effectiveness of the overall system.

The techniques used to develop the COMPASS paradigm have been exploratory in nature and represent general classes of approaches that are appropriate for future efforts. The field of datamining uses many approaches such as cluster analysis and decision trees in addition to the neural network evaluations performed here. These areas, and others, certainly provide appropriate approaches for future development and improvement of the COMPASS approach.

\subsection{The COMPASS Paradigm.}

The COMPASS paradigm has allowed the presentation of a hypothesis that postulates that decreasing the amount of time required to perform individual tasks in a TOC based work environment will result in more effective operator performance up to the point of operator overload. This paradigm has the potential to investigate many other issues in this work domain. 
Future research will cover other performance areas. Some of these performance areas could include changes in the decision maker's battlefield situation awareness through increased volume of communication traffic or through better structured communications traffic. Analyses that look at the significance of the contribution of the activities of individual operators to overall mission success could be performed. Finally, the ability to provide the medium for the investigation for, as yet, undefined performance areas from a pre-existing and growing knowledge base is perhaps the biggest potential contribution of the COMPASS paradigm. All of this current and future research directly contributes to the U.S. Army's efforts to improve the integration of the human system component into overall socio-technical work system designs. Ongoing initiatives that can benefit from research of this type, such as the Army MANPRINT program, are critical to the effectiveness of tomorrow's C2 systems. These benefits can only be achieved if new and innovative research programs are pursued that can address the complexities of the work systems involved.

The COMPASS paradigm has proved to be a viable framework to examine TOC performance at the system level. The demands that war places on the complex battlefield of today means that the systems and subsystems designed to assist human operators in their attempts to control that battlefield must effectively allow an improved performance of the operator and subsequently the overall system. This is a complicated performance arena where the effects of new automation, communications, and organizational structures cannot be readily ascertained. One trend of modern $\mathrm{C} 2$ is to employ new automation to enable smaller groups of individuals to exercise direct control over greatly expanded forces and terrain. Previous studies with the CoHOST model have mirrored subsequent field observations that show that just providing a more efficient communications capability can force a data overload state on the TOC operators and decision makers with less, not more, efficiency in their decision making abilities.

It is in response to this complicated performance environment that has directed the development of the COMPASS approach. The evaluation of human cognitive performance is a challenge in any work environment, but military $\mathrm{C} 2$ TOCs perform at a level of sophistication that is hard to detect by the initiated observer and processes, but far surpasses any conceivable peacetime effort. The heart of the COMPASS approach is that it is not a theoretical estimation of TOC performance, but is rooted in direct observation of actual performing systems over time to build a knowledge base of what is and is not significant within the TOC work system. The 
key to this approach is the establishment of the ability to take the results of factor screening methods, such as this use of the JNNS, and apply the resultant significant factors to simulations of human system performance such as the CoHOST TOC simulation.

The significance of this research is the development of the COMPASS paradigm. This is a two phase procedure that consists of naturalistic observation of multiple TOC performance over time along with a neural network evaluation of the data and simulation studies of the performance of the TOC using a discrete event computer simulation that utilizes a task network modeling approach whose performance was shaped by the models from the neural network simulation. This paradigm is illustrated in Figure 39.

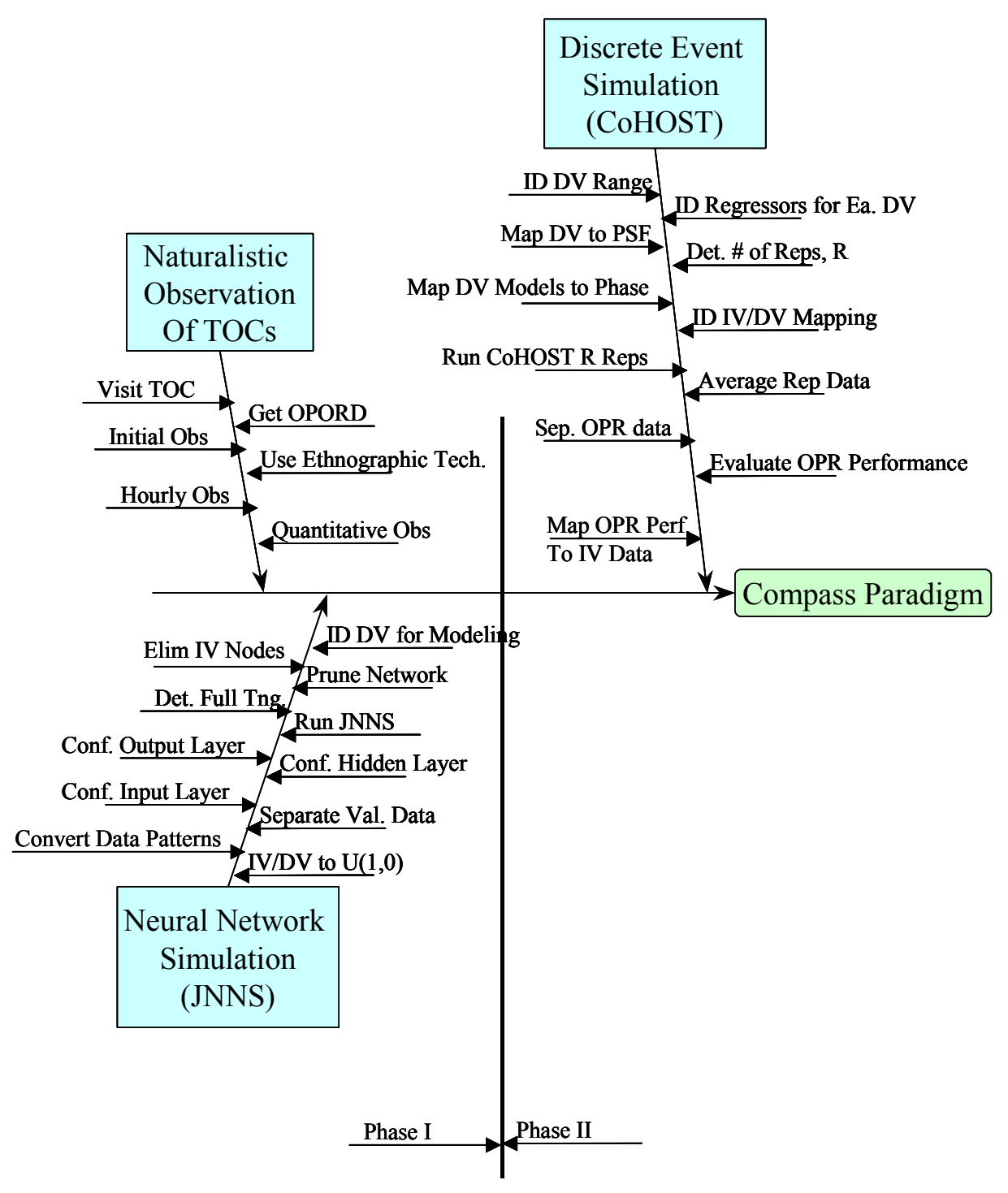

Figure 39 - Block Diagram of the COMPASS Paradigm 
The naturalistic observation and data gathering performed here looked at three TOC deployments. These data provided a knowledge set sufficient to develop and test COMPASS and to provide preliminary results that describe some of the factors that significantly affect $\mathrm{C} 2$ performance in TOCs. Following this dissertation effort, over the next several years, it is envisioned that this research will be extended to the observation of many future TOC deployments and the generation of a knowledge base from the observation of 40 or more such events. As this knowledge set grows, so will the ability to investigate performance relationships among the combined data that will describe those significant elements that determine efficient TOC operation while transcending the interpersonal differences in the areas of task and team performance that exist within each individual TOC exercise.

A series of guidelines, derived from these methods, is presented to formalize the COMPASS concept. These guidelines are identified in Table 40. It is noted that these guidelines are for the JNNS and CoHOST simulations. The process may be altered slightly if other neural network and discrete event simulations are employed.

Table 40 - Guidelines For The Use Of The COMPASS Paradigm

\begin{tabular}{|l|c|l|}
\hline \multicolumn{1}{|c|}{ Compass Phase } & $\#$ & \multicolumn{1}{|c|}{ Description } \\
\hline Naturalistic Observation & 1 & Visit TOC before start of the exercise to become familiar with its layout. \\
\hline Naturalistic Observation & 2 & Obtain copy of OPORD before start of the exercise to identify scenario(s). \\
\hline Naturalistic Observation & 3 & $\begin{array}{l}\text { Spend the first few hours after start of the exercise just watching to ascertain } \\
\text { flow of activity in the TOC. }\end{array}$ \\
\hline Naturalistic Observation & 4 & $\begin{array}{l}\text { After start of the exercise do not initiate communication or try to elicit } \\
\text { information from personnel in TOC engaged in the exercise. }\end{array}$ \\
\hline Naturalistic Observation & 5 & $\begin{array}{l}\text { Readily and immediately respond to communication attempts initiated by TOC } \\
\text { personnel. After answering their query, use the opportunity to objectively } \\
\text { discuss the exercise and to query them about what they think about what is } \\
\text { happening. }\end{array}$ \\
\hline Naturalistic Observation & 6 & $\begin{array}{l}\text { Treat all personnel with equal respect and enthusiasm regardless of rank or team } \\
\text { assignment. }\end{array}$ \\
\hline Naturalistic Observation & 7 & $\begin{array}{l}\text { Regardless of personal opinion, always display a positive opinion about what is } \\
\text { happening, then make objective observations about the real situation. Do not } \\
\text { share raw data from observations with TOC personnel, however, be frank and } \\
\text { honest about it if asked. Never lie or misrepresent any aspect of the project, or } \\
\text { the data. }\end{array}$ \\
\hline Naturalistic Observation & 8 & $\begin{array}{l}\text { Capitalize on personal military background or other experience to } \\
\text { ethnographically relate to TOC personnel to gain temporary acceptance to the } \\
\text { work group environment. }\end{array}$ \\
\hline Naturalistic Observation & 9 & $\begin{array}{l}\text { Longitudinally record observations according to a set time interval, such as } \\
\text { hourly, and focus on consistency and repeatability on type and kind of }\end{array}$ \\
\hline
\end{tabular}




\begin{tabular}{|c|c|c|}
\hline Compass Phase & \# & Description \\
\hline & & observations. \\
\hline Naturalistic Observation & 10 & $\begin{array}{l}\text { Record all observations quantitatively using direct automated input, if possible, } \\
\text { to preclude possible transcription and interpretation errors after the end of the } \\
\text { exercise. }\end{array}$ \\
\hline Neural Network Simulation & 1 & $\begin{array}{l}\text { Convert all independent and dependent measures to either binary or } \\
\text { standardized }(0,1) \text { IV and DV as appropriate. }\end{array}$ \\
\hline Neural Network Simulation & 2 & $\begin{array}{l}\text { Reorganize IV and DV into input and output patterns of data where each pattern } \\
\text { is one set of observations. }\end{array}$ \\
\hline Neural Network Simulation & 3 & $\begin{array}{l}\text { Randomly select } 15 \%-25 \% \text { of the pattern data and separate it into a validation } \\
\text { pattern set. Reclassify the remaining pattern data as the training pattern data } \\
\text { set. }\end{array}$ \\
\hline Neural Network Simulation & 4 & Configure the input layer of the neural network with one node per IV. \\
\hline Neural Network Simulation & 5 & $\begin{array}{l}\text { Configure the hidden layer(s) of the neural network with the minimum number } \\
\text { of layers and nodes in each layer that will support successful training. This may } \\
\text { require trial and error test runs to determine the optimum configuration. Start } \\
\text { with one hidden layer and a number of nodes equal to approximately } 1 / 2 \text { of the } \\
\text { number of input nodes. }\end{array}$ \\
\hline Neural Network Simulation & 6 & Configure the output layer of the neural network with one node per DV. \\
\hline Neural Network Simulation & 7 & $\begin{array}{l}\text { Set the training threshold parameters and run the neural network simulation for } \\
\text { a fixed number of epochs (start with } 100 \text { and increase until full training is } \\
\text { achieved). Observe the error curve from the training and validation data sets. } \\
\text { When the validation curve turns up the point of full training is indicated. Do } \\
\text { not overtrain. If the curves flatten out and do not turn up, the network is stable } \\
\text { and will not overtrain. }\end{array}$ \\
\hline Neural Network Simulation & 8 & $\begin{array}{l}\text { If full training is not indicated, increase the number of epochs and rerun until it } \\
\text { is. }\end{array}$ \\
\hline Neural Network Simulation & 9 & $\begin{array}{l}\text { Once full training is indicated, set the pruning parameters and perform pruning } \\
\text { of the IV nodes. The nodes removed by pruning are predicted to have less } \\
\text { significance to the development of the output than those nodes remaining. }\end{array}$ \\
\hline Neural Network Simulation & 10 & $\begin{array}{l}\text { By examination of the network diagram and comparison of the pre and post } \\
\text { pruning network results files, identify those input nodes that are remaining as } \\
\text { candidates for development of mathematical models of their response. }\end{array}$ \\
\hline Neural Network Simulation & 11 & $\begin{array}{l}\text { Examine the training dataset and identify those output parameters that have a } \\
\text { sufficient number of observations to support development of models. A } \\
\text { subjective number of } 10 \text { was chosen as the minimum number of observations } \\
\text { that would support model generation. }\end{array}$ \\
\hline Neural Network Simulation & 12 & $\begin{array}{l}\text { Using traditional linear regression analysis procedures, perform a stepwise } \\
\text { linear regression analysis of each DV model. Begin each DV model with all of } \\
\text { the IV remaining after pruning. Eliminate IV from each model that are not } \\
\text { significant after each regression run }(\alpha=.05) \text { and rerun the regression analysis. } \\
\text { Continue repeating the regression runs with stepwise elimination until each } \\
\text { model only contains significant IV. }\end{array}$ \\
\hline Neural Network Simulation & 13 & $\begin{array}{l}\text { Identify the significant DV models for use in the interface to the discrete event } \\
\text { simulation. }\end{array}$ \\
\hline Discrete Event Simulation & 1 & $\begin{array}{l}\text { For each DV model, determine the range of its response by setting all regressors } \\
\text { to first } 0 \text {, and then } 1 \text {. Record the results. }\end{array}$ \\
\hline Discrete Event Simulation & 2 & $\begin{array}{l}\text { Identify response points in the range for correlation to performance points in the } \\
\text { discrete event simulation (for example, } 25 \%, 50 \% \text {, and } 75 \% \text {.) }\end{array}$ \\
\hline Discrete Event Simulation & 3 & $\begin{array}{l}\text { For each DV model, iteratively adjust the values of the regressors ( } 0 \text { or } 1 \text { for } \\
\text { binary IV, and }(0,1) \text { for standardized IV) until a set of regressors is identified } \\
\text { for each DV model that contributes to the } 0 \%, 25 \%, 50 \%, 75 \% \text {, and } 100 \% \\
\text { response of the model. }\end{array}$ \\
\hline Discrete Event Simulation & 4 & $\begin{array}{l}\text { Using SME input, identify discrete event simulation performance shaping } \\
\text { factors (PSF) that correlate to response points of the DV models. For example, }\end{array}$ \\
\hline
\end{tabular}




\begin{tabular}{|c|c|c|}
\hline Compass Phase & $\#$ & Description \\
\hline & & $\begin{array}{l}\text { identify a PSF that causes an overall reduction in task time performance by a } \\
\text { fixed percentile. Then, identify a range of these PSFs that correlate to observed } \\
\text { IV utilization in the DV models. The values used in this dissertation are: } \\
\text { The } 75 \% \text { DV level equates to a PSF = approximately }-30 \% \text {. } \\
\text { The } 50 \% \text { DV level equates to a PSF = approximately }-20 \% \text {. } \\
\text { The } 25 \% \text { DV level equates to a PSF = approximately }-10 \% \text {. } \\
\text { The } 0 \% \text { DV level equates to a PSF }=0 \% \text { (default). } \\
\text { For example, a } 75 \% \text { increase in performance of a DV model causes a } 30 \% \\
\text { decrease in task time performance in the discrete event simulation. }\end{array}$ \\
\hline Discrete Event Simulation & 5 & $\begin{array}{l}\text { Determine the minimum number of replications, } R \text {, required to run the discrete } \\
\text { event simulation to account for the effects of random variability using Banks' } \\
\text { procedures. }\end{array}$ \\
\hline Discrete Event Simulation & 6 & $\begin{array}{l}\text { For each phase of the scenario, use SME input to identify those DV models that } \\
\text { are applicable to the performance of that scenario phase. For example in this } \\
\text { dissertation, during the scenario phase Movement to Contact, it was determined } \\
\text { that the performance model for DV TA3 (Movement To The Line Of } \\
\text { Departure) applied to this combat activity. }\end{array}$ \\
\hline Discrete Event Simulation & 7 & $\begin{array}{l}\text { For each DV model associated with a combat scenario phase, list its IV as } \\
\text { factors whose utilization contributes to the performance of that DV during the } \\
\text { combat phase. For all the DV that apply to each combat phase, sum all of the } \\
\text { applying IV to compile a list of IV whose level of utilization contributes to the } \\
\text { performance of operators during that combat phase. }\end{array}$ \\
\hline Discrete Event Simulation & 8 & $\begin{array}{l}\text { Run the discrete event simulation for the requisite number of runs. A run is } \\
\text { defined as the simulation executing the full scenario for a PSF factor for R } \\
\text { replications. Make a separate run for each PSF factor. }\end{array}$ \\
\hline Discrete Event Simulation & 9 & Average the results for all the replications in each run. \\
\hline Discrete Event Simulation & 10 & $\begin{array}{l}\text { For operators of interest, separate out the results data for that operator and } \\
\text { display it in a suitable format to allow evaluation of workload/taskload and } \\
\text { utilization profiles, and numbers of tasks that were queued, dropped, and } \\
\text { interrupted by that operator during the run. }\end{array}$ \\
\hline Discrete Event Simulation & 11 & $\begin{array}{l}\text { Evaluate the operator performance during each combat phase according to } \\
\text { taskload, utilization, and task performance. Compare the operator performance } \\
\text { characteristics during each combat phase to the IV identified in step 7. Derive } \\
\text { conclusions on how operator performance can be improved to this level through } \\
\text { actions that can be taken to utilize the IV to that performance level. }\end{array}$ \\
\hline
\end{tabular}

\subsection{Considerations Regarding The COMPASS Paradigm.}

It can be generally stated that, in any exploratory research, there are issues and discussion points that may not be fully resolved at the beginning of the research effort and will involve some investigation during the course of the research in order to resolve them. Two such areas have been identified during the development of the COMPASS paradigm. The first is the deviation from traditional ethnographic qualitative techniques that have been taken while still operating under the guise of using ethnographic methodologies. The second is the potential in this multi-simulation process to generate a phenomenon that is described as "stacking of errors." This is a situation where the inherent error induced by each stage of the process from data 
collection to neural network simulation to discrete event simulation is carried forward to the next stage to the extent that the error is significant in the final result. This is a challenge to the validation of the overall process.

\subsubsection{Challenges to Validation.}

While the COMPASS framework provides for controlling the error generated at each stage of the process, there is some concern that cumulative error across the entire framework may become unmanageable. Current strategies for controlling error during the COMPASS process begin at the data collection stage. There is a strict adherence to quantitative recording of observations in the field. For those events that are binary or categorical in nature it is a simple matter of determining if the event is active or which category of a set of options is appropriate at the time of observation. For those events that are recorded as continuous variables many are completely objective in nature, however some are subjective. For example, ambient temperature and noise levels can be recorded exactly (at least to the accuracy of the recording instruments.) However, for those events that require a subjective assessment of the observer there is the increased risk of error. For example, if the observer has to judge the level of usage of a communications system on a scale of $0-100 \%$ at the time of observation, then error might be induced where another observer might make a different determination. This type of error is minimized in COMPASS by requiring the assessment to be made on the spot at the time of observation when all the sensory input conditions of the environment can be experienced by the observer. This is as opposed to making the judgment at a later time from the analysis of a video recording of the events when only the video image is available to the observer in order to make the judgment.

During the neural network analysis error can be induced from the fact that the exact nature of the architecture of the network is up to the subjective choice of the analyst. In this case a multi-layer perceptron approach was chosen as being one of the most widely accepted architectures for this type of analysis. This mandated the use of one input and one output layer with the number of nodes in each exactly matching the IV and DV of the observed data. However, the number of hidden layers and number of nodes within the hidden layer is purely up to the discretion of the analyst. The desire is to have as few a number of hidden layers with each hidden layer containing as few number of nodes required to achieve successful training. For the 
number of hidden layers the logical starting point for the COMPASS analysis was one. For the number of nodes within it the number of 90 was chosen as being between $25-50 \%$ of the number of input nodes which agrees with opinion stated in the literature. An excessive number of hidden layers or hidden nodes can increase the computational result of having the network attempt to model noise within the data. Two few hidden nodes or layers can prevent the network from being able to successfully train itself because of insufficient computational capability which resides primarily within the hidden nodes. In many cases the determination of numbers of hidden nodes and layers can only be determined through iterative network simulation runs where the hidden node numbers are varied between runs. For the analysis presented thus far in this research successful training was achieved with very low epoch and pruning replication counts. The results supported the conclusion that the network was operating successfully with no overtraining with its resultant potential for modeling error through network noise.

During the discrete event simulation phase error can be induced through the effects of random variability from the random number generation process. These effects were countered in COMPASS through a numerical determination of the number of replication cycles required to run the simulation in order to achieve a confidence interval of $95 \%$.

While attempts to control individual error effects have been carefully managed during this process, the cumulative effects of error that is insignificant at each stage can combine with error generated during subsequent stages to produce a cumulative error effect, also described as "stacking of errors," that is significant. This is an issue for future investigation and analysis. For the current effort this effect has been minimized through as close as possible adherence to procedures at each computational stage.

\subsubsection{Ethnography Challenges.}

Conventional ethnography theory, as formulated by Nardi and others (Borman et al., 1986; Lawlor and Mattingly, 2001; LeCompte and Goetz, 1982; Nardi, 1997b; Petty, 1997; Segall, 1991; Uzzell, 2000), is highly qualitative in nature where the observer becomes immersed in the culture of the ethnic group being observed while maintaining as objective an opinion as possible in interpreting the meaning and nature of observations made. Those espousing this discipline would also postulate that all situations are different and should be considered differently even if they involve individuals performing like activities. For example, if baseball 
teams were being observed to try to ascertain what contributes to success on the field, they might be observed playing during the day or at night, and in different stadiums of varying quality of the facilities. True adherence to ethnographic principles would consider each of these conditions as unique and require a separate analysis for each case. Other researchers might take a different approach with the view that baseball is baseball and that it can be observed as such regardless of the circumstances.

The COMPASS paradigm takes a modified approach to this discipline by promulgating a responsible approach that adheres to ethnographic constructs as closely as possible while evaluating a domain that is so complex as to preclude the ability to assess the meaning of hundreds of qualitative assessments for each possible condition that might be observed. In order to reduce potential field observational error as previously described, a strict quantitative approach to observation recording is utilized that requires on the spot determination of the values of not only digital and interval variables, but also continuous measures of performance. The key to the ethnographic approach in COMPASS is not the way data is recorded, but the way it is gathered. Achieving the confidence and respect of the target audience is one important aspect. This is achieved by having an observer that is knowledgeable in the activities being performed to the extent that they can speak the technical dialect of the group that will almost always exist especially in military circles. They must be able to demonstrate that the group does not have to deal with them at a level of explaining the basic nature of the environment, but can communicate with them as perceived peers to reflect why and how they do the things they do. The successful integration of ethnographic considerations into a field observation situation that is only temporary in nature with a high degree of complexity forces more stringent data recording techniques. This is one of the things that makes the COMPASS framework unique.

\subsection{Topics For Future Research.}

This project has the potential to assist the Army in many different ways with the new COMPASS paradigm forming the basis for continued investigative work. The focus chosen for this dissertation is the first logical step to achieve this overall objective of providing a foundation for a variety of future scientific research efforts not only for the Army, but also for investigating complex, time critical $\mathrm{C} 2 \mathrm{Ss}$, in general. 
As the work started by this dissertation continues in the future it is anticipated that other research areas that have potential contribution to the overall project of developing ways and means to improve TOC performance will be identified as candidates for further investigation. Some of these anticipated topics relate to physiological and psychophysical stressors related to trying to perform HCI tasks while bouncing around in the back of a tracked command post vehicle that is moving over open terrain. Other research could focus on training issues related to fewer numbers of operators and decision makers attempting to control greater numbers of resources on the battlefield. Another area that has been previously evaluated is the effect on human operators from prolonged exertion in confined environments such as the inside of military tactical vehicles (Hicks, 1973; Hicks, 1960a; Hicks, 1960b; Hicks, 1961a; Hicks, 1961b; Hicks, 1961c; Hicks, 1962; Hicks, 1963; Hicks, 1964a; Hicks, 1964b). This work could be expanded with a look at the efficiency of HCI performance under these conditions that could also include sleep deprivation and exposure to temperature extremes. Gender issues from opposite-sex operators working in close quarters for extended periods of time could also be the basis for TOC performance changes and ways to alleviate these issues could be investigated.

Other, more traditional techniques, that could be used to refine the COMPASS framework include the selection of designs that are either consist of pure factorial designs or some combination of factorial, blocked, or central composite methodologies (Clark and Williges, 1972a; Clark and Williges, 1972b; Simon, 1970; Williges, 1976; Williges and Baron, 1972; Williges and Mills, 1972). The linear and higher order components of the IV responses could then be considered according to their response surfaces for a more complete look at overall system response (Simon, 1968; Simon, 1976; Williges and Simon, 1970; Williges and Simon, 1971).

\subsubsection{Future Enhancements to COMPASS.}

This dissertation is considered just the first phase and what is to be a long term research effort investigating the performance of C2Ss. While this effort has used tools such as JNNS for neural network analyses and CoHOST for TOC evaluation, there are more sophisticated and newer technology approaches that could not be explored here due to time and budget constraints. Future work will hopefully be able to capitalize on more sophisticated neural network simulations and next generation discrete event approaches to TOC simulation. Considerations in these two topical areas are: 


\subsubsection{Improving the Neural Network Interface.}

While performing the neural network phase there were two neural network simulation packages that were evaluated. The freeware JNNS simulation used here has been discussed previously. While it performs well during the network training and pruning stages, it has no ability to directly translate its internal programming to a form that can be used as an interface to other simulations. In addition, it is very manually intensive to use JNNS as all of the data translation, separation of data into training and validation data sets, and layout of the network must be performed separately and put into the required format necessary to interface with JNNS. Other packages allow for the generation of $\mathrm{C}++$ language code that describe the relationships between the input and output nodes and can be used as models of that relationship.

One software product that provides this capability is the Enterprise Miner (EM) program from SAS Institute. EM is a suite of datamining modules that includes regression analysis, cluster analysis, decision tree analysis, neural network analysis, and principle components analysis. There are also automated tools that allow for partitioning, attribute specification and validation of data. Each module is invoked and linked to the other modules through the use of a GUI based tools diagram. Figure 40 shows an example of an EM tool control diagram that was used in its evaluation here. 


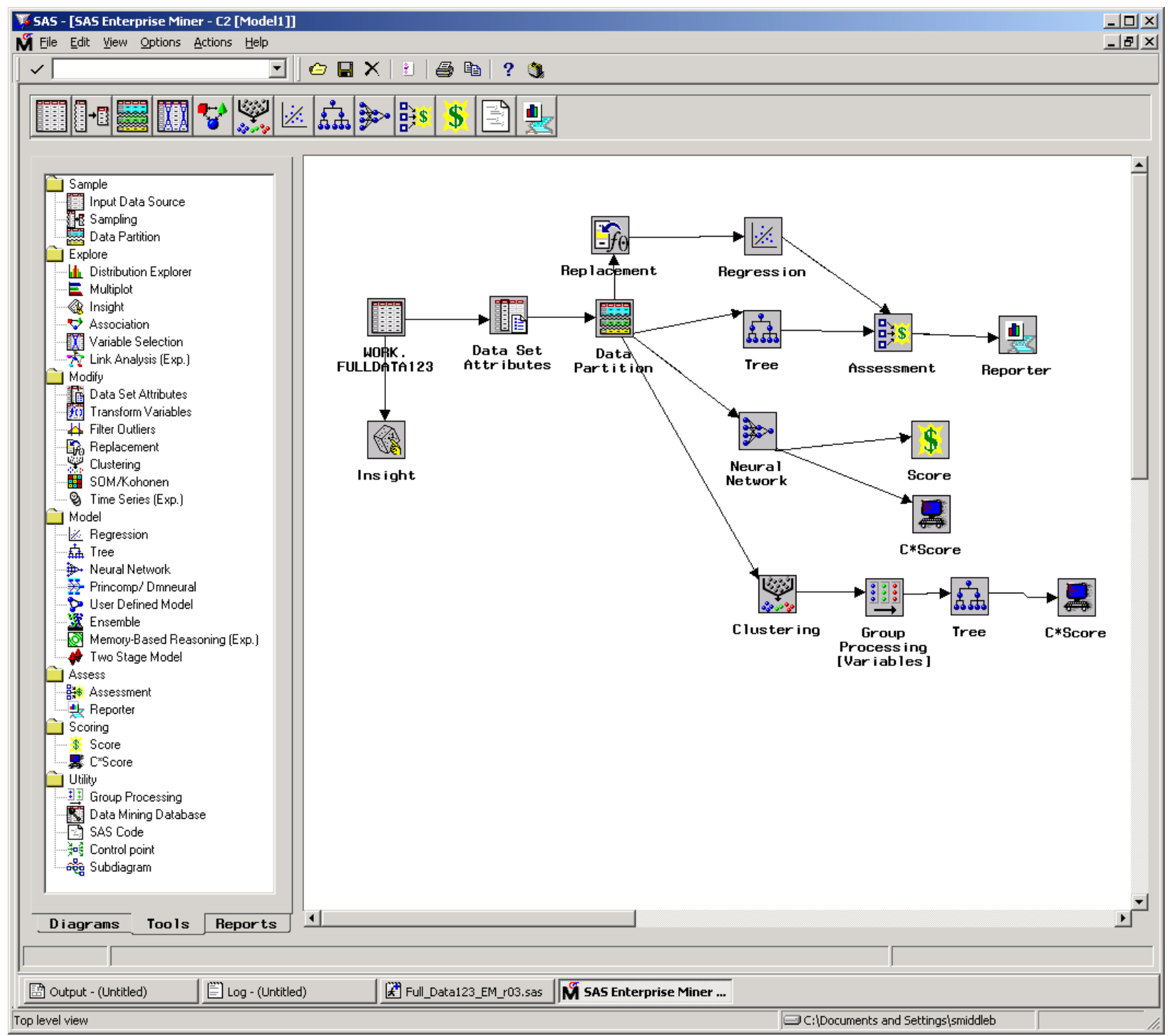

Figure 40 - Enterprise Miner Tools Diagram

To use this simulation, parameters are entered for each module and the simulation performs a full preparation and analysis of the data during each run. Thus, this type of system could have great potential for applications, like the COMPASS paradigm, where the database is constantly growing with each exercise observation. The neural network is represented in a diagram very similar to JNNS as shown in Figure 41, however, EM creates this diagram automatically from the dataset while JNNS requires that the diagram be manually entered and configured to establish the network. 


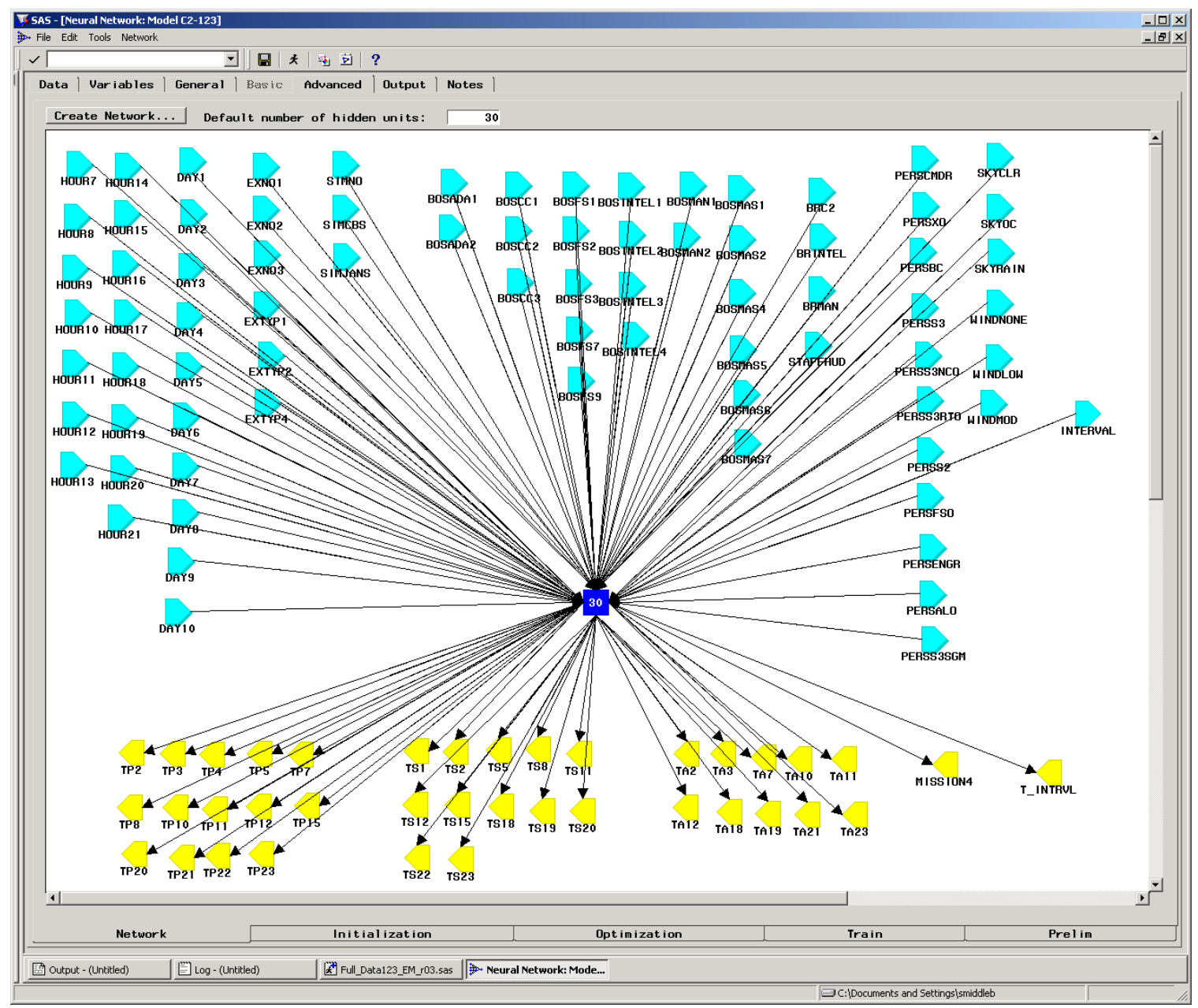

Figure 41 - EM Neural Network Diagram

EM also determines the point of full training automatically and stops the simulation at that point thus preventing any possibility of overtraining. An error curve of the training process is provided as shown in Figure 42, however, it is for informational purposes only as no interpretation is required of the analyst to determine the number of epochs required to achieve full training. 


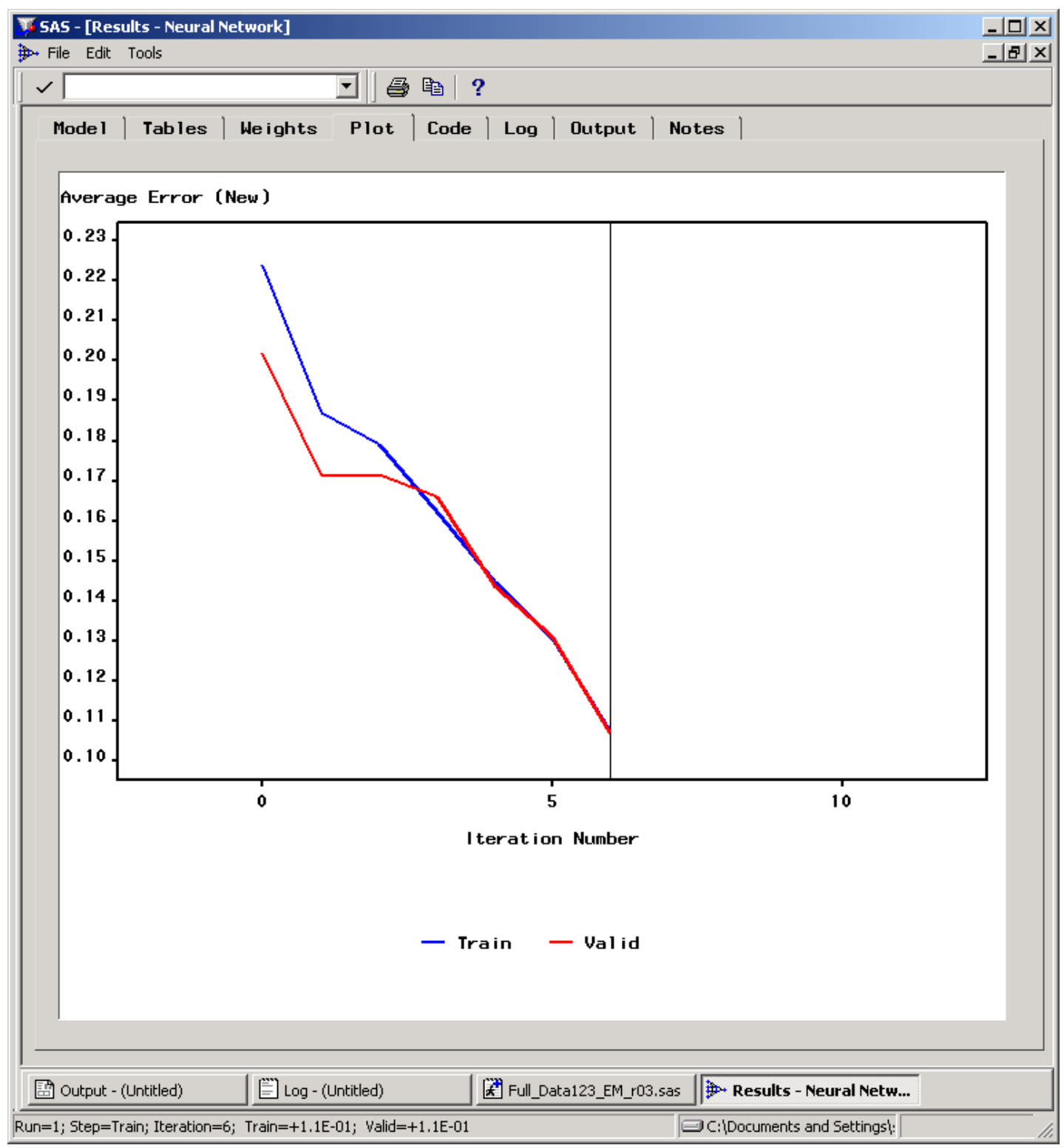

Figure 42 - EM Error Graph

Once the full datamining procedure is coded into EM, then an analysis can be performed after each exercise observation with minimal manual preparation required. However, the power of this simulation also includes the ability to translate the logic from the neural network, or any other simulation module, into SAS language code or $\mathrm{C}++$ code using the Score and $\mathrm{C}^{*}$ Score nodes.

The SAS EM package was not selected at this time because of technical difficulties with its use that could not be resolved in time to support this analysis and because of the cost of the program as limited funding was available. However, its potential for future analyses is 
significant and could become the neural network of choice to support ongoing studies involving a constantly growing database.

\subsubsection{Improving the CoHOST Operator Interface-C3 TRACE.}

The CoHOST discrete event simulation was the product of a multi-year effort involving considerable expense and manpower as previously described. However, it is a very structured simulation model not well suited for evaluation of system changes. In 2001, recognizing the need for a rapid turnaround capability to examine TOC operations and structures, the U.S. Army developed a GUI shell to reside on top of the Micro Saint ${ }^{\mathrm{TM}}$ discrete event programming language that provides an ability to quickly configure and test TOC structures using a building block approach for the identification of operators, operator performance characteristics, and TOC system elements. This GUI interface is called Command, Control and CommunicationTechniques for Reliable Assessment of Concept Execution (C3-TRACE) (Plott, 2002). The CoHOST simulation has already been rehosted into C3-TRACE and, once testing and validation is complete, is expected to support future COMPASS efforts.

Operator workload is determined by a visual, auditory, cognitive, and psychomotor (VACP) (McCracken and Aldrich, 1984) assessment of operator activity multiplied by the amount of time that the tasks are being performed. The C3 TRACE graphical user interface (GUI) to CoHOST-R allows for the definition of PSF factors such as aptitude, age, and level of training that the simulation then uses to modify actual task performance times. These embedded factors can be used in the PSF definition or user defined factors may be established and used. By taking the results of the regression analysis of the JNNS products and mapping them to task time and follow-on workload on the operator a set of user defined PSF factors can be defined that will cause the amount of time required to perform tasks across the TOC model. The simulation's work and task load performance then becomes responsive to $\mathrm{C} 2$ system mission task demands as they result in subsequent operator task time performance.

Figure 43 shows an example of the GUI window used to define the operators and sections of a TOC using C3 TRACE. Parameters for the operators and other structures of the TOC and configured with similar graphical windows. The scenario is provided through a series of communications events that are loaded into a data table. 


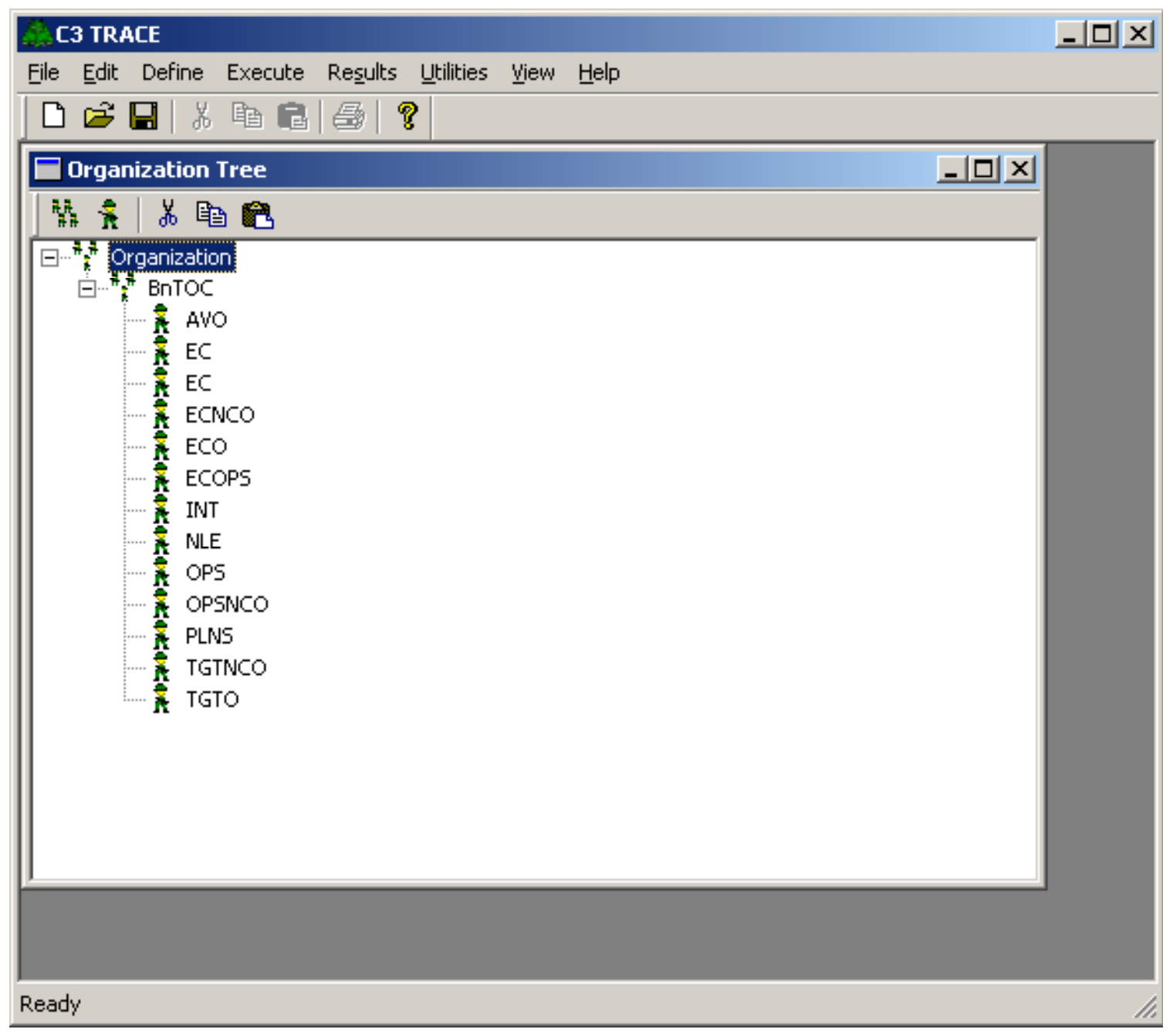

Figure 43 - C3 TRACE Organization Tree Window

After all the data structures have been identified the performance logic is entered as a Micro Saint ${ }^{\mathrm{TM}}$ like logic flow diagram that identifies performance activities for the TOC as shown in Figure 44. 


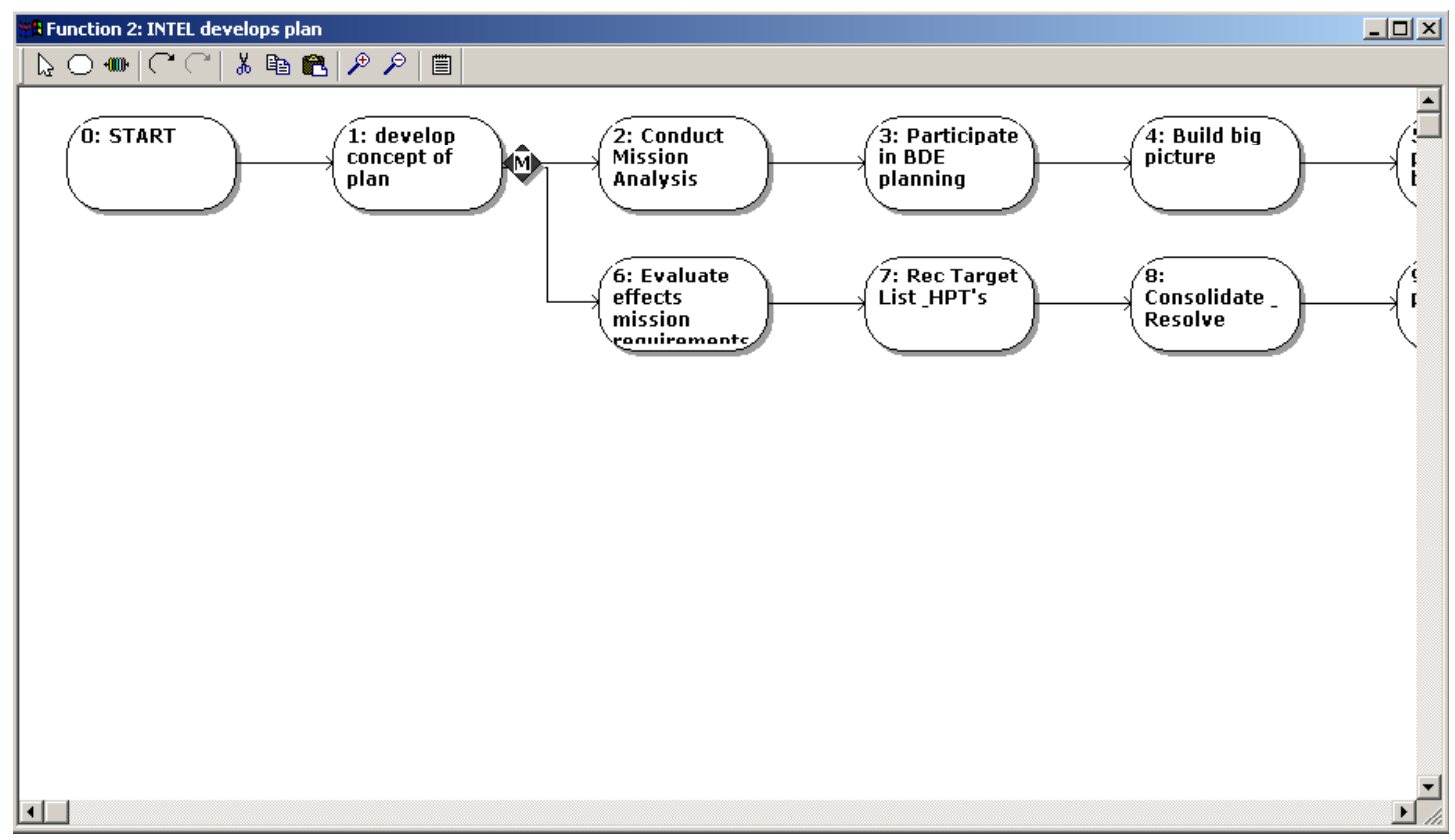

Figure 44 - C3 TRACE Task Definitions

Upon execution C3 TRACE automatically generates a Micro Saint ${ }^{\mathrm{TM}}$ simulation model of the TOC based on the C3 TRACE definitions and executes it with an embedded Micro Saint ${ }^{\text {TM }}$ simulation engine. Thus, conceptual changes can be made in the C3 TRACE definitions and a new model can be generated and executed that provides data for immediate analysis.

C3 TRACE was evaluated for use in this dissertation effort, however, being a new product still in the final stages of development it could not provide reliable models in time for this study. However, its potential for support of evolving COMPASS studies where the discrete event simulations need to have the ability for rapid modification to support TOC analyses is significant. Future work in TOC simulations will be able to greatly benefit from rapid generation development packages such as C3 TRACE.

\subsubsection{Future Evolution of the TOC Knowledge Base.}

This research has developed the COMPASS paradigm with data gathered from the observation of three TOC deployments. These three deployments have provided an opportunity to observe each of the three major types of brigade training exercises. These three types have been previously described as a test exercise, an evaluated exercise, and a pure training exercise. One of these was a field training exercise while the other two were conducted using computer 
simulation to replicate the movement of forces on the battlefield. While all three of these exercises were conducted at Fort Hood, Texas, it is envisioned that future TOC observations will continue to expand the data on each of these exercise types with TOC deployments across the continental United States. Brigades are in constant rotation through the National Training Center (NTC) at Fort Irwin, California conducting full scale maneuver exercises that are evaluated. National Guard brigades across the nation are continually conducting evaluated exercises using computer simulation where evaluator teams from the Battle Command Training Program's (BCTP) Operations Group C deploys from Fort Leavenworth, Kansas, along with the computer simulation equipment, to conduct evaluated exercises at the National Guard brigade's home location. In addition to Fort Hood, there are major combat forces stationed at posts around the country. BCTP exercises are in continued execution with Operations Groups A and B for them as well as exercises that they conduct in preparation for NTC rotations.

All of these exercise events provide a rich environment for observation of future TOC deployments. It is the goal of this research project to observe 30 to 40 or more TOCs over the next few years and grow the TOC observation knowledge base to be truly capable of supporting studies on what is and is not important to TOC operation and design.

\subsection{Conclusions.}

This dissertation has developed a procedural and analytical framework that allows for the quantitative description of the complete $\mathrm{C} 2 \mathrm{~S}$. While individual and team performance is a major component of the overall system, it is by no means the only component. Although the literature acknowledges the existence of a command and control "system," the research predominately focuses on the human computer interface ( $\mathrm{HCI})$ and team performance. The military decision making process is a structured approach to problem solving, however, there are situations where it does not work very well and research that just focuses on this process does not account for other variables that can induce stress and affect the situation awareness of the decision makers. The COMPASS approach to analysis of the TOC and the way it operates, encompasses all these internal variabilities as it considers this system from a top level viewpoint while still maintaining significant levels of detail concerning the processes that are going on between humans and other humans and between humans and the system as represented by the HCI interface. 
The overall goal of this study has been to develop an observation and analysis technique that will enable a better understanding of the systemic performance of U.S. Army TOCs. This technique is validated using exploratory observations of selected TOCs in operation that generates data that is be evaluated in an existing computer simulation chosen for its ability to address the different activities present in a modern day TOC. Each future round of observation, analysis, and simulation will serve to refine and update the computer simulation and provide an evolving evaluative tool that will be utilized to investigate future TOC systems while they are still in the design stage. 
This page left intentionally blank. 


\section{References.}

Adams, M. J., Tenney, Y. J., \& Pew, R. W. (1991). Strategic Workload and the Cognitive Management of Advanced Multi-Task Systems. Wright Patterson Air Force Base, OH: SOAR Crew System Ergonomics Information Analysis Center (CSERIAC). Technical Report 91-6. BBN Report No. 7650.

Adams, M. J., Tenney, Y. J., \& Pew, R. W. (1995). Situation Awareness and the Cognitive Management of Complex Systems. Human Factors, 37(1), 85-104.

Adelman, L., Leedom, D. K., Murphy, J., \& Killam, B. (1998). Description of Brigade C2 Decision Process. Human Research and Engineering Division, U.S. Army Research Laboratory, Aberdeen Proving Ground, MD 21005.

Adelson, M. (1961). Human Decisions in Command Control Centers. Annals of the New York Academy of Sciences, $\underline{89}$, 726-731.

AGARD. (1998). A Designer's Guide to Human Performance Modelling. Advisory Group For Aerospace Research \& Development (AGARD), North Atlantic Treaty Organization (NATO). AGARD-AR-356. DTIC ADA359405.

Ainslie, F. M., Leibrecht, B. C., \& Atwood, N. K. (1991). Combat Vehicle Command and Control Systems: III. Simulation-Based Company Evaluation of the Soldier-Machine Interface (SMI). U.S. Army Research Institute for the Behavioral and Social Sciences.

Ainsworth, L. (2001). Task Analysis. In W. Karwowski (Ed.), International Encyclopedia of Ergonomics and Human Factors (Vol. 1). New York: Taylor \& Francis.

Allender, L., Kelley, T., Archer, S., \& Adkins, R. (1994). IMPRINT - the Transition and Further Development of a Soldier System Analysis Tool. Human Research and Engineering Division, U.S. Army Research Laboratory, Aberdeen Proving Ground, MD 21005.

Anderson, D., \& McNeill, G. (1992). Artificial Neural Networks Technology. Rome Laboratory, Griffiss AFB, NY.

Anonymous. (1997). Major Datamining Technolgoies. Datamation, 43(2), 54.

Archer, R. D., Lewis, G. W., \& Lockett, J. (1996). Human Performance Modeling of Reduced Manning Concepts for Navy Ships. In Human Factors and Egronomics Society Proceedings.

Archer, R. D., \& Lockett, J. F., III. (1997). WINCREW- a Tool for Analyzing Performance, Mental Workload and Function Allocation among Operators. In Proceedings of 1997 Allocations of Functions Conference. Galway, Ireland.

Army. (1983). Army Regulation 310-25. Dictionary of United States Army Terms (Short Title: Ad). Headquarters, Department of the Army.

Army. (1991). Army Regulation 602-1. Human Factors Engineering Program. Headquarters, Department of the Army. Washington, D.C.

Army. (1993). FM 100-5. Operations. U.S. Army Field Manual.

Army. (1995). FM 34-25-3. All-Source Analysis System and the Analysis and Control Element. U.S. Army Field Manual. 
Army. (1996). FM 100-15. Corps Operations. U.S. Army Field Manual.

Army. (1997). FM 101-5. Staff Organization and Operations. U.S. Army Field Manual. Fort Leavenworth, KS.

Army. (1998). FM 100-11. Force Integration. U.S. Army Field Manual.

Army. (2001a). Army Regulation 602-2. Manpower and Personnel Integration (MANPRINT) in the System Acquisition Process. Headquarters, Department of the Army.

Army. (2001b). FM 1. The Army. U.S. Army Field Manual.

Army. (2001c). FM 3-0. Operations. U.S. Army Field Manual.

Army. (2002a). ABCS-LRG 6.2. Army Battle Command System Leader's Reference Guide. U.S. Army Special Text. Fort Leavenworth, KS.

Army. (2002b). United States Army White Paper: Concepts for the Objective Force. http://www.army.mil/features/WhitePaper/ObjectiveForceWhitePaper.pdf.

Army, \& Marines. (1997). FM 101-5-1. MCRP 5-2a. Operational Terms and Graphics. U.S. Army Field Manual. U.S. Marine Corps Reference Publication.

Asbrand, D. (1997). Is Datamining Ready for the Masses? Datamation, 43(11), 66-71.

Baker, J. D. (1972). Quantitative Modeling of Human Performance in Information Systems. U.S. Army Behavior And Systems Research Laboratory, Support Systems Research Division, 1300 Wilson Boulevard, Arlington, VA. 22209.

Banks, A. P., \& Millward, L. J. (2000). Running Shared Mental Models as a Distributed Cognitive Process. British Journal of Psychology, 91(4), 513-531.

Banks, J., Carson, J. S., II, \& Nelson, B. L. (1996). Discrete-Event System Simulation. (2 ed.). Upper Saddle River, New Jersey 07458: Prentice Hall.

Barber, J. L., Ching, H. L. F., Jones, R. E., Jr., \& Miles, J. L., Jr. (1990). MANPRINT Handbook for RFP Development. (2nd. Ed.). U.S. Army Research Institute for the Behavioral and Social Sciences.

Barila, T. B. (1987). MANPRINT on - Line. Soldier Support Center - National Capital Region.

Baron, S., Kruser, D. S., \& Huey, B. M. (Eds.). (1990). Quantitative Modeling of Human Performance in Complex, Dynamic Systems. Washington, D.C.: National Academy Press.

BCTP. (2002). Midpoint after Action Review Briefing (Powerpoint) Given to 1st Brigade, 1st Cavalry Division Following Bctp Warfighter Exercise Phantom Thunder, 28 January - 8 February 2002. Fort Hood, Texas.

BDM. (1985). Army Manpower Cost System (AMCOS) Economic and Budget Cost Models. Naval Postgraduate School.

Benel, D. C. R., \& Avery, L. W. (1985). Display, Control, and Software Evaluation Requirements for Emerging Tactical Army Computer Systems. In Human Factors Society 29th Annual Meeting Proceedings (pp. 1161-1166).

Bent, J. E., III. (1983). Computer Simulation of a Combat Model Which Uses Command and 
Control. Unpublished Thesis, Naval Postgraduate School, Monterey, Ca. Ad-a127-8902.

Bethmann, R. C., Malloy, K. A., \& Hoever, M. H. (1989). Command and Control: An Introduction. Naval Postgraduate School, Monterey, CA 93943-5000.

Biles, W. E., \& Kleijnen, J. P. C. (1999). Java-Based Simulation Manager for Optimization and Response Surface Methodology in Multiple-Response Parallel Simulation. In 1999 Winter Simulation Conference Proceedings (WSC) (pp. 513-517). Phoenix, AZ, USA: IEEE (http://ieeexplore.ieee.org/iel5/6629/17684/00823117.pdf).

Biles, W. E., \& Ozmen, H. T. (1987). Optimization of Simulation Responses in a Multicomputing Environment. In Proceedings of the 1987 conference on Winter simulation (pp. 402-408)ACM.

Biles, W. E., \& Swain, J. J. (1979). Mathematical Programming and the Optimization of Computer Simulations. In M. Avriel and R.S. Dembo (Eds.), Mathematical Programming Study 11: Engineering Optimization. Amsterdam: North-Holland.

Blackwood, W. O., \& Dice, J. W. (Eds.). (1988). MANPRINT Primer. Alexandria, VA: HQDA ODCSPER. Office of the Deputy Chief of Staff for Personnel, MANPRINT, Research and Studies Directorate. ADA197681.

Bogner, M. S. (1989). Catalog of MANPRINT Methods. U.S. Army Research Institute for the Behavioral and Social Sciences. ARI Research Product 89-09. ADA208236.

Bolte, P. L., Black, B. A., \& Mendel, R. M. (1991). Review of Armor Battalion and Below Automated Command and Control (C2) Soldier Performance Requirements. U.S. Army Research Institute for the Behavioral and Social Sciences.

Booher, H. R. (1990). Preface. In H.R. Booher (Ed.), MANPRINT: An Approach to Systems Integration. New York, NY: Van Nostrand Reinhold.

Borman, K. M., LeCompte, M. D., \& Goetz, J. P. (1986). Ethnographic and Qualitative Research Design and Why It Doesn't Work. American Behavioral Scientist, 30(1), 42-57.

Boucsein, W. (2000). The Use of Psychophysiology for Evaluating Stress-Strain Processes in Human-Computer Interaction. In R.W. Backs and W. Boucsein (Eds.), Engineering Psychophysiology: Issues and Applications. Mahwah, NJ: Lawrence Erlbaum Associates.

Bowers, C. A., Braun, C. C., \& Morgan, B. B., Jr. (1997). Team Workload: Its Meaning and Measurement. In M.T. Brannick and E. Salas (Eds.), Team Performance Assessment and Measurement: Theory, Methods, and Applications. Series in Applied Psychology (Vol. 12(4) Jul-Aug 1982). Mahwah, NJ, US: Lawrence Erlbaum Associates, Inc., Publishers; Lawrence Erlbaum Associates, Inc., Publishers.

Bowers, C. A., Jentsch, F., \& Morgan, B. B., Jr. (2001). Team Performance. In W. Karwowski (Ed.), International Encyclopedia of Ergonomics and Human Factors (Vol. 2). New York: Taylor \& Francis.

Box, M. J. (1965). A New Method of Constrained Optimization and a Comparison with Other Methods. The Computer Journal, $\underline{8}(1), 42-52$.

Braun, J., Lichtenstein, E., Long, J., Le Prell, G., Martha, H., Beach, G., Cobb, P., Melcher, D., 
Milas, C., Cruz, V., \& Voronka, N. (1999). Human Performance Based Measurement System. Unpublished SIBR Report, Human Research and Engineering Directorate, U.S. Army Research Laboratory. SIBR DAAL01-98-C-0031, Aberdeen Proving Ground, MD.

Brenner, T., Sheehan, K., Arthur, W., Jr., \& Bennett, W., Jr. (1999). Behavioral and Cognitive Task Analysis Integration for Assessing Individual and Team Work Activities. Navy Education And Training Professional Development And Technology Center. DTIC AQI99-07-1455.

Brown, B. (2002). Powerpoint Briefing: Operations and Training at the Infantry School. Presented at the U.S. Army Infantry Conference. June 12, 2002. Fort Benning, GA.

Buckalew, L. W. (1990). Soldier Performance as a Function of Stress and Load: A Review. U.S. Army Research Institute for the Behavioral and Social Sciences. Fort Hood, TX. ARI Research Report 1545.

Campion, J., Brander, G., \& Koritsas, E. (1998). Establishing Common Ground and Competence in Team Performance. In M.C. Walker (Ed.), Collaborative Crew Performance in Complex Operational Systems. Cedex, France: Papers Presented At The NATO Research And Technology Organization Human Factors And Medicine Panel Symposium Held In Edinburgh, United Kingdom, 20-22 April 1998.

Casali, J. G. (2001). Sound Instrumentation and Measurement. Course Notes: ISE 5644Auditory Systems. Virginia Polytechnic Institute and State University. Blacksburg, VA.

Centric, J. H., \& Salter, M. S. (1999). Division Level Military Decision-Making Process (MDMP): Design and Development of a Prototype Computer-Based Training Product. U.S. Army Research Institute for the Behavioral and Social Sciences. Alexandria, VA.

Ceruti, M. G., \& McCarthy, S. J. (2000). Establishing a Data-Mining Environment for Wartime Event Prediction with an Object-Oriented Command and Control Database. Space and Naval Warfare Systems Command, San Diego, CA. Third IEEE International Symposium on Object - Oriented Real - Time Distributed Computing, ISOR2K, pp. 174179, March 15-17, 2000, Newport Beach, CA, USA.

Charlton, J. W. (1997). Digitized Chaos: Is Our Military Decision Making Process Ready for the Information Age. Unpublished Monograph, Army Command and General Staff College, Fort Leavenworth, KS. School of Advanced Military Studies. Ad-a339-521-7-xab.

Christiansen, E. (1997). Tamed by a Rose: Computers as Tools in Human Activity. In B.A. Nardi (Ed.), Context and Consciousness: Activity Theory and Human- Computer Interaction. Cambridge, MA: The MIT Press.

Cioppa, T. M. (2002). Efficient Nearly Orthogonal and Space-Filling Experimental Designs for High-Dimensional Complex Models. Unpublished Dissertation, Naval Postgraduate School, Monterey, CA.

Clark, C., \& Williges, R. C. (1972a). Central Composite Response Surface Methodology Design and Analysis. University of Illinois at Urbana-Champaign Aviation Research Laboratory, Institute of Aviation.

Clark, C., \& Williges, R. C. (1972b). Response Surface Methodology Central Composite Design 
Modifications for Human Performance Research. In R.C. Williges and B.H. Williges (Eds.), Selected Papers on Response Surface Methodology. Savoy, Ill: University of Illinois at Urbana-Champaign Aviation Research Laboratory, Institute of Aviation for the Life Sciences Program, Air Force Office of Scientific Research. Technical Report ARL72-20/AFOSR-72-9.

Clark, C., \& Williges, R. C. (1973). Response Surface Methodology Central-Composite Design Modifications for Human Performance Research. Human Factors, 15(4), 295-310.

Clausewitz, C. v. (1832). On War: Translated by Colonel J.J. Graham in 1874 With An Introduction and Notes by Colonel F.N. Maude, C.B. Project Gutenberg electronic archive: ftp://ibiblio.org/pub/docs/books/gutenberg/etext99/1 onwr10.txt.

Clayton, E. R., Weber, W. E., \& Taylor, B. W. I. (1982). A Goal Programming Approach to the Optimization of Multiresponse Simulation Models. IIE Transactions, 14(4).

Clifton, C., \& Thuraisingham, B. (2001). Emerging Standards for Data Mining. Computer Standards \& Interfaces, 23, 187-193.

Cohen, M. S., \& Freeling, A. N. (1981). The Impact of Information on Decisions: Command and Control System Evaluation. Catalog of Selected Documents in Psychology. DARPA Technical Report 81-1. ADA097220, 11 MS. 2397, p94.

Colton, R. A., \& Ganze, R. H. (1993). Including the Human Element in Design of Command and Control Decision Support Systems: The Koalas Concept. Naval Research Laboratory, Washington, DC. NRL/MR/5534-93-7309. Ad-a265-010-9-xab.

Cook, T. M., Leedom, D. K., Grynovicki, J. O., \& Golden, M. G. (2000). Cognitive Representations of Battlespace Complexity: Six Fundamental Variables of Combat. Human Research and Engineering Division, U.S. Army Research Laboratory, Aberdeen Proving Ground, MD 21005.

Cooke, N. J. (1999). Knowledge Elicitation. In F.T. Durso (Ed.), Handbook of Applied Cognition. New York, NY: John Wiley \& Sons, Inc.

Cooke, N. J., Stout, R. J., \& Salas, E. (2001). A Knowledge Elicitation Approach to the Measurement of Team Situation Awareness. In M.D. McNeese, E. Salas, \& M.R. Endsley (Eds.), New Trends in Cooperative Activities (pp. 96-113): HFES.

Cooper, M., Shiflett, S., \& Crotkin, A. L. (1984). Command and Control Teams: Techniques for Assessing Team Performance. Air Force Human Resources Laboratory.

Cooper, R. A. (1991). System Identification of Human Performance Models. IEEE Transactions on Systems, Man \& Cybernetics, 21(1).

Corker, K. M., Cramer, N. L., Henry, E. H., \& Smoot, D. E. (1990). Methodology for Evaluation of Automation Impacts on Tactical Command and Control (C2) Systems. U.S. Air Force Human Resources Laboratory. AFHRL-TR-90-91. ADA 245573.

Costa, P. T., \& McCrae, R. R. (1995). Domains and Facets: Hierarchical Personality Assessment Using the Revised Neo Personality Inventory. Journal of Personality Assessment.

Cothier, P. H., \& Levis, A. H. (1986). Timeliness and Measures of Effectiveness in Command and Control. IEEE Transactions on Systems, Man \& Cybernetics, 16(6) Nov-Dec 1986. 
Cowings, P. S., Toscano, W. B., DeRoshia, C., \& Tauson, R. A. (1999). The Effects of the Command and Control Vehicle (C2V) Operational Environment on Soldier Health and Performance. Human Research and Engineering Division, U.S. Army Research Laboratory, Aberdeen Proving Ground, MD 21005.

Crumley, L. M., \& Sherman, M. B. (1990). Review of Command and Control Models and Theory. U.S. Army Research Institute for the Behavioral and Social Sciences, Ft. Leavenworth FE.

Czaja, S. J. (1997). Systems Design and Evaluation. In G. Salvendy (Ed.), Handbook of Human Factors and Ergonomics (2 ed., pp. 17-39). NY, New York: John Wiley \& Sons, Inc.

Dahl, S., Laughery, R., Hahler, B., Lockett, J., \& Thein, B. (1991). CREWCUT - a Computer Modeling Tool for Studying Dynamic Human Performance under Conditions of High Workload. In International Ergonomics Society Proceedings.

D'Angelo, H. (1980). A Quantitive Approach to Aggregation in the Modeling of Tactical Command, Control, and Communication Systems. Air Force Office of Scientific Research.

Darling, C. B. (1997). Datamining for the Masses. Datamation, 43(2), 52-56.

DePuy, W. E., \& Bonder, S. (1982). Integration of Mpt Supply and Demand and the System Acquisition Process. U.S. Army Institute for the Behavioral and Social Sciences.

Diamond, W. J. (1981). Introduction to Experiment Design: Fundamental Concepts, Practical Experiment Designs for Engineers and Scientists. Belmont, CA: Lifetime Learning Publications.

DOD. (1999). Department of Defense Handbook: Human Engineering Program Process and Procedures. Mil-Hdbk-46855a.

Dryer, D. (1998). Decision Support Rendering Tools (Dsrts) for Battlespace Command and Control. U.S. Army Communications - Electronics Command, Command / Control \& Systems Integration Directorate, Attn: AMSEL-RD-C2-BV-M-2, Ft. Monmouth, NJ 07703-5203.

Durso, F. T., \& Gronlund, S. D. (1999). Situation Awareness. In F.T. Durso (Ed.), Handbook of Applied Cognition. New York, NY: John Wiley \& Sons, Inc.

Endsley, M. R. (1988). Design and Evaluation for Situation Awareness Enhancement. Proceedings of the Human Factors Society 32nd Annual Meeting. Santa Monica, CA, 97-102.

Endsley, M. R. (1995a). Measurement of Situation Awareness in Dynamic Systems. Human Factors, 37(1), 65-84.

Endsley, M. R. (1995b). Toward a Theory of Situation Awareness in Dynamic Systems. Human Factors, 37(1), 32-64.

Endsley, M. R. (2000). Theoretical Underpinnings of Situation Awareness: A Critical Review. In M.R. Endsley and D.J. Garland (Eds.), Situation Awareness Analysis and Measurement. Mahwah, NJ: Lawrence Erlbaum Associates.

Fallesen, J. J., Lussier, J. W., \& Michel, R. R. (1992a). Tactical Command and Control Process. 
U.S. Army Research Institute for the Behavioral and Social Sciences. ARI Research Product 92-06. Ad-a255-036-6-xab.

Fallesen, J. J., Lussier, J. W., \& Michel, R. R. (1992b). Tactical Command and Control Process. Army Research Institute For the Behavioral and Social Sciences, Alexandria, Va. ARI Research Product 92-06. Ad-a255-036-6-xab.

Fallesen, J. J., \& Quinkert, K. (1990). Workspace Design Handbook for Standardized Command Posts. U.S. Army Research Institute for the Behavioral and Social Sciences. Fort Leavenworth, KS.

Farrell, R. L., Bonder, S., Proegler, L. D., Miller, G., \& Thompson, D. E. (1986). Capturing Expertise: Some Approaches to Modeling Command Decisionmaking in Combat Analysis. IEEE Transactions on Systems, Man \& Cybernetics, 16(6).

Fischer, I., Hennecke, F., Bannes, C., \& Zell, A. (2001). Java Neural Network Simulator (JNNS), User Manual, Version 1.1. University of Tübingen, Wilhelm Schickard Institute For Computer Science, Department of Computer Architecture, Tubingen, Germany. http://www-ra.informatik.uni-tuebingen.de/downloads/JavaNNS/.

Flanagan, J. C. (1954). The Critical Incident Technique. Psychological Bulletin, Vol. 51, No. 4.

Fleishman, E. A. (1975). Toward a Taxonomy of Human Performance. American Psychologist, pp. $1127-1149$.

Fleishman, E. A. (1978). Relating Individual Differences to the Dimensions of Human Tasks. Ergonomics, Vol. 21, No. 12, pp 1007-1019.

Fleishman, E. A. (1984). Systems for Linking Job Tasks to Personnel Requirements. Public Personnel Management Journal, pp 395-408.

Fleishman, E. A. (1988). Some New Frontiers in Personnel Selection Research. Personnel Psychology, Vol 41.

Fleishman, E. A., \& Quaintance, M. K. (1984). Taxonomies of Human Performance - the Description of Human Tasks. Bethesda, Maryland: Management Research Institute, Inc.

Fleishman, E. A., \& Zaccaro, S. J. (1992). Toward a Taxonomy of Team Performance Functions. In R.W. Swezey and E. Salas (Eds.), Teams: Their Training and Performance. Norwood, NJ: Ablex Publishing Corp.

Ford, J. P., Mullen, W. J. I., \& Keesling, J. W. (1997). Analysis of Command and Control Battlefield Functions as Performed in the Armored Brigade. U.S. Army Research Institute for the Behavioral and Social Sciences, Alexandria, VA.

Garrett, T., Schendel, J., \& Walker, L. (1991). Methodology to Incorporate Human Factor Variables into Army Combat Models. U.S. Army Training and Doctrine Command (TRADOC). Contract DABT60-87-D-3873. ADA239906.

Gawron, V. J., Travale, D. J., \& Neal, J. G. (1989). A Human Performance Consultant System and Some Applications. In S.A. Murtaugh (Ed.), Human Behavior and Performance as Essential Ingredients in Realistic Modeling of Combat - MORIMOC II. Proceedings of the Military Operations Research Society Mini-Symposium Held in Alexandria, Virginia on 22-24 February 1989. Volume 1 (pp. 360). Alexandria, Virginia: Military Operations 
Research Society. DTIC ADA227841.

Gibbings, L. G. (1991). U.S. Army Training and Doctrine Command (TRADOC) Pamphlet 11-9: Blueprint of the Battlefield. U.S. Army Research Institute for the Behavioral and Social Sciences. Alexandria, VA. Report Number E-17541-Rev.

Gilman, A. W. (1990). An Alternative to 'Suppression': Modeling Methodology for Assessing Indirect Effects of Weapons and Human Performance Degradation. Ballistic Research Laboratory. Aberdeen Proving Ground, MD. BRL-TR-3131. ADA226518.

Glover, F., Taillard, E., \& de Werra, D. (1993). A User's Guide to TABU Search. In P.L. Hammer (Ed.), Annals of Operations Research: TABU Search (Vol. 41). Basel, SW: J.C. Baltzer, AG.

Glovier, D. A. (1998). Study of Human Factors Variables in Battle Outcome Prediction Models. Unpublished Dissertation, Old Dominion University.

Godowski, J. C., King, G. F., Ronco, P. G., \& Askren, W. B. (1978). Integration and Application of Human Resource Technolgoies in Weapon System Design: Consolidated Data Base Functional Specification. Air Force Human Research Laboratory. AFRL Technical Report AFHRK-TR-78-6 (III). ADA059298.

Goedkoop, T. R. (1988). Task Force Tactical Operations Center: An Organization for Success. Unpublished Monograph, Army Command and General Staff College, Fort Leavenworth, Ks. School of Advanced Military Studies. Ad-a208-320-2-xab.

Golden, M. G., Cook, T. M., Grynovicki, J. O., Kysor, K. P., \& Leedom, D. K. (2000). ARL Insights from the Battle Command Re-Engineering III Concept Experimentation Program. Human Research and Engineering Division, U.S. Army Research Laboratory, Aberdeen Proving Ground, MD 21005.

Gopher, D., \& Braune, R. (1984). On the Psychophysics of Workload: Why Bother with Subjective Measures? Human Factors, 26(5), 519-532.

Gopher, D., \& Donchin, E. (1986a). Cognitive Processes and Performance, Ch. 41, Workload an Examination of the Concept, Handbook of Perception and Human Performance (Edition ed., Vol. II). City: John Wiley \& Sons.

Gopher, D., \& Donchin, E. (1986b). Workload - an Examination of the Concept. In K.R. Boff, L. Kaufman, \& J.P. Thomas (Eds.), Handbook of Perception and Human Performance: Cognitive Processes and Performance (Vol. II). New York, NY: John Wiley \& Sons, Inc.

Gordon, D. E. (1973). Induced Stress, Artificial Environment, Simulated Tactical Operations Center Model. Unpublished Thesis, Army Command and General Staff College. Ada090-875-6.

Green, C. A. (1995). Development and Validation of a Methodology for Comprehensive Performance Assessment of Complex Tasks. Unpublished Dissertation, Virginia Polytechnic Institute and State University, Blacksburg, VA.

Grossberg, S., \& Mingolla, E. (1986). Computer Simulation of Neural Networks for Perceptual Psychology. Behavior Research Methods, Instruments, \& Computers, 18(6).

Guerrier, J. H., Lowry, J. C., Jones, R. E., Jr., Guthrie, J. L., Barber, J. L., \& Miles, J. L., Jr. 
(1991). Handbook for Conducting Analysis of the Manpower, Personnel, and Training Elements for a MANPRINT Assessment. U.S. Army Research Institute for the Behavioral and Social Sciences.

Hahler, B., Dahl, S., Laughery, R., Lockett, J., \& Thein, B. (1991). CREWCUT - a Tool for Modeling the Effects of High Workload on Human Performance. In $\underline{35 \text { th Human Factors }}$ and Egronomics Society Proceedings (pp. 1210-1215).

Halpin, S. M. (1992). Army Command and Control Evaluation System (Acces): A Brief Description. U.S. Army Research Institute for the Behavioral and Social Sciences, Ft. Leavenworth FE. ARI Working Paper LVN 92-01.

Hamilton, D. B., Bierbaum, C. R., \& Fulford, L. A. (1991). Task Analysis / Workload (TAWL) User's Guide: Version 4.0. U.S. Army Research Institute for the Behavioral and Social Sciences.

Han, S. H., Williges, B. H., \& Williges, R. C. (1997). A Paradigm for Sequential

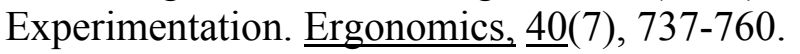

Hancock, P. A., \& Caird, J. K. (1993). Experimental Evaluation of a Model of Mental Workload. Human Factors, 35(3), 413-429.

Hancock, P. A., \& Meshkati, N. (Eds.). (1988). Human Mental Workload. (Vol. 52). Amsterdam: North Holland: Tavistock Publications.

Harper, W. R. (1974). Human Factors in Command and Control of the Los Angeles Fire Department. Applied Ergonomics, 5(1), 26-35.

Hart, S. G., \& Wickens, C. D. (1993). Workload Assessment and Prediction. In R.W. Pew and P. Green (Eds.), Human Factors Engineering Short Course Notes (34 ed.). Ann Arbor, Michigan: The University of Michigan, Chrysler Center for Continuing Engineering Education. MANPRINT: An Approach to Systems Integration. Harold R. Booher (Ed.). Van Nostrand Reinhold, New York.

Hausrath, A. H. (1971). Venture Simulation in War, Business, and Politics. New York, NY: McGraw-Hill, Inc.

Hedgepeth, W. O. (1996). Comparing Traditional Statistical Models with Neural Network Models: The Case of the Relation of Human Performance Factors to the Outcomes of Military Combat. Dissertation Abstracts International: Section B: the Sciences \& Engineering, 57(3-B), 1-20.

Hendrick, H. W., \& Kleiner, B. M. (2001). Macroergonomics: In Introduction to Work System Design. Santa Monica, CA: Human Factors and Ergonomics Society.

Hendy, K. C., Hamilton, K. M., \& Landry, L. N. (1993). Measuring Subjective Workload: When Is One Scale Better Than Many? Human Factors, 35, 579.

Hicks, C. R. (1973). Fundamental Concepts in the Design of Experiments. (2 ed.). New York: Holt, Rinehart and Winston.

Hicks, S. A. (1960a). The Effects of Eight Hours Confinement in Mobile Armored Personnel Carriers on Selected Combat Relevant Skills: Study 2 of 8. U.S. Army Human Engineering Laboratory, Aberdeen Proving Ground, MD 21005. 
Hicks, S. A. (1960b). The Effects of Four Hours Confinement in Mobile Armored Personnel Carriers on Selected Combat Relevant Skills: A Pilot Study - Study 1 of 8. U.S. Army Human Engineering Laboratory, Aberdeen Proving Ground, MD 21005.

Hicks, S. A. (1961a). The Effects of Twelve Hours Confinement in Mobile Armored Personnel Carriers on Selected Combat Relevant Skills: Study 4 of 8. U.S. Army Human Engineering Laboratory, Aberdeen Proving Ground, MD 21005.

Hicks, S. A. (1961b). The Effects of Twelve Hours Confinement in Static Armored Personnel Carriers on Selected Combat Relevant Skills: Study 3 of 8. U.S. Army Human Engineering Laboratory, Aberdeen Proving Ground, MD 21005.

Hicks, S. A. (1961c). The Effects of Twenty-Four Hours Confinement in Mobile Armored Personel Carriers on Selected Combat Relevant Skills: Study 5 of 8. U.S. Army Human Engineering Laboratory, Aberdeen Proving Ground, MD 21005.

Hicks, S. A. (1962). The Effects of Twenty-Four Hours Confinement in Mobile Armored Personnel Carriers on Selected Combat Relevant Skills: A Followup- Study 6 of 8 . U.S. Army Human Engineering Laboratory, Aberdeen Proving Ground, MD 21005.

Hicks, S. A. (1963). The Effects of Repeated Confinement on the Performance of Men in a HotWet Climate; Study 7 of 8. U.S. Army Human Engineering Laboratory, Aberdeen Proving Ground, MD 21005.

Hicks, S. A. (1964a). The Effects of Confinement on the Performance of Combat Relevant Skills: Summary Report. U.S. Army Human Engineering Laboratory, Aberdeen Proving Ground, MD 21005.

Hicks, S. A. (1964b). The Effects of Repeated Confinement on the Performance of Men in a Temperate Environment; Study 8 of 8. U.S. Army Human Engineering Laboratory, Aberdeen Proving Ground, MD 21005.

Hiemstra, M., Korzym, D., Barila, T., \& Imbs, D. (1987). MANPRINT Risk Assessment. Soldier Support Center - National Capital Region. ADA185995.

Hill, R. R., Miller, J. O., \& McIntyre, G. A. (2001). Applications of Discrete Event Simulation Modeling to Military Problems. In Winter Simulation Conference Proceedings- ACM.

Hoffman, R. R., Hayes, P., \& Ford, K. M. (2001). Human Centered Computing: Thinking in and out of the Box. IEEE Intelligent Systems, September / October 2001.

Hoffman, R. R., Klein, G., \& Laughery, K. R., Jr. (2002). The State of Cognitive Systems Engineering. IEEE Intelligent Systems, January / February 2002.

Hood, S. J., \& Welch, P. D. (1993). Response Surface Methodology and Its Application in Simulation. In Proceedings of the 1993 Winter Simulation Conference (pp. 115-122) ACM.

Howell, D. C. (1997). Statistical Methods for Psychology. (4 ed.). Belmont, California: Duxbury Press.

Huron, M. A. (1997). The Army Tactical Command and Control System. Naval Postgraduate School, Monterey, CA 93943-5000.

Jablunovsky, G., Dorman, C., \& Yoworsky, P. (2000). A Neural Network Sub-Model as an 
Abstraction Tool: Relating Network Performance to Combat Outcome. In Enabling Technology for Simulation Science IV, Proceedings of SPIE. Data Mining and Knowledge Discovery: Theory, Tools, and Technology II : 24-25 April, 2000. Vol. 4026 (pp. 192-200). Orlando, FL: Bellingham, Washington : SPIE.

JCS. (1994). Department of Defense Dictionary of Military and Associated Terms. Joint Publication 1-02. Joint Chiefs of Staff.

Johnson, K. M., Rossmeissl, P., Kracov, W., \& Shields, J. L. (1988). MANPRINT Handbook for Nondevelopmental Item (NDI) Acquisition. U.S. Army Research Institute for the Behavioral and Social Sciences.

Johnson, K. M., \& Wright, C. L. (1990). MANPRINT Practitioner's Guide. MANPRINT Policy Office, Office of the Deputy Chief of Staff for Personnel, The Pentagon.

Joyce, G. F., Abarbanel, H., Callan, C., Dally, W., \& Dyson, F. (2000). Data Mining and the Human Genome. Mitre Corp Mclean Va Jason Program Office.

Kac, M. (1969). Some Mathematical Models in Science. Science, 166(3906), 695-699.

Kaempf, G. L., Klein, G., Thordsen, M. L., \& Wolf, S. (1996). Decision Making in Complex Naval Command-and-Control Environments. Human Factors, 38(2), 220-231.

Kay, G. G., \& Dolgin, D. L. (1998). Team Compatibility as a Predictor of Team Performance: Picking the Best Team. In M.C. Walker (Ed.), Collaborative Crew Performance in Complex Operational Systems. Cedex, France: Papers Presented At The NATO Research And Technology Organization Human Factors And Medicine Panel Symposium Held In Edinburgh, United Kingdom, 20-22 April 1998.

Keane, J. (1992). Human Computer Interface Style Guide (Version 1.0, February 12,1992). Defense Information Systems Agency, Center For Information Management.

Kelton, W. D. (1995). A Tutorial on Design and Analysis of Simulation Experiments. In Proceedings of the 1995 Winter Simulation Conference. ACM.

Kieras, D. E., \& Meyer, D. E. (2000). The Role of Cognitive Task Analysis in the Application of Predictive Models of Human Performance. In J.M. Schraagen, S.F. Chipman, \& V.L. Shalin (Eds.), Cognitive Task Analysis. Mahwah, NJ: Lawrence Erlbaum Associates.

Kilmer, R. A. (1994). Artificial Neural Network Metamodels of Stochastic Computer Simulations. Unpublished Dissertation, University of Pittsburg, Pittsburg, PA.

King, D. W., King, L. A., Foy, D. W., \& Gudanowski, D. M. (1996). Prewar Factors in CombatRelated Posttraumatic Stress Disorder: Structural Equation Modeling with a National Sample of Female and Male Vietnam Veterans. Journal of Consulting \& Clinical Psychology, 64(3).

Kleijnen, J. P. C. (1998). Experimental Design for Sensitivity Analysis, Optimization, and Validation of Simulation Models. In J. Banks (Ed.), Handbook of Simulation: Principles, Methodology, Advances, Applications, and Practice (pp. 849). New York: John Wiley and Sons, Inc.

Klein, G. (1993). Naturalistic Decision Making: Implications for Design. In Gateway: Volume 4, Number 3. Crew System Ergonomics Information Analysis Center (CSERIAC). DTIC 
ADA386200.

Klein, G. (1998). Sources of Power- How People Make Decisions. Cambridge, Massachusetts: The MIT Press.

Klein, G. (2000a). Analysis of Situation Awareness from Critical Incident Reports. In M.R. Endsley and D.J. Garland (Eds.), Situation Awareness Analysis and Measurement. Mahwah, NJ: Lawrence Erlbaum Associates.

Klein, G. (2000b). Cognitive Task Analysis of Teams. In J.M. Schraagen, S.F. Chipman, \& V.L. Shalin (Eds.), Cognitive Task Analysis (pp. 417-429). Mahwah, NJ: Lawrence Erlbaum Associates.

Klein, G., \& Crandall, B. (1996). Recognition-Primed Decision Strategies. U.S. Army Research Institute for the Behavioral and Social Sciences. ARI Research Note 96-36. DTIC ADA3095700.

Kleiner, B. M. (1997). An Integrative Framework for Measuring and Evaluating Information Management Performance. International Journal of Computers And Industrial Engineering, 32(3), 545-555.

Kleinman, D. L., Luh, P. B., Pattipati, K. R., \& Serfaty, D. (1992). Mathematical Models of Team Performance: A Distributed Decision-Making Approach. In R.W. Swezey and E. Salas (Eds.), Teams: Their Training and Performance. Norwood, NJ: Ablex Publishing Corp.

Knapp, B. G. (1996a). Job Comparison and Analysis Tool (Jcat) Instruction Booklet - 96b Case. Human Research and Engineering Division, U.S. Army Research Laboratory, Aberdeen Proving Ground, MD 21005.

Knapp, B. G. (1996b). Job Comparison and Analysis Tool (Jcat) Instruction Booklet - Nursing Case. Human Research and Engineering Division, U.S. Army Research Laboratory, Aberdeen Proving Ground, MD 21005.

Knapp, B. G., Johnson, J., Barnette, D. B., Wojciechowski, J., Kilduff, P., \& Swoboda, J. (1997a). Modeling Maneuver Battalion C2 Operations of a Current Army Command Post for a Force on Force Scenario- Baseline Model. Human Research and Engineering Division, U.S. Army Research Laboratory, Aberdeen Proving Ground, MD 21005.

Knapp, B. G., Johnson, J., Barnette, D. B., Wojciechowski, J., Kilduff, P., \& Swoboda, J. (1997b). Modeling Maneuver Battalion C2 Operations of a Force XXI Equipped Army Command Post for a Force on Force Scenario-Traditional Model. Human Research and Engineering Division, U.S. Army Research Laboratory, Aberdeen Proving Ground, MD 21005.

Knapp, B. G., Johnson, J., Barnette, D. B., Wojciechowski, J., Kilduff, P. S., J., Bird, S., \& Plott, B. (1997c). Modeling Maneuver Battalion C2 Operations of a Force XXI Equipped Army Command Post for a Force on Force Scenario- Integrated Model. Human Research and Engineering Division, U.S. Army Research Laboratory, Aberdeen Proving Ground, MD 21005.

Kozycki, R. W., Faughn, J. A., Leiter, K. L., \& Lockett, J. F., III. (1997). Applying Virtual $\underline{\text { Reality and Human Figure Modeling Tools to Explore Crew Manning Configurations of }}$ 
the U.S. Navy DDG Class Bridge. Human Research and Engineering Division, U.S. Army Research Laboratory, Aberdeen Proving Ground, MD 21005.

Kubala, A. L., \& Warnick, W. L. (1979). A Review of Selected Literature on Stresses Affecting Soldiers in Combat. U.S. Army Research Institute for the Behavioral and Social Sciences. Alexandria, VA. ARI Technical Report TR-79-A14.

Kumashiro, M. (1995). Practical Measurement of Psychophysiological Functions for Determining Workloads. In J.R. Wilson and E.N. Corlett (Eds.), Evaluation of Human Work: A Practical Ergonomics Methodology (2 ed.). Bristol, PA: Taylor \& Francis.

Kwahk, J. (2002). Neural Network and Data Mining. Unpublished Monograph, Virginia Polytechnic Institute and State University. Blacksburg, VA, Blacksburg, VA.

Lanchester, F. W. (1916). Aircraft in Warfare: The Dawn of the Fourth Arm. London, UK: Constable and Company Limited.

Laughery, K. R., \& Drury, C. G. (1979). Human Performance and Strategy in a Two-Variable Optimization Task. Ergonomics, 22(4).

Laughery, K. R., Jr. (1989a). Task Network Modeling as a Basis for Analyzing Operator Workload. In Proceedings of the Human Factors Society 33th Annual Meeting (pp. 110114) HFES.

Laughery, K. R., Jr. (1989b). Task Network Modeling Constructs as Applied to Modeling Human Performance in Combat. In S.A. Murtaugh (Ed.), Human Behavior and Performance as Essential Ingredients in Realistic Modeling of Combat - MORIMOC II. Proceedings of the Military Operations Research Society Mini-Symposium Held in Alexandria, Virginia on 22-24 February 1989. Volume 1 (pp. 360). Alexandria, Virginia: Military Operations Research Society. DTIC ADA227841.

Laughery, K. R., Jr. (1999). Modeling Human Performance During System Design. In E. Salas (Ed.), Human/Technology Interaction in Complex Systems (Vol. 9, pp. 147-174): JAI Press, Inc.

Laughery, K. R., Jr., \& Corker, K. (1997). Computer Modeling and Simulation. In G. Salvendy (Ed.), Handbook of Human Factors and Ergonomics (2 ed., pp. 1375-1408). NY, New York: John Wiley \& Sons, Inc.

Law, A. M. (1997). How to Select Simulation Software: Seminar Presented to the U.S. Army Research Laboratory. Averill M. Law \& Associates.

Law, A. M., \& Kelton, W. D. (2000). Simulation Modeling and Analysis. (3 ed.). NY, New York: McGraw-Hill, Inc.

Lawlor, M. C., \& Mattingly, C. F. (2001). Beyond the Unobtrusive Observer: Reflections on Researcher-Informant Relationships in Urban Ethnography. American Journal of Occupational Therapy, 55(2), 147-154.

LeCompte, M. D., \& Goetz, J. P. (1982). Ethnographic Data Collection in Evaluation Research. Educational Evaluation \& Policy Analysis, 4(3), 387-400.

Lee, J. D. (1999). Information Dominance in Military Decision Making. Unpublished Thesis, Army Command and General Staff College. Fort Leavenworth, KS. Ada367899-xab, 
Fort Leavenworth, KS.

Leedom, D. K., Adelman, L., \& Murphy, J. (1998). Critical Indicators in Naturalistic Decision Making. In Fourth Conference on Natualistic Decision Making.

Leedom, D. K., \& Fallesen, J. (1998). Initial Insights from Prairie Warrior 98. Human Research and Engineering Division, U.S. Army Research Laboratory, Aberdeen Proving Ground, MD 21005.

Leedom, D. K., Murphy, J., \& Adelman, L. (1998a). A Cognitive Engineering Analysis in Support of Future Task Force Tactical Operations Center (TOC) Concept Experimentation. Human Research and Engineering Division, U.S. Army Research Laboratory, Aberdeen Proving Ground, MD 21005.

Leedom, D. K., Murphy, J., \& Adelman, L. (1998b). Cognitive Engineering of the Human Computer Interface for ABCS. Human Research and Engineering Division, U.S. Army Research Laboratory, Aberdeen Proving Ground, MD 21005.

Levis, A. H., \& Athans, M. (1986). Command and Control Theory. Laboratory for Information and Decision systems, Massachusetts Institute of Technology, Cambridge, Mass., 02139.

Lickteig, C. W. (1987). Training Requirements for the Battlefield Management System (BMS): A Preliminary Analysis. U.S. Army Institute for the Behavioral and Social Sciences. ARI Research Product 87-18. ADA185468.

Littlefield, T. K. (1998). Military Decision Process - Overlooked by the Revolution in Military Affairs. Unpublished Monograph, U.S. Army War College. Carlisle Barracks, PA. Ada344-635-8-xab.

Lopes, C., Pacheco, M., Vellasco, M., \& Passos, E. (1999). Rule-Evolver: An Evolutionary Approach for Data Mining. In N. Zhong, A. Skowron, \& S. Ohsuga (Eds.), International Workship New Directions in Rough Sets, Data Mining \& Granular-Soft Computing. (7th International Workshop, Rsfdgrc'99). Data Mining and Knowledge Discovery Workshop. Yamaguchi, Japan.

Luczak, H., \& Stahl, J. (2001). Task Analysis in Industry. In W. Karwowski (Ed.), International Encyclopedia of Ergonomics and Human Factors (Vol. 3). New York: Taylor \& Francis.

Lynch, R. (2001). Commanding a Digital Brigade Combat Team: Tactics, Techniques, and Procedures. Center For Army Lessons Learned (CALL). Fort Leavenworth, KS. CALL Special Edition Newsletter No. 01-21. http://call.army.mil/products/spc edtn/01-21/0121 toc.htm.

Lysaght, R. J., Hill, S. G., Dick, A. O., Plamondon, B. D., Linton, P. M., Wierwille, W. W., Zaklad, A. L., Bittner, A. C., Jr., \& Wherry, R. J., Jr. (1989). Operator Workload: Comprehensive Review and Evaluation of Operator Workload Methodologies. U.S. Army Institute for the Behavioral and Social Sciences.

MA\&D. (1996). MicroSaint User's Manual for Windows, Version 2.0. Boulder, CO: Micro Analysis and Design, Inc.

Mackie, R. R., Wylie, C. D., \& Smith, M. J. (1985). Comparative Effects of 19 Stressors on Task Performance: Critical Literature Review (What We Appear to Know, Don't Know, and Should Know). In Human Factors Society 29th Annual Meeting Proceedings (pp. 462- 
465).

Maillefert, C. W. (1975). Command and Control: A Contemporary Perspective. Naval War College, Newport, Rhode Island.

Marshall, C. W. (1965). Probabilistic Models in the Theory of Combat. Transactions of the New York Academy of Sciences, 27(5), 477-487.

Mason, C. R., \& Moffat, J. (2000). Representing the C2 Process in Simulations: Modelling the Human Decision-Maker. In Winter Simulation Conference Proceedings- ACM.

Massaro, D. W. (1988). Some Criticisms of Connectionist Models of Human Performance. Journal of Memory \& Language, 27(2).

Mathieu, J. E., Heffner, T. S., Goodwin, G. F., Salas, E., \& Cannon-Bowers, J. A. (2000). The Influence of Shared Mental Models on Team Process and Performance. Journal of Applied Psychology, 85(2), 273-283.

McCann, C. A. (1990). Human Cognitive Processes in Command and Control Planning. 1: Identification of Research Issues. The Netherlands Organization For Applied Scientific Research, TNO Institute for Perception, TNO Division of National Defence Research, Group: Cognitive Psychology.

McCoy, M. S. (1996). A Rule-Based Pilot Performance Model. Dissertation Abstracts International: Section B: the Sciences \& Engineering, 56 (8-B).

McCoy, M. S., \& Levary, R. R. (1988). Augmenting Knowledge Acquisition Processes of Expert Systems with Human Performance Modeling Techniques. IEEE Transactions on Systems, Man \& Cybernetics, 18(3).

McCracken, J. H., \& Aldrich, T. B. (1984). Analyses of Selected LHX Mission Functions: Implications for Operator Workload and System Automation Goals. U.S. Army Research Institute Aviation Research and Development Activity, Fort Rucker, AL. ARI Research Report ASI479-024-84. AD-a232 330.

McDermott, P., Klein, G., Thordsen, M., Ransom, S., \& Paley, M. (2000). Representing the Cognitive Demands of New Systems: A Decision-Centered Design Approach. Human Effectiveness Directorate, Crew system Interface Division, U.S. Air Force Research Laboratory. AFRL-HE-TR-2000-0023. DTIC ADA385318.

McGlynn, R. P., \& Pierce, L. G. (1997). Development of a Team Performance Task Battery to Evaluate Performance of the Command and Control Vehicle (C2V) Crew. Human Research and Engineering Division, U.S. Army Research Laboratory, Aberdeen Proving Ground, MD 21005 / Texas Tech University.

McMillan, G. R., Beevis, D., Stein, W., Strub, M. H., Salas, E., Sutton, R., \& Reynolds, K. C. (1991). A Directory of Human Performance Models for System Design. Defense Research Section, NATO Headquarters, B-1110 Brussels, Belgium.

McMillan, G. R., \& Martin, E. A. (1989). An Overview of Human Performance Models and Potential Applications to Combat Simulation. In S.A. Murtaugh (Ed.), Human Behavior and Performance as Essential Ingredients in Realistic Modeling of Combat - MORIMOC II. Proceedings of the Military Operations Research Society Mini-Symposium Held in Alexandria, Virginia on 22-24 February 1989. Volume 1 (pp. 360). Alexandria, Virginia: 
Military Operations Research Society. DTIC ADA227841.

McNeese, M. D., \& Rentsch, J. R. (2001). Identifying the Social and Cognitive Requirements of Teamwork Using Collaborative Task Analysis. In M.D. McNeese, E. Salas, \& M.R. Endsley (Eds.), New Trends in Cooperative Activities (pp. 96-113): HFES.

Mee, A. (2000). Distance Matters. In G.M. Olson and J.S. Olson (Eds.), Human Computer Interaction. From "the Pleasure Telephone" (1898). The Strand Magazine, 16, 339-345. (Vol. 15, pp. 139-178): Lawrence Erlbaum Associates, Inc., Publishers.

Megaputer. (2002). Data Mining. Http://Www.Megaputer.Com/Dm/Dm101.Php3.

Middlebrooks, S. E. (1991). An Evaluation of Current and Future Capabilities for Wartime Mission Support from the Corps Battle Simulation (CBS) System. Unpublished Monograph, V (U.S. Army) Corps Headquarters, Frankfurt, Germany.

Middlebrooks, S. E. (2001). Experimental Interrogation of Network Simulation Models of Human Task and Workload Performance in a U.S. Army Tactical Operations Center. Unpublished Thesis, Virginia Polytechnic Institute and State University, Blacksburg, VA.

Middlebrooks, S. E., Knapp, B. G., Barnette, B. D., Bird, C. A., Johnson, J. M., Kilduff, P. W., Schipani, S. P., Swoboda, J. C., Wojciechowski, J. Q., Tillman, B. W., Ensing, A. R., Archer, S. G., Archer, R. D., \& Plott, B. M. (1999a). CoHOST - Computer Simulation Models to Investigate Human Performance Task and Workload Conditions in a U.S. Army Heavy Maneuver Battalion Tactical Operations Center. In Proceedings of the HFES 43rd Annual Meeting (pp. 242-246). Houston, TX: Human Factors and Ergonomics Society.

Middlebrooks, S. E., Knapp, B. G., Barnette, B. D., Bird, C. A., Johnson, J. M., Kilduff, P. W., Schipani, S. P., Swoboda, J. C., Wojciechowski, J. Q., Tillman, B. W., Ensing, A. R., Archer, S. G., Archer, R. D., \& Plott, B. M. (1999b). CoHOST (Computer Modeling of Human Operator System Tasks) Computer Simulation Models to Investigate Human Performance Task and Workload Conditions in a U.S. Army Heavy Maneuver Battalion Tactical Operations Center. Human Research and Engineering Directorate, U.S. Army Research Laboratory, Aberdeen Proving Ground, MD 21005. ARL Technical Report ARL-TR-1994; DTIC ADA368587.

Militello, L. G., Kyne, M. M., Klein, G., Getchell, K., \& Thordsen, M. (1999). A Synthesized Model of Team Performance. International Journal of Cognitive Ergonomics, $\underline{3}$ (2), 131158.

Monguillet, J., \& Levis, A. H. (1988). Modeling and Evaluation of Variable Structure Command and Control Organizations. Massachusetts Institute of Technology.

Moray, N. (1982). Subjective Mental Workload. Human Factors, 24(1), 25-40.

Moscovic, S. A. (1992). Development of Time and Workload Methodologies for Micro Saint Models of Visual Display and Control Systems. Unpublished Dissertation, Virginia Polytechnic Institute and State University, Blacksburg, VA.

Murphy, J., Adelman, L., Leedom, D., Grynovicki, J., Golden, M., \& Kysor, K. P. (1998). Cognitive Engineering of the Battalion-Brigade Decision Process. Human Research and Engineering Division, U.S. Army Research Laboratory, Aberdeen Proving Ground, MD 
21005.

Myers, L. B., Tijerina, L., \& Geddie, J. C. (1987). Proposed Military Standard for Task Analysis. U.S. Army Human Engineering Laboratory, Aberdeen Proving Ground, MD 21005. USA HEL Technical Memorandum 13-87.

Nardi, B. A. (1997a). Activity Theory and Human - Computer Interaction. In B.A. Nardi (Ed.), Context and Consciousness: Activity Theory and Human- Computer Interaction. Cambridge, MA: The MIT Press.

Nardi, B. A. (1997b). The Use of Ethnographic Methods in Design and Evaluation. In M.G. Helander, T.K. Landauer, \& P.V. Prabhu (Eds.), Handbook of Human-Computer Interaction (2 ed., pp. 361 - 366). Amsterdam, Holland: Elsevier.

Newell, A., \& Simon, H. A. (1972). Human Problem Solving. Englewood Cliffs, NJ: Prentice Hall.

Nieva, V. F., Fleishman, E. A., \& Rieck, A. (1985). Team Dimensions: Their Identify, Their Measurement and Their Relationships. U.S. Army Research Institute for the Behavioral and Social Sciences.

Ntuen, C. A., \& Li, R. (2000). A Neural Network Model for Human Workload Simulation in Complex Human- Machine System. North Carolina Agricultural and Technical State University. Greensboro, NC. U.S. Air Force Research Laboratory, Wright Patterson AFB, OH. AFRL-HE-WP-SR-2000-0009.

O'Brien, T. G. (1985). Human Factors Engineering Test and Evaluation in the US Military. In Human Factors Society 29th Annual Meeting Proceedings (pp. 499-503).

O'Donnell, R. D., \& Eggemeier, F. T. (1994). Cognitive Processes and Performance, Ch. 42Workload Assessment Methodology. In K.R. Boff, L. Kaufman, \& J.P. Thomas (Eds.), Handbook of Perception and Human Performance. Volume II: Cognitive Processes and Performance. Harry G. Armstrong Aerospace Medical Research Laboratory, Human Engineering Division, Wright Patterson AFB, Ohio.

Olmstead, J. A., Christensen, H. E., \& Lackey, L. L. (1973). Components of Organizational Competence: Test of a Conceptual Framework. Human Resources Research Organization. Department of the Army, Office of the Chief of Research and Development, Fort Benning, GA. HumRRO Technical Report 73-19.

Olsen, L. M. (1991). The Battle of Britan, a Study in Command and Control. U.S. Army War College, Carlisle Barracks, PA 17013-5050.

Olson, G. M., \& Olson, J. S. (2000). Distance Matters. Human Computer Interaction, 15, 139178.

Olson, J. R., \& Olson, G. M. (1995). The Growth of Cognitive Modeling in Human Computer Interaction since GOMS. In R.W. Pew and P. Green (Eds.), Human Factors Engineering Short Course Notes (36 ed.). Ann Arbor, Michigan: The University of Michigan, Chrysler Center for Continuing Engineering Education. Human Computer Interaction. 1990. Vol. 5. pp. 221-265.

Orrell, D. H. (1997). Performance Assessment Methodology: Task Dependent Evaluation of Display Systems. Unpublished Dissertation, Virginia Polytechnic Institute and State 
University, Blacksburg, VA.

Palmer, C. R., \& Faloutsos, C. (1999). Density Biased Sampling: An Improved Method for Data Mining and Clustering. Carnegie-Mellon Univ Pittsburgh Pa School Of Computer Science.

Parasuraman, R., \& Riley, V. (1997). Humans and Automation: Use, Misuse, Disuse, Abuse. Human Factors, 39(2), 230-253.

Parry, S., Collins, D. D., \& VanNostrand, S. J. (1990). Complex Environment Models in Systems Integration. In H.R. Booher (Ed.), MANPRINT: An Approach to Systems Integration. New York, NY: Van Nostrand Reinhold.

Patterson, E. S., Roth, E. M., \& Woods, D. D. (2002). Predicting Vulnerabilities in Computer Supported Inferential Analysis under Data Overload. Cognition, Technology, and Work (In Press).

Pegden, C. D., Shannon, R. E., \& Sadowski, R. P. (1995). Introduction to Simulation Using Siman. (2 ed.). New York: McGraw-Hill, Inc.

Pendergrass, W. S., \& Hughes, W. P. J. (1993). Combat Analysis for Command, Control and Communications: A Primer, 1993 Edition. Naval Postgraduate School, Monterey, CA 93943-5000.

Perdu, D. M. (1988). Distributed Process Coordination in Adaptive Command and Control Teams. Unpublished Dissertation, George Mason University, Fairfax, Virginia.

Peters, J. F. (1997). An Empirical Correlation of Maslow's Hierarchy of Human Needs Levels and Team Performance. Unpublished Dissertation, The University of Alabama in Huntsville, Huntsville, Alabama.

Petty, R. (1997). Everything Is Different Now: Surviving Ethnography Research. In E. Stringer, M.F. Agnello, S.C. Baldwin, L.M. Christensen, D.L.P. Henry, K.I. Henry, T.P. Katt, P.G. Nason, V. Newman, R. Petty, \& P.S. Tinsley-Batson (Eds.), Community-Based Ethnography: Breaking Traditional Boundaries of Research, Teaching, and Learning (pp. 53-67). Mahwah, NJ: Lawrence Erlbaum Associates, Inc., Publishers.

Pew, R. W., \& Mavor, A. S. (Eds.). (1997). Representing Human Behavior in Military Simulations. Washington, D.C.: National Academy Press.

Pew, R. W., \& Mavor, A. S. (1998a). Behavior Moderators. In R.W. Pew and A.S. Mavor (Eds.), Modeling Human and Organizational Behavior. Application to Military Simulations. Washington, D.C.: National Academy Press.

Pew, R. W., \& Mavor, A. S. (1998b). Decision Making. In R.W. Pew and A.S. Mavor (Eds.), Modeling Human and Organizational Behavior. Application to Military Simulations. Washington, D.C.: National Academy Press.

Pew, R. W., \& Mavor, A. S. (1998c). Human Behavior Representation: Military Requirements and Current Models. In R.W. Pew and A.S. Mavor (Eds.), Modeling Human and Organizational Behavior. Application to Military Simulations. Washington, D.C.: National Academy Press.

Pew, R. W., \& Mavor, A. S. (1998d). Integrative Architectures for Modeling the Individual 
Combatant. In R.W. Pew and A.S. Mavor (Eds.), Modeling Human and Organizational Behavior. Application to Military Simulations. Washington, D.C.: National Academy Press.

Pew, R. W., \& Mavor, A. S. (1998e). Memory and Learning. In R.W. Pew and A.S. Mavor (Eds.), Modeling Human and Organizational Behavior. Application to Military Simulations. Washington, D.C.: National Academy Press.

Pew, R. W., \& Mavor, A. S. (1998f). Modeling of Behavior at the Unit Level. In R.W. Pew and A.S. Mavor (Eds.), Modeling Human and Organizational Behavior. Application to Military Simulations. Washington, D.C.: National Academy Press.

Pew, R. W., \& Mavor, A. S. (1998g). Planning. In R.W. Pew and A.S. Mavor (Eds.), Modeling Human and Organizational Behavior. Application to Military Simulations. Washington, D.C.: National Academy Press.

Pew, R. W., \& Mavor, A. S. (1998h). Situation Awareness. In R.W. Pew and A.S. Mavor (Eds.), Modeling Human and Organizational Behavior. Application to Military Simulations. Washington, D.C.: National Academy Press.

Pierreval, H., Tautou, L., \& Bzeznik, B. (1995). Parallel Evolutionary Algorithms for Simulation Optimization. Conference Proceedings of EUROSIM '95 Conducted in Vienna, Austria, 225-230.

Pirolli, P. (1999). Cognitive Engineering Models and Cognitive Architectures in HumanComputer Interaction. In F.T. Durso (Ed.), Handbook of Applied Cognition. New York, NY: John Wiley \& Sons, Inc.

Plott, B. (2002). Software User's Manual For: C3 TRACE (Command, Control and Communication - Techniques for Reliable Assessment of Concept Execution). U.S. Army Research Laboratory, Human Research and Engineering Directorate, Aberdeen Proving Ground, MD.

Pond, D. J., DesRochers, D. L., \& Driskell, J. E. (1986). Human Performance Task Batteries and Models: An Abilities-Based Directory. Naval Training Systems Center, Human Factors Division. Technical Report NTSC TR86-020. ADA180751.

Price, H. E., Sawyer, C. R., \& Kidd, J. S. (1983). A Review of Major Issues Relating to Human Machine Integration in the Development of Military Systems. U.S. Army Institute for the Behavioral and Social Sciences.

Prietula, M. J., Feltovich, P. J., \& Marchak, F. (2000). Factors Influencing Analysis of Complex Cognitive Tasks: A Framework and Example from Industrial Process Control. Human Factors, $\underline{42}(1), 56-74$.

Pucik, T. A., Curry, T. F., Dziuban, S. T., \& Senseny, P. E. (1999). The Use of Experimental Design in Large Scale Finite Element Simulations. Headquarters, Defense Nuclear Agency, Washington, D.C. 22310.

Rabbitt, P. M. A., \& Maylor, E. A. (1991). Investigating Models of Human Performance. British Journal of Psychology, 82(3).

Randel, J. M., Pugh, L., \& Wyman, B. G. (1996). Methods for Conducting Cognitive Task Analysis for a Decision Making Task. Navy Personnel Research and Development 
Center. NPRDC - TN-96-10.

Rasker, P. C., Post, W. M., \& Schraagen, J. M. C. (2000). Effects of Two Types of Intra-Team Feedback on Developing a Shared Mental Model in Command \& Control Teams. Ergonomics, 43(8), 1167-1189.

Reid, G. B., Potter, S. S., \& Bressler, J. R. (1986). Subjective Workload Assessment Technique (SWAT): A User's Guide. Armstrong Aerospace Medical Research Laboratory. DTIC ADA215405.

Reilly, D. L., \& Cooper, L. N. (1995). An Overview of Neural Networks: Early Models to Real World Systems. In S.F. Zornetzer and J.L. Davis (Eds.), An Introduction to Neural and Electronic Networks (2nd Ed.). Neural Networks: Foundations to Applications (pp. 229250). San Diego, CA: Academic Press, Inc; Academic Press, Inc.

Reynolds, C. (1997). Doctrinal Use of the Command and Control Vehicle (C2V) at Brigade and Below. USAARMC DFD.

Romesburg, H. C. (1984). Cluster Analysis for Researchers. Belmont, CA: Lifetime Learning Publications.

Rouse, W. B., Edwards, S. L., \& Hammer, J. M. (1992). Workload and Human Performance in Complex Systems. Wright Laboratory, Air Force Systems Command, Wright Patterson AFT, OH 45433-6553.

Rouse, W. B., Edwards, S. L., \& Hammer, J. M. (1993). Modeling the Dynamics of Mental Workload and Human Performance in Complex Systems. IEEE Transactions on Systems, Man \& Cybernetics, 23(6).

Runals, S. E. (1985). Command and Control: Does Current U.S. Army Tactical Command and Control Doctrine Meet the Requriement for Today's High Intensity Battlefield? School of Advanced Military Studies, U.S. Army Command and General Staff College, Fort Leavenworth, Kansas.

Ryan, J. L., Bailey, T. G., Moore, J. T., \& Carlton, W. B. (1998). Reactive TABU Search in Unmanned Aerial Reconnaissance Simulations. In 1998 Winter Simulation Conference ACM.

Salas, E., Dickinson, T. L., Converse, S. A., \& Tannenbaum, S. I. (1992). Toward an Understanding of Team Performance and Training. In R.W. Swezey and E. Salas (Eds.), Teams: Their Training and Performance. Norwood, NJ: Ablex Publishing Corp.

Sanders, A. F. (1997). A Summary of Resource Theories from a Behavioral Perspective. Biological Psychology, Vol. 45, Issue 1-3, pp 5-18.

Sanders, M. S., \& McCormick, E. J. (1993). Human Factors in Engineering and Design. (7 ed.). NY, New York: McGraw-Hill, Inc.

Schaafstal, A., Schraagen, J. M., \& van Berlo, M. (2000). Cognitive Task Analysis and Innovation of Training: The Case of Structured Troubleshooting. Human Factors, $\underline{42}$ (1), 75-86.

Schaafstal, A. M., \& Schraagen, J. M. C. (1992). Method for Cognitive Task Analysis. Institute for Perception Rvo-Tno, Soesterberg (Netherlands). Technisch Documentatie En 
Informatie Centrum Voor De Krijgsmacht, the Hague (Netherlands). TNO Institute for Perception. TNO Report IZF 1992 B-5. Ad-a256-550-5-xab.

Schipani, S. P., Knapp, B. G., Johnson, J., Barnette, D. B., Wojciechowski, J., Kilduff, P., Swoboda, J., Bird, S., Middlebrooks, S. E., \& Plott, B. (1998). Modeling Maneuver Battalion C2 Operations of a Force XXI Equipped Army Command Post for a Force on Force Scenario. Human Research and Engineering Division, U.S. Army Research Laboratory, Aberdeen Proving Ground, MD 21005.

Schraagen, J. M., Chipman, S. F., \& Shalin, V. L. (Eds.). (2000a). Cognitive Task Analysis. Mahwah, NJ: Lawrence Erlbaum Associates.

Schraagen, J. M., Chipman, S. F., \& Shalin, V. L. (2000b). Introduction to Cognitive Task Analysis. In J.M. Schraagen, S.F. Chipman, \& V.L. Shalin (Eds.), Cognitive Task Analysis. Mahwah, NJ: Lawrence Erlbaum Associates.

Schraagen, J. M., Chipman, S. F., \& Shute, V. J. (2000). State-of-the-Art Review of Cognitive Task Analysis Techniques. In J.M. Schraagen, S.F. Chipman, \& V.L. Shalin (Eds.), Cognitive Task Analysis. Mahwah, NJ: Lawrence Erlbaum Associates.

Schvaneveldt, R. W., Reid, G. B., Gomez, R. L., \& Rice, S. (1997). Modeling Mental Workload. Air Force Research Laboratory, Human Effectiveness Directorate, Crew system Interface Division, Wright-Patterson AFT, OH. AFRL-HE-WP-SR-2000-0010. International Journal of Cognitive Technology, 1998, (3)1, p. 19-31.

Seamster, T. L., Redding, R. E., \& Kaempf, G. L. (2000). A Skill Based Cognitive Task Analysis Framework. In J.M. Schraagen, S.F. Chipman, \& V.L. Shalin (Eds.), Cognitive Task Analysis. Mahwah, NJ: Lawrence Erlbaum Associates.

Sebok, A. (2000). Team Performance in Process Control: Influences of Interface Design and Staffing Levels. Ergonomics, $\underline{43}$ (8), 1210-1236.

Segall, R. G. (1991). A Multimedia Research Tool for Ethnographic Investigation. In I. Harel and S. Papert (Eds.), Constructionism (pp. 467-497). Stamford, CT, US: Ablex Publishing Corp.

Sejnowski, T. J. (1998). Memory and Neural Networks. In P. Fara and K. Patterson (Eds.), Memory. The Darwin College Lectures. Cambridge, MA: Cambridge University Press.

Sellen, A. J. (1995). Remote Conversations: The Effects of Mediating Talk with Technology. Human Computer Interaction, 10, 401-444.

Seven, S., Akman, A., Muckler, F., Knapp, B. G., \& Burnstein, D. (1991). Development and Application of a Military Intelligence (Mi) Job Comparison and Analysis Tool (Jcat). U.S. Army Research Institute for the Behavioral and Social Sciences.

Sheridan, T. B., \& Ferrell, W. R. (1974). Ch. 1- Man Machine Systems and Models. In T.B. Sheridan and W.R. Ferrell (Eds.), Man-Machine Systems; Information, Control, and Decision Models of Human Performance (pp. 1-20). Cambridge, MA, US: The MIT Press.

Simon, C. W. (1968). A Human Factors Study to Investigate the Response Surface Methodology. Aerospace Group, Display Systems And Human Factors Dept., Hughes Aircraft Company, Technical Report No. AFOSR-70-6. 
Simon, C. W. (1970). The Use of Central Composite Designs in Human Factors Engineering Experiments. Office of Scientific Research Research, AFSC, United States Air Force Contract to: Aerospace Group, Display Systems And Human Factors Dept., Hughes Aircraft Company, Technical Report No. AFOSR-70-6.

Simon, C. W. (1976). Response Surface Methodology Revisited: A Commentary on Research Strategy. U.S. Air Force Office of Scientific Research (AFSC). Contract to: Canyon Research Group Inc. Technical Report CWS-01-76.

Simon, C. W. (1977a). Design, Analysis, and Interpretation of Screening Studies for Human Factors Engineering Research. U.S. Air Force Office of Scientific Research (AFSC). Contract to Canyon Research Group, Inc. Technical Report No. CWS-03-77.

Simon, C. W. (1977b). New Research Paradigm for Applied Experimental Psychology: A System Approach. U.S. Air Force Office Of Scientific Research (AFSC). Contract to: Canyon Research Group, Inc. Technical Report No. CWS-04-77.

Smith, D. E., \& Mauro, C. A. (1981). The Problem of Experimental Design in Simulation. Office of Naval Research Contract No. N00014-79-C-0650, Task No. NR 274-317; Technical Report No. 113-4.

Smith, K., \& Hancock, P. A. (1995). Situation Awareness Is Adaptive, Externally Directed Consciousness. Human Factors, 37(1), 137-148.

Smith, K. A., \& Gupta, J. N. D. (2000). Neural Networks in Business: Techniques and Applications for the Operations Researcher. Computers \& Operations Research, 27, 1023-1044.

Snyder, F. M. (1993). Command and Control: The Literature and Commentaries. National Defense University, Washington, D.C.

StatSoft. (2002). Cluster Analysis. StatSoft, Inc. http://www.statsoftinc.com/textbook/stcluan.html.

Streufert, S., \& Nogami, G. (1992). Cognitive Complexity and Team Decision Making. In R.W. Swezey and E. Salas (Eds.), Teams: Their Training and Performance. Norwood, NJ: Ablex Publishing Corp.

Sutherland, J. W. (1990). Model-Base Structures to Support Adaptive Planning in Command/Control Systems. IEEE Transactions on Systems, Man \& Cybernetics, 20(1) $\underline{\text { Jan-Feb } 1990 .}$.

Sutten, C. G., \& Hervey, R. F. (1986). Command and Control at the Operational Level. U.S. Army War College, Carlisle Barracks, PA 17013-5050.

Swanson, J. T., \& Gibson, J. H. (1990). Combat Modeling for Command, Control, and Communications: A Primer. Unpublished Thesis, Naval Postgraduate School, Monterey, CA 93943-5000.

Swezey, R. W., \& Llaneras, R. E. (1997). Models in Training and Instruction. In G. Salvendy (Ed.), Handbook of Human Factors and Ergonomics (2 ed., pp. 514-560). NY, New York: John Wiley \& Sons, Inc.

Tan, G. P. (1991). Modeling a CIM System with MicroSaint. Unpublished Thesis, Virginia 
Polytechnic Institute and State University, Blacksburg, VA.

Thompson, M. M. (1998). Individual Differences in Decision Making: Establishing Cognitive Style: Normative Values for a Military Sample. Defence and Civil Institute of Environmental Medicine, Department of National Defence- Canada, Toronto, Ontario, Canada.

Thruraisingham, B., Clifton, C., Maurer, J., \& Ceruti, M. G. (2001). Real Time Data Mining of Multimedia Objects. Space and Naval Warfare Systems Center. IEEE. DTIC ADA397147.

Thurmond, P. E., \& Collins, D. D. (1988). MANPRINT Analysis Methodology: Victory through Design. Headquarters, Department of the Army, The Directorate for MANPRINT Research and Studies. ADA230494.

Tijerina, L., Kiger, S. M., Rockwell, T. H., \& Tornow, C. (1995). Workload Assessment of in$\mathrm{Cab}$ Text Message System and Cellular Phone Use by Heavy Vehicle Drivers on the Road. In Proceedings of the Human Factors and Ergonomics Society 39th Annual Meeting. Santa Monica, CA.

Tsang, P., \& Wilson, G. F. (1997). Mental Workload. In G. Salvendy (Ed.), Handbook of Human Factors and Ergonomics (2 ed., pp. 417-449). NY, New York: John Wiley \& Sons, Inc.

Tzu, S. (1910). The Art of War. In L. Giles (Ed.): Sun Tzu On The Art Of War: The Oldest Military Treatise In The World. Translated from the Chinese with Introduction and Critical Notes by Lionel Giles, M.A., Assistant in the Department of Oriental Printed Books and MSS in the British Museum. From The 2400 Year Old Work: British Museum - Project Gutenburg Electronic Text.

Uzzell, D. (2000). Ethnographic and Action Research. In G.M. Breakwell and S. Hammond (Eds.), Research Methods in Psychology (2 ed., pp. 326-337). London, England: Sage Publications Ltd.

Vandivier, P. L. (1990). Soldier Dimensions in Combat Models. Army TRADOC Analysis Command. ADA240079.

Vicente, K. J. (2000). Work Domain Analysis and Task Analysis: A Difference That Matters. In J.M. Schraagen, S.F. Chipman, \& V.L. Shalin (Eds.), Cognitive Task Analysis. Mahwah, NJ: Lawrence Erlbaum Associates.

Vidulich, M. A., \& Tsang, P. S. (1985). Assessing Subjective Workload Assessment: A Comparison of SWAT and the NASA-Bipolar Methods. In Human Factors Society 29th Annual Meeting Proceedings (pp. 71-75).

Vidulich, M. A., Ward, G. F., \& Schueren, J. (1991). Using the Subjective Workload Dominance (SWORD) Technique for Projective Workload Assessment. Human Factors, 33(6), 677691.

Walker, J. R., Reimer, J., Brown, J. D., \& Kloecker, R. L. (1984). Information Requirements for Command and Control (IRC2), Phase 1a. U.S. Army Human Engineering Laboratory, Aberdeen Proving Ground, MD 21005.

Walker, L. (1997). A Handbook for MANPRINT in Acquisition. Headquarters, Depart of the Army, Office of the Deputy Chief of Staff for Personnel, Personnel Technologies 
Directorate.

Warren, C. A., Stern, J. A., Eddy, D. R., Horst, R. L., Kramer, A. F., Parasuraman, R., Sanquist, T. F., \& Wilson, G. F. (1985). The Role of Event-Related Potentials in Human-Machine Applications. In Human Factors Society 29th Annual Meeting Proceedings (pp. 981985).

Webster. (1979). Webster's New Collegiate Dictionary. Springfield, MA: A Merriam Webster.

Weick, K. E. (1983). Managerial Thought in the Context of Action. In S. Srivastva (Ed.), The Executive Mind (pp. 221-242). San Francisco, CA: Jossey-Bass Inc, Publishers.

Weisz, J. D. (1989). Human Engineering Laboratory Support of MANPRINT. U.S. Army Human Engineering Laboratory. HEL-TN-1-89. DTIC AD-a205 830.

Whicker, M. L., \& Sigelman, L. (1991). The Art and Science of Simulation, Computer Simulation Applications: An Introduction. Applied Social Research Methods Series (Vol. 25, pp. 130-137). Newbury Park, CA: Sage Publications.

Whitaker, L. A., Oatman, L. C., \& Shank, M. D. (1987). Measuring Mental Workload: A Performance Battery. U.S. Army Human Engineering Laboratory, Aberdeen Proving Ground, MD 21005.

Wickens, C. D. (1984). The Multiple Resources Model of Human Performance: Implications for Display Design. University of Illinois/Urbana-Champaign. NTIS ADP004516.

Wickens, C. D. (1995). Ch. 9. Attention, Time Sharing and Workload. In R.W. Pew and P. Green (Eds.), Human Factors Engineering Short Course Notes (36 ed.). Ann Arbor, Michigan: The University of Michigan, Chrysler Center for Continuing Engineering Education. Engineering Psychology and Human Performance. C. Wickens. 1991. Harper-Collins.

Wickens, C. D., Sandry, D. L., \& Vidulich, M. (1983). Compatibility and Resource Competition between Modalities of Input, Central Processing, and Output. Human Factors, 25 (2), 227248.

Wierwille, W. W., \& Casali, J. G. (1983). A Validated Rating Scale for Global Mental Workload Measurement Applications. In Human Factors Society 27th Annual Meeting Proceedings (pp. 95-99).

Wierwille, W. W., Rahimi, M., \& Casali, J. G. (1986). Evaluation of 16 Measures of Mental Workload Using a Simulated Flight Task Emphasizing Mediational Activity. In R.W. Pew and P. Green (Eds.), Human Factors Engineering Short Course Notes (27 ed.). Ann Arbor, Michigan: The University of Michigan, Chrysler Center for Continuing Engineering Education. Human Factors. Vol 27(5). 1985. pp 489-502.

Wildenberg, J. T. (1987). Command and Control Architecture of the Future. U.S. Army War College, Carlisle Barracks, PA 17013-5050.

Williams, R. M., Jr. (1984). Field Observations and Surveys in Combat Zones. Social Psychology Quarterly, 47(2), 186-192.

Williges, B. H., \& Williges, R. C. (1981). User Considerations in Computer-Based Information System. Performer: Virginia Polytechnic Inst. and State Univ., Blacksburg. Computer 
Science, Industrial Engineering and Operations Research.

Williges, R. C. (1976). Research Note: Modified Orthogonal Central Composite Designs. Human Factors, 18(1), 95-98.

Williges, R. C. (1981). Development and Use of Research Methodologies for Complex System / Simulation Experimentation. In M.J. Moraal and K.F. Kraiss (Eds.), Manned System Design. New York: Plenum Press.

Williges, R. C. (1987). The Use of Models in Human Computer Interface Design. Ergonomics, 30(3), 491-502. ADA191876.

Williges, R. C. (1990). Foreword. In H.R. Booher (Ed.), MANPRINT: An Approach to Systems Integration. New York, NY: Van Nostrand Reinhold.

Williges, R. C. (2001). S.E. Middlebrooks' Class Notes, Ise5616 - Research Design II. Blacksburg, VA: Virginia Polytechnic Institute and State University.

Williges, R. C., \& Baron, M. L. (1972). Transfer Assessment Using a between Subjects Central Composite Design. In R.C. Williges and B.H. Williges (Eds.), Selected Papers on Response Surface Methodology. Savoy, Ill: University of Illinois at Urbana-Champaign Aviation Research Laboratory, Institute of Aviation for the Life Sciences Program, Air Force Office of Scientific Research. Technical Report ARL-72-20/AFOSR-72-9.

Williges, R. C., Ehrich, R. W., Williges, B. H., Hartson, H. R., \& Greenstein, J. S. (1984). Human-Computer Interactions and Decision Behavior. Performer: Virginia Polytechnic Institute and State University, Blacksburg, VA. Computer Science, Industrial Engineering and Operations Research. January 1984. 65p. Report: CSIE-83-16. NTIS, SPRINGFIELD, VA (USA), 1984, 65 pp.

Williges, R. C., Johnston, W. A., \& Briggs, G. E. (1966). Role of Verbal Communication in Teamwork. Journal of Applied Psychology, 50(6), 473-478.

Williges, R. C., \& Mills, R. G. (1972). Predictive Validity of Central Composite Design Regression Equations. In R.C. Williges and B.H. Williges (Eds.), Selected Papers on Response Surface Methodology. Savoy, Ill: University of Illinois at Urbana-Champaign Aviation Research Laboratory, Institute of Aviation for the Life Sciences Program, Air Force Office of Scientific Research. Technical Report ARL-72-20/AFOSR-72-9.

Williges, R. C., \& Simon, C. W. (1970). Response Surface Methodology Related to Problems of Target Acquisition. Life Sciences Program, Office of Scientific Research, U.S. Air Force Systems Command. Technical Report Number AFOSR-70-4.

Williges, R. C., \& Simon, C. W. (1971). Applying Response Surface Methodology to Problems of Target Acquisition. Human Factors, 13(6), 511-519.

Williges, R. C., \& Wierwille, W. W. (1979). Behavioral Measures of Aircrew Mental Workload. Human Factors, 21(5), 549-574.

Williges, R. C., \& Williges, B. H. (1989). Integrated Research Paradigm for Complex Experimentation. In Proceedings of the Human Factors and Ergonomics Society 33rd Annual Meeting (pp. 060-610) Human Factors and Ergonomics Society.

Williges, R. C., Williges, B. H., \& Han, S. H. (1992). Developing Quantitative Guidelines Using 
Integrated Data from Sequential Experiments. Human Factors, 34(4), 399-408.

Williges, R. C., Williges, B. H., \& Han, S. H. (1993). Sequential Experimentation in Human Computer Interface Design. In H.R. Hartson and D. Hix (Eds.), Advances in Human Computer Interaction (Vol. 4, pp. 1-30). Norwood, New Jersey: Ablex Publishing Corporation.

Wilson, G. F., \& Eggemier, F. T. (2001). Mental Workload Measurement. In W. Karwowski (Ed.), International Encyclopedia of Ergonomics and Human Factors (Vol. 1). New York: Taylor \& Francis.

Wilson, J. R. (1987). Future Directions in Response Surface Methodology for Simulation. In Proceedings of the 1987 Winter Simulation Conference (pp. 115-122) ACM.

Winer, B. J., Brown, D. R., \& Michels, K. M. (1991). Statistical Principles in Experimental Design. (3 ed.). New York: McGraw-Hill, Inc.

Wisner, S. (1999). The Realities of Datamining. Catalog Age, 16(1), 72-73.

Witus, G., \& Blum, R. (1992). Information Flow Management for Distributed Tactical Command Control Systems. IEEE Transactions on Systems, Man \& Cybernetics, 12(4) Jul-Aug 1982.

Wohl, J. G., Entin, E. E., \& Eterno, J. S. (1983). Modeling Human Decision Processes in Command and Control. Office of Naval Research, Department of the Navy, Arlington, Virginia 22217.

Woods, D. D., Patterson, E. S., \& Roth, E. M. (2002). Can We Ever Escape from Data Overload? A Cognitive Systems Diagnosis. Cognition, Technology, and Work (In Press).

Woolf, H. B. (Ed.). (1979). Webster's New Collegiate Dictionary. Springfield, MA: A Merriam Webster.

Xie, B. (1997). A Methodology for Modeling and Predicting Mental Workload in Single and Multi-Task Environments. Unpublished Dissertation, Purdue University.

Young, M. S., \& Stanton, N. A. (2001). Mental Workload: Theory, Measurement and Application. In W. Karwowski (Ed.), International Encyclopedia of Ergonomics and Human Factors (Vol. 1). New York: Taylor \& Francis.

Zachary, W. W., Ryder, J. M., \& Hicinbothom, J. H. (1998). Cognitive Task Analysis and Modeling of Decision Making in Complex Environments. In J.A. Cannon-Bowers and E. Salas (Eds.), Making Decisions under Stress: Implications for Individual and Team Training. Mahwah, NJ: Lawrence Erlbaum Associates.

Zaki, M. J., Parthasarathy, S., Li, W., \& Ogihara, M. (1996). Evaluation of Sampling for Data Mining of Association Rules. Rochester Univ Ny Dept Of Computer Science.

Zimm, A. D. (1998). Modeling Maneuver Warfare: Human Factors, Decisionmaking, and Attrition Calculations. Johns Hopkins University, Applied Physics Laboratory. Unpublished Manuscript.

Zubal, O., \& Steinberg, S. (1989). Information Requirements for Command and Control (IRC2), Phase 1b. U.S. Army Human Engineering Laboratory, Aberdeen Proving Ground, MD 
and Industrieanlagen-Betreibsgesellschaft (IABG). 
This page left intentionally blank. 
Appendices 
This page left intentionally blank. 
Appendix A - TOC Data Collection Spreadsheet.

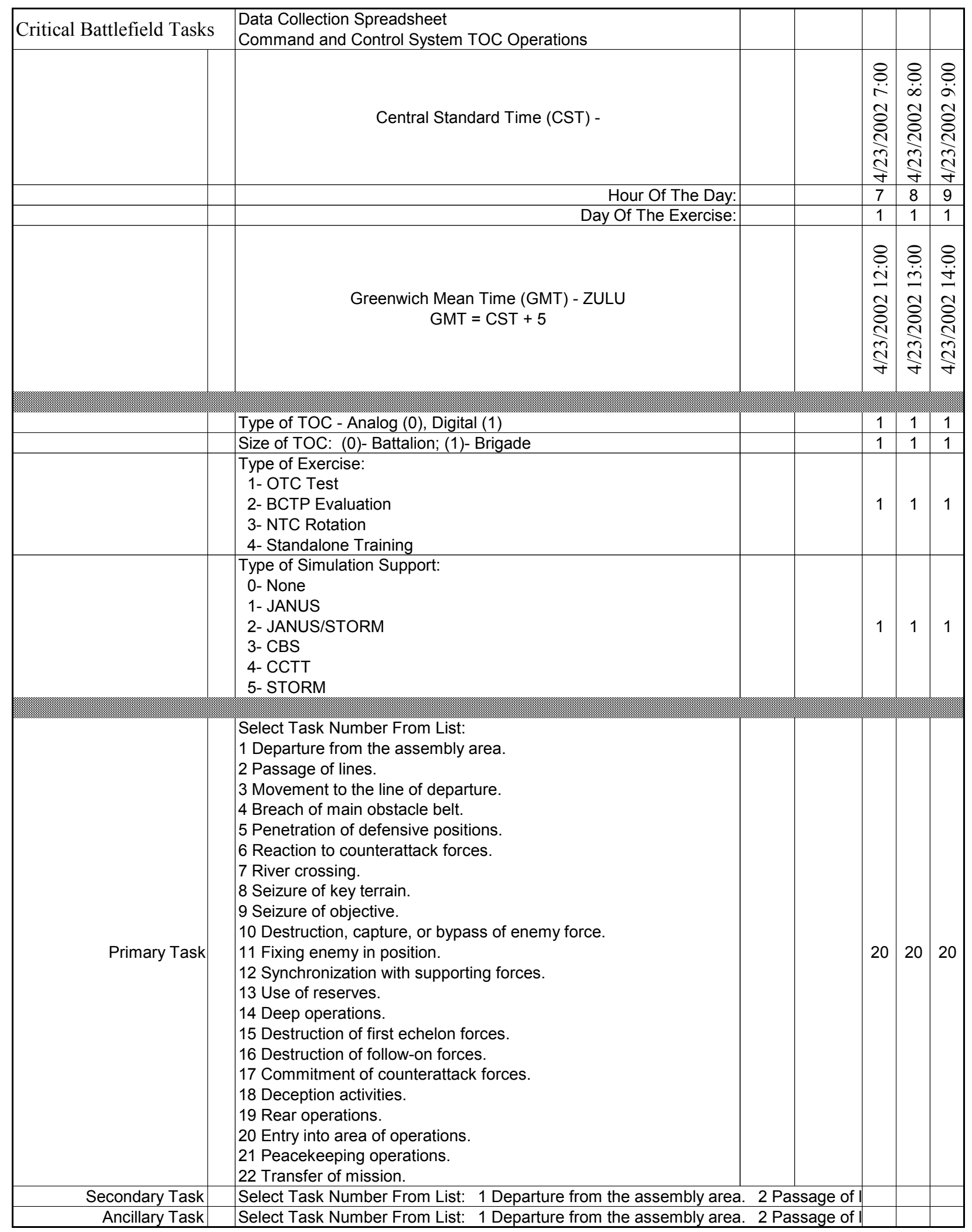




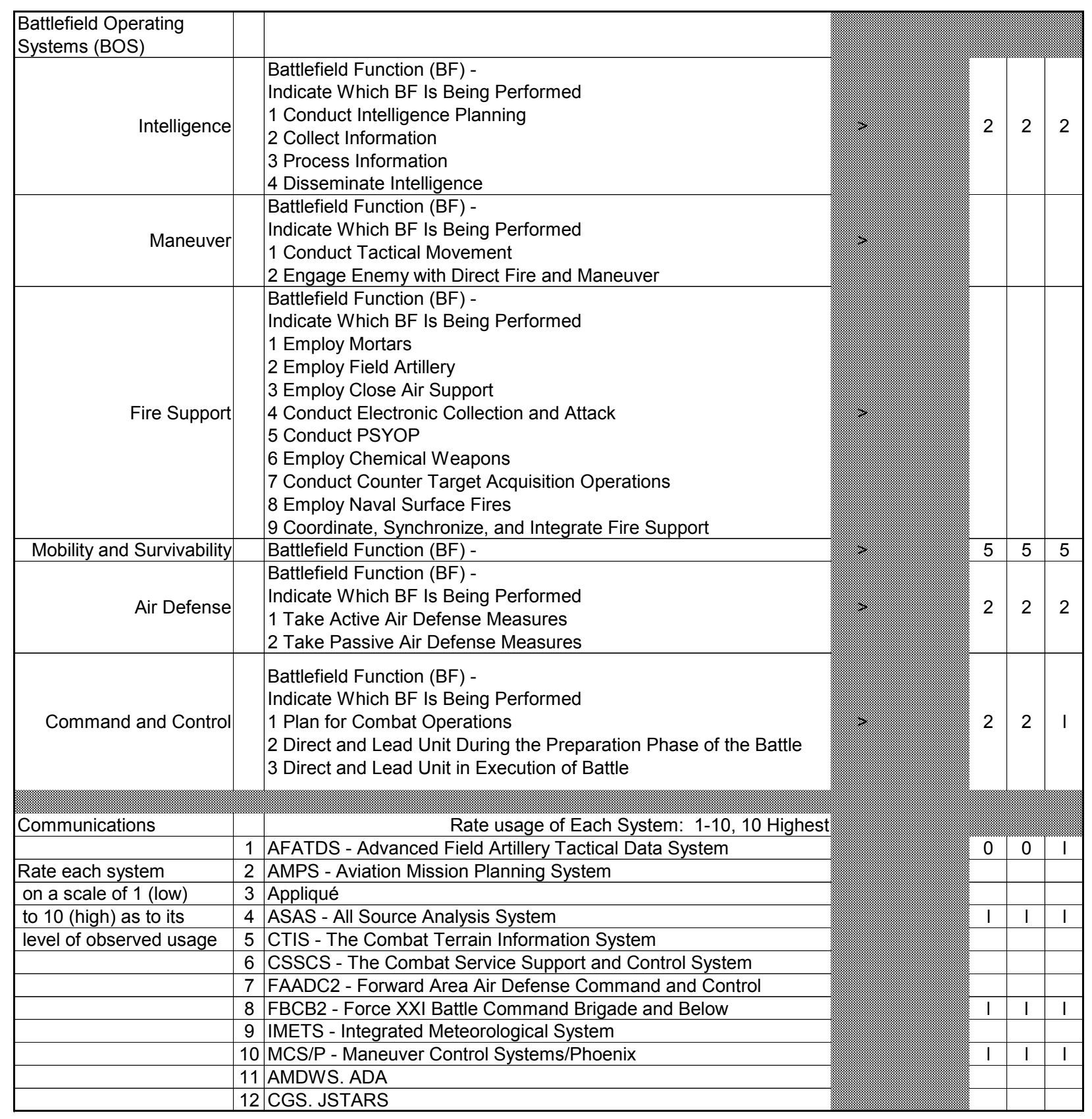




\begin{tabular}{|c|c|c|c|c|}
\hline \multirow{2}{*}{\multicolumn{5}{|c|}{ Rate usage of Each System: $1-10,10$ Highest }} \\
\hline & & & & \\
\hline & 1 TUAV - Tactical Unmanned Aerial Vehicle & 3 & 3 & 3 \\
\hline & 2 JSTARS - Joint \& Strategic Tactical Airborne Radar System & I & I & I \\
\hline & 3 GSR - Ground Surveillance Radar & & & \\
\hline \multicolumn{5}{|l|}{ Other Factors } \\
\hline \multicolumn{5}{|c|}{ Rate usage of Each System: $1-10,10$ Highest } \\
\hline & \begin{tabular}{l|l|}
1 & Battle Timing \\
\end{tabular} & & & \\
\hline & 2 Battle Tempo & I & I & I \\
\hline & \begin{tabular}{l|l}
3 & Reconnaissance Operations
\end{tabular} & 2 & 2 & \\
\hline & 4 Information Operations & 2 & 2 & I \\
\hline & \begin{tabular}{l|l}
5 & Tactics \\
\end{tabular} & & & \\
\hline & \begin{tabular}{l|l}
6 & Observed Activity / Stress Level in TOC
\end{tabular} & I & I & I \\
\hline \multicolumn{5}{|c|}{ g t } \\
\hline \multicolumn{5}{|c|}{ Indicate Present (I) or Absent (0) in TOC - } \\
\hline & Commanding Officer - Col. Campbell & 0 & 0 & 0 \\
\hline & Executive Officer & 0 & 0 & 0 \\
\hline & Battle Captain & I & I & I \\
\hline & S3 - Major Winkle & 0 & 0 & 0 \\
\hline & S3 RTO & & & \\
\hline & S2 - Cpt. Boone / Cpt. Briggman & I & I & 0 \\
\hline & FSO - Cpt Ikena & I & I & I \\
\hline & Engineer & I & I & I \\
\hline & ALO & 1 & 1 & 1 \\
\hline \multicolumn{5}{|l|}{ Environmental Conditions } \\
\hline \multirow[t]{8}{*}{ Record Actual Readings } & Sky Condition: Clear-1; Overcast-2; Rain-3 & I & I & I \\
\hline & Wind Condition: 0 - none; 1 - low; 2- moderate; 3- high & 0 & 0 & 0 \\
\hline & Light Level, Foot Lamberts - Background at $20 \mathrm{ft}$. & 2 & 2 & 2 \\
\hline & Light Level, Foot Lamberts - Map Display (Rear Projection) at $20 \mathrm{ft}$. & 20 & 20 & 10 \\
\hline & Noise, $\mathrm{dbA}$ & 66 & 66 & 66 \\
\hline & Dry Bulb Temperature, degrees $\mathrm{F}$ & 74 & 74 & 77 \\
\hline & Wet Bulb Temperature, degrees $\mathrm{F}$ & 70 & 70 & 71 \\
\hline & Relative Humidity, \% & 82 & 82 & 75 \\
\hline
\end{tabular}


Appendix B - Glossary Of U.S. Army

ABF

Acronyms.

(D)

(M)

(T)

"Heart Beat"

1BCT

1BCT/4ID

$1 \mathrm{LT}$

$1 \mathrm{SG}$

2S1

2S6

96 hours

A/DACG

$\mathrm{A} / \mathrm{L}$

A/SPOD

A2

$\mathrm{A} 2 \mathrm{C} 2$

$\mathrm{A} 2 \mathrm{C} 2 \mathrm{~S}$

AA

AA

AAC

AAC

AADC

AAE

AAMMP

AAO

AAR

AASA

AASLT

AAV

AAVs

AAW

AAW

AB2

$\mathrm{Abb}$

ABCS

$\mathrm{ABE}$
Draft

(Mechanized)

Towed

Futuristic Concept Of

Monitoring The Body Vitals Of

A Soldier.

1st Brigade Combat Team

1st Brigade Combat Team (4th

Infantry Division, Ft. Hood, TX)

1st Lieutenant

First Sergeant

Russian Built Self -Propelled

Light Artillery

Russian Built Self-Propelled

Medium Artillery

After 96 Hours, The FCS Must

Be Fully Deployed.

Arrival/Departure Airtleld

Control Group

Administrative And Logistics

Aerial/Sea Ports Of Debarkation

Antiarmor

Army Airspace Command And

Control

Army Aviation Command And

Control System

Assembly Areas

Active Army

Anti-Aircraft

Army Acquisition Corps

Area Air Defense Commander

Army Acquisition Executive

Active Army Military Manpower

Program

Army Acquisition Objective

After-Action Review

Administrative Assistant To The

Secretary Of The Army

Air Assault

Army Aviation

Autonomous Air Vehicle (See

Also FAAV)

(1) Antiair Warfare; (2) Army

Acquisition Workforce

Analysis And Wargaming

Army Brigade And Below

Abbreviated, In This Use, A

Report With Only Minimal

Information

Army Battle Command System

Assistant Brigade Engineer
ABGD

ABMS

$\mathrm{ABN}$

$\mathrm{AC}$

$\mathrm{AC}$

ACA

ACA

ACAT

$\mathrm{ACC}$

$\mathrm{ACC}$

$\mathrm{ACC}$

$\mathrm{ACE}$

ACE

ACMR

$\mathrm{ACO}$

ACR

ACR

ACSIM

ACT

ACT II

ACTD

ACTS

ACTY

ACU

ACUS

$\mathrm{AD}$

$\mathrm{AD}$

ADA

ADADO

ADAM

ADAM/RAAM

$\mathrm{ADC}$

ADCOORD

ADCSOPS

ADDS

ADE

ADE

ADHPM

ADJ

ADM

ADM

ADMIN

ADMS
Attack By Fire

Air Base Ground Defense

Assault Breach Marking System

Airborne

Active Component

Aircraft

Airspace Control Authority

Air Coordination Area

Acquisition Category

Air Component Commander

Army Commanders' Conference

Army Component Commander

Analysis Control Element;

Advanced Collaborative

Environment

Air Control Measure Request

Airspace Control Order

Armored Cavalry Regiment

Armored Cavalry Regiment

Assistant Chief Of Staff For

Installation Management

Analysis Control Team

Advanced Concepts \&

Technology Program II

Advanced Concept Technology

Demonstration

Army Criteria Tracking System

Activity

Area Common User

Army Common User System

Air Defense

Air Defense Or Armored

Division (Depending On Usage)

Air Defense Artillery

Assistant Division Air Defense

Officer

Air Defense Air Management

Aerial Denial Artillery

Munition/Remote Antiarmor

Minefield

Area Damage Control

Air Defense Coordinator

Assistant DCSOPS

Automatic Data Distribution

System

Air Defense Element

Assistant Division Engineer

Artillery-Delivered, High-

Precision Munitions

Adjustment

Analytical Decision-Making

Model

Acquisition Decision

Memorandum

Administration

Area Denial Munition System 


\begin{tabular}{|c|c|c|c|}
\hline ADOCS & $\begin{array}{l}\text { Automated Deep Operations } \\
\text { Coordination System }\end{array}$ & AMEDDC\&S & $\begin{array}{l}\text { Army Medical Department } \\
\text { Center And School }\end{array}$ \\
\hline ADT & Active Duty For Training & AMIM & Army Modernization Information \\
\hline \multirow[t]{2}{*}{ ADWCS } & Air Defense Weapons Control & & Memorandum \\
\hline & Status & AMO & Aviation Medical Officer \\
\hline $\mathrm{AE}$ & Aerial Exploitation & AMOPES & Army Mobilization And \\
\hline $\mathrm{AF}$ & US Air Force & & Operations Planning And \\
\hline AFAS & Advanced Field Artillery System & & Execution System \\
\hline AFATDS & $\begin{array}{l}\text { Advanced Field Artillery } \\
\text { Tactical Data System }\end{array}$ & AMOPES & $\begin{array}{l}\text { Army Mob. And Ops Planning } \\
\text { And Execution System }\end{array}$ \\
\hline $\mathrm{AFC}$ & Army Fiscally Constrained & AMOPES & Army Mobilization And \\
\hline $\mathrm{AFCF}$ & Army Fiscally Constrained Force & & Operations Planning And \\
\hline AFCS & Automatic Fire Control System & & Execution System \\
\hline AFH & Army Family Housing & AMP & Army Modernization Plan; Army \\
\hline AFHO & Family Housing, Army & & Mobilization Plan \\
\hline AFPDA & $\begin{array}{l}\text { (Operations) } \\
\text { Army Force Planning Data And } \\
\text { Assumptions }\end{array}$ & $\begin{array}{l}\text { AMPMOD } \\
\text { AMRD }\end{array}$ & $\begin{array}{l}\text { Army Materiel Plan Modified } \\
\text { Army Modernization Reference } \\
\text { Data }\end{array}$ \\
\hline AFSE & Automated Fire Support Element & AMSAA & Army Materiel Systems Analysis \\
\hline AFV & Airborne Fighting Vehicle & & Agency \\
\hline AG & Adjutant General & AMSCO & Army Management Structure \\
\hline \multirow[t]{2}{*}{ AGCCS } & Army Global Command And & & Code \\
\hline & Control System & AMT & Army Modernization Training \\
\hline AGL & Above Ground Level & ANAL & Analysis \\
\hline AGMB & Advance Guard Main Body & ANGLICO & Air And Naval Gunfire Liaison \\
\hline AGR & Active Guard Reserve & & Company \\
\hline AGS & Armored Gun System & $\mathrm{AO}$ & Area Of Operations \\
\hline $\mathrm{AH}$ & Attack Helicopter & Ao & Operational Availability \\
\hline AH-64 & Apache Attack Helicopter (US) & $\mathrm{AOC}$ & Air Operations Center \\
\hline AI & Air Interdiction & AOLCM & Army Organizational Life Cycle \\
\hline AI & Additional Issue & & Model \\
\hline \multirow[t]{2}{*}{ AID } & Agency For International & AOP & Army Order Of Precedence \\
\hline & Development & AOR & Area Of Responsibility \\
\hline AIN & Army Interoperability Network & AOS & Accoustic Overwatching Sensor \\
\hline AIS & Automated Information System & APADS & Advanced Precision Airborne \\
\hline \multirow[t]{2}{*}{ AIT } & Automatic Identification & & Delivery System \\
\hline & Technology & APB & Acquisition Program Baseline \\
\hline AIT & Advanced Individual Training & APC & Armored Personnel Carriers \\
\hline \multirow[t]{2}{*}{ ALACV } & Advanced Light-Armament For & APERS & Antipersonnel \\
\hline & Combat Vehicles & APG & Army Program Guidance \\
\hline ALBE & Airland Battlefield Environment & APGM & Army Program Guidance \\
\hline ALBM & Airland Battle Management & & Memorandum \\
\hline ALCC & Airlift Control Center & APICM & Armor-Piercing Improved \\
\hline ALO & Air Liaison Officer & & Conventional Munition \\
\hline \multirow[t]{2}{*}{ ALO } & Authorized Level Of & APKWS & 2.75 Rocket \\
\hline & Organization & APOBS & Antipersonnel Obstacle \\
\hline ALOC & Air Line Of Communications & & Breaching System \\
\hline ALOC & $\begin{array}{l}\text { Administrative \& Logisitics } \\
\text { Operations Center }\end{array}$ & APPG & $\begin{array}{l}\text { Army Preliminary Programming } \\
\text { Guidance }\end{array}$ \\
\hline ALRPG & $\begin{array}{l}\text { Army Long Range Planning } \\
\text { Guidance }\end{array}$ & APPIS & $\begin{array}{l}\text { Army POM Preparation } \\
\text { Instructions Supplement }\end{array}$ \\
\hline AMC & U.S. Army Materiel Command & Apr & April \\
\hline \multirow[t]{2}{*}{ AMC } & United States Army Materiel & APR & Army Procurement Requirement \\
\hline & Command & APS & Active Protection System; \\
\hline AMEDD & Army Medical Department & & Advanced Photographic System \\
\hline
\end{tabular}


APS

APSES

APSOP

APSS

APU

AQF

AR

ARB

ARBSG

ARCOM

ARCOM

ARDEC

ARES

ARFOR

ARFPC

ARI

ARL

ARLO

ARM

ARM

ARMT

ARNG

ARNG-TSP

ARNGUS

ARO

AROC

ARPA

ARPERCEN

ARPRINT

ARSEC

ARSTAF

ARSTRUC

ART

ARTEP

ARTY

AS

ASA

$\mathrm{ASA}(\mathrm{CW})$
Army Planning System; Army

Prepositioned Stocks

Army Prepositioned Stocks

Equipment Sets

Army Prepositioned Stocks

Operational Project

Army Prepositioned Stocks

Sustainment

Auxiliary Power Unit

Advanced Quick Fix

Army Regulation

Army Resources Board

Army Resources Board Support

Group

Army Command

United States Army Reserve

Command

Armament Research,

Development, \& Engineering

Center

Advanced Robotic Engagement

System

Army Force

Army Reserve Forces Policy

Committee

Army Research Institute

(1) Army Research Laboratory;

(2) Airborne Reconnaisance Low

Air Reconnaissance Liaison

Officer

Antiradiation Missile

Anti-Radiation Missile

Armament

Army National Guard

Army National Guard-Troop

Structure Program

Army National Guard Of The

United States

Army Research Office

Army Requirements Oversight

Council

Advanced Research Projects

Agency

Army Reserve Personnel Center

Army Program For Individual

Training

Army Secretariat

Army Staff

Army Structure Message

Army Reserve Technician

Army Training And Evaluation

Program

Artillery

Acquisition Strategy

Assistant Secretary Of The Army

ASA (Civil Works)
ASA(FM\&C) ASA (Financial Management \&

Comptroller)

ASA(IL\&E) ASA (Installations, Logistics,

And Environment)

ASA(M\&RA) ASA (Manpower And Reserve Affairs)

ASA(RDA) ASA (Research, Development, And Acquisition)

ASAP

ASARC

ASARC

ASAS

ASB

ASCC

ASCC

ASD

$\operatorname{ASD}(\mathrm{C} 3 \mathrm{I})$

ASD(FMP)

$\operatorname{ASD}(\mathrm{HA})$

ASD(RA)

$\mathrm{ASD}(\mathrm{S} \& \mathrm{R})$

ASI

ASIOE

ASIOEP

ASIP

ASL

ASLT

ASM

ASOC

ASPS

ASST

AST

ASTAG

A-STAMIDS

ASTMIS

ASTMP

AT

AT

AT-5
As Soon As Possible

Assistant Sectary Of The Army

Review Committee

Army Systems Acquisition

Review Council

All-Source Analysis System

Army Science Board

Army Service Component

Command

Army Service Component

Commander

Assistant Secretary Of Defense

ASD (Command, Control,

Communications, And

Intelligence)

ASD For Force Management

Policy

ASD For Health Affairs

ASD (Reserve Affairs)

ASD For Strategy And

Requirements

Additional Skill Identifier

Associated Support Items Of

Equipment

Associated Support Items Of

Equipment And Personnel

Army Stationing And Installation

Plan

Authorized Stockage List

Assault

Armored Systems Modernization

Air Support Operations Center

All-Source Production System

Advanced Sensor/Submunition

Technology

ATEC System Team

Army Science And Techonology

Advisory Group

Airborne Standoff Mine-

Detection System

Army Science And Techonology

Management Information System

Army Science And Technology

Master Plan

Antitank

Annual Training

Russian-Built Anti-Tank Guided Missile 


\begin{tabular}{|c|c|c|c|}
\hline ATACMS & Army Tactical Missile Systems & AWE & Advanced Warfighting \\
\hline ATACMS & Army Tactical Missile System & & Experiment \\
\hline ATAS & Air-To-Air Stinger & AWIS & Army Wwmces Information \\
\hline ATC & Army Training Center & & System \\
\hline ATC & Ammunition Team Chief & AWP & Annual Work Plan \\
\hline ATC & Army Training Center & AWR & Army War Reserve \\
\hline ATCAS & $\begin{array}{l}\text { Advanced Towed Cannon } \\
\text { System }\end{array}$ & AWRSA & $\begin{array}{l}\text { Army War Reserve Stocks For } \\
\text { Allies }\end{array}$ \\
\hline ATCCS & $\begin{array}{l}\text { Army Tactical Command And } \\
\text { Control System }\end{array}$ & AWRSI & $\begin{array}{l}\text { Army War Reserve Secondary } \\
\text { Items }\end{array}$ \\
\hline ATD & $\begin{array}{l}\text { Advanced Technology } \\
\text { Demonstration }\end{array}$ & AWWG & $\begin{array}{l}\text { Advanced Warfighting Working } \\
\text { Group }\end{array}$ \\
\hline $\mathrm{ATD} / \mathrm{C}$ & $\begin{array}{l}\text { Aided Target } \\
\text { Detection/Classification }\end{array}$ & $\mathrm{B} 2 \mathrm{C} 2$ & $\begin{array}{l}\text { Battalion And Below Command } \\
\text { And Control }\end{array}$ \\
\hline ATEC & $\begin{array}{l}\text { United States Army Test And } \\
\text { Evaluation Command }\end{array}$ & BAAV & $\begin{array}{l}\text { Brigade/Battalion Autonomous } \\
\text { Air Vehicle }\end{array}$ \\
\hline ATEC & $\begin{array}{l}\text { Army Test And Evaluation } \\
\text { Command }\end{array}$ & Backward Capability & $\begin{array}{l}\text { ty How Will This System } \\
\text { (New Technology) Be Used By }\end{array}$ \\
\hline ATGM & Anti-Tank Guided Missile & & Legacy Units \\
\hline ATK & Attack & BAE 155 & Name Of The South African \\
\hline ATK & Attack Position Or Attack & & Artillery Piece FCS Is Reviewing \\
\hline ATLS & Advanced Trauma Life Support & & For NLOS. \\
\hline ATMCT & $\begin{array}{l}\text { Air Terminal Movement Control } \\
\text { Team }\end{array}$ & $\begin{array}{l}\text { BAS } \\
\text { BAT }\end{array}$ & $\begin{array}{l}\text { Battlespace Awareness Module } \\
\text { Brilliant Anti-Tank }\end{array}$ \\
\hline ATMDE & $\begin{array}{l}\text { Army Theater Missile Defense } \\
\text { Element }\end{array}$ & $\begin{array}{l}\mathrm{BC} \\
\mathrm{BC}\end{array}$ & $\begin{array}{l}\text { Bradley Commander } \\
\text { Battle Captain (Officer In Charge }\end{array}$ \\
\hline ATO & Air Tasking Order & & Of A Command Post) \\
\hline ATP & $\begin{array}{l}\text { Allied Tactical Publication; } \\
\text { Ammunition Transfer Point }\end{array}$ & $\begin{array}{l}\mathrm{BC} \\
\mathrm{BCC}\end{array}$ & $\begin{array}{l}\text { Battle Command } \\
\text { Battlefield Circulation Control }\end{array}$ \\
\hline ATRRS & $\begin{array}{l}\text { Army Training Requirements } \\
\text { And Resources System }\end{array}$ & $\mathrm{BCD}$ & $\begin{array}{l}\text { Battle Coordination Detachment } \\
\text { (Formerly Called BCE, See }\end{array}$ \\
\hline ATSA & ATEC Threat Support Activity & & Above) \\
\hline ATTN & Attention & $\mathrm{BCE}$ & Battlefield Coordination Element \\
\hline $\begin{array}{l}\text { AUEL } \\
\text { AUG }\end{array}$ & $\begin{array}{l}\text { Automated Unit Equipment List } \\
\text { August }\end{array}$ & BCIS & $\begin{array}{l}\text { Battlefield Combat Identification } \\
\text { System }\end{array}$ \\
\hline AUGTDA & Augmentation TDA & BCIS & Battlefield Combat Identification \\
\hline AURS & Automated Unit Reference Sheet & & System \\
\hline AUTH & Authorization & BCOTM & Battle Command On The Move \\
\hline AUTMV & Automotive & $\mathrm{BCT}$ & Brigade Combat Team \\
\hline AUTO & Automation & ВСТР & Battle Command Training \\
\hline AUTS & $\begin{array}{l}\text { Automatic Update Transaction } \\
\text { System }\end{array}$ & $\mathrm{BCV}$ & $\begin{array}{l}\text { Program } \\
\text { Bradley Command Vehicle }\end{array}$ \\
\hline AV & Avenger & $\mathrm{BCV}$ & Battle Command Vehicle \\
\hline AV & Air Vehicle & BD & Battlefield Distribution \\
\hline AVCSA & $\begin{array}{l}\text { Assistant Vice Chief Of Staff, } \\
\text { United States Army }\end{array}$ & BDA & $\begin{array}{l}\text { Battle Damage Assessment / } \\
\text { Bomb Damage Assessment }\end{array}$ \\
\hline AVIM & $\begin{array}{l}\text { Aviation Intermediate } \\
\text { Maintenance }\end{array}$ & $\begin{array}{l}\text { BDCST } \\
\text { BDE }\end{array}$ & $\begin{array}{l}\text { Broadcast } \\
\text { Brigade }\end{array}$ \\
\hline AVLB & $\begin{array}{l}\text { Armored Vehicle Launched } \\
\text { Bridge }\end{array}$ & $\begin{array}{l}\mathrm{BDO} \\
\mathrm{BDP}\end{array}$ & $\begin{array}{l}\text { Battle Dress Overgarment } \\
\text { Battlefield Development Plan }\end{array}$ \\
\hline AVN & Aviation & BDU & Battle Dress Uniform \\
\hline AVO & Air Vehicle Operator & $\mathrm{BE}$ & Budget Estimate \\
\hline AVUM & Aviation Unit Maintenance & BES & Budget Estimate Supplement \\
\hline AWACS & Airborne Warning And Control & BES & Budget Estimates Submission \\
\hline AWCF & $\begin{array}{l}\text { System } \\
\text { Army Working Capital Fund }\end{array}$ & BEWSS & $\begin{array}{l}\text { Battlefield Environment Weapon } \\
\text { System Simulation }\end{array}$ \\
\hline
\end{tabular}




\begin{tabular}{|c|c|c|c|}
\hline BFA & Battlefield Functional Area & BY & Budget Year \\
\hline BFIST & Bradley Fire Support Team & $\mathrm{C} 2$ & Command And Control \\
\hline BFIST-V & $\begin{array}{l}\text { Bradley Fire Support Team } \\
\text { Vehicle }\end{array}$ & $\mathrm{C} 21$ & $\begin{array}{l}\text { Command, Control, And } \\
\text { Intelligence }\end{array}$ \\
\hline BFM & Battle-Scale Forecast Model & $\mathrm{C} 2 \mathrm{~V}$ & Command And Control Vehicle \\
\hline BFV & Bradley Fighting Vehicle & $\mathrm{C} 2 \mathrm{~W}$ & Command And Control Warfare \\
\hline BHL & Battle Handover Line & $\mathrm{C} 3$ & Command, Control, And \\
\hline \multirow[t]{2}{*}{ BIDS } & Biological Integrated Detection & & Communications \\
\hline & System & $\mathrm{C} 31$ & Command, Control, \\
\hline BII & Basic Issue Items & & Communications, And \\
\hline BIM & Battlefield Information Module & & Intelligence \\
\hline BIOL & Biological & C3OTM & Command, Control, And \\
\hline BIP & Battlefield Imaging Projectile & & Communications On The Move \\
\hline BIT & Built In Test & $\mathrm{C} 4$ & Command, Control, \\
\hline BITE & Built-In Test Equipment & & Communications, And \\
\hline BL & Battle Lab & & Computers \\
\hline \multirow[t]{4}{*}{ BLK I, BLK II } & Various Fiscal Year (FY) Dates & $\mathrm{C} 4 \mathrm{I}$ & Command, Control, \\
\hline & That Indicate When A Product & & Communications, Computers, \\
\hline & Should Be Ready (For Example: & & And Intelligence \\
\hline & $\begin{array}{l}\text { BLK } 12010 \text { - TRL6 By } 03 \text { BLK } \\
\text { 1) }\end{array}$ & C4ISR & $\begin{array}{l}\text { Command, Control, } \\
\text { Communications, Computers, }\end{array}$ \\
\hline BLOS & Beyond Line Of Sight & & Intelligence, Surveillance, And \\
\hline \multirow[t]{2}{*}{ BLRSI } & Battle Lab Reconfigurable & & Reconnaissance \\
\hline & Simulator Initiative & CA & Civil Affairs; Combined Arms \\
\hline BLSE & Battle Lab Support Element & $\mathrm{CA}$ & Civil Affairs \\
\hline BLT & Battalion Landing Team & CAA & Concepts Analysis Agency \\
\hline BLUFOR & Blue Force & CAAM & Computer-Assisted Artillery \\
\hline \multirow[t]{2}{*}{ BLWE } & Battle Lab Warfighting & & Meteorological \\
\hline & Experiment & CAAV & Company Autonomous Air \\
\hline \multirow[t]{2}{*}{ BMDO } & Ballistic Missile Defense & & Vehicle \\
\hline & Organization & $\mathrm{CAB}$ & Combat Aviation Brigade \\
\hline BMO & Battalion Maintenance Officer & CAD & Course Administrative Data \\
\hline $\mathrm{BN}$ & Battalion & CAE & Component Acquisition \\
\hline \multirow[t]{3}{*}{ BOEING } & Designated Authors Of The & & Executive \\
\hline & System Of Systems Architecture & CAL & Caliber \\
\hline & (SOSA) & CAOC & Combined Air Operations Center \\
\hline BOIP & Basis-Of-Issue Plan & CAP & Combat Air Patrol; Crisis-Action \\
\hline BOS & Battlefield Operating Systems & & Planning \\
\hline BP & Battle Position & CAPCES & Construction Appropriation, \\
\hline BR & Branch; British & & Programming, Control, And \\
\hline BRAC & Base Realignment And Closure & & Execution System \\
\hline BRAG & Brigade Artillery Group & CAPS & Counteractive Protection System \\
\hline \multirow[t]{3}{*}{ BRDM } & Russian-Built Four-Wheeled & CAR & Chief, Army Reserve \\
\hline & Armored Car, Used For Recon & CARD & Concept Analysis And \\
\hline & And Anti-Tank Rocket Launcher & & Requirements Determination \\
\hline BRT & Brigade Reconnaissance Troop & CAS & Close Air Support \\
\hline BSA & Brigade Support Area & CASTFOREM & Combined Arms And Support \\
\hline BSC & Battle Simulation Center & & Task Force Evaluation Model \\
\hline \multirow[t]{2}{*}{ BSFV-E } & Bradley Stinger Fighting & CAT & Catalog \\
\hline & Vehicle-Enhanced & CATS & Combined Arms Training \\
\hline BT & Basic Training & & Strategy \\
\hline BTOE & Base TOE & САTТВ & Combined Arms Tank Test Bed \\
\hline \multirow[t]{2}{*}{ BTR } & Russian Built Eight-Wheeled & CAV & Cavalry \\
\hline & Armored Personnel Carrier & $\mathrm{CBE}$ & Command Budget Estimate \\
\hline Btry & Battery (Basic Artillery Unit) & $\mathrm{CBO}$ & Congressional Budget Office \\
\hline BV-ACTD & Battlefield Visualization ACTD & & \\
\hline
\end{tabular}




\begin{tabular}{|c|c|c|c|}
\hline CBR & $\begin{array}{l}\text { Chemical, Biological And } \\
\text { Radiological }\end{array}$ & $\begin{array}{l}\mathrm{CH} ; \mathrm{CHAP} \\
\text { CHS }\end{array}$ & $\begin{array}{l}\text { Cargo Helicopter Chaplain } \\
\text { Combat Health Support }\end{array}$ \\
\hline \multirow[t]{2}{*}{ CBRS } & Combat-Based Requirements & $\mathrm{CI}$ & Counterintelligence \\
\hline & System & CIA & Central Intelligence Agency \\
\hline CBS & Corps Battle Simulation & $\mathrm{CIC}$ & Combat Information Center \\
\hline CBS-X & $\begin{array}{l}\text { Continuing Balance System- } \\
\text { Expanded }\end{array}$ & $\begin{array}{l}\text { CID } \\
\text { CINC }\end{array}$ & $\begin{array}{l}\text { Combat Intelligence Division } \\
\text { Commander-In-Chief }\end{array}$ \\
\hline CBT & Combat & CINC's & Commanders - In - Chief \\
\hline CBTDEV & Combat Developer; Combat & CIR & Critical Information Requirement \\
\hline CBU & Cluster Bomb Unit & CITV & Commander's Independent \\
\hline $\mathrm{CC}$ & Closed Caption & & Thermal Viewer \\
\hline $\mathrm{CCD}$ & Camouflage, Concealment, And & $\begin{array}{l}\text { CJCS } \\
\text { CICS }\end{array}$ & Chairman, Joint Chiefs Of Staff \\
\hline $\mathrm{CCH}$ & Close Combat Heavy & & Staff \\
\hline $\mathrm{CCH}$ & Chief Of Chaplains & CJCSI & Chairman Of The Joint Chiefs Of \\
\hline \multirow[t]{2}{*}{ CCIR } & Commander's Critical & & Staff Instruction \\
\hline & Information Requirements & CJTF & Commander, Joint Task Force \\
\hline CCSS & $\begin{array}{l}\text { Commodity Command Standard } \\
\text { System }\end{array}$ & CJTFEX & $\begin{array}{l}\text { Combined Joint Task Force } \\
\text { Exercise }\end{array}$ \\
\hline ССТT & Close Combat Tactical Trainer & C-KEM & Compact Kinetic Energy Missile \\
\hline $\mathrm{CD}$ & Cavalry Division & CLL & Chief Of Legislative Liaison \\
\hline CDR & Commander & CLS & Contractor Logistics Support \\
\hline CDS & Container Delivery System & $\mathrm{CM}$ & Command Manager \\
\hline CDS & $\begin{array}{l}\text { Congressional Descriptive } \\
\text { Summaries }\end{array}$ & $\mathrm{CM}(\mathrm{FS})$ & $\begin{array}{l}\text { Command Manager (Force } \\
\text { Structure) }\end{array}$ \\
\hline $\mathrm{CE}$ & Command Element & $\mathrm{CM}(\mathrm{PBG})$ & Command Manager (Program \\
\hline $\mathrm{CE}$ & Chemical Energy & & Budget Guidance) \\
\hline \multirow[t]{2}{*}{ CECOM } & Communications \& Electronics & $\mathrm{CMC}$ & Civil-Military Cell \\
\hline & Command (U.S. Army) & $\mathrm{CMCC}$ & Corps Movement Control Center \\
\hline CED & Concept Exploration Design & $\mathrm{CMD}$ & Command \\
\hline CEM & Common Engagement Module & CMISE & Corps Military \\
\hline CENTAF & Central Command Air Force & & Intelligencesupport Element \\
\hline CENTCOM & Central Command & CML & Chemical \\
\hline CEOI & Communications-Electronics & CMMC & Corps Materiel Management \\
\hline CEP & Concept Evaluation Program & & Center \\
\hline \multirow[t]{2}{*}{ CEP } & (1) Circular Error Probable; (2) & $\mathrm{CMO}$ & Civil-Military Operations \\
\hline & Concept Exploration Program & $\mathrm{CMOC}$ & Civil-Military Operations Center \\
\hline CEP & Circular Error Probable & CMTC & Combat Maneuver Training \\
\hline CEP & Concept Evaluation Proposal & & Center \\
\hline \multirow[t]{4}{*}{ CEPCSS } & Centralized Equipment & CNGB & Chief, NGB \\
\hline & Procurement Conversion & CNR & Combat Net Radio \\
\hline & Capability System Operating & $\mathrm{CO}$ & Company \\
\hline & Instructions & $\mathrm{CO}$ & Commanding Officer \\
\hline \multirow[t]{2}{*}{ CEWI } & Combat Electronic Warfare & $\mathrm{COA}$ & Course Of Action \\
\hline & Intelligence & $\mathrm{COB}$ & Carrier Onboard \\
\hline CFA & Covering Force Area & $\mathrm{COC}$ & Council Of Colonels \\
\hline $\mathrm{CFC}$ & Chairman Fiscally Constrained & COCOM & Combatant Command \\
\hline CFST & Critical Fire Support Task & $\mathrm{COE}$ & Command Operating \\
\hline CFT & Captive Flight Test & & Environment \\
\hline \multirow[t]{2}{*}{$\mathrm{CG}$} & Commanding General, & $\mathrm{COE}$ & Common Operating Environment \\
\hline & Chairman's Guidance & $\mathrm{COE}$ & Contemporary Operational \\
\hline CGS & Common Ground Station & & Environment \\
\hline CGSC & $\begin{array}{l}\text { Command Group; Commanding } \\
\text { General U.S. Army Command } \\
\text { And General Staff College }\end{array}$ & $\mathrm{COE}$ & $\begin{array}{l}\text { Corps Of Engineers; Chief Of } \\
\text { Engineers }\end{array}$ \\
\hline
\end{tabular}




\begin{tabular}{|c|c|c|c|}
\hline \multirow[t]{2}{*}{ COEA } & Cost And Operational & CRS & Chairman's Readiness System \\
\hline & Effectiveness Analysis & $\mathrm{CS}$ & Combat Support \\
\hline $\mathrm{COI}$ & Critical Operational Issue & CSA & Chief Of Staff, U.S. Army \\
\hline \multirow[t]{2}{*}{ COLT } & Combat Observation \& Lasing & CSAR & Combat Search And Rescue \\
\hline & Team & CSB & Corps Support Battalion \\
\hline COS & Chief Of Staff & $\mathrm{CSC}$ & Combat Stress Control \\
\hline COMARFOR & Commander, ARFOR & CSE & Combat Support Equipment \\
\hline COMDT & Commandant & CSG & Corps Support Group \\
\hline COMINT & Communications Intelligence & $\mathrm{CSH}$ & Combat Support Hospital \\
\hline \multirow[t]{2}{*}{ Commo } & Communications, May Be Of & CSM & Command Sergeant Major \\
\hline & Any Type; Voice, Digital, Etc. & CSMA & Carrier Sense Multiple Access \\
\hline \multirow[t]{2}{*}{ Common Missile } & One Missile For Many Different & CSP & Cost Schedule Performance \\
\hline & Vehicles-Tri-Mode Warhead. & CSS & Combat Service Support \\
\hline COMMZ & Communications Zone & CSSCS & Combat Service Support Control \\
\hline COMPO & Component & & System \\
\hline COMPT & Comptroller & CSSE & Combat Service Support Element \\
\hline COMSAT & Communications Satellite & $\mathrm{CT}$ & Customer Test \\
\hline COMSEC & Communications Security & CTA & Cased Telescoped Ammunition \\
\hline $\mathrm{CON}$ & Control & CTA & Common Table Of Allowances \\
\hline CONPLAN & Concept Plan; Contingency Plan & CTAPS & Contingency Theater Automated \\
\hline CONUS & Continental United States & & Planning System \\
\hline CONUSA & Continental United States Army & СTC & Combat Training Center \\
\hline CONUSA & Continental U.S. Army & CTCP & Combat Trains Command Post \\
\hline COP & Common Operating Picture & CTD & Concept And Technology \\
\hline \multirow[t]{2}{*}{ CORDS } & Civil Operations Revolutionary & & Demonstration \\
\hline & Development Support & CTIL & Commander's Tracked Item List \\
\hline COSCOM & Corps Support Command & CTOC & Corps Tactical Operations Center \\
\hline COTS & Commercial-Off-The-Shelf & CTSF & Central Technical Support \\
\hline \multirow[t]{2}{*}{ CP } & Command Post; Computer & & Facility \\
\hline & Program & CTU & Consolidated TOE Update \\
\hline $\mathrm{CP}$ & Command Post & $\mathrm{CV}$ & Commander's Vehicle \\
\hline \multirow[t]{2}{*}{$\mathrm{CP}$} & Depending On Usage, Command & $\mathrm{CVC}$ & Combat Vehicle Communication \\
\hline & Post Or Check Point & CWRP & Chemical Warfare Request \\
\hline \multirow[t]{3}{*}{ CPA } & Chairman's Program & & Procedures \\
\hline & Assessment; Chief Of Public & $\mathrm{CX}$ & Categorical Exclusion \\
\hline & Affairs & CY & Current Year \\
\hline CPG & Contingency Planning Guidance & $\mathrm{D} \& \mathrm{O}$ & Doctrine And Operational \\
\hline CPLAN & Command Plan & D\&SA & Depth \& Simultaneous Attack \\
\hline CPM & Critical Path Method & D3A & Decide, Detect, Deliver \& Assess \\
\hline \multirow[t]{2}{*}{ CPR } & Chairman's Program & DA & Department Agriculture \\
\hline & Recommendation & DA & (1) Department Of The Army; \\
\hline CPSE & Corps Psyop Support Element & & (2) Decision Aid \\
\hline CPT & Captain & DAAG & Data-At-A-Glance \\
\hline CPU & Central Processing Unit & DAB & Defense Acquisition Board \\
\hline CPX & Command Post Exercise & DAB & Director Of The Army Budget \\
\hline CRA & Continuing Resolution Authority & & (Used To Refer To The Deputy \\
\hline \multirow{2}{*}{ CRAMM } & Complete Responsive Accurate & & Assistant Secretary Of The Army \\
\hline & Mission Module-Manned & & For Budget) Defense Acquisition \\
\hline CRC & Control And Reporting Center & & Board \\
\hline \multirow[t]{4}{*}{ CROP } & Command Research Objectives & DAE & Department (Of Defense) \\
\hline & Plan; Common Relevant & & Acquisition Executive \\
\hline & Operating Picture; Container & DAG & Division Artillery Group \\
\hline & Roll-In/Roll-Out Platform & DAG & Dynamic Airspace Management \\
\hline & Combat Reconnaissance Patrol & & System \\
\hline \multirow[t]{2}{*}{ CRRC } & Construction Requirements & DAG & Data Authentication Group \\
\hline & Review Committee & DALSO & DA Logistics Systems Officer \\
\hline
\end{tabular}




\begin{tabular}{|c|c|c|c|}
\hline \multirow{3}{*}{$\begin{array}{l}\text { DAMPL } \\
\text { DARI }\end{array}$} & DA Master Priority List & DGCS & Downsized Ground Control \\
\hline & Detect, Acquire, Recognize, And & & Station \\
\hline & Identify & DI & Document Integrator \\
\hline \multirow[t]{2}{*}{ DARNG } & Director Of The Army National & DIA & Defense Intelligence Agency \\
\hline & Guard & DII & Defense Information \\
\hline \multirow[t]{2}{*}{ DARPA } & Defense Advanced Research & & Infrastructure \\
\hline & Projects Agency & DIR & Director \\
\hline DAS & Director Of The Army Staff & DIRCM & Directional Infrared \\
\hline \multirow[t]{2}{*}{ DASC } & Department Of The Army Direct & & Countermeasure \\
\hline & Air Support Center & DIS & Distributed Interactive \\
\hline \multirow[t]{2}{*}{ DA-WAM } & Deep-Attack Wide-Area & & Simulation \\
\hline & Munitions & DISA & Defense Information Systems \\
\hline \multirow[t]{2}{*}{ DBSL } & Deep Battle Synchronization & & Agency \\
\hline & Line & DISC4 & Director Of Information Systems \\
\hline DBST & $\begin{array}{l}\text { Digital Battle-Staff Sustainment } \\
\text { Trainer }\end{array}$ & & $\begin{array}{l}\text { For Command, Control, } \\
\text { Communications, And }\end{array}$ \\
\hline DCA & Defensive Counter Air & & Computers \\
\hline $\mathrm{DCG}$ & Deputy Commanding General & DISC4 & Director Of Information Systems \\
\hline DCS & Deputy Chief Of Staff & & For Command, Control, \\
\hline \multirow[t]{3}{*}{ DCSCD } & Deputy Chief Of Staff For & & Communications, And \\
\hline & Combat Developments, & & Computers \\
\hline & TRADOC & DISCOM & Division Support Command \\
\hline DCSINT & $\begin{array}{l}\text { Deputy Chief Of Staff For } \\
\text { Intelligence }\end{array}$ & DISE & $\begin{array}{l}\text { Deployable Intelligence Support } \\
\text { Element }\end{array}$ \\
\hline DCSLOG & DCS For Logistics & DISP & Disposal \\
\hline DCSOPS & DCS For Operations And Plans & DIV & Division \\
\hline \multirow[t]{2}{*}{ DCSPER } & Deputy Chief Of Staff For & DIV CAV & Division Cavalry Squadron \\
\hline & Personnel & DLA & Defense Logistics Agency \\
\hline \multirow[t]{2}{*}{ DCT } & Digital Communications & $\mathrm{DM}$ & Director Of Management \\
\hline & Terminal & DMA & Defense Mapping Agency \\
\hline $\mathrm{DCX}$ & Division Capstone Exercise & DMAIN & Division Main Command Post \\
\hline \multirow[t]{2}{*}{ DDR\&E } & Director, Defense Research And & DMD & Digital Message Device \\
\hline & Engineering & DME & Displace, Move, Emplace \\
\hline $\mathrm{DE}$ & Directed Energy & DMMF & Division Mobile Maintenance \\
\hline $\mathrm{DEC}$ & December & & Facility \\
\hline DECON & Decontamination & DOCC & Deep Operations Coordination \\
\hline DEF & Defense & & Cell \\
\hline \multirow[t]{2}{*}{$\mathrm{DEH}$} & Directorate Of Engineering And & DOD & Department Of Defense \\
\hline & Housing (Now Known As DPW) & DOD & Department Of Defense \\
\hline DEN & Dental & DODAAC & Department Of Defense Activity \\
\hline DEP & Deputy & & Address Code \\
\hline DEPSECDEF & Deputy SECDEF & DODD & DOD Directive \\
\hline DET & Detachment & DoE & Department Of Energy \\
\hline DET & Displaced Equipment Training & DOS & Days Of Supply; Department Of \\
\hline \multirow[t]{2}{*}{ DETP } & Displaced Equipment Training & & State \\
\hline & Plan & DOTLMS & Doctrine, Organization, Training, \\
\hline DEUCE & $\begin{array}{l}\text { Deployable Universal Combat } \\
\text { Earthmover }\end{array}$ & & $\begin{array}{l}\text { Leader Development, Materiel, } \\
\text { And Soldiers }\end{array}$ \\
\hline DEW & Direct-Energy Weapons & DP & Dual Purpose \\
\hline DF & Direction Finding & DPAE & Director Of Program Analysis \\
\hline \multirow[t]{2}{*}{ DFAS } & Defense Finance And & & And Evaluation \\
\hline & Accounting Service & DPAMMH & Direct Productive Annual \\
\hline \multirow[t]{2}{*}{ DFBS } & Defense Finance Battlefield & & Maintenance Man-Hours \\
\hline & System & DPAS & Defense Priorities And \\
\hline DFSCOORD & Deputy Fire Support Coordinator & & Allocation System \\
\hline
\end{tabular}




\begin{tabular}{|c|c|c|c|}
\hline \multirow[t]{2}{*}{ DPDA } & Defense Property Disposal & $\mathrm{EBC}$ & Enroute Battle Command \\
\hline & Agency & $\mathrm{EBC}$ & Embedded Battle Command \\
\hline DPG & Defense Planning Guidance & $\mathrm{EC}$ & Emerging Concepts \\
\hline \multirow[t]{2}{*}{ DPICM } & Dual-Purpose Improved & ECB & Engineer Combat Battalion \\
\hline & Conventional Munition & ECBRS & Enhanced Concept Based \\
\hline DPM & Decision And Planning Module & & Requirements System \\
\hline DPP & Dedicated Procurement Program & ECM & Electronic Countermeasures \\
\hline DPRG & $\begin{array}{l}\text { Defense Planning And Resources } \\
\text { Board }\end{array}$ & ECP & $\begin{array}{l}\text { Emergency Command } \\
\text { Precedence }\end{array}$ \\
\hline DPW & Director Of Public Works & EDAS & Enlisted Distribution And \\
\hline DRB & Division Ready Brigade & & Assignment System \\
\hline DRB & Defense Resources Board & E-date & Effective Date \\
\hline DRMO & $\begin{array}{l}\text { Defense Reutilization And } \\
\text { Marketing Office }\end{array}$ & $\begin{array}{l}\text { EDI } \\
\text { EDSS }\end{array}$ & $\begin{array}{l}\text { Electronic Data Interchange } \\
\text { Equipment Distribution }\end{array}$ \\
\hline DRU & Dynamic Reference Unit & & Sequence System \\
\hline DRVT & $\begin{array}{l}\text { Downsized Remote Video } \\
\text { Terminal }\end{array}$ & EEFI & $\begin{array}{l}\text { Essential Elements Of Friendly } \\
\text { Information }\end{array}$ \\
\hline DS & Direct Support & EELS & Early Entry Lethality And \\
\hline DS FA & $\begin{array}{l}\text { Direct Support Field Artillery } \\
\text { (Principal Supporting Batteries) }\end{array}$ & EFAMS & $\begin{array}{l}\text { Survivability } \\
\text { Enhanced Fuel Armament }\end{array}$ \\
\hline DSA & Division Support Area & & Management Subsystem \\
\hline DSI & Defense Simulation Internet & EFFECTS & New Term For Artillery Support. \\
\hline DSMAC & $\begin{array}{l}\text { Digital Scene Matching And } \\
\text { Area Correlation }\end{array}$ & EFOG-M & $\begin{array}{l}\text { Enhanced Fiber Optic Guided } \\
\text { Missile }\end{array}$ \\
\hline DT & Developmental Testing & EFP & Explosively Formed Penatrator \\
\hline DT\&E & $\begin{array}{l}\text { Development Test And } \\
\text { Evaluation }\end{array}$ & EGI & $\begin{array}{l}\text { Embedded Global } \\
\text { Positioning/Inertial Navigation }\end{array}$ \\
\hline DTAC & Division Tactical Command Post & & System \\
\hline DTG & Date-Time Group & EIS & Environmental Impact Statement \\
\hline \multirow[t]{3}{*}{ DTLOMS } & Doctrine, Training, Leader & ELCT & Electronics \\
\hline & Development, Organizations, & ELIM & Enlisted Loss Inventory Model \\
\hline & Materiel, And Soldiers & ELIM-COMPLIP & Enlisted Loss Inventory Model- \\
\hline DTSS & $\begin{array}{l}\text { Digital Topographic Support } \\
\text { System }\end{array}$ & & $\begin{array}{l}\text { Computation Of Manpower } \\
\text { Using Linear Programming }\end{array}$ \\
\hline DTT & Doctrine And Tactics Training & ELINT & Electronic Intelligence \\
\hline DTV & Driver's Thermal Viewer & EMC & Electro-Magnetic Compatible \\
\hline DU & Depleted Uranium & EMFCS & Enhanced Mortar Fire Control \\
\hline DY & Design Year & & System \\
\hline $\mathrm{DZ}$ & Drop Zone & EMG & Electro-Magnetic Gun \\
\hline \multirow[t]{2}{*}{ E3 } & Electromagnetic Environmental & EMI & Eloctromagnetic Interference \\
\hline & Effects & EMI & Electro-Magnetic Interference \\
\hline E4 & Specialist & EMP & Electromagnetic Pulse \\
\hline E5 & Sergeant & EMV & Eloctromagnetic Vulnerability \\
\hline EA & Engagement Area & EN & Engineers, Both Letters \\
\hline EA & Environmental Assessment & & Capitalized. \\
\hline \multirow[t]{2}{*}{ EA } & Electronic Attack; Engagement & ENGR & Engineer \\
\hline & Area; Each & ENSCD & Enemy Situation Correlation \\
\hline \multirow[t]{2}{*}{ EAC } & Eastern Area Command; & & Division \\
\hline & Echelons Above Corps & EOD & Explosive Ordnance Disposal \\
\hline EAC & Echelon-Above-Corps & EOM & End Of Mission \\
\hline \multirow[t]{2}{*}{ EAC } & Echelons Above Corps; & EOM & End Of Message \\
\hline & Evaluation Analysis Center & EOTAS & Electro-Optical Target \\
\hline EAD & Echelon-Above-Division & & Acquisition System \\
\hline EAD & Echelons Above Division & EP & Electronic Protection \\
\hline \multirow[t]{2}{*}{ EADSIM } & Extended Air Defense & EPDS & Electronic Processing And \\
\hline & Simulation & & Dissemination System \\
\hline
\end{tabular}




\begin{tabular}{|c|c|c|c|}
\hline \multirow{3}{*}{$\begin{array}{l}\text { EPG } \\
\text { EPLRS }\end{array}$} & Electronic Proving Ground & FBCB2 & Force XXI Battle Command \\
\hline & Enhanced Position Location & & Battalion/Brigade And Below \\
\hline & Reporting System & FCS & Future Combat System \\
\hline \multirow[t]{2}{*}{ EPR } & Environmental Program & FCS LSI & FCS-Lead System Integrator \\
\hline & Requirement & FCS-ARCMS & FCS-Advanced Robotic \\
\hline EPW & Enemy Prisoner Of War & & Countermine System \\
\hline EQUIP & Equipment & FCS-AREMS & FCS-Advanced Robotic \\
\hline ERA & Explosive Reactive Armor & & Engagement Mortar System \\
\hline ERC & Equipment Readiness Codes & FCS-ARERS & FCS-Advanced Robotic \\
\hline ERC & Equipment Readiness Code & & Engagement Rocket System \\
\hline ERC/DAMPL & $\begin{array}{l}\text { Equip. Readiness Codes \& The } \\
\text { DA Master Pri. List }\end{array}$ & FCS-ARES & $\begin{array}{l}\text { FCS-Advanced Robotic } \\
\text { Engagement System }\end{array}$ \\
\hline ERGM & $\begin{array}{l}\text { Extended Range Guided } \\
\text { Munition }\end{array}$ & FCS-ARRS & $\begin{array}{l}\text { FCS-Advanced Robotic } \\
\text { Reconnaissance System }\end{array}$ \\
\hline ER-MLRS & $\begin{array}{l}\text { Extended Range Multiple } \\
\text { Launch Rocket System }\end{array}$ & FCS-ARSS & $\begin{array}{l}\text { FCS-Advanced Robotic } \\
\text { Sustainment System }\end{array}$ \\
\hline ERPS & $\begin{array}{l}\text { Equipment Release Priority } \\
\text { System }\end{array}$ & FCS-ARTAS & $\begin{array}{l}\text { FCS-Advance Robotic Target } \\
\text { Acquisition System }\end{array}$ \\
\hline ES & Electronic Warfare Support & FCS-CBT & FCS-Combat \\
\hline ES & End Strength & FCS-FC2V & FCS-Future Command And \\
\hline ESL & Enhanced Single Laser & & Control Vehicle (System) \\
\hline ET & Embedded Training & FCS-FCDR & FCS-Future Commander's \\
\hline ETC & Electro-Thermal Chemical & & (System) \\
\hline EUCOM & U.S. Army European Command & FCS-FICV & FCS-Future Infantry Carrier \\
\hline EVAC & Evacuation & & Vehicle (And Light (L)) \\
\hline EVD & Early-Version Demonstration & FCS-FIFV & FCS-Future Infantry Fighting \\
\hline EW & Electronic Warfare & & Vehicle \\
\hline EWO & Electronic Warfare Officer & FCS-FMV & FCS-Future Medical Vehicle \\
\hline EXCOM & Executive Committee & & (System) \\
\hline EXEVAL & External Evaluation & FCS-FRMV & FCS-Future Recovery And \\
\hline EXEVAL & External Evaluation & & Maintenance Vehicle (System) \\
\hline EXFOR & Experimental Force & FCS-FRS & FCS-Future Resupply System \\
\hline FA & Field Artillery & FCS-FRV & FCS-Future Reconnaissance \\
\hline \multirow{2}{*}{$\mathrm{FA}(\mathrm{R})$} & (Indicates A Reinforcing Field & & Vehicle \\
\hline & Artillery Unit) & FCS-FSPH & FCS-Future Self-Propelled \\
\hline FAA & Forward Assembly Area & & Howitzer \\
\hline FAA & Functional Area Assessment & FCS-FUV & FCS-Future Utility Vehicle \\
\hline FAAD & Forward Area Air Defense & FD & Fire Direction Or Forward \\
\hline \multirow[t]{3}{*}{ FAAD-C2I } & Forward Area Air Defense & & Detachment (Depending On \\
\hline & Command, Control, And & & Usage) \\
\hline & Intelligence System & $\mathrm{FD} / \mathrm{SC}$ & Failure Definition/Scoring \\
\hline \multirow[t]{2}{*}{ FAASV } & Field Artillery Ammunition & & Criteria \\
\hline & Supply Vehicle & FDC & Fire Direction Center \\
\hline \multirow{2}{*}{ FAAV } & Family Of Autonomous Air & FDD & First Digitized Division \\
\hline & Vehicles (AAV) & FDD & Force Design Directorate, \\
\hline FAB & Field Artillery Brigade & & DCSCD, TRADOC \\
\hline FAC & Forward Air Controller & FDO & Fire Direction Officer \\
\hline FAIO & $\begin{array}{l}\text { Field Artillery Intelligence } \\
\text { Officer }\end{array}$ & FDTE & $\begin{array}{l}\text { Force Development Test And } \\
\text { Experimentation }\end{array}$ \\
\hline FAR & Federal Acquisition Requirement & FDU & Force Design Update \\
\hline \multirow[t]{2}{*}{ FARP } & Forward Arming And Refueling & FEB & February \\
\hline & Point & FEBA & Forward Edge Of The Battle \\
\hline & Family Of Scatterable Mines & & Area \\
\hline \multirow[t]{2}{*}{ FASTALS } & Force Analysis Simulation Of & FEBA & Forward Edge Of Battle Area \\
\hline & Theater Administrative And & FED & Forward Entry Device \\
\hline FB & Finance Battalion & & \\
\hline
\end{tabular}


FEMA

FFR

FG

FI

FIA

FID

FIFA

FIN

FIST

FISTDA

FIST-V

FLB

FLIR

FLO

FLOT

FLS

FM

FM;CFF

FMBT

FMF

FMS

FMSP

FMTI

FMTV

FNSI

FO

FOA

FOB

FOC

FOCA

FOG-M

FORMDEPS

FORMS

FORSCOM

FOS

FOT

FOV

FP

FPS

FRAGO

FS

FS

FSA

FSAC

FSB
Federal Emergency Management

Agency

Force Feasibility Review

Finance Group

Force Integrator

Force Integration Analysis

Foreign Internal Defense

Force Integration Functional

Area

Finance

Fire Support Team

Full Time Support TDA

Fire Support Team Vehicle

Forward Logistics Base

Forward-Looking Infrared

Fighter Liaison Officer

Forward Line Of Own Troops

Future Leaders And Soldiers

Field Manual; Financial

Management

Fire Mission;Call-For-Fire

Future Main Battle Tank

Fleet Marine Forces

Foreign Military Sales

Foreign Military Sales Program

Future Missile Technology

Institution

Family Of Medium Tactical

Vehicles

Finding Of No Significant

Impact

Forward Observer

Field Operating Agency

Forward Operating Base

Future Army Operational

Capability

Future Operational Capabilities

Assessment

Fiber-Optic Guided Missile

Forscom Mobilization And

Deployment Planning System

Forward Observer Ranging \&

Marking System

U.S. Army Forces Command

Forward Observer System

Follow On Operational Test

Field Of View

Firing Position

Facility Planning System

Fragmentary Order

(1) Future Systems; (2) Fire

Support

Force Structure

Force Structure Allowance

Fire Support Armaments Center

Forward Support Battalion
FSC

FSCC

FSCL

FSCM

FSCOORD

FSD

FSE

FSE

FSNCO

FSO

FSR

FSSG

FTC

FTS

FTSMC

FTSTDA

FTX

FUE

FUED

FVC

FWAAV

FWD

FY

FYDP

G\&C

G/VLLD

G1

G2

G2

G3

G3

G4

G5

Gal.

GAO

GBCS

GBR
Forward Support Company

Fire Support Coordination Center Fire Support Coordination Line Fire Support Coordination Measures

Fire Support Coordinator

Full Scale Development

Fire Support Element

Fire Support Element

Fire Support Non-Commissioned Officer

Fire Support Officer

Field Service Regulation

Force Service Support Group

Forward Test Center

Full-Time Support

Full Time Support Management

Center

Full Time Support Table Of

Distribution And Allowance

Field Training Exercise

First Unit Equipped

First Unit Equipped Date

Force Validation Committee

Fixed-Wing Autonomous Air

Vehicle

Forward

Fiscal Year

Future Years Defense Program

Guidance \& Control

Ground/Vehicular Laser Locator

Designator

Army Component Manpower Or

Personnel Staff Officer (Army

Division Or Higher Staff)

Army Component Intelligence

Staff Officer (Army Division Or

Higher Staff)

Division Level Intelligence

Office/Officer

Division Level Plans \&

Operations Office/Officer

Army Component Operations

Staff Officer (Army Division Or

Higher Staff)

Army Component Logistics Staff

Officer (Army Division Or

Higher Staff)

Assistant Chief Of Staff, Civil

Affairs (Army Division Or

Higher Staff)

Gallons

General Accounting Office

Ground-Based Common Sensor

Ground-Based Radar 


\section{GCCS}

GCE

GCS

GDT

GED

GEN

GENESIS

GIE

GIS

GLO

GLPS

GMTI

GO

GOCOM

GOSC

GP

GPS

GRCS

GS

GSA

GSM

GSR

GSR

GS-R

GSTAMIDS

GT

GY

HAC

HBC

HE

HEAT

HEDP

HEL

HEMTT

HEP

HEP

HEP-T

HERO

HESD

HF

HFE

HG

$\mathrm{HH}$

HHC

HHD
Global Command And Control

System

Ground Combat Element

Ground Control Station

Ground Data Terminal

General Equivalency Diploma

General

Generic Smart Indirect Fire

Simulation

Global Information Environment

Geographic Information System

Ground Liaison Officer

Gun-Laying And Positioning

System

Ground Moving-Target Indicator

General Officer

General Officer Command

General Officer Steering

Committee

Group

Global Positioning System

Guardrail Common Sensor

General Support

General Services Administration

Ground Station Module

General Support Reinforcing

Ground Surveillance Radar

General Support-Reinforcing

Ground Standoff Mine Detection

System

General Test

Guidance Year

House Appropriations

Committee

House Budget Committee

High Explosive

High Explosive Anti-Tank

High Explosive Dual Purpose

Helicopter

Heavy Enhanced Mobility

Tactical Truck

High Explosive Plastic

Hybrid Electric Propulsion

High-Explosive Tracer

Hazards Of Electromagnetic

Radiation To Ordnance

Helicopter Electrostatic

Discharge

High Frequency

Human Factors Engineering

Hydrogen Generator

Health Hazard

Headquarters And Headquarters

Company

Headquarters And Headquarters

Detachment
HIDACZ

HIMAD

HIMARS

HIMARS

His

HIST

HL-UAV

HMD

HMEE

HMMWV

HN

HNSC

HOMES

HOW;MSN

HPC

HPCWG

HPSS

HPT

HPTL

HQ

HQDA

HQIFS

HQISR

HQRPLANS

HR

Hrs

HSIP

HSV

HT

HTI

HTTB

HUD

HUD

HUMINT

HVA

HVT

HVTL

HVY

HYEX

I2

IADS
High-Density Airspace Control

Zone

High- To Medium-Altitude Air Defense

High Mobility Artillery Rocket System

High-Mobility Rocket System

Human System Integration

Historian

Hand-Launched Unmanned

Aerial Vehicle

Helmet Mounted Display

High-Mobility Excavation

Equipment

High Mobility Multipurpose

Wheeled Vehicle

Host Nation

House National Security

Committee

Housing Operations Management

System

Howitzer;Mission

High Performance Computing

High Performance Computing

Working Group

Helmet Position Sensing System

High-Payoff Target

High Pay-Off Target List

Headquarters

Headquarters, Department Of

The Army

Headquarters Integrated

Facilities System

Headquarters Installation Status

Report

Headquarters Real Property

Planning And Analysis System

Hour

Hours

Human System Integration Plan

Hunter Surrogate Vehicle

Highway Traffic

Horizonatal Technology

Integration

High Technology Test Bed

Heads-Up Display

Department Of Housing And

Urban Development

Human Intelligence

High Value Asset

High-Value Target

High Value Target List

Heavy

Hydraulic Excavator

Image Intensification

Integrated Air Defense System 


\begin{tabular}{|c|c|c|c|}
\hline IADT & Initial Active Duty For Training & INC & Internet Controller \\
\hline IAP & Initial Aiming Point & INDIV & Individual \\
\hline IAW & In Accordance With & $\mathrm{INF}$ & Infantry \\
\hline IB & International Border & INFO & Information \\
\hline \multirow[t]{2}{*}{ IC3 } & Integrated Combat Command & ING & Inactive Army National Guard \\
\hline & And Control & INS & Inertial Navigation System \\
\hline ICD & Initial Concept Design & INTEL & Intelligence \\
\hline ICNIA & $\begin{array}{l}\text { Integrated Communications } \\
\text { Navigation Identification } \\
\text { Avionics }\end{array}$ & Interoperability & $\begin{array}{l}\text { New Definition Includes Joint, } \\
\text { Legacy, And Coalition } \\
\text { Interoperability. }\end{array}$ \\
\hline ICP & Incremental Change Package & INVT & Inventory \\
\hline ID & Infantry Division & $\mathrm{IOC}$ & (1) Initial Operational Capability; \\
\hline ID & Identification & & (2) Industrial Operations \\
\hline ID (L) & Infantry Division (Light) & IOT & Initial Operational Test \\
\hline $\mathrm{ID}(\mathrm{M})$ & Infantry Division (Mechanized) & IP & Intervention Point \\
\hline IDA & Institute For Defense Analysis & IP & Initial Point \\
\hline \multirow[t]{2}{*}{ IDAD } & Internal Defense And & IP & Internet Protocol \\
\hline & Development & IPA & Integrated Program Assessment \\
\hline IDG & Installation Design Guide & IPB & Intelligence Preparation Of The \\
\hline IDM & Improved Data Modem & & Battlefield \\
\hline IDT & Inactive Duty Training & IPB & Installation Planning Board \\
\hline IER & $\begin{array}{l}\text { Information Exchange } \\
\text { Requirement }\end{array}$ & IPB & $\begin{array}{l}\text { Intelligence Preparation Of The } \\
\text { Battlefield }\end{array}$ \\
\hline IEW & Intelligence Electronic Warfare & IPL & Integrated Priority List \\
\hline IEW & $\begin{array}{l}\text { Intelligence \& Electronic } \\
\text { Warfare }\end{array}$ & IPPT & $\begin{array}{l}\text { Integrated Process And Product } \\
\text { Team }\end{array}$ \\
\hline \multirow[t]{2}{*}{ IEWTD } & Intelligence Electronic Warfare & IPR & In Process Review \\
\hline & Test Directorate & IPS & Integrated Program Summary \\
\hline IFCS & Improved Fire Control System & IPT & Integrated Product/Process Team \\
\hline IFDC & Improved Field Data Collector & IR & Infrared \\
\hline IFF & Identification, Friend Or Foe & IR\&D & Independent Research \& \\
\hline IFS & Integrated Facilities System & & Development \\
\hline \multirow[t]{2}{*}{ IFSAS } & Initial Fire Support Automation & IRR & Individual Ready Reserve \\
\hline & System & IS & Information Superiority \\
\hline IFV & Infantry Fighting Vehicle & ISA & International Standardization \\
\hline IG & Inspector General & & Agreements \\
\hline \multirow[t]{2}{*}{ I-HMMWV } & Improved High-Mobility & ISB & Intermediate Staging Base \\
\hline & Multipurpose Wheeled Vehicle & ISR & Intelligence, Surveillance, And \\
\hline IIQ & Initial Issue Quantity & & Reconnaissance \\
\hline IKP & Instructor And Key Personnel & ISR & Installation Status Report \\
\hline \multirow[t]{2}{*}{ ILIR } & Independent Laboratory In- & IT & Information Technology \\
\hline & House Research & ITAADS & Installation The Army \\
\hline ILMS & $\begin{array}{l}\text { Improved Launcher Mechanical } \\
\text { System }\end{array}$ & & $\begin{array}{l}\text { Authorization Documents } \\
\text { System }\end{array}$ \\
\hline ILS & Integrated Logistics Support & ITOE & Intermediate $\mathrm{TOE}$ \\
\hline ILSP & Integrated Logistics Support Plan & ITP & Individual Training Plan \\
\hline \multirow[t]{2}{*}{ IMA } & Individual Mobilization & ITV & In-Transit Visibility \\
\hline & Augmenters & IVIS & Intervehicular Information \\
\hline IMA & Information Mission Area & & System/Radio Interface Unit \\
\hline IMETP & $\begin{array}{l}\text { International Military Education } \\
\text { And Training Program }\end{array}$ & $\mathrm{J} 1$ & $\begin{array}{l}\text { Manpower And Personnel } \\
\text { Directorate Of A Joint Staff }\end{array}$ \\
\hline IMF & Intelligent Minefield & $\mathrm{J} 2$ & Intelligence Directorate Of A \\
\hline IMINT & Imagery Intelligence & & Joint Staff \\
\hline IMP & Installation Master Plan & $\mathrm{J} 3$ & Operations Directorate Of A \\
\hline IMS & Integrated Master Schedule & & Joint Staff \\
\hline IN & Inch & & \\
\hline
\end{tabular}




\begin{tabular}{|c|c|c|c|}
\hline $\mathrm{J} 4$ & $\begin{array}{l}\text { Logistics Directorate Of A Joint } \\
\text { Staff }\end{array}$ & Joint STARS & $\begin{array}{l}\text { Joint Surveillance Target Attack } \\
\text { Radar System }\end{array}$ \\
\hline J5 & $\begin{array}{l}\text { Plans Directorate Of A Joint } \\
\text { Staff }\end{array}$ & JOPES & $\begin{array}{l}\text { Joint Operations Planning And } \\
\text { Execution System }\end{array}$ \\
\hline J5 & Strategic Plans And Policy & JP & Joint Publication \\
\hline & Directorate, The Joint Staff & JPD & Joint Planning Document \\
\hline J6 & Command, Control, & JPO & Joint Petroleum Office \\
\hline & Communications, And Computer & JPOC & Joint Project Optic Cobra \\
\hline & $\begin{array}{l}\text { Systems Directorate Of A Joint } \\
\text { Staff }\end{array}$ & JPOTF & $\begin{array}{l}\text { Joint Psychological Operations } \\
\text { Task Force }\end{array}$ \\
\hline $\mathrm{J} 8$ & $\begin{array}{l}\text { Force Structure Resources And } \\
\text { Assessments Directorate, The }\end{array}$ & JPO-UAV & $\begin{array}{l}\text { Joint Program Office For } \\
\text { Unmanned Aerial Vehicles }\end{array}$ \\
\hline & Joint Staff & JPS & Joint Precision Strikes \\
\hline JAAT & Joint Air Attack Team & JRB & JROC Review Board \\
\hline JANUS & $\begin{array}{l}\text { Joint Army, Navy Uniform } \\
\text { Simulation }\end{array}$ & $\mathrm{JRCC}$ & $\begin{array}{l}\text { Joint Rescue Coordination } \\
\text { Center }\end{array}$ \\
\hline $\mathrm{JCCC}$ & $\begin{array}{l}\text { Joint Communications Control } \\
\text { Center }\end{array}$ & $\begin{array}{l}\text { JROC } \\
\text { JROC }\end{array}$ & $\begin{array}{l}\text { Joint Research Oversight Council } \\
\text { Joint Requirements Oversight }\end{array}$ \\
\hline JCDB & Joint Common Database & & Council \\
\hline $\mathrm{JCM}$ & Joint Countermine & JRTC & Joint Readiness Training Center \\
\hline JCMEB & $\begin{array}{l}\text { Joint Civil-Military Engineering } \\
\text { Board }\end{array}$ & $\begin{array}{l}\text { JSCP } \\
\text { J-SEAD }\end{array}$ & $\begin{array}{l}\text { Joint Strategic Capabilities Plan } \\
\text { Joint Suppression Of Enemy Air }\end{array}$ \\
\hline JCMOTF & Joint Civil-Military Operations & & Defenses \\
\hline $\mathrm{ICS}$ & $\begin{array}{l}\text { Task Force } \\
\text { Joint Chiefs Of Staff }\end{array}$ & JSOTF & $\begin{array}{l}\text { Joint Special Operations Task } \\
\text { Force }\end{array}$ \\
\hline JCSE & $\begin{array}{l}\text { Joint Communications Support } \\
\text { Element }\end{array}$ & $\begin{array}{l}\text { JSPS } \\
\text { JSR }\end{array}$ & $\begin{array}{l}\text { Joint Strategic Planning System } \\
\text { Joint Strategy Review }\end{array}$ \\
\hline JDEC & $\begin{array}{l}\text { Joint Documents Exploitation } \\
\text { Center }\end{array}$ & JSTARS & $\begin{array}{l}\text { Joint Surveillance Target Attack } \\
\text { Radar System }\end{array}$ \\
\hline JFACC & $\begin{array}{l}\text { Joint Force Air Component } \\
\text { Commander }\end{array}$ & JSTARS & $\begin{array}{l}\text { Joint Surveillance Target Attack } \\
\text { Radar System }\end{array}$ \\
\hline $\begin{array}{l}\text { JFC } \\
\text { JFLCC }\end{array}$ & $\begin{array}{l}\text { Joint Force Commander } \\
\text { Joint Force Land Component }\end{array}$ & JTA-A & $\begin{array}{l}\text { Joint Technical Architecture- } \\
\text { Army }\end{array}$ \\
\hline & Commander & JTCB & Joint Targeting Coordination \\
\hline JFUB & Joint Facilities Utilization Board & & Board \\
\hline $\mathrm{JIC}$ & Joint Intelligence Center & JTCG/ME & Joint Technical Coordinating \\
\hline JIF & Joint Interrogation Facility & & Group/Munitions Effectiveness \\
\hline JIPC & $\begin{array}{l}\text { Joint Imagery Production } \\
\text { Complex }\end{array}$ & JTDA & $\begin{array}{l}\text { Joint / Defense Table Of } \\
\text { Distribution And Allowance }\end{array}$ \\
\hline JLOTS & Joint Logistics Over The Shore & JTF & Joint Task Force \\
\hline JMAO & Joint Mortuary Affairs Office & JTIDS & Joint Tactical Information \\
\hline JMBPO & $\begin{array}{l}\text { Joint Military Blood Program } \\
\text { Office }\end{array}$ & JTRS & $\begin{array}{l}\text { Distriubtion System } \\
\text { Joint Training System }\end{array}$ \\
\hline $\mathrm{JMC}$ & Joint Movement Center & JTRS & Joint Tactical Radio System \\
\hline JMCIS & $\begin{array}{l}\text { Joint Maritime Command } \\
\text { Information System }\end{array}$ & JTTP & $\begin{array}{l}\text { Joint Tactics, Techniques, And } \\
\text { Procedures }\end{array}$ \\
\hline JMEC & $\begin{array}{l}\text { Joint Materiel Exploitation } \\
\text { Center }\end{array}$ & $\begin{array}{l}\text { JVMF } \\
\text { JWCA }\end{array}$ & $\begin{array}{l}\text { Joint Variable Message Format } \\
\text { Joint Warfighting Capabilities }\end{array}$ \\
\hline JMEM & Joint Munitions Effectiveness & & Assessment \\
\hline & Manual & JWID & Joint Warfighting \\
\hline JMFU & Joint Meteorological Forecasting & & Interoperability Demonstration \\
\hline & Unit & KB & Kilobyte \\
\hline JMRO & Joint Medical Regulating Office & $\mathrm{KE}$ & Kenetic Energy \\
\hline JMRR & Joint Monthly Readiness Review & KIA & Killed In Action \\
\hline JOA & Joint Operations Area & KM & Kilometer \\
\hline $\mathrm{JOC}$ & Joint Operations Center & $\mathrm{Km}(\mathrm{s})$ & Kilometer(S) \\
\hline
\end{tabular}




\begin{tabular}{|c|c|c|c|}
\hline KPH & Kilometers Per Hour & LRRDAP & Long Range Research \\
\hline KPP & Key Performance Parameter & & Development And Acquisition \\
\hline KS & Kansas & & Plan \\
\hline KTI & Key Technical Issue & LRS & Long-Range Surveillance \\
\hline $\mathrm{L} / \mathrm{R}$ & Launch And Recovery & LRSU & Long-Range Surveillance Units \\
\hline Labs & Laboratories & LRU & Line Replaceable Unit \\
\hline LAM-A & Loitering Attack Munitions & LSI & Lead System Integrator \\
\hline LAN & Local Area Network & $\mathrm{LT}$ & Light \\
\hline \multirow[t]{2}{*}{ LAPES } & Low-Altitude Parachute & LTHF & Lighten The Heavy Force \\
\hline & Extraction System & LTOE & Living TOE \\
\hline LAV & Light Armored Vehicle & LUT 2 & Limited User Test 2 \\
\hline LBS & Pounds & LWR & Laser Warning Receiver \\
\hline $\mathrm{LC}$ & Line Of Contact & LWTB & Land Warrior Test Bed \\
\hline $\mathrm{LCC}$ & Land Component Commander & $\mathrm{LZ}$ & Landing Zone \\
\hline LCPK & Low-Cost Precision Kill & $\mathrm{M}$ & Meter \\
\hline LCR & Light Cavalry Regiment & M1 & Abrahams Main Battle Tank \\
\hline LCSMM & $\begin{array}{l}\text { Life Cycle System Management } \\
\text { Model }\end{array}$ & M1A2 SEP & $\begin{array}{l}\text { M1A2 System Enhancement } \\
\text { Program }\end{array}$ \\
\hline LCU & Lightweight Computer Unit & M2 & Bradley Fighting Vehicle (BFV) \\
\hline LCX & Logistics Coordination Exercise & M3V & Mobile Medical Mentoring \\
\hline LD & Line Of Departure & & Vehicle \\
\hline \multirow[t]{2}{*}{ LEAP } & Lightweight Exoatmospheric & M4 Carbine & M4 Carbine \\
\hline & Projectile & M88 & Heavy Recover Vehicle (US) \\
\hline LER & Loss Exchange Ratio & MAC & Maintenance Allocation Chart \\
\hline LGM & Laser Guided Munition & MACOM & Major Army Command \\
\hline LIF & Logistics Intelligence File & MACV & Military Assistance Command, \\
\hline LIM & Logistic Information System & & Vietnam \\
\hline LIN & Line Item Number & MAE & Mission Accomplishment \\
\hline LINEDIT & LIN-Edit & & Estimate \\
\hline \multirow[t]{2}{*}{ LLDR } & Lightweight Laser Designator & MAG & Marine Aircraft Group \\
\hline & Rangefinder & MAGTF & Marine Air-Ground Task Force \\
\hline \multirow[t]{2}{*}{ LLDR } & Lightweight Laser Designator & MAINT & Maintenance \\
\hline & Range Finder & MAIS & Mobile Army Instrumentation \\
\hline LLTR & Low-Level Transit Route & & System \\
\hline LMG & Light Machinegun & MAISRC & Major Automated Information \\
\hline LNO & Liaison Officer & & Systems Review Council \\
\hline LO & Liaision Officer & MANPRINT & Manpower And Personnel \\
\hline LOA & Limit Of Advance & & Integration \\
\hline LOC & Line Of Communications & MANSCEN & Maneuver Support Center \\
\hline LOG & Logistics & MAPS & Modular Azimuth Positioning \\
\hline \multirow{2}{*}{ LOGCAP } & Logistics Civil Augmentation & & System \\
\hline & Program & Mar & March \\
\hline LOGPAC & Logistics Package & MARC & Manpower Requirements Criteria \\
\hline \multirow[t]{2}{*}{ LOGSACS } & Logistics Structure And & MARDIV & Marine Division \\
\hline & Composition System & MARFOR & Marine Force \\
\hline LOM & Loitering Attack Munitions & MAT & Materiel \\
\hline LOS & Line Of Sight & MAT CMD & Materiel Command \\
\hline LOSAT & Line-Of-Sight Antitank & MATCH & TAA Comparison Report \\
\hline LOTS & Logistics-Over-The-Shore & MATDEV & Materiel Developer; Materiel \\
\hline LP & Listening Post & & Development \\
\hline LR & Long Range & MAW & Marine Aircraft Wings \\
\hline LRC & Lesser Regional Contingency & MBA & Main Battle Area \\
\hline LRC & Long-Range Component & MBI & Major Budget Issue \\
\hline LRF & Laser Range Finder & MBT & Main Battle Tank \\
\hline LRIP & Low Rate Initial Production & $\mathrm{MC}$ & Mobilization Component \\
\hline
\end{tabular}




\begin{tabular}{|c|c|c|c|}
\hline MC4 & $\begin{array}{l}\text { Medical Communication And } \\
\text { Combat Casualty Care }\end{array}$ & MI-8 & $\begin{array}{l}\text { Russian-Built Heavy Transport } \\
\text { Helicopter }\end{array}$ \\
\hline MCA & Military Construction, Army & MIBN & Mechanized Infantry Battalion \\
\hline MCAGCC & $\begin{array}{l}\text { Marine Corps Air Ground } \\
\text { Combat Center }\end{array}$ & MICAD & $\begin{array}{l}\text { Multipurpose Integrated } \\
\text { Chemical Agent Detector }\end{array}$ \\
\hline $\mathrm{MCC}$ & Movement Control Center & MICLIC & Mine Clearing Line Charge (A \\
\hline MCDM & $\begin{array}{l}\text { Military Construction, Defense } \\
\text { Medical }\end{array}$ & & $\begin{array}{l}\text { String Of Explosives Pulled By } \\
\text { A Rocket To Clear Mines, Wire, }\end{array}$ \\
\hline $\mathrm{MCOO}$ & $\begin{array}{l}\text { Modified Combined Obstacle } \\
\text { Overlay }\end{array}$ & MICOM & $\begin{array}{l}\text { Etc.) } \\
\text { Missile Command }\end{array}$ \\
\hline $\begin{array}{l}\text { MCS } \\
\text { MCS-P }\end{array}$ & $\begin{array}{l}\text { Maneuver Control System } \\
\text { Maneuver Control System- }\end{array}$ & MICV & $\begin{array}{l}\text { Mechanized Infantry Combat } \\
\text { Vehicle }\end{array}$ \\
\hline & Phoenix & MIES & Modernized Imagery \\
\hline MCT & Movement Control Team & & Exploitation System \\
\hline MDAP & $\begin{array}{l}\text { Major Defense Acquisition } \\
\text { Program }\end{array}$ & $\begin{array}{l}\text { MILCON } \\
\text { MILDEP }\end{array}$ & $\begin{array}{l}\text { Military Construction } \\
\text { Military Deputy }\end{array}$ \\
\hline $\begin{array}{l}\text { MDEP } \\
\text { MDL }\end{array}$ & $\begin{array}{l}\text { Management Decision Package } \\
\text { Mission Data Loader }\end{array}$ & MILES & $\begin{array}{l}\text { Multiple Integrated Laser } \\
\text { Engagement System }\end{array}$ \\
\hline MDM & Medium & MIN & Minute \\
\hline MDMP & $\begin{array}{l}\text { Military Decision Making } \\
\text { Process }\end{array}$ & $\begin{array}{l}\text { MIP } \\
\text { MITT }\end{array}$ & $\begin{array}{l}\text { Met Improvement Plan } \\
\text { Mobile Integrated Tactical }\end{array}$ \\
\hline MDR & Milestone Decision Review & & Terminal \\
\hline MDS & Meteorological Data System & MLRS & Multiple-Launch Rocket System \\
\hline MEB & Marine Expeditionary Brigade & MM & Millimeter \\
\hline $\mathrm{MECH}$ & Mechanized & MMC & Materiel Management Center \\
\hline MED & Medical & MMEWR & Minimum Mission Essential \\
\hline MEF & Marine Expeditionary Force & & Wartime Requirements \\
\hline MEGAJULE & $\begin{array}{l}\text { Amount Of Energy Used In } \\
\text { EMG Velocity/Force } \\
\text { Measurements. }\end{array}$ & $\begin{array}{l}\text { MMR } \\
\text { MMS }\end{array}$ & $\begin{array}{l}\text { Multi-Mission Radar } \\
\text { Meteorological Measuring } \\
\text { System }\end{array}$ \\
\hline MEL & Master Events List & MNS & Mission Need Statement \\
\hline MELIOS & $\begin{array}{l}\text { Mini-Eye-Safe Laser Infrared } \\
\text { Observation Set }\end{array}$ & $\begin{array}{l}\text { Mnv Bn FS } \\
\text { MOA }\end{array}$ & $\begin{array}{l}\text { Maneuver Battalion Fire Support } \\
\text { Memorandum Of Agreement }\end{array}$ \\
\hline MEMS & $\begin{array}{l}\text { Micro-Electrical Munitions } \\
\text { Systems }\end{array}$ & $\begin{array}{l}\text { MOBTDA } \\
\text { MOC }\end{array}$ & $\begin{array}{l}\text { Mobilization TDA } \\
\text { Management Of Change }\end{array}$ \\
\hline MERM & $\begin{array}{l}\text { Medium-Extended Range } \\
\text { Munitions }\end{array}$ & $\begin{array}{l}\text { MODPATH } \\
\text { MODSAF }\end{array}$ & $\begin{array}{l}\text { Modernization Path } \\
\text { Modular Semi-Automated Force }\end{array}$ \\
\hline Met & Meteorological & MOE & Measure Of Effectiveness \\
\hline METL & Mission Essential Task List & $\mathrm{MON}$ & Memorandum Of Notification \\
\hline METT-T & $\begin{array}{l}\text { Mission, Enemy, Terrain, } \\
\text { Troops, And Time Available }\end{array}$ & $\begin{array}{l}\text { MOP } \\
\text { MOPP }\end{array}$ & $\begin{array}{l}\text { Memorandum Of Policy } \\
\text { Mission-Oriented Protection }\end{array}$ \\
\hline MEU & Marine Expeditionary Unit & & Posture \\
\hline MFA & Materiel Fielding Agreement & MOPP-4 & Mission-Oriented Protection \\
\hline MFCS & Mortar Fire Control System & & Posture Level 4 \\
\hline MFDC & Multifunctional Data Collector & MOS & Military Occupational Specialty \\
\hline MFO & $\begin{array}{l}\text { Multinational Force Of } \\
\text { Observers }\end{array}$ & MOSLS & $\begin{array}{l}\text { Military Occupational Specialty } \\
\text { Level System }\end{array}$ \\
\hline MFOM & $\begin{array}{l}\text { MLRS Family Of } \\
\text { Munitions/Submunitions }\end{array}$ & MOUT & $\begin{array}{l}\text { Military Operations On Urban } \\
\text { Terrain }\end{array}$ \\
\hline MFORCE & Master Force & MOV & Movement \\
\hline MFP & Materiel Fielding Plan & MP & Military Police \\
\hline MG & Machine Gun & MPDI & MACOM POM Development \\
\hline MGB & Medium Girder Bridge & & Instructions \\
\hline MGMT & Management & MPES & Mobilization Planning And \\
\hline MI & Military Intelligence & & Execution System \\
\hline & & MPO & Mission Payload Operator \\
\hline
\end{tabular}




\begin{tabular}{|c|c|}
\hline MPU & Mobile Power Unit \\
\hline MRA & Manpower Reserve Affairs \\
\hline \multirow[t]{2}{*}{ MRAAS } & Multi-Role Armament \\
\hline & Ammunition System \\
\hline MRB & Motorized Rifle Battalion \\
\hline MRC & Major Regional Contingency \\
\hline MRC & $\begin{array}{l}\text { (1) Motorized Rifle Company; } \\
\text { (2) Major Regional Conflict; (3) }\end{array}$ \\
\hline MRD & Mechanized Rifle Division \\
\hline \multirow[t]{2}{*}{ MRIS } & Modernization Resource \\
\hline & Information Submission \\
\hline MRL & Mobile Rocket Launcher \\
\hline MRL & Materiel Requirements List \\
\hline MRMC & $\begin{array}{l}\text { Medical Research And Materiel } \\
\text { Command }\end{array}$ \\
\hline MRR & Minimum-Risk Route \\
\hline MRR & Motorized Rifle Regiment \\
\hline MRSI & $\begin{array}{l}\text { Multiple-Round Simultaneous } \\
\text { Impact }\end{array}$ \\
\hline MRSI (mission) & $\begin{array}{l}\text { Multi-Rounds Simultaneous } \\
\text { Impact }\end{array}$ \\
\hline MRT & Movement Regulating Team \\
\hline MS & Microsoft \\
\hline MS & Milestone \\
\hline MS “B” & $\begin{array}{l}\text { Milestone "B" = April 15, } 2003= \\
\text { Target Date For FCS Efforts. }\end{array}$ \\
\hline MSC & $\begin{array}{l}\text { Military Sealift Command; } \\
\text { Major Subordinate Command }\end{array}$ \\
\hline MSE & Mobile Subscriber Equipment \\
\hline MSF & Mobile Strike Force \\
\hline MSF95 & Mobile Strike Force 95 \\
\hline MSG & Multi Source Group \\
\hline MSIP & Multispectral Imagery Processor \\
\hline MSL & Missile \\
\hline MSP & Met Sensing Package \\
\hline MSP & Mission Support Plan \\
\hline MSR & Main Supply Route \\
\hline MSS & Mission Support System \\
\hline MSTAR & MLRS Smart Tactical Rocket \\
\hline \multirow[t]{2}{*}{ MSTAR } & A New Form Of Precision \\
\hline & Deliverable Munitions. \\
\hline MT & Mechanical Time \\
\hline MTBSA & $\begin{array}{l}\text { Mean-Time-Between-System- } \\
\text { Abort }\end{array}$ \\
\hline MTF & Medical Treatment Facility \\
\hline MTI & Moving-Target Indicator \\
\hline MTLD & $\begin{array}{l}\text { Man-Portable Target Locating } \\
\text { Device }\end{array}$ \\
\hline \multirow[t]{2}{*}{ MTMC } & U.S. Army Military Traffic \\
\hline & Management Command \\
\hline MTOE & Modification TOE \\
\hline \multirow[t]{2}{*}{ MTOE } & Modification Table Of \\
\hline & Organization And Equipment \\
\hline MTP & Materiel Transfer Plan \\
\hline Mtr & Mortar \\
\hline MTS & Marine Tactical System \\
\hline
\end{tabular}

\begin{tabular}{|c|c|}
\hline MTSQ & Mechanical Time, Superquick \\
\hline MTT & Mobile Training Teams \\
\hline MTTR & Mean-Time-To-Repair \\
\hline MTW & Major Theater War \\
\hline MULE & $\begin{array}{l}\text { Modular Universal Laser } \\
\text { Equipment }\end{array}$ \\
\hline MULE & $\begin{array}{l}\text { Wheeled/Tracked Robotic } \\
\text { Transport Vehicle. }\end{array}$ \\
\hline MUN & Munition \\
\hline MUTA & $\begin{array}{l}\text { Multiple Unit Training } \\
\text { Assemblies (Utas) }\end{array}$ \\
\hline MUTA-4 & $\begin{array}{l}\text { Four Utas Conducted Back-To- } \\
\text { Back (Normally One Weekend } \\
\text { MUTA) }\end{array}$ \\
\hline MWR & Morale, Welfare, And Recreation \\
\hline NAF & Numbered Air Force \\
\hline NAI & Named Area Of Interest \\
\hline NATO & $\begin{array}{l}\text { North Atlantic Treaty } \\
\text { Organization }\end{array}$ \\
\hline NAVAID & Navigational AID \\
\hline $\mathrm{NBC}$ & $\begin{array}{l}\text { Nuclear, Biological, And } \\
\text { Chemical }\end{array}$ \\
\hline NCA & National Command Authorities \\
\hline $\mathrm{NCO}$ & Noncommissioned Officer \\
\hline NDM & $\begin{array}{l}\text { Naturalistic Decision Making } \\
\text { Model }\end{array}$ \\
\hline NEA & Northeast Asia \\
\hline NEO & $\begin{array}{l}\text { Noncombatant Evacuation } \\
\text { Operation }\end{array}$ \\
\hline NEPA & $\begin{array}{l}\text { National Environmental Policy } \\
\text { Act Of } 1969\end{array}$ \\
\hline NET & New Equipment Training \\
\hline NET FIRES & $\begin{array}{l}\text { Intelligence Integrated Artillery } \\
\text { Fires System. }\end{array}$ \\
\hline NETP & New Equipment Training Plan \\
\hline NETT & New Equipment Training Team \\
\hline NG & National Guard \\
\hline $\mathrm{NG}$ & Next Generation \\
\hline NGB & National Guard Bureau \\
\hline NGF & Naval Gunfire \\
\hline NGFS & Naval Gunfire Fire Support \\
\hline NGLO & Naval Gunfire Liaison Officer \\
\hline $\mathrm{NGO}$ & Nongovernment Organization \\
\hline $\mathrm{NICP}$ & National Inventory Control Point \\
\hline NITF & $\begin{array}{l}\text { National Imagery Transmission } \\
\text { Format }\end{array}$ \\
\hline $\mathrm{NLO}$ & Naval Liaison Officer \\
\hline NLOS & Non-Line Of Sight \\
\hline NLT & Not Later Than \\
\hline NM & Nautical Mile \\
\hline NMCM & $\begin{array}{l}\text { Not Mission Capable For } \\
\text { Maintenance }\end{array}$ \\
\hline NMP & National Maintenance Point \\
\hline NMS & National Military Strategy \\
\hline NMSD & $\begin{array}{l}\text { National Military Strategy } \\
\text { Document }\end{array}$ \\
\hline
\end{tabular}




\begin{tabular}{|c|c|c|c|}
\hline \multirow[t]{2}{*}{ NODE } & Any Place Where Encryption & $\mathrm{OFC}$ & Office \\
\hline & And Decryption Occurs. & OFCD & Objective Force Concept Design \\
\hline NOE & Nap Of The Earth & OFF & Officer \\
\hline NOF & Notional Force & OFT & Operational Feasibility Test \\
\hline NOI & Notice Of Intent & $\mathrm{OH}$ & Observation Helicopter \\
\hline NOT & New Organization Training & OI & Organization Integrator; \\
\hline NOV & November & & Organizational Integration \\
\hline NRC & National Research Council & OICW & Objective Individual Combat \\
\hline NRT & Near-Real Time & & Weapon \\
\hline NSC & National Security Council & OJCS & Office Of The Joint Chiefs Of \\
\hline \multirow[t]{2}{*}{ NSCS } & National Security Council & & Staff \\
\hline & System & OLOS & Organic LOS \\
\hline NSD & National Security Directive & OMA & Operation And Maintenance, \\
\hline NSFS & Naval Surface Fire Support & & Army \\
\hline NSG & North Seeking Gyroscope & OMB & Office Of Management And \\
\hline NSR & National Security Review & & Budget \\
\hline NSS & Node Switching Sites & OMFTS & Operational Maneuver From The \\
\hline NSTO & New System Training Office & & Sea \\
\hline NTC & National Training Center & OMS/MP & Operational Mode \\
\hline NTDR & Near-Term Digital Radio & & Summary/Mission Profile \\
\hline NV & Night Vision & OOD & Out-Of-DAMPL Sequence \\
\hline NVPS & Night Vision Pilotage System & OODA & Observe, Orient, Decide, Act \\
\hline NWC & Naval Weapons Center & OOTW & Operations Other Than War \\
\hline O\&I & Operations And Intelligence & OP & Observation Post \\
\hline $\mathrm{O} \& \mathrm{O}$ & Organizational And Operational & OPALS & Officer Projection Aggregrate \\
\hline O\&S & Operations And Support & & Level System \\
\hline $\mathrm{O} / \mathrm{C}$ & Observer/Controller & OPALS & Officer Projection Aggregate \\
\hline $\mathrm{OA}$ & Operational Architecture & & Level System \\
\hline $\mathrm{OA}$ & Obligation Authority & OPCOM & Operational Command \\
\hline OAS & Organization Of American States & OPCON & Operational Control \\
\hline OAV & Organic Air Vehicle & OPFAC & Operational Facilities \\
\hline OBJ & Objective & OPFOR & Opposing Force \\
\hline $\mathrm{OC}$ & Observer-Controller & OPLAN & Operation Plan \\
\hline OCA & Offensive Counter Air & OPLANS & Operations Plans \\
\hline \multirow[t]{2}{*}{ OCAR } & Office Of The Chief, Army & OPM & Office Of Personnel \\
\hline & Reserve & & Management \\
\hline \multirow[t]{2}{*}{ OCD } & Operational Concept & OPORD & Operation Order \\
\hline & Demonstration & OPS & Operations \\
\hline \multirow[t]{2}{*}{ OCONUS } & Outside The Continental United & OPSEC & Operations Security \\
\hline & States & OPTEC & Operational Test \& Evaluation \\
\hline \multirow[t]{2}{*}{ OCR } & Operational Capability & & Command \\
\hline & Requirement & OPTEMPO & Operating Tempo \\
\hline \multirow[t]{2}{*}{ OCSW } & $\begin{array}{l}\text { Objective Crew-Served Weapon; } \\
\text { Objective Crew-Served Weapon }\end{array}$ & ORD & $\begin{array}{l}\text { Operational Requirements } \\
\text { Document }\end{array}$ \\
\hline & TRL4 & ORF & Operational Readiness Float \\
\hline ODCSLOG & $\begin{array}{l}\text { Office Of The Deputy Chief Of } \\
\text { Staff, Logistics }\end{array}$ & ORSA & $\begin{array}{l}\text { Operations Research And } \\
\text { Systems Analysts }\end{array}$ \\
\hline \multirow[t]{2}{*}{ ODCSOPS } & Office Of The Deputy Chief Of & OS & Operating Strength \\
\hline & Staff, Operations And Plans & OSA & Office Of The Secretary Of The \\
\hline \multirow[t]{2}{*}{ ODCSPER } & Office Of The Deputy Chief Of & & Army \\
\hline & Staff, Personnel & OSD & Office Of The Secretary Of \\
\hline ODH VI & Operation Desert Hammer VI & & Defense \\
\hline \multirow[t]{2}{*}{ OEC } & Operational Evaluation & OSDe & Operating Strength Deviation \\
\hline & Command & OSUT & One Station Unit Training \\
\hline OER & Officer Efficiency Report & OT & Operational Testing \\
\hline $\mathrm{OF}$ & Objective Force & OT & Operating Time \\
\hline
\end{tabular}




\begin{tabular}{|c|c|c|c|}
\hline \multirow[t]{2}{*}{ OT\&E } & Operational Testing And & PHTK & Precision Hit To Kill \\
\hline & Evaluation & PIP & Product Improvement Program \\
\hline OTC & Operational Test Command & PIR & Priority Information \\
\hline OTM & On-Line Training Module & & Requirements \\
\hline OTM & On The Move & PKO & Peace-Keeping Operations \\
\hline OTN & Own-The-Night & PL & Phase Line \\
\hline OTOE & Objective TOE & PLC & Pulsed Logistic Concept \\
\hline OTRR & $\begin{array}{l}\text { Operational Test Readiness } \\
\text { Review }\end{array}$ & PLGR & $\begin{array}{l}\text { Precision Lightweight Global } \\
\text { Positioning System Receiver }\end{array}$ \\
\hline OTT & October & PLL & Prescribed Load List \\
\hline $\mathrm{OV}$ & Orbiting Vehicle & PLS & Palletized Loading System \\
\hline P3I & Preplanned Product Improvement & PLT & Platoon \\
\hline PA & Public Affairs & PM & Provost Marshal \\
\hline PA & Precision Attack & PM & (1) Project Manager; (2) Program \\
\hline \multirow[t]{2}{*}{ PAC-3 } & Patriot Advanced Capability & & Manager \\
\hline & Level-3 & PMAD & Personnel Management \\
\hline PACOM & US Army Pacific Command & & Authorization Document \\
\hline \multirow[t]{2}{*}{ PADS } & Position And Azimuth & $\mathrm{PMC}$ & Personnel Management Center \\
\hline & Determining System & PMCS & Preventive Maintenance Checks \\
\hline \multirow[t]{2}{*}{ PAED } & Program Analysis And & & And Services \\
\hline & Evaluation Directorate & PMJ & Professional Military Judgment \\
\hline PAM & Pamphlet & PO & Project Office \\
\hline PAM & Precision Attack Munitions & POC & Platoon Operations Center \\
\hline PAO & Public Affairs Officer & POC & Point Of Contact \\
\hline PB & President's Budget & POD & Port Of Debarkation \\
\hline \multirow[t]{2}{*}{ PBAC } & Program Budget Advisory & POE & Port Of Embarkation \\
\hline & Committee & POI & Program Of Instruction \\
\hline \multirow{2}{*}{ PBC } & Program Budget Committee Of & POL & Petroleum, Oils, And Lubricants \\
\hline & PPBES & POM & Program Objective Memorandum \\
\hline PBC & Program And Budget Committee & POS/NAV & Position Navigation \\
\hline PBD & Program/Budget Decision & POSC-edit & Personnel Occupational Specialty \\
\hline PBG & Program And Budget Guidance & & Code-Edit File \\
\hline PBG & Program Budget Guidance & POSNAV & Position Navigation \\
\hline PCS & Permanent Change Of Station & POTF & Psychological Operations Task \\
\hline PD & Point Detonating & & Force \\
\hline PDD & Presidential Decision Directive & $\mathrm{PP} \& \mathrm{O}$ & Plans, Programs, And Operations \\
\hline PDM & Program Decision Memorandum & PPAR & Purpose, Priority, Allocation And \\
\hline PE & Program Element & & Restrictions \\
\hline \multirow{3}{*}{ PEGS } & Program Evaluation Group & PPBERS & Program Perf. And Budget Exec. \\
\hline & Program Evaluation Groups Of & & Rev. Sys. \\
\hline & PPBES & PPBES & Planning, Programming, \\
\hline PEO & Peace Enforcement Operations & & Budgeting, And Execution \\
\hline PEO & Program Executive Officer & & System \\
\hline PERS & Personnel & PPBS & Planning, Programming, And \\
\hline \multirow[t]{2}{*}{ PERSACS } & Personnel Structure And & & Budgeting System \\
\hline & Composition System & PQT & Production Qualification Test \\
\hline PERSCOM & Total Army Personnel Command & PR & Personnel Readiness \\
\hline PERSO & Personnel Systems Staff Officer & PRB & Program Review Board \\
\hline \multirow[t]{2}{*}{ PERT } & Program Evaluation And Review & PRD & Presidential Review Decision \\
\hline & Technique & PREPO & Prepositioned Sets Of Equipment \\
\hline PESD & Personnel Electrostatic Discharge & PRG & Program Review Group \\
\hline PETRI & Petroleum & PRGM & Program \\
\hline PG & Personnel Group & PRMD & Personnel Readiness \\
\hline & Portable Ground Control Station & & Management Division \\
\hline \multirow[t]{2}{*}{ PGMM } & Precision Guided Mortar & PRO & Procedures \\
\hline & Munitions & & \\
\hline
\end{tabular}




\begin{tabular}{|c|c|c|c|}
\hline PROBE & $\begin{array}{l}\text { Program Optimization And } \\
\text { Budget Evaluation }\end{array}$ & RCEM & $\begin{array}{l}\text { Regional Contingency } \\
\text { Engineering Manager }\end{array}$ \\
\hline PROC & Processing & $\mathrm{RCF}$ & Repair Cycle Float \\
\hline PROC & Procurement Appropriation & RCP & Relevant Common Picture \\
\hline PROP & Property & $\mathrm{RD}(\mathrm{S})$ & Round(S) \\
\hline PS & Personnel Support & RDA & Research, Development, And \\
\hline PSA & Port Support Activity & & Acquisition \\
\hline PSB & Personnel Service Battalion & RDAP & Research, Development, And \\
\hline \multirow[t]{2}{*}{ PSG } & Prioritization Steering Group Of & & Acquisition Plan \\
\hline & PPBES & RDCOMP & Round(S) Complete \\
\hline PSG & Prioritization Steering Group & RDD & Required Delivery Date \\
\hline PSYOP & Psychological Operations & RDD & Requirements Documentation \\
\hline PSYOPS & Psychological Operations & & Directorate, USAFMSA \\
\hline PT & Physical Training & RDEC & Research Development And \\
\hline PTS & Parts & & Engineering Center \\
\hline PY & Program Year; Prior Year & RDF & Radio Direction Finding \\
\hline PZ & Pick-Up Zone & RDS & Requirements Documentation \\
\hline QA & Quality Assurance & & System \\
\hline QAPR & $\begin{array}{l}\text { Quarterly Army Performance } \\
\text { Review }\end{array}$ & RDTE & $\begin{array}{l}\text { Research, Development, Test, } \\
\text { And Evaluation }\end{array}$ \\
\hline $\mathrm{QC}$ & Quality Control & REC & Record Of Environmental \\
\hline QE & Quadrant Elevation & & Consideration \\
\hline QF & Quick Fire & RECON & Reconnaissance \\
\hline QFD & Quality Function Deployment & Redcon & Readiness Condition; 1 = Ready \\
\hline QMP & $\begin{array}{l}\text { Qualitative Management } \\
\text { Program }\end{array}$ & & $\begin{array}{l}\text { To Move Now, } 2=\text { Ready To } \\
\text { Move In } 15 \text { Minutes, } 3=\text { Ready }\end{array}$ \\
\hline QQPRI & $\begin{array}{l}\text { Quan. \& Qual. Personnel Reqr } \\
\text { Info. }\end{array}$ & & $\begin{array}{l}\text { To Move In } 60 \text { Minutes, } 4= \\
\text { Ready To Move In One Hour }\end{array}$ \\
\hline \multirow[t]{3}{*}{ QQPRI } & Qualitative And Quantitative & REFORGER & Reinforce Germany \\
\hline & Personnel Requirements & REGT & Regiment \\
\hline & Information & REP & Representative \\
\hline \multirow{2}{*}{ QRMP } & Quick-Response Multicolor & REPLMT & Replacement \\
\hline & Printer & REQUEST & Recruit Quota System \\
\hline \multirow[t]{2}{*}{ QSTAG } & Quadripartite Standardization & REQVAL & Requisition Validation \\
\hline & Agreement & RETAIN & Reenlistment, Reclassification \\
\hline QuickLook & $105 \mathrm{~mm}$ Tube-Launched UAV. & & And Assignment System \\
\hline $\mathrm{R}$ & Reinforcing & RFL & Restricted Fire Line \\
\hline$R \& D$ & Research And Development & RFP & Request For Proposal \\
\hline $\mathrm{R} \& \mathrm{M}$ & Reliability And Maintainability & RFPB & Reserve Forces Policy Board \\
\hline \multirow[t]{2}{*}{$\mathrm{R} \& \mathrm{~S}$} & Reconnaissance And & RFPI & Rapid Force Projection Initiative \\
\hline & Surveillance & RGR & Ranger \\
\hline \multirow[t]{2}{*}{ RAD } & Requirements Authorization & RHU & Replacement Holding Unit \\
\hline & Docuement & RIMS & Research And Development \\
\hline \multirow[t]{2}{*}{ RAM } & Reliability, Availability, And & & Information Management System \\
\hline & Maintainability & RISTA & Reconnaissance, Intelligence, \\
\hline \multirow[t]{2}{*}{ RAM2000 } & Reliability, Availability, And & & Surveillance, And Target \\
\hline & Maintainability 2000 & & Acquisition \\
\hline \multirow[t]{2}{*}{ RAMM } & Responsive Accurate Mission & RLO & Reconnaissance Liaison Officer \\
\hline & Module & RLT & Regimental Landing Team \\
\hline RAOC & Rear Area Operations Center & RMS & Risk Management Strategy \\
\hline RAP & Rocket-Assisted Projectile & RMU & Resource Management Update \\
\hline RAP & Revised Approved Program & ROA & Restricted Operations Area \\
\hline & Reserve Component & ROC-V & Robotic Countermine Vehicle \\
\hline \multirow[t]{3}{*}{$\mathrm{RCCC}$} & Reserve Component & ROD & Record Of Decision \\
\hline & Coordination Council & ROE & Rules Of Engagement \\
\hline & & ROM & Refuel On The Move \\
\hline
\end{tabular}




\begin{tabular}{|c|c|c|c|}
\hline ROTC & Reserve Officers' Training Corps & SADM & System Acquisition Decision \\
\hline ROZ & Restricted Operations Zone & & Memorandum \\
\hline RP & Release Point & SAF & Semi-Automated Forces \\
\hline RPD & $\begin{array}{l}\text { Recognition Primed Decision- } \\
\text { Making Model }\end{array}$ & SAG & $\begin{array}{l}\text { Senior Advisory Group; Study } \\
\text { Advisory Group }\end{array}$ \\
\hline RPI & Real Property Inventory & SALUTE & Size, Activity, Location, Unit, \\
\hline RPIP & Real Property Investment Plan & & Time, Equipment (Message) \\
\hline \multirow[t]{2}{*}{ RPLANS } & Real Property Planning And & SAM & Surface-To-Air Missile \\
\hline & Analysis System & SAMAS & Structure And Manpower \\
\hline RPM & Real Property Maintenance & & Allocation System \\
\hline RPMA & $\begin{array}{l}\text { Real Property Maintenance } \\
\text { Activities }\end{array}$ & $\begin{array}{l}\text { SAPO } \\
\text { SAR }\end{array}$ & $\begin{array}{l}\text { Subarea Petroleum Office } \\
\text { Synthetic Aperture Radar }\end{array}$ \\
\hline RPMP & Real Property Master Plan & SARDA & Office Of The Assistant \\
\hline RPMS & $\begin{array}{l}\text { Real Property Management } \\
\text { System }\end{array}$ & & $\begin{array}{l}\text { Secretary Of The Army } \\
\text { (Research, Development, and }\end{array}$ \\
\hline RPPB & Real Property Planning Board & & Analysis) \\
\hline RPV & Remotely Piloted Vehicle & SARDB & Survivable Armed \\
\hline RPWS & Remote Player Workstation & & Reconnaissance On The Digital \\
\hline \multirow[t]{2}{*}{ RSC } & Army Reserve Regional Support & & Battlefield \\
\hline & Command & SASC & Senate Armed Services \\
\hline \multirow{2}{*}{ RSOI } & Reception, Staging, Onward- & & Committee \\
\hline & Movement, And Integration & SASO & Stability And Support Operations \\
\hline \multirow[t]{2}{*}{ RSOP } & Readiness Standing Operating & SAT & Systems Approach To Training \\
\hline & Procedures & SATP & Security Assistance Training \\
\hline \multirow[t]{2}{*}{ RSS } & Reconnaissance Surveillance & & Program \\
\hline & And Security & SAW & Squad Automatic Weapon \\
\hline \multirow[t]{2}{*}{ RSTA } & Reconnaissance, Surveillance, & SBC & Senate Budget Committee \\
\hline & And Target Acquisition & SBF & Support By Fire \\
\hline RSV & Resupply Vehicle & SBIR & Small Business Innovation \\
\hline RTCA & Real-Time Casualty Assessment & & Research Program \\
\hline RTO & Radio-Telephone Operator & SCIPS & Standardized Integrated \\
\hline \multirow[t]{2}{*}{ RUDE } & Receive, Understand, & & Command Post System \\
\hline & Disseminate, Execute & $\mathrm{SCP}$ & Survey Control Point \\
\hline RVT & Remote Video Terminal & Sct & Scout \\
\hline \multirow[t]{2}{*}{ RWAAV } & Rotary Wing Autonomous Air & SDD & System Design Demonstration \\
\hline & Vehicle & SDD & System Development Definition \\
\hline RWS & Remote Workstation & SEAD & Suppression Of Enemy Air \\
\hline S\&I & Science And Infrastructure & & Defenses \\
\hline S\&T & Science And Technology & SEC & Section \\
\hline \multirow[t]{2}{*}{ S1 } & Battalion Or Brigade Personnel & SECDEF & Secretary Of Defense \\
\hline & Officer & SELCOM & Select Committee Of PPBES \\
\hline S2 & $\begin{array}{l}\text { Battalion Or Brigade Intelligence } \\
\text { Officer }\end{array}$ & SEMA & $\begin{array}{l}\text { Special Electronics Mission } \\
\text { Aircraft }\end{array}$ \\
\hline \multirow[t]{2}{*}{ S3 } & Battalion Or Brigade Operations & SEP & September \\
\hline & And Staff Officer & SEP & System Evaluation Plan \\
\hline \multirow[t]{2}{*}{ S4 } & Battalion Or Brigade Logistics & SERV & Service \\
\hline & Officer & SESIL & System Electronics And \\
\hline SA & Situational Awareness & & Software Integration Laboratory \\
\hline SA & Secretary Of The Army & $\mathrm{SF}$ & Special Forces \\
\hline \multirow[t]{2}{*}{ SAC } & Senate Appropriations & $\mathrm{SF}$ & Sustained Fire \\
\hline & Committee & SFC & Sergeant First Class \\
\hline \multirow[t]{2}{*}{ SACC } & Supporting Arms Coordination & SFM & Sensor Fused Munitions \\
\hline & Center & SFOA & Special Forces Operational Area \\
\hline \multirow[t]{2}{*}{ SACS } & Structure And Composition & SFOB & Special Forces Operational Base \\
\hline & System & SFOD & Special Forces Operational \\
\hline SADARM & Sense And Destroy Armor & & Detachment \\
\hline
\end{tabular}




\begin{tabular}{|c|c|c|c|}
\hline \multirow[t]{2}{*}{ SGA } & Standards Of Grade & SOV & Staff Operations Vehicle \\
\hline & Authorization & SOW & Statement Of Work \\
\hline SGS & Secretary Of The General Staff & SP & Self-Propelled \\
\hline SHOT & Round(S) Fired & SP & (1) Self-Propelled; (2) Solid \\
\hline SHSM & Soldier Health Status Monitor & & Propellent; (3) Single Purpose \\
\hline SI & Systems Integrator & SP & Start Point \\
\hline SIB & Separate Infantry Brigade & SPC & Strategy Planning Committee Of \\
\hline \multirow[t]{2}{*}{ SIDPERS } & Standard Installation Division & & PPBES \\
\hline & Personnel System & SPCE & Survey Planning And \\
\hline SIG & Signal & & Coordination Element \\
\hline SIGINT & Signal Intelligence & SPH & Self-Propelled Howitzer \\
\hline SIGINT & Signals Intelligence & SPLASH & Round(S) Impacting In About 5 \\
\hline SIMOS & Space Imbalanced MOS & & Seconds \\
\hline \multirow[t]{2}{*}{ SINCGARS } & Single Channel Ground And & SPLL & Self-Propelled Launcher-Loader \\
\hline & Airborne Radio System & SPNV & Special Project Night Vector \\
\hline \multirow[t]{2}{*}{ SIO’s } & Missions Of Standard & SPT & Support \\
\hline & Installation Organizations & SQD & Squad \\
\hline \multirow[t]{2}{*}{ SIPC } & Stationing And Installation & SQDN & Squadron \\
\hline & Planning Committee & SR & Short Range \\
\hline SIRDAP & $\begin{array}{l}\text { Science And Infrastructure RDA } \\
\text { Plan }\end{array}$ & $\mathrm{SRC}$ & $\begin{array}{l}\text { Standard Requirement Code; } \\
\text { Short-Range Component }\end{array}$ \\
\hline \multirow[t]{2}{*}{ SISA } & Science And Infrastructure & SRP & Soldier Readiness Processing \\
\hline & Support Analysis & SS & System Safety \\
\hline Sit Rpt, Sitrep & Situation Report & $\mathrm{SSC}$ & Small-Scale Contingencies \\
\hline SJA & Staff Judge Advocate & SSM & Surface-To-Surface Missile \\
\hline \multirow[t]{2}{*}{ SKA } & Skills, Knowledge, And & SSP & System Support Package \\
\hline & Attributes & SSv & Soldier Survivability \\
\hline SLAM & Standoff Land Attack Missile & ST & Standby Time \\
\hline \multirow[t]{2}{*}{ SLID } & System-Level Integration & ST & Sustainment Training \\
\hline & Demonstration & STACCS & Standard Theater Army \\
\hline \multirow[t]{2}{*}{ SMART Mines } & Self-Healing Mines That Will & & Command And Control System \\
\hline & Follow An Opponent. & STAFF & Smart Target Activated Fire Anc \\
\hline \multirow[t]{2}{*}{ SMDR } & Structure \& Manning Decision & & Forget \\
\hline & Review & STANAG & Standardized North Atlantic \\
\hline SME & Subject Matter Experts & & Treaty Organization Agreement \\
\hline SMI & Soldier-Machine Interface & STANAG & Standardization Agreement \\
\hline SO & Special Operations & STAR & System Threat Assessment \\
\hline SOC & Special Operations Command & & Report \\
\hline \multirow[t]{2}{*}{ SOCCE } & Special Operations Command & STARC & State Area Command \\
\hline & And Control Element & STAW & Smart Top Attack Weapon \\
\hline \multirow[t]{2}{*}{$\mathrm{SOCCH}$} & Special Operations Command & STD & System Technology \\
\hline & And Control Headquarters & & Demonstration \\
\hline SOCOM & Special Operations Command & STO & Science \& Technology \\
\hline SOCOORD & Special Operations Coordinator & & Objectives \\
\hline SOE & Statement Of Equipment & STO & Science And Technology \\
\hline SOF & Special Operations Forces & & Objective \\
\hline SOFA & Status Of Forces Agreement & STOL & Short Take-Off And Landing \\
\hline SOI & Signal Operations Instructions & STORM & Simulation, Testing, Operation, \\
\hline $\mathrm{SOO}$ & Statement Of Objectives & & Rehearsal Model \\
\hline SOP & Standing Operating Procedures & STOW & Synthetic Theater Of War \\
\hline \multirow[t]{2}{*}{ SORC } & System's Operations & STP & System Training Plan \\
\hline & Requirements And Capabilities & STP's & Short Term Projects \\
\hline \multirow[t]{2}{*}{ SORTS } & Status Of Resources And & STRAC & Standards In Weapons Training \\
\hline & Training System & STRAP & System Training Plan \\
\hline SOS & Speed Of Service & STRICOM & Simulation, Training, And \\
\hline SOSA & System Of Systems Architecture & & Instrumentation Command \\
\hline
\end{tabular}




\begin{tabular}{|c|c|c|c|}
\hline \multirow{3}{*}{$\begin{array}{l}\text { STRIKWARN } \\
\text { STTE }\end{array}$} & Strike Warning & TAG & $\begin{array}{l}\text { The Adjutant General Of A State } \\
\text { Or Territory }\end{array}$ \\
\hline & Special Tools And Test & & Or Territory \\
\hline & Equipment & TAI & Target Areas Of Interest \\
\hline STTR & $\begin{array}{l}\text { Small Business Technology } \\
\text { Transfer Pilot Program }\end{array}$ & TAIS & $\begin{array}{l}\text { Tactical Airspace Integration } \\
\text { System }\end{array}$ \\
\hline STX & Situational Training Exercise & TALDT & Total Administrative And \\
\hline SU & Situational Understanding & & Logistics Delay Time \\
\hline S-UAV & $\begin{array}{l}\text { Shot Unattended Vehicle (Tube- } \\
\text { Launched } 105 \mathrm{~mm} \text { ) }\end{array}$ & $\begin{array}{l}\text { TALO } \\
\text { TALS }\end{array}$ & $\begin{array}{l}\text { Tactical Airlift Liaison Ofllcer } \\
\text { Tactical Automatic Landing }\end{array}$ \\
\hline SUBS & Subsistence & & System \\
\hline SUP & Supply & TAP & The Army Plan \\
\hline SURG & Surgeon & TAPDB & Total Army Personnel Database \\
\hline SVC & Service & TAPDB-AE & Total Army Personnel Data \\
\hline SVP's & Special Visibility Programs & & Base-Active Enlisted \\
\hline SWA & Southwest Asia & TAPDB-AE & TAPDB-Active Enlisted \\
\hline SWG & $\begin{array}{l}\text { Seminar Wargame (For This } \\
\text { Iteration Caspian Sea Scenario) }\end{array}$ & TAPDB-AO & $\begin{array}{l}\text { Total Army Personnel Data } \\
\text { Base-Active Officer }\end{array}$ \\
\hline SWO & Staff Weather Officer & TAPDB-AO & TAPDB-Active Officer \\
\hline \multirow[t]{2}{*}{ SWOE } & Smart Weapons Operability & TAR & Tactical Air Reconnaissance \\
\hline & Enhancement & TARABS & Tactical Air Reconnaissance And \\
\hline SYNJAM & Synthetic Jammers & & Aerial Battlefield Surveillance \\
\hline SYS & Systems & TASOSC & Theater Army Special Operations \\
\hline T\&E & Testing And Evaluation & & Support Command \\
\hline \multirow[t]{2}{*}{ T\&E IPT } & Test And Evaluation Integrated & TAV & Total Asset Visibility \\
\hline & Process Team & TBEP & Training Base Expansion Plan \\
\hline $\mathrm{T}-80$ & Russian Built Tank & TBM & Tactical Ballistic Missile \\
\hline TA & Theater Army & TBMD & Tactical Ballistic Missile \\
\hline TA & Target Acquisition & & Defense \\
\hline TAA & Tactical Assembly Area & TCACCIS & Transportation Coordinator \\
\hline TAA & Total Army Analysis & & Automated Command And \\
\hline \multirow[t]{2}{*}{ TAADS } & The Army Authorization & & Control Information System \\
\hline & Documents System & TCC & Test Control Center \\
\hline \multirow[t]{2}{*}{ TAADS-R } & The Army Authorization & TCF & Tactical Combat Force \\
\hline & Document System³/4 Re-Design & TCM & Total Corrective Maintenance \\
\hline TAC & Tactical & TCP & Traffic Control Plan \\
\hline TAC & Tactical Command Post & TCP & Transmission Control Protocol \\
\hline TACAIR & Tactical Air & TDA & Table Of Distribution And \\
\hline TACC & Tactical Air Control Center & & Allowances \\
\hline TACFIRE & Tactical Fire & TDA & Target Damage Assessment \\
\hline TACFIRE & Tactical Fire Direction System & TDA & Table Of Distribution And \\
\hline TACMS & Tactical Missile System (Navy) & & Allowances \\
\hline TACOM & Tactical Command & TDR & Test Data Report \\
\hline \multirow[t]{2}{*}{ TACOM } & Tank-Automotive \& Armaments & $\mathrm{TE}$ & Tactical Exploitation \\
\hline & Command & TECOM & Test And Evaluation Command \\
\hline TACON & Tactical Control & TEL & Transporter/Erector/Launcher \\
\hline TACP & Tactical Air Control Party & TELE-MED & Television Medicine \\
\hline TACSAT & Tactical Satellite & TEM/OPS & Terrain Evaluation \\
\hline TACSOP & $\begin{array}{l}\text { Tactical Standing Operating } \\
\text { Procedure }\end{array}$ & & $\begin{array}{l}\text { Module/Obstacle Planning } \\
\text { System }\end{array}$ \\
\hline TADARS & $\begin{array}{l}\text { Target Acquisition, Designation } \\
\text { And Reconnaissance System }\end{array}$ & TENCAP & $\begin{array}{l}\text { Tactical Exploitation Of National } \\
\text { Capabilities }\end{array}$ \\
\hline TAEDP & Total Army Equipment & TEXCOM & Test And Experimentation \\
\hline \multirow[t]{2}{*}{ TAFSM } & $\begin{array}{l}\text { Target Acquisition Fire Support } \\
\text { Model }\end{array}$ & TEXCOM & $\begin{array}{l}\text { U.S. Army Test And } \\
\text { Experimentation Command }\end{array}$ \\
\hline & & $\mathrm{TF}$ & Task Force \\
\hline
\end{tabular}




\begin{tabular}{|c|c|c|c|}
\hline \multirow{3}{*}{$\begin{array}{l}\text { TF FSE } \\
\text { TFAWE }\end{array}$} & Task Force Fire Support Element & TRAC-WSMR & TRADOC Requirements \\
\hline & Taskforce, Advanced & & Analysis Center - White Sands \\
\hline & Warfighting Experiment & & Missile Range (White Sands, \\
\hline TFC & Traffic & & NM) \\
\hline THAAD & $\begin{array}{l}\text { Theater High-Altitude Area } \\
\text { Defense }\end{array}$ & TRADOC & $\begin{array}{l}\text { U.S. Army Training And } \\
\text { Doctrine Command }\end{array}$ \\
\hline TI & Tactical Internet & TRANS & Transportation \\
\hline TIG & The Inspector General & TRANSCOM & U.S. Army Transportation \\
\hline \multirow[t]{2}{*}{ TIMS } & Tactical Internet Management & & Command \\
\hline & System & TRAS & Training Requirements Analysis \\
\hline TIR & Test Incident Report & & System \\
\hline TJAG & The Judge Advocate General & TRC & Type Requisition Code \\
\hline TLAM & Tactical Land Attack Missile & TRICAP & Triple Capabilities \\
\hline TLAM & Tomahawk Land Attack Missile & TRL & Technology Research Levels (1- \\
\hline TLP & Troop Leading Procedure & & 7) \\
\hline \multirow[t]{2}{*}{ TM } & (1) Theater Missile; (2) & TRN & Terrain \\
\hline & Technical Manual & TRP & Troop \\
\hline $\mathrm{TM}$ & Team & TRW & Thompson Ramo Wooldridge \\
\hline \multirow[t]{3}{*}{ TMAS } & (1) Thermal Mine Avoidance & & Company \\
\hline & System; (2) Tank Main & TSCD & Targeting Station Control And \\
\hline & Armament System & & Display \\
\hline TMD & Tactical Munitions Dispenser & TSFO & Training Set Fire Observation \\
\hline TMD & Theater Missile Defense & TSG & The Surgeon General \\
\hline \multirow[t]{2}{*}{ TMDE } & Test, Measurement, And & TSM & TRADOC System Manager \\
\hline & Diagnostic Equipment & TSMO & Threat Support Management \\
\hline TMM & Target Management Matrix & & Office \\
\hline TMS & Technology Maturation Strategy & TSOP & Tactical Standing Operating \\
\hline TNG & Training & & Procedure \\
\hline \multirow[t]{2}{*}{ TNGDEV } & Training Developer; Training & TSS & Topographic Support Systems \\
\hline & Development & TSS & Target Selection Standards \\
\hline TOA & Transfer Of Authority & TSTAR & Tomahak Stops The Attacking \\
\hline TOA & Total Obligational Authority & & Regiment \\
\hline TOC & Tactical Operations Center & TTHS & Transients, Trainees, Holdees, \\
\hline \multirow[t]{2}{*}{ TOE } & Table Of Organization And & & And Students \\
\hline & Equipment & TTL & Time To Live \\
\hline TOM-D & $\begin{array}{l}\text { Training, Operation, } \\
\text { Mobilization, And Deployment }\end{array}$ & TTP & $\begin{array}{l}\text { Tactics, Techniques, And } \\
\text { Procedures }\end{array}$ \\
\hline \multirow[t]{2}{*}{ TOPMIS } & Total Officer Personnel & TU & Traversing Unit \\
\hline & Management Information System & TUAV & Tactical Unmanned Aerial \\
\hline TOPO & Topographical & & Vehicle \\
\hline \multirow[t]{2}{*}{ TOPSS } & The Officer Projection Specialty & TUAV & Tactical Unattended Vehicle \\
\hline & System & U.S. & United States \\
\hline TOR & Terms Of Reference & UAD & Updated Authorizations \\
\hline \multirow[t]{2}{*}{ TOW } & Tube-Launched, Optically & & Document \\
\hline & Tracked, Wire-Guided Missile & UAM & Unattended Munitions \\
\hline TPF & Total Package Fielding & UAV & Unmanned Aerial Vehicle \\
\hline \multirow[t]{2}{*}{ TPFDD } & Time-Phased Force Deployment & UAV-CR & Unmanned Aerial Vehicle \\
\hline & Data & UAV-E & Unmanned Aerial Vehicle \\
\hline TPFDL & $\begin{array}{l}\text { Time-Phased Force Deployment } \\
\text { List }\end{array}$ & UAV-SR & $\begin{array}{l}\text { Unmanned Aerial Vehicle-Short } \\
\text { Range }\end{array}$ \\
\hline TPG & Troop Program Guidance & UAV-SR & Unmanned Aerial Vehicle \\
\hline TPM & Total Preventive Maintenance & $\mathrm{UCP}$ & Unified Command Plan \\
\hline TPS & Target Processing Section & UDP & User Datagram Protocol \\
\hline TPU & Troop Program Unit & UGS & Unmanned Ground Systems \\
\hline \multirow[t]{2}{*}{ TRAC } & TRADOC Analysis Command & UGS (BUGS) & Bug-Sized UGS \\
\hline & (U.S. Army) & UGS (MUGS) & Mobile UGS \\
\hline
\end{tabular}




\begin{tabular}{|c|c|c|c|}
\hline UGS (SLUGS) & Stationary UGS & USD(A\&T) & Under Secretary Of Defense For \\
\hline UGV & Unmanned Ground Vehicles & & Acquisition And Technology \\
\hline UH & Utility Helicopter & USD(Comptroller) & Under Secretary Of Defense For \\
\hline UIC & Unit Identification Code & & Comptroller \\
\hline UK & United Kingdom & USD(P\&R) & Under Secretary Of Defense For \\
\hline ULLS & Unit Level Logistics System & & Personnel And Readiness \\
\hline UML & Unified Modeling Language & $\mathrm{USD}(\mathrm{P})$ & Under Secretary Of Defense For \\
\hline \multirow[t]{2}{*}{ UMMCA } & Unspecified Minor Military & & Policy \\
\hline & Construction, Army & USEUCOM & U.S. European Command \\
\hline UMS & Unmanned Systems & USMC & U.S. Marine Corps \\
\hline UN & United Nations & USMTF & U.S. Message Text Format \\
\hline UNAAF & Unified Action Armed Forces & USMTF & U.S. Message Text Format \\
\hline \multirow[t]{2}{*}{ UPH } & Unaccompanied Personnel & USN & U.S. Navy \\
\hline & Housing & USPACOM & U.S. Pacific Command \\
\hline URN & Unit Reference Number & USPFO & U.S. Property And Fiscal Officer \\
\hline URS & Unit Reference Sheet & USPHS & U.S. Public Health Service \\
\hline US & United States & USR & Unit Status Report \\
\hline USA & U.S. Army & UTA & Unit Training Assembly \\
\hline USA & Under Secretary Of The Army & UTM & Universal Transverse Mercator \\
\hline \multirow[t]{2}{*}{ USAARMC } & U.S. Army Armor Center And & UTR & Unit Task Reorganization \\
\hline & School & UW & Unconventional Warfare \\
\hline USACE & U.S. Army Corps Of Engineers & VAAV & Vehicle Autonomous Air Vehicle \\
\hline USACGSC & $\begin{array}{l}\text { U.S. Army Command And } \\
\text { General Staff College }\end{array}$ & VCJCS & $\begin{array}{l}\text { Vice Chairman, Joint Chiefs Of } \\
\text { Staff }\end{array}$ \\
\hline USACMLS & U. S. Army Chemical School & VCSA & Vice Chief Of Staff, Army \\
\hline USAEC & U.S. Army Evaluation Center & VEH & Vehicle \\
\hline USAF & U.S. Air Force & VET & Veterinarian \\
\hline USAFAS & U.S. Army Field Artillery School & VGAS & Vertical Gun For Advanced \\
\hline \multirow[t]{2}{*}{ USAFMSA } & U.S. Army Force Management & & Ships (Navy) \\
\hline & Support Agency & VHSIC & Very High-Speed Integrated \\
\hline \multirow[t]{2}{*}{ USAIC\&FH } & U.S. Army Intelligence Center & & Circuit \\
\hline & And Fort Huachuca & VIC & Vector In Command \\
\hline \multirow[t]{2}{*}{ USAINSCOM } & U.S. Army Intelligence And & VLS & Vertical Launch System \\
\hline & Security Command & VMF & Variable Message Format \\
\hline \multirow[t]{2}{*}{ USAISC } & U. S. Army Information Systems & VMS & Virtual Memory System \\
\hline & Command & VSEL & Vickers Shipbuilding \& \\
\hline USAMEDCOM & U.S. Army Medical Command & & Engineering Limited \\
\hline \multirow[t]{2}{*}{ USAOTC } & U.S. Army Operational Test & VSTOL & Vertical Short Takeoffllanding \\
\hline & Command & VT & Variable Time \\
\hline \multirow[t]{2}{*}{ USAOTC } & U.S. Army Operational Test & VTOL & Vertical Take-Off And Landing \\
\hline & Command & VV\&A & Verification, Validation, And \\
\hline USAR & U.S. Army Reserve & & Accreditation \\
\hline USARC & U.S. Army Reserve Command & $\mathrm{w} /$ & With \\
\hline USAREC & U.S. Army Recruiting Command & WAM & Wide Area Mine \\
\hline USAREUR & U.S. Army Europe & WARF & Wartime Active Replacement \\
\hline USARF & U.S. Army Reserve Force & & Factors \\
\hline USARPAC & U.S. Army Pacific & WARLINK & Warrior Link \\
\hline \multirow[t]{2}{*}{ USASMDC } & U.S. Army Space And Missile & WARNO & Warning Order \\
\hline & Defense Command & WARSIM 2000 & Warfighter Simulation 2000 \\
\hline \multirow[t]{2}{*}{ USASOC } & U.S. Army Special Operations & WECM & Warfighter Electronic Collection \\
\hline & Command & & And Mapping (Operational \\
\hline \multirow[t]{2}{*}{ USATIC } & U. S. Army Technology & & Concept) \\
\hline & Integration Center & WFLA & Warfighting Lens Analysis \\
\hline USC & U.S. Code & WHL & Wheeled \\
\hline USCINCPAC & $\begin{array}{l}\text { U.S. Commander-In-Chief, } \\
\text { Pacific Command }\end{array}$ & WIA & Wounded In Action \\
\hline
\end{tabular}




$\begin{array}{ll}\text { WIDD } & \begin{array}{l}\text { Warfighting Integration \& } \\ \text { Development Directorate }\end{array} \\ \text { WIN } & \text { WWMCCS Intercomputer } \\ & \text { Network } \\ \text { WINS } & \text { Wireless Intelligence Network } \\ \text { WINT-T } & \text { Warfighter Information Network } \\ & - \text { Tactical } \\ \text { WMD } & \text { Weapons Of Mass Destruction } \\ \text { WOC } & \text { Wing Operation Center } \\ \text { WOF } & \text { Worthy Of Fielding } \\ \text { WP } & \text { White Phosphorous } \\ \text { WPNS } & \text { Weapons } \\ \text { WRAP } & \text { Warfighter Rapid Acquisition } \\ & \text { Program } \\ \text { WRSI } & \text { War Reserve Secondary Items } \\ \text { WSMR } & \text { White Sands Missile Range } \\ & \text { (White Sands, NM) } \\ \text { WSRO } & \text { Weapon System Replacement } \\ & \text { Operation } \\ \text { WUIS } & \text { Work Unit Information } \\ & \text { Summaries } \\ \text { WWI } & \text { World War I } \\ \text { WWII } & \text { World War II } \\ \text { WWMCCS } & \text { Worldwide Military Command } \\ \text { XM30 LCPK } & \text { And Control System } \\ \text { XM984 } & \text { Missile Low-Cost Precision Kill } \\ & \text { Extended-Range Mortar } \\ \text { XO } & \text { Cartridge } \\ \text { Z } & \text { Executive Officer } \\ & \text { Zulu } \\ & \end{array}$


Appendix C - U.S. Army BOS Diagrams.

This appendix contains a graphical representation (Gibbings, 1991) of the subfunction structure of each of the seven U.S. Army BOS's. The BOS do not represent Army branches or proponents, but rather, any type organization that relates to one or more of the seven BOS. 


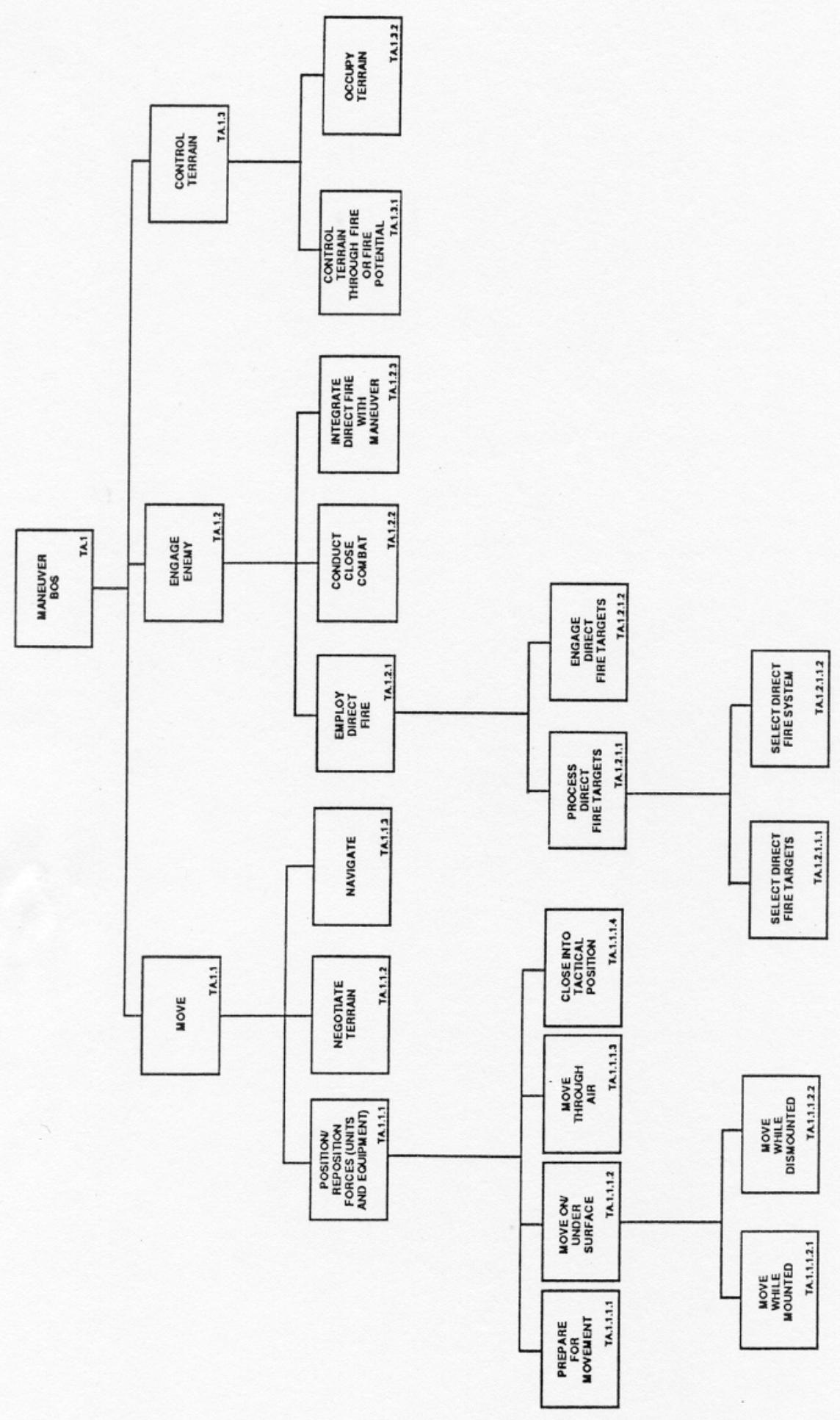

Figure 45 - Maneuver BOS 


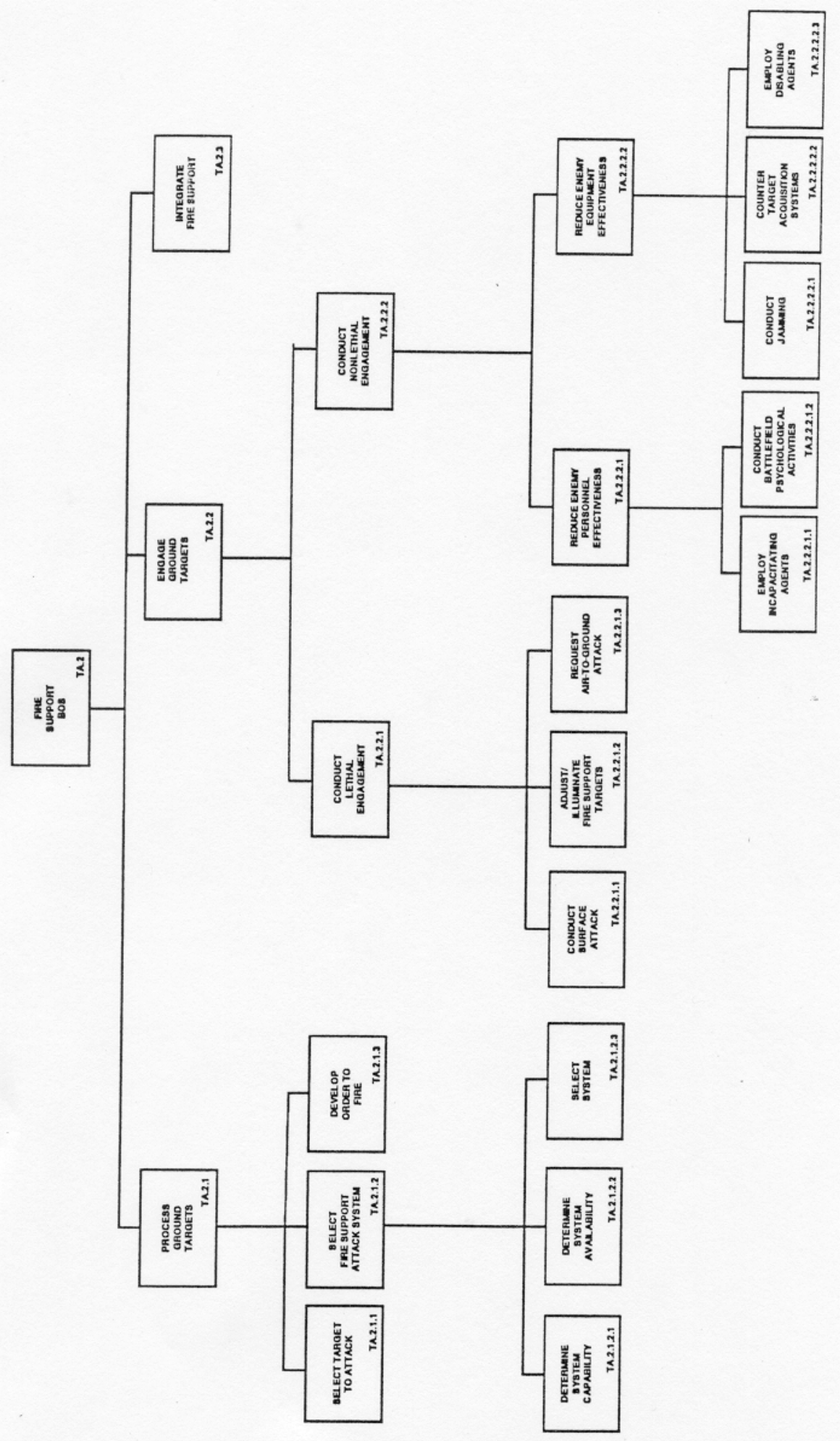

Figure 46 - Fire Support BOS 


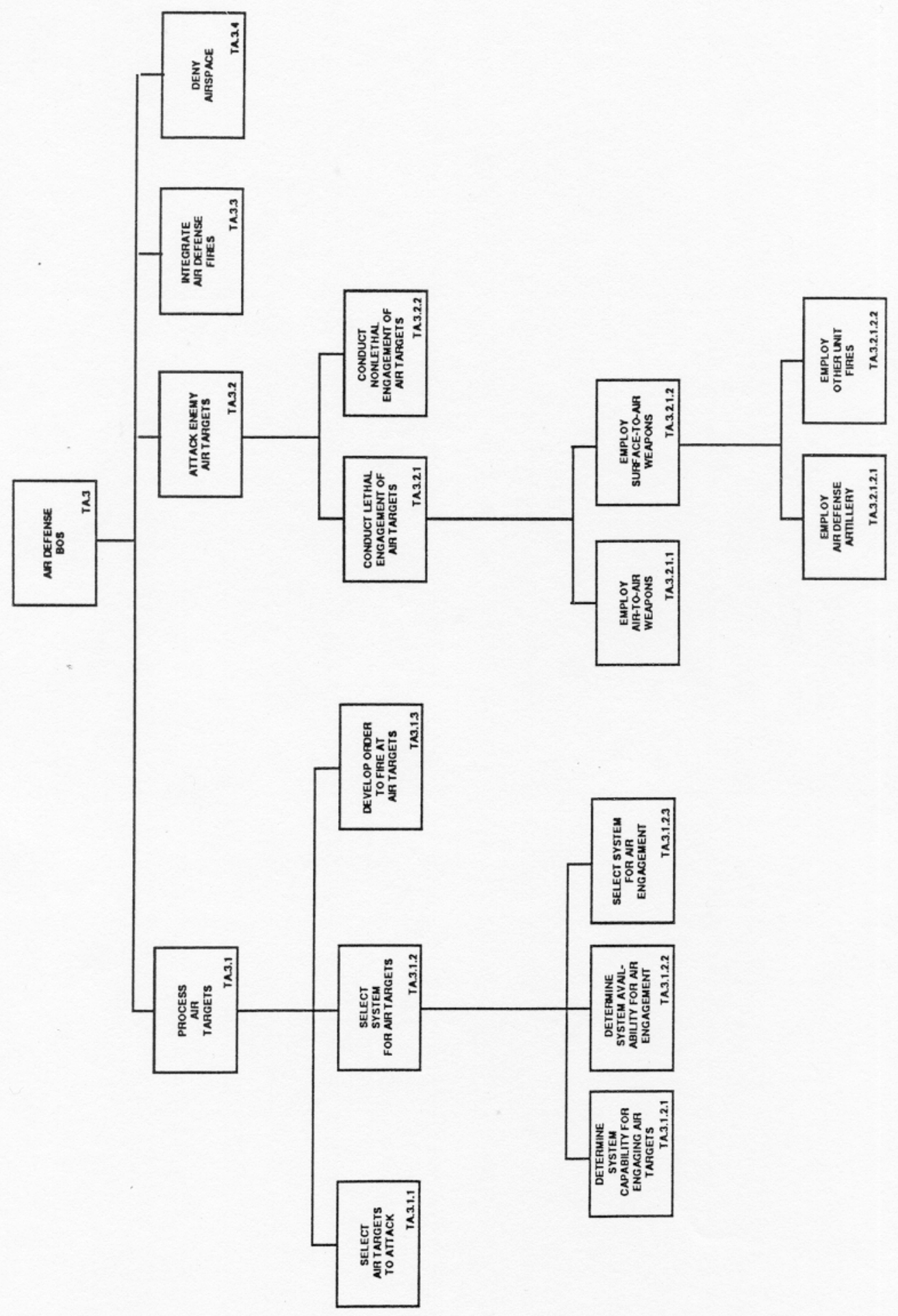

Figure 47 - Air Defense BOS 


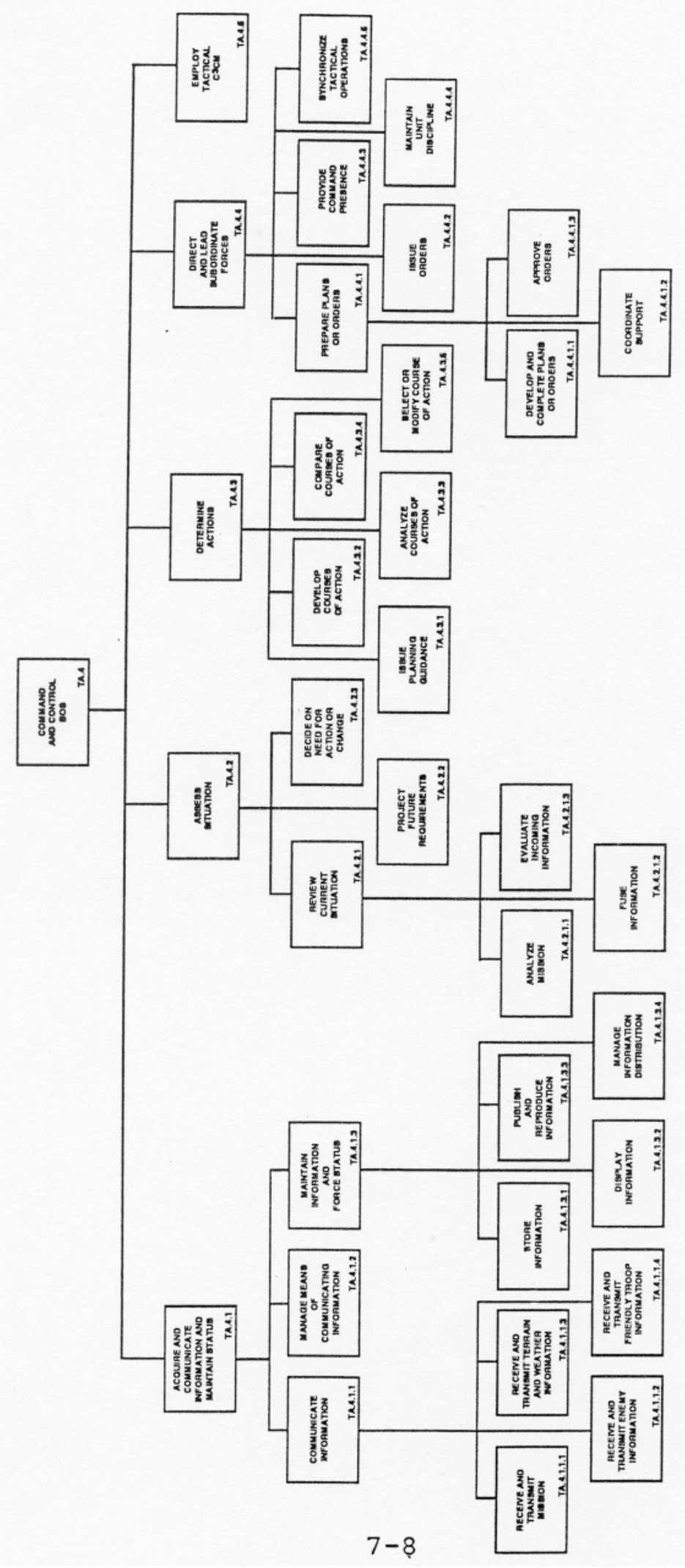

Figure 48 - Command And Control BOS 


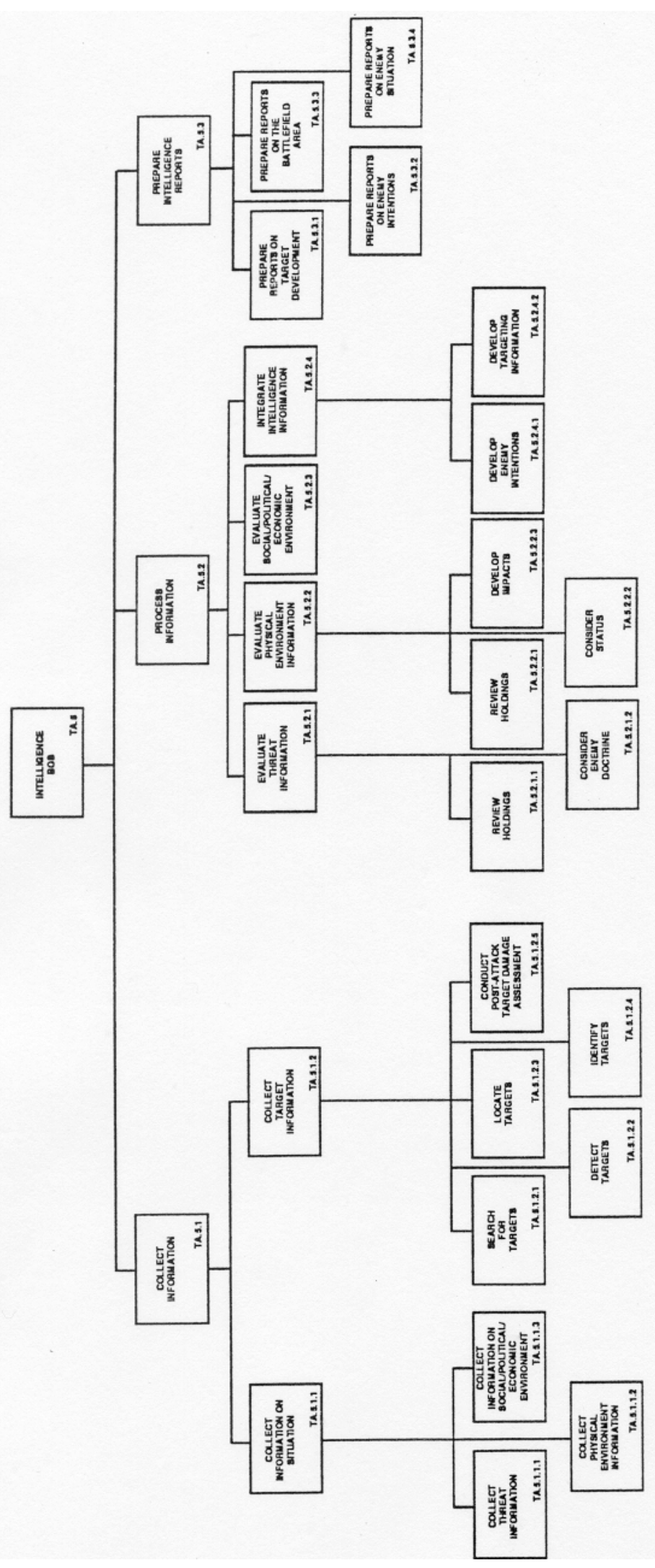

Figure 49 - Intelligence BOS 


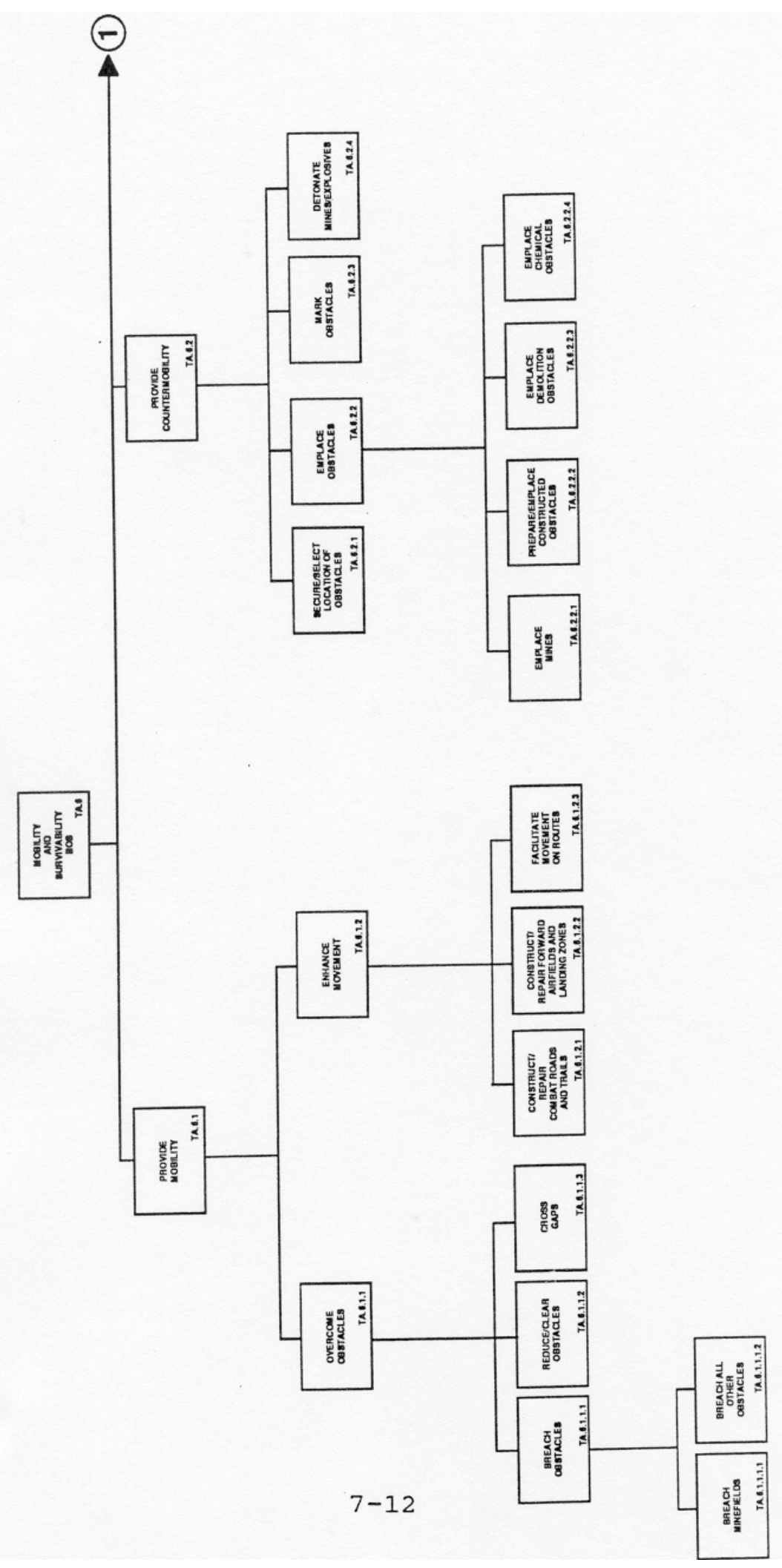

Figure 50 - Mobility And Survivability BOS - Part 1 


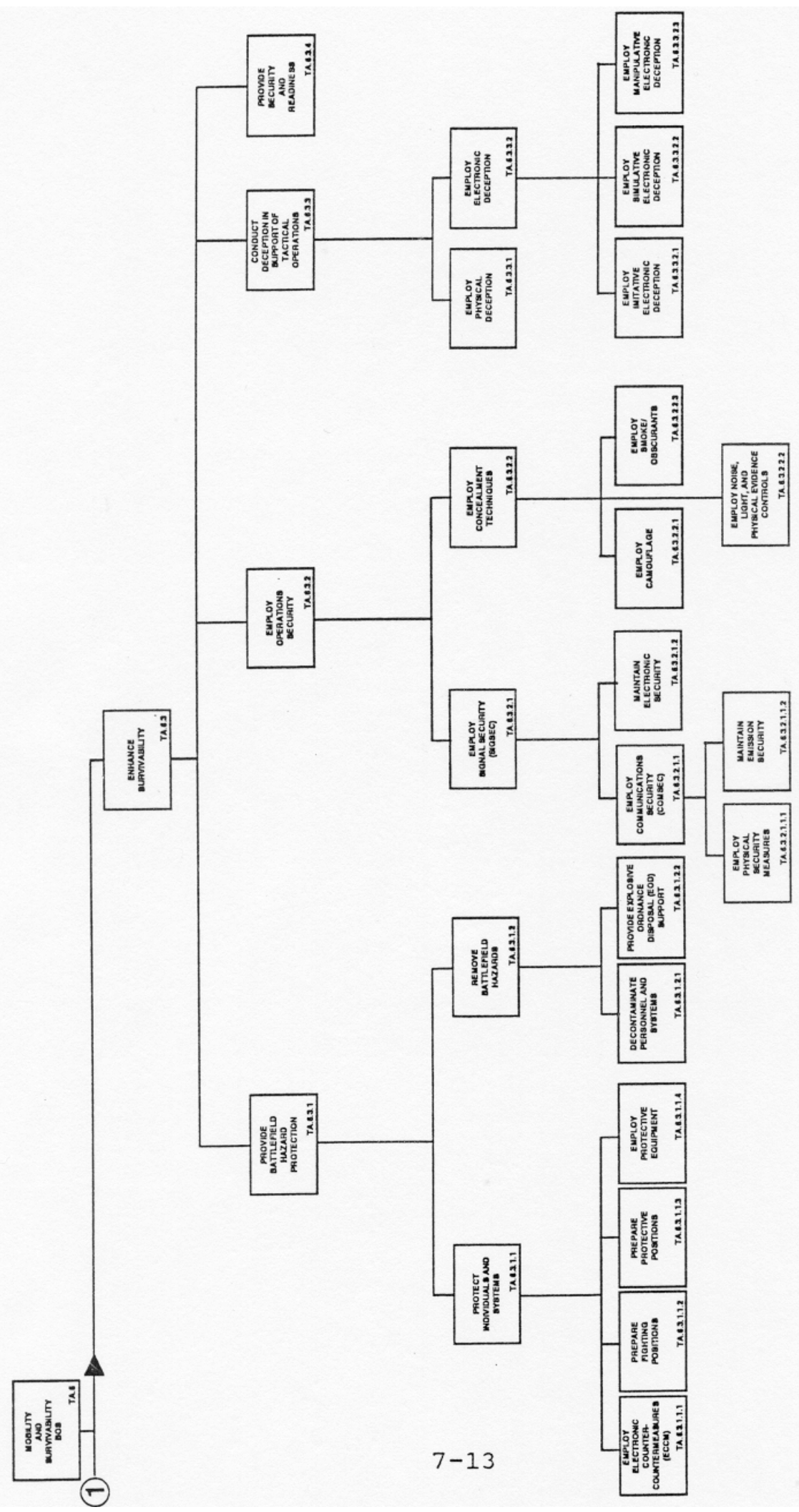

Figure 51 - Mobility And Survivability BOS - Part 2 


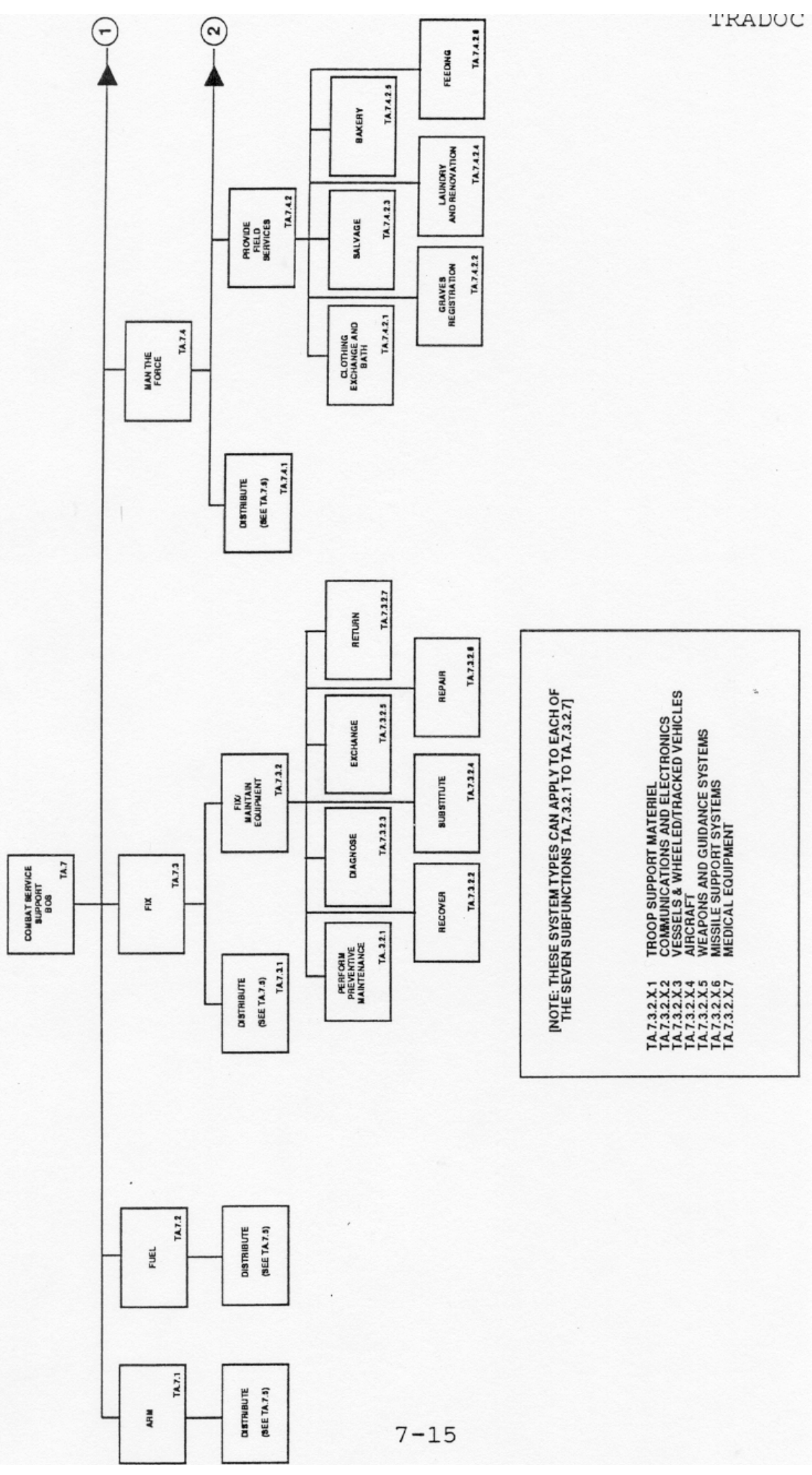

Figure 52 - Combat Service Support BOS - Part 1 


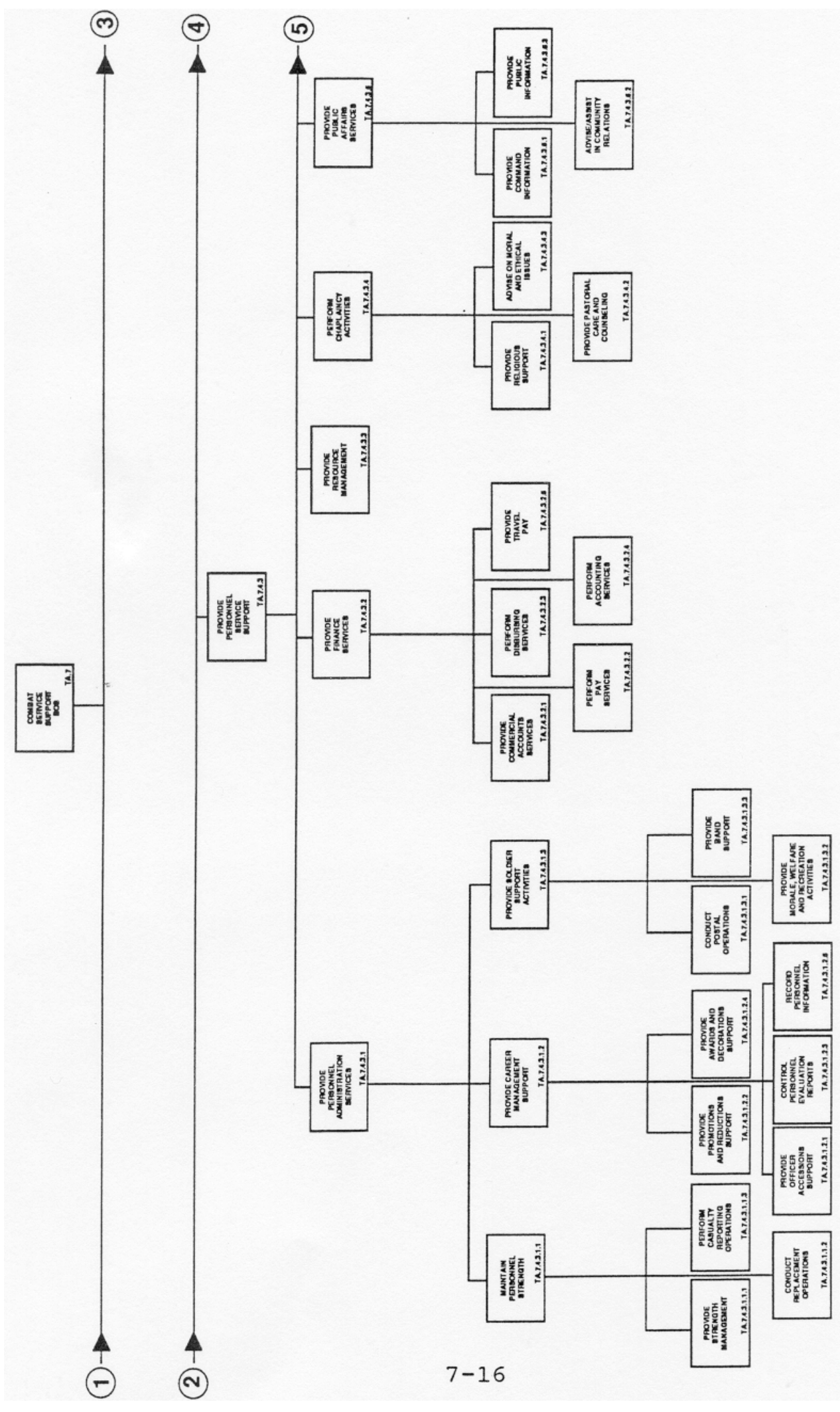

Figure 53 - Combat Service Support BOS - Part 2 


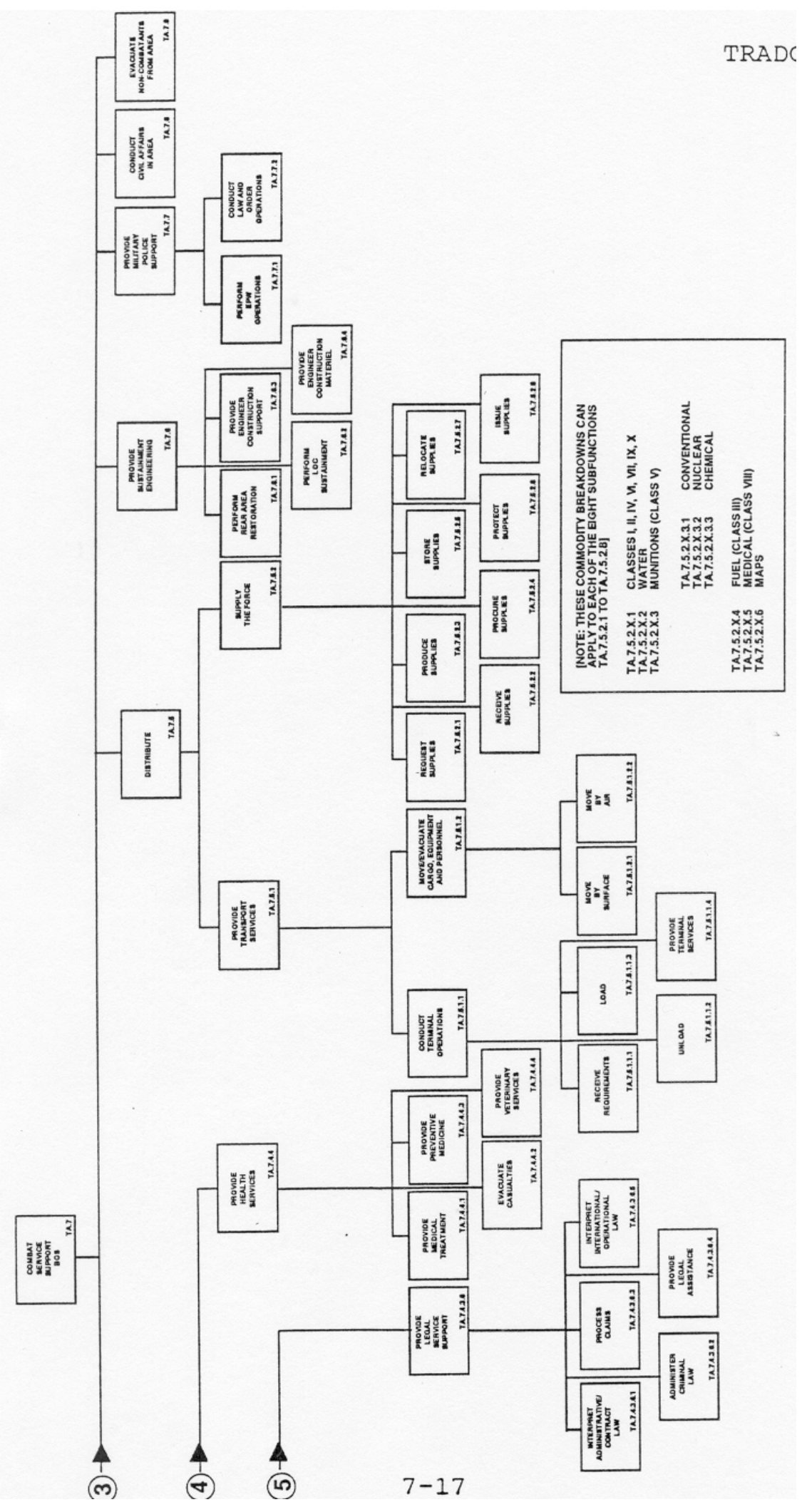

Figure 54 - Combat Service Support BOS - Part 3 
Appendix D - Critical Incident Interview Guide.

This interview guide provides a suggested framework for conducting interviews of system operators following the performance of a critical incident (Randel et al., 1996). It is useful in developing data as part of a cognitive task analysis while investigating intensive cognitive tasks such as decision making.

\section{Critical Incident Interview Guide}

(Randel et al., 1996)

A. Describe the purpose of the interview: "We want to learn how you make decisions while working on your job. Please respond accurately and completely and take your time. I would like to use the tape recorder in case I can't write everything down, if that is OK with you. No one outside this research group will hear the tape."

B. Give the interviewees some paper and a pencil in case they want to make a diagram.

C. Start the interview.

1. Select incident.

"I would like you to discuss some difficult decisions or events that occurred while you were performing your job. This could be a situation where an error was likely, or did in fact occur. Let's start with one such event."

2. Obtain unstructured incident account in the form of a time line.

'describe the incident from start to completion. I would like to reconstruct the events in the form of a time line that establishes the sequence of each event."

Repeat the incident back to the interviewee to make sure you have it correct.

3. Decision point identification.

"Now I would like you to help me identify specific decision points on the time line. A decision point occurs when someone else might have slchosen to act differently."

4. Decision point probing.

For each decision point, use the following probes.

a. Errors. "If an error occurred, what was the error?"

Hypothetical. "If an error occurred, how should the incident have proceeded?"

Missing data. "What would have helped you make the decision? What data or information was missing?" 
Error avoidance. "How could the error have been avoided?"

b. Cues/Situation Awareness. "What were you seeing or hearing that caused you to make this particular decision?"

c. Comparison to novice (if interviewing an expert). "How might someone with less experience have responded?"

d. Knowledge. "What information did you use in making this decision, and how was it obtained?"

e. Decision options.

(1) "What other choices did you consider or were available to you?"

\{If none, do not proceed with options.\}

List other choices:

(a) Restate first rejected option.

"What was the reason for rejecting it?"

(b) Restate each rejected option and ask the reason for rejecting it.

(2) Classification of the types of options elicited. "Were the options you considered standard or constructed?"

(a) Standard - ones you are commonly taught to consider.

(b) Constructed - created by you or changed from the standard in some way. (If so, "What experience was necessary to generate this option \{personal operator experience, special training, etc.\}?")

f. Rule of thumb. "If a new operator was standing beside you during this incident and asked you what rule (advice) you were following when you made this decision, what would you say?"

5. Additional incidents. "Can you recall another incident that was difficult or where an error might likely have occurred?" If so, repeat steps 2-5. 
Appendix E - Example of A Brigade Operations Order.

This is an example of a brigade operations order that was used in one of the exercises observed by this dissertation project. Except for the sanitization of names and locations reflecting fictional units, countries, and locations that was done by the unit to allow the exercise to be conducted in an unclassified arena, this is representative of the type of written commands that are generated prior to actual combat missions where the commander's guidance and intent is transmitted using the basic 5 paragraph operations order format.

Copy __ of _ copies
1BCT Headquarters, 9AD
FT HOOD, TX
211000 MAR 02

\section{$1^{\mathrm{ST}}$ BCT OPLAN 02-03-01}

Task Organization: See Annex A: Task Organization.

\section{SITUATION.}

a. Enemy Forces.

(1) Current Situation: Intelligence reports indicate that Gordian SPF UW elements from Gordo have infiltrated Kazar. The approximately 800 infiltrated personnel have attacked government buildings, conducted arson, kidnappings, and ethnic cleansing in the region. Several large 3 caches of small arms, communication equipment, and mines have been discovered The Gordian conventional military forces are capable of attacking with five brigade-size elements from $1^{\text {st }}$ Corps within three to six days. The Gordian conventional forces have made no move from their current positions, but intelligence sources indicate that there has been increased communication activity between Unconventional Forces within Kazar to elements in Gordo military units.

(2) Enemy most likely Course of action. TBP

b. Friendly Forces.

(1) JTF.

(a) Mission. Joint Task Force KAZAR deploys to Kazar and conducts military operations to restore stability to Kazar. On order, conduct hand over to United Nations Peacekeeping forces.

(b) Intent. Neutralize Gordian UW activities and ethnic Skandian hostilities directed against ethnic Gordians in Kazar, and be prepared to defeat cross-border incursions by Gordian Forces. 
(c) Concept. Omitted

\section{(2) ARFOR.}

(a) Mission. On order, the ARFOR deploys to conduct operations in KAZAR, conducts limited reception, staging, onward movement and integration (RSOI) defeats Gordian unconventional forces and deters employment of Gordian conventional forces, in order to stabilize KAZAR. On order, conducts hand-over to U.N. Peacekeeping forces and deploys.

(b) Intent. I intend to deploy ARFOR, in conjunction with the JTF, as quickly as possible to stabilize the situation in Kazar. I will use the $1 / 325^{\text {th }}$ Parachute Infantry Regiment to secure Pristina (Georgetown) Airfield. $1^{\text {st }}$ BCT will secure the city of Pristina and immediate environs, eliminating all aspects of the paramilitary threat. Follow-on forces will operate in the West and North, eliminating insurgent forces and deterring possible Gordian incursions in Kazar. Once I am satisfied that the situation is stabilized, I will recommend to the JTF the ARFOR transfer control of Kazar to U.N. forces and redeploy to home station.

(c) Concept of operations. This is a four phase operation:

- Phase I Entry Operations

Attack

- Phase II Defeat Gordian SPF and deter Gordian Conventional

- Phase III Transfer Control to Coalition Peacekeeping Forces

- Phase IV Redeployment

(d) Assumptions.

1. SPF activity remains low in AOR \#5 (Brigade Rear Area.

2. IBCT and $1^{\text {st }}$ KAZAR are able to protect West and East flanks.

(3) Adjacent Units.

(a) Left/West. IBCT: Remaining units of IBCT move north from Skopje (Austin) assembly areas to Kosovska/Mitrovica, establish presence, defeat SPF in sector, and prepare to counter incursions by 1 st Corps along the international border on a line generally from VUCIFRN PV0546, Kosovska/Mitrovica NV7838, and Colorado River NV5329.

(b) Right/East. $1^{\text {st }}$ Kazarian Brigade

(c) Front/North. Phase II: 1-10 CAV Relocate assets to Pec Airfield in order to support IBCT along the international border (IB). Conduct zone recon forward of Phase Line Kansas to GORDO/KAZAR IB to identify threat forces in zone, and to provide early warning to deploying brigades. Upon consolidation of objective (OBJ) JACK by IBCT, provide recon of IB between VUCIFRN on the east and the Colorado River on the west. Prepare to engage advancing GORDO units in IBCT sector. Priority of support is to IBCT. 
(d) Rear/South. Phase II: 2 BCT Upon arrival at Skopje, prepare to move north to Pristina and assume control from 1/325th PIR. Prepare to assume overwatch over area of approach (AA)1 and AA 2. Prepare to conduct operations to defeat Gordian SPF units in sector.

\section{MISSION. On order, $1^{\text {st }}$ BCT occupies AO Raider and defeats GORDIAN Special Forces units in sector in order to deter GORDIAN conventional forces. Be prepared to conduct defensive operations to defeat Gordian conventional forces within AO Raider. On order, conducts hand-over to U.N. Peacekeeping forces and re-deploy.}

\section{EXECUTION.}

Commander's Intent.

PURPOSE: The purpose of this operation is to stabilize the situation in AO Raider.

KEY TASKS: Occupy AO Raider

Defeat Gordian Special Police Force (SPF) units

Deter Gordian conventional attack

Be prepared to defeat conventional forces in sector.

ENDSTATE: Gordian SPF units rendered in-effective, situation stabilized in AO Raider. $1^{\text {st }}$ BCT prepared to defend against a Gordian military attack, United Nations Peacekeeping units in sector preparing for battle handover.

a. Concept of the Operation. The decisive point of this operation is completing the Brigade's show of force by occupying AO Raider. On order, $1^{\text {st }}$ BCT, $4^{\text {th }}$ ID (M) secures KAZAR City and establishes AORs (Areas of Responsibility) in sector in order to defeat Gordian SPF units and deter elements of Gordian conventional forces. The brigade occupies KAZAR city by establishing task force AORs with two Task Forces forward and one back. CLOSE: G/10 CAV occupies the $1 \mathrm{KM}$ buffer zone to identify Gordian SPF and insurgent forces and provide early warning to the BCT. 4th MP (-) $\left(1^{\text {st }}\right.$ and $2^{\text {nd }}$ Platoon supports civil authorities in the Brigade's exclusion zone in conjunction with the local police in order to prevent civil disturbances in sector. TF 1-22, the main effort, occupies AOR Regular in sector in order deter elements of Gordo from conducting insurgent operations. TF 1-66, supporting effort 1, occupies AOR IRON KNIGHT in sector in order to protect the flank of the Brigade main effort and protect the Brigade Support Area. 3-66 AR, supporting effort 2, occupies AOR LANCER and protects the eastern flank of the ME. Fires suppress SPF's to allow freedom of movement to the BCT's within AO Raider. REAR: A/95 ${ }^{\text {th }}$ MP protects the Brigade logistics and lines of communication in order to prevent insurgent activities in the Brigade Rear Areas.

(1) Scheme of Maneuver. 
(a) Phase I (Entry Operations). This phase begins when the Brigade moves north to Pristina to secure the city and immediate environs, eliminate all aspects of paramilitary threat and prepare for follow-on missions. This phase ends with the completed battle hand-over with the $1^{\text {st }} / 325$ PIR. (COMPLETED as of 24 MAR 02)

(b) Phase II (Defeat Gordian SPF and deter a GORDIAN conventional attack). This phase begins with the Brigade's occupation of AO Raider. Initially, the BRT occupies OPs along the $1 \mathrm{KM}$ buffer zone along the international boundary between KAZAR and GORDO. The Brigade occupies AO Raider in order to defeat GORDIAN SPF and deter a GORDIAN conventional attack. DEEP: The brigade employs information operations to isolate, disrupt, and demoralize insurgent forces in zone and influence GORDIAN military leadership to believe that ARFOR is capable of defeating attacks into KAZAR. Each TF has a CA/PSYOP/CI team in order to conduct information operations in their TF AOR. TFs will gain the confidence of local officials to include village mayors and leaders, police chiefs, religious leaders, business leaders, media and local citizens. TFs will identify NGOs (Non Governmental Organizations) and PVOs (Private Volunteer Organizations) in their AORs and provide reasonable support as appropriate. CLOSE: AO Raider consist of Task Forces occupying AORs. The TFs will defeat GORDIAN SPF teams by patrolling designated routes, establishing check points as necessary, seizing contraband, clearing routes of mines, and preventing crimes against humanity. Each TF will maintain one company positioned on camps near the international border as a show of force in order to deter GORDIAN attack and at least one tank or mech platoon as a QRF. TFs will patrol designated routes twice each day. Mission Essential Vulnerable Areas (MEVAs) will be checked at least twice a day. At all times, TFs will detain black (wanted for war crimes and terrorist activity) and grey (criminal activity) list personnel in order to stabilize the region. TF 122, the main effort, occupies AOR REGULAR north of KAZAR CITY in order to defeat GORDAIN SPF and deter GORDIAN conventional attack. TF 1-66, supporting effort, occupies AOR IRON KNIGHT in order to protect the flank of the Brigade main effort and protect the Brigade Support Area. 3-66 AR, supporting effort, occupies AOR LANCER to protect the eastern flank of the ME. The BRT occupies designated OPS along the $1 \mathrm{KM}$ zone to observe NAIs IOT provide early warning of cross-border invasion routes. The brigade accepts risks in the south with only A/95 MP CO to patrol MSRs in AOR 5 (Brigade Rear Area). TF 1-66 is prepared to provide a QRF to reinforce A/95 MPs as needed in AOR 5. The 4th MP (-) conducts multinational patrols with local police forces in OBJ KING (KAZAR CITY). TFs will have a howitzer battery in their AOR and maintain a minimum of platoon fire base ( 3 guns) and one hot gun (ready to fire immediately) at all times. TFs have an engineer company in their AOR to conduct mobility operations to clear routes and areas of mines and sustain LOCs and survivability operations to improve force protection measures in camps. The brigade is to be prepared to execute defensive CONPLANS in order to defeat GORDIAN conventional aggression. REAR: Each TF will maintain their FSE in their TF base camp. This phase ends with the transfer of control to the U.N. Peacekeeping forces.

(c) Phase III (Transfer Control to Coalition Peacekeeping Force). This phase begins with the transfer of control of KAZAR city to the U.N. peacekeeping forces. This phase ends with the completion of transfer of control to U.N. peacekeeping forces. 
(d) Phase IV (Redeployment). This phase begins with the redeployment of $1^{\text {st }}$ BCT soldiers and equipment. This phase ends with the safe return of all $1^{\text {st }}$ BCT soldiers and equipment to the continental U.S.

(2) Fires. See Annex D: Fire Support.

(3) Engineer. Engineers support all phases with mobility, survivability, and sustainment engineering operations. Engineer companies task organized with maneuver battalions ensure freedom of maneuver by clearing mines and obstacles off routes and conducting limited repairs to road surfaces and fording sites as required. High Value Assets (HVAs) are supported with survivability assets. Host nation contracted horizontal construction assets under the control of the $299 \mathrm{EN} \mathrm{BN} \mathrm{HQ}$ are prepared to conduct airfield and MSR repair operations. Engineers will employ the $759^{\text {th }}$ ORD (EOD) to identify, mark, report, and O/O destroy unexploded ordnance (UXOs) and coordinate for EOD support. All identified obstacles and UXOs not cleared will be turned over to A/307 EN (ABN). Engineers are prepared to transition to countermobility operations in the event Gordian conventional forces are identified crossing the border into KAZAR.

Essential Mobility Survivability Tasks:

T: Provide mobility to 1BCT forces.

P: Facilitate northward movement into AO Raider.

M: Clearing and maintaining open routes in AO Raider.

E: SPF don"t impede 1BCT occupation of AO Raider and support positive relations with local Kazarians.

(4) Reconnaissance \& Surveillance. See Annex L: R\&S.

(5) Air Defense. A/1-44 ADA (+) integrates and deploys with $1^{\text {st }}$ BCT, 4ID (M) to the Province of KAZAR and provides air defense, surveillance and general support throughout the established task force AORs. One Linebacker Platoon (1/A/1-44 ADA) with four Linebacker fire units provides direct support to TF 1-22 in AOR Regular. Fire units deploy with supported $\mathrm{CO} / \mathrm{TMs}$ and monitor AAAs within AOR Regular. Air defense coverage in AOR Regular is weighted forward and to the east monitoring the International Border and buffer zone in order to detect possible RW insurgency into the Province of KAZAR. One Linebacker Platoon (2/A/1-44 ADA) with four Linebacker fire units provides direct support to 3-66 in AOR Lancer. Air defense coverage in AOR Lancer is weighted to the northeast along AAAs 2a (along E Range Road heading south into KAZAR) and $2 b$ (moving through the Belton Reservoir from the northeast to the southwest) and in the east along 2c (following US Hwy 190 from the I-35 corridor to vic KAZAR CITY). One Avenger section (1/3/A/1-44 ADA) provides GS AD coverage vic BSA and Robert Gray Army Airfield (RGAAF) to TF 1-66 located in AOR IRON KNIGHT. One Avenger section (2/3/A/1-44 ADA) remains under BCT control and provides GS AD for HVTs (Killeen Municipal Airport and US HWY 190, Hood Army Airfield, BCT TOC) vic KAZAR CITY and the BCT Exclusion Zone. Sentinel 1 sets vicinity West Fort Hood and provides EW coverage vic AORs REGULAR and IRON KNIGHT and associated AAAs. Sentinel 2 sets vicinity Hood Army Airfield and Airfield Lake and provides EW coverage vic AOR LANCER, the BCT exclusion zone and associated AAAs. 


\begin{tabular}{|c|c|c|}
\hline UNIT & SUPPORTED UNIT & LOCATION \\
\hline \multirow[t]{3}{*}{ 1/A/1-44 ADA } & TF $1-22$ & 1 LB DS B/3-66 AOR Regular \\
\hline & & 2 LB DS A/1-22 AOR Regular \\
\hline & & 1 LB DS C/1-66 AOR Regular \\
\hline \multirow[t]{2}{*}{ 2/A/1-44 ADA } & $3-66$ & $\begin{array}{l}\text { 1/2/A/1-44 (2 LB) DS B/3-66 } \\
\text { AOR Lancer }\end{array}$ \\
\hline & & $\begin{array}{l}\text { 2/2/A/1-44 (2 LB) DS A/3-66 } \\
\text { AOR Lancer }\end{array}$ \\
\hline \multirow[t]{3}{*}{ 1/3/A/1-44 ADA } & TF 1-66 & 1 AVENGER VIC PV 092388 \\
\hline & & $\begin{array}{l}\text { 1 AVENGER VIC PV } 116405 \\
\text { (Crossville Mtn) }\end{array}$ \\
\hline & & $\begin{array}{l}\text { 1 AVENGER VIC PV } 088362 \\
\text { (Radar Hill) }\end{array}$ \\
\hline \multirow[t]{3}{*}{ 2/3/A/1-44 ADA } & $\begin{array}{l}\text { BCT, KAZAR } \\
\text { CITY }\end{array}$ & $\begin{array}{l}\text { 1 AVENGER VIC PV } 179488 \\
\text { (Blackwell Gap) }\end{array}$ \\
\hline & & $\begin{array}{l}\text { 1 AVENGER VIC PV } 262463 \\
\text { (Hood AAF) }\end{array}$ \\
\hline & & $\begin{array}{l}\text { 1 AVENGER VIC PV } 315379 \\
\text { (Hill 258) }\end{array}$ \\
\hline SENTINEL 1 & BCT & PV 156412 \\
\hline SENTINEL 2 & BCT & PV 241464 \\
\hline
\end{tabular}

b. Tasks to Subordinate Units.

(1) TF 1-22 (ME)

(a) Phase I.

1. Occupy Pristina in order to support stability operations.

2. $\mathrm{O} / \mathrm{O}$ conduct battle-hand over with $1^{\text {st }} / 325^{\text {th }}$ PIR of Pristina

Airfield.

(b) Phase II

activities.

1. Occupy AOR REGULAR in order to deter Gordo insurgent

2. Establish Task Force Base Camp within AOR REGULAR.

3. Establish CPs and Patrol Bases within AOR REGULAR as

necessary.

4. Conduct operations to defeat Gordian SPF units in sector.

5. Conduct platoon size patrols within AOR REGULAR.

6. Establish checkpoints in AOR REGULAR as required.

bases.

7. Limit civilian access to Task Force Base Camps, CPs, and patrol 
8. Stop and check civilians at all checkpoints IAW Annex E.

9. Seize contraband IAW Annex E.

10. Prevent crimes against humanity IAW Annex E.

11. Employ information operations to isolate, disrupt, and

demoralize insurgent forces in zone.

12. Gain confidence of the local officials to include village mayors and leaders, police chiefs, religious leaders, business leaders, media and local citizens.

13. Identify NGOs and PVOs in AORs and provide reasonable support as appropriate.

$(\mathrm{R} \& \mathrm{~S})$

14. Clear ROUTES BLUE and WHITE in AORs IAW Annex L

region.

15. Detain all black and grey list personnel in order stabilize the

REGULAR

16. Provide Platoon QRF to destroy SPF forces in AOR

17. BPT defeat conventional Gordian Forces in sector in EA METZ, BERLIN, and DRAGOON IOT deter a Gordian Conventional Attack.

(c) Phase III. O/O conduct hand over of KAZAR city with U.N.

Peacekeeping Forces

(d) Phase IV. O/O conduct redeployment.

(2) TF 1-66 (SE 1)

(a) Phase I. Occupy Pristina in order to support stability operations.

(b) Phase II

1. Occupy AOR IRON KNIGHT in order to deter Gordo insurgent

activities.

2. Establish Task Force Base Camp within AOR IRON KNIGHT.

3. Establish CPs and Patrol Bases within AOR IRON KNIGHT as

necessary.

4. Conduct operations to defeat Gordian SPF units in sector.

5. Conduct platoon size patrols within AOR IRON KNIGHT.

6. Establish checkpoints in AOR IRON KNIGHT as required.

bases.

7. Limit civilian access to Task Force Base Camps, CPs, and patrol

8. Stop and check civilians at all checkpoints IAW Annex E.

9. Seize contraband IAW Annex E.

10. Prevent crimes against humanity IAW Annex E.

11. Employ information operations to isolate, disrupt, and

demoralize insurgent forces in zone.

12. Gain confidence of the local officials to include village mayors and leaders, police chiefs, religious leaders, business leaders, media and local citizens.

support as appropriate.

13. Identify NGOs and PVOs in AORs and provide reasonable 
$(\mathrm{R} \& \mathrm{~S})$

14. Patrol ROUTES Yellow in AORs LANCER IAW Annex L

region.

15. Detain all black and grey list personnel in order stabilize the 16. BPT defeat conventional Gordian Forces in EA SICILY and EZ ANZIO IOT deter a Gordian Conventional Attack.

Peacekeeping Forces

(c) Phase III. O/O conduct hand over of KAZAR city with U.N.

(d) Phase IV. O/O conduct redeployment.

(3) 3-66 (SE 2)

(a) Phase I Occupy Pristina in order to support stability operations.

(b) Phase II

activities.

1. Occupy AOR LANCER in order to deter Gordo insurgent

2. Establish Task Force Base Camp within AOR LANCER.

3. Establish CPs and Patrol Bases within AOR LANCER as

necessary.

4. Conduct operations to defeat Gordian SPF units in sector.

5. Conduct platoon size patrols within AOR LANCER.

6. Establish checkpoints in AOR LANCER as required.

bases.

7. Limit civilian access to Task Force Base Camps, CPs, and patrol

8. Stop and check civilians at all checkpoints IAW Annex E.

9. Seize contraband IAW Annex E.

10. Prevent crimes against humanity IAW Annex E.

11. Employ information operations to isolate, disrupt, and demoralize insurgent forces in zone.

12. Gain confidence of the local officials to include village mayors and leaders, police chiefs, religious leaders, business leaders, media and local citizens.

13. Identify NGOs and PVOs in AORs and provide reasonable support as appropriate.

IAW Annex L (R\&S)

region.

14. Patrol ROUTES ORANGE and GREEN in AOR LANCER

15. Detain all black and grey list personnel in order stabilize the

16. BPT defeat conventional Gordian Forces in EA OMAHA IOT defeat a Gordian conventional attack into AO RAIDER. 
Peacekeeping Forces

(c) Phase III. O/O conduct hand over of KAZAR city with U.N.

(d) Phase IV. O/O conduct redeployment.

(4) G/10 CAV

(a) Phase I. Conduct zone reconnaissance north of PL KANSAS.

(b) Phase II.

1. Occupy OP and observe NAIs from along the $1 \mathrm{KM}$ buffer zone IOT provide early warning to the BCT.

2. Conduct platoon size patrols along ROUTE RED.

3. Gain confidence of the local officials to include village mayors and leaders, police chiefs, religious leaders, business leaders, media and local citizens.

support as appropriate.

4. Identify NGOs and PVOs in AORs and provide reasonable

5. Occupy OPs IAW Annex L (R\&S)

6. Observe NAIs IAW Annex L (R\&S)

7. BPT screen the KAZAR/GORDO border in the event of

GORDO conventional attack.

(c) Phase III. O/O conduct hand over of KAZAR city with U.N.

Peacekeeping Forces

(d) Phase IV. O/O conduct redeployment.

c. Task to Combat Support.

(1) 4-42 FA.

(a) Provide DS fires to $1^{\text {st }} \mathrm{BCT}$ in order to allow freedom of movement

with AO Raider.

(b) Provide 1 Paladin Battery to each Task Force AOR.

(2) $299 \mathrm{EN}$.

(a) Provide mobility support to maneuver battalions to include limited MSR and ford site repairs.

(b) Provide survivability support to HVAs (Q-36, Q-37).

(c) $\mathrm{O} / \mathrm{O}$ direct Host Nation horizontal construction assets to conduct

airfield and MSR repair.

(d) Identify, mark, record, report, and O/O destroy all UXOs.

UXOs not cleared.

(e) BPT conduct obstacle turn-over to A/307 EN BN on all obstacles and

(f) Coordinate for an EOD team and provide support to their operations. 
all necessary repairs.

(g) Track status of all MSRs, ASRs, and stream crossings and coordinate

(3) A/95th MP. Protect the Brigade logistics and lines of communication in order to prevent insurgent activities.

(4) A/104 ${ }^{\text {th }}$ MI. Provide FM RETRANS between GCS's at the BCT TOC and the TUAV launch/recovery site.

(5) $1 / 4^{\text {th }}$ MP. Conduct supports of civil authorities in the Brigade's exclusion zone in conjunction with the local police in order to prevent civil disturbances.

c. Coordinating Instructions.

(1) Task organization effective 250900 MAR 01

(2) This OPLAN is effective upon receipt.

(3) CCIR.

(a) PIR.

\begin{tabular}{|l|l|l|l|}
\hline \multicolumn{1}{|c|}{ PIR } & STATUS & $\begin{array}{c}\text { START / } \\
\text { STOP }\end{array}$ & \multicolumn{1}{|c|}{ INDICATORS / REMARKS } \\
\hline $\begin{array}{l}\text { 1. Where will SPF attempt to disrupt 1BCT } \\
\text { freedom of movement? }\end{array}$ & & & $\begin{array}{l}\text { riots, illegal checkpoints, sabotage, SPF HQ } \\
\text { vic KAZAR }\end{array}$ \\
\hline $\begin{array}{l}\text { 2. Will the 43d attack across the } \\
\text { International Border vic NAI R201, R203, } \\
\text { R132, R133, R133a, R136, R138, R217, } \\
\text { R217a? }\end{array}$ & & $\begin{array}{l}\text { 8 or more CBT armored vehicles moving } \\
\text { along route Black, greater than a Battery, } 5 \text { or } \\
\text { MICLICS/ACES, 4 or plows moving East }\end{array}$ \\
\hline $\begin{array}{l}\text { 3. Is the 54th repositioning vic KURSUMLIJA } \\
\text { NAI R301? }\end{array}$ & & & $30(+)$ APC, M60RCGL, M80ATGL \\
\hline $\begin{array}{l}\text { 4. Is the 25th repositiong South East of } \\
\text { KURSUMLIJA vic NAI R316, R311, R329? }\end{array}$ & & & \\
\hline
\end{tabular}

(b) EEFI.

1. Location of TUAV Launch and Recovery sites.

2. Location of Brigade TOC

(c) FFIR.

operations in sector?

1. Permission from ARFOR commander to transition to defensive

operations?

2. Are 2 of the $3 \mathrm{TF}$ able to transition from SASO to defensive

(d) CSIR. 
1. Any loss of a sensitive item.

2. SBF or terrorist incident in AO Raider.

3. Serious incidents or death of a BDE soldier

4. TF OR rate below $90 \%$.

5. Demonstration of 500 people or more in AO Raider.

6. Crash of ARFOR aircraft in AO Raider.

7. ROUTES not patrolled twice daily.

8. Closure of an MSR for more than 2 hours.

9. Any attack on a MEVA.

10. Any route not patrolled within required standard.

11. Arrival of any ARFOR or higher VIP.

12. Loss of contact of a patrol for more than 1 hour.

(5) Risk reduction and control Measures. ARFOR ground forces (less reconnaissance) will conduct operations in no smaller than platoon size elements. Platoon $=3$ vehicles with mounted automatic weapons. See Annex W.

(6) ROE. See Annex E.

(7) Force Protection. The ARFOR HQ will coordinate for host nation support (HNS) to defend APODs and SPODs. Unit commanders should conduct risk assessment that consider operational as well as environmental factors.

(8) System administrators and commanders at all levels will ensure compliance with policies and procedures to maintain Information Assurance.

(9) Commanders and staffs at all levels will ensure compliance with ARFOR Information Management Plan to support development of the common operating picture and facilitation of operational understanding of the battlespace.

(10) Commander, Joint Task Force (CJTF) retains authority for emplacement of all obstacles, conventional or FASCAM.

(11) All requests for obstacle emplacement to the CJTF must include specific details on the NAI, purpose, trigger, exact location, composition, decision points, and duration.

(12) Signal assets attached to maneuver units are OPCON'd for movement, security, and sustainment. Signal units remain under technical control (TECHCON) of the ARFOR signal battalion.

(13) Provide additions to graphic control measures or any changes in OPS to Raider Mike on Brigade Command.

(14) Units will have to make extensive use of their organic RETRANS teams due to the severe terrain. Ensure comprehensive PCI's are conducted prior to deploying these teams. 
(15) All units will test all digital systems during the BCT connectivity exercise during RSOI (Script TBP).

(16) Status of comms systems is due from all units to the Brigade S6 at the 1BCT TOC NLT 0300 and 1500 hrs daily.

(17) MOPP level 0 in effect 251900 MAR 02.

(18) ADA defense warning is White.

(19) Weapons control Status is Hold.

\section{SERVICE SUPPORT.}

a. Support Concept- $4^{\text {th }}$ FSB with attachments provides uninterrupted CSS to the 1 BCT to facilitate SASO operations and transition immediately to combat operations dependant on METT-T. Support to civilian personnel is limited to lifesaving medical treatment, emergency water and food only in the most extreme of circumstances.

(1) Support Area Locations

-BSA Location

-ALT BSA Location

-TFSA s are located in Maneuver BN Headquarters Base

(2) ARFOR Priority of Support: IBC, 1BCT, 1/325 PIR, and 2BCT(upon arrival)

(3) Priority of Support (SASO)- TF 1-22, TF 1-66, 3-66 AR

M2s, FA, and ADA

Maintenance- BRT/MP HMMWVs, CSS, Signal, ENG Equipment, M1s, Movement-MSR Patrols, Resupply Convoys, ENG, and CBT Vehicles Supply-CL I/Water, CL VIII, CL IX, CL III and CL V

(4) Priority of Support(Combat)- No Change

Maintenance-M1s, M2s, ENG, ADA, BRT, Signal, and CSS

Movement to FLOT-M1/M2, FA, ADA, ENG, Smoke, and CSS

Movement to Rear-Medical, empty fuelers and cargo vehicles, maintenance evacuation.

Priority of Supply-CL III, CL V, CL VIII, CL IX and CL I/Water

(5) Unique Support requirements

Man-Personnel replacements are processed through BDE ALCO and pushed forward to unit LRP. 
Arm-UBL on hand in each unit. Resupply vicinity the BSA

Fuel-Pushed to LRP vicinity the BSA by the $7^{\text {th }}$ CSG

Fix-CRTs with companies

Move-No transportation units available to augment

Sustain: Ration cycle (SASO) A-M-A, (Combat first 5 days) M-M-M, Bulk water available at BSA collocated with HDC.

(6) Host Nation Support - The ARFOR contracting officer is still negotiating HNS.

b. Material and Services

(1) Mortuary Affairs-One MA Team from the $7^{\text {th }}$ CSG is located in the BSA in the HDC area. Units are responsible for the transportation of remains to the BSA. Host nation remains are not processed by military personnel and are not to be transported in US Army vehicles

c. Medical Evacuation and Hospitalization

(1) Casualty Estimate

-SASO: 32 Per Day

-Combat: 55 Per Day

(2) Evacuation (SASO)- No AXPs active. Unit's will evacuate injured personnel to the nearest Level I facility located with a Maneuver BN HQs, Level II is located in BSA. Level III treatment facility, 134 CHS is located at the Port.

(3) Evacuation (Combat)-Two AXPs will be pushed out from BSA with one initially located at the intersection of MSR Pea and ASR Apple south of Kazar City and the other at the intersection of MSR Squash and MSR Brocolli.

(4) Evacuation Air- Attached FST has three MEDEVAC helicopters on call. BDE AL F403 Call Sign Dust Off. Use Army 9 line MEDEVAC Request. Aerial MEDVAC is used only to prevent loss of life, limb or eye-sight.

d. Personnel Support-A combined personnel/finance detachment from the $13^{\text {th }}$ PSB is located in the BSA for finance and administrative actions.

\section{COMMAND AND SIGNAL.}

a. Command.

(1) 1BCT TOC will be located at (PV 179467).

(2) 1BCT ALOC will be located at (PV 110390). 
(3) Command succession. TF 1-22 Cdr, TF 1-66 Cdr, 3-66 Cdr.

b. Signal.

(1) Signal Task Organization. See Annex A.

(2) ARFOR signal operating instructions (SOI) edition $\mathrm{xx}$ is in effect.

(3) All higher headquarter orders, OPLANS, FRAGOS, etc., will be posted on the ARFOR web page accessible via tactical network. 1BCT will post all brigade products to this web site.

(4) Tactical communications systems are the primary means of communication. (FM, FBCB2, MSE, ATCCS, Single-channel TACSAT). The host nation communications infrastructure can be used, but tactical communications will remain the primary means during all phases.

\section{ACKNOWLEDGE:}

\section{CAMPBELL}

COL

\section{OFFICIAL:}

WINKIE

S3
ANNEXES:
A - Task Organization
$\mathrm{B}$ - Intelligence
C - Operations Overlay
D - Fire Support
E - ROE
L - Reconnaissance \& Surveillance
$\mathrm{O}$ - Airspace Command and Control
$\mathrm{Q}-\mathrm{OPSEC}$
$\mathrm{U}$ - Civil Military Operations
W - Risk Assessment 
Appendix F - Checklist Procedure for Executing the Java Neural Network Simulation.

Using Java Neural Network Simulator (JNNS).

- The type of NNS being used here is a Multi-Layer Perceptron (MLP).

- There are three layers:

○ Input.

- One Hidden with 30 nodes.

○ Output.

- The learning algorithm is feed forward with back propagation causing the error value to propagate back up the network.

- The state learning parameters are:

- Learning rate (Dmax).

$\circ$ Level of error.

- Number of epochs.

- The method of pruning is to eliminate the non-contributing parameters.

1. To start JNNS - Execute JavaNNS.bat in whatever directory that JavaNNS-Win is set up in, for example:

C: \Documents and Settings\All Users $\backslash$ JavaNNS-Win $\backslash$ JavaNNS.bat

First a DOS window will appear and then the JavaNNS window.

2. Select $\underline{\text { View }}$ Network to display the network sub window.

3. Either open and existing file with the extension .net or create a new network data file.

4. Use Tools - Create - Layers to display the Create Layers sub window to create the nodes in the layers. The layers must be created in the following order:

a. Input.

b. Hidden.

c. Output.

5. In the Create layers sub window leave the width and height set to 1 . Set the Top left position to the coordinate on the network window where the new node will be placed when created. Set Unit type to either Input, Output or Hidden for the layer being created. Leave the defaults for Activation function and Output function. Set the Layer number to the number for the layer that the node is being created for (this must be set each time a node is created as the default will always be wrong). Use the following layer numbering assignments:

a. Input -1 .

b. Hidden - 2. Additional hidden layers may be 2,3,4, etc and then the output layer would be the last hidden layer plus 1 . By definition only one hidden layer is required. Some complex problems can be better solved with multiple hidden layers. There is no guidance on the number of hidden layers required. The rule is try one first, then try to increase the number of nodes in the hidden layer. If the 
network still does not train effectively then try increasing the number of hidden layers.

c. Output -3 . (One greater than the highest hidden layer).

6. Once the nodes have been created they can be named by right clicking on the node icon and selecting Edit Units... The edit units sub window appears where parameters for the node can be modified including the default name.

7. The information displayed above and below the node icon (Top label and Base label) can be set by selecting the Units and Links tab on the View - Display Settings pull down menu

8. To create the connections between all the nodes select Tools - Create - Connections. In the Create links sub window select the Connect feed - forward and not the With shortcut connections check boxes. Click the Connect button to execute the create connections command. Each link is created with a default link value of zero " 0 ".

9. Next need to load the data set. Go to the file open menu and select the pattern dataset with the extension of .pat. Check to see if it is loaded by Tools - control panel and the patterns tab. The dataset will show in both the Training set and the Validation set windows. If a different Validation set is to be used then load it and select which one is the training and which one is the validation set.

a. The .pat file was previously created from the observation data.

b. To create a validation file make a random selection of about $10-15 \%$ of the observation data and save it into a separate .pat file. Remove the validation patterns from the remaining training patterns. Renumber the pattern sequence for both the training and validation pattern files. Read them both in and indicate which one is for training and which one is for validation under the Learning tab in the control panel.

10. Next set the learning parameters.

a. Select tools - control panel and the learning tab.

b. Set learning function as desired - Backpropagation is typical when there are no direct links.

c. Set parameter $n$, the learning rate, typically to .1 or lower but greater than zero. If set too high then it learns fast then it could be stuck in local maximums and could not reach the true optimal value.

d. Set parameter dmax, the range of fluxuactions. If it is small then the learning curve becomes smooth. Typically set at .1 .

e. Set cycles (epochs) to 500 as a start to test the network- then increase to 1000 or even 10000. Always save before starting training in case it hangs up. Setting the cycles small gives a test to see if it will run. This works in conjunction with repeated uses of the learn all button.

f. Set steps to $1-$ steps are the number of cycles before the connection weights are updated. Always set to 1.

g. Check the shuffle check box. This changes the presentation order of the data patterns randomly for each cycle - randomizes the data order for each cycle. Always check this box. This may not be used for datasets that may contain time series data sets. Do not check for the learning function Backprop thru time.

h. Turn on the error graph with the selection View - Error Graph. 
i. Initialize the connection weights in the network by selecting the Init button in the control panel This assigns random initial weights to each connection. The weights will show on the network diagram.

j. Start the learning by selecting the learn all button in the control panel. Look at the error graph and if the end of the graph is still going down then repeat the learn all selection until the graph starts to flatten out. Can zoom in and out of the graph to look at the graph.

$\mathrm{k}$. If a validation dataset is used then there will be 2 curves on the error graph. The training set may continue to go down while the validation set will turn up. At the point of intersection is an indicator of where the network is starting to overtrain.

1. A validation dataset is created by randomly selecting datapoints from the training dataset. The validation set typically has about $10-20 \%$ of the original dataset. A validation set may also be created by collecting additional data.

$\mathrm{m}$. One iteration is running the network with a set of random initial values. Additional iterations can be run with different initial values. Each iteration will show as a separate line on the error graph. Can also change $\mathrm{n}$, the learning rate, to a lower number such as .05, and also dmax to a larger (as in .2) or smaller (.01) values and try again. It is important to try out multiple variations.

n. If an acceptable level of error cannot be achieved through multiple iterations then should increase the number of nodes in the hidden layer and repeat the process.

o. Once an acceptable level of error has been achieved with a flat line this indicates possible overtraining then the network is ready for pruning. A better method is to use a validation dataset and see where the graphs cross in the training and validation curves.

i. The first purpose of pruning is to eliminate the overtrained part of the network. If the response line being modeled has many curves then removing some of the nodes may cause a smoother response in the regression like model. This is analogous to removing regressors from a regression model in an attempt to smooth out the regression model.

ii. The second purpose is to eliminate noncontributing units (nodes) from the trained network. The first purpose is automatically achieved by doing this second purpose.

11. Pruning.

a. Save the network before pruning so additional pruning iterations can be run.

b. Select pruning tab in the control panel.

c. Select Method: Non - contributing Units as the typical selection. Can also use Magnitude based where one node is randomly thrown out and the network is tested to see if the functions call still occur.

d. Set Maximum error increase (\%): set to .001 - real low so that the network is not damaged. If set too high then most of the connections will be lost.

e. Set Accepted error: set to .001 - this is the level as shown on the error graph.

f. Set Cycles for retraining - start at 100 - this is the number of epochs that will be used in the pruning. Try higher numbers.

g. Set Minimum error to train - set to .001-

h. Check boxes:

i. Recreate last element - check - 
ii. Refresh display - check - the display will show animation during the pruning process.

iii. Prune input units - check - prunes input units / nodes.

iv. Prune hidden units - typically do not check if you are only interested in eliminating input nodes only to see their effect.

i. Select the Prune button. This step cannot be reversed so be sure to save the unpruned network before pruning so different input combinations can be tested.

\section{Questions:}

- Can additional nodes be added after the network has already been built and the connections made?

Nodes must be entered in the order input-hidden-output. For example to increase nodes in the input layer first delete the layers hidden and output and add them. Then recreate the hidden and output layers.

- Can they be added after the network has been trained? After it has been pruned? Yes, using the same rules and above

- Can node levels and other information be changed after a node has been created? Yes - use the edit units box and change the Unit type and layer number.

- What is the purpose of the shortcut connections and should they be used?

This type of neural network model is called multi-layer perceptron. Its purpose is to solve XOR problems is where two binary numbers exist with the rule that only when either is 1 then output is 1 - the exclusive or problem. Before the XOR problem could not be solved hence this type of network model. Direct connections of input to output help to solve the XOR problem. Generally do not use this option because it causes a much more complex network. If direct connections are used use the quick propagation learning algorithm. If direct connections are not used then use back propagation or back propagation with momentum which is the most popular one. 
Appendix G - Trained JNNS Output Node Values For TOC63 Simulation.

\begin{tabular}{|c|c|c|c|c|c|c|c|c|c|c|c|c|}
\hline Pattern & $\begin{array}{c}\text { Observed } \\
\text { 1- PT11 }\end{array}$ & $\begin{array}{c}\text { Observed } \\
\text { 2- РT20 }\end{array}$ & $\begin{array}{c}\text { Observed } \\
\text { 3- PT } 21\end{array}$ & $\begin{array}{c}\text { Observed } \\
\text { 4- ST } 12\end{array}$ & $\begin{array}{c}\text { Observed } \\
\text { 5- ST } 19\end{array}$ & $\begin{array}{c}\text { Observed } \\
6-\text { AT } 21\end{array}$ & $\begin{array}{c}\text { Calculated } \\
\text { 1- PT11 }\end{array}$ & $\begin{array}{c}\text { Calculated } \\
\text { 2- PT20 }\end{array}$ & $\begin{array}{c}\text { Calculated } \\
\text { 3- PT } 21\end{array}$ & $\begin{array}{c}\text { Calculated } \\
\text { 4- ST } 12\end{array}$ & $\begin{array}{c}\text { Calculated } \\
\text { 5- ST } 19\end{array}$ & $\begin{array}{c}\text { Calculated } \\
\text { 6- AT } 21\end{array}$ \\
\hline$\# 1$ & 0 & 0 & 1 & 0 & 0 & 0 & 0.0022 & 0.0011 & 0.9984 & 0.0045 & 0.0010 & 0.0066 \\
\hline$\# 2$ & 0 & 0 & 1 & 0 & 0 & 0 & 0.0031 & 0.0156 & 0.9829 & 0.0001 & 0.0151 & 0.0063 \\
\hline$\# 3$ & 1 & 0 & 0 & 1 & 0 & 1 & 0.9846 & 0.0363 & 0.0041 & 0.9642 & 0.0265 & 0.9821 \\
\hline$\# 4$ & 0 & 0 & 1 & 0 & 0 & 0 & 0.0124 & 0.0035 & 0.9919 & 0.0008 & 0.0129 & 0.0051 \\
\hline$\# 5$ & 0 & 0 & 1 & 0 & 0 & 0 & 0.0027 & 0.0326 & 0.9801 & 0.0011 & 0.0073 & 0.0025 \\
\hline$\# 6$ & 1 & 0 & 0 & 1 & 0 & 1 & 0.9912 & 0.0020 & 0.0160 & 0.9754 & 0.0138 & 0.9909 \\
\hline$\# 7$ & 1 & 0 & 0 & 1 & 0 & 1 & 0.9940 & 0.0014 & 0.0173 & 0.9890 & 0.0064 & 0.9895 \\
\hline$\# 8$ & 0 & 0 & 1 & 0 & 0 & 0 & 0.0062 & 0.0021 & 0.9961 & 0.0008 & 0.0060 & 0.0061 \\
\hline$\# 9$ & 0 & 0 & 1 & 0 & 0 & 0 & 0.0069 & 0.0062 & 0.9940 & 0.0153 & 0.0008 & 0.0052 \\
\hline$\# 10$ & 0 & 0 & 1 & 0 & 0 & 0 & 0.0055 & 0.0013 & 0.9994 & 0.0006 & 0.0057 & 0.0066 \\
\hline \#11 & 0 & 0 & 1 & 0 & 0 & 0 & 0.0074 & 0.0047 & 0.9887 & 0.0030 & 0.0030 & 0.0100 \\
\hline$\# 12$ & 0 & 0 & 1 & 0 & 0 & 0 & 0.0063 & 0.0053 & 0.9908 & 0.0068 & 0.0014 & 0.0066 \\
\hline \#13 & 1 & 0 & 0 & 1 & 0 & 1 & 0.9934 & 0.0020 & 0.0108 & 0.9654 & 0.0383 & 0.9921 \\
\hline$\# 14$ & 0 & 0 & 1 & 0 & 0 & 0 & 0.0043 & 0.0041 & 0.9966 & 0.0005 & 0.0060 & 0.0040 \\
\hline \#15 & 0 & 0 & 1 & 0 & 0 & 0 & 0.0099 & 0.0025 & 0.9925 & 0.0217 & 0.0022 & 0.0159 \\
\hline$\# 16$ & 1 & 0 & 0 & 1 & 0 & 1 & 0.9851 & 0.0024 & 0.0228 & 0.9820 & 0.0032 & 0.9836 \\
\hline$\# 17$ & 1 & 0 & 0 & 1 & 0 & 1 & 0.9952 & 0.0012 & 0.0139 & 0.9931 & 0.0059 & 0.9894 \\
\hline \#18 & 0 & 0 & 1 & 0 & 0 & 0 & 0.0028 & 0.0261 & 0.9854 & 0.0009 & 0.0054 & 0.0016 \\
\hline \#19 & 0 & 0 & 1 & 0 & 0 & 0 & 0.0061 & 0.0254 & 0.9709 & 0.0025 & 0.0115 & 0.0078 \\
\hline$\# 20$ & 0 & 1 & 0 & 0 & 0 & 0 & 0.0204 & 0.9403 & 0.0616 & 0.0012 & 0.0164 & 0.0111 \\
\hline$\# 21$ & 0 & 1 & 0 & 0 & 0 & 0 & 0.0170 & 0.9414 & 0.0380 & 0.0040 & 0.0038 & 0.0267 \\
\hline$\# 22$ & 1 & 0 & 0 & 1 & 0 & 1 & 0.9873 & 0.0044 & 0.0144 & 0.9579 & 0.0543 & 0.9908 \\
\hline$\# 23$ & 0 & 0 & 1 & 0 & 0 & 0 & 0.0022 & 0.0014 & 0.9983 & 0.0063 & 0.0010 & 0.0058 \\
\hline$\# 24$ & 0 & 0 & 1 & 0 & 0 & 0 & 0.0037 & 0.0143 & 0.9719 & 0.0042 & 0.0010 & 0.0038 \\
\hline$\# 25$ & 1 & 0 & 0 & 1 & 0 & 1 & 0.9827 & 0.0178 & 0.0018 & 0.9960 & 0.0018 & 0.9882 \\
\hline$\# 26$ & 0 & 0 & 1 & 0 & 0 & 0 & 0.0063 & 0.0057 & 0.9828 & 0.0007 & 0.0096 & 0.0082 \\
\hline$\# 27$ & 0 & 0 & 1 & 0 & 0 & 0 & 0.0091 & 0.0033 & 0.9963 & 0.0006 & 0.0216 & 0.0036 \\
\hline$\# 28$ & 0 & 0 & 1 & 0 & 0 & 0 & 0.0152 & 0.0078 & 0.9792 & 0.0115 & 0.0038 & 0.0199 \\
\hline$\# 29$ & 0 & 1 & 0 & 0 & 0 & 0 & 0.0199 & 0.9534 & 0.0434 & 0.0277 & 0.0008 & 0.0186 \\
\hline \#30 & 1 & 0 & 0 & 1 & 0 & 1 & 0.9905 & 0.0018 & 0.0083 & 0.9895 & 0.0064 & 0.9918 \\
\hline
\end{tabular}




\begin{tabular}{|c|c|c|c|c|c|c|c|c|c|c|c|c|}
\hline Pattern & $\begin{array}{l}\text { Input 1- } \\
\text { PT11 }\end{array}$ & $\begin{array}{c}\text { Input 2- } \\
\text { PT20 }\end{array}$ & $\begin{array}{c}\text { Input 3- } \\
\text { PT } 21\end{array}$ & $\begin{array}{c}\text { Input 4- } \\
\text { ST } 12\end{array}$ & $\begin{array}{l}\text { Input 5- } \\
\text { ST } 19\end{array}$ & $\begin{array}{c}\text { Input 6- } \\
\text { AT } 21\end{array}$ & $\begin{array}{l}\text { Output 1- } \\
\text { PT11 }\end{array}$ & $\begin{array}{l}\text { Output 2- } \\
\text { PT20 }\end{array}$ & $\begin{array}{l}\text { Output 3- } \\
\text { PT } 21\end{array}$ & $\begin{array}{l}\text { Output 4- } \\
\text { ST } 12\end{array}$ & $\begin{array}{l}\text { Output 5- } \\
\text { ST } 19\end{array}$ & $\begin{array}{c}\text { Output 6- } \\
\text { AT } 21\end{array}$ \\
\hline \#31 & 0 & 0 & 1 & 0 & 0 & 0 & 0.0125 & 0.0007 & 0.9959 & 0.0255 & 0.0011 & 0.0082 \\
\hline \#32 & 0 & 1 & 0 & 0 & 0 & 0 & 0.0189 & 0.9744 & 0.0194 & 0.0021 & 0.0108 & 0.0257 \\
\hline \#33 & 0 & 0 & 1 & 0 & 0 & 0 & 0.0040 & 0.0076 & 0.9916 & 0.0014 & 0.0034 & 0.0020 \\
\hline \#34 & 0 & 1 & 0 & 0 & 0 & 0 & 0.0137 & 0.9538 & 0.0415 & 0.0228 & 0.0006 & 0.0140 \\
\hline \#35 & 1 & 0 & 0 & 1 & 0 & 1 & 0.9880 & 0.0173 & 0.0014 & 0.9926 & 0.0072 & 0.9946 \\
\hline$\# 36$ & 0 & 0 & 1 & 0 & 0 & 0 & 0.0035 & 0.0065 & 0.9901 & 0.0053 & 0.0013 & 0.0090 \\
\hline \#37 & 0 & 0 & 1 & 0 & 0 & 0 & 0.0056 & 0.0025 & 0.9973 & 0.0017 & 0.0029 & 0.0055 \\
\hline \#38 & 1 & 0 & 0 & 1 & 0 & 1 & 0.9925 & 0.0060 & 0.0042 & 0.9964 & 0.0034 & 0.9903 \\
\hline$\# 39$ & 1 & 0 & 0 & 0 & 1 & 1 & 0.9709 & 0.0114 & 0.0169 & 0.0632 & 0.9267 & 0.9763 \\
\hline$\# 40$ & 0 & 0 & 1 & 0 & 0 & 0 & 0.0035 & 0.0167 & 0.9809 & 0.0017 & 0.0032 & 0.0042 \\
\hline$\# 41$ & 0 & 0 & 1 & 0 & 0 & 0 & 0.0032 & 0.0187 & 0.9820 & 0.0158 & 0.0005 & 0.0056 \\
\hline$\# 42$ & 1 & 0 & 0 & 1 & 0 & 1 & 0.9860 & 0.0046 & 0.0053 & 0.9910 & 0.0028 & 0.9898 \\
\hline$\# 43$ & 0 & 0 & 1 & 0 & 0 & 0 & 0.0017 & 0.0023 & 0.9973 & 0.0009 & 0.0028 & 0.0075 \\
\hline$\# 44$ & 0 & 0 & 1 & 0 & 0 & 0 & 0.0061 & 0.0767 & 0.9322 & 0.0018 & 0.0022 & 0.0080 \\
\hline$\# 45$ & 0 & 0 & 1 & 0 & 0 & 0 & 0.0092 & 0.0012 & 0.9983 & 0.0008 & 0.0237 & 0.0102 \\
\hline$\# 46$ & 0 & 0 & 1 & 0 & 0 & 0 & 0.0008 & 0.0078 & 0.9984 & 0.0005 & 0.0070 & 0.0013 \\
\hline$\# 47$ & 0 & 0 & 1 & 0 & 0 & 0 & 0.0110 & 0.0032 & 0.9867 & 0.0066 & 0.0023 & 0.0178 \\
\hline$\# 48$ & 1 & 0 & 0 & 1 & 0 & 1 & 0.9813 & 0.0057 & 0.0123 & 0.9615 & 0.0280 & 0.9791 \\
\hline$\# 49$ & 0 & 0 & 1 & 0 & 0 & 0 & 0.0022 & 0.0066 & 0.9958 & 0.0003 & 0.0199 & 0.0041 \\
\hline$\# 50$ & 1 & 0 & 0 & 1 & 0 & 1 & 0.9792 & 0.0028 & 0.0175 & 0.9734 & 0.0028 & 0.9768 \\
\hline \#51 & 0 & 0 & 1 & 0 & 0 & 0 & 0.0065 & 0.0046 & 0.9902 & 0.0108 & 0.0011 & 0.0070 \\
\hline$\# 52$ & 0 & 0 & 1 & 0 & 0 & 0 & 0.0081 & 0.0050 & 0.9874 & 0.0004 & 0.0217 & 0.0023 \\
\hline$\# 53$ & 0 & 0 & 1 & 0 & 0 & 0 & 0.0049 & 0.0050 & 0.9948 & 0.0054 & 0.0012 & 0.0058 \\
\hline$\# 54$ & 1 & 0 & 0 & 1 & 0 & 1 & 0.9901 & 0.0078 & 0.0081 & 0.9473 & 0.0573 & 0.9878 \\
\hline$\# 55$ & 1 & 0 & 0 & 0 & 1 & 1 & 0.9796 & 0.0154 & 0.0227 & 0.0691 & 0.9078 & 0.9732 \\
\hline$\# 56$ & 0 & 0 & 1 & 0 & 0 & 0 & 0.0198 & 0.0279 & 0.9619 & 0.0122 & 0.0021 & 0.0123 \\
\hline \#57 & 1 & 0 & 0 & 1 & 0 & 1 & 0.9919 & 0.0039 & 0.0100 & 0.9949 & 0.0050 & 0.9893 \\
\hline$\# 58$ & 1 & 0 & 0 & 1 & 0 & 1 & 0.9936 & 0.0043 & 0.0031 & 0.9771 & 0.0417 & 0.9899 \\
\hline \#59 & 1 & 0 & 0 & 1 & 0 & 1 & 0.9957 & 0.0012 & 0.0112 & 0.9917 & 0.0063 & 0.9945 \\
\hline
\end{tabular}




\section{Appendix H - SAS Pearson Correlation Analysis of JNNS Observed to Trained DV Data.}

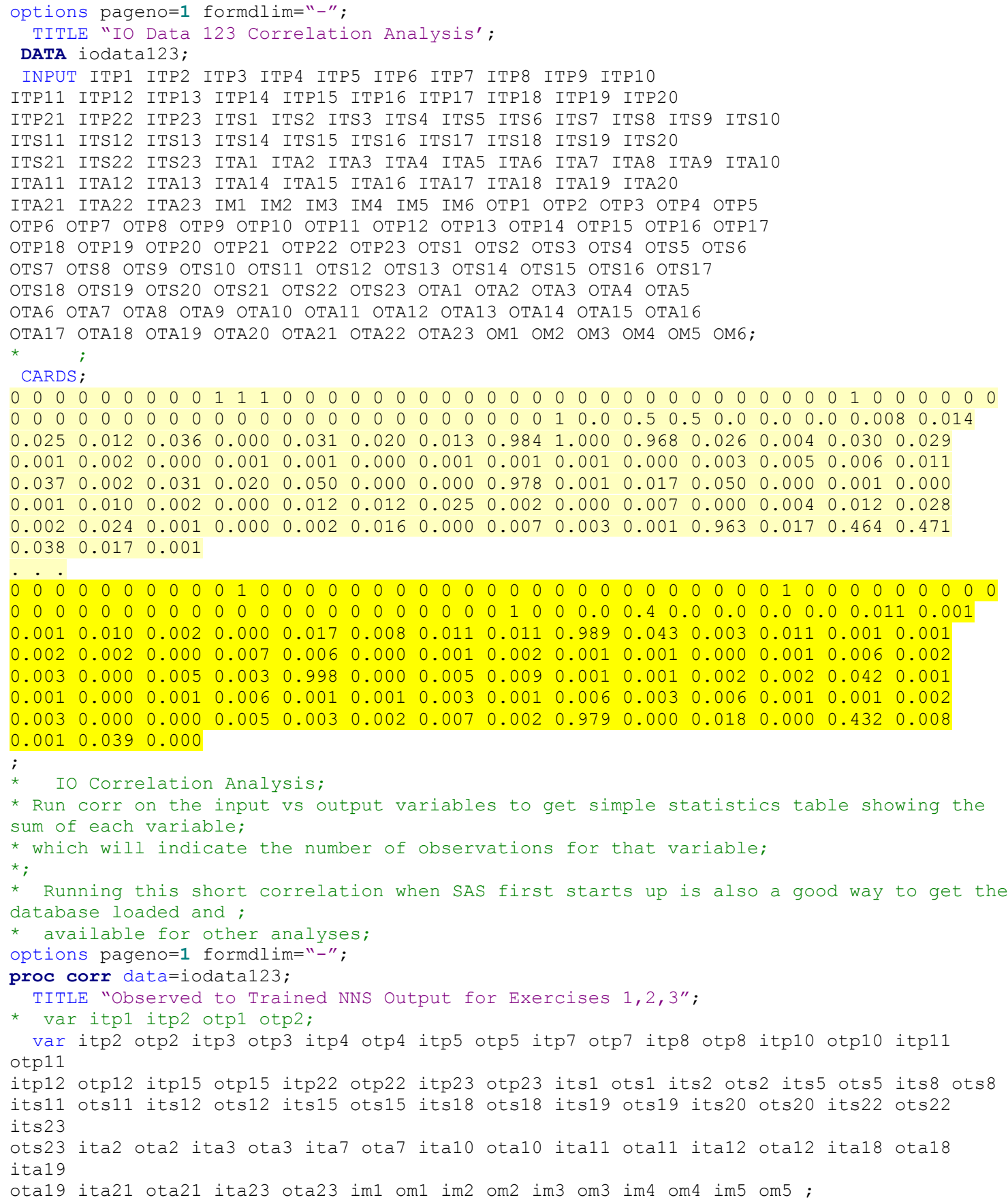


Output:

\begin{tabular}{lllllll}
\multicolumn{2}{c}{ Observed to Trained NNS Output for Exercises $1,2,3$} \\
$07: 43$ Wednesday, January 15,2003
\end{tabular}




\begin{tabular}{|c|c|c|c|c|c|c|}
\hline Variable & $\mathrm{N}$ & Mean & Std Dev & Sum & Minimum & Maximum \\
\hline ITP2 & 139 & 0.00719 & 0.08482 & 1.00000 & 0 & 1.00000 \\
\hline 0TP2 & 139 & 0.00553 & 0.00918 & 0.76900 & 0 & 0.05000 \\
\hline ITP3 & 139 & 0.04317 & 0.20396 & 6.00000 & 0 & 1.00000 \\
\hline ОTP3 & 139 & 0.04994 & 0.19439 & 6.94200 & 0 & 0.97300 \\
\hline ITP4 & 139 & 0.00719 & 0.08482 & 1.00000 & 0 & 1.00000 \\
\hline OTP4 & 139 & 0.01293 & 0.07550 & 1.79700 & 0 & 0.88400 \\
\hline ITP5 & 139 & 0.04317 & 0.20396 & 6.00000 & 0 & 1.00000 \\
\hline 0TP5 & 139 & 0.04859 & 0.19338 & 6.75400 & 0 & 0.96200 \\
\hline ITP7 & 139 & 0.05755 & 0.23374 & 8.00000 & 0 & 1.00000 \\
\hline 0TP7 & 139 & 0.06871 & 0.22446 & 9.55100 & 0 & 0.99400 \\
\hline ITP8 & 139 & 0.01439 & 0.11952 & 2.00000 & 0 & 1.00000 \\
\hline 0TP8 & 139 & 0.02480 & 0.11118 & 3.44700 & 0.00100 & 0.94500 \\
\hline ITP10 & 139 & 0.10791 & 0.31139 & 15.00000 & 0 & 1.00000 \\
\hline OTP10 & 139 & 0.11249 & 0.30231 & 15.63600 & 0 & 0.99900 \\
\hline ITP11 & 139 & 0.43165 & 0.49710 & 60.00000 & 0 & 1.00000 \\
\hline OTP11 & 139 & 0.43596 & 0.47717 & 60.59900 & 0 & 1.00000 \\
\hline ITP12 & 139 & 0.08633 & 0.28187 & 12.00000 & 0 & 1.00000 \\
\hline 0TP12 & 139 & 0.09551 & 0.26966 & 13.27600 & 0 & 0.99600 \\
\hline ITP15 & 139 & 0.09353 & 0.29222 & 13.00000 & 0 & 1.00000 \\
\hline OTP15 & 139 & 0.09894 & 0.28060 & 13.75200 & 0 & 0.99700 \\
\hline ITP22 & 139 & 0.05036 & 0.21948 & 7.00000 & 0 & 1.00000 \\
\hline оTP22 & 139 & 0.05629 & 0.21142 & 7.82500 & 0 & 0.99800 \\
\hline ITP23 & 139 & 0.10791 & 0.31139 & 15.00000 & 0 & 1.00000 \\
\hline 0TP23 & 139 & 0.11085 & 0.29980 & 15.40800 & 0 & 0.99800 \\
\hline ITS1 & 139 & 0.00719 & 0.08482 & 1.00000 & 0 & 1.00000 \\
\hline OTS1 & 139 & 0.01226 & 0.07887 & 1.70400 & 0 & 0.92800 \\
\hline ITS2 & 139 & 0.04317 & 0.20396 & 6.00000 & 0 & 1.00000 \\
\hline OTS2 & 139 & 0.05004 & 0.19682 & 6.95500 & 0 & 0.99000 \\
\hline ITS5 & 139 & 0.04317 & 0.20396 & 6.00000 & 0 & 1.00000 \\
\hline OTS5 & 139 & 0.04932 & 0.19380 & 6.85500 & 0 & 0.97900 \\
\hline ITS8 & 139 & 0.00719 & 0.08482 & 1.00000 & 0 & 1.00000 \\
\hline OTS8 & 139 & 0.00738 & 0.01001 & 1.02600 & 0 & 0.05000 \\
\hline ITS11 & 139 & 0.03597 & 0.18689 & 5.00000 & 0 & 1.00000 \\
\hline OTS11 & 139 & 0.04899 & 0.17669 & 6.81000 & 0 & 0.96800 \\
\hline ITS12 & 139 & 0.20144 & 0.40253 & 28.00000 & 0 & 1.00000 \\
\hline OTS12 & 139 & 0.20658 & 0.38491 & 28.71400 & 0 & 0.99800 \\
\hline ITS15 & 139 & 0.02878 & 0.16778 & 4.00000 & 0 & 1.00000 \\
\hline OTS15 & 139 & 0.03504 & 0.16019 & 4.87100 & 0 & 0.97800 \\
\hline ITS18 & 139 & 0.10072 & 0.30205 & 14.00000 & 0 & 1.00000 \\
\hline OTS18 & 139 & 0.10267 & 0.28929 & 14.27100 & 0 & 0.98100 \\
\hline ITS19 & 139 & 0.08633 & 0.28187 & 12.00000 & 0 & 1.00000 \\
\hline OTS19 & 139 & 0.09393 & 0.26919 & 13.05600 & 0 & 0.99200 \\
\hline ITS20 & 139 & 0.01439 & 0.11952 & 2.00000 & 0 & 1.00000 \\
\hline OTS20 & 139 & 0.02371 & 0.10744 & 3.29600 & 0 & 0.91900 \\
\hline ITS22 & 139 & 0.01439 & 0.11952 & 2.00000 & 0 & 1.00000 \\
\hline OTS22 & 139 & 0.02383 & 0.11025 & 3.31200 & 0 & 0.92800 \\
\hline ITS23 & 139 & 0.12950 & 0.33696 & 18.00000 & 0 & 1.00000 \\
\hline OTS23 & 139 & 0.13570 & 0.32150 & 18.86200 & 0 & 0.99000 \\
\hline ITA2 & 139 & 0.00719 & 0.08482 & 1.00000 & 0 & 1.00000 \\
\hline OTA2 & 139 & 0.01717 & 0.08006 & 2.38600 & 0 & 0.94300 \\
\hline ITA3 & 139 & 0.08633 & 0.28187 & 12.00000 & 0 & 1.00000 \\
\hline ОТАЗ & 139 & 0.09373 & 0.27009 & 13.02800 & 0 & 0.99100 \\
\hline ITA7 & 139 & 0.04317 & 0.20396 & 6.00000 & 0 & 1.00000 \\
\hline
\end{tabular}




Variable
OTA7
ITA10
OTA10
ITA11
OTA11
ITA12
OTA12
ITA18
OTA18
ITA19
OTA19
ITA21
OTA21
ITA23
OTA23
IM1
OM1
IM2
OM2
IM3
OM3
IM4
OM4
IM5
OM5

$\mathrm{N}$
139
139
139
139
139
139
139
139
139
139
139
139
139
139
139
139
139
139
139
139
139
139
139
139
139

\begin{tabular}{ccc}
\multicolumn{3}{c}{ Simple Statistics } \\
0.05236 & Std Dev & Sum \\
0.01439 & 0.19442 & 7.27800 \\
0.02231 & 0.11952 & 2.00000 \\
0.05036 & 0.21948 & 7.10100 \\
0.05843 & 0.20806 & 8.12200 \\
0.02158 & 0.14584 & 3.00000 \\
0.02882 & 0.13936 & 4.00600 \\
0.05036 & 0.21948 & 7.00000 \\
0.05845 & 0.20810 & 8.12500 \\
0.02158 & 0.14584 & 3.00000 \\
0.03160 & 0.13681 & 4.39300 \\
0.15827 & 0.36632 & 22.00000 \\
0.16578 & 0.35213 & 23.04400 \\
0.19424 & 0.39705 & 27.00000 \\
0.19943 & 0.38212 & 27.72100 \\
0.16835 & 0.33211 & 23.40000 \\
0.17009 & 0.32012 & 23.64300 \\
0.22158 & 0.20913 & 30.80000 \\
0.23159 & 0.19982 & 32.19100 \\
0.08417 & 0.22320 & 11.70000 \\
0.09353 & 0.21926 & 13.00000 \\
0.00576 & 0.04781 & 0.80000 \\
0.01485 & 0.04307 & 2.06400 \\
0.07050 & 0.21551 & 9.80000 \\
0.07935 & 0.20647 & 11.02900
\end{tabular}

$\begin{array}{cc}\text { Minimum } & \text { Maximum } \\ 0 & 0.97800 \\ 0 & 1.00000 \\ 0 & 0.98700 \\ 0 & 1.00000 \\ 0 & 0.97800 \\ 0 & 1.00000 \\ 0 & 0.96900 \\ 0 & 1.00000 \\ 0 & 0.97100 \\ 0 & 1.00000 \\ 0 & 0.95000 \\ 0 & 1.00000 \\ 0 & 0.99400 \\ 0 & 1.00000 \\ 0 & 0.99800 \\ 0 & 1.00000 \\ 0 & 0.99200 \\ 0 & 0.60000 \\ 0 & 0.64400 \\ 0 & 0.85000 \\ 0 & 0.89800 \\ 0 & 0.40000 \\ 0 & 0.36300 \\ 0 & 0.90000 \\ 0 & 0.92800\end{array}$


07:43 Wednesday, January 15, 2003

The CORR Procedure

Pearson Correlation Coefficients, $N=139$

Prob $>|r|$ under $\mathrm{HO}$ : Rho $=0$

\begin{tabular}{|c|c|c|c|c|c|c|c|c|c|}
\hline & ITP2 & 0TP2 & ITP3 & ОТР3 & ITP4 & OTP4 & ITP5 & 0TP5 & ITP7 \\
\hline \multirow[t]{2}{*}{ ITP2 } & 1.00000 & -0.05150 & 0.40078 & 0.39557 & -0.00725 & -0.00897 & -0.01808 & -0.01351 & -0.02104 \\
\hline & & 0.5471 & $<.0001$ & $<.0001$ & 0.9325 & 0.9165 & 0.8327 & 0.8745 & 0.8058 \\
\hline \multirow[t]{2}{*}{ 0TP2 } & -0.05150 & 1.00000 & 0.25475 & 0.25682 & -0.04219 & -0.04770 & 0.26637 & 0.28439 & 0.32342 \\
\hline & 0.5471 & & 0.0025 & 0.0023 & 0.6219 & 0.5771 & 0.0015 & 0.0007 & 0.0001 \\
\hline \multirow[t]{2}{*}{ ITP3 } & 0.40078 & 0.25475 & 1.00000 & 0.99832 & -0.01808 & -0.02615 & 0.47744 & 0.48325 & -0.05249 \\
\hline & $<.0001$ & 0.0025 & & $<.0001$ & 0.8327 & 0.7599 & $<.0001$ & $<.0001$ & 0.5394 \\
\hline \multirow[t]{2}{*}{ 0TP3 } & 0.39557 & 0.25682 & 0.99832 & 1.00000 & -0.02151 & -0.03016 & 0.47562 & 0.48256 & -0.05846 \\
\hline & $<.0001$ & 0.0023 & $<.0001$ & & 0.8016 & 0.7245 & $<.0001$ & $<.0001$ & 0.4943 \\
\hline \multirow[t]{2}{*}{ ITP4 } & 0.00725 & 0.04219 & 0.01808 & 0.02151 & 1.00000 & 0.98570 & 0.01808 & 0.00283 & -0.02104 \\
\hline & 0.9325 & 0.6219 & 0.8327 & 0.8016 & & $<.0001$ & 0.8327 & 0.9736 & 0.8058 \\
\hline \multirow[t]{2}{*}{ OTP4 } & -0.00897 & 0.04770 & 0.02615 & 0.03016 & 0.98570 & 1.00000 & 0.03408 & 0.05530 & -0.01126 \\
\hline & 0.9165 & 0.5771 & 0.7599 & 0.7245 & $<.0001$ & & 0.6904 & 0.5179 & 0.8953 \\
\hline \multirow[t]{2}{*}{ ITP5 } & -0.01808 & 0.26637 & 0.47744 & 0.47562 & 0.01808 & 0.03408 & 1.00000 & 0.99784 & 0.05249 \\
\hline & 0.8327 & 0.0015 & $<.0001$ & $<.0001$ & 0.8327 & 0.6904 & & $<.0001$ & 0.5394 \\
\hline \multirow[t]{2}{*}{ TP5 } & -0.01351 & 0.28439 & 0.48325 & 0.48256 & 0.00283 & 0.05530 & 0.99784 & 1.00000 & -0.05125 \\
\hline & 0.8745 & 0.0007 & $<.0001$ & $<.0001$ & 0.9736 & 0.5179 & $<.0001$ & & 0.5490 \\
\hline \multirow[t]{2}{*}{ ITP7 } & -0.02104 & 0.32342 & 0.05249 & .05846 & 0.02104 & 0.01126 & 0.05249 & 0.05125 & 1.00000 \\
\hline & 0.8058 & 0.0001 & 0.5394 & 0.4943 & 0.8058 & 0.8953 & 0.5394 & 0.5490 & \\
\hline \multirow[t]{2}{*}{ 0TP7 } & -0.00750 & 0.33248 & 0.05528 & 0.06153 & 0.01435 & 0.00278 & 0.05069 & 0.04843 & 0.99782 \\
\hline & 0.9301 & $<.0001$ & 0.5180 & 0.4718 & 0.8668 & 0.9741 & 0.5534 & 0.5713 & $<.0001$ \\
\hline \multirow[t]{2}{*}{ ITP8 } & -0.01029 & 0.05328 & 0.02566 & 0.02180 & 0.01029 & 0.03465 & -0.02566 & 0.02451 & 0.48893 \\
\hline & 0.9043 & 0.5333 & 0.7643 & 0.7990 & 0.9043 & 0.6855 & 0.7643 & 0.7746 & $<.0001$ \\
\hline \multirow[t]{2}{*}{ 0TP8 } & 0.01860 & 0.05224 & 0.02454 & 0.02049 & 0.01598 & 0.02968 & -0.04020 & 0.03806 & 0.48285 \\
\hline & 0.8280 & 0.5414 & 0.7743 & 0.8108 & 0.8519 & 0.7287 & 0.6385 & 0.6564 & $<.0001$ \\
\hline \multirow[t]{2}{*}{ ITP10 } & -0.02961 & 0.37279 & -0.07387 & 0.05221 & 0.02961 & 0.02124 & -0.07387 & -0.05088 & -0.08595 \\
\hline & 0.7294 & $<.0001$ & 0.3874 & 0.5416 & 0.7294 & 0.8040 & 0.3874 & 0.5519 & 0.3144 \\
\hline \multirow[t]{2}{*}{ 0TP10 } & -0.03066 & 0.38401 & -0.05664 & -0.03495 & -0.03094 & -0.02144 & -0.06357 & -0.04053 & -0.07896 \\
\hline & 0.7201 & $<.0001$ & 0.5078 & 0.6829 & 0.7177 & 0.8022 & 0.4572 & 0.6357 & 0.3555 \\
\hline \multirow[t]{2}{*}{ ITP11 } & 0.09768 & 0.00486 & 0.02931 & 0.03685 & -0.07419 & -0.04975 & -0.18510 & -0.16481 & -0.09063 \\
\hline & 0.2526 & 0.9548 & 0.7320 & 0.6667 & 0.3854 & 0.5608 & 0.0291 & 0.0525 & 0.2887 \\
\hline
\end{tabular}


The CORR Procedure

07:43 Wednesday, January 15, 2003

Pearson Correlation Coefficients, $N=139$

Prob $>|r|$ under $\mathrm{HO}$ : Rho=0

\begin{tabular}{|c|c|c|c|c|c|c|c|c|c|}
\hline & 0TP7 & ITP8 & 0TP8 & ITP10 & 0TP10 & ITP11 & 0TP11 & ITP12 & 0TP12 \\
\hline \multirow[t]{2}{*}{ ITP2 } & .00750 & 0.01029 & 0.01860 & 0.02961 & 0.03066 & 0.09768 & .09203 & -0.02617 & -0.02709 \\
\hline & 0.9301 & 0.9043 & 0.8280 & 0.7294 & 0.7201 & 0.2526 & 0.2812 & 0.7598 & 0.7516 \\
\hline \multirow[t]{2}{*}{ OTP2 } & 0.33248 & -0.05328 & 0.05224 & 0.37279 & 0.38401 & 0.00486 & 0.00611 & -0.04311 & 0.03612 \\
\hline & $<.0001$ & 0.5333 & 0.5414 & $<.0001$ & $<.0001$ & 0.9548 & 0.9431 & 0.6143 & 0.6729 \\
\hline \multirow[t]{2}{*}{ ITP3 } & -0.05528 & -0.02566 & 0.02454 & 0.07387 & 0.05664 & 0.02931 & 0.02823 & -0.06529 & -0.05126 \\
\hline & 0.5180 & 0.7643 & 0.7743 & 0.3874 & 0.5078 & 0.7320 & 0.7414 & 0.4451 & 0.5490 \\
\hline \multirow[t]{2}{*}{ OTP3 } & -0.06153 & -0.02180 & 0.02049 & 0.05221 & 0.03495 & 0.03685 & 0.03577 & -0.04170 & -0.02768 \\
\hline & 0.4718 & 0.7990 & 0.8108 & 0.5416 & 0.6829 & 0.6667 & 0.6759 & 0.6260 & 0.7463 \\
\hline \multirow[t]{2}{*}{ ITP4 } & -0.01435 & 0.01029 & 0.01598 & -0.02961 & 0.03094 & 0.07419 & 0.06910 & -0.02617 & -0.02994 \\
\hline & 0.8668 & 0.9043 & 0.8519 & 0.7294 & 0.7177 & 0.3854 & 0.4189 & 0.7598 & 0.7264 \\
\hline \multirow[t]{2}{*}{ OTP4 } & -0.00278 & 0.03465 & 0.02968 & -0.02124 & 0.02144 & 0.04975 & 0.04418 & -0.03614 & -0.04023 \\
\hline & 0.9741 & 0.6855 & 0.7287 & 0.8040 & 0.8022 & 0.5608 & 0.6056 & 0.6727 & 0.6382 \\
\hline \multirow[t]{2}{*}{ ITP5 } & -0.05069 & -0.02566 & -0.04020 & -0.07387 & 0.06357 & 0.18510 & 0.17942 & -0.06529 & -0.06193 \\
\hline & 0.5534 & 0.7643 & 0.6385 & 0.3874 & 0.4572 & 0.0291 & 0.0346 & 51 & 0.4689 \\
\hline \multirow[t]{2}{*}{ 0TP5 } & -0.04843 & -0.02451 & -0.03806 & -0.05088 & 0.04053 & -0.16481 & 0.15918 & -0.04973 & -0.04601 \\
\hline & 0.5713 & 0.7746 & 0.6564 & 0.5519 & 0.6357 & 0.0525 & 0.0613 & 10 & 07 \\
\hline \multirow[t]{2}{*}{ ITP7 } & 0.99782 & 0.48893 & 0.48285 & -0.08595 & -0.07896 & -0.09063 & 0.08769 & -0.07596 & -0.07175 \\
\hline & $<.0001$ & $<.0001$ & $<.0001$ & 0.3144 & 0.3555 & 0.2887 & 0.3047 & 0.3741 & 0.4013 \\
\hline \multirow[t]{2}{*}{ 0TP7 } & 1.00000 & 0.47503 & 0.47245 & -0.06404 & -0.05762 & -0.06350 & -0.06008 & -0.06191 & -0.05742 \\
\hline & & $<.0001$ & $<.0001$ & 0.4539 & 0.5005 & 0.4577 & 0.4824 & 0.4690 & 0.5019 \\
\hline \multirow[t]{2}{*}{ ITP8 } & 0.47503 & 1.00000 & 0.99383 & -0.04202 & -0.02567 & 0.13864 & 0.14321 & -0.03714 & -0.02789 \\
\hline & $<.0001$ & & $<.0001$ & 0.6233 & 0.7642 & 0.1036 & 0.0926 & 0.6642 & 0.7445 \\
\hline \multirow[t]{2}{*}{ 0TP8 } & 0.47245 & 0.99383 & 1.00000 & 0.00273 & 0.01751 & 0.16469 & 0.16978 & -0.03852 & -0.02867 \\
\hline & $<.0001$ & $<.0001$ & & 0.9746 & 0.8379 & 0.0527 & 0.0457 & 0.6526 & 0.7376 \\
\hline \multirow[t]{2}{*}{ ITP10 } & -0.06404 & -0.04202 & 0.00273 & 1.00000 & 0.99906 & 0.39909 & 0.40622 & 0.22333 & 0.22699 \\
\hline & 0.4539 & 0.6233 & 0.9746 & & $<.0001$ & $<.0001$ & $<.0001$ & 0.0082 & 0.0072 \\
\hline \multirow[t]{2}{*}{ OTP10 } & -0.05762 & -0.02567 & 0.01751 & 0.99906 & 1.00000 & 0.40421 & 0.41139 & 0.22656 & 0.23082 \\
\hline & 0.5005 & 0.7642 & 0.8379 & $<.0001$ & & $<.0001$ & $<.0001$ & 0.0073 & 0.0063 \\
\hline \multirow[t]{2}{*}{ ITP11 } & -0.06350 & 0.13864 & 0.16469 & 0.39909 & 0.40421 & 1.00000 & 0.99938 & 0.35272 & 0.37205 \\
\hline & 0.4577 & 0.1036 & 0.0527 & $<.0001$ & $<.0001$ & & $<.0001$ & $<.0001$ & $<.0001$ \\
\hline
\end{tabular}


07:43 Wednesday, January 15, 2003

The CORR Procedure

Pearson Correlation Coefficients, $N=139$

Prob $>|r|$ under HO: Rho $=0$

\begin{tabular}{|c|c|c|c|c|c|c|c|c|c|}
\hline & ITP15 & 0TP15 & ТР22 & 22 & ITP23 & 0ТР23 & ITS1 & OTS1 & TS2 \\
\hline \multirow[t]{2}{*}{ ITP2 } & .02734 & .02951 & .01960 & .01952 & .02961 & .01763 & -0.00725 & 0.01111 & -0.01808 \\
\hline & .7493 & .7302 & 8188 & 8196 & 0.7294 & .8368 & 9325 & 8967 & 327 \\
\hline \multirow[t]{2}{*}{ DTP2 } & 0.38661 & 0.40411 & 0.09092 & .09377 & 0.00757 & .00247 & 0.16261 & 0.17476 & 0186 \\
\hline & $<.0001$ & $<.0001$ & 0.2871 & 0.2722 & 0.9295 & 0.9770 & 0.0558 & 0.0396 & 0001 \\
\hline \multirow[t]{2}{*}{ ITP3 } & -0.06822 & 0.05921 & 0.04891 & 0.05189 & 0.07387 & 0.05381 & 0.01808 & 0.00696 & 0.04511 \\
\hline & 0.4249 & 0.4887 & 0.5675 & 0.5441 & 0.3874 & 0.5292 & 0.8327 & 0.9352 & 0.5980 \\
\hline \multirow[t]{2}{*}{ DTP3 } & -0.06267 & 0.05357 & 0.05649 & 0.05975 & 0.07005 & 0.04972 & 0.01492 & 0.01022 & 0.05422 \\
\hline & 0.4636 & 0.5 & 0.5089 & 0.4847 & 0.4126 & 0.5611 & 0.8616 & 0.9050 & 0.5261 \\
\hline \multirow[t]{2}{*}{ ITP4 } & -0.02734 & 0.01764 & 0.01960 & 0.02275 & 0.02961 & 0.03073 & -0.00725 & 0.00570 & 0.01808 \\
\hline & 0.7493 & 0.8367 & 0.8188 & 04 & 0.7294 & 0.7195 & 0.9325 & 0.9469 & 8327 \\
\hline \multirow[t]{2}{*}{ OTP4 } & 0.00622 & 0.01526 & 0.03564 & 0.03942 & 0.04282 & 0.04346 & -0.00558 & -0.00285 & -0.03321 \\
\hline & 0.9421 & 0.8585 & 0.6770 & 0.6449 & 0.6167 & 0.6115 & 0.9481 & 0.9735 & 0.6980 \\
\hline \multirow[t]{2}{*}{ ITP5 } & -0.06822 & -0.05389 & 0.04891 & 0.05508 & -0.07387 & -0.06069 & -0.01808 & -0.01241 & -0.04511 \\
\hline & 0.4249 & 0.5287 & 0.5675 & 0.5196 & 0.3874 & 0.4779 & 0.8327 & 0.8847 & 0.5980 \\
\hline \multirow[t]{2}{*}{ 0TP5 } & -0.05664 & -0.04166 & 0.05756 & -0.06406 & -0.07387 & -0.06031 & -0.02102 & -0.01454 & -0.04437 \\
\hline & 0.5078 & 0.6263 & 0.5009 & 0.4538 & 0.3875 & 0.4806 & 0.8059 & 0.8651 & 0.6040 \\
\hline \multirow[t]{2}{*}{ ITP7 } & -0.07938 & -0.07485 & 0.05691 & 0.05372 & -0.08595 & -0.08260 & -0.02104 & -0.03422 & 0.85949 \\
\hline & 0.3530 & 0.3812 & 0.5058 & 0.5299 & 0.3144 & 0.3337 & 0.8058 & 8992 & $<.0001$ \\
\hline \multirow[t]{2}{*}{ 0TP7 } & -0.06996 & -0.06522 & 0.05634 & 0.05338 & 0.09649 & 0.09339 & -0.01892 & 0.03176 & 0.86513 \\
\hline & 0.4131 & 0.44 & 0.5101 & 0.5326 & 0.2585 & 0.2 & 0.8250 & 06 & $<.0001$ \\
\hline \multirow[t]{2}{*}{ ITP8 } & -0.03881 & -0.03843 & 0.02782 & 0.03114 & 0.04202 & 0.04261 & -0.01029 & 0.01270 & 0.02566 \\
\hline & 0.6501 & 0.6533 & 0.7451 & 59 & 0.6233 & 0.6184 & 9043 & 8821 & 0.7643 \\
\hline \multirow[t]{2}{*}{ 0TP8 } & -0.02908 & -0.02823 & -0.03433 & 0.03780 & -0.05776 & 0.05850 & 630 & 0.00443 & 0.02901 \\
\hline & 0.7340 & 0.7415 & 0.6883 & 0.6587 & 0.4994 & 0.4939 & 0.9413 & 0.9587 & 0.7346 \\
\hline \multirow[t]{2}{*}{ ITP10 } & 0.60499 & 0.61974 & 0.08009 & 0.08524 & 0.12097 & 0.12286 & -0.02961 & -0.04599 & -0.07387 \\
\hline & $<.0001$ & $<.0001$ & 0.3486 & 0.3184 & 0.1560 & 0.1496 & 0.7294 & 0.5908 & 0.3874 \\
\hline \multirow[t]{2}{*}{ OTP10 } & 0.61657 & 0.63150 & -0.08578 & 0.09107 & 0.11388 & 0.11547 & 0.03179 & 0.04796 & 0.07544 \\
\hline & $<.0001$ & $<.0001$ & 0.3154 & 0.2863 & 0.1819 & 0.1759 & 0.7103 & 0.5750 & 0.3774 \\
\hline \multirow[t]{2}{*}{ ITP11 } & 0.16903 & 0.16322 & -0.20069 & 0.22076 & -0.30311 & 0.30084 & 0.07419 & 0.08678 & 0.18510 \\
\hline & 0.0467 & 0.0549 & 0.0178 & 0.0090 & 0.0003 & 0.0003 & 0.3854 & 0.3097 & 0.0291 \\
\hline
\end{tabular}


The CORR Procedure

07:43 Wednesday, January 15, 2003

Pearson Correlation Coefficients, $N=139$ Prob $>|r|$ under HO: Rho=0

\begin{tabular}{|c|c|c|c|c|c|c|c|c|c|}
\hline & 0TS2 & ITS5 & 0TS5 & ITS8 & OTS8 & ITS11 & OTS11 & ITS12 & OTS12 \\
\hline \multirow[t]{2}{*}{ ITP2 } & -0.01825 & -0.01808 & -0.02174 & -0.00725 & -0.00325 & -0.01644 & -0.02320 & -0.04275 & -0.03941 \\
\hline & 0.8312 & 0.8327 & 0.7995 & 0.9325 & 0.9697 & 0.8476 & 0.7863 & 0.6173 & 0.6450 \\
\hline \multirow[t]{2}{*}{ 0TP2 } & 0.40547 & -0.07043 & -0.07346 & -0.03288 & 0.24461 & 0.44504 & 0.46239 & -0.11163 & -0.11793 \\
\hline & $<.0001$ & 0.4100 & 0.3901 & 0.7008 & 0.0037 & $<.0001$ & $<.0001$ & 0.1908 & 0.1668 \\
\hline \multirow[t]{2}{*}{ ITP3 } & -0.04968 & -0.04511 & -0.05259 & -0.01808 & 0.40703 & 0.52926 & 0.53223 & -0.10668 & -0.10517 \\
\hline & 0.5614 & 0.5980 & 0.5386 & 0.8327 & $<.0001$ & $<.0001$ & $<.0001$ & 0.2113 & 0.2179 \\
\hline \multirow[t]{2}{*}{ ОТР3 } & -0.05921 & -0.05038 & -0.05832 & -0.01843 & 0.42105 & 0.52643 & 0.53092 & -0.11876 & -0.11722 \\
\hline & 0.4887 & 0.5559 & 0.4953 & 0.8295 & $<.0001$ & $<.0001$ & $<.0001$ & 0.1638 & 0.1694 \\
\hline \multirow[t]{2}{*}{ ITP4 } & -0.01347 & 0.40078 & 0.39748 & -0.00725 & -0.04592 & -0.01644 & -0.00725 & -0.04275 & -0.04186 \\
\hline & 0.8749 & $<.0001$ & $<.0001$ & 0.9325 & 0.5914 & 0.8476 & 0.9325 & 0.6173 & 0.6247 \\
\hline \multirow[t]{2}{*}{ ОТР4 } & -0.02732 & 0.45948 & 0.46063 & 0.09287 & -0.06368 & -0.00341 & 0.00622 & -0.02837 & -0.02631 \\
\hline & 0.7496 & $<.0001$ & $<.0001$ & 0.2769 & 0.4564 & 0.9682 & 0.9420 & 0.7402 & 0.7585 \\
\hline \multirow[t]{2}{*}{ ITP5 } & -0.04119 & 0.04511 & 0.03280 & 0.40078 & 0.31478 & 0.52926 & 0.54912 & -0.10668 & -0.10822 \\
\hline & 0.6302 & 0.5980 & 0.7015 & $<.0001$ & 0.0002 & $<.0001$ & $<.0001$ & 0.2 & 0.2048 \\
\hline \multirow[t]{2}{*}{ 0TP5 } & -0.04024 & 0.03262 & 0.02106 & 0.39867 & 0.33548 & 0.53133 & 0.55314 & -0.11036 & -0.11165 \\
\hline & 0.6381 & 0.7031 & 0.8056 & $<.0001$ & $<.0001$ & $<.0001$ & $<.0$ & 59 & 0.1907 \\
\hline \multirow[t]{2}{*}{ ITP7 } & 0.85715 & 0.05249 & 0.05591 & -0.02104 & -0.11781 & -0.04774 & 0.06315 & 0.02992 & 0.02492 \\
\hline & $<.0001$ & 0.5394 & 0.5133 & 0.8058 & 0.1672 & 0.5768 & 0.4602 & 0.7266 & 0.7709 \\
\hline \multirow[t]{2}{*}{ 0TP7 } & 0.86334 & 0.05481 & -0.05733 & -0.01740 & -0.11295 & -0.05106 & -0.06678 & 0.03786 & 0.03352 \\
\hline & $<.0001$ & 0.5216 & 0.5026 & 0.8389 & 0.1855 & 0.5506 & 0.4348 & 0.6581 & 0.6952 \\
\hline \multirow[t]{2}{*}{ ITP8 } & -0.02805 & 0.02566 & 0.03054 & -0.01029 & -0.03490 & -0.02334 & -0.03294 & 0.24057 & 0.23909 \\
\hline & 0.7430 & 0.7643 & 0.7211 & 0.9043 & 0.6834 & 0.7851 & 0.7003 & 0.0043 & 0.0046 \\
\hline \multirow[t]{2}{*}{ 0ТР8 } & -0.03211 & 0.04275 & 0.04763 & -0.01521 & -0.04860 & -0.03871 & -0.04955 & 0.25610 & 0.25514 \\
\hline & 0.7074 & 0.6173 & 0.5776 & 0.8589 & 0.5699 & 0.6510 & 0.5624 & 0.0023 & 0.0024 \\
\hline \multirow[t]{2}{*}{ ITP10 } & -0.08082 & -0.07387 & -0.07442 & -0.02961 & 0.08665 & -0.06718 & -0.05003 & -0.05906 & -0.05759 \\
\hline & 0.3443 & 0.3874 & 0.3840 & 0.7294 & 0.3105 & 0.4320 & 0.5586 & 0.4898 & 0.5007 \\
\hline \multirow[t]{2}{*}{ 0TP10 } & -0.08227 & -0.06992 & -0.07090 & -0.03151 & 0.09610 & -0.05200 & -0.03418 & -0.05894 & -0.05750 \\
\hline & 0.3357 & 0.4134 & 0.4069 & 0.7127 & 0.2604 & 0.5432 & 0.6896 & 0.4907 & 0.5014 \\
\hline \multirow[t]{2}{*}{ ITP11 } & -0.18384 & 0.02931 & 0.03400 & -0.07419 & 0.18362 & -0.16834 & -0.16934 & 0.43145 & 0.44024 \\
\hline & 0.0303 & 0.7320 & 0.6911 & 0.3854 & 0.0305 & 0.0476 & 0.0463 & $<.0001$ & $<.0001$ \\
\hline
\end{tabular}


07:43 Wednesday, January 15, 2003

The CORR Procedure

Pearson Correlation Coefficients, $\mathrm{N}=139$

Prob $>|r|$ under $\mathrm{HO}$ : Rho=0

\begin{tabular}{|c|c|c|c|c|c|c|c|c|c|}
\hline & ITS15 & OTS15 & ITS18 & OTS18 & ITS19 & OTS19 & ITS20 & OTS20 & ITS22 \\
\hline \multirow[t]{2}{*}{ ITP2 } & -0.01465 & -0.01709 & 0.25436 & 0.25053 & .02617 & .02917 & .01029 & -0.00454 & -0.01029 \\
\hline & 0.8641 & 0.8417 & 0.0025 & 0.0029 & 0.7598 & 0.7332 & 0.9043 & 0.9577 & 0.9043 \\
\hline \multirow[t]{2}{*}{ 0TP2 } & 0.04645 & 0.07270 & 0.25239 & 0.25693 & -0.14956 & -0.15260 & 0.03261 & 0.04463 & 0.01279 \\
\hline & 0.5871 & 0.3950 & 0.0027 & 0.0023 & 0.0789 & 0.0729 & 0.7032 & 0.6019 & 0.8813 \\
\hline \multirow[t]{2}{*}{ ITP3 } & -0.03656 & -0.01869 & 0.28179 & 0.28246 & -0.06529 & -0.05564 & -0.02566 & -0.03944 & -0.02566 \\
\hline & 0.6692 & 0.8272 & 0.0008 & 0.0008 & 0.4451 & 0.5153 & 0.7643 & 0.6448 & 0.7643 \\
\hline \multirow[t]{2}{*}{ 0TP3 } & -0.01683 & 0.00124 & 0.29556 & 0.29705 & -0.05916 & -0.04984 & -0.02554 & -0.04066 & -0.02772 \\
\hline & 0.8441 & 0.9885 & 0.0004 & 0.0004 & 0.4891 & 0.5601 & 0.7654 & 0.6346 & 0.7460 \\
\hline \multirow[t]{2}{*}{ ITP4 } & -0.01465 & 0.00322 & 0.02849 & .02766 & 0.02617 & 0.02949 & 0.01029 & 0.01931 & 0.01029 \\
\hline & 0.8641 & 0.9700 & 0.7392 & 0.7465 & 0.7598 & 0.7304 & 0.9043 & 0.8215 & 0.9043 \\
\hline \multirow[t]{2}{*}{ OTP4 } & -0.00956 & 0.00679 & 0.03559 & 0.03467 & 0.04738 & -0.05147 & 0.02180 & 0.05759 & 0.02260 \\
\hline & 0.9111 & 0.9368 & 0.6775 & 0.6853 & 0.5797 & 0.5474 & 0.7989 & 0.5007 & 0.7917 \\
\hline \multirow[t]{2}{*}{ ITP5 } & 0.17519 & 0.20176 & 0.07108 & 0.06914 & 0.06529 & 0.06105 & 0.02566 & 0.00902 & 0.27160 \\
\hline & 0.0391 & 0.0172 & 0.4057 & 0.4186 & 0.4451 & 0.4753 & 0.7643 & 0.9161 & 0.0012 \\
\hline \multirow[t]{2}{*}{ 0TP5 } & 0.19556 & 0.22374 & .06231 & 0.05935 & 0.07233 & 0.06891 & 0.01918 & 0.00293 & 0.28086 \\
\hline & 0.0210 & 0.0081 & 0.4662 & 0.4877 & 0.3975 & 0.4202 & 0.8227 & 0.9726 & 0.0008 \\
\hline \multirow[t]{2}{*}{ ITP7 } & -0.04254 & 0.05096 & 0.08270 & 0.08395 & 0.07596 & -0.07917 & 0.02986 & 0.00211 & 0.02986 \\
\hline & 0.6190 & 0.5513 & 0.3331 & 0.3258 & 0.3741 & 0.3542 & 0.7271 & 0.9804 & 0.7271 \\
\hline \multirow[t]{2}{*}{ 0TP7 } & -0.03595 & 0.04261 & 0.07492 & 0.07575 & 0.08974 & -0.09378 & 0.01497 & 0.01774 & 0.01119 \\
\hline & 0.6744 & 0.6185 & 0.3807 & 0.3755 & 0.2934 & 0.2722 & 0.8611 & 0.8358 & 0.8960 \\
\hline \multirow[t]{2}{*}{ ITP8 } & -0.02080 & 0.02312 & 0.04044 & 0.03842 & 0.03714 & -0.04231 & 0.01460 & 0.00653 & 0.01460 \\
\hline & 0.8080 & 0.7870 & 0.6365 & 0.6534 & 0.6642 & 0.6209 & 0.8646 & 0.9392 & 0.8646 \\
\hline \multirow[t]{2}{*}{ 0TP8 } & -0.02377 & 0.02528 & -0.01903 & -0.01660 & 0.05956 & -0.06558 & 0.00840 & 0.02994 & 0.01113 \\
\hline & 0.7812 & 0.7677 & 0.8241 & 0.8462 & 0.4861 & 0.4431 & 0.9218 & 0.7264 & 0.8966 \\
\hline \multirow[t]{2}{*}{ ITP10 } & 0.35622 & 0.38893 & 0.57700 & 0.59381 & -0.10691 & -0.11972 & 0.04202 & 0.06187 & 0.15268 \\
\hline & $<.0001$ & $<.0001$ & $<.0001$ & $<.0001$ & 0.2103 & 0.1604 & 0.6233 & 0.4693 & 0.0727 \\
\hline \multirow[t]{2}{*}{ 0TP10 } & 0.35459 & 0.38835 & 0.59005 & 0.60711 & -0.09804 & -0.11073 & -0.04512 & -0.06502 & 0.15062 \\
\hline & $<.0001$ & $<.0001$ & $<.0001$ & $<.0001$ & 0.2509 & 0.1944 & 0.5979 & 0.4470 & 0.0767 \\
\hline \multirow[t]{2}{*}{ ITP11 } & 0.11063 & 0.12989 & 0.38401 & 0.39031 & -0.11274 & -0.11478 & -0.10530 & -0.09846 & 0.01667 \\
\hline & 0.1948 & 0.1275 & $<.0001$ & $<.0001$ & 0.1864 & 0.1785 & 0.2173 & 0.2488 & 0.845 \\
\hline
\end{tabular}


The CORR Procedure

07:43 Wednesday, January 15, 2003

$\begin{array}{rrrrrrr}\text { Pearson Correlation Coefficients, N=139 } & & & \\ \text { Prob }>|r| \text { under H0: Rho=0 } & & & \\ \text { 0TS23 } & \text { ITA2 } & \text { OTA2 } & \text { ITA3 } & \text { 0TA3 } & \text { ITA7 } & \text { OTA7 } \\ -0.03579 & -0.00725 & -0.01618 & -0.02617 & -0.02933 & -0.01808 & -0.00411 \\ 0.6757 & 0.9325 & 0.8500 & 0.7598 & 0.7318 & 0.8327 & 0.9617 \\ 0.03119 & 0.05090 & 0.04540 & 0.33787 & 0.34112 & 0.09990 & 0.08701 \\ 0.7155 & 0.5518 & 0.5957 & <.0001 & <.0001 & 0.2420 & 0.3084 \\ -0.07738 & -0.01808 & -0.04393 & -0.06529 & -0.07082 & -0.04511 & -0.04699 \\ 0.3653 & 0.8327 & 0.6076 & 0.4451 & 0.4074 & 0.5980 & 0.5828 \\ -0.08184 & -0.02195 & -0.04917 & -0.07331 & -0.07931 & -0.04855 & -0.05102 \\ 0.3382 & 0.7976 & 0.5654 & 0.3911 & 0.3533 & 0.5703 & 0.5508 \\ -0.03606 & -0.00725 & -0.01725 & -0.02617 & -0.02395 & -0.01808 & -0.01422 \\ 0.6734 & 0.9325 & 0.8403 & 0.7598 & 0.7796 & 0.8327 & 0.8680 \\ -0.05231 & -0.01463 & -0.02954 & -0.03342 & -0.02824 & -0.00780 & -0.00181 \\ 0.5408 & 0.8643 & 0.7300 & 0.6961 & 0.7414 & 0.9274 & 0.9832 \\ -0.07174 & -0.01808 & -0.04571 & -0.06529 & -0.05793 & -0.04511 & -0.04115 \\ 0.4013 & 0.8327 & 0.5932 & 0.4451 & 0.4982 & 0.5980 & 0.6306 \\ -0.06086 & -0.02147 & -0.05248 & -0.05000 & -0.04232 & -0.04640 & -0.04266 \\ 0.4766 & 0.8019 & 0.5395 & 0.5589 & 0.6208 & 0.5876 & 0.6181 \\ -0.08636 & -0.02104 & -0.01639 & 0.58396 & 0.59002 & -0.05249 & -0.04319 \\ 0.3121 & 0.8058 & 0.8481 & <.0001 & <.0001 & 0.5394 & 0.6136 \\ -0.07178 & -0.02387 & -0.02068 & 0.59610 & 0.60279 & -0.04373 & -0.03458 \\ 0.4011 & 0.7803 & 0.8091 & <.0001 & <.0001 & 0.6092 & 0.6861 \\ -0.04100 & -0.01029 & -0.02146 & -0.03714 & -0.02682 & -0.02566 & -0.01301 \\ 0.6318 & 0.9043 & 0.8020 & 0.6642 & 0.7540 & 0.7643 & 0.8792 \\ -0.05763 & -0.01675 & -0.02881 & -0.04060 & -0.03138 & -0.01048 & 0.00141 \\ 0.5004 & 0.8448 & 0.7364 & 0.6351 & 0.7139 & 0.9026 & 0.9868 \\ -0.14067 & -0.02961 & -0.05304 & -0.10691 & -0.11252 & -0.07387 & -0.09197 \\ 0.0986 & 0.7294 & 0.5352 & 0.2103 & 0.1872 & 0.3874 & 0.2815 \\ -0.14485 & -0.03151 & -0.05410 & -0.11148 & -0.11676 & -0.07932 & -0.09796 \\ 0.0889 & 0.7127 & 0.5271 & 0.1914 & 0.1711 & 0.3533 & 0.2513 \\ 0.17076 & -0.07419 & -0.11160 & 0.04242 & 0.04471 & -0.18510 & -0.19289 \\ 0.0444 & 0.3854 & 0.1909 & 0.6201 & 0.6012 & 0.0291 & 0.0229\end{array}$


Observed to Trained NNS Output for Exercises 1,2,3

07:43 Wednesday, January 15, 2003

The CORR Procedure

Pearson Correlation Coefficients, $N=139$

Prob $>|r|$ under $\mathrm{HO}$ : Rho $=0$

\begin{tabular}{|c|c|c|c|c|c|c|c|c|c|}
\hline & ITA10 & OTA10 & ITA11 & OTA11 & ITA12 & OTA12 & ITA18 & OTA18 & ITA19 \\
\hline \multirow[t]{2}{*}{ ITP2 } & -0.01029 & 0.01502 & 0.01960 & .02153 & .01264 & .00501 & .01960 & -0.02194 & -0.01264 \\
\hline & 0.9043 & 0.8607 & 0.8188 & 0.8014 & 0.8826 & 0.9533 & 0.8188 & 0.7976 & 0.8826 \\
\hline \multirow[t]{2}{*}{ 0TP2 } & 0.16474 & 0.19269 & 0.09092 & 0.11518 & 0.43531 & 0.45616 & -0.06377 & -0.05739 & -0.03572 \\
\hline & 0.0526 & 0.0231 & 0.2871 & 0.1770 & $<.0001$ & $<.0001$ & 0.4558 & 0.5022 & 0.6764 \\
\hline \multirow[t]{2}{*}{ ITP3 } & -0.02566 & 0.02056 & -0.04891 & 0.05099 & 0.69926 & 0.71382 & -0.04891 & -0.04246 & -0.03155 \\
\hline & 0.7643 & 0.8102 & 0.5675 & 0.5511 & $<.0001$ & $<.0001$ & 0.5675 & 0.6197 & 0.7124 \\
\hline \multirow[t]{2}{*}{ 0TP3 } & -0.03115 & 0.02677 & 0.05513 & 0.05682 & 0.70014 & 0.71661 & -0.04596 & -0.03912 & -0.03293 \\
\hline & 0.7158 & 0.7544 & 0.5192 & 0.5064 & $<.0001$ & $<.0001$ & 0.5911 & 0.6475 & 0.7004 \\
\hline \multirow[t]{2}{*}{ ITP4 } & 0.01029 & 0.00273 & 0.01960 & 0.00552 & .01264 & 01644 & 0.01960 & -0.02318 & 0.01264 \\
\hline & 0.9043 & 0.9746 & 0.8188 & 0.9486 & 0.8826 & 0.8477 & 0.8188 & 0.7865 & 0.8826 \\
\hline \multirow[t]{2}{*}{ OTP4 } & 0.01537 & 0.03010 & 0.00197 & 0.01766 & 0.01697 & 0.01798 & 0.03520 & 0.03919 & 0.02421 \\
\hline & 0.8574 & 0.7250 & 0.9816 & 0.8365 & 0.8428 & 0.8336 & 0.6808 & 0.6469 & 0.7773 \\
\hline \multirow[t]{2}{*}{ ITP5 } & 0.02566 & 0.00211 & 0.11296 & 0.11977 & 0.69926 & 0.71229 & 0.04891 & 0.04536 & 0.03155 \\
\hline & 0.7643 & 0.9804 & 0.1855 & 0.1602 & $<.0001$ & $<.0001$ & 0.5675 & 0.5959 & 0.7124 \\
\hline \multirow[t]{2}{*}{ 0TP5 } & -0.02420 & 0.00003 & 0.12478 & 0.13312 & 0.70071 & 0.71754 & 0.04475 & 0.04129 & 0.03720 \\
\hline & 0.7774 & 0.9997 & 0.1433 & 0.1182 & $<.0001$ & $<.0001$ & 0.6009 & 0.6294 & 0.6638 \\
\hline \multirow[t]{2}{*}{ ITP7 } & -0.02986 & .01381 & .05691 & .05550 & .03670 & 0.03950 & 0.05691 & -0.06162 & 0.03670 \\
\hline & 0.7271 & 0.8718 & 0.5058 & 0.5164 & 0.6680 & 0.6443 & 0.5058 & 0.4711 & 0.6680 \\
\hline \multirow[t]{2}{*}{ 0TP7 } & -0.02740 & 0.01093 & 0.06487 & 0.06281 & 0.04297 & 0.04421 & 0.04530 & 0.05025 & 0.03633 \\
\hline & 0.7489 & 0.8984 & 0.4481 & 0.4626 & 0.6154 & 0.6053 & 0.5964 & 0.5569 & 0.6711 \\
\hline \multirow[t]{2}{*}{ ITP8 } & -0.01460 & 0.02079 & 0.02782 & 0.03376 & 0.01795 & 0.01507 & 0.02782 & -0.02853 & 0.01795 \\
\hline & 0.8646 & 0.8081 & 0.7451 & 0.6931 & 0.8339 & 0.8602 & 0.7451 & 0.7389 & 0.8339 \\
\hline \multirow[t]{2}{*}{ 0TP8 } & -0.02378 & .03040 & .04413 & 0.05067 & 0.03012 & 0.02484 & 0.04116 & -0.04256 & -0.02699 \\
\hline & 0.7812 & 0.7224 & 0.6060 & 0.5536 & 0.7249 & 0.7716 & 0.6305 & 0.6188 & 0.7525 \\
\hline \multirow[t]{2}{*}{ ITP10 } & -0.04202 & 0.05128 & -0.08009 & 0.06504 & 0.05166 & 0.01141 & 0.08009 & -0.08787 & -0.05166 \\
\hline & 0.6233 & 0.5488 & 0.3486 & 0.4469 & 0.5459 & 0.8940 & 0.3486 & 0.3037 & 0.5459 \\
\hline \multirow[t]{2}{*}{ OTP10 } & -0.03750 & 0.04649 & 0.07431 & -0.05941 & 0.03591 & 0.00438 & -0.07835 & -0.08608 & -0.04922 \\
\hline & 0.6612 & 0.5868 & 0.3846 & 0.4873 & 0.6747 & 0.9592 & 0.3592 & 0.3137 & 0.5650 \\
\hline \multirow[t]{2}{*}{ ITP11 } & -0.10530 & -0.12774 & -0.20069 & -0.20528 & -0.12944 & -0.09835 & 0.19782 & 0.19185 & -0.02948 \\
\hline & 0.2173 & 0.1340 & 0.0178 & 0.0153 & 0.1289 & 0.2494 & 0.0196 & 0.0237 & 0.730 \\
\hline
\end{tabular}


The CORR Procedure

07:43 Wednesday, January 15, 2003

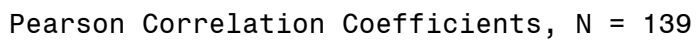

\begin{tabular}{|c|c|c|c|c|c|c|c|c|c|}
\hline & OTA19 & ITA21 & OTA21 & ITA23 & OTA23 & IM1 & OM1 & IM2 & OM2 \\
\hline \multirow[t]{2}{*}{ ITP2 } & -0.01536 & 0.03691 & 0.03925 & 0.17338 & .16871 & -0.04331 & -0.04246 & 0.03204 & 0.01129 \\
\hline & 0.8575 & 0.6662 & 0.6464 & 0.0412 & 0.0471 & 0.6127 & 0.6197 & 0.7081 & 0.8950 \\
\hline \multirow[t]{2}{*}{ 0TP2 } & -0.04824 & -0.23864 & 0.24296 & 0.21602 & .23115 & 0.13301 & 0.13620 & 0.43835 & 0.43662 \\
\hline & 0.5728 & 0.0047 & 0.0040 & 0.0106 & 0.0062 & 0.1185 & 0.1099 & $<.0001$ & $<.0001$ \\
\hline \multirow[t]{2}{*}{ ITP3 } & -0.04665 & -0.09210 & 0.09673 & 0.16415 & .16721 & -0.10805 & -0.10083 & 0.14789 & 0.14001 \\
\hline & 0.5856 & 0.2809 & 0.2573 & 0.0535 & 0.0491 & 0.2055 & 0.2376 & 0.0823 & 0.1002 \\
\hline \multirow[t]{2}{*}{ 0TP3 } & -0.04836 & -0.10682 & -0.11145 & 0.18041 & .18360 & -0.11261 & -0.10517 & 0.14638 & 0.13864 \\
\hline & 0.5718 & 0.2107 & 0.1915 & 0.0336 & 0.0305 & 0.1869 & 0.2179 & 855 & 0 . \\
\hline \multirow[t]{2}{*}{ ITP4 } & -0.01911 & -0.03691 & -0.03949 & 0.17338 & 0.17117 & -0.04331 & -0.04406 & 0.15459 & 0.13656 \\
\hline & 0.8233 & 0.6662 & 0.6444 & 0.0412 & 0.0439 & 0.6127 & 0.6065 & 0.0692 & 0.1089 \\
\hline \multirow[t]{2}{*}{ OTP4 } & -0.03077 & -0.03234 & -0.03571 & 0.23133 & 0.22953 & -0.02963 & -0.03090 & 0.14522 & 0.13066 \\
\hline & 0.7191 & 0.7055 & 0.6764 & 0.0061 & 0.0066 & 0.7292 & 0.7180 & 0.0881 & 0.1252 \\
\hline \multirow[t]{2}{*}{ ITP5 } & -0.04223 & -0.09210 & 0.09743 & 0.07467 & .07851 & -0.10805 & -0.09773 & 0.13090 & 0.14534 \\
\hline & 0.6216 & 0.2809 & 0.2538 & 0.3823 & 0.3583 & 055 & 524 & 246 & 0.0878 \\
\hline \multirow[t]{2}{*}{ 0TP5 } & -0.05006 & -0.09994 & -0.10599 & 0.09853 & 10295 & -0.09185 & 191 & 966 & 3436 \\
\hline & 0.5584 & 0.2418 & 0.2143 & 0.2485 & 0.2278 & 0.2822 & 0.3378 & 0.1606 & 0.1148 \\
\hline \multirow[t]{2}{*}{ ITP7 } & -0.03146 & -0.10716 & -0.09748 & 0.03483 & 0.03542 & 0.04231 & 0.04612 & 0.26279 & 0.26696 \\
\hline & 0.7131 & 0.2093 & 0.2536 & 0.6840 & 0.6789 & 0.6209 & 0.5898 & 0.0018 & 0.0015 \\
\hline \multirow[t]{2}{*}{ 0TP7 } & -0.03120 & -0.09489 & -0.08540 & 0.05088 & 0.05198 & 0.04942 & 0.05378 & 0.27007 & -0.27422 \\
\hline & 0.7154 & 0.2665 & 0.3175 & 0.5519 & 0.5434 & 0.5634 & 0.5295 & 0.0013 & 0.0011 \\
\hline \multirow[t]{2}{*}{ ITP8 } & -0.01915 & -0.05239 & -0.04263 & 0.24608 & .24676 & 147 & 0.06367 & 848 & 0.12142 \\
\hline & 0.8230 & 0.5402 & 0.6183 & 0.0035 & 0.0034 & 0.4722 & 0.4565 & 317 & 0.1545 \\
\hline \multirow[t]{2}{*}{ 0TP8 } & -0.02973 & -0.04369 & -0.03306 & 0.27618 & 0.27674 & 0.06920 & -0.07130 & 0.11983 & -0.11394 \\
\hline & 0.7283 & 0.6095 & 0.6992 & 0.0010 & 0.0010 & 0.4183 & 0.4042 & 0.1600 & 0.1817 \\
\hline \multirow[t]{2}{*}{ ITP10 } & -0.07604 & -0.15082 & -0.15086 & 0.70837 & 0.71743 & 0.17694 & -0.18147 & 0.14730 & -0.15091 \\
\hline & 0.3736 & 0.0764 & 0.0763 & $<.0001$ & $<.0001$ & 0.0372 & 0.0325 & 0.0836 & 0.0762 \\
\hline \multirow[t]{2}{*}{ 0TP10 } & -0.07362 & -0.15245 & -0.15254 & 0.71315 & 0.72296 & 0.18469 & -0.18903 & -0.15906 & -0.16208 \\
\hline & 0.3890 & 0.0732 & 0.0730 & $<.0001$ & $<.0001$ & 0.0295 & 0.0258 & 0.0615 & 0.0566 \\
\hline \multirow[t]{2}{*}{ ITP11 } & -0.04457 & 0.49757 & 0.49300 & 0.45325 & 0.46531 & 0.12287 & 0.10598 & -0.08330 & -0.07137 \\
\hline & 0.6024 & $<.0001$ & $<.0001$ & $<.0001$ & $<.0001$ & 0.1496 & 0.2143 & 0.3296 & 0.4037 \\
\hline
\end{tabular}


Observed to Trained NNS Output for Exercises 1,2,3

07:43 Wednesday, January 15, 2003

The CORR Procedure

Pearson Correlation Coefficients, $\mathrm{N}=139$

Prob $>|r|$ under HO: Rho=0

\begin{tabular}{|c|c|c|c|c|c|c|}
\hline & IM3 & OM3 & IM4 & OM4 & IM5 & OM5 \\
\hline \multirow[t]{2}{*}{ ITP2 } & -0.03222 & -0.02047 & -0.01029 & -0.02747 & -0.02795 & -0.02869 \\
\hline & 0.7065 & 0.8110 & 0.9043 & 0.7482 & 0.7440 & 0.7374 \\
\hline \multirow[t]{2}{*}{ 0TP2 } & 0.30661 & 0.32194 & 0.16474 & 0.19645 & 0.50923 & 0.52162 \\
\hline & 0.0002 & 0.0001 & 0.0526 & 0.0205 & $<.0001$ & $<.0001$ \\
\hline \multirow[t]{2}{*}{ ITP3 } & -0.08039 & -0.06549 & -0.02566 & -0.02400 & -0.06974 & -0.07882 \\
\hline & 0.3469 & 0.4437 & 0.7643 & 0.7792 & 0.4146 & 0.3563 \\
\hline \multirow[t]{2}{*}{ 0TP3 } & -0.06992 & -0.05523 & -0.03115 & -0.02958 & -0.06982 & -0.07931 \\
\hline & 0.4134 & 0.5184 & 0.7158 & 0.7296 & 0.4141 & 0.3534 \\
\hline \multirow[t]{2}{*}{ ITP4 } & -0.03222 & -0.01969 & -0.01029 & 0.01617 & -0.02795 & -0.01256 \\
\hline & 0.7065 & 0.8181 & 0.9043 & 0.8502 & 0.7440 & 0.8834 \\
\hline \multirow[t]{2}{*}{ OTP4 } & -0.00039 & 0.01392 & 0.01537 & 0.04454 & -0.01982 & -0.00119 \\
\hline & 0.9964 & 0.8708 & 0.8574 & 0.6026 & 0.8169 & 0.9889 \\
\hline \multirow[t]{2}{*}{ ITP5 } & 0.05491 & 0.06009 & -0.02566 & 0.05601 & -0.06974 & -0.06781 \\
\hline & 0.5209 & 0.4823 & 0.7643 & 0.5125 & 0.4146 & 0.4277 \\
\hline \multirow[t]{2}{*}{ 0TP5 } & 0.07472 & 0.08105 & -0.02420 & 0.06085 & -0.07042 & -0.06745 \\
\hline & 0.3820 & 0.3429 & 0.7774 & 0.4768 & 0.4101 & 0.4302 \\
\hline \multirow[t]{2}{*}{ ITP7 } & -0.09353 & -0.08854 & -0.02986 & -0.03800 & 0.63814 & 0.64104 \\
\hline & 0.2735 & 0.3000 & 0.7271 & 0.6570 & $<.0001$ & $<.0001$ \\
\hline \multirow[t]{2}{*}{ 0TP7 } & -0.07470 & -0.07010 & -0.02740 & -0.03786 & 0.64759 & 0.65306 \\
\hline & 0.3821 & 0.4122 & 0.7489 & 0.6581 & $<.0001$ & $<.0001$ \\
\hline \multirow[t]{2}{*}{ ITP8 } & -0.04573 & -0.02435 & -0.01460 & -0.04180 & 0.35421 & 0.33721 \\
\hline & 0.5930 & 0.7760 & 0.8646 & 0.6251 & $<.0001$ & $<.0001$ \\
\hline \multirow[t]{2}{*}{ 0TP8 } & -0.02145 & -0.00084 & -0.02378 & -0.05718 & 0.35747 & 0.34426 \\
\hline & 0.8021 & 0.9921 & 0.7812 & 0.5038 & $<.0001$ & $<.0001$ \\
\hline \multirow[t]{2}{*}{ ITP10 } & 0.73371 & 0.73487 & -0.04202 & -0.04092 & 0.40412 & 0.42151 \\
\hline & $<.0001$ & $<.0001$ & 0.6233 & 0.6325 & $<.0001$ & $<.0001$ \\
\hline \multirow[t]{2}{*}{ OTP10 } & 0.73920 & 0.74111 & -0.03750 & -0.03707 & 0.41659 & 0.43320 \\
\hline & $<.0001$ & $<.0001$ & 0.6612 & 0.6649 & $<.0001$ & $<.0001$ \\
\hline \multirow[t]{2}{*}{ ITP11 } & 0.21223 & 0.21630 & -0.10530 & -0.16277 & 0.13324 & 0.14285 \\
\hline & 0.0121 & 0.0105 & 0.2173 & 0.0556 & 0.1179 & 0.0934 \\
\hline
\end{tabular}


The CORR Procedure

07:43 Wednesday, January 15, 2003

\begin{tabular}{|c|c|c|c|c|c|c|c|c|c|}
\hline \multicolumn{10}{|c|}{$\begin{array}{l}\text { Pearson Correlation Coefficients, } N=139 \\
\text { Prob }>|r| \text { under HO: Rho=0 }\end{array}$} \\
\hline & ITP2 & 0TP2 & ITP3 & 0TP3 & ITP4 & OTP4 & ITP5 & 0TP5 & ITP7 \\
\hline \multirow[t]{2}{*}{ 0TP11 } & 0.09203 & 0.00611 & 0.02823 & 0.03577 & -0.06910 & -0.04418 & -0.17942 & -0.15918 & -0.08769 \\
\hline & 0.2812 & 0.9431 & 0.7414 & 0.6759 & 0.4189 & 0.6056 & 0.0346 & 0.0613 & 0.3047 \\
\hline \multirow[t]{2}{*}{ ITP12 } & -0.02617 & -0.04311 & -0.06529 & -0.04170 & -0.02617 & -0.03614 & -0.06529 & -0.04973 & -0.07596 \\
\hline & 0.7598 & 0.6143 & 0.4451 & 0.6260 & 0.7598 & 0.6727 & 0.4451 & 0.5610 & 0.3741 \\
\hline \multirow[t]{2}{*}{ 0TP12 } & -0.02709 & -0.03612 & -0.05126 & -0.02768 & -0.02994 & -0.04023 & -0.06193 & -0.04601 & -0.07175 \\
\hline & 0.7516 & 0.6729 & 0.5490 & 0.7463 & 0.7264 & 0.6382 & 0.4689 & 0.5907 & 0.4013 \\
\hline \multirow[t]{2}{*}{ ITP15 } & -0.02734 & 0.38661 & -0.06822 & -0.06267 & -0.02734 & 0.00622 & -0.06822 & -0.05664 & -0.07938 \\
\hline & 0.7493 & $<.0001$ & 0.4249 & 0.4636 & 0.7493 & 0.9421 & 0.4249 & 0.5078 & 0.3530 \\
\hline \multirow[t]{2}{*}{ OTP15 } & -0.02951 & 0.40411 & -0.05921 & -0.05357 & -0.01764 & 0.01526 & -0.05389 & -0.04166 & -0.07485 \\
\hline & 0.7302 & $<.0001$ & 0.4887 & 0.5311 & 0.8367 & 0.8585 & 0.5287 & 0.6263 & 0.3812 \\
\hline \multirow[t]{2}{*}{ ITP22 } & -0.01960 & 0.09092 & -0.04891 & -0.05649 & -0.01960 & -0.03564 & -0.04891 & -0.05756 & -0.05691 \\
\hline & 0.8188 & 0.2871 & 0.5675 & 0.5089 & 0.8188 & 0.6770 & 0.5675 & 0.5009 & 0.5058 \\
\hline \multirow[t]{2}{*}{ 0TP22 } & -0.01952 & 0.09377 & -0.05189 & -0.05975 & -0.02275 & -0.03942 & -0.05508 & -0.06406 & -0.05372 \\
\hline & 0.8196 & 0.2722 & 0.5441 & 0.4847 & 0.7904 & 0.6449 & 0.5196 & 0.4538 & 0.5299 \\
\hline \multirow[t]{2}{*}{ ITP23 } & -0.02961 & -0.00757 & -0.07387 & -0.07005 & -0.02961 & -0.04282 & -0.07387 & -0.07387 & -0.08595 \\
\hline & 0.7294 & 0.9295 & 0.3874 & 0.4126 & 0.7294 & 0.6167 & 0.3874 & 0.3875 & 0.3144 \\
\hline \multirow[t]{2}{*}{ 0ТР23 } & -0.01763 & 0.00247 & -0.05381 & -0.04972 & -0.03073 & -0.04346 & -0.06069 & -0.06031 & -0.08260 \\
\hline & 0.8368 & 0.9770 & 0.5292 & 0.5611 & 0.7195 & 0.6115 & 0.4779 & 0.4806 & 0.3337 \\
\hline \multirow[t]{2}{*}{ ITS1 } & -0.00725 & 0.16261 & -0.01808 & -0.01492 & -0.00725 & -0.00558 & -0.01808 & -0.02102 & -0.02104 \\
\hline & 0.9325 & 0.0558 & 0.8327 & 0.8616 & 0.9325 & 0.9481 & 0.8327 & 0.8059 & 0.8058 \\
\hline \multirow[t]{2}{*}{ OTS1 } & -0.01111 & 0.17476 & 0.00696 & 0.01022 & -0.00570 & -0.00285 & -0.01241 & -0.01454 & -0.03422 \\
\hline & 0.8967 & 0.0396 & 0.9352 & 0.9050 & 0.9469 & 0.9735 & 0.8847 & 0.8651 & 0.6892 \\
\hline \multirow[t]{2}{*}{ ITS2 } & -0.01808 & 0.40186 & 0.04511 & 0.05422 & -0.01808 & -0.03321 & -0.04511 & -0.04437 & 0.85949 \\
\hline & 0.8327 & $<.0001$ & 0.5980 & 0.5261 & 0.8327 & 0.6980 & 0.5980 & 0.6040 & $<.0001$ \\
\hline \multirow[t]{2}{*}{ OTS2 } & -0.01825 & 0.40547 & -0.04968 & -0.05921 & -0.01347 & -0.02732 & -0.04119 & -0.04024 & 0.85715 \\
\hline & 0.8312 & $<.0001$ & 0.5614 & 0.4887 & 0.8749 & 0.7496 & 0.6302 & 0.6381 & $<.0001$ \\
\hline \multirow[t]{2}{*}{ ITS5 } & -0.01808 & -0.07043 & -0.04511 & -0.05038 & 0.40078 & 0.45948 & -0.04511 & -0.03262 & -0.05249 \\
\hline & 0.8327 & 0.4100 & 0.5980 & 0.5559 & $<.0001$ & $<.0001$ & 0.5980 & 0.7031 & 0.5394 \\
\hline \multirow[t]{2}{*}{ 0TS5 } & -0.02174 & -0.07346 & -0.05259 & -0.05832 & 0.39748 & 0.46063 & -0.03280 & -0.02106 & -0.05591 \\
\hline & 0.7995 & 0.3901 & 0.5386 & 0.4953 & $<.0001$ & $<.0001$ & 0.7015 & 0.8056 & 0.5133 \\
\hline
\end{tabular}


Observed to Trained NNS Output for Exercises 1,2,3

07:43 Wednesday, January 15, 2003

The CORR Procedure

Pearson Correlation Coefficients, $N=139$

Prob $>|r|$ under $\mathrm{HO}$ : Rho=0

\begin{tabular}{|c|c|c|c|c|c|c|c|c|c|}
\hline & 0TP7 & ITP8 & 0TP8 & ITP10 & 0TP10 & ITP11 & OTP11 & ITP12 & OTP12 \\
\hline \multirow[t]{2}{*}{ 0TP11 } & -0.06008 & 0.14321 & 0.16978 & 0.40622 & 0.41139 & 0.99938 & 1.00000 & 0.35551 & 0.37533 \\
\hline & 0.4824 & 0.0926 & 0.0457 & $<.0001$ & $<.0001$ & $<.0001$ & & $<.0001$ & $<.0001$ \\
\hline \multirow[t]{2}{*}{ ITP12 } & -0.06191 & .03714 & 0.03852 & 0.22333 & .22 & 0.35272 & 1 & 1.00000 & 0.99855 \\
\hline & 0.4690 & 0.6642 & 0.6526 & 0.0082 & 0.0 & $<.0001$ & $<.0$ & & $<.0001$ \\
\hline \multirow[t]{2}{*}{ OTP12 } & -0.05742 & -0.02789 & 0.02867 & 0.22699 & 0.23082 & 0.37205 & 0.37533 & 0.99855 & 1.00000 \\
\hline & 0.5019 & 0.7445 & 0.7376 & 0.0072 & 0.0063 & $<.0001$ & $<.0001$ & $<.0001$ & \\
\hline \multirow[t]{2}{*}{ ITP15 } & -0.06996 & -0.03881 & -0.02908 & 0.60499 & 0.61657 & 0.16903 & 0.16845 & -0.09874 & -0.09947 \\
\hline & 0.4131 & 0.6501 & 0.7340 & $<.0001$ & $<.0001$ & 0.0467 & 0.0474 & 0.2475 & 0.2440 \\
\hline \multirow[t]{2}{*}{ OTP15 } & -0.06522 & -0.03843 & 0.02823 & 0.61974 & 0.63150 & 0.16322 & 0.16282 & -0.09585 & -0.09679 \\
\hline & 0.4456 & 0.6533 & 0.7415 & $<.0001$ & $<.0001$ & 0.0549 & 0.0555 & 0.2617 & 0.2570 \\
\hline \multirow[t]{2}{*}{ ITP22 } & -0.05634 & -0.02782 & 0.03433 & .08009 & .08578 & 0.20069 & 0.20459 & 0.07079 & 0.07831 \\
\hline & 0.5101 & 0.7451 & 0.6883 & 0.3486 & 0.3154 & 0.0178 & 0.0157 & 0.4076 & 0.3595 \\
\hline \multirow[t]{2}{*}{ 0TP22 } & -0.05338 & -0.03114 & 0.03780 & 0.08524 & 0.09107 & 0.22076 & -0.22521 & -0.07911 & -0.08744 \\
\hline & 0.5326 & 0.7159 & 0.6587 & 0.3184 & 0.2863 & 0.0090 & 0.0077 & 0.3546 & 0.3060 \\
\hline \multirow[t]{2}{*}{ ITP23 } & -0.09649 & 0.04202 & 0.05776 & 0.12097 & 0.11388 & 0.30311 & 0.30619 & 0.10691 & 0.11026 \\
\hline & 0.2585 & 0.6233 & 0.4994 & 0.1560 & 0.1819 & 0.0003 & 0.0002 & 0.2103 & 0.1963 \\
\hline \multirow[t]{2}{*}{ 0TP23 } & -0.09339 & 0.04261 & 850 & 0.12286 & 7 & 0.30 & 0.30 & -0.10 & 0.10812 \\
\hline & 0.2742 & 0.6184 & 0.4939 & 0.1496 & 0.1 & 0.0003 & 0.0003 & 0.2184 & 0.2052 \\
\hline \multirow[t]{2}{*}{ ITS1 } & -0.01892 & 0.01029 & 0.00630 & 0.02961 & 0.03 & 0.07419 & 0.07412 & -0.02617 & -0.02963 \\
\hline & 0.8250 & 0.9043 & 0.9413 & 0.7294 & 0.7 & 0.3854 & 0.3859 & 0.7598 & 0.7292 \\
\hline \multirow[t]{2}{*}{ OTS1 } & -0.03176 & 0.01270 & 0.00443 & 0.04599 & 0.04 & 0.08678 & 0.08750 & -0.03784 & -0.04139 \\
\hline & 0.7106 & 0.8821 & 0.9587 & 0.5908 & 0.5 & 0.3097 & 0.3057 & 0.6583 & 0.6285 \\
\hline \multirow[t]{2}{*}{ ITS2 } & 0.86513 & -0.02566 & 0.02901 & 0.07387 & 0.07 & 0.18510 & 0.18441 & -0.06529 & -0.06588 \\
\hline & $<.0001$ & 0.7643 & 0.7346 & 0.3874 & 0.3 & 0.0291 & 0.0298 & 0.4451 & 0.4410 \\
\hline \multirow[t]{2}{*}{ OTS2 } & 0.86334 & -0.02805 & 0.03211 & 0.08082 & 0.08 & 0.18384 & -0.18370 & -0.06354 & -0.06456 \\
\hline & $<.0001$ & 0.7430 & 0.7074 & 0.3443 & 0.3357 & 0.0303 & 0.0304 & 0.4574 & 0.4502 \\
\hline \multirow[t]{2}{*}{ ITS5 } & -0.05481 & -0.02566 & -0.04275 & 0.07387 & 0.06992 & 0.02931 & 0.02414 & -0.06529 & -0.07471 \\
\hline & 0.5216 & 0.7643 & 0.6173 & 0.3874 & 0.4134 & 0.7320 & 0.7779 & 0.4451 & 0.3821 \\
\hline \multirow[t]{2}{*}{ OTS5 } & -0.05733 & -0.03054 & -0.04763 & -0.07442 & 0.07090 & 0.03400 & 0.02878 & -0.07439 & -0.08443 \\
\hline & 0.5026 & 0.7211 & 0.5776 & 0.3840 & 0.4069 & 0.6911 & 0.7366 & 0.3841 & 0.3231 \\
\hline
\end{tabular}


The CORR Procedure

07:43 Wednesday, January 15, 2003

Pearson Correlation Coefficients, $N=139$ Prob $>|r|$ under HO: Rho=0

\begin{tabular}{|c|c|c|c|c|c|c|c|c|c|}
\hline & ITP15 & 0TP15 & ITP22 & 0TP22 & ITP23 & ОТР23 & ITS1 & OTS1 & ITS2 \\
\hline \multirow[t]{2}{*}{ OTP11 } & 0.16845 & .16282 & .20459 & 0.22521 & 30619 & .30417 & .07412 & -0.08750 & -0.18441 \\
\hline & 0.0474 & 0.0555 & 0.0157 & 077 & 0.0002 & 0.0003 & 0.3859 & 0.3057 & 298 \\
\hline \multirow[t]{2}{*}{ ITP12 } & .09874 & .09585 & 0.07079 & 0.07911 & 0.10691 & 0.10506 & 0.02617 & 0.03784 & 0.06529 \\
\hline & 0.2475 & 0.2617 & 0.4076 & 0.3546 & 0.2103 & 0.2184 & 0.7598 & 0.6583 & 0.4451 \\
\hline \multirow[t]{2}{*}{ 0TP12 } & -0.09947 & -0.09679 & -0.07831 & -0.08744 & 0.11026 & -0.10812 & -0.02963 & -0.04139 & -0.06588 \\
\hline & 0.2440 & 0.2570 & 0.3595 & 0.3060 & 0.1963 & 0.2052 & 0.7292 & 0.6285 & 0.4410 \\
\hline \multirow[t]{2}{*}{ ITP15 } & 1.00000 & 0.99877 & -0.07397 & -0.07481 & 0.11172 & -0.11696 & -0.02734 & -0.03187 & -0.06822 \\
\hline & & $<.0001$ & 0.3868 & 0.3814 & 0.1904 & 0.1703 & 0.7493 & 0.7096 & 0.4249 \\
\hline \multirow[t]{2}{*}{ 0TP15 } & 0.99877 & 1.00000 & -0.06584 & -0.06661 & 0.10607 & -0.11090 & -0.02890 & -0.03355 & -0.06326 \\
\hline & $<.0001$ & & 0.4413 & 0.4359 & 0.2139 & 0.1937 & 0.7355 & 0.6950 & 0.4594 \\
\hline \multirow[t]{2}{*}{ ITP22 } & 0.07397 & 0.06584 & 1.00000 & 0.99806 & 0.08009 & -0.06794 & 0.36966 & 0.38979 & -0.04891 \\
\hline & 0.3868 & 0.4413 & & $<.0001$ & 0.3486 & 0.4268 & $<.0001$ & $<.0001$ & 0.5675 \\
\hline \multirow[t]{2}{*}{ 0TP22 } & 0.07481 & 0.06661 & 0.99806 & 1.00000 & -0.05739 & -0.04604 & 0.35832 & 0.38215 & -0.04332 \\
\hline & 0.3814 & 0.4359 & $<.0001$ & & 0.5022 & 0.5904 & $<.0001$ & $<.0001$ & 0.6126 \\
\hline \multirow[t]{2}{*}{ ITP23 } & -0.11172 & -0.10607 & 0.08009 & 0.05739 & 1.00000 & 0.99886 & 0.02961 & 0.00144 & 0.07387 \\
\hline & 0.19 & 0.2139 & 0.3486 & 0.5022 & & $<.0001$ & 0.7294 & 0.9866 & 0.3874 \\
\hline \multirow[t]{2}{*}{ 0TP23 } & 0.116 & 0.11090 & -0.06794 & -0.0 & 0.99886 & 1.00000 & 63 & 87 & 0.06969 \\
\hline & 0.17 & 0.1937 & 0.4268 & 0.5904 & $<.0001$ & & 0.8368 & 0.9082 & 0.4149 \\
\hline \multirow[t]{2}{*}{ ITS1 } & 0.02734 & 0.02890 & 0.36966 & 0.35832 & 0.02961 & 0.01 & 1.00000 & 0.99189 & -0.01808 \\
\hline & 0.74 & 0.7355 & $<.0001$ & $<.0001$ & 0.7294 & 0.8368 & & $<.0001$ & 0.8327 \\
\hline \multirow[t]{2}{*}{ OTS1 } & -0.03187 & 0.03355 & 0.38979 & 0.38215 & -0.00144 & 0.00987 & 0.99189 & 1.00000 & -0.03178 \\
\hline & 0.70 & 0.6950 & $<.0001$ & $<.0001$ & 0.9866 & 0.9082 & $<.0001$ & & 0.7103 \\
\hline \multirow[t]{2}{*}{ ITS2 } & -0.06822 & 0.06326 & 0.04891 & .04332 & 0.07387 & 0.06969 & 0.01808 & 0.03178 & 1.00000 \\
\hline & 0.4249 & 0.4594 & 0.5675 & 0.6126 & 0.3874 & 0.4149 & 0.8327 & 0.7103 & \\
\hline \multirow[t]{2}{*}{ OTS2 } & -0.07112 & -0.06589 & -0.03309 & -0.02696 & -0.05965 & 0.05495 & 0.02085 & 0.03398 & 0.99872 \\
\hline & 0.4054 & 0.4409 & 0.6990 & 0.7527 & 0.4854 & 0.5205 & 0.8075 & 0.6913 & $<.0001$ \\
\hline \multirow[t]{2}{*}{ ITS5 } & 0.17 & 0.17275 & -0.04891 & 0.04785 & -0.07387 & -0.07088 & 0.01808 & 0.02007 & -0.04511 \\
\hline & 0.0394 & 0.0420 & 0.5675 & 0.5759 & 0.3874 & 0.4070 & 0.8327 & 0.8146 & 0.5980 \\
\hline \multirow[t]{2}{*}{ OTS5 } & 0.18 & 0.17797 & 0.04672 & -0.04612 & -0.08150 & -0.07864 & 0.01248 & -0.01383 & -0.04618 \\
\hline & 0.0338 & 0.0361 & 0.5850 & 0.5898 & 0.3402 & 0.3575 & 0.8840 & 0.8717 & 0.589 \\
\hline
\end{tabular}


The CORR Procedure

Pearson Correlation Coefficients, $\mathrm{N}=139$

Prob > $|r|$ under $\mathrm{HO}$ : Rho=0

\begin{tabular}{|c|c|c|c|c|c|c|c|c|c|}
\hline & OTS2 & ITS5 & OTS5 & ITS8 & OTS8 & ITS11 & OTS11 & ITS12 & OTS12 \\
\hline \multirow[t]{2}{*}{ 0TP11 } & -0.18370 & 0.02414 & 0.02878 & .06928 & 0.18595 & -0.16656 & -0.16791 & 0.43666 & 0.44566 \\
\hline & 0.0304 & 0.7779 & 0.7366 & 0.4177 & 0.0284 & 00 & 0.0482 & $<.0001$ & $<.0$ \\
\hline \multirow[t]{2}{*}{ ITP12 } & -0.06354 & .06529 & 0.07439 & 0.02617 & .49150 & -0.05 & -0.04233 & 0.09 & 0.08622 \\
\hline & 0.4574 & 0.4451 & 0.3841 & 0.7598 & $<.0001$ & 0.4875 & 0.6 & 0.2 & 0 . \\
\hline \multirow[t]{2}{*}{ OTP12 } & -0.06456 & -0.07471 & 0.08443 & -0.02963 & 0.50524 & -0.05529 & -0.03800 & -0.07653 & -0.07197 \\
\hline & 0.4502 & 0.3821 & 0.3231 & 0.7292 & $<.0001$ & 0.5180 & 0.6570 & 0.3706 & 0.3998 \\
\hline \multirow[t]{2}{*}{ ITP15 } & -0.07112 & 0.17493 & 0.18014 & -0.02734 & 0.06429 & 0.20332 & 0.22105 & -0.16133 & -0.16225 \\
\hline & 0.4054 & 0.0394 & 0.0338 & 0.7493 & 0.4521 & 0.0164 & 0.0089 & 0.0578 & 0.0564 \\
\hline \multirow[t]{2}{*}{ OTP15 } & -0.06589 & 0.17275 & 0.17797 & 0.02951 & 0.06000 & 0.21602 & 0.23475 & -0.16335 & -0.16445 \\
\hline & 0.4409 & 0.0420 & 0.0361 & 0.7302 & 0.4829 & 0.0106 & 0.0054 & 0.0547 & 0.0531 \\
\hline \multirow[t]{2}{*}{ ITP22 } & -0.03309 & 0.04891 & 0.04672 & 0.01960 & 0.14070 & 0.04448 & 0.04876 & 0.11566 & 0.11692 \\
\hline & 0.6990 & 0.5675 & 0.5850 & 0.8188 & 0.0985 & 0.6031 & 0.5687 & 0.1751 & 0.1705 \\
\hline \multirow[t]{2}{*}{ 0TP22 } & -0.02696 & 0.04785 & 0.04612 & 0.02275 & 0.14998 & -0.04135 & -0.04611 & -0.12715 & -0.12850 \\
\hline & 0.7527 & 0.5759 & 0.5898 & 0.7904 & 0.0780 & 0.6289 & 0.5899 & 0.1358 & 0.1317 \\
\hline \multirow[t]{2}{*}{ ITP23 } & -0.05965 & 0.07387 & 0.08150 & 0.02961 & 0.12718 & 0.06718 & 0.04937 & 0.05656 & 0.05413 \\
\hline & 0.4854 & 0.3874 & 0.3402 & 0.7294 & 0.1357 & 0.4320 & 0.5638 & 0.5084 & 0.5268 \\
\hline \multirow[t]{2}{*}{ 0TP23 } & -0.05495 & -0.07088 & 0.07864 & 0.02560 & 0. & 36 & 0.04 & 0.05 & 0.0 \\
\hline & 0.5205 & 0.4070 & 0.3575 & 0.7648 & 0.1633 & 0.4950 & 0.6376 & 0.5558 & 0.5757 \\
\hline \multirow[t]{2}{*}{ ITS1 } & -0.02085 & 0.01808 & 0.01248 & 0.00725 & 0.02885 & 0.0 & -0.00580 & 0.04 & 0.04274 \\
\hline & 0.8075 & 0.8327 & 0.8840 & 0.9325 & 0.7360 & 0.8476 & 0.9460 & 0.6173 & 0.6173 \\
\hline \multirow[t]{2}{*}{ OTS1 } & -0.03398 & 0.02007 & 0.01383 & 0.00895 & 0.03029 & 0.00330 & 0.01448 & 0.04251 & -0.04193 \\
\hline & 0.6913 & 0.8146 & 0.8717 & 0.9168 & 0.7234 & 0.9693 & 0.8656 & 0.6193 & 0.6241 \\
\hline \multirow[t]{2}{*}{ ITS2 } & 0.99872 & -0.04511 & 0.04618 & 0.01808 & 0.11457 & 0.04103 & -0.05307 & -0.10668 & -0.11154 \\
\hline & $<.0001$ & 0.5980 & 0.5893 & 0.8327 & 0.1793 & 0.6315 & 0.5349 & 0.2113 & 0.1911 \\
\hline \multirow[t]{2}{*}{ OTS2 } & 1.00000 & -0.03867 & -0.03935 & 0.01130 & 0.11766 & -0.04 & -0.05587 & -0.10702 & -0.11182 \\
\hline & & 0.6513 & 0.6456 & 0.8950 & 0.1677 & 0.6057 & 0.5136 & 0.2099 & 0.1900 \\
\hline \multirow[t]{2}{*}{ ITS5 } & -0.03867 & 1.00000 & 0.99818 & 0.01808 & -0.12876 & -0.04103 & -0.03880 & -0.10668 & -0.11200 \\
\hline & 0.6513 & & $<.0001$ & 0.8327 & 0.1309 & 0.6315 & 0.6502 & 0.2113 & 0.1893 \\
\hline \multirow[t]{2}{*}{ OTS5 } & -0.03935 & 0.99818 & 1.00000 & 0.00014 & -0.14173 & -0.03773 & -0.03576 & -0.10077 & -0.10587 \\
\hline & 0.6456 & $<.0001$ & & 0.9987 & 0.0960 & 0.6592 & 0.6760 & 0.2379 & 0.2148 \\
\hline
\end{tabular}


The CORR Procedure

07:43 Wednesday, January 15, 2003

Pearson Correlation Coefficients, $N=139$ Prob > $|r|$ under $\mathrm{HO}: \mathrm{Rho}=0$

\begin{tabular}{|c|c|c|c|c|c|c|c|c|c|}
\hline & ITS15 & OTS15 & ITS18 & OTS18 & ITS19 & OTS19 & ITS20 & OTS20 & s22 \\
\hline \multirow[t]{2}{*}{ OTP11 } & 0.11270 & .13275 & .38154 & 0.38810 & .11958 & .12173 & .10634 & -0.09934 & 932 \\
\hline & 0.1865 & 0.1193 & $<.0001$ & 01 & 0.1 & 0.1534 & & & \\
\hline \multirow[t]{2}{*}{ ITP12 } & 0.40676 & .43082 & 0.06736 & 08780 & 0.09449 & 0.10163 & 0.03 & 0.05 & 0.03714 \\
\hline & $<.0001$ & $<.0001$ & 0.4308 & 0.3041 & 0.2 & 0.2339 & 0.6 & 17 & 42 \\
\hline \multirow[t]{2}{*}{ 0TP12 } & 0.39927 & .42460 & 0.07312 & 0.09375 & 0.09602 & -0.10303 & -0.04250 & -0.05865 & 0.03193 \\
\hline & $<.0001$ & $<.0001$ & 0.3923 & 0.2723 & 0.2608 & 0.2274 & 0.6194 & 0.4928 & 0.7090 \\
\hline \multirow[t]{2}{*}{ ITP15 } & -0.05529 & 0.02238 & 0.63140 & 0.63125 & 0.09874 & -0.11064 & -0.03881 & -0.05822 & 0.03881 \\
\hline & 0.5180 & 0.7937 & $<.0001$ & $<.0001$ & 0.2475 & 0.1948 & 0.6501 & 0.4960 & 0.6501 \\
\hline \multirow[t]{2}{*}{ 0TP15 } & -0.03798 & 0.00463 & 0.62909 & 0.62985 & -0.09576 & -0.10782 & -0.04275 & -0.06239 & 0.03065 \\
\hline & 0.6571 & 0.9569 & $<.0001$ & $<.0001$ & 0.2621 & 0.2064 & 0.6173 & 0.4656 & 0.7202 \\
\hline \multirow[t]{2}{*}{ ITP22 } & 0.03964 & 0.04829 & 0.07707 & 0.08065 & 0.07079 & 0.05611 & -0.02782 & 0.01076 & 0.02782 \\
\hline & 0.6431 & 0.5724 & 0.3672 & 0.3453 & 0.4076 & 0.5118 & 0.7451 & 0.9000 & 0.7451 \\
\hline \multirow[t]{2}{*}{ 0TP22 } & 0.04600 & 0.05568 & 0.08104 & 0.08476 & -0.06135 & -0.04654 & -0.00533 & 0.03139 & 0.02369 \\
\hline & 0.5908 & 0.5150 & 0.3430 & 0.3211 & 0.4731 & 0.5865 & 0.9503 & 0.7138 & 0.7820 \\
\hline \multirow[t]{2}{*}{ ITP23 } & 0.05987 & 0.07578 & 0.11640 & -0.10771 & 0.63612 & 0.63971 & 0.34739 & 0.36068 & -0.04202 \\
\hline & 0.4839 & 0.3753 & 0.1724 & 0.2069 & $<.0001$ & $<.0001$ & $<.0001$ & $<.0001$ & 0.6233 \\
\hline \multirow[t]{2}{*}{ 0TP23 } & 0.05927 & 07490 & 0.10 & -0.10 & 0.63 & 0.64 & 0.3 & 0.3 & 998 \\
\hline & 0.4883 & 0.3808 & 0.1986 & 0.2370 & $<.0001$ & $<.0001$ & $<.0$ & $<.0001$ & 0.6403 \\
\hline \multirow[t]{2}{*}{ ITS1 } & 0.01 & 0.01816 & 0.02849 & 0.02 & 0.02 & $0.02 \varepsilon$ & 0.0 & 0.0 & 0.01029 \\
\hline & 0.8641 & 0.8320 & 0.7392 & 0.7 & 0.7 & 0.7387 & 43 & 0.3 & 0.9043 \\
\hline \multirow[t]{2}{*}{ OTS1 } & 0.02411 & 0.02825 & 0.02300 & 0.02301 & 0.03752 & 0.03913 & 0.06 & 0.15366 & -0.01270 \\
\hline & 0.7781 & 0.7413 & 0.7881 & 0.7 & 0.6 & 0.6474 & 0.4 & 0.0 & 0.8821 \\
\hline \multirow[t]{2}{*}{ ITS2 } & 0.03656 & 0.04486 & 0.07108 & 0.07369 & 0.06529 & 0.06593 & 0.02566 & 0.00141 & 0.02566 \\
\hline & 0.6692 & 0.6000 & 0.4057 & 0.3886 & 0.4 & 0.4406 & 0.7643 & 0.9868 & 0.7643 \\
\hline \multirow[t]{2}{*}{ OTS2 } & 0.04107 & 0.04939 & 0.07308 & 0.07577 & -0.05505 & 0.05506 & 0.01 & 0.00981 & -0.01727 \\
\hline & 0.6312 & 0.5637 & 0.3926 & 0.3753 & 0.5198 & 0.5197 & 0.8513 & 0.9088 & 0.8401 \\
\hline \multirow[t]{2}{*}{ ITS5 } & 0.03656 & -0.01691 & 0.07108 & -0.073 & -0.06529 & -0.06105 & -0.02566 & -0.00836 & -0.02566 \\
\hline & 0.6692 & 0.8433 & 0.4057 & 0.3871 & 0.44 & 0.4753 & 0.7643 & 0.9222 & 0.7643 \\
\hline \multirow[t]{2}{*}{ OTS5 } & 0.03259 & 0.01315 & 0.07383 & 0.07700 & -0.07 & -0.06640 & -0.01647 & 0.00250 & -0.01647 \\
\hline & 0.7033 & 0.8779 & 0.3877 & 0.3676 & 0.4119 & 0.4374 & 0.8474 & 0.9767 & 0.847 \\
\hline
\end{tabular}


07:43 Wednesday, January 15, 2003

The CORR Procedure

Pearson Correlation Coefficients, $N=139$

Prob $>|r|$ under HO: Rho $=0$

\begin{tabular}{|c|c|c|c|c|c|c|c|c|c|}
\hline & OTS22 & ITS23 & 0TS23 & ITA2 & ОТА2 & ITA3 & ОТАЗ & ITA7 & A7 \\
\hline \multirow[t]{2}{*}{ 0TP11 } & 0.02635 & 0.17944 & 0.16700 & 0.07788 & -0.11595 & 0.04043 & 0.04249 & -0.19044 & -0.19848 \\
\hline & .7582 & 0.0345 & 0.0494 & 0.3622 & 0.1740 & 0.6365 & 0.6195 & 0.0247 & 0.0192 \\
\hline \multirow[t]{2}{*}{ ITP12 } & -0.01724 & 0.33921 & 0.34278 & -0.02617 & -0.02536 & 0.09449 & -0.09097 & -0.06529 & -0.08189 \\
\hline & 0.8404 & $<.0001$ & $<.0001$ & 0.7598 & 0.7669 & 0.2685 & 0.2868 & 0.4451 & 0.3379 \\
\hline \multirow[t]{2}{*}{ 0TP12 } & -0.01151 & 0.34546 & 0.34868 & -0.03026 & -0.03125 & -0.08972 & -0.08642 & -0.07497 & -0.09246 \\
\hline & 0.8930 & $<.0001$ & $<.0001$ & 0.7236 & 0.7149 & 0.2935 & 0.3118 & 0.3804 & 0.2790 \\
\hline \multirow[t]{2}{*}{ ITP15 } & -0.03278 & .12389 & .12604 & 0.02734 & -0.03288 & -0.09874 & -0.09112 & -0.06822 & -0.08325 \\
\hline & 0.7016 & 0.1462 & 0.1393 & 0.7493 & 0.7008 & 0.2475 & 0.2861 & 0.4249 & 0.3299 \\
\hline \multirow[t]{2}{*}{ TP15 } & -0.02265 & 0.12468 & 0.12669 & 0.01825 & -0.02235 & 0.09814 & 0.09047 & 0.06933 & -0.08471 \\
\hline & 0.7913 & 0.1436 & 0.1372 & 0.8312 & 0.7940 & 0.2504 & 0.2895 & 0.4173 & 0.3214 \\
\hline \multirow[t]{2}{*}{ ITP22 } & -0.01371 & 0.49908 & 0.50055 & 0.36966 & 0.42595 & 0.07079 & 0.05575 & 0.59858 & 0.61349 \\
\hline & 0.8727 & $<.0001$ & $<.0001$ & $<.0001$ & $<.0001$ & 0.4076 & 0.5145 & $<.0001$ & $<.0001$ \\
\hline \multirow[t]{2}{*}{ 0TP22 } & -0.00881 & 0.49188 & 0.49454 & 0.37610 & 0.43439 & 0.07230 & 0.05731 & 0.61256 & 0.62827 \\
\hline & 0.9180 & $<.0001$ & $<.0001$ & $<.0001$ & $<.0001$ & 0.3977 & 0.5028 & $<.0001$ & $<.0001$ \\
\hline \multirow[t]{2}{*}{ ITP23 } & -0.03196 & 0.13415 & .13329 & .02961 & 0.01468 & 0.10691 & -0.11028 & 0.15431 & 1838 \\
\hline & 0.7088 & 0.1154 & 0.1178 & 0.7294 & 0.8638 & 0.2103 & 0.1962 & 0.0697 & 0.0813 \\
\hline \multirow[t]{2}{*}{ TP23 } & -0.02866 & 0.12856 & .12765 & .02703 & 0.01738 & 0.10052 & -0.10354 & 0.15760 & 0.1 \\
\hline & 0.7377 & 0.1315 & 0.1342 & 0.7521 & 0.8391 & 0.2390 & 0.2251 & 0.0639 & 0.0743 \\
\hline \multirow[t]{2}{*}{ ITS1 } & -0.01304 & 0.03283 & 0.02277 & 0.00725 & 0.00196 & 0.02617 & 0.02933 & 0.40078 & 0.39446 \\
\hline & 0.8789 & 0.7012 & 0.7902 & 0.9325 & 0.9818 & 0.7598 & 0.7318 & $<.0001$ & $<.0001$ \\
\hline \multirow[t]{2}{*}{ OTS1 } & -0.01535 & 0.02908 & 0.01834 & 0.00570 & 0.00272 & 0.04078 & -0.04353 & 0.46189 & 0.45602 \\
\hline & 0.8576 & 0.7340 & 0.8303 & 0.9469 & 0.9746 & 0.6336 & 0.6109 & $<.0001$ & $<.0001$ \\
\hline \multirow[t]{2}{*}{ ITS2 } & 0.00388 & -0.08192 & 0.07494 & 0.01808 & 0.00621 & 0.69097 & 0.69187 & 0.04511 & 0.04188 \\
\hline & 0.9639 & 0.3377 & 0.3806 & 0.8327 & 0.9422 & $<.0001$ & $<.0001$ & 0.5980 & 0.6245 \\
\hline \multirow[t]{2}{*}{ OTS2 } & 0.01415 & -0.06497 & 0.05800 & 0.00002 & 0.01335 & 0.69667 & 0.69838 & 0.03253 & -0.02874 \\
\hline & 0.8687 & 0.4473 & 0.4976 & 0.9999 & 0.8760 & $<.0001$ & $<.0001$ & 0.7038 & 0.7370 \\
\hline \multirow[t]{2}{*}{ ITS5 } & -0.02674 & -0.08192 & -0.07925 & 0.01808 & -0.04038 & 0.06529 & 0.04859 & 0.04511 & -0.03493 \\
\hline & 0.7547 & 0.3377 & 0.3537 & 0.8327 & 0.6370 & 0.4451 & 0.5700 & 0.5980 & 0.6831 \\
\hline \multirow[t]{2}{*}{ OTS5 } & -0.01821 & -0.08641 & -0.08389 & 0.02174 & -0.04515 & 0.06484 & -0.04782 & 0.04013 & -0.02968 \\
\hline & 0.8315 & 0.3118 & 0.3262 & 0.7995 & 0.5977 & 0.4482 & 0.5762 & 0.6391 & 0.7287 \\
\hline
\end{tabular}


The CORR Procedure

07:43 Wednesday, January 15, 2003

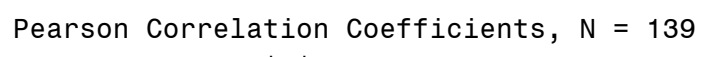

\begin{tabular}{|c|c|c|c|c|c|c|c|c|c|}
\hline & ITA10 & OTA10 & ITA11 & OTA11 & ITA12 & OTA12 & ITA18 & OTA18 & ITA 19 \\
\hline \multirow[t]{2}{*}{ 0TP11 } & .11028 & 0.13301 & 0.20244 & .20742 & . 12307 & .09206 & 19819 & 0.19229 & 0.02758 \\
\hline & 0.1962 & 0.1185 & 0.0168 & 0.0143 & 0.1489 & 0.2811 & 0.0193 & 0.0233 & 0.7472 \\
\hline \multirow[t]{2}{*}{ ITP12 } & 0.03714 & 0.04176 & 0.07079 & 0.07181 & .04565 & 01934 & .63202 & .64432 & 0.13062 \\
\hline & 0.6642 & 0.6254 & 0.4076 & 0.4009 & 0.5936 & 0.8212 & $<.0001$ & & 0.1254 \\
\hline \multirow[t]{2}{*}{ 0TP12 } & -0.04205 & 0.04712 & 0.07537 & 0.07691 & 0.03640 & 0.00898 & .63967 & 0.65225 & 0.12888 \\
\hline & 0.6231 & 0.5818 & 0.3779 & 0.3682 & 0.6 & 0.9164 & $<.0$ & $<.0001$ & \\
\hline \multirow[t]{2}{*}{ ITP15 } & 0.37616 & 0.38135 & 0.15200 & 0.17072 & 0.04771 & 0.03482 & 0.07397 & 0.08900 & 0.04771 \\
\hline & $<.0001$ & $<.0001$ & 0.0741 & 0.0445 & 0.5 & 0.6841 & 0.3868 & 0.2975 & 0.5771 \\
\hline \multirow[t]{2}{*}{ 0TP15 } & 0.37837 & 0.38461 & 0.15796 & 0.17756 & 0.03 & -0.01963 & 0.08055 & 0.09536 & 0.04388 \\
\hline & $<.0001$ & $<.0001$ & 0.0633 & 0.0365 & 0.6975 & 0.8186 & 0.3459 & 0.2641 & 0.6080 \\
\hline \multirow[t]{2}{*}{ ITP22 } & -0.02782 & -0.00748 & -0.05303 & -0.03666 & -0.03420 & -0.04448 & 0.05303 & -0.03477 & 0.41857 \\
\hline & 0.7451 & 0.9304 & 0.5353 & 0.6683 & 0.6894 & 0.6031 & 0.5353 & 0.6845 & $<.0001$ \\
\hline \multirow[t]{2}{*}{ 0TP22 } & -0.01881 & 0.00186 & -0.05014 & -0.03324 & -0.03758 & -0.04833 & 0.05295 & -0.03475 & 0.41107 \\
\hline & 0.8260 & 0.9827 & 0.5578 & 0.6977 & 0.6 & 0.5721 & 0.5359 & 47 & $<.0001$ \\
\hline \multirow[t]{2}{*}{ ITP23 } & -0.04202 & 0.05350 & 0.34402 & 0.35003 & -0.05 & -0.04447 & 0.02594 & 0.04 & 0.05166 \\
\hline & 0.6233 & 0.5317 & $<.0001$ & $<.0001$ & 0.5 & 0.6032 & 0.76 & 0.6313 & 0.5459 \\
\hline \multirow[t]{2}{*}{ 0TP23 } & -0.04484 & 0.05584 & 0.33899 & 0.34594 & -0.03804 & -0.02990 & 0.02247 & 0.03855 & 0.03970 \\
\hline & 0.6002 & 0.5138 & $<.0001$ & $<.0001$ & 0.6566 & 0.7268 & 0.7929 & 0.6523 & 0.6426 \\
\hline \multirow[t]{2}{*}{ ITS1 } & -0.01029 & -0.00466 & 0.01960 & 0.01907 & -0.01264 & -0.01522 & 0.01960 & 0.02112 & 0.01264 \\
\hline & 0.9043 & 0.9565 & 0.8188 & 0.8237 & 0.8826 & 0.8589 & 0.8188 & 0.8050 & 0.8826 \\
\hline \multirow[t]{2}{*}{ OTS1 } & 0.00652 & 0.01316 & 0.01290 & 0.01023 & 0.00112 & 0.00274 & 0.03299 & 0.03272 & 0.00868 \\
\hline & 0.9393 & 0.8778 & 0.8802 & 0.9048 & 0.9 & 0.9744 & 0.6998 & 0.7022 & 0.9192 \\
\hline \multirow[t]{2}{*}{ ITS2 } & -0.02566 & 0.00365 & 0.04891 & 0.04381 & -0.03155 & 0.03643 & 0.04891 & 0.05390 & 0.03155 \\
\hline & 0.7643 & 0.9660 & 0.5675 & 0.6086 & 0.7124 & 0.6702 & 0.5675 & 0.5286 & 0.7124 \\
\hline \multirow[t]{2}{*}{ OTS2 } & -0.02590 & 0.00279 & 0.04232 & -0.03613 & -0.03537 & -0.03972 & 0.04651 & 0.05077 & 0.01921 \\
\hline & 0.7622 & 0.9740 & 0.6209 & 0.6728 & 0.6793 & 0.6425 & 0.5867 & 0.5528 & 0.8224 \\
\hline \multirow[t]{2}{*}{ ITS5 } & -0.02566 & 0.00611 & 0.27484 & 0.27635 & -0.03155 & -0.04128 & 0.04891 & 0.05578 & -0.03155 \\
\hline & 0.7643 & 0.9431 & 0.0011 & 0.0010 & 0.7124 & 0.6295 & 0.5675 & 0.5143 & 0.7124 \\
\hline \multirow[t]{2}{*}{ 0TS5 } & 0.01302 & 0.00646 & 0.27032 & 0.27271 & -0.03767 & -0.04768 & 0.05864 & -0.06529 & -0.02768 \\
\hline & 0.8790 & 0.9398 & 0.0013 & 0.0012 & 0.6597 & 0.5773 & 0.4929 & 0.4451 & 0.746 \\
\hline
\end{tabular}


The CORR Procedure

Pearson Correlation Coefficients, $\mathrm{N}=139$

Prob $>|r|$ under $\mathrm{HO}$ : Rho=0

\begin{tabular}{|c|c|c|c|c|c|c|c|c|c|}
\hline & OTA19 & ITA21 & 0TA21 & ITA23 & 0TA23 & IM1 & OM1 & IM2 & OM2 \\
\hline \multirow[t]{2}{*}{ OTP11 } & .04284 & 0.49863 & 49441 & 0.45576 & .46819 & 0.11369 & .09697 & -0.07614 & 6492 \\
\hline & 0.6165 & .0001 & $<.0001$ & $<.0001$ & $<.0001$ & 0.1826 & 561 & 730 & 477 \\
\hline \multirow[t]{2}{*}{ ITP12 } & 0.14164 & .13329 & .13363 & 0.10807 & 11436 & 0.11456 & 403 & 3101 & 7939 \\
\hline & 0963 & 0.1177 & 0.1168 & 0.2054 & 0.1801 & 0.1793 & 1814 & 431 & 3529 \\
\hline \multirow[t]{2}{*}{ TP12 } & 0.13933 & 0.12304 & 0.12352 & 0.11412 & 0.12094 & 0.11384 & 0.11307 & -0.08561 & -0.08389 \\
\hline & 0.1019 & 0.1490 & 0.1474 & 0.1810 & 0.1561 & 0.1821 & 0.1851 & 0.3163 & 0.3262 \\
\hline \multirow[t]{2}{*}{ ITP15 } & -0.07012 & 0.13929 & 0.14424 & 0.40438 & 0.42087 & 0.16341 & 0.17044 & -0.34157 & -0.32634 \\
\hline & 0.4121 & 0.1020 & 0.0903 & $<.0001$ & $<.0001$ & 0.0546 & 0.0449 & $<.0001$ & $<.0001$ \\
\hline \multirow[t]{2}{*}{ DTP15 } & -0.06661 & 0.15175 & 0.15671 & 0.41657 & 0.43307 & 0.16848 & -0.17523 & -0.34973 & -0.33504 \\
\hline & 0.4359 & 0.0745 & 0.0654 & $<.0001$ & $<.0001$ & 0.0474 & 0.0391 & $<.0001$ & $<.0001$ \\
\hline \multirow[t]{2}{*}{ ITP22 } & 0.42976 & 0.09986 & 0.10562 & .11307 & 0.11639 & 0.32028 & 0.33523 & -0.24488 & -0.25447 \\
\hline & $<.0001$ & 0.2422 & 0.2159 & 0.1851 & 0.1724 & 0.0001 & $<.0001$ & 0.0037 & 0.0025 \\
\hline \multirow[t]{2}{*}{ 0TP22 } & 0.42370 & -0.11467 & 0.12064 & 0.11930 & 0.12293 & 0.32660 & 0.34211 & -0.25571 & -0.26610 \\
\hline & $<.0001$ & 0.1789 & 0.1572 & 0.1619 & 0.1494 & $<.0001$ & $<.0001$ & 0.0024 & 0.0015 \\
\hline \multirow[t]{2}{*}{ ITP23 } & -0.01821 & -0.15082 & -0.15324 & -0.17077 & -0.16476 & 0.12436 & 0.13802 & -0.36985 & -0.38208 \\
\hline & 0.8315 & 0.0764 & 0.0717 & 0.0444 & 0.0526 & 0.1447 & 0.1052 & $<.0001$ & $<.0001$ \\
\hline \multirow[t]{2}{*}{ 0TP23 } & -0.00709 & -0.16012 & -0.16281 & -0.16047 & -0.15458 & 0.13814 & 0.15197 & -0.37864 & -0.39128 \\
\hline & 0.9340 & 0.0597 & 0.0555 & 0.0592 & 0.0692 & 49 & 741 & 001 & $<.0001$ \\
\hline \multirow[t]{2}{*}{ ITS1 } & -0.01849 & -0.03691 & 0.03707 & 0.04180 & 0.04191 & 0.18822 & 492 & 052 & -0.09474 \\
\hline & 0.8290 & 0.6662 & 0.6648 & 0.6252 & 0.6243 & 265 & 293 & 392 & 673 \\
\hline \multirow[t]{2}{*}{ OTS1 } & -0.01466 & -0.04356 & -0.04465 & 0.04396 & -0.04365 & 0.21460 & 288 & 0.10116 & -0.10501 \\
\hline & 0.8640 & 0.6106 & 0.6017 & 0.6073 & 0.6099 & 112 & 119 & 360 & 0.2186 \\
\hline \multirow[t]{2}{*}{ ITS2 } & -0.02483 & -0.09210 & -0.08674 & 0.10429 & -0.10400 & 0.08450 & 0.09017 & -0.22586 & -0.23478 \\
\hline & 0.7717 & 0.2809 & 0.3100 & 0.2218 & 0.2231 & 0.3226 & 0.2912 & 0.0075 & 0.0054 \\
\hline \multirow[t]{2}{*}{ OTS2 } & -0.01116 & -0.09154 & -0.08653 & -0.10719 & 0.10679 & 0.10121 & 0.10755 & -0.24373 & 0.25258 \\
\hline & 0.8962 & 0.2838 & 0.3111 & 0.2091 & 0.2108 & 0.2358 & 0.2076 & 0.0038 & 0.0027 \\
\hline \multirow[t]{2}{*}{ ITS5 } & -0.03081 & -0.09210 & -0.09915 & 0.25363 & 0.25498 & 0.21288 & 0.20448 & -0.12393 & -0.11886 \\
\hline & 0.7188 & 0.2809 & 0.2455 & 0.0026 & 0.0025 & 0.0119 & 0.0158 & 0.1461 & 0.1634 \\
\hline \multirow[t]{2}{*}{ OTS5 } & -0.02679 & -0.08053 & -0.08794 & 0.26080 & 0.26187 & 0.21200 & 0.20347 & 0.11782 & -0.11104 \\
\hline & 0.7542 & 0.3460 & 0.3033 & 0.0019 & 0.0018 & 0.0122 & 0.0163 & 0.1672 & 0.1931 \\
\hline
\end{tabular}


Observed to Trained NNS Output for Exercises 1,2,3 20

$07: 43$ Wednesday, January 15, 2003

The CORR Procedure

Pearson Correlation Coefficients, $N=139$

Prob > $|r|$ under $\mathrm{HO}:$ Rho=0

\begin{tabular}{|c|c|c|c|c|c|c|}
\hline & IM3 & OM3 & IM4 & OM4 & IM5 & OM5 \\
\hline \multirow[t]{2}{*}{ OTP11 } & 0.21655 & 0.22033 & -0.11028 & -0.16731 & 0.13747 & 0.14753 \\
\hline & 0.0105 & 0.0092 & 0.1962 & 0.0490 & 0.1066 & 0.0831 \\
\hline \multirow[t]{2}{*}{ ITP12 } & 0.05643 & 0.05777 & -0.03714 & -0.04488 & -0.10093 & -0.10461 \\
\hline & 0.5094 & 0.4994 & 0.6642 & 0.5999 & 0.2371 & 0.2204 \\
\hline \multirow[t]{2}{*}{ OTP12 } & 0.06014 & 0.06122 & -0.04205 & -0.05127 & -0.09762 & -0.10158 \\
\hline & 0.4819 & 0.4741 & 0.6231 & 0.5489 & 0.2529 & 0.2341 \\
\hline \multirow[t]{2}{*}{ ITP15 } & 0.66723 & 0.67079 & 0.37616 & 0.37133 & 0.44686 & 0.45886 \\
\hline & $<.0001$ & $<.0001$ & $<.0001$ & $<.0001$ & $<.0001$ & $<.0001$ \\
\hline \multirow[t]{2}{*}{ OTP15 } & 0.67870 & 0.68346 & 0.37837 & 0.37528 & 0.45410 & 0.46644 \\
\hline & $<.0001$ & $<.0001$ & $<.0001$ & $<.0001$ & $<.0001$ & $<.0001$ \\
\hline \multirow[t]{2}{*}{ ITP22 } & 0.03858 & 0.04929 & -0.02782 & -0.06281 & -0.07561 & -0.07107 \\
\hline & 0.6521 & 0.5645 & 0.7451 & 0.4626 & 0.3763 & 0.4058 \\
\hline \multirow[t]{2}{*}{ 0TP22 } & 0.03259 & 0.04365 & -0.01881 & -0.05400 & -0.07461 & -0.07019 \\
\hline & 0.7033 & 0.6099 & 0.8260 & 0.5278 & 0.3827 & 0.4116 \\
\hline \multirow[t]{2}{*}{ ITP23 } & -0.13163 & -0.12607 & -0.04202 & -0.06793 & -0.11420 & -0.11882 \\
\hline & 0.1224 & 0.1392 & 0.6233 & 0.4269 & 0.1807 & 0.1636 \\
\hline \multirow[t]{2}{*}{ 0TP23 } & -0.13598 & -0.12950 & -0.04484 & -0.06992 & -0.11936 & -0.12378 \\
\hline & 0.1105 & 0.1287 & 0.6002 & 0.4134 & 0.1616 & 0.1466 \\
\hline \multirow[t]{2}{*}{ ITS1 } & -0.03222 & -0.01696 & -0.01029 & -0.02747 & -0.02795 & -0.01463 \\
\hline & 0.7065 & 0.8429 & 0.9043 & 0.7482 & 0.7440 & 0.8643 \\
\hline \multirow[t]{2}{*}{ 0TS1 } & -0.04447 & -0.02743 & 0.00652 & -0.01459 & -0.04499 & -0.03056 \\
\hline & 0.6032 & 0.7486 & 0.9393 & 0.8647 & 0.5989 & 0.7210 \\
\hline \multirow[t]{2}{*}{ ITS2 } & -0.08039 & -0.08720 & -0.02566 & -0.01905 & 0.52375 & 0.53703 \\
\hline & 0.3469 & 0.3074 & 0.7643 & 0.8239 & $<.0001$ & $<.0001$ \\
\hline \multirow[t]{2}{*}{ 0TS2 } & -0.08270 & -0.08893 & -0.02590 & -0.01882 & 0.52285 & 0.53640 \\
\hline & 0.3331 & 0.2979 & 0.7622 & 0.8259 & $<.0001$ & $<.0001$ \\
\hline \multirow[t]{2}{*}{ ITS5 } & 0.19020 & 0.20511 & -0.02566 & 0.03622 & -0.06974 & -0.05903 \\
\hline & 0.0249 & 0.0154 & 0.7643 & 0.6721 & 0.4146 & 0.4900 \\
\hline \multirow[t]{2}{*}{ 0TS5 } & 0.19385 & 0.20878 & -0.01302 & 0.04953 & -0.06895 & -0.05732 \\
\hline & 0.0222 & 0.0136 & 0.8790 & 0.5625 & 0.4199 & 0.5027 \\
\hline
\end{tabular}


07:43 Wednesday, January 15, 2003

The CORR Procedure

Pearson Correlation Coefficients, $N=139$

Prob $>|r|$ under $\mathrm{HO}$ : Rho=0

\begin{tabular}{|c|c|c|c|c|c|c|c|c|c|}
\hline & ITP2 & 0TP2 & ITP3 & 0ТР3 & ITP4 & OTP4 & ITP5 & 0TP5 & ITP7 \\
\hline \multirow[t]{2}{*}{ ITS8 } & -0.00725 & -0.03288 & -0.01808 & 0.01843 & .00725 & 0.09287 & 0.40078 & .39867 & -0.02104 \\
\hline & 0.9325 & 0.7008 & 0.8327 & 0.8295 & 0.9325 & 0.2769 & $<.0001$ & $<.0001$ & 0.8058 \\
\hline \multirow[t]{2}{*}{ 0TS8 } & -0.00325 & 0.24461 & 0.40703 & 0.42105 & -0.04592 & -0.06368 & 0.31478 & 0.33548 & -0.11781 \\
\hline & 0.9697 & 0.0037 & $<.0001$ & $<.0001$ & 0.5914 & 0.4564 & 0.0002 & $<.0001$ & 0.1672 \\
\hline \multirow[t]{2}{*}{ ITS11 } & -0.01644 & 0.44504 & 0.52926 & 0.52643 & -0.01644 & -0.00341 & 0.52926 & 0.53133 & -0.04774 \\
\hline & 0.8476 & $<.0001$ & $<.0001$ & $<.0001$ & 0.8476 & 0.9682 & $<.0001$ & $<.0001$ & 0.5768 \\
\hline \multirow[t]{2}{*}{ OTS11 } & -0.02320 & 0.46239 & 0.53223 & 0.53092 & 0.00725 & 0.00622 & 0.54912 & 0.55314 & -0.06315 \\
\hline & 0.7863 & $<.0001$ & $<.0001$ & $<.0001$ & 0.9325 & 0.9420 & $<.0001$ & $<.0001$ & 0.4602 \\
\hline \multirow[t]{2}{*}{ ITS12 } & -0.04275 & .11163 & 0.10668 & . 11876 & 0.04275 & 0.02837 & 0.10668 & 0.11036 & 0.02992 \\
\hline & 0.6173 & 0.1908 & 0.2113 & 0.1638 & 0.6173 & 0.7402 & 0.2113 & 0.1959 & 0.7266 \\
\hline \multirow[t]{2}{*}{ OTS12 } & -0.03941 & 0.11793 & 0.10517 & 0.11722 & 0.04186 & 0.02631 & 0.10822 & 0.11165 & 0.02492 \\
\hline & 0.6450 & 0.1668 & 0.2179 & 0.1694 & 0.6247 & 0.7585 & 0.2048 & 0.1907 & 0.7709 \\
\hline \multirow[t]{2}{*}{ ITS15 } & -0.01465 & 0.04645 & 0.03656 & 0.01683 & 0.01465 & 0.00956 & 0.17519 & 0.19556 & 0.04254 \\
\hline & 0.8641 & 0.5871 & 0.6692 & 0.8441 & 0.8641 & 0.9111 & 0.0391 & 0.0210 & 0.6190 \\
\hline \multirow[t]{2}{*}{ OTS15 } & -0.01709 & 0.07270 & .01869 & 0.00124 & 0.00322 & 0.00679 & 0.20176 & 0.22374 & 0.05096 \\
\hline & 0.8417 & 0.3950 & 0.8272 & 0.9885 & 0.9700 & 0.9368 & 0.0172 & 0.0081 & 0.5513 \\
\hline \multirow[t]{2}{*}{ ITS18 } & 0.25436 & 0.25239 & 0.28179 & 0.29556 & 0.02849 & 0.03559 & 0.07108 & 0.06231 & 0.08270 \\
\hline & 0.0025 & 0.0027 & 0.0008 & 0.0004 & 0.7392 & 0.6775 & 0.4057 & 0.4662 & 0.3331 \\
\hline \multirow[t]{2}{*}{ OTS18 } & 0.25053 & 0.25693 & 0.28246 & 0.29705 & 0.02766 & 0.03467 & 0.06914 & 0.05935 & 0.08395 \\
\hline & 0.0029 & 0.0023 & 0.0008 & 0.0004 & 0.7465 & 0.6853 & 0.4186 & 0.4877 & 0.3258 \\
\hline \multirow[t]{2}{*}{ ITS19 } & -0.02617 & 0.14956 & 0.06529 & 0.05916 & 0.02617 & 0.04738 & 0.06529 & 0.07233 & 0.07596 \\
\hline & 0.7598 & 0.0789 & 0.4451 & 0.4891 & 0.7598 & 0.5797 & 0.4451 & 0.3975 & 0.3741 \\
\hline \multirow[t]{2}{*}{ OTS19 } & -0.02917 & 0.15260 & .05564 & 0.04984 & 0.02949 & 0.05147 & 0.06105 & 0.06891 & -0.07917 \\
\hline & 0.7332 & 0.0729 & 0.5153 & 601 & 0.7304 & 0.5474 & 0.4753 & 0.4202 & 0.3542 \\
\hline \multirow[t]{2}{*}{ ITS20 } & -0.01029 & 0.03261 & -0.02566 & 0.02554 & 0.01029 & 0.02180 & 0.02566 & 0.01918 & 0.02986 \\
\hline & 0.9043 & 0.7032 & 0.7643 & 0.7654 & 0.9043 & 0.7989 & 0.7643 & 0.8227 & 0.7271 \\
\hline \multirow[t]{2}{*}{ OTS20 } & -0.00454 & 0.04463 & 0.03944 & -0.04066 & 0.01931 & 0.05759 & 0.00902 & 0.00293 & 0.00211 \\
\hline & 0.9577 & 0.6019 & 0.6448 & 0.6346 & 0.8215 & 0.5007 & 0.9161 & 0.9726 & 0.9804 \\
\hline \multirow[t]{2}{*}{ ITS22 } & -0.01029 & 0.01279 & -0.02566 & -0.02772 & -0.01029 & 0.02260 & 0.27160 & 0.28086 & -0.02986 \\
\hline & 0.9043 & 0.8813 & 0.7643 & 0.7460 & 0.9043 & 0.7917 & 0.0012 & 0.0008 & 0.7271 \\
\hline
\end{tabular}


The CORR Procedure

07:43 Wednesday, January 15, 2003

\begin{tabular}{|c|c|c|c|c|c|c|c|c|c|}
\hline & & & $\begin{array}{r}\text { arson } \mathrm{Co} \\
\mathrm{Pr}\end{array}$ & $\begin{array}{l}\text { elation } \\
>|r|\end{array}$ & $\begin{array}{l}\text { effici } \\
\text { ler } \mathrm{HO} \text { : }\end{array}$ & $\begin{array}{l}5, N= \\
b=0\end{array}$ & & & \\
\hline & 0TP7 & ITP8 & 0ТР8 & ITP10 & 0TP10 & ITP11 & 0TP11 & ITP12 & 0TP12 \\
\hline ITS8 & -0.01740 & -0.01029 & -0.01521 & -0.02961 & -0.03151 & -0.07419 & -0.06928 & -0.02617 & -0.02963 \\
\hline & 0.8389 & 0.9043 & 0.8589 & 0.7294 & 0.7127 & 0.3854 & 0.4177 & 0.7598 & 0.7292 \\
\hline 0TS8 & -0.11295 & -0.03490 & -0.04860 & 0.08665 & 0.09610 & 0.18362 & 0.18595 & 0.49150 & 0.50524 \\
\hline & 0.1855 & 0.6834 & 0.5699 & 0.3105 & 0.2604 & 0.0305 & 0.0284 & $<.0001$ & $<.0001$ \\
\hline ITS11 & -0.05106 & -0.02334 & -0.03871 & -0.06718 & -0.05200 & -0.16834 & -0.16656 & -0.05938 & -0.05529 \\
\hline & 0.5506 & 0.7851 & 0.6510 & 0.4320 & 0.5432 & 0.0476 & 0.0500 & 0.4875 & 0.5180 \\
\hline OTS11 & -0.06678 & -0.03294 & -0.04955 & -0.05003 & -0.03418 & -0.16934 & -0.16791 & -0.04233 & -0.03800 \\
\hline & 0.4348 & 0.7003 & 0.5624 & 0.5586 & 0.6896 & 0.0463 & 0.0482 & 0.6208 & 0.6570 \\
\hline ITS12 & 0.03786 & 0.24057 & 0.25610 & -0.05906 & -0.05894 & 0.43145 & 0.43666 & -0.09052 & -0.07653 \\
\hline & 0.6581 & 0.0043 & 0.0023 & 0.4898 & 0.4907 & $<.0001$ & $<.0001$ & 0.2893 & 0.3706 \\
\hline OTS12 & 0.03352 & 0.23909 & 0.25514 & -0.05759 & -0.05750 & 0.44024 & 0.44566 & -0.08622 & -0.07197 \\
\hline & 0.6952 & 0.0046 & 0.0024 & 0.5007 & 0.5014 & $<.0001$ & $<.0001$ & 0.3129 & 0.3998 \\
\hline ITS15 & -0.03595 & -0.02080 & -0.02377 & 0.35622 & 0.35459 & 0.11063 & 0.11270 & 0.40676 & 0.39927 \\
\hline & 0.6744 & 0.8080 & 0.7812 & $<.0001$ & $<.0001$ & 0.1948 & 0.1865 & $<.0001$ & $<.0001$ \\
\hline OTS15 & -0.04261 & -0.02312 & -0.02528 & 0.38893 & 0.38835 & 0.12989 & 0.13275 & 0.43082 & 0.42460 \\
\hline & 0.6185 & 0.7870 & 0.7677 & $<.0001$ & $<.0001$ & 0.1275 & 0.1193 & $<.0001$ & $<.0001$ \\
\hline ITS18 & -0.07492 & -0.04044 & -0.01903 & 0.57700 & 0.59005 & 0.38401 & 0.38154 & 0.06736 & 0.07312 \\
\hline & 0.3807 & 0.6365 & 0.8241 & $<.0001$ & $<.0001$ & $<.0001$ & $<.0001$ & 0.4308 & 0.3923 \\
\hline OTS18 & -0.07575 & -0.03842 & -0.01660 & 0.59381 & 0.60711 & 0.39031 & 0.38810 & 0.08780 & 0.09375 \\
\hline & 0.3755 & 0.6534 & 0.8462 & $<.0001$ & $<.0001$ & $<.0001$ & $<.0001$ & 0.3041 & 0.2723 \\
\hline ITS19 & -0.08974 & -0.03714 & -0.05956 & -0.10691 & -0.09804 & -0.11274 & -0.11958 & -0.09449 & -0.09602 \\
\hline & 0.2934 & 0.6642 & 0.4861 & 0.2103 & 0.2509 & 0.1864 & 0.1609 & 0.2685 & 0.2608 \\
\hline OTS19 & -0.09378 & -0.04231 & -0.06558 & -0.11972 & -0.11073 & -0.11478 & -0.12173 & -0.10163 & -0.10303 \\
\hline & 0.2722 & 0.6209 & 0.4431 & 0.1604 & 0.1944 & 0.1785 & 0.1534 & 0.2339 & 0.2274 \\
\hline ITS20 & -0.01497 & -0.01460 & 0.00840 & -0.04202 & -0.04512 & -0.10530 & -0.10634 & -0.03714 & -0.04250 \\
\hline & 0.8611 & 0.8646 & 0.9218 & 0.6233 & 0.5979 & 0.2173 & 0.2128 & 0.6642 & 0.6194 \\
\hline OTS20 & 0.01774 & 0.00653 & 0.02994 & -0.06187 & -0.06502 & -0.09846 & -0.09934 & -0.05349 & -0.05865 \\
\hline & 0.8358 & 0.9392 & 0.7264 & 0.4693 & 0.4470 & 0.2488 & 0.2446 & 0.5317 & 0.4928 \\
\hline ITS22 & -0.01119 & -0.01460 & 0.01113 & 0.15268 & 0.15062 & 0.01667 & 0.01932 & -0.03714 & -0.03193 \\
\hline & 0.8960 & 0.8646 & 0.8966 & 0.0727 & 0.0767 & 0.8456 & 0.8214 & 0.6642 & 0.7090 \\
\hline
\end{tabular}


The CORR Procedure

Pearson Correlation Coefficients, $N=139$

Prob > $|r|$ under $\mathrm{HO}$ : Rho=0

\begin{tabular}{|c|c|c|c|c|c|c|c|c|c|}
\hline & ITP15 & OTP15 & ITP22 & 0TP22 & ITP23 & 0ТР23 & ITS1 & OTS1 & s2 \\
\hline \multirow[t]{2}{*}{ ITS8 } & -0.02734 & 02951 & 0.01960 & .02275 & .02961 & .02560 & .00725 & -0.00895 & 0.01808 \\
\hline & 0.7493 & 0.7302 & 0.8188 & 0.7904 & 0.7294 & 0.7648 & 0.9325 & 0.9168 & 0.8327 \\
\hline \multirow[t]{2}{*}{ 0TS8 } & -0.06429 & -0.06000 & -0.14070 & 0.14998 & -0.12718 & -0.11891 & -0.02885 & -0.03029 & -0.11457 \\
\hline & 0.4521 & 0.4829 & 0.0985 & 0.0780 & 0.1357 & 0.1633 & 0.7360 & 0.7234 & 0.1793 \\
\hline \multirow[t]{2}{*}{ ITS11 } & 0.20332 & 0.21602 & -0.04448 & 0.04135 & 0.06718 & -0.05836 & -0.01644 & 0.00330 & -0.04103 \\
\hline & 0.0164 & 0.0106 & 0.6031 & 0.6289 & 0.4320 & 0.4950 & 0.8476 & 0.9693 & 0.6315 \\
\hline \multirow[t]{2}{*}{ OTS11 } & 0.22105 & 0.23475 & -0.04876 & 0.04611 & 0.04937 & -0.04030 & 0.00580 & 0.01448 & -0.05307 \\
\hline & 0.0089 & 0.0054 & 0.5687 & 0.5899 & 0.5638 & 0.6376 & 0.9460 & 0.8656 & 0.5349 \\
\hline \multirow[t]{2}{*}{ ITS12 } & 0.16133 & .16335 & 0.11566 & .12715 & 0.05656 & 0.05039 & 0.04275 & 0.04251 & 0.10668 \\
\hline & 0.0578 & 0.0547 & 0.1751 & 0.1358 & 0.5084 & 0.5558 & 0.6173 & 0.6193 & 0.2113 \\
\hline \multirow[t]{2}{*}{ OTS12 } & -0.16225 & 0.16445 & 0.11692 & 0.12850 & 0.05413 & 0.04788 & 0.04274 & 0.04193 & 0.11154 \\
\hline & 0.0564 & 0.0531 & 0.1705 & 0.1317 & 0.5268 & 0.5757 & 0.6173 & 0.6241 & 0.1911 \\
\hline \multirow[t]{2}{*}{ ITS15 } & -0.05529 & 0.03798 & 0.03964 & 0.04600 & 0.05987 & 0.05927 & 0.01465 & 0.02411 & 0.03656 \\
\hline & 0.5180 & 0.6571 & 0.6431 & 0.5908 & 0.4839 & 0.4883 & 0.8641 & 0.7781 & 0.6692 \\
\hline \multirow[t]{2}{*}{ OTS15 } & -0.02238 & .00463 & 0.04829 & 0.05568 & 0.07578 & 0.07490 & 0.01816 & 0.02825 & 0.04486 \\
\hline & 0.7937 & 0.9569 & 0.5724 & 0.5150 & 0.3753 & 0.3808 & 0.8320 & 7413 & 0.6000 \\
\hline \multirow[t]{2}{*}{ ITS18 } & 0.63140 & 0.62909 & 0.07707 & 0.08104 & 0.11640 & 0.10970 & 0.02849 & 0.02300 & 0.07108 \\
\hline & $<.0001$ & $<.0001$ & 0.3672 & 0.3430 & 0.1724 & 0.1986 & 0.7392 & 0.7881 & 0.4057 \\
\hline \multirow[t]{2}{*}{ OTS18 } & 0.63125 & 0.62985 & 0.08065 & 0.08476 & 0.10771 & -0.10096 & 0.02825 & 0.02301 & 0.07369 \\
\hline & $<.0001$ & $<.0001$ & 0.3453 & 0.3211 & 0.2069 & 0.2370 & 0.7413 & 0.7880 & 0.3886 \\
\hline \multirow[t]{2}{*}{ ITS19 } & -0.09874 & 0.09576 & 0.07079 & 0.06135 & 0.63612 & 0.63910 & 0.02617 & -0.03752 & 0.06529 \\
\hline & 0.2475 & 0.2621 & 0.4076 & 0.4731 & $<.0001$ & $<.0001$ & 0.7598 & 0.6610 & 0.4451 \\
\hline \multirow[t]{2}{*}{ OTS19 } & -0.11064 & .10782 & 0.05611 & -0.04654 & 0.63971 & 0.64333 & 0.02854 & -0.03913 & -0.06593 \\
\hline & 0.1948 & 0.2064 & 0.5118 & 0.5865 & $<.0001$ & $<.0001$ & 0.7387 & 6474 & 0.4406 \\
\hline \multirow[t]{2}{*}{ ITS20 } & -0.03881 & 0.04275 & 0.02782 & 0.00533 & 0.34739 & 0.34023 & 0.01029 & 0.06648 & 0.02566 \\
\hline & 0.6501 & 0.6173 & 0.7451 & 0.9503 & $<.0001$ & $<.0001$ & 0.9043 & 0.4368 & 0.7643 \\
\hline \multirow[t]{2}{*}{ OTS20 } & -0.05822 & 0.06239 & 0.01076 & 0.03139 & 0.36068 & 0.35564 & 0.07974 & 0.15366 & 0.00141 \\
\hline & 0.4960 & 0.4656 & 0.9000 & 0.7138 & $<.0001$ & $<.0001$ & 0.3507 & 0.0709 & 0.9868 \\
\hline \multirow[t]{2}{*}{ ITS22 } & -0.03881 & -0.03065 & -0.02782 & -0.02369 & 0.04202 & -0.03998 & 0.01029 & -0.01270 & -0.02566 \\
\hline & 0.6501 & 0.7202 & 0.7451 & 0.7820 & 0.6233 & 0.6403 & 0.9043 & 0.8821 & 0.764 \\
\hline
\end{tabular}


The CORR Procedure

07:43 Wednesday, January 15, 2003

Pearson Correlation Coefficients, $N=139$ Prob > $|r|$ under HO: Rho=0

\begin{tabular}{|c|c|c|c|c|c|c|c|c|c|}
\hline & 0TS2 & ITS5 & 0TS5 & ITS8 & OTS8 & ITS11 & OTS11 & ITS12 & OTS12 \\
\hline \multirow[t]{2}{*}{ ITS8 } & 0.01130 & 0.01808 & -0.00014 & 1.00000 & -0.04592 & -0.01644 & -0.00048 & -0.04275 & -0.03475 \\
\hline & 0.8950 & 0.8327 & 0.9987 & & 0.5914 & 0.8476 & 0.9955 & 0.6173 & 0.6846 \\
\hline \multirow[t]{2}{*}{ 0TS8 } & -0.11766 & -0.12876 & -0.14173 & .04592 & 1.00000 & 0.36050 & 0.38931 & -0.26012 & -0.26099 \\
\hline & 0.1677 & 0.1309 & 0.0960 & 0.5914 & & $<.0001$ & $<.0001$ & 0.0020 & 0.0019 \\
\hline \multirow[t]{2}{*}{ ITS11 } & -0.04416 & -0.04103 & -0.03773 & -0.01644 & 0.36050 & 1.00000 & 0.99624 & -0.09702 & -0.09891 \\
\hline & 0.6057 & 0.6315 & 0.6592 & 0.8476 & $<.0001$ & & $<.0001$ & 0.2559 & 0.2467 \\
\hline \multirow[t]{2}{*}{ OTS11 } & -0.05587 & 0.03880 & 0.03576 & 0.00048 & 0.38931 & 0.99624 & 1.00000 & -0.11928 & -0.12146 \\
\hline & 0.5136 & 0.6502 & 0.6760 & 0.9955 & $<.0001$ & $<.0001$ & & 0.1619 & 0. \\
\hline \multirow[t]{2}{*}{ ITS12 } & -0.10702 & .10668 & 0.10077 & 0.04275 & .26012 & 0.09702 & 0.11928 & 1.0 & 0.99904 \\
\hline & 0.2099 & 0.2113 & 0.2379 & 0.6173 & 0.0020 & 0.2559 & 0.1619 & & $<.0001$ \\
\hline \multirow[t]{2}{*}{ OTS12 } & -0.11182 & 0.11200 & 0.10587 & 0.03475 & 0.26099 & 0.09891 & 0.12 & 0.99904 & 1.0 \\
\hline & 0.1900 & 0.1893 & 0.2148 & 0.6846 & 0.0019 & 0.2467 & 0.1 & $<.0001$ & \\
\hline \multirow[t]{2}{*}{ ITS15 } & -0.04107 & 0.03656 & 0.03259 & 0.01465 & 0.18321 & 0.03325 & 0.01 & 0.08645 & 0.0 \\
\hline & 0.6312 & 0.6692 & 0.7033 & 0.8641 & 0.0309 & 0.6976 & $0 . \varepsilon$ & 0.3116 & 0.3303 \\
\hline \multirow[t]{2}{*}{ OTS15 } & 0.04939 & 0.01691 & 0.01315 & 0.00798 & 0.21938 & 0.01361 & 0.0 & -0.09161 & 0.08858 \\
\hline & 0.5637 & 0.8433 & 0.8779 & 0.9257 & 0.0095 & 0.8737 & 0.5 & 0.2835 & 0.2998 \\
\hline \multirow[t]{2}{*}{ ITS18 } & -0.07308 & 0.07108 & 0.07383 & .02849 & 0.02794 & 0.06465 & 0.04 & 0.16808 & 0.16343 \\
\hline & 0.3926 & 0.4057 & 0.3877 & 0.7392 & 0.7440 & 0.4496 & 0.5 & 0.0479 & 0.0546 \\
\hline \multirow[t]{2}{*}{ OTS18 } & -0.07577 & 0.07393 & 0.07700 & 0.02943 & 0.03989 & 0.06384 & 0.04787 & 0.16906 & 0.16425 \\
\hline & 0.3753 & 0.3871 & 0.3676 & 0.7309 & 0.6410 & 0.4553 & 0.5758 & 0.0466 & 0.0533 \\
\hline \multirow[t]{2}{*}{ ITS19 } & -0.05505 & 0.06529 & 0.07015 & 0.02617 & 0.12216 & 0.05938 & 0.04 & 0.15439 & 0.15127 \\
\hline & 0.5198 & 0.4451 & 0.4119 & 0.7598 & 0.1520 & 0.4875 & 24 & 0.0696 & 0.0755 \\
\hline \multirow[t]{2}{*}{ OTS19 } & -0.05506 & -0.06105 & -0.06640 & 0.02981 & 0.13051 & 0.05324 & 0.03 & -0.14191 & -0.13952 \\
\hline & 0.5197 & 0.4753 & 0.4374 & 0.7276 & 0.1257 & 0.5336 & 0.6 & 0.0956 & 0.1014 \\
\hline \multirow[t]{2}{*}{ ITS20 } & -0.01604 & 0.02566 & -0.01647 & 0.01029 & 0.05912 & -0.02334 & -0.03191 & -0.06068 & 0.05279 \\
\hline & 0.8513 & 0.7643 & 0.8474 & 0.9043 & 0.4894 & 0.7851 & 0.7092 & 0.4779 & 0.5371 \\
\hline \multirow[t]{2}{*}{ OTS20 } & 0.00981 & -0.00836 & 0.00250 & 0.01295 & -0.10248 & -0.04170 & -0.05069 & -0.01356 & 0.00510 \\
\hline & 0.9088 & 0.9222 & 0.9767 & 0.8797 & 0.2300 & 0.6260 & 0.5535 & 0.8741 & 0.9525 \\
\hline \multirow[t]{2}{*}{ ITS22 } & -0.01727 & -0.02566 & 0.01647 & -0.01029 & -0.06517 & -0.02334 & -0.01338 & -0.06068 & -0.05421 \\
\hline & 0.8401 & 0.7643 & 0.8474 & 0.9043 & 0.4459 & 0.7851 & 0.8758 & 0.4779 & 0.526 \\
\hline
\end{tabular}


The CORR Procedure

Pearson Correlation Coefficients, $N=139$

Prob > $|r|$ under $\mathrm{HO}$ : Rho=0

\begin{tabular}{|c|c|c|c|c|c|c|c|c|c|}
\hline & TS15 & OTS15 & ITS18 & OTS18 & ITS19 & OTS19 & ITS20 & OTS20 & ITS22 \\
\hline \multirow[t]{2}{*}{ ITS8 } & 01465 & .00798 & -0.02849 & 0.02943 & .02617 & -0.02981 & -0.01029 & 0.01295 & -0.01029 \\
\hline & 0.8641 & 0.9257 & 0.7392 & 0.7309 & 0.7598 & 0.7276 & 0.9043 & 97 & 0.9043 \\
\hline \multirow[t]{2}{*}{ 0TS8 } & 0.18321 & .21938 & 0.02794 & 0.03989 & 0.12216 & -0.13051 & -0.05912 & 0.10248 & -0.06517 \\
\hline & 0.0309 & 0.0095 & 0.7440 & 0.6410 & 0.1520 & 0.1257 & 0.4894 & 0.2300 & 0.4459 \\
\hline \multirow[t]{2}{*}{ ITS11 } & -0.03325 & 0.01361 & -0.06465 & -0.06384 & -0.05938 & -0.05324 & -0.02334 & -0.04170 & -0.02334 \\
\hline & 0.6976 & 0.8737 & 0.4496 & 0.4553 & 0.4875 & 0.5336 & 0.7851 & 0.6260 & 0.7851 \\
\hline \multirow[t]{2}{*}{ OTS11 } & -0.01588 & 0.00579 & -0.04982 & -0.04787 & -0.04335 & -0.03845 & -0.03191 & -0.05069 & -0.01338 \\
\hline & 0.8528 & 0.9461 & 0.5603 & 0.5758 & 0.6124 & 0.6531 & 0.7092 & 0.5535 & 0.8758 \\
\hline \multirow[t]{2}{*}{ ITS12 } & -0.08645 & 0.09161 & -0.16808 & 0.16906 & -0.15439 & -0.14191 & -0.06068 & -0.01356 & -0.06068 \\
\hline & 0.3116 & 0.2835 & 0.0479 & 0.0466 & 0.0696 & 0.0956 & 0.4779 & 0.8741 & 0.4779 \\
\hline \multirow[t]{2}{*}{ OTS12 } & -0.08318 & 0.08858 & -0.16343 & 0.16425 & 0.15127 & -0.13952 & -0.05279 & 0.00510 & -0.05421 \\
\hline & 0.3303 & 0.2998 & 0.0546 & 0.0533 & 0.0755 & 0.1014 & 0.5371 & 0.9525 & 0.5262 \\
\hline \multirow[t]{2}{*}{ ITS15 } & 1.00000 & 0.99642 & -0.05761 & -0.04131 & 0.05291 & -0.05980 & -0.02080 & 0.02567 & -0.02080 \\
\hline & & $<.0001$ & 0.5006 & 0.6292 & 0.5362 & 0.4844 & 0.8080 & 0.7643 & 0.8080 \\
\hline \multirow[t]{2}{*}{ OTS15 } & 0.99642 & 1.00 & -0.04053 & 0.02353 & 0.06 & 0.07 & 0.0 & 0.03 & 0.01479 \\
\hline & $<.0001$ & & 0.6357 & 0.7833 & 0.4332 & 0.3808 & 0.7667 & 0.7135 & 0.8628 \\
\hline \multirow[t]{2}{*}{ ITS18 } & -0.05761 & 0.040 & 1.00000 & 0.99919 & -0.10 & -0.10 & -0.0 & -0.0 & -0.04044 \\
\hline & 0.5006 & 0.6357 & & $<.0001$ & 0.2282 & 0.2109 & 0.6 & 0.5747 & 0.6365 \\
\hline \multirow[t]{2}{*}{ OTS18 } & -0.04131 & .02353 & 0.999 & 1.00000 & -0.09713 & -0.10 & -0.04 & -0.04976 & -0.02962 \\
\hline & 0.6292 & 0.7833 & $<.0001$ & & 0.2553 & 0.23 & 0.6 & 0.5607 & 0.7292 \\
\hline \multirow[t]{2}{*}{ ITS19 } & -0.05291 & -0.06701 & 0.10287 & 0.09713 & 1.00000 & 0.99836 & -0.03714 & -0.03770 & -0.03714 \\
\hline & 0.5362 & 0.4332 & 0.2282 & 0.2553 & & $<.0001$ & 0.6642 & 0.6595 & 0.6642 \\
\hline \multirow[t]{2}{*}{ OTS19 } & -0.05980 & 0.07491 & 0.10677 & 0.10137 & 0.99836 & 1.00000 & -0.03961 & -0.03918 & -0.04164 \\
\hline & 0.4844 & 0.3808 & 0.2109 & 0.2351 & $<.0001$ & & 0.6434 & 0.6470 & 0.6265 \\
\hline \multirow[t]{2}{*}{ ITS20 } & -0.02080 & -0.02539 & -0.040 & -0.04178 & -0.03714 & -0.03961 & 1.00000 & 0.98841 & -0.01460 \\
\hline & 0.8080 & 0.7667 & 0.63 & 0.6253 & 0.6642 & 0.6434 & & $<.0001$ & 0.8646 \\
\hline \multirow[t]{2}{*}{ OTS20 } & -0.02567 & -0.03142 & $-0.04 \varepsilon$ & -0.04976 & -0.03770 & -0.03918 & 0.98 & 1.00000 & 0.00597 \\
\hline & 0.7643 & 0.7135 & 0.5747 & 0.5607 & 0.6595 & 0.6470 & $<.0001$ & & 0.9444 \\
\hline \multirow[t]{2}{*}{ ITS22 } & -0.02080 & -0.01479 & -0.04044 & -0.02962 & -0.03714 & -0.04164 & -0.01460 & 0.00597 & 1.00000 \\
\hline & 0.8080 & 0.8628 & 0.6365 & 0.7292 & 0.6642 & 0.6265 & 0.8646 & 0.9444 & \\
\hline
\end{tabular}


The CORR Procedure

07:43 Wednesday, January 15, 2003

\begin{tabular}{|c|c|c|c|c|c|c|c|c|c|}
\hline & & & On & $>|r|$ & er $\mathrm{HO}$ : & $\begin{array}{l}, \quad N \\
=0\end{array}$ & & & \\
\hline & OTS22 & ITS23 & 0TS23 & ITA2 & ОТА2 & ITA3 & ОТАЗ & ITA7 & OTA7 \\
\hline TS8 & 0.03811 & 0.03283 & 0.03579 & -0.00725 & -0.01832 & -0.02617 & -0.01605 & -0.01808 & -0.00104 \\
\hline & 0.6561 & 0.7012 & 0.6757 & 0.9325 & 0.8305 & 0.7598 & 0.8513 & 0.8327 & 0.9903 \\
\hline 0TS8 & -0.05492 & .42770 & 0.43397 & -0.06298 & -0.10011 & 0.17312 & 0.17259 & -0.11457 & -0.13544 \\
\hline & 0.5208 & $<.0001$ & $<.0001$ & 0.4614 & 0.2410 & 0.0415 & 0.0422 & 793 & 119 \\
\hline ITS11 & -0.00181 & 0.07450 & -0.05831 & -0.01644 & -0.01880 & -0.05938 & -0.05019 & -0.04103 & -0.05121 \\
\hline & 0.9832 & 0.3834 & 0.4953 & 0.8476 & 0.8261 & 0.4875 & 0.5573 & 0.6315 & 0.5493 \\
\hline OTS11 & 0.01043 & -0.06145 & -0.04527 & -0.01789 & -0.02060 & -0.06051 & -0.05130 & -0.04443 & -0.05600 \\
\hline & 0.9030 & 0.4724 & 0.5967 & 0.8345 & 0.8098 & 0.4792 & 0.5487 & 0.6035 & 0.5126 \\
\hline ITS12 & -0.06502 & -0.19371 & -0.20340 & -0.04275 & -0.05816 & -0.15439 & -0.15233 & -0.10668 & -0.10307 \\
\hline & 0.4470 & 0.0223 & 0.0163 & 0.6173 & 0.4965 & 0.0696 & 0.0734 & 0.2113 & 0.2273 \\
\hline OTS12 & -0.05922 & -0.19501 & -0.20512 & -0.04452 & -0.06023 & -0.15956 & -0.15777 & -0.10563 & -0.10205 \\
\hline & 0.4887 & 0.0214 & 0.0154 & 0.6028 & 0.4812 & 0.0606 & 0.0636 & 0.2159 & 0.2319 \\
\hline ITS15 & -0.01305 & 0.06639 & 0.06351 & -0.01465 & -0.03704 & -0.05291 & -0.05116 & -0.03656 & -0.04608 \\
\hline & 0.8788 & 0.4374 & 0.4576 & 0.8641 & 0.6651 & 0.5362 & 0.5498 & 0.6692 & 0.5901 \\
\hline OTS15 & -0.00437 & 0.05206 & 0.04955 & 0.01869 & 0.04477 & -0.05513 & -0.05256 & -0.04574 & -0.05616 \\
\hline & 0.9592 & 0.5428 & 0.5624 & 0.8271 & 0.6007 & 0.5192 & 0.5389 & 0.5928 & 0.5114 \\
\hline ITS18 & -0.01166 & -0.12908 & -0.13430 & 0.02849 & -0.03216 & -0.10287 & -0. & -0.07 & -0.08219 \\
\hline & 0.8916 & 0.1299 & 0.1150 & 0.7392 & 0.7070 & 0.2282 & 0.1945 & 0.4057 & 0.3361 \\
\hline OTS18 & -0.00009 & 0.12704 & 0.13237 & 0.02943 & -0.03288 & 0.10797 & -0.11585 & -0.07406 & -0.08588 \\
\hline & 0.9991 & 0.1361 & 0.1203 & 0.7309 & 0.7008 & 0.2058 & 0.1745 & 0.3863 & 0.3148 \\
\hline ITS19 & -0.02750 & 0.11856 & 0.12326 & 0.02617 & 0.02280 & -0.09449 & -0.09763 & -0.06529 & -0.06946 \\
\hline & 0.7479 & 0.1645 & 0.1483 & 0.7598 & 0.7899 & 0.2685 & 0.2529 & 0.4451 & 0.4165 \\
\hline OTS19 & -0.03144 & -0.11909 & 0.12387 & -0.01394 & 0.03688 & -0.10144 & -0.10435 & -0.05524 & -0.05877 \\
\hline & 0.7133 & 0.1626 & 0.1463 & 0.8706 & 0.6665 & 0.2348 & 0.2215 & 0.5183 & 0.4920 \\
\hline ITS20 & -0.02346 & -0.04660 & -0.04741 & -0.01029 & -0.02146 & -0.03714 & -0.04163 & 0.56886 & 0.56923 \\
\hline & 0.7840 & 0.5859 & 0.5794 & 0.9043 & 0.8020 & 0.6642 & 0.6265 & $<.0001$ & $<.0001$ \\
\hline OTS20 & 0.00009 & -0.06781 & -0.06761 & -0.01011 & -0.02008 & -0.03506 & -0.03838 & 0.59543 & 0.59775 \\
\hline & 0.9992 & 0.4276 & 0.4291 & 0.9060 & 0.8145 & 0.6820 & 0.6537 & $<.0001$ & $<.0001$ \\
\hline ITS22 & 0.99121 & -0.04660 & -0.04496 & -0.01029 & -0.02373 & -0.03714 & -0.03580 & -0.02566 & -0.01956 \\
\hline & $<.0001$ & 0.5859 & 0.5992 & 0.9043 & 0.7816 & 0.6642 & 0.6757 & 0.7643 & 0.8192 \\
\hline
\end{tabular}


Observed to Trained NNS Output for Exercises 1,2,3

07:43 Wednesday, January 15, 2003

The CORR Procedure

Pearson Correlation Coefficients, $N=139$

Prob > $|r|$ under $\mathrm{HO}$ : Rho=0

\begin{tabular}{|c|c|c|c|c|c|c|c|c|c|}
\hline & ITA10 & OTA10 & ITA11 & OTA11 & ITA12 & OTA12 & ITA18 & OTA18 & ITA19 \\
\hline \multirow[t]{2}{*}{ ITS8 } & -0.01029 & .00495 & 0.01960 & .00510 & .01264 & .00931 & .01960 & -0.02359 & -0.01264 \\
\hline & 0.9043 & 0.9539 & 0.8188 & 0.9524 & 0.8826 & 0.9134 & 0.8188 & 0.7828 & 0.8826 \\
\hline \multirow[t]{2}{*}{ 0TS8 } & -0.07728 & 0.07463 & -0.11102 & 0.11147 & 0.52530 & .55539 & 0.43636 & 0.45005 & -0.08507 \\
\hline & 0.3658 & 0.3826 & 0.1932 & 0.1914 & $<.0001$ & $<.0001$ & $<.0001$ & $<.0001$ & 0.3194 \\
\hline \multirow[t]{2}{*}{ ITS11 } & 0.62549 & 0.63502 & -0.04448 & 0.03320 & 0.76888 & 0.76173 & -0.04448 & -0.03824 & -0.02869 \\
\hline & $<.0001$ & $<.0001$ & 0.6031 & 0.6980 & $<.0001$ & $<.0001$ & 0.6031 & 0.6549 & 0.7374 \\
\hline \multirow[t]{2}{*}{ OTS11 } & 0.61868 & 0.63026 & -0.02466 & -0.01232 & 0.76965 & .76631 & -0.02596 & -0.02017 & -0.04021 \\
\hline & $<.0001$ & $<.0001$ & 0.7733 & 0.8856 & $<.0001$ & $<.0001$ & 0.7616 & 0.8137 & 0.6384 \\
\hline \multirow[t]{2}{*}{ ITS12 } & .06068 & 0.07177 & 0.21243 & 0.19779 & .07459 & 08331 & 0.11566 & -0.11684 & -0.07459 \\
\hline & 0.4779 & 0.4011 & 0.0120 & 0.0196 & 0.3828 & 0.3295 & 0.1751 & 0.1707 & 0.3828 \\
\hline \multirow[t]{2}{*}{ OTS12 } & -0.05752 & 0.06926 & 0.20415 & 0.18986 & 0.07961 & 0.08776 & 0.11117 & 0.11268 & 0.07148 \\
\hline & 0.5012 & 0.4178 & 0.0159 & 0.0252 & 0.3515 & 0.3043 & 0.1926 & 0.1866 & 0.4031 \\
\hline \multirow[t]{2}{*}{ ITS15 } & -0.02080 & 0.02289 & 0.15714 & 0.16446 & 0.02557 & 0.00332 & 0.03964 & 0.04043 & 0.02557 \\
\hline & 0.8080 & 0.7891 & 0.0647 & 0.0530 & 0.7651 & 0.9690 & 0.6431 & 0.6365 & 0.7651 \\
\hline \multirow[t]{2}{*}{ OTS15 } & -0.02198 & 0.02193 & 0.16544 & 0.17343 & 0.00058 & 0.03173 & 0.01676 & 0.01764 & 0.02951 \\
\hline & 0.7973 & 0.7978 & 0.0516 & 0.0412 & 0.9946 & 0.7108 & 0.8448 & 0.8367 & 0.7302 \\
\hline \multirow[t]{2}{*}{ ITS18 } & -0.04044 & .05426 & .07707 & .06562 & .04971 & 0.02212 & 0.07707 & 0.08097 & 0.11479 \\
\hline & 0.6365 & 0.5258 & 0.3672 & 0.4428 & 0.5612 & 0.7961 & 0.3672 & 0.3434 & 0.1784 \\
\hline \multirow[t]{2}{*}{ OTS18 } & -0.04262 & 0.05660 & 0.07472 & 0.06308 & 0.04689 & 0.01795 & 0.06684 & 0.07059 & 0.11267 \\
\hline & 0.6184 & 0.5081 & 0.3820 & 0.4607 & 0.5836 & 0.8339 & 0.4343 & 0.4089 & 0.1867 \\
\hline \multirow[t]{2}{*}{ ITS19 } & -0.03714 & 0.05111 & 0.07079 & 0.07354 & 0.04565 & 0.04775 & 0.04635 & 0.04467 & 0.04565 \\
\hline & 0.6642 & 0.5502 & 0.4076 & 0.3896 & 0.5936 & 0.5767 & 0.5880 & 0.6016 & 0.5936 \\
\hline \multirow[t]{2}{*}{ OTS19 } & -0.04209 & 05620 & 0.07022 & 0.07357 & 0.03374 & 0.03713 & 0.03600 & 0.03521 & -0.03983 \\
\hline & 0.6228 & 0.5111 & 0.4114 & 0.3894 & 0.6934 & 0.6644 & 0.6740 & 0.6807 & 0.6416 \\
\hline \multirow[t]{2}{*}{ ITS20 } & -0.01460 & -0.02131 & 0.02782 & 0.01249 & 0.01795 & 0.00811 & 0.02782 & 0.01920 & 0.01795 \\
\hline & 0.8646 & 0.8033 & 0.7451 & 0.8840 & 0.8339 & 0.9245 & 0.7451 & 0.8225 & 0.8339 \\
\hline \multirow[t]{2}{*}{ OTS20 } & -0.02563 & 0.03182 & -0.00983 & 0.00632 & 0.03243 & 0.02276 & 0.03226 & 0.02373 & 0.00410 \\
\hline & 0.7645 & 0.7100 & 0.9086 & 0.9411 & 0.7047 & 0.7903 & 0.7062 & 0.7816 & 0.9618 \\
\hline \multirow[t]{2}{*}{ ITS22 } & -0.01460 & -0.00924 & -0.02782 & -0.02415 & -0.01795 & 0.00103 & -0.02782 & -0.03086 & -0.01795 \\
\hline & 0.8646 & 0.9140 & 0.7451 & 0.7778 & 0.8339 & 0.9904 & 0.7451 & 0.7184 & 0.833 \\
\hline
\end{tabular}


The CORR Procedure

07:43 Wednesday, January 15, 2003

\begin{tabular}{|c|c|c|c|c|c|c|c|c|c|}
\hline & & & $\begin{array}{r}\text { rson } \mathrm{Co} \\
\mathrm{Pr}\end{array}$ & $\begin{array}{l}\text { elation } \\
>|r|\end{array}$ & $\begin{array}{l}\text { effici } \\
\text { ler } \mathrm{HO} \text { : }\end{array}$ & $\begin{array}{l}N \\
=0\end{array}$ & & & \\
\hline & OTA19 & ITA21 & OTA21 & ITA23 & ОТА23 & IM1 & OM1 & IM2 & OM2 \\
\hline ITS8 & -0.01974 & -0.03691 & -0.03925 & 0.17338 & 0.16781 & -0.04331 & -0.04192 & 0.15459 & 0.14811 \\
\hline & 0.8176 & 0.6662 & 0.6464 & 0.0412 & 0.0483 & 0.6127 & 0.6241 & 0.0692 & 0.0819 \\
\hline 0TS8 & -0.09422 & -0.25958 & -0.26642 & -0.00783 & 0.00156 & 0.19804 & 0.19974 & -0.08978 & -0.08760 \\
\hline & 0.2699 & 0.0020 & 0.0015 & 0.9271 & 0.9854 & 0.0194 & 0.0184 & 0.2932 & 0.3051 \\
\hline ITS11 & -0.04450 & -0.08376 & -0.08885 & -0.09484 & -0.08708 & -0.09827 & -0.09356 & -0.03855 & -0.02424 \\
\hline & 0.6029 & 0.3269 & 0.2983 & 0.2667 & 0.3081 & 0.2498 & 0.2733 & 0.6523 & 0.7769 \\
\hline OTS11 & -0.05741 & -0.10914 & -0.11484 & -0.07786 & -0.06959 & -0.09488 & -0.09042 & -0.05457 & -0.03994 \\
\hline & 0.5021 & 0.2009 & 0.1783 & 0.3623 & 0.4156 & 0.2666 & 0.2898 & 0.5234 & 0.6407 \\
\hline ITS12 & -0.07868 & 0.71595 & 0.71767 & -0.01990 & -0.00994 & -0.14710 & -0.16070 & 0.16319 & 0.16545 \\
\hline & 0.3572 & $<.0001$ & $<.0001$ & 0.8162 & 0.9075 & 0.0840 & 0.0588 & 0.0549 & 0.0516 \\
\hline OTS12 & -0.07536 & 0.72609 & 0.72782 & -0.01733 & -0.00742 & -0.15012 & -0.16354 & 0.17068 & 0.17297 \\
\hline & 0.3779 & $<.0001$ & $<.0001$ & 0.8395 & 0.9309 & 0.0777 & 0.0544 & 0.0445 & 0.0417 \\
\hline ITS15 & -0.03202 & -0.07464 & -0.08035 & 0.24181 & 0.23897 & -0.08757 & -0.08653 & 0.12674 & 0.12506 \\
\hline & 0.7083 & 0.3825 & 0.3471 & 0.0041 & 0.0046 & 0.3053 & 0.3111 & 0.1371 & 0.1424 \\
\hline OTS15 & -0.03778 & -0.08816 & -0.09422 & 0.27193 & 0.27043 & -0.08905 & -0.08796 & 0.11546 & 0.11493 \\
\hline & 0.6588 & 0.3020 & 0.2699 & 0.0012 & 0.0013 & 0.2972 & 0.3032 & 0.1759 & 0.1779 \\
\hline ITS18 & 0.10198 & -0.14512 & -0.14798 & 0.56076 & 0.57329 & -0.13414 & -0.13664 & -0.20674 & -0.20978 \\
\hline & 0.2322 & 0.0883 & 0.0821 & $<.0001$ & $<.0001$ & 0.1154 & 0.1087 & 0.0146 & 0.0132 \\
\hline OTS18 & 0.09965 & -0.15281 & -0.15571 & 0.57345 & 0.58613 & -0.13623 & -0.13878 & -0.21099 & -0.21426 \\
\hline & 0.2431 & 0.0725 & 0.0672 & $<.0001$ & $<.0001$ & 0.1098 & 0.1032 & 0.0127 & 0.0113 \\
\hline ITS19 & -0.02880 & 0.07725 & 0.07918 & -0.15093 & -0.15422 & -0.08671 & -0.07911 & -0.17936 & -0.18617 \\
\hline & 0.7365 & 0.3661 & 0.3541 & 0.0761 & 0.0699 & 0.3101 & 0.3546 & 0.0346 & 0.0282 \\
\hline OTS19 & -0.02224 & 0.08991 & 0.09189 & -0.16048 & -0.16382 & -0.07948 & -0.07172 & -0.17727 & -0.18527 \\
\hline & 0.7949 & 0.2925 & 0.2820 & 0.0591 & 0.0540 & 0.3524 & 0.4015 & 0.0368 & 0.0290 \\
\hline ITS20 & -0.00497 & -0.05239 & -0.05399 & -0.05932 & -0.05678 & 0.26715 & 0.28785 & -0.12848 & -0.12233 \\
\hline & 0.9537 & 0.5402 & 0.5279 & 0.4879 & 0.5067 & 0.0015 & 0.0006 & 0.1317 & 0.1514 \\
\hline OTS20 & 0.01805 & -0.01817 & -0.02012 & -0.04862 & -0.04584 & 0.29435 & 0.31437 & -0.14223 & -0.13618 \\
\hline & 0.8330 & 0.8319 & 0.8142 & 0.5698 & 0.5921 & 0.0004 & 0.0002 & 0.0949 & 0.1099 \\
\hline ITS22 & -0.02402 & -0.05239 & -0.05485 & 0.24608 & 0.24152 & -0.06147 & -0.05420 & 0.04547 & 0.05942 \\
\hline & 0.7789 & 0.5402 & 0.5213 & 0.0035 & 0.0042 & 0.4722 & 0.5262 & 0.5951 & 0.4872 \\
\hline
\end{tabular}


Observed to Trained NNS Output for Exercises 1,2,3 29

$07: 43$ Wednesday, January 15, 2003

The CORR Procedure

Pearson Correlation Coefficients, $N=139$

Prob $>|r|$ under HO: Rho $=0$

\begin{tabular}{|c|c|c|c|c|c|c|}
\hline & IM3 & OM3 & IM4 & OM4 & IM5 & OM5 \\
\hline \multirow[t]{2}{*}{ ITS8 } & -0.03222 & -0.03254 & -0.01029 & 0.00823 & -0.02795 & -0.01380 \\
\hline & 0.7065 & 0.7037 & 0.9043 & 0.9234 & 0.7440 & 0.8719 \\
\hline \multirow[t]{2}{*}{ OTS8 } & -0.00295 & -0.00652 & -0.07728 & -0.02725 & -0.09180 & -0.11183 \\
\hline & 0.9725 & 0.9392 & 0.3658 & 0.7501 & 0.2824 & 0.1900 \\
\hline \multirow[t]{2}{*}{ ITS11 } & -0.07311 & -0.06536 & 0.62549 & 0.62542 & -0.06342 & -0.05967 \\
\hline & 0.3924 & 0.4446 & $<.0001$ & $<.0001$ & 0.4582 & 0.4853 \\
\hline \multirow[t]{2}{*}{ OTS11 } & -0.05124 & -0.04234 & 0.61868 & 0.62405 & -0.06073 & -0.05784 \\
\hline & 0.5492 & 0.6207 & $<.0001$ & $<.0001$ & 0.4776 & 0.4988 \\
\hline \multirow[t]{2}{*}{ ITS12 } & -0.13363 & -0.13356 & -0.06068 & -0.11150 & -0.04796 & -0.03101 \\
\hline & 0.1168 & 0.1170 & 0.4779 & 0.1913 & 0.5751 & 0.7170 \\
\hline \multirow[t]{2}{*}{ OTS12 } & -0.13463 & -0.13426 & -0.05752 & -0.10965 & -0.05295 & -0.03528 \\
\hline & 0.1141 & 0.1151 & 0.5012 & 0.1988 & 0.5359 & 0.6801 \\
\hline \multirow[t]{2}{*}{ ITS15 } & 0.38956 & 0.39136 & -0.02080 & 0.06679 & -0.05652 & -0.05342 \\
\hline & $<.0001$ & $<.0001$ & 0.8080 & 0.4347 & 0.5087 & 0.5322 \\
\hline \multirow[t]{2}{*}{ OTS15 } & 0.42516 & 0.42722 & -0.02198 & 0.06767 & -0.04620 & -0.04271 \\
\hline & $<.0001$ & $<.0001$ & 0.7973 & 0.4287 & 0.5891 & 0.6176 \\
\hline \multirow[t]{2}{*}{ ITS18 } & 0.45376 & 0.46258 & -0.04044 & -0.07680 & 0.42447 & 0.42530 \\
\hline & $<.0001$ & $<.0001$ & 0.6365 & 0.3688 & $<.0001$ & $<.0001$ \\
\hline \multirow[t]{2}{*}{ OTS18 } & 0.46271 & 0.47196 & -0.04262 & -0.07809 & 0.42462 & 0.42580 \\
\hline & $<.0001$ & $<.0001$ & 0.6184 & 0.3609 & $<.0001$ & $<.0001$ \\
\hline \multirow[t]{2}{*}{ ITS19 } & -0.11634 & -0.12291 & -0.03714 & -0.09561 & -0.10093 & -0.11644 \\
\hline & 0.1726 & 0.1494 & 0.6642 & 0.2629 & 0.2371 & 0.1722 \\
\hline \multirow[t]{2}{*}{ OTS19 } & -0.13068 & -0.13724 & -0.04209 & -0.10290 & -0.11262 & -0.12831 \\
\hline & 0.1252 & 0.1072 & 0.6228 & 0.2280 & 0.1868 & 0.1322 \\
\hline \multirow[t]{2}{*}{ ITS20 } & -0.04573 & -0.03734 & -0.01460 & -0.03899 & -0.03967 & -0.02546 \\
\hline & 0.5930 & 0.6625 & 0.8646 & 0.6486 & 0.6429 & 0.7661 \\
\hline \multirow[t]{2}{*}{ OTS20 } & -0.05556 & -0.04474 & -0.02563 & -0.05475 & -0.03398 & -0.01561 \\
\hline & 0.5159 & 0.6010 & 0.7645 & 0.5221 & 0.6913 & 0.8553 \\
\hline \multirow[t]{2}{*}{ ITS22 } & 0.14442 & 0.15788 & -0.01460 & 0.01028 & -0.03967 & -0.02516 \\
\hline & 0.0899 & 0.0634 & 0.8646 & 0.9044 & 0.6429 & 0.7687 \\
\hline
\end{tabular}


The CORR Procedure

07:43 Wednesday, January 15, 2003

\begin{tabular}{|c|c|c|c|c|c|c|c|c|c|}
\hline & & & $\begin{array}{r}\operatorname{arson} \mathrm{C} \\
\mathrm{P}\end{array}$ & $\begin{array}{l}\text { elation } \\
>|r|\end{array}$ & $\begin{array}{l}\text { effici } \\
\text { ler HO: }\end{array}$ & $\begin{array}{l}5, N= \\
b=0\end{array}$ & & & \\
\hline & ITP2 & отР2 & ITP3 & 0ТР3 & ITP4 & OTP4 & ITP5 & 0TP5 & ITP7 \\
\hline 0TS22 & 0.01021 & 0.06024 & 0.00420 & 0.00266 & -0.00297 & 0.03463 & 0.31647 & 0.32699 & 0.01192 \\
\hline & 0.9051 & 0.4812 & 0.9609 & 0.9752 & 0.9724 & 0.6857 & 0.0001 & $<.0001$ & 0.8893 \\
\hline ITS23 & -0.03283 & 0.01972 & -0.08192 & -0.08618 & -0.03283 & -0.04891 & -0.08192 & -0.07091 & -0.09531 \\
\hline & 0.7012 & 0.8177 & 0.3377 & 0.3131 & 0.7012 & 0.5675 & 0.3377 & 0.4068 & 0.2644 \\
\hline 0TS23 & -0.03579 & 0.03119 & -0.07738 & -0.08184 & -0.03606 & -0.05231 & -0.07174 & -0.06086 & -0.08636 \\
\hline & 0.6757 & 0.7155 & 0.3653 & 0.3382 & 0.6734 & 0.5408 & 0.4013 & 0.4766 & 0.3121 \\
\hline ITA2 & -0.00725 & 0.05090 & -0.01808 & -0.02195 & -0.00725 & -0.01463 & -0.01808 & -0.02147 & -0.02104 \\
\hline & 0.9325 & 0.5518 & 0.8327 & 0.7976 & 0.9325 & 0.8643 & 0.8327 & 0.8019 & 0.8058 \\
\hline ОТА2 & -0.01618 & 0.04540 & -0.04393 & -0.04917 & -0.01725 & -0.02954 & -0.04571 & -0.05248 & -0.01639 \\
\hline & 0.8500 & 0.5957 & 0.6076 & 0.5654 & 0.8403 & 0.7300 & 0.5932 & 0.5395 & 0.8481 \\
\hline ITA3 & -0.02617 & 0.33787 & -0.06529 & -0.07331 & -0.02617 & -0.03342 & -0.06529 & -0.05000 & 0.58396 \\
\hline & 0.7598 & $<.0001$ & 0.4451 & 0.3911 & 0.7598 & 0.6961 & 0.4451 & 0.5589 & $<.0001$ \\
\hline ОТАЗ & -0.02933 & 0.34112 & -0.07082 & -0.07931 & -0.02395 & -0.02824 & -0.05793 & -0.04232 & 0.59002 \\
\hline & 0.7318 & $<.0001$ & 0.4074 & 0.3533 & 0.7796 & 0.7414 & 0.4982 & 0.6208 & $<.0001$ \\
\hline ITA7 & -0.01808 & 0.09990 & -0.04511 & -0.04855 & -0.01808 & -0.00780 & -0.04511 & -0.04640 & -0.05249 \\
\hline & 0.8327 & 0.2420 & 0.5980 & 0.5703 & 0.8327 & 0.9274 & 0.5980 & 0.5876 & 0.5394 \\
\hline OTA7 & -0.00411 & 0.08701 & -0.04699 & -0.05102 & -0.01422 & -0.00181 & -0.04115 & -0.04266 & -0.04319 \\
\hline & 0.9617 & 0.3084 & 0.5828 & 0.5508 & 0.8680 & 0.9832 & 0.6306 & 0.6181 & 0.6136 \\
\hline ITA10 & -0.01029 & 0.16474 & -0.02566 & -0.03115 & -0.01029 & 0.01537 & -0.02566 & -0.02420 & -0.02986 \\
\hline & 0.9043 & 0.0526 & 0.7643 & 0.7158 & 0.9043 & 0.8574 & 0.7643 & 0.7774 & 0.7271 \\
\hline OTA10 & -0.01502 & 0.19269 & -0.02056 & -0.02677 & 0.00273 & 0.03010 & -0.00211 & 0.00003 & -0.01381 \\
\hline & 0.8607 & 0.0231 & 0.8102 & 0.7544 & 0.9746 & 0.7250 & 0.9804 & 0.9997 & 0.8718 \\
\hline ITA11 & -0.01960 & 0.09092 & -0.04891 & -0.05513 & -0.01960 & 0.00197 & 0.11296 & 0.12478 & -0.05691 \\
\hline & 0.8188 & 0.2871 & 0.5675 & 0.5192 & 0.8188 & 0.9816 & 0.1855 & 0.1433 & 0.5058 \\
\hline OTA11 & -0.02153 & 0.11518 & -0.05099 & -0.05682 & -0.00552 & 0.01766 & 0.11977 & 0.13312 & -0.05550 \\
\hline & 0.8014 & 0.1770 & 0.5511 & 0.5064 & 0.9486 & 0.8365 & 0.1602 & 0.1182 & 0.5164 \\
\hline ITA12 & -0.01264 & 0.43531 & 0.69926 & 0.70014 & -0.01264 & -0.01697 & 0.69926 & 0.70071 & -0.03670 \\
\hline & 0.8826 & $<.0001$ & $<.0001$ & $<.0001$ & 0.8826 & 0.8428 & $<.0001$ & $<.0001$ & 0.6680 \\
\hline OTA12 & 0.00501 & 0.45616 & 0.71382 & 0.71661 & -0.01644 & -0.01798 & 0.71229 & 0.71754 & -0.03950 \\
\hline & 0.9533 & $<.0001$ & $<.0001$ & $<.0001$ & 0.8477 & 0.8336 & $<.0001$ & $<.0001$ & 0.6443 \\
\hline
\end{tabular}


Observed to Trained NNS Output for Exercises 1,2,3

07:43 Wednesday, January 15, 2003

The CORR Procedure

Pearson Correlation Coefficients, $N=139$

Prob $>|r|$ under $\mathrm{HO}$ : Rho $=0$

\begin{tabular}{|c|c|c|c|c|c|c|c|c|c|}
\hline & 0TP7 & ITP8 & 0TP8 & & 0TP10 & & 0TP11 & ITP12 & 0TP12 \\
\hline \multirow[t]{2}{*}{ OTS22 } & 03107 & 01669 & 04193 & 0.16582 & 0.16595 & 0.02372 & 02635 & 0.01724 & -0.01151 \\
\hline & .7166 & 0.8454 & 241 & .0511 & 509 & & 0.7582 & 0.8404 & 930 \\
\hline \multirow[t]{2}{*}{ ITS23 } & .08008 & .04660 & .06274 & . 13415 & .13849 & 0.18300 & 17944 & 0.33921 & 34546 \\
\hline & 0.3487 & 859 & 4631 & 1154 & .1040 & .0311 & .0345 & $<.0001$ & $<.0001$ \\
\hline \multirow[t]{2}{*}{ 0TS23 } & -0.07178 & 0.04100 & 0.05763 & 0.14067 & 0.14485 & 0.17076 & 0.16700 & 0.34278 & 0.34868 \\
\hline & 0.4011 & 0.6318 & 0.5004 & 0.0986 & 0.0889 & 0.0444 & 0.0494 & $<.0001$ & $<.0001$ \\
\hline \multirow[t]{2}{*}{ ITA2 } & -0.02387 & 0.01029 & 0.01675 & 0.02961 & 0.03151 & 0.07419 & 0.07788 & 0.02617 & 0.03026 \\
\hline & 0.7803 & & 0.8448 & 0.7294 & 0.7127 & 0.3854 & 0.3622 & 0.7598 & 0.7236 \\
\hline \multirow[t]{2}{*}{ OTA2 } & -0.02068 & 0.02146 & 0.02881 & 0.05304 & 0.05410 & 0.11160 & 0.11595 & 0.02536 & 0.03125 \\
\hline & 0.8091 & 0.8020 & 364 & 0.5352 & 0.5271 & 0.1909 & 0.1740 & 0.7669 & 0.7149 \\
\hline \multirow[t]{2}{*}{ ITA3 } & 0.59610 & 0.03714 & 0.04060 & -0.10691 & 0.11148 & 0.04242 & 0.04043 & 0.09449 & -0.08972 \\
\hline & $<.0001$ & 0.6642 & 0.6351 & 0.2103 & 0.1914 & 0.6201 & 0.6365 & 0.2685 & 0.2935 \\
\hline \multirow[t]{2}{*}{ ОТАЗ } & 0.60279 & 0.02682 & -0.03138 & -0.11252 & -0.11676 & 0.04471 & 0.04249 & -0.09097 & 0.08642 \\
\hline & $<.0001$ & 0.7540 & 0.7139 & 0.1872 & 0.1711 & 0.6012 & 0.6195 & 0.2868 & 0.3118 \\
\hline \multirow[t]{2}{*}{ ITA7 } & -0.04373 & -0.02566 & -0.01048 & -0.07387 & -0.07932 & 0.18510 & 0.19044 & -0.06529 & -0.07497 \\
\hline & 0.6092 & 0.7643 & 0.9026 & 0.3874 & 0.3533 & 0.0291 & 0.0247 & 0.4451 & 0.3804 \\
\hline \multirow[t]{2}{*}{ OTA7 } & -0.03458 & 0.01301 & 0.00141 & -0.09197 & 0.09796 & 0.19289 & 0.19848 & -0.08189 & -0.09246 \\
\hline & 0.6861 & 0.8792 & 0.9868 & 0.2815 & 0.2513 & 0.0229 & 0.0192 & 379 & 0.2790 \\
\hline \multirow[t]{2}{*}{ ITA10 } & -0.02740 & 0.01460 & 0.02378 & -0.04202 & 0.03750 & 0.10530 & 0.11028 & -0.03714 & 0.04205 \\
\hline & 0.7489 & 0.8646 & 0.7812 & 0.6233 & 0.6612 & 0.2173 & 0.1962 & 42 & 0.6231 \\
\hline \multirow[t]{2}{*}{ OTA10 } & -0.01093 & 0.02079 & -0.03040 & -0.05128 & 0.04649 & -0.12774 & 0.13301 & -0.04176 & 0.04712 \\
\hline & 0.8984 & 0.8081 & 0.7224 & 0.5488 & 0.5868 & 0.1340 & 0.1185 & 0.6254 & 0.5818 \\
\hline \multirow[t]{2}{*}{ ITA11 } & -0.06487 & -0.02782 & -0.04413 & -0.08009 & -0.07431 & 0.20069 & 0.20244 & 0.07079 & -0.07537 \\
\hline & 0.4481 & 0.7451 & 0.6060 & 0.3486 & 0.3846 & 0.0178 & 0.0168 & 0.4076 & 0.3779 \\
\hline \multirow[t]{2}{*}{ OTA11 } & -0.06281 & -0.03376 & -0.05067 & 0.06504 & 0.05941 & 0.20528 & 0.20742 & 0.07181 & 0.07691 \\
\hline & 0.4626 & 0.6931 & 0.5536 & 0.4469 & 0.4873 & 0.0153 & 0.0143 & 0.4009 & 0.3682 \\
\hline \multirow[t]{2}{*}{ ITA12 } & -0.04297 & 0.01795 & 0.03012 & -0.05166 & -0.03591 & -0.12944 & -0.12307 & -0.04565 & -0.03640 \\
\hline & 0.6154 & 0.8339 & 0.7249 & 0.5459 & 0.6747 & 0.1289 & 0.1489 & 0.5936 & 0.6706 \\
\hline \multirow[t]{2}{*}{ OTA12 } & -0.04421 & 0.01507 & 0.02484 & -0.01141 & 0.00438 & -0.09835 & -0.09206 & -0.01934 & -0.00898 \\
\hline & 0.6053 & 0.8602 & 0.7716 & 0.8940 & 0.9592 & 0.2494 & 0.2811 & 0.8212 & 0.916 \\
\hline
\end{tabular}


The CORR Procedure

07:43 Wednesday, January 15, 2003

\begin{tabular}{|c|c|c|c|c|c|c|c|c|c|}
\hline \multicolumn{10}{|c|}{$\begin{array}{l}\text { Pearson Correlation Coefficients, } N=139 \\
\text { Prob }>|r| \text { under HO: Rho=0 }\end{array}$} \\
\hline & ITP15 & 0TP15 & ITP22 & 0TP22 & ITP23 & 0TP23 & ITS1 & OTS1 & ITS2 \\
\hline \multirow[t]{2}{*}{ 0TS22 } & -0.03278 & -0.02265 & -0.01371 & -0.00881 & -0.03196 & -0.02866 & -0.01304 & -0.01535 & 0.00388 \\
\hline & 0.7016 & 0.7913 & 0.8727 & 0.9180 & 0.7088 & 0.7377 & 0.8789 & 0.8576 & 0.9639 \\
\hline \multirow[t]{2}{*}{ ITS23 } & -0.12389 & -0.12468 & 0.49908 & 0.49188 & -0.13415 & -0.12856 & -0.03283 & -0.02908 & -0.08192 \\
\hline & 0.1462 & 0.1436 & $<.0001$ & $<.0001$ & 0.1154 & 0.1315 & 0.7012 & 0.7340 & 0.3377 \\
\hline \multirow[t]{2}{*}{ 0TS23 } & -0.12604 & -0.12669 & 0.50055 & 0.49454 & -0.13329 & -0.12765 & -0.02277 & -0.01834 & -0.07494 \\
\hline & 0.1393 & 0.1372 & $<.0001$ & $<.0001$ & 0.1178 & 0.1342 & 0.7902 & 0.8303 & 0.3806 \\
\hline \multirow[t]{2}{*}{ ITA2 } & -0.02734 & -0.01825 & 0.36966 & 0.37610 & -0.02961 & -0.02703 & -0.00725 & -0.00570 & -0.01808 \\
\hline & 0.7493 & 0.8312 & $<.0001$ & $<.0001$ & 0.7294 & 0.7521 & 0.9325 & 0.9469 & 0.8327 \\
\hline \multirow[t]{2}{*}{ 0TA2 } & -0.03288 & -0.02235 & 0.42595 & 0.43439 & 0.01468 & 0.01738 & 0.00196 & 0.00272 & -0.00621 \\
\hline & 0.7008 & 0.7940 & $<.0001$ & $<.0001$ & 0.8638 & 0.8391 & 0.9818 & 0.9746 & 0.9422 \\
\hline \multirow[t]{2}{*}{ ITA3 } & -0.09874 & -0.09814 & -0.07079 & -0.07230 & -0.10691 & -0.10052 & -0.02617 & -0.04078 & 0.69097 \\
\hline & 0.2475 & 0.2504 & 0.4076 & 0.3977 & 0.2103 & 0.2390 & 0.7598 & 0.6336 & $<.0001$ \\
\hline \multirow[t]{2}{*}{ ОТАЗ } & -0.09112 & -0.09047 & -0.05575 & -0.05731 & -0.11028 & -0.10354 & -0.02933 & -0.04353 & 0.69187 \\
\hline & 0.2861 & 0.2895 & 0.5145 & 0.5028 & 0.1962 & 0.2251 & 0.7318 & 0.6109 & $<.0001$ \\
\hline \multirow[t]{2}{*}{ ITA7 } & -0.06822 & -0.06933 & 0.59858 & 0.61256 & 0.15431 & 0.15760 & 0.40078 & 0.46189 & -0.04511 \\
\hline & 0.4249 & 0.4173 & $<.0001$ & $<.0001$ & 0.0697 & 0.0639 & $<.0001$ & $<.0001$ & 0.5980 \\
\hline \multirow[t]{2}{*}{ OTA7 } & -0.08325 & -0.08471 & 0.61349 & 0.62827 & 0.14838 & 0.15186 & 0.39446 & 0.45602 & -0.04188 \\
\hline & 0.3299 & 0.3214 & $<.0001$ & $<.0001$ & 0.0813 & 0.0743 & $<.0001$ & $<.0001$ & 0.6245 \\
\hline \multirow[t]{2}{*}{ ITA10 } & 0.37616 & 0.37837 & 0.02782 & -0.01881 & -0.04202 & -0.04484 & -0.01029 & 0.00652 & -0.02566 \\
\hline & $<.0001$ & $<.0001$ & 0.7451 & 0.8260 & 0.6233 & 0.6002 & 0.9043 & 0.9393 & 0.7643 \\
\hline \multirow[t]{2}{*}{ OTA10 } & 0.38135 & 0.38461 & -0.00748 & 0.00186 & -0.05350 & -0.05584 & -0.00466 & 0.01316 & -0.00365 \\
\hline & $<.0001$ & $<.0001$ & 0.9304 & 0.9827 & 0.5317 & 0.5138 & 0.9565 & 0.8778 & 0.9660 \\
\hline \multirow[t]{2}{*}{ ITA11 } & 0.15200 & 0.15796 & -0.05303 & -0.05014 & 0.34402 & 0.33899 & -0.01960 & -0.01290 & -0.04891 \\
\hline & 0.0741 & 0.0633 & 0.5353 & 0.5578 & $<.0001$ & $<.0001$ & 0.8188 & 0.8802 & 0.5675 \\
\hline \multirow[t]{2}{*}{ OTA11 } & 0.17072 & 0.17756 & -0.03666 & -0.03324 & 0.35003 & 0.34594 & -0.01907 & -0.01023 & -0.04381 \\
\hline & 0.0445 & 0.0365 & 0.6683 & 0.6977 & $<.0001$ & $<.0001$ & 0.8237 & 0.9048 & 0.6086 \\
\hline \multirow[t]{2}{*}{ ITA12 } & -0.04771 & -0.03325 & -0.03420 & -0.03758 & -0.05166 & -0.03804 & -0.01264 & -0.00112 & -0.03155 \\
\hline & 0.5771 & 0.6975 & 0.6894 & 0.6605 & 0.5459 & 0.6566 & 0.8826 & 0.9896 & 0.7124 \\
\hline \multirow[t]{2}{*}{ OTA12 } & -0.03482 & -0.01963 & -0.04448 & -0.04833 & -0.04447 & -0.02990 & -0.01522 & -0.00274 & -0.03643 \\
\hline & 0.6841 & 0.8186 & 0.6031 & 0.5721 & 0.6032 & 0.7268 & 0.8589 & 0.9744 & 0.6702 \\
\hline
\end{tabular}


07:43 Wednesday, January 15, 2003

The CORR Procedure

Pearson Correlation Coefficients, $\mathrm{N}=139$

Prob $>|r|$ under $\mathrm{HO}$ : Rho=0

\begin{tabular}{|c|c|c|c|c|c|c|c|c|c|}
\hline & OTS2 & ITS5 & OTS5 & ITS8 & OTS8 & ITS11 & OTS11 & ITS12 & $S 12$ \\
\hline \multirow[t]{2}{*}{ 0TS22 } & 0.01415 & -0.02674 & 0.01821 & 0.03811 & -0.05492 & -0.00181 & .01043 & -0.06502 & -0.05922 \\
\hline & 0.8687 & 0.7547 & 0.8315 & 0.6561 & 0.5208 & 0.9832 & 0.9030 & 0.4470 & 0.4887 \\
\hline \multirow[t]{2}{*}{ ITS23 } & -0.06497 & -0.08192 & -0.08641 & 0.03283 & 0.42770 & -0.07450 & -0.06145 & -0.19371 & -0.19501 \\
\hline & 0.4473 & 0.3377 & 0.3118 & 0.7012 & $<.0001$ & 0.3834 & 0.4724 & 0.0223 & 0.0214 \\
\hline \multirow[t]{2}{*}{ 0TS23 } & -0.05800 & -0.07925 & -0.08389 & 0.03579 & 0.43397 & -0.05831 & -0.04527 & -0.20340 & -0.20512 \\
\hline & 0.4976 & 0.3537 & 0.3262 & 0.6757 & $<.0001$ & 0.4953 & 0.5967 & 0.0163 & 0.0154 \\
\hline \multirow[t]{2}{*}{ ITA2 } & -0.00002 & -0.01808 & 0.02174 & 0.00725 & 0.06298 & -0.01644 & -0.01789 & -0.04275 & -0.04452 \\
\hline & 0.9999 & 0.8327 & 0.7995 & 0.9325 & 0.4614 & 0.8476 & 0.8345 & 0.6173 & 0.6028 \\
\hline \multirow[t]{2}{*}{ ОTA2 } & 0.01335 & 0.04038 & 0.04515 & 0.01832 & 0.10011 & 0.01880 & 0.02060 & 0.05816 & 0.06023 \\
\hline & 0.8760 & 0.6370 & 0.5977 & 0.8305 & 0.2410 & 0.8261 & 0.8098 & 0.4 & 0.4812 \\
\hline \multirow[t]{2}{*}{ ITA3 } & 0.69667 & -0.06529 & 0.06484 & 0.02617 & 0.17312 & -0.05938 & 0.06051 & 0.15 & 0.15956 \\
\hline & $<.0001$ & 0.4451 & 0.4482 & 0.7598 & 0.0415 & 0.4875 & 0.4792 & 0.0696 & 0.0606 \\
\hline \multirow[t]{2}{*}{ ОТАЗ } & 0.69838 & 0.04859 & 0.04782 & 0.01605 & 0.17259 & -0.05019 & 0.05130 & 0.15233 & 0.15777 \\
\hline & $<.0001$ & 0.5700 & 0.5762 & 0.8513 & 0.0422 & 0.5573 & 0.5487 & 0.0734 & 0.0636 \\
\hline \multirow[t]{2}{*}{ ITA7 } & 0.03253 & 0.04511 & 0.04013 & 0.01808 & .11457 & -0.04 & 0.04443 & 0.10668 & -0.10563 \\
\hline & 0.7038 & 0.5980 & 0.6391 & 0.8327 & 0.1793 & 0.6 & 0.6035 & 0.2 & 0.2159 \\
\hline \multirow[t]{2}{*}{ TA7 } & 0.02874 & 0.03493 & 0.02968 & 0.00104 & 0.13544 & -0.05 & -0.05600 & -0.10 & -0.10205 \\
\hline & 0.7370 & 0.6831 & 0.7287 & 0.9903 & 0.1119 & 0.5493 & 0.5126 & 0.2273 & 0.2319 \\
\hline \multirow[t]{2}{*}{ ITA10 } & -0.02590 & 0.02566 & 0.01302 & 0.01029 & 0.07728 & 0.62549 & 0.61868 & 0.06068 & -0.05752 \\
\hline & 0.7622 & 0.7643 & 0.8790 & 0.9043 & 0.3658 & $<.0001$ & $<.0001$ & 0.4779 & 0.5012 \\
\hline \multirow[t]{2}{*}{ OTA10 } & -0.00279 & -0.00611 & 0.00646 & 0.00495 & 0.07463 & 0.63502 & 0.63026 & -0.07177 & -0.06926 \\
\hline & 0.9740 & 0.9431 & 0.9398 & 0.9539 & 0.3826 & $<.0001$ & $<.0001$ & 0.4011 & 0.4178 \\
\hline \multirow[t]{2}{*}{ ITA11 } & -0.04232 & 0.27484 & 0.27032 & 0.01960 & 0.11102 & 0.04448 & -0.02466 & 0.21243 & 0.20415 \\
\hline & 0.6209 & 0.0011 & 13 & 0.8188 & 0.1932 & 31 & 0.7733 & 0.0120 & 0.0159 \\
\hline \multirow[t]{2}{*}{ OTA11 } & -0.03613 & 0.27635 & 0.27271 & 0.00510 & 0.11147 & -0.03320 & -0.01232 & 0.19779 & 0.18986 \\
\hline & 0.6728 & 0.0010 & 0.0012 & 0.9524 & 0.1914 & 0.6980 & 0.8856 & 0.0196 & 0.0252 \\
\hline \multirow[t]{2}{*}{ ITA12 } & -0.03537 & 0.03155 & 0.03767 & -0.01264 & 0.52530 & 0.76888 & 0.76965 & 0.07459 & 0.07961 \\
\hline & 0.6793 & 0.7124 & 0.6597 & 0.8826 & $<.0001$ & $<.0001$ & $<.0001$ & 0.3828 & 0.3515 \\
\hline \multirow[t]{2}{*}{ OTA12 } & -0.03972 & 0.04128 & 0.04768 & 0.00931 & 0.55539 & 0.76173 & 0.76631 & -0.08331 & -0.08776 \\
\hline & 0.6425 & 0.6295 & 0.5773 & 0.9134 & $<.0001$ & $<.0001$ & $<.0001$ & 0.3295 & 0.3043 \\
\hline
\end{tabular}


The CORR Procedure

07:43 Wednesday, January 15, 2003

Pearson Correlation Coefficients, $N=139$ Prob > $|r|$ under $\mathrm{HO}$ : Rho=0

\begin{tabular}{|c|c|c|c|c|c|c|c|c|c|}
\hline & & OTS15 & ITS18 & OTS18 & ITS19 & OTS19 & ITS20 & OTS20 & S22 \\
\hline \multirow[t]{2}{*}{ OTS22 } & .01305 & 0.00437 & .01166 & 0.00009 & .02750 & .03144 & .02346 & 0.00009 & 121 \\
\hline & 0.8788 & 0.9592 & 0.8916 & 0.9991 & 0.7479 & 0.7133 & 40 & 92 & \\
\hline \multirow[t]{2}{*}{ ITS23 } & -0.06639 & 05206 & 0.12 & 0.12704 & 0.11856 & 0.1 & 0.04 & 0.06781 & 0.0 \\
\hline & 0.4374 & 0.5428 & 0.1 & 0.1361 & 0.1645 & 0.1626 & 0.5859 & 0.4 & \\
\hline \multirow[t]{2}{*}{ OTS23 } & -0.06351 & -0.04955 & -0.13430 & -0.13237 & -0.12326 & -0.12387 & -0.04741 & -0.06761 & 0.04496 \\
\hline & 0.4576 & 0.5624 & 0.1150 & 0.1203 & 0.1483 & 0.1463 & 0.5794 & 0.4291 & 0.5992 \\
\hline \multirow[t]{2}{*}{ ITA2 } & -0.01465 & -0.01869 & -0.02849 & -0.02943 & -0.02617 & -0.01394 & -0.01029 & -0.01011 & 0.01029 \\
\hline & 0.8641 & 0.8271 & 0.7392 & 0.7309 & 0.7598 & 0.8706 & 0.9043 & 0.9060 & 0.9043 \\
\hline \multirow[t]{2}{*}{ OTA2 } & -0.03704 & -0.04477 & -0.03216 & -0.03288 & 0.02280 & 0.03688 & -0.02146 & -0.02008 & 0.02373 \\
\hline & 0.6651 & 0.6007 & 0.7070 & 0.7008 & 0.7899 & 0.6665 & 0.8020 & 0.8145 & 0.7816 \\
\hline \multirow[t]{2}{*}{ ITA3 } & -0.05291 & 0.05513 & -0.10287 & -0.10797 & 0.09449 & 0.10144 & -0.03714 & 0.03506 & 0.03714 \\
\hline & 0.5362 & 0.5192 & 0.2282 & 0.2058 & 0.2685 & 0.2348 & 0.6642 & 0.6820 & 0.6642 \\
\hline \multirow[t]{2}{*}{ ОТАЗ } & -0.05116 & -0.05256 & -0.11069 & 0.11585 & 0.09763 & 0.10435 & -0.04163 & 0.03838 & 0.03580 \\
\hline & 0.54 & 0.5389 & 0.1945 & 0.1745 & 0.2529 & 0.2215 & 0.6265 & 0.6537 & 0.6757 \\
\hline \multirow[t]{2}{*}{ ITA7 } & -0.03656 & 0.04574 & 0.07108 & 0.07406 & 0.06529 & 0.05524 & 0.56886 & 0.59543 & 0.02566 \\
\hline & 0.66 & 0.5928 & 0.4057 & 0.3863 & 0.4451 & 0.5183 & $<.0001$ & $<.0001$ & 0.7643 \\
\hline \multirow[t]{2}{*}{ OTA7 } & -0.04608 & -0.05 & -0.08 & 0.08588 & 0.06 & 0.0 & 0.5 & 0.5 & 0.01956 \\
\hline & 0.5 & 0.5114 & 0.3361 & 0.3148 & 0.4165 & 0.4920 & $<.0001$ & $<.0001$ & 0.8192 \\
\hline \multirow[t]{2}{*}{ ITA10 } & -0.020 & 0.02198 & 0.0 & 0.04262 & 0.03 & 0.0 & 0.0 & 0.0 & 0.01460 \\
\hline & 0.80 & 0.7973 & 0.6 & 0.6184 & 0.6 & 0.6 & 0.8 & 0.7 & 0.8646 \\
\hline \multirow[t]{2}{*}{ OTA10 } & -0.02289 & 0.02193 & 0.05426 & 0.05660 & 0.051 & 0.05620 & 0.02131 & 0.03182 & 0.00924 \\
\hline & $0.7 \varepsilon$ & 0.7978 & 0.5 & 0.5081 & 0.5 & 0.5 & 0.8033 & 0.7 & 0.9140 \\
\hline \multirow[t]{2}{*}{ ITA11 } & 0.157 & 0.16544 & 0.07707 & 0.07472 & -0.07079 & -0.07022 & -0.02782 & 0.00983 & 0.02782 \\
\hline & 0.06 & 0.0516 & 0.3672 & 0.3820 & 0.4076 & 0.4 & 0.7451 & 0.9086 & 0.7451 \\
\hline \multirow[t]{2}{*}{ OTA11 } & 0.16446 & 0.17343 & -0.06562 & -0.06308 & -0.07354 & 0.07 & 0.01249 & 0.00632 & -0.02415 \\
\hline & 0.0530 & 0.0412 & 0.4428 & 0.4607 & 0.38 & 0.3 & 0.8840 & 0.9411 & 0.7778 \\
\hline \multirow[t]{2}{*}{ ITA12 } & -0.025 & 0.00058 & 0.04971 & 0.04689 & -0.04 & -0.03 & -0.01795 & 0.03243 & -0.01795 \\
\hline & $0.7 €$ & 0.9946 & 0.5612 & 0.5836 & 0.5 & 0.6 & 0.8339 & 0.7047 & 0.8339 \\
\hline \multirow[t]{2}{*}{ OTA12 } & 0.00 & 0.03173 & -0.02212 & 0.01795 & -0.04775 & -0.03713 & -0.00811 & -0.02276 & 0.00103 \\
\hline & 0.9690 & 0.7108 & 0.7961 & 0.8339 & 0.5767 & 0.6644 & 0.9245 & 0.7903 & 0.990 \\
\hline
\end{tabular}


07:43 Wednesday, January 15, 2003

The CORR Procedure

Pearson Correlation Coefficients, $N=139$

Prob $>|r|$ under $\mathrm{HO}$ : Rho $=0$

\begin{tabular}{|c|c|c|c|c|c|c|c|c|c|}
\hline & оTS22 & ITS23 & 0TS23 & ITA2 & ОТА2 & ITA3 & ОТАЗ & ITA7 & OTA7 \\
\hline \multirow[t]{2}{*}{ 0TS22 } & 1.00000 & -0.04036 & 0.03772 & 0.01098 & -0.00020 & -0.03006 & -0.02709 & -0.02255 & -0.01582 \\
\hline & & 0.6371 & 0.6593 & 0.8979 & 0.9981 & 0.7253 & 0.7515 & 0.7922 & 0.8533 \\
\hline \multirow[t]{2}{*}{ ITS23 } & -0.04036 & 1.00000 & 0.99863 & 0.22071 & 0.24176 & 0.33921 & 0.35416 & 0.23438 & 0.23622 \\
\hline & 0.6371 & & $<.0001$ & 0.0090 & 0.0041 & $<.0001$ & $<.0001$ & 0.0055 & 0.0051 \\
\hline \multirow[t]{2}{*}{ 0TS23 } & -0.03772 & 0.99863 & 1.00000 & 0.21666 & 0.23839 & 0.34230 & 0.35765 & 0.24022 & 0.24236 \\
\hline & 0.6593 & $<.0001$ & & 0.0104 & 0.0047 & $<.0001$ & $<.0001$ & 0.0044 & 0.0040 \\
\hline \multirow[t]{2}{*}{ ITA2 } & 0.01098 & 0.22071 & 0.21666 & 1.00000 & 0.98799 & -0.02617 & -0.01921 & -0.01808 & -0.00104 \\
\hline & 0.8979 & 0.0090 & 0.0104 & & $<.0001$ & 0.7598 & 0.8224 & 0.8327 & 0.9903 \\
\hline \multirow[t]{2}{*}{ ОTA2 } & -0.00020 & 0.24176 & 0.23839 & 0.98799 & 1.00000 & -0.03403 & -0.02717 & 0.02264 & 0.03877 \\
\hline & 0.9981 & 0.0041 & 0.0047 & $<.0001$ & & 0.6908 & 0.7509 & 0.7914 & 0.6505 \\
\hline \multirow[t]{2}{*}{ ITA3 } & -0.03006 & 0.33921 & 0.34230 & 0.02617 & 0.03403 & 1.00000 & 0.99872 & 0.06529 & 0.06576 \\
\hline & 0.7253 & $<.0001$ & $<.0001$ & 0.7598 & 0.6908 & & $<.0001$ & 0.4451 & 0.4418 \\
\hline \multirow[t]{2}{*}{ ОТАЗ } & -0.02709 & 0.35416 & 0.35765 & 0.01921 & 0.02717 & 0.99872 & 1.00000 & 0.05937 & 0.05910 \\
\hline & 0.7515 & $<.0001$ & $<.0001$ & 0.8224 & 0.7509 & $<.0001$ & & 0.4875 & 0.4895 \\
\hline \multirow[t]{2}{*}{ ITA7 } & -0.02255 & 0.23438 & 0.24022 & 0.01808 & 0.02264 & 0.06529 & -0.05937 & 1.00000 & 0.99755 \\
\hline & 0.7922 & 0.0055 & 0.0044 & 0.8327 & 0.7914 & 0.4451 & 0.4875 & & $<.0001$ \\
\hline \multirow[t]{2}{*}{ TA7 } & -0.01582 & 0.23622 & 0.24236 & 0.00104 & 0.03877 & 0.06576 & -0.05910 & 0.99755 & 1.00000 \\
\hline & 0.8533 & 0.0051 & 0.0040 & 0.9903 & 0.6505 & 0.4418 & 0.4895 & $<.0001$ & \\
\hline \multirow[t]{2}{*}{ ITA10 } & -0.01411 & 0.04660 & 0.03572 & 0.01029 & 0.00959 & 0.03714 & 0.02053 & 0.02566 & 0.03203 \\
\hline & 0.8691 & 0.5859 & 0.6764 & 0.9043 & 0.9107 & 0.6642 & 0.8104 & 0.7643 & 0.7081 \\
\hline \multirow[t]{2}{*}{ OTA10 } & -0.00501 & 0.03174 & 0.02017 & 0.00421 & 0.02449 & 0.02129 & -0.00352 & 0.00949 & -0.01565 \\
\hline & 0.9533 & 0.7107 & 0.8136 & 0.9608 & 0.7747 & 0.8035 & 0.9672 & 0.9117 & 0.8549 \\
\hline \multirow[t]{2}{*}{ ITA11 } & -0.02030 & 0.08882 & 0.08143 & 0.01960 & 0.02687 & 0.07079 & -0.06297 & -0.04891 & -0.05477 \\
\hline & 0.8125 & 0.2985 & 0.3406 & 0.8188 & 0.7535 & 0.4076 & 0.4615 & 0.5675 & 0.5219 \\
\hline \multirow[t]{2}{*}{ OTA11 } & -0.01497 & -0.07739 & 0.06998 & 0.00346 & 0.01136 & 0.05859 & -0.04992 & -0.04040 & -0.04534 \\
\hline & 0.8611 & 0.3652 & 0.4130 & 0.9677 & 0.8944 & 0.4933 & 0.5595 & 0.6368 & 0.5961 \\
\hline \multirow[t]{2}{*}{ ITA12 } & 0.00925 & -0.05728 & -0.04545 & -0.01264 & -0.03196 & -0.04565 & -0.04750 & -0.03155 & -0.03938 \\
\hline & 0.9140 & 0.5030 & 0.5952 & 0.8826 & 0.7088 & 0.5936 & 0.5787 & 0.7124 & 0.6453 \\
\hline \multirow[t]{2}{*}{ OTA12 } & 0.03204 & -0.04163 & -0.03024 & -0.01644 & -0.03933 & -0.03576 & -0.03741 & -0.03159 & -0.03997 \\
\hline & 0.7080 & 0.6266 & 0.7238 & 0.8477 & 0.6457 & 0.6760 & 0.6620 & 0.7120 & 0.6403 \\
\hline
\end{tabular}


The CORR Procedure

07:43 Wednesday, January 15, 2003

Pearson Correlation Coefficients, $N=139$ Prob > $|r|$ under $\mathrm{HO}: \mathrm{RhO}=0$

\begin{tabular}{|c|c|c|c|c|c|c|c|c|c|}
\hline & ITA10 & OTA10 & ITA11 & 0TA11 & ITA12 & OTA12 & ITA18 & OTA18 & ITA19 \\
\hline \multirow[t]{2}{*}{ 0TS22 } & -0.01411 & -0.00501 & -0.02030 & -0.01497 & 0.00925 & 0.03204 & -0.01761 & -0.02030 & -0.00292 \\
\hline & 0.8691 & 0.9533 & 0.8125 & 0.8611 & 0.9140 & 0.7080 & 0.8370 & 0.8125 & 0.9728 \\
\hline \multirow[t]{2}{*}{ ITS23 } & -0.04660 & -0.03174 & -0.08882 & -0.07739 & 0.05728 & 0.04163 & 0.49908 & 0.52030 & 0.23762 \\
\hline & 0.5859 & 0.7107 & 0.2985 & 0.3652 & 0.5030 & 0.6266 & $<.0001$ & $<.0001$ & 0.0049 \\
\hline \multirow[t]{2}{*}{ 0TS23 } & -0.03572 & -0.02017 & -0.08143 & -0.06998 & -0.04545 & -0.03024 & 0.50672 & 0.52825 & 0.23922 \\
\hline & 0.6764 & 0.8136 & 0.3406 & 0.4130 & 0.5952 & 0.7238 & $<.0001$ & $<.0001$ & 0.0046 \\
\hline \multirow[t]{2}{*}{ ITA2 } & -0.01029 & 0.00421 & -0.01960 & -0.00346 & -0.01264 & -0.01644 & -0.01960 & -0.02153 & -0.01264 \\
\hline & 0.9043 & 0.9608 & 0.8188 & 0.9677 & 0.8826 & 0.8477 & 0.8188 & 0.8013 & 0.8826 \\
\hline \multirow[t]{2}{*}{ OTA2 } & 0.00959 & 0.02449 & -0.02687 & -0.01136 & -0.03196 & -0.03933 & -0.01450 & -0.01537 & 0.03631 \\
\hline & 0.9107 & 0.7747 & 0.7535 & 0.8944 & 0.7088 & 0.6457 & 0.8655 & 0.8575 & 0.6713 \\
\hline \multirow[t]{2}{*}{ ITA3 } & -0.03714 & -0.02129 & -0.07079 & -0.05859 & -0.04565 & -0.03576 & -0.07079 & -0.06466 & -0.04565 \\
\hline & 0.6642 & 0.8035 & 0.4076 & 0.4933 & 0.5936 & 0.6760 & 0.4076 & 0.4495 & 0.5936 \\
\hline \multirow[t]{2}{*}{ ОТАЗ } & -0.02053 & -0.00352 & -0.06297 & -0.04992 & -0.04750 & -0.03741 & -0.06248 & -0.05634 & -0.04087 \\
\hline & 0.8104 & 0.9672 & 0.4615 & 0.5595 & 0.5787 & 0.6620 & 0.4650 & 0.5100 & 0.6328 \\
\hline \multirow[t]{2}{*}{ ITA7 } & -0.02566 & -0.00949 & -0.04891 & -0.04040 & -0.03155 & -0.03159 & -0.04891 & -0.03171 & -0.03155 \\
\hline & 0.7643 & 0.9117 & 0.5675 & 0.6368 & 0.7124 & 0.7120 & 0.5675 & 0.7110 & 0.7124 \\
\hline \multirow[t]{2}{*}{ 0TA7 } & -0.03203 & -0.01565 & -0.05477 & -0.04534 & -0.03938 & -0.03997 & -0.05341 & -0.03697 & -0.01689 \\
\hline & 0.7081 & 0.8549 & 0.5219 & 0.5961 & 0.6453 & 0.6403 & 0.5323 & 0.6657 & 0.8436 \\
\hline \multirow[t]{2}{*}{ ITA10 } & 1.00000 & 0.99663 & -0.02782 & -0.00637 & -0.01795 & -0.02464 & -0.02782 & -0.03260 & -0.01795 \\
\hline & & $<.0001$ & 0.7451 & 0.9407 & 0.8339 & 0.7734 & 0.7451 & 0.7032 & 0.8339 \\
\hline \multirow[t]{2}{*}{ OTA10 } & 0.99663 & 1.00000 & -0.00576 & 0.01635 & -0.00298 & -0.00928 & -0.02491 & -0.02966 & -0.02706 \\
\hline & $<.0001$ & & 0.9463 & 0.8485 & 0.9722 & 0.9137 & 0.7710 & 0.7289 & 0.7519 \\
\hline \multirow[t]{2}{*}{ ITA11 } & -0.02782 & -0.00576 & 1.00000 & 0.99750 & -0.03420 & -0.03050 & -0.05303 & -0.04271 & -0.03420 \\
\hline & 0.7451 & 0.9463 & & $<.0001$ & 0.6894 & 0.7215 & 0.5353 & 0.6177 & 0.6894 \\
\hline \multirow[t]{2}{*}{ OTA11 } & -0.00637 & 0.01635 & 0.99750 & 1.00000 & -0.03732 & -0.03194 & -0.05634 & -0.04568 & -0.01894 \\
\hline & 0.9407 & 0.8485 & $<.0001$ & & 0.6627 & 0.7090 & 0.5101 & 0.5933 & 0.8249 \\
\hline \multirow[t]{2}{*}{ ITA12 } & -0.01795 & -0.00298 & -0.03420 & -0.03732 & 1.00000 & 0.99632 & -0.03420 & -0.02229 & -0.02206 \\
\hline & 0.8339 & 0.9722 & 0.6894 & 0.6627 & & $<.0001$ & 0.6894 & 0.7945 & 0.7966 \\
\hline \multirow[t]{2}{*}{ OTA12 } & -0.02464 & -0.00928 & -0.03050 & -0.03194 & 0.99632 & 1.00000 & -0.01984 & -0.00800 & -0.02940 \\
\hline & 0.7734 & 0.9137 & 0.7215 & 0.7090 & $<.0001$ & & 0.8167 & 0.9256 & 0.7312 \\
\hline
\end{tabular}


07:43 Wednesday, January 15, 2003

The CORR Procedure

Pearson Correlation Coefficients, $N=139$

Prob $>|r|$ under $\mathrm{HO}$ : Rho $=0$

\begin{tabular}{|c|c|c|c|c|c|c|c|c|c|}
\hline & OTA19 & ITA21 & OTA21 & ITA23 & ОТА23 & IM1 & OM1 & IM2 & OM2 \\
\hline \multirow[t]{2}{*}{ 0TS22 } & -0.00813 & 0.07594 & .07832 & 0.27988 & .27608 & .06453 & -0.05654 & -0.00031 & 0.01418 \\
\hline & 0.9244 & 0.3743 & 0.3594 & 0.0008 & 0.0010 & 0.4504 & 0.5085 & 0.9971 & 0.8684 \\
\hline \multirow[t]{2}{*}{ ITS23 } & 0.25435 & -0.16725 & 0.17626 & 0.18937 & 0.18469 & 0.61320 & 0.61940 & -0.41015 & -0.40504 \\
\hline & 0.0025 & 0.0491 & 0.0379 & 0.0256 & 0.0295 & $<.0001$ & $<.0001$ & $<.0001$ & $<.0001$ \\
\hline \multirow[t]{2}{*}{ 0TS23 } & 0.25667 & -0.18357 & -0.19281 & 0.19436 & 0.18975 & 0.62502 & 0.63081 & -0.42246 & -0.41752 \\
\hline & 0.0023 & 0.0305 & 0.0230 & 0.0219 & 0.0253 & $<.0001$ & $<.0001$ & $<.0001$ & $<.0001$ \\
\hline \multirow[t]{2}{*}{ ITA2 } & 0.01149 & -0.03691 & 0.03925 & 0.04180 & 0.04437 & 0.05959 & 0.05976 & 0.09052 & -0.09559 \\
\hline & 0.8932 & 0.6662 & 0.6464 & 0.6252 & 0.6040 & 0.4859 & 0.4847 & 0.2892 & 0.2630 \\
\hline \multirow[t]{2}{*}{ OTA2 } & 0.06429 & -0.04760 & 0.04962 & .08354 & 0.08621 & 0.06940 & 086 & 0.13153 & 0.13913 \\
\hline & 0.4521 & 0.5779 & 0.5619 & 0.3282 & 0.3129 & 0.4169 & 072 & 0.1227 & 0.1024 \\
\hline \multirow[t]{2}{*}{ ITA3 } & 0.04289 & -0.13329 & 0.13509 & 0.15093 & 0.14621 & 742 & 383 & 0.32688 & 0.33181 \\
\hline & 0.6161 & 0.1177 & 0.1128 & 0.0761 & 0.0859 & $<.0001$ & $<.0001$ & $<.0001$ & $<.0001$ \\
\hline \multirow[t]{2}{*}{ ОТАЗ } & 0.03814 & 0.13513 & 0.13724 & 0.14512 & 0.14050 & 989 & 130 & 0.34356 & 0.34817 \\
\hline & 0.6558 & 0.1127 & 0.1072 & 0.0883 & 0.0990 & $<.0001$ & $<.0001$ & $<.0001$ & $<.0001$ \\
\hline \multirow[t]{2}{*}{ ITA7 } & 0.02224 & -0.09210 & 0.09572 & 0.10429 & 0.10493 & 62 & 0.48826 & 0.22586 & -0.22625 \\
\hline & 0.7950 & 0.2809 & 0.2623 & 0.2218 & 0.2189 & 001 & 001 & 075 & 0.0074 \\
\hline \multirow[t]{2}{*}{ TA7 } & 0.00727 & 0.08821 & 0.09182 & 0.10145 & 0.10298 & 0.47616 & 508 & 761 & -0.21899 \\
\hline & 0.9323 & 0.3018 & 0.2824 & 0.2347 & 0.2277 & $<.0001$ & $<.0001$ & 0.0101 & 0.0096 \\
\hline \multirow[t]{2}{*}{ ITA10 } & -0.02757 & -0.05239 & 0.05692 & 0.05932 & 0.06043 & 0.06147 & 0.06424 & 0.12848 & -0.11232 \\
\hline & 0.7473 & 0.5402 & 0.5057 & 0.4879 & 0.4798 & 0.4722 & 0.4525 & 0.1317 & 0.1880 \\
\hline \multirow[t]{2}{*}{ OTA10 } & -0.03742 & -0.07033 & 0.07495 & .06402 & 0.06495 & 0.05273 & 0.05537 & 0.14305 & -0.12593 \\
\hline & 0.6618 & 0.4107 & 0.3806 & 0.4540 & 0.4475 & 0.5376 & 0.5174 & 0.0930 & 0.1396 \\
\hline \multirow[t]{2}{*}{ ITA11 } & -0.02202 & -0.09986 & 0.10834 & 0.11307 & 0.09824 & 191 & 624 & -0.2 & -0.23861 \\
\hline & 0.7970 & 0.2422 & 0.2043 & 0.1851 & 0.2499 & 242 & 719 & 037 & 0.0047 \\
\hline \multirow[t]{2}{*}{ OTA11 } & -0.00761 & -0.12060 & -0.12980 & 0.09277 & 07801 & 897 & 383 & -0.2 & -0.25819 \\
\hline & 0.9292 & 0.1573 & 0.1278 & 0.2774 & 0.3614 & 0.4905 & 0.5291 & 0.0017 & 0.0021 \\
\hline \multirow[t]{2}{*}{ ITA12 } & -0.03443 & -0.06440 & -0.06721 & -0.07292 & 0.06206 & 0.07556 & 0.06725 & 0.05589 & 0.06098 \\
\hline & 0.6874 & 0.4513 & 0.4318 & 0.3936 & 0.4680 & 0.3767 & 0.4315 & 0.5134 & 0.4758 \\
\hline \multirow[t]{2}{*}{ OTA12 } & -0.04370 & -0.08290 & -0.08644 & -0.02778 & -0.01655 & 0.06050 & -0.05219 & 0.03783 & 0.04333 \\
\hline & 0.6094 & 0.3319 & 0.3116 & 0.7454 & 0.8467 & 0.4793 & 0.5417 & 0.6584 & 0.6125 \\
\hline
\end{tabular}


Observed to Trained NNS Output for Exercises 1,2,3

07:43 Wednesday, January 15, 2003

The CORR Procedure

Pearson Correlation Coefficients, $N=139$

Prob $>|r|$ under $\mathrm{HO}:$ Rho $=0$

\begin{tabular}{|c|c|c|c|c|c|c|}
\hline & IM3 & OM3 & IM4 & OM4 & IM5 & OM5 \\
\hline \multirow[t]{2}{*}{ 0TS22 } & 0.16219 & 0.17703 & -0.01411 & 0.00910 & 0.00390 & 0.01816 \\
\hline & 0.0564 & 0.0371 & 0.8691 & 0.9153 & 0.9636 & 0.8320 \\
\hline \multirow[t]{2}{*}{ ITS23 } & -0.06408 & -0.06438 & -0.04660 & -0.05256 & -0.12664 & -0.13865 \\
\hline & 0.4536 & 0.4514 & 0.5859 & 0.5388 & 0.1374 & 0.1036 \\
\hline \multirow[t]{2}{*}{ 0TS23 } & -0.06662 & -0.06628 & -0.03572 & -0.04017 & -0.12625 & -0.13848 \\
\hline & 0.4358 & 0.4382 & 0.6764 & 0.6387 & 0.1386 & 0.1040 \\
\hline \multirow[t]{2}{*}{ ITA2 } & -0.03222 & -0.03216 & -0.01029 & -0.02549 & -0.02795 & -0.02994 \\
\hline & 0.7065 & 0.7071 & 0.9043 & 0.7658 & 0.7440 & 0.7265 \\
\hline \multirow[t]{2}{*}{ OTA2 } & -0.05865 & -0.05807 & 0.00959 & -0.01645 & -0.02038 & -0.02262 \\
\hline & 0.4928 & 0.4971 & 0.9107 & 0.8476 & 0.8118 & 0.7915 \\
\hline \multirow[t]{2}{*}{ ITA3 } & -0.11634 & -0.12420 & -0.03714 & 0.00586 & 0.32853 & 0.33294 \\
\hline & 0.1726 & 0.1452 & 0.6642 & 0.9454 & $<.0001$ & $<.0001$ \\
\hline \multirow[t]{2}{*}{ ОТАЗ } & -0.11014 & -0.11768 & -0.02053 & 0.02242 & 0.32882 & 0.33406 \\
\hline & 0.1968 & 0.1677 & 0.8104 & 0.7934 & $<.0001$ & $<.0001$ \\
\hline \multirow[t]{2}{*}{ ITA7 } & -0.08039 & -0.06305 & -0.02566 & -0.05864 & -0.06974 & -0.05800 \\
\hline & 0.3469 & 0.4609 & 0.7643 & 0.4929 & 0.4146 & 0.4976 \\
\hline \multirow[t]{2}{*}{ OTA7 } & -0.09234 & -0.07481 & -0.03203 & -0.06591 & -0.07437 & -0.06258 \\
\hline & 0.2796 & 0.3814 & 0.7081 & 0.4407 & 0.3842 & 0.4643 \\
\hline \multirow[t]{2}{*}{ ITA10 } & -0.04573 & -0.03790 & 1.00000 & 0.96609 & -0.03967 & -0.02458 \\
\hline & 0.5930 & 0.6578 & $<.0001$ & $<.0001$ & 0.6429 & 0.7740 \\
\hline \multirow[t]{2}{*}{ OTA10 } & -0.03407 & -0.02596 & 0.99663 & 0.96785 & -0.03161 & -0.01580 \\
\hline & 0.6905 & 0.7616 & $<.0001$ & $<.0001$ & 0.7118 & 0.8536 \\
\hline \multirow[t]{2}{*}{ ITA11 } & 0.29004 & 0.30151 & -0.02782 & 0.06673 & -0.07561 & -0.07634 \\
\hline & 0.0005 & 0.0003 & 0.7451 & 0.4351 & 0.3763 & 0.3717 \\
\hline \multirow[t]{2}{*}{ OTA11 } & 0.30219 & 0.31496 & -0.00637 & 0.09081 & -0.06615 & -0.06532 \\
\hline & 0.0003 & 0.0002 & 0.9407 & 0.2877 & 0.4391 & 0.4448 \\
\hline \multirow[t]{2}{*}{ ITA12 } & -0.05621 & -0.05270 & -0.01795 & 0.00975 & -0.04877 & -0.05632 \\
\hline & 0.5110 & 0.5378 & 0.8339 & 0.9093 & 0.5686 & 0.5102 \\
\hline \multirow[t]{2}{*}{ OTA12 } & -0.02649 & -0.02210 & -0.02464 & 0.00623 & -0.04551 & -0.05207 \\
\hline & 0.7569 & 0.7962 & 0.7734 & 0.9420 & 0.5947 & 0.5427 \\
\hline
\end{tabular}


The CORR Procedure

Pearson Correlation Coefficients, $N=139$

Prob $>|r|$ under $\mathrm{HO}$ : Rho=0

\begin{tabular}{|c|c|c|c|c|c|c|c|c|c|}
\hline & ITP2 & 0TP2 & ITP3 & TP3 & ITP4 & OTP4 & ITP5 & 0TP5 & ITP7 \\
\hline \multirow[t]{2}{*}{ ITA18 } & .01960 & .06377 & .04891 & .04596 & .01960 & .03520 & -0.04891 & -0.04475 & -0.05691 \\
\hline & 0.8188 & .4558 & 0.5675 & 0.5911 & 0.8188 & 0.6808 & 5675 & 009 & 058 \\
\hline \multirow[t]{2}{*}{ OTA18 } & -0.02194 & 0.05739 & 0.04246 & 0.03912 & 0.02318 & 0.03919 & -0.04536 & -0.04129 & -0.06162 \\
\hline & 0.7976 & 0.5022 & 0.6197 & 0.6475 & 0.7865 & 0.6469 & 0.5959 & 0.6294 & .4711 \\
\hline \multirow[t]{2}{*}{ ITA19 } & -0.01264 & 0.03572 & 0.03155 & 0.03293 & 0.01264 & 0.02421 & -0.03155 & 0.03720 & 0.03670 \\
\hline & 0.8826 & 0.6764 & 0.7124 & 0.7004 & 0.8826 & 0.7773 & 0.7124 & 0.6638 & 0.6680 \\
\hline \multirow[t]{2}{*}{ OTA19 } & -0.01536 & 0.04824 & 0.04665 & 0.04836 & 0.01911 & 0.03077 & -0.04223 & -0.05006 & -0.03146 \\
\hline & 0.8575 & 0.5728 & 0.5856 & 0.5718 & 0.8233 & 0.7191 & 0.6216 & 0.5584 & 0.7131 \\
\hline \multirow[t]{2}{*}{ ITA21 } & -0.03691 & 0.23864 & 0.09210 & 0.10682 & 0.03691 & 0.03234 & -0.09210 & -0.09994 & -0.10716 \\
\hline & 0.6662 & 0.0047 & 0.2809 & 0.2107 & 0.6662 & 0.7055 & 0.2809 & 0.2418 & 0.2093 \\
\hline \multirow[t]{2}{*}{ 0TA21 } & -0.03925 & 0.24296 & 0.09673 & 0.11145 & 0.03949 & 0.03571 & -0.09743 & -0.10599 & -0.09748 \\
\hline & 0.6464 & 0.0040 & 0.2573 & 0.1915 & 0.6444 & 0.6764 & 0.2538 & 0.2143 & 0.2536 \\
\hline \multirow[t]{2}{*}{ ITA23 } & 0.17338 & 0.21602 & 0.16415 & 0.18041 & 0.17338 & 0.23133 & 0.07467 & 0.09853 & 0.03483 \\
\hline & 0.0412 & 0.0106 & 0.0535 & 0.0336 & 0.0412 & 0.0061 & 0.3823 & 0.2485 & 0.6840 \\
\hline \multirow[t]{2}{*}{ OTA23 } & 0.16871 & 0.23115 & 0.16721 & 0.18360 & 0.17117 & 0.22953 & 0.07851 & 0.10295 & 0.03542 \\
\hline & 0.0471 & 0.0062 & 0.0491 & 0.0305 & 0.0439 & 0.0066 & 0.3583 & 0.2278 & 0.6789 \\
\hline \multirow[t]{2}{*}{ IM1 } & -0.04331 & 0.13301 & 0.10805 & 0.11261 & 0.04331 & -0.02963 & 0.10805 & -0.09185 & 0.04231 \\
\hline & 0.6127 & 0.1185 & 0.2055 & 0.1869 & 0.6127 & 0.7292 & 0.2055 & 0.2822 & 0.6209 \\
\hline \multirow[t]{2}{*}{ OM1 } & -0.04246 & 0.13620 & 0.10083 & 0.10517 & 0.04406 & 0.03090 & -0.09773 & -0.08191 & 0.04612 \\
\hline & 0.6197 & 0.1099 & 0.2376 & 0.2179 & 0.6065 & 0.7180 & 0.2524 & 38 & 0.5898 \\
\hline \multirow[t]{2}{*}{ IM2 } & 0.03204 & 0.43835 & 0.14789 & 0.14638 & 0.15459 & 0.14522 & 0.13090 & 0.11966 & 0.26279 \\
\hline & 0.7081 & $<.0001$ & 0.0823 & 0.0855 & 0.0692 & 0.0881 & 0.1246 & 0.1606 & 0.0018 \\
\hline \multirow[t]{2}{*}{ OM2 } & 0.01129 & 0.43662 & 0.14001 & 0.13864 & 0.13656 & 0.13066 & 0.14534 & 0.13436 & -0.26696 \\
\hline & 0.8950 & $<.0001$ & 0.1002 & 0.1036 & 0.1089 & 0.1252 & 0.0878 & 0.1148 & 0.0015 \\
\hline \multirow[t]{2}{*}{ IM3 } & 0.03222 & 0.30661 & 0.08039 & 0.06992 & 0.03222 & -0.00039 & 0.05491 & 0.07472 & -0.09353 \\
\hline & 0.7065 & 0.0002 & 0.3469 & 0.4134 & 0.7065 & 0.9964 & 0.5209 & 0.3820 & 0.2735 \\
\hline \multirow[t]{2}{*}{ OM3 } & 0.02047 & 0.32194 & 0.06549 & 0.05523 & 0.01969 & 0.01392 & 0.06009 & 0.08105 & 0.08854 \\
\hline & 0.8110 & 0.0001 & 0.4437 & 0.5184 & 0.8181 & 0.8708 & 0.4823 & 0.3429 & 0.3000 \\
\hline \multirow[t]{2}{*}{ IM4 } & 0.01029 & 0.16474 & 0.02566 & 0.03115 & 0.01029 & 0.01537 & 0.02566 & 0.02420 & 0.02986 \\
\hline & 0.9043 & 0.0526 & 0.7643 & 0.7158 & 0.9043 & 0.8574 & 0.7643 & 0.7774 & 0.7271 \\
\hline
\end{tabular}


The CORR Procedure

07:43 Wednesday, January 15, 2003

Pearson Correlation Coefficients, $N=139$

\begin{tabular}{|c|c|c|c|c|c|c|c|c|c|}
\hline & & & & $>|r|$ & er $\mathrm{HO}$ : & & & & \\
\hline & 0TP7 & ITP8 & 0TP8 & ITP10 & 0TP10 & ITP11 & 0TP11 & ITP12 & 0TP12 \\
\hline ITA18 & -0.04530 & -0.02782 & -0.04116 & -0.08009 & -0.07835 & 0.19782 & 0.19819 & 0.63202 & 0.63967 \\
\hline & 0.5964 & 0.7451 & 0.6305 & 0.3486 & 0.3592 & 0.0196 & 0.0193 & $<.0001$ & $<.0001$ \\
\hline OTA18 & -0.05025 & -0.02853 & -0.04256 & -0.08787 & -0.08608 & 0.19185 & 0.19229 & 0.64432 & 0.65225 \\
\hline & 0.5569 & 0.7389 & 0.6188 & 0.3037 & 0.3137 & 0.0237 & 0.0233 & $<.0001$ & $<.0001$ \\
\hline ITA19 & -0.03633 & -0.01795 & -0.02699 & -0.05166 & -0.04922 & -0.02948 & -0.02758 & 0.13062 & 0.12888 \\
\hline & 0.6711 & 0.8339 & 0.7525 & 0.5459 & 0.5650 & 0.7304 & 0.7472 & 0.1254 & 0.1305 \\
\hline OTA19 & -0.03120 & -0.01915 & -0.02973 & -0.07604 & -0.07362 & -0.04457 & -0.04284 & 0.14164 & 0.13933 \\
\hline & 0.7154 & 0.8230 & 0.7283 & 0.3736 & 0.3890 & 0.6024 & 0.6165 & 0.0963 & 0.1019 \\
\hline ITA21 & -0.09489 & -0.05239 & -0.04369 & -0.15082 & -0.15245 & 0.49757 & 0.49863 & -0.13329 & -0.12304 \\
\hline & 0.2665 & 0.5402 & 0.6095 & 0.0764 & 0.0732 & $<.0001$ & $<.0001$ & 0.1177 & 0.1490 \\
\hline 0TA21 & -0.08540 & -0.04263 & -0.03306 & -0.15086 & -0.15254 & 0.49300 & 0.49441 & -0.13363 & -0.12352 \\
\hline & 0.3175 & 0.6183 & 0.6992 & 0.0763 & 0.0730 & $<.0001$ & $<.0001$ & 0.1168 & 0.1474 \\
\hline ITA23 & 0.05088 & 0.24608 & 0.27618 & 0.70837 & 0.71315 & 0.45325 & 0.45576 & 0.10807 & 0.11412 \\
\hline & 0.5519 & 0.0035 & 0.0010 & $<.0001$ & $<.0001$ & $<.0001$ & $<.0001$ & 0.2054 & 0.1810 \\
\hline 0TA23 & 0.05198 & 0.24676 & 0.27674 & 0.71743 & 0.72296 & 0.46531 & 0.46819 & 0.11436 & 0.12094 \\
\hline & 0.5434 & 0.0034 & 0.0010 & $<.0001$ & $<.0001$ & $<.0001$ & $<.0001$ & 0.1801 & 0.1561 \\
\hline IM1 & 0.04942 & -0.06147 & -0.06920 & -0.17694 & -0.18469 & 0.12287 & 0.11369 & 0.11456 & 0.11384 \\
\hline & 0.5634 & 0.4722 & 0.4183 & 0.0372 & 0.0295 & 0.1496 & 0.1826 & 0.1793 & 0.1821 \\
\hline OM1 & 0.05378 & -0.06367 & -0.07130 & -0.18147 & -0.18903 & 0.10598 & 0.09697 & 0.11403 & 0.11307 \\
\hline & 0.5295 & 0.4565 & 0.4042 & 0.0325 & 0.0258 & 0.2143 & 0.2561 & 0.1814 & 0.1851 \\
\hline IM2 & -0.27007 & -0.12848 & -0.11983 & -0.14730 & -0.15906 & -0.08330 & -0.07614 & -0.08101 & -0.08561 \\
\hline & 0.0013 & 0.1317 & 0.1600 & 0.0836 & 0.0615 & 0.3296 & 0.3730 & 0.3431 & 0.3163 \\
\hline OM2 & -0.27422 & -0.12142 & -0.11394 & -0.15091 & -0.16208 & -0.07137 & -0.06492 & -0.07939 & -0.08389 \\
\hline & 0.0011 & 0.1545 & 0.1817 & 0.0762 & 0.0566 & 0.4037 & 0.4477 & 0.3529 & 0.3262 \\
\hline IM3 & -0.07470 & -0.04573 & -0.02145 & 0.73371 & 0.73920 & 0.21223 & 0.21655 & 0.05643 & 0.06014 \\
\hline & 0.3821 & 0.5930 & 0.8021 & $<.0001$ & $<.0001$ & 0.0121 & 0.0105 & 0.5094 & 0.4819 \\
\hline OM3 & -0.07010 & -0.02435 & -0.00084 & 0.73487 & 0.74111 & 0.21630 & 0.22033 & 0.05777 & 0.06122 \\
\hline & 0.4122 & 0.7760 & 0.9921 & $<.0001$ & $<.0001$ & 0.0105 & 0.0092 & 0.4994 & 0.4741 \\
\hline IM4 & -0.02740 & -0.01460 & -0.02378 & -0.04202 & -0.03750 & -0.10530 & -0.11028 & -0.03714 & -0.04205 \\
\hline & 0.7489 & 0.8646 & 0.7812 & 0.6233 & 0.6612 & 0.2173 & 0.1962 & 0.6642 & 0.6231 \\
\hline
\end{tabular}


07:43 Wednesday, January 15, 2003

The CORR Procedure

Pearson Correlation Coefficients, $N=139$

Prob $>|r|$ under $\mathrm{HO}$ : Rho=0

\begin{tabular}{|c|c|c|c|c|c|c|c|c|c|}
\hline & ITP15 & OTP15 & ITP22 & 0TP22 & ITP23 & 0ТР23 & ITS1 & OTS1 & 32 \\
\hline \multirow[t]{2}{*}{ ITA18 } & -0.07397 & 0.08055 & 0.05303 & .05295 & 0.02594 & .02247 & -0.01960 & -0.03299 & -0.04891 \\
\hline & 0.3868 & 0.3459 & 0.5353 & 0.5359 & 0.7618 & 0.7929 & 0.8188 & 0.6998 & 0.5675 \\
\hline \multirow[t]{2}{*}{ OTA18 } & -0.08900 & -0.09536 & -0.03477 & -0.03475 & 0.04106 & 0.03855 & -0.02112 & -0.03272 & -0.05390 \\
\hline & 0.2975 & 0.2641 & 0.6845 & 0.6847 & 0.6313 & 0.6523 & 0.8050 & 0.7022 & 0.5286 \\
\hline \multirow[t]{2}{*}{ ITA19 } & -0.04771 & -0.04388 & 0.41857 & 0.41107 & 0.05166 & 0.03970 & -0.01264 & -0.00868 & -0.03155 \\
\hline & 0.5771 & 0.6080 & $<.0001$ & $<.0001$ & 0.5459 & 0.6426 & 0.8826 & 0.9192 & 0.7124 \\
\hline \multirow[t]{2}{*}{ OTA19 } & -0.07012 & -0.06661 & 0.42976 & 0.42370 & 0.01821 & 0.00709 & 0.01849 & -0.01466 & -0.02483 \\
\hline & 0.4121 & 0.4359 & $<.0001$ & $<.0001$ & 0.8315 & 0.9340 & 0.8290 & 0.8640 & 0.7717 \\
\hline \multirow[t]{2}{*}{ ITA21 } & -0.13929 & -0.15175 & 0.09986 & .11467 & 0.15082 & 0.16012 & 0.03691 & -0.04356 & 0.09210 \\
\hline & 0.1020 & 0.0745 & 0.2422 & 0.1789 & 0.0764 & 0.0597 & 0.6662 & 0.6106 & 0.2809 \\
\hline \multirow[t]{2}{*}{ OTA21 } & -0.14424 & 0.15671 & 0.10562 & 0.12064 & 0.15324 & 0.16281 & 0.03707 & 0.04465 & 0.08674 \\
\hline & 0.0903 & 0.0654 & 0.2159 & 0.1572 & 0.0717 & 0.0555 & 0.6648 & 0.6017 & 0.3100 \\
\hline \multirow[t]{2}{*}{ ITA23 } & 0.40438 & 0.41657 & 0.11307 & 0.11930 & 0.17077 & 0.16047 & 0.04180 & 0.04396 & 0.10429 \\
\hline & $<.0001$ & $<.0001$ & 0.1851 & 0.1619 & 0.0444 & 0.0592 & 0.6252 & 0.6073 & 0.2218 \\
\hline \multirow[t]{2}{*}{ OTA23 } & 0.42087 & 0.43307 & 0.11639 & 0.12293 & 0.16476 & 0.15458 & 0.04191 & -0.04365 & 0.10400 \\
\hline & $<.0001$ & $<.0001$ & 0.1724 & 0.1494 & 0.0526 & 0.0692 & 0.6243 & 0.6099 & 0.2231 \\
\hline \multirow[t]{2}{*}{ IM1 } & -0.16341 & 0.16848 & 0.32028 & 0.32660 & 0.12436 & 0.13814 & 0.18822 & 0.21460 & 0.08450 \\
\hline & 0.0546 & 0.0474 & 0.0001 & $<.0001$ & 0.1447 & 0.1049 & 0.0265 & 0.0112 & 0.3226 \\
\hline \multirow[t]{2}{*}{ OM1 } & -0.17044 & 0.17523 & 0.33523 & 0.34211 & 0.13802 & 0.15197 & 0.18492 & 0.21288 & 0.09017 \\
\hline & 0.0449 & 0.0391 & $<.0001$ & $<.0001$ & 0.1052 & 0.0741 & 0.0293 & 0.0119 & 0.2912 \\
\hline \multirow[t]{2}{*}{ IM2 } & -0.34157 & 0.34973 & 0.24488 & .25571 & 0.36985 & 0.37864 & 0.09052 & 0.10116 & 0.22586 \\
\hline & $<.0001$ & $<.0001$ & 0.0037 & 0.0024 & $<.0001$ & $<.0001$ & 0.2892 & 0.2360 & 0.0075 \\
\hline \multirow[t]{2}{*}{ OM2 } & -0.32634 & 0.33504 & 0.25447 & 0.26610 & 0.38208 & 0.39128 & 0.09474 & -0.10501 & 0.23478 \\
\hline & $<.0001$ & $<.0001$ & 0.0025 & 0.0015 & $<.0001$ & $<.0001$ & 0.2673 & 0.2186 & 0.0054 \\
\hline \multirow[t]{2}{*}{ IM3 } & 0.66723 & 0.67870 & 0.03858 & 0.03259 & 0.13163 & 0.13598 & 0.03222 & -0.04447 & -0.08039 \\
\hline & $<.0001$ & $<.0001$ & 0.6521 & 0.7033 & 0.1224 & 0.1105 & 0.7065 & 0.6032 & 0.3469 \\
\hline \multirow[t]{2}{*}{ ОМ3 } & 0.67079 & 0.68346 & 0.04929 & 0.04365 & -0.12607 & -0.12950 & 0.01696 & -0.02743 & 0.08720 \\
\hline & $<.0001$ & $<.0001$ & 0.5645 & 0.6099 & 0.1392 & 0.1287 & 0.8429 & 0.7486 & 0.3074 \\
\hline \multirow[t]{2}{*}{ IM4 } & 0.37616 & 0.37837 & -0.02782 & -0.01881 & -0.04202 & -0.04484 & -0.01029 & 0.00652 & -0.02566 \\
\hline & $<.0001$ & $<.0001$ & 0.7451 & 0.8260 & 0.6233 & 0.6002 & 0.9043 & 0.9393 & 0.764 \\
\hline
\end{tabular}


The CORR Procedure

07:43 Wednesday, January 15, 2003

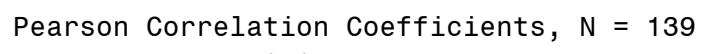

\begin{tabular}{|c|c|c|c|c|c|c|c|c|c|}
\hline & oTs2 & ITS5 & 0TS5 & ITS8 & OTS8 & ITS11 & OTS11 & ITS12 & оTS12 \\
\hline \multirow[t]{2}{*}{ ITA18 } & -0.04651 & -0.04891 & -0.05864 & -0.01960 & 0.43636 & -0.04448 & -0.02596 & -0.11566 & -0.11117 \\
\hline & 0.5867 & 0.5675 & 0.4929 & 0.8188 & $<.0001$ & 0.6031 & 0.7616 & 0.1751 & 0.1926 \\
\hline \multirow[t]{2}{*}{ OTA18 } & -0.05077 & -0.05578 & -0.06529 & -0.02359 & 0.45005 & -0.03824 & -0.02017 & -0.11684 & -0.11268 \\
\hline & 0.5528 & 0.5143 & 0.4451 & 0.7828 & $<.0001$ & 0.6549 & 0.8137 & 0.1707 & 0.1866 \\
\hline \multirow[t]{2}{*}{ ITA19 } & -0.01921 & -0.03155 & -0.02768 & -0.01264 & -0.08507 & -0.02869 & -0.04021 & -0.07459 & -0.07148 \\
\hline & 0.8224 & 0.7124 & 0.7464 & 0.8826 & 0.3194 & 0.7374 & 0.6384 & 0.3828 & 0.4031 \\
\hline \multirow[t]{2}{*}{ OTA19 } & -0.01116 & -0.03081 & -0.02679 & -0.01974 & -0.09422 & -0.04450 & -0.05741 & -0.07868 & -0.07536 \\
\hline & 0.8962 & 0.7188 & 0.7542 & 0.8176 & 0.2699 & 0.6029 & 0.5021 & 0.3572 & 0.3779 \\
\hline \multirow[t]{2}{*}{ ITA21 } & -0.09154 & -0.09210 & -0.08053 & -0.03691 & -0.25958 & -0.08376 & -0.10914 & 0.71595 & 0.72609 \\
\hline & 0.2838 & 0.2809 & 0.3460 & 0.6662 & 0.0020 & 0.3269 & 0.2009 & $<.0001$ & $<.0001$ \\
\hline \multirow[t]{2}{*}{ OTA21 } & -0.08653 & -0.09915 & -0.08794 & -0.03925 & -0.26642 & -0.08885 & -0.11484 & 0.71767 & 0.72782 \\
\hline & 0.3111 & 0.2455 & 0.3033 & 0.6464 & 0.0015 & 0.2983 & 0.1783 & $<.0001$ & $<.0001$ \\
\hline \multirow[t]{2}{*}{ ITA23 } & -0.10719 & 0.25363 & 0.26080 & 0.17338 & -0.00783 & -0.09484 & -0.07786 & -0.01990 & 0.01733 \\
\hline & 0.2091 & 0.0026 & 0.0019 & 0.0412 & 0.9271 & 0.2667 & 0.3623 & 0.8162 & 0.8395 \\
\hline \multirow[t]{2}{*}{ 0TA23 } & -0.10679 & 0.25498 & 0.26187 & 0.16781 & 0.00156 & -0.08708 & -0.06959 & -0.00994 & -0.00742 \\
\hline & 0.2108 & 0.0025 & 0.0018 & 0.0483 & 0.9854 & 0.3081 & 0.4156 & 0.9075 & 0.9309 \\
\hline \multirow[t]{2}{*}{ IM1 } & 0.10121 & 0.21288 & 0.21200 & -0.04331 & 0.19804 & -0.09827 & -0.09488 & -0.14710 & -0.15012 \\
\hline & 0.2358 & 0.0119 & 0.0122 & 0.6127 & 0.0194 & 0.2498 & 0.2666 & 0.0840 & 0.0777 \\
\hline \multirow[t]{2}{*}{ OM1 } & 0.10755 & 0.20448 & 0.20347 & -0.04192 & 0.19974 & -0.09356 & -0.09042 & -0.16070 & -0.16354 \\
\hline & 0.2076 & 0.0158 & 0.0163 & 0.6241 & 0.0184 & 0.2733 & 0.2898 & 0.0588 & 0.0544 \\
\hline \multirow[t]{2}{*}{ IM2 } & -0.24373 & -0.12393 & -0.11782 & 0.15459 & -0.08978 & -0.03855 & -0.05457 & 0.16319 & 0.17068 \\
\hline & 0.0038 & 0.1461 & 0.1672 & 0.0692 & 0.2932 & 0.6523 & 0.5234 & 0.0549 & 0.0445 \\
\hline \multirow[t]{2}{*}{ OM2 } & -0.25258 & -0.11886 & -0.11104 & 0.14811 & -0.08760 & -0.02424 & -0.03994 & 0.16545 & 0.17297 \\
\hline & 0.0027 & 0.1634 & 0.1931 & 0.0819 & 0.3051 & 0.7769 & 0.6407 & 0.0516 & 0.0417 \\
\hline \multirow[t]{2}{*}{ IM3 } & -0.08270 & 0.19020 & 0.19385 & -0.03222 & -0.00295 & -0.07311 & -0.05124 & -0.13363 & -0.13463 \\
\hline & 0.3331 & 0.0249 & 0.0222 & 0.7065 & 0.9725 & 0.3924 & 0.5492 & 0.1168 & 0.1141 \\
\hline \multirow[t]{2}{*}{ OM3 } & -0.08893 & 0.20511 & 0.20878 & -0.03254 & -0.00652 & -0.06536 & -0.04234 & -0.13356 & -0.13426 \\
\hline & 0.2979 & 0.0154 & 0.0136 & 0.7037 & 0.9392 & 0.4446 & 0.6207 & 0.1170 & 0.1151 \\
\hline \multirow[t]{2}{*}{ IM4 } & -0.02590 & -0.02566 & -0.01302 & -0.01029 & -0.07728 & 0.62549 & 0.61868 & -0.06068 & -0.05752 \\
\hline & 0.7622 & 0.7643 & 0.8790 & 0.9043 & 0.3658 & $<.0001$ & $<.0001$ & 0.4779 & 0.5012 \\
\hline
\end{tabular}


Observed to Trained NNS Output for Exercises 1,2,3

07:43 Wednesday, January 15, 2003

The CORR Procedure

Pearson Correlation Coefficients, $N=139$

Prob $>|r|$ under $\mathrm{HO}$ : Rho=0

\begin{tabular}{|c|c|c|c|c|c|c|c|c|c|}
\hline & ITS15 & OTS15 & ITS18 & 8 & ITS19 & OTS19 & ITS20 & 0TS20 & 522 \\
\hline \multirow[t]{2}{*}{ ITA18 } & .03964 & 01676 & .07707 & .06684 & .04635 & 03600 & .02782 & -0.03226 & -0.02782 \\
\hline & .6431 & .8448 & .3672 & 4343 & .5880 & .6740 & 0.7451 & 7062 & 7451 \\
\hline \multirow[t]{2}{*}{ 0TA18 } & 0.04043 & 0.01764 & 0.08097 & 0.07059 & 04467 & 03521 & 0.01920 & 0.02373 & 0.03086 \\
\hline & 0.6365 & 0.8367 & 0.3434 & 0.4089 & 0.6016 & 0.6807 & 0.8225 & 0.7816 & 0.7184 \\
\hline \multirow[t]{2}{*}{ ITA19 } & -0.02557 & 0.02951 & 0.11479 & 0.11267 & .04565 & 0.03983 & 0.01795 & 0.00410 & 0.01795 \\
\hline & 0.7651 & 0.7302 & 0.1784 & 0.1867 & 0.5936 & 0.6416 & 0.8339 & 0.9618 & 0.8339 \\
\hline \multirow[t]{2}{*}{ OTA19 } & -0.03202 & 0.03778 & 0.10198 & 0.09965 & 0.02880 & 0.02224 & -0.00497 & 0.01805 & 0.02402 \\
\hline & 0.7083 & 0.6588 & 0.2322 & 0.2431 & 0.7365 & 0.7949 & 0.9537 & 0.8330 & 0.7789 \\
\hline \multirow[t]{2}{*}{ ITA21 } & -0.07464 & 0.08816 & 0.14512 & 0.15281 & 0.07725 & 0.08991 & 0.05239 & 0.01817 & 0.05239 \\
\hline & 0.3825 & 0.3 & 0. & 725 & 0.3661 & 0.2925 & 0.5402 & 0.8319 & 0.5402 \\
\hline \multirow[t]{2}{*}{ 0TA21 } & -0.08035 & 0.09422 & 0.14798 & .15571 & 0.07918 & 0.09189 & 0.05399 & 0.02012 & 0.05485 \\
\hline & 0.3471 & 0.2699 & 0.0821 & 0.0672 & 0.3541 & 0.2820 & 0.5279 & 0.8142 & 0.5213 \\
\hline \multirow[t]{2}{*}{ ITA23 } & 0.24181 & 0.27193 & 0.56076 & 0.57345 & 0.15093 & 0.16048 & -0.05932 & -0.04862 & 0.24608 \\
\hline & 0.0041 & 0.0012 & $<.0001$ & $<.0001$ & 0.0761 & 0.0591 & 0.4879 & 0.5698 & 0.0035 \\
\hline \multirow[t]{2}{*}{ OTA23 } & 0.23897 & 0.27043 & 0.57329 & 0.58613 & 0.15422 & -0.16382 & -0.05678 & -0.04584 & 0.24152 \\
\hline & 0.0046 & 0.0013 & $<.0001$ & $<.0001$ & 0.0699 & 0.0540 & 0.5067 & 0.5921 & 0.0042 \\
\hline \multirow[t]{2}{*}{ IM1 } & -0.08757 & -0.08905 & 0.13414 & 0.13623 & 0.08671 & 0.07948 & 0.26715 & 0.29435 & -0.06147 \\
\hline & 0.3053 & 0.2972 & 0.11 & 0.1098 & 0.3101 & 0.3524 & 0.0015 & 0.0004 & 0.4722 \\
\hline \multirow[t]{2}{*}{ OM1 } & -0.08653 & -0.08796 & 0.13664 & 0.13878 & 0.07911 & 0.07172 & 0.28785 & 0.31437 & 0.05420 \\
\hline & 0.3111 & 0.3032 & 0.1 & 0.1032 & 0.3546 & 0.4015 & 0.0006 & 0.0002 & 0.5262 \\
\hline \multirow[t]{2}{*}{ IM2 } & 0.12674 & 0.11546 & 0.20674 & 0.21099 & 0.17936 & 0.17727 & 0.12848 & 0.14223 & 0.04547 \\
\hline & 0.1371 & 0.1759 & 0.0 & 0.0127 & 0.0346 & 0.0368 & 0.1317 & 0.0949 & 0.5951 \\
\hline \multirow[t]{2}{*}{ OM2 } & 0.12506 & 0.11493 & -0.20978 & -0.21426 & 0.18617 & 0.18527 & 0.12233 & -0.13618 & 0.05942 \\
\hline & 0.1424 & 0.1779 & 0.0 & 0.0113 & 0.0282 & 0.0290 & 0.1514 & 0.1099 & 0.4872 \\
\hline \multirow[t]{2}{*}{ IM3 } & 0.38956 & 0.42516 & 0.45376 & 0.46271 & 0.11634 & 0.13068 & 0.04573 & -0.05556 & 0.14442 \\
\hline & $<.0001$ & $<.0001$ & $<.0001$ & $<.0001$ & 0.1726 & 0.1252 & 0.5930 & 0.5159 & 0.0899 \\
\hline \multirow[t]{2}{*}{ OM3 } & 0.39136 & 0.42722 & 0.46258 & 0.47196 & -0.12291 & 0.13724 & 0.03734 & 0.04474 & 0.15788 \\
\hline & $<.0001$ & $<.0001$ & $<.0001$ & $<.0001$ & 0.1494 & 0.1072 & 0.6625 & 0.6010 & 0.0634 \\
\hline \multirow[t]{2}{*}{ IM4 } & 0.02080 & 0.02198 & 0.04044 & 0.04262 & 0.03714 & 0.04209 & 0.01460 & 0.02563 & 0.01460 \\
\hline & 0.8080 & 0.7973 & 0.6365 & 0.6184 & 0.6642 & 0.6228 & 0.8646 & 0.7645 & 0.8646 \\
\hline
\end{tabular}


The CORR Procedure

07:43 Wednesday, January 15, 2003

\begin{tabular}{|c|c|c|c|c|c|c|c|c|c|}
\hline \multicolumn{10}{|c|}{$\begin{array}{l}\text { Pearson Correlation Coefficients, } N=139 \\
\text { Prob }>|r| \text { under HO: Rho=0 }\end{array}$} \\
\hline & 0TS22 & ITS23 & 0TS23 & ITA2 & 0TA2 & ITA3 & ОТАЗ & ITA7 & OTA7 \\
\hline \multirow[t]{2}{*}{ ITA18 } & -0.01761 & 0.49908 & 0.50672 & -0.01960 & -0.01450 & -0.07079 & -0.06248 & -0.04891 & -0.05341 \\
\hline & 0.8370 & $<.0001$ & $<.0001$ & 0.8188 & 0.8655 & 0.4076 & 0.4650 & 0.5675 & 0.5323 \\
\hline \multirow[t]{2}{*}{ OTA18 } & -0.02030 & 0.52030 & 0.52825 & -0.02153 & -0.01537 & -0.06466 & -0.05634 & -0.03171 & -0.03697 \\
\hline & 0.8125 & $<.0001$ & $<.0001$ & 0.8013 & 0.8575 & 0.4495 & 0.5100 & 0.7110 & 0.6657 \\
\hline \multirow[t]{2}{*}{ ITA19 } & -0.00292 & 0.23762 & 0.23922 & -0.01264 & 0.03631 & -0.04565 & -0.04087 & -0.03155 & -0.01689 \\
\hline & 0.9728 & 0.0049 & 0.0046 & 0.8826 & 0.6713 & 0.5936 & 0.6328 & 0.7124 & 0.8436 \\
\hline \multirow[t]{2}{*}{ OTA19 } & -0.00813 & 0.25435 & 0.25667 & 0.01149 & 0.06429 & -0.04289 & -0.03814 & -0.02224 & -0.00727 \\
\hline & 0.9244 & 0.0025 & 0.0023 & 0.8932 & 0.4521 & 0.6161 & 0.6558 & 0.7950 & 0.9323 \\
\hline \multirow[t]{2}{*}{ ITA21 } & -0.07594 & 0.16725 & 0.18357 & 0.03691 & 0.04760 & -0.13329 & -0.13513 & -0.09210 & -0.08821 \\
\hline & 0.3743 & 0.0491 & 0.0305 & 0.6662 & 0.5779 & 0.1177 & 0.1127 & 0.2809 & 0.3018 \\
\hline \multirow[t]{2}{*}{ 0TA21 } & -0.07832 & 0.17626 & -0.19281 & -0.03925 & 0.04962 & -0.13509 & -0.13724 & -0.09572 & -0.09182 \\
\hline & 0.3594 & 0.0379 & 0.0230 & 0.6464 & 0.5619 & 0.1128 & 0.1072 & 0.2623 & 0.2824 \\
\hline \multirow[t]{2}{*}{ ITA23 } & 0.27988 & 0.18937 & -0.19436 & 0.04180 & 0.08354 & -0.15093 & -0.14512 & -0.10429 & -0.10145 \\
\hline & 0.0008 & 0.0256 & 0.0219 & 0.6252 & 0.3282 & 0.0761 & 0.0883 & 0.2218 & 0.2347 \\
\hline \multirow[t]{2}{*}{ 0TA23 } & 0.27608 & -0.18469 & -0.18975 & -0.04437 & -0.08621 & -0.14621 & -0.14050 & -0.10493 & -0.10298 \\
\hline & 0.0010 & 0.0295 & 0.0253 & 0.6040 & 0.3129 & 0.0859 & 0.0990 & 0.2189 & 0.2277 \\
\hline \multirow[t]{2}{*}{ IM1 } & -0.06453 & 0.61320 & 0.62502 & 0.05959 & 0.06940 & 0.44742 & 0.45989 & 0.46962 & 0.47616 \\
\hline & 0.4504 & $<.0001$ & $<.0001$ & 0.4859 & 0.4169 & $<.0001$ & $<.0001$ & $<.0001$ & $<.0001$ \\
\hline \multirow[t]{2}{*}{ OM1 } & -0.05654 & 0.61940 & 0.63081 & 0.05976 & 0.07086 & 0.44883 & 0.46130 & 0.48826 & 0.49508 \\
\hline & 0.5085 & $<.0001$ & $<.0001$ & 0.4847 & 0.4072 & $<.0001$ & $<.0001$ & $<.0001$ & $<.0001$ \\
\hline \multirow[t]{2}{*}{ IM2 } & -0.00031 & 0.41015 & 0.42246 & 0.09052 & 0.13153 & 0.32688 & 0.34356 & 0.22586 & 0.21761 \\
\hline & 0.9971 & $<.0001$ & $<.0001$ & 0.2892 & 0.1227 & $<.0001$ & $<.0001$ & 0.0075 & 0.0101 \\
\hline \multirow[t]{2}{*}{ OM2 } & 0.01418 & 0.40504 & 0.41752 & 0.09559 & 0.13913 & -0.33181 & -0.34817 & -0.22625 & -0.21899 \\
\hline & 0.8684 & $<.0001$ & $<.0001$ & 0.2630 & 0.1024 & $<.0001$ & $<.0001$ & 0.0074 & 0.0096 \\
\hline \multirow[t]{2}{*}{ IM3 } & 0.16219 & 0.06408 & 0.06662 & -0.03222 & -0.05865 & -0.11634 & -0.11014 & -0.08039 & -0.09234 \\
\hline & 0.0564 & 0.4536 & 0.4358 & 0.7065 & 0.4928 & 0.1726 & 0.1968 & 0.3469 & 0.2796 \\
\hline \multirow[t]{2}{*}{ OM3 } & 0.17703 & -0.06438 & -0.06628 & -0.03216 & -0.05807 & -0.12420 & -0.11768 & -0.06305 & -0.07481 \\
\hline & 0.0371 & 0.4514 & 0.4382 & 0.7071 & 0.4971 & 0.1452 & 0.1677 & 0.4609 & 0.3814 \\
\hline \multirow[t]{2}{*}{ IM4 } & -0.01411 & -0.04660 & -0.03572 & -0.01029 & 0.00959 & -0.03714 & -0.02053 & -0.02566 & -0.03203 \\
\hline & 0.8691 & 0.5859 & 0.6764 & 0.9043 & 0.9107 & 0.6642 & 0.8104 & 0.7643 & 0.7081 \\
\hline
\end{tabular}


Observed to Trained NNS Output for Exercises 1,2,3

07:43 Wednesday, January 15, 2003

The CORR Procedure

Pearson Correlation Coefficients, $N=139$

Prob > $|r|$ under $\mathrm{HO}$ : Rho=0

\begin{tabular}{|c|c|c|c|c|c|c|c|c|c|}
\hline & ITA10 & OTA10 & ITA11 & OTA11 & ITA12 & OTA12 & ITA18 & OTA18 & ITA19 \\
\hline \multirow[t]{2}{*}{ ITA18 } & -0.02782 & .02491 & 0.05303 & 0.05634 & -0.03420 & -0.01984 & 1.00000 & 0.99792 & -0.03420 \\
\hline & 0.7451 & 0.7710 & 0.5353 & 0.5101 & 0.6894 & 0.8167 & & $<.0001$ & 0.6894 \\
\hline \multirow[t]{2}{*}{ OTA18 } & -0.03260 & 0.02966 & 0.04271 & 0.04568 & -0.02229 & -0.00800 & 22 & 1.00000 & -0.01513 \\
\hline & 0.7032 & 0.7289 & 0.6177 & 0.5933 & 0.7945 & 0.9256 & $<.0$ & & 0.8597 \\
\hline \multirow[t]{2}{*}{ ITA19 } & -0.01795 & -0.02706 & -0.03420 & -0.01894 & -0.02206 & -0.02940 & -0.03420 & -0.01513 & 1.00000 \\
\hline & 0.8339 & 0.7519 & 0.6894 & 0.8249 & 0.7966 & 0.7312 & 0.6894 & 0.8597 & \\
\hline \multirow[t]{2}{*}{ OTA19 } & -0.02757 & -0.03742 & -0.02202 & -0.00761 & -0.03443 & -0.04370 & -0.01357 & 0.00748 & 0.99483 \\
\hline & 0.7473 & 0.6618 & 0.7970 & 0.9292 & 0.6874 & 0.6094 & 0.8740 & 0.9304 & $<.0001$ \\
\hline \multirow[t]{2}{*}{ ITA21 } & -0.05239 & -0.07033 & -0.09986 & -0.12060 & -0.06440 & -0.08290 & -0.09986 & -0.11720 & -0.06440 \\
\hline & 0.5402 & 0.4107 & 0.2422 & 0.1573 & 0.4513 & 0.3319 & 0.2422 & 0.1694 & 0.4513 \\
\hline \multirow[t]{2}{*}{ OTA21 } & -0.05692 & -0.07495 & -0.10834 & -0.12980 & -0.06721 & -0.08644 & -0.10131 & -0.11899 & -0.06848 \\
\hline & 0.5057 & 0.3806 & 0.2043 & 0.1278 & 0.4318 & 0.3116 & 0.2354 & 0.1630 & 0.4231 \\
\hline \multirow[t]{2}{*}{ ITA23 } & -0.05932 & -0.06402 & -0.11307 & 0.09277 & -0.07292 & -0.02778 & 0.11307 & 0.12043 & 0.07292 \\
\hline & 0.4879 & 0.4540 & 0.1851 & 0.2774 & 0.3936 & 0.7454 & 0.1851 & 0.1579 & 0.3936 \\
\hline \multirow[t]{2}{*}{ 0TA23 } & -0.06043 & -0.06495 & 0.09824 & 0.07801 & 0.06206 & 0.01655 & 0.10879 & 0.11571 & 0.07038 \\
\hline & 0.4798 & 0.4475 & 0.2499 & 0.3614 & 0.4680 & 0.8467 & 0.2024 & 0.1749 & 0.4103 \\
\hline \multirow[t]{2}{*}{ IM1 } & -0.06147 & -0.05273 & 0.04191 & 0.05897 & -0.07556 & -0.06 & 0.27057 & 0.29428 & 0.05909 \\
\hline & 0.4722 & 0.5376 & 0.6242 & 0.4905 & 0.3767 & 0.4793 & 0.0013 & 0.0004 & 0.4896 \\
\hline \multirow[t]{2}{*}{ OM1 } & -0.06424 & -0.05537 & 0.03624 & 0.05383 & -0.06725 & -0.05219 & 0.26479 & 0.28952 & 0.06623 \\
\hline & 0.4525 & 0.5174 & 0.6719 & 0.5291 & 0.4315 & 0.5417 & 0.0016 & 0.0005 & 0.4385 \\
\hline \multirow[t]{2}{*}{ IM2 } & -0.12848 & -0.14305 & 0.24488 & 0.26375 & 0.05589 & 0.03783 & 0.24488 & 0.26341 & -0.15794 \\
\hline & 0.1317 & 0.0930 & 0.0037 & 0.0017 & 0.5134 & 0.6584 & 0.0037 & 0.0017 & 0.0633 \\
\hline \multirow[t]{2}{*}{$\mathrm{OM} 2$} & -0.11232 & -0.12593 & 0.23861 & 0.25819 & 0.06098 & 0.04333 & -0.22952 & -0.24863 & -0.16356 \\
\hline & 0.1880 & 0.1396 & 0.0047 & 0.0021 & 0.4758 & 0.6125 & 0.0066 & 0.0032 & 0.0544 \\
\hline \multirow[t]{2}{*}{ IM3 } & -0.04573 & -0.03407 & 0.29004 & 0.30219 & -0.05621 & -0.02649 & -0.08716 & -0.09458 & 0.13300 \\
\hline & 0.5930 & 0.6905 & 0.0005 & 0.0003 & 0.5110 & 0.7569 & 0.3076 & 0.2681 & 0.1186 \\
\hline \multirow[t]{2}{*}{ OM3 } & -0.03790 & -0.02596 & 0.30151 & 0.31496 & 0.05270 & -0.02210 & -0.08729 & -0.09437 & 0.13153 \\
\hline & 0.6578 & 0.7616 & 0.0003 & 0.0002 & 0.5378 & 0.7962 & 0.3069 & 0.2691 & 0.1227 \\
\hline \multirow[t]{2}{*}{ IM4 } & 1.00000 & 0.99663 & 0.02782 & 0.00637 & -0.01795 & -0.02464 & -0.02782 & -0.03260 & -0.01795 \\
\hline & $<.0001$ & $<.0001$ & 0.7451 & 0.9407 & 0.8339 & 0.7734 & 0.7451 & 0.7032 & 0.8339 \\
\hline
\end{tabular}


The CORR Procedure

07:43 Wednesday, January 15, 2003

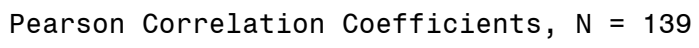

\begin{tabular}{|c|c|c|c|c|c|c|c|c|c|}
\hline & OTA19 & ITA21 & OTA21 & ITA23 & ОТА23 & IM1 & OM1 & IM2 & OM2 \\
\hline \multirow[t]{2}{*}{ ITA18 } & -0.01357 & -0.09986 & -0.10131 & -0.11307 & -0.10879 & 0.27057 & 0.26479 & -0.24488 & -0.22952 \\
\hline & 0.8740 & 0.2422 & 0.2354 & 0.1851 & 0.2024 & 0.0013 & 0.0016 & 0.0037 & 0.0066 \\
\hline \multirow[t]{2}{*}{ OTA18 } & 0.00748 & -0.11720 & -0.11899 & -0.12043 & -0.11571 & 0.29428 & 0.28952 & -0.26341 & -0.24863 \\
\hline & 0.9304 & 0.1694 & 0.1630 & 0.1579 & 0.1749 & 0.0004 & 0.0005 & 0.0017 & 0.0032 \\
\hline \multirow[t]{2}{*}{ ITA19 } & 0.99483 & -0.06440 & -0.06848 & -0.07292 & -0.07038 & 0.05909 & 0.06623 & -0.15794 & -0.16356 \\
\hline & $<.0001$ & 0.4513 & 0.4231 & 0.3936 & 0.4103 & 0.4896 & 0.4385 & 0.0633 & 0.0544 \\
\hline \multirow[t]{2}{*}{ OTA19 } & 1.00000 & -0.07567 & -0.07978 & -0.09729 & -0.09478 & 0.07723 & 0.08592 & -0.18505 & -0.19121 \\
\hline & & 0.3760 & 0.3505 & 0.2545 & 0.2671 & 0.3662 & 0.3146 & 0.0292 & 0.0241 \\
\hline \multirow[t]{2}{*}{ ITA21 } & -0.07567 & 1.00000 & 0.99931 & -0.21291 & -0.20752 & 0.22060 & 50 & 0.37129 & 0.38074 \\
\hline & 0.3760 & & $<.0001$ & 0.0119 & 0.0142 & 0.0091 & 063 & $<.0001$ & $<.0001$ \\
\hline \multirow[t]{2}{*}{ OTA21 } & -0.07978 & 0.99931 & 1.00000 & -0.21510 & -0.21000 & 0.23219 & 0.24200 & 0.37826 & 0.38746 \\
\hline & 0.3505 & $<.0001$ & & 0.0110 & 0.0131 & 0.0060 & 0.0041 & $<.0001$ & $<.0001$ \\
\hline \multirow[t]{2}{*}{ ITA23 } & -0.09729 & 0.21291 & 0.21510 & 1.00000 & 0.99915 & 0.06294 & 0.06970 & 0.07704 & 0.08082 \\
\hline & 0.2545 & 0.0119 & 0.0110 & & $<.0001$ & 0.4617 & 0.4149 & 0.3674 & 0.3442 \\
\hline \multirow[t]{2}{*}{ TA23 } & -0.09478 & 0.20752 & 0.21000 & 0.99915 & 1.00000 & 0.05978 & -0.06638 & 0.09373 & -0.09655 \\
\hline & 0.2671 & 0.0142 & 0.0131 & $<.0001$ & & 0.4845 & 375 & 724 & 0.2582 \\
\hline \multirow[t]{2}{*}{ IM1 } & 0.07723 & 0.22060 & 0.23219 & 0.06294 & 0.05978 & 1.00000 & 813 & 0.54099 & -0.53359 \\
\hline & 0.3662 & 0.0091 & 0.0060 & 0.4617 & 0.4845 & & $<.0001$ & $<.0001$ & $<.0001$ \\
\hline \multirow[t]{2}{*}{ OM1 } & 0.08592 & -0.23050 & 0.24200 & 0.06970 & -0.06638 & 0.99813 & 1.00000 & -0.54553 & -0.53881 \\
\hline & 0.3146 & 0.0063 & 0.0041 & 0.4149 & 0.4375 & $<.0001$ & & $<.0001$ & $<.0001$ \\
\hline \multirow[t]{2}{*}{ IM2 } & 0.18505 & 0.37129 & 0.37826 & 0.07704 & -0.09373 & 0.54099 & -0.54553 & 1.00000 & 0.99073 \\
\hline & 0.0292 & $<.0001$ & $<.0001$ & 0.3674 & 0.2724 & $<.0001$ & $<.0001$ & & $<.0001$ \\
\hline \multirow[t]{2}{*}{ OM2 } & -0.19121 & 0.38074 & 0.38746 & 0.08082 & -0.09655 & 0.53359 & -0.53881 & 0.99073 & 1.00000 \\
\hline & 0.0241 & $<.0001$ & $<.0001$ & 0.3442 & 0.2582 & $<.0001$ & $<.0001$ & $<.0001$ & \\
\hline \multirow[t]{2}{*}{ IM3 } & 0.11391 & -0.16412 & 0.17002 & 0.49283 & 0.50568 & -0.19254 & 0.19479 & 0.28604 & 0.27657 \\
\hline & 0.1818 & 0.0535 & 0.0454 & $<.0001$ & $<.0001$ & 0.0232 & 0.0216 & 0.0006 & 0.0010 \\
\hline \multirow[t]{2}{*}{ OM3 } & 0.11265 & -0.17688 & -0.18324 & 0.51463 & 0.52754 & 0.17748 & -0.18001 & 0.30037 & -0.29162 \\
\hline & 0.1867 & 0.0373 & 0.0308 & $<.0001$ & $<.0001$ & 0.0366 & 0.0340 & 0.0003 & 0.0005 \\
\hline \multirow[t]{2}{*}{ IM4 } & -0.02757 & -0.05239 & -0.05692 & -0.05932 & -0.06043 & 0.06147 & -0.06424 & 0.12848 & -0.11232 \\
\hline & 0.7473 & 0.5402 & 0.5057 & 0.4879 & 0.4798 & 0.4722 & 0.4525 & 0.1317 & 0.1880 \\
\hline
\end{tabular}


Observed to Trained NNS Output for Exercises 1,2,3

07:43 Wednesday, January 15, 2003

The CORR Procedure

Pearson Correlation Coefficients, $N=139$

Prob $>|r|$ under HO: Rho $=0$

\begin{tabular}{|c|c|c|c|c|c|c|}
\hline & IM3 & ОМ3 & IM4 & OM4 & IM5 & OM5 \\
\hline \multirow[t]{2}{*}{ ITA18 } & -0.08716 & -0.08729 & -0.02782 & -0.06205 & -0.07561 & -0.08818 \\
\hline & 0.3076 & 0.3069 & 0.7451 & 0.4681 & 0.3763 & 0.3020 \\
\hline \multirow[t]{2}{*}{ OTA18 } & -0.09458 & -0.09437 & -0.03260 & -0.06592 & -0.08944 & -0.10245 \\
\hline & 0.2681 & 0.2691 & 0.7032 & 0.4407 & 0.2951 & 0.2301 \\
\hline \multirow[t]{2}{*}{ ITA19 } & 0.13300 & 0.13153 & -0.01795 & -0.04562 & -0.04877 & -0.04790 \\
\hline & 0.1186 & 0.1227 & 0.8339 & 0.5938 & 0.5686 & 0.5755 \\
\hline \multirow[t]{2}{*}{ OTA19 } & 0.11391 & 0.11265 & -0.02757 & -0.05787 & -0.05167 & -0.05165 \\
\hline & 0.1818 & 0.1867 & 0.7473 & 0.4986 & 0.5458 & 0.5459 \\
\hline \multirow[t]{2}{*}{ ITA21 } & -0.16412 & -0.17688 & -0.05239 & -0.13258 & -0.14238 & -0.12681 \\
\hline & 0.0535 & 0.0373 & 0.5402 & 0.1197 & 0.0945 & 0.1368 \\
\hline \multirow[t]{2}{*}{ OTA21 } & -0.17002 & -0.18324 & -0.05692 & -0.13914 & -0.13463 & -0.11924 \\
\hline & 0.0454 & 0.0308 & 0.5057 & 0.1024 & 0.1141 & 0.1621 \\
\hline \multirow[t]{2}{*}{ ITA23 } & 0.49283 & 0.51463 & -0.05932 & -0.04022 & 0.36385 & 0.38104 \\
\hline & $<.0001$ & $<.0001$ & 0.4879 & 0.6383 & $<.0001$ & $<.0001$ \\
\hline \multirow[t]{2}{*}{ 0TA23 } & 0.50568 & 0.52754 & -0.06043 & -0.04124 & 0.37475 & 0.39208 \\
\hline & $<.0001$ & $<.0001$ & 0.4798 & 0.6298 & $<.0001$ & $<.0001$ \\
\hline \multirow[t]{2}{*}{ IM1 } & -0.19254 & -0.17748 & -0.06147 & -0.04654 & -0.16704 & -0.16173 \\
\hline & 0.0232 & 0.0366 & 0.4722 & 0.5864 & 0.0494 & 0.0572 \\
\hline \multirow[t]{2}{*}{ OM1 } & -0.19479 & -0.18001 & -0.06424 & -0.04906 & -0.16462 & -0.15939 \\
\hline & 0.0216 & 0.0340 & 0.4525 & 0.5663 & 0.0528 & 0.0609 \\
\hline \multirow[t]{2}{*}{ IM2 } & -0.28604 & -0.30037 & -0.12848 & -0.10535 & -0.34915 & -0.34768 \\
\hline & 0.0006 & 0.0003 & 0.1317 & 0.2171 & $<.0001$ & $<.0001$ \\
\hline \multirow[t]{2}{*}{ OM2 } & -0.27657 & -0.29162 & -0.11232 & -0.09127 & -0.34945 & -0.34892 \\
\hline & 0.0010 & 0.0005 & 0.1880 & 0.2852 & $<.0001$ & $<.0001$ \\
\hline \multirow[t]{2}{*}{ IM3 } & 1.00000 & 0.99664 & -0.04573 & -0.00021 & 0.30960 & 0.32215 \\
\hline & & $<.0001$ & 0.5930 & 0.9980 & 0.0002 & 0.0001 \\
\hline \multirow[t]{2}{*}{ OM3 } & 0.99664 & 1.00000 & -0.03790 & 0.00645 & 0.31790 & 0.33056 \\
\hline & $<.0001$ & & 0.6578 & 0.9399 & 0.0001 & $<.0001$ \\
\hline \multirow[t]{2}{*}{ IM4 } & -0.04573 & -0.03790 & 1.00000 & 0.96609 & -0.03967 & -0.02458 \\
\hline & 0.5930 & 0.6578 & & $<.0001$ & 0.6429 & 0.7740 \\
\hline
\end{tabular}


The CORR Procedure

07:43 Wednesday, January 15, 2003

\begin{tabular}{|c|c|c|c|c|c|c|c|c|c|}
\hline \multicolumn{10}{|c|}{$\begin{array}{l}\text { Pearson Correlation Coefficients, } N=139 \\
\text { Prob }>|r| \text { under HO: Rho=0 }\end{array}$} \\
\hline & ITP2 & 0TP2 & ITP3 & 0ТР3 & ITP4 & OTP4 & ITP5 & 0TP5 & ITP7 \\
\hline \multirow[t]{2}{*}{ OM4 } & -0.02747 & 0.19645 & -0.02400 & -0.02958 & 0.01617 & 0.04454 & 0.05601 & 0.06085 & -0.03800 \\
\hline & 0.7482 & 0.0205 & 0.7792 & 0.7296 & 0.8502 & 0.6026 & 0.5125 & 0.4768 & 0.6570 \\
\hline \multirow[t]{2}{*}{ IM5 } & -0.02795 & 0.50923 & -0.06974 & -0.06982 & -0.02795 & -0.01982 & -0.06974 & -0.07042 & 0.63814 \\
\hline & 0.7440 & $<.0001$ & 0.4146 & 0.4141 & 0.7440 & 0.8169 & 0.4146 & 0.4101 & $<.0001$ \\
\hline \multirow[t]{4}{*}{ OM5 } & -0.02869 & 0.52162 & -0.07882 & -0.07931 & -0.01256 & -0.00119 & -0.06781 & -0.06745 & 0.64104 \\
\hline & 0.7374 & $<.0001$ & 0.3563 & 0.3534 & 0.8834 & 0.9889 & 0.4277 & 0.4302 & $<.0001$ \\
\hline & \multicolumn{9}{|c|}{$\begin{array}{l}\text { Pearson Correlation Coefficients, } N=139 \\
\text { Prob }>|r| \text { under HO: Rho }=0\end{array}$} \\
\hline & 0TP7 & ITP8 & 0TP8 & ITP10 & 0TP10 & ITP11 & 0TP11 & ITP12 & 0TP12 \\
\hline \multirow[t]{2}{*}{ OM4 } & -0.03786 & -0.04180 & -0.05718 & -0.04092 & -0.03707 & -0.16277 & -0.16731 & -0.04488 & -0.05127 \\
\hline & 0.6581 & 0.6251 & 0.5038 & 0.6325 & 0.6649 & 0.0556 & 0.0490 & 0.5999 & 0.5489 \\
\hline \multirow[t]{2}{*}{ IM5 } & 0.64759 & 0.35421 & 0.35747 & 0.40412 & 0.41659 & 0.13324 & 0.13747 & -0.10093 & -0.09762 \\
\hline & $<.0001$ & $<.0001$ & $<.0001$ & $<.0001$ & $<.0001$ & 0.1179 & 0.1066 & 0.2371 & 0.2529 \\
\hline \multirow[t]{4}{*}{ OM5 } & 0.65306 & 0.33721 & 0.34426 & 0.42151 & 0.43320 & 0.14285 & 0.14753 & -0.10461 & -0.10158 \\
\hline & $<.0001$ & $<.0001$ & $<.0001$ & $<.0001$ & $<.0001$ & 0.0934 & 0.0831 & 0.2204 & 0.2341 \\
\hline & \multicolumn{9}{|c|}{$\begin{array}{l}\text { Pearson Correlation Coefficients, } N=139 \\
\text { Prob }>|r| \text { under HO: Rho }=0\end{array}$} \\
\hline & ITP15 & ОTP15 & ITP22 & 0ТР22 & ITP23 & 0ТР23 & ITS1 & OTS1 & ITS2 \\
\hline \multirow[t]{2}{*}{ OM4 } & 0.37133 & 0.37528 & -0.06281 & -0.05400 & -0.06793 & -0.06992 & -0.02747 & -0.01459 & -0.01905 \\
\hline & $<.0001$ & $<.0001$ & 0.4626 & 0.5278 & 0.4269 & 0.4134 & 0.7482 & 0.8647 & 0.8239 \\
\hline \multirow[t]{2}{*}{ IM5 } & 0.44686 & 0.45410 & -0.07561 & -0.07461 & -0.11420 & -0.11936 & -0.02795 & -0.04499 & 0.52375 \\
\hline & $<.0001$ & $<.0001$ & 0.3763 & 0.3827 & 0.1807 & 0.1616 & 0.7440 & 0.5989 & $<.0001$ \\
\hline \multirow[t]{2}{*}{ OM5 } & 0.45886 & 0.46644 & -0.07107 & -0.07019 & -0.11882 & -0.12378 & -0.01463 & -0.03056 & 0.53703 \\
\hline & $<.0001$ & $<.0001$ & 0.4058 & 0.4116 & 0.1636 & 0.1466 & 0.8643 & 0.7210 & $<.0001$ \\
\hline
\end{tabular}


Observed to Trained NNS Output for Exercises 1,2,3

07:43 Wednesday, January 15, 2003

The CORR Procedure

Pearson Correlation Coefficients, $N=139$

Prob > $|r|$ under HO: Rho=0

\begin{tabular}{|c|c|c|c|c|c|c|c|c|c|}
\hline & OTS2 & ITS5 & 0TS5 & ITS8 & OTS8 & ITS11 & OTS11 & ITS12 & OTS12 \\
\hline \multirow[t]{2}{*}{ OM4 } & -0.01882 & 0.03622 & 0.04953 & 0.00823 & -0.02725 & 0.62542 & 0.62405 & -0.11150 & -0.10965 \\
\hline & 0.8259 & 0.6721 & 0.5625 & 0.9234 & 0.7501 & $<.0001$ & $<.0001$ & 0.1913 & 0.1988 \\
\hline \multirow[t]{2}{*}{ IM5 } & 0.52285 & -0.06974 & -0.06895 & -0.02795 & -0.09180 & -0.06342 & -0.06073 & -0.04796 & -0.05295 \\
\hline & $<.0001$ & 0.4146 & 0.4199 & 0.7440 & 0.2824 & 0.4582 & 0.4776 & 0.5751 & 0.5359 \\
\hline \multirow[t]{4}{*}{ OM5 } & 0.53640 & -0.05903 & -0.05732 & -0.01380 & -0.11183 & -0.05967 & -0.05784 & -0.03101 & -0.03528 \\
\hline & $<.0001$ & 0.4900 & 0.5027 & 0.8719 & 0.1900 & 0.4853 & 0.4988 & 0.7170 & 0.6801 \\
\hline & \multicolumn{9}{|c|}{$\begin{array}{l}\text { Pearson Correlation Coefficients, } N=139 \\
\text { Prob }>|r| \text { under HO: Rho }=0\end{array}$} \\
\hline & ITS15 & OTS15 & ITS18 & OTS18 & ITS19 & OTS19 & ITS20 & OTS20 & ITS22 \\
\hline \multirow[t]{2}{*}{ OM4 } & 0.06679 & 0.06767 & -0.07680 & -0.07809 & -0.09561 & -0.10290 & -0.03899 & -0.05475 & 0.01028 \\
\hline & 0.4347 & 0.4287 & 0.3688 & 0.3609 & 0.2629 & 0.2280 & 0.6486 & 0.5221 & 0.9044 \\
\hline \multirow[t]{2}{*}{ IM5 } & -0.05652 & -0.04620 & 0.42447 & 0.42462 & -0.10093 & -0.11262 & -0.03967 & -0.03398 & -0.03967 \\
\hline & 0.5087 & 0.5891 & $<.0001$ & $<.0001$ & 0.2371 & 0.1868 & 0.6429 & 0.6913 & 0.6429 \\
\hline \multirow[t]{4}{*}{ OM5 } & -0.05342 & -0.04271 & 0.42530 & 0.42580 & -0.11644 & -0.12831 & -0.02546 & -0.01561 & -0.02516 \\
\hline & 0.5322 & 0.6176 & $<.0001$ & $<.0001$ & 0.1722 & 0.1322 & 0.7661 & 0.8553 & 0.7687 \\
\hline & \multicolumn{9}{|c|}{$\begin{array}{l}\text { Pearson Correlation Coefficients, } N=139 \\
\text { Prob }>|r| \text { under HO: Rho=0 }\end{array}$} \\
\hline & 0TS22 & ITS23 & 0TS23 & ITA2 & OTA2 & ITA3 & ОТАЗ & ITA7 & OTA7 \\
\hline \multirow[t]{2}{*}{ OM4 } & 0.00910 & -0.05256 & -0.04017 & -0.02549 & -0.01645 & 0.00586 & 0.02242 & -0.05864 & -0.06591 \\
\hline & 0.9153 & 0.5388 & 0.6387 & 0.7658 & 0.8476 & 0.9454 & 0.7934 & 0.4929 & 0.4407 \\
\hline \multirow[t]{2}{*}{ IM5 } & 0.00390 & -0.12664 & -0.12625 & -0.02795 & -0.02038 & 0.32853 & 0.32882 & -0.06974 & -0.07437 \\
\hline & 0.9636 & 0.1374 & 0.1386 & 0.7440 & 0.8118 & $<.0001$ & $<.0001$ & 0.4146 & 0.3842 \\
\hline \multirow[t]{2}{*}{ OM5 } & 0.01816 & -0.13865 & -0.13848 & -0.02994 & -0.02262 & 0.33294 & 0.33406 & -0.05800 & -0.06258 \\
\hline & 0.8320 & 0.1036 & 0.1040 & 0.7265 & 0.7915 & $<.0001$ & $<.0001$ & 0.4976 & 0.4643 \\
\hline
\end{tabular}


The CORR Procedure

07:43 Wednesday, January 15, 2003

\begin{tabular}{|c|c|c|c|c|c|c|c|c|c|c|}
\hline \multicolumn{11}{|c|}{$\begin{array}{l}\text { Pearson Correlation Coefficients, } N=139 \\
\text { Prob }>|r| \text { under HO: Rho=0 }\end{array}$} \\
\hline & & ITA10 & OTA10 & ITA11 & OTA11 & ITA12 & OTA12 & ITA18 & OTA18 & ITA19 \\
\hline \multirow[t]{2}{*}{ OM4 } & & 0.96609 & 0.96785 & 0.06673 & 0.09081 & 0.00975 & 0.00623 & -0.06205 & -0.06592 & -0.04562 \\
\hline & & $<.0001$ & $<.0001$ & 0.4351 & 0.2877 & 0.9093 & 0.9420 & 0.4681 & 0.4407 & 0.5938 \\
\hline \multirow[t]{2}{*}{ IM5 } & & 0.03967 & -0.03161 & -0.07561 & -0.06615 & -0.04877 & -0.04551 & -0.07561 & -0.08944 & -0.04877 \\
\hline & & 0.6429 & 0.7118 & 0.3763 & 0.4391 & 0.5686 & 0.5947 & 0.3763 & 0.2951 & 0.5686 \\
\hline \multirow[t]{2}{*}{ OM5 } & & 0.02458 & -0.01580 & -0.07634 & -0.06532 & -0.05632 & -0.05207 & -0.08818 & -0.10245 & -0.04790 \\
\hline & & 0.7740 & 0.8536 & 0.3717 & 0.4448 & 0.5102 & 0.5427 & 0.3020 & 0.2301 & 0.5755 \\
\hline \multicolumn{11}{|c|}{$\begin{array}{l}\text { Pearson Correlation Coefficients, } N=139 \\
\text { Prob }>|r| \text { under } \mathrm{HO}: \mathrm{RhO}=0\end{array}$} \\
\hline \multirow{3}{*}{ OM4 } & & OTA19 & ITA21 & OTA21 & ITA23 & ОТА23 & IM1 & OM1 & IM2 & $\mathrm{OM} 2$ \\
\hline & & 0.05787 & -0.13258 & -0.13914 & -0.04022 & -0.04124 & -0.04654 & -0.04906 & -0.10535 & -0.09127 \\
\hline & & 0.4986 & 0.1197 & 0.1024 & 0.6383 & 0.6298 & 0.5864 & 0.5663 & 0.2171 & 0.2852 \\
\hline \multirow[t]{2}{*}{ IM5 } & & 0.05167 & -0.14238 & -0.13463 & 0.36385 & 0.37475 & -0.16704 & -0.16462 & -0.34915 & -0.34945 \\
\hline & & 0.5458 & 0.0945 & 0.1141 & $<.0001$ & $<.0001$ & 0.0494 & 0.0528 & $<.0001$ & $<.0001$ \\
\hline \multirow[t]{2}{*}{ OM5 } & & 0.05165 & -0.12681 & -0.11924 & 0.38104 & 0.39208 & -0.16173 & -0.15939 & -0.34768 & -0.34892 \\
\hline & & 0.5459 & 0.1368 & 0.1621 & $<.0001$ & $<.0001$ & 0.0572 & 0.0609 & $<.0001$ & $<.0001$ \\
\hline \multicolumn{11}{|c|}{$\begin{array}{l}\text { Pearson Correlation Coefficients, } N=139 \\
\text { Prob }>|r| \text { under HO: Rho }=0\end{array}$} \\
\hline \multirow{3}{*}{\multicolumn{2}{|c|}{ OM4 }} & & IM3 & OM3 & \multicolumn{2}{|c|}{ IM4 } & OM4 & IM5 & & OM5 \\
\hline & & \multicolumn{2}{|c|}{$\begin{array}{r}\text { IM3 } \\
-0.00021\end{array}$} & 0.00645 & & .00000 & -0.04896 & \\
\hline & & \multicolumn{2}{|c|}{0.9980} & 0.9399 & \multicolumn{2}{|c|}{$<.0001$} & & 0.5670 & \multicolumn{2}{|c|}{$\begin{array}{r}-0.03560 \\
0.6774\end{array}$} \\
\hline \multicolumn{4}{|c|}{0.30960} & 0.31790 & \multicolumn{2}{|c|}{0.03967} & .04896 & 1.00000 & \multicolumn{2}{|c|}{0.99683} \\
\hline \multicolumn{4}{|c|}{0.0002} & 0.0001 & \multicolumn{2}{|c|}{0.6429} & 0.5670 & & \multicolumn{2}{|c|}{$<.0001$} \\
\hline \multicolumn{4}{|c|}{0.32215} & 0.33056 & -0.02 & & .03560 & 0.99683 & \multirow{2}{*}{\multicolumn{2}{|c|}{1.00000}} \\
\hline \multicolumn{4}{|c|}{0.0001} & $<.0001$ & \multicolumn{2}{|c|}{0.7740} & 0.6774 & $<.0001$ & & \\
\hline
\end{tabular}




\section{Appendix I - SAS Run Showing Simple Statistics For All DV}

SAS Code:

options pageno=1 formdlim="-";

TITLE "Training Data 123 Regression Analysis';

DATA Tngdata123;

INPUT Day1 Day2 Day3 Day4 Day5 Day6 Day7 Day8 Day9 Day10 Day11 Day12 Day13 Day14 Day15 Day16

Day17 Day18 Day19 Day20 Hour1 Hour2 Hour3 Hour4 Hour5 Hour6 Hour7 Hour8 Hour9 Hour10 Hour11

Hour12 Hour13 Hour14 Hour15 Hour16 Hour17 Hour18 Hour19 Hour20 Hour21 Hour22 Hour23 Hour24 ToCAn ToCDg TOCBn TOCBde ExTyp1 ExTyp2 ExTyp3 ExTyp4 ExNo1 ExNo2 ExNo3 ExNo4 ExNo5 ExNo6 ExNo7 ExNo8 ExNo9 ExNo10 ExNo11 ExNo12 ExNo13 ExNo14 ExNo15 ExNo16 ExNo17 ExNo18 ExNo19 ExNo20 SimNo SimJANS SimJAST SimCBS SimCCTT SimSTRM BOSIntel1 BOSIntel2 BOSIntel3 BOSIntel4 BOSMan1 BOSMan2 BOSFS1 BOSES2 BOSFS3 BOSFS4 BOSFS5 BOSFS 6 BOSFS7 BOSFS 8 BOSFS 9 BOSMAS1 BOSMAS2 BOSMAS 3 BOSMAS4 BOSMAS5 BOSMAS 6 BOSMAS7 BOSADA1 BOSADA2 BOSCC1 BOSCC2 BOSCC3 BRIntel BRMan BRFS BRMAS BRADA BRCSS BRC2 COMAFATDS COMAMPS COMASAS COMCTIS COMCSSCS COMFAADC2 COMFBCB2 COMIMETS COMMCS COMAMDWS COMJSTARS COMEPLARS INTELTUAV INTELJSTARS INTELGSR MISCBattleTiming MISCBattleTempo

MISCReconOps MISCInfoOps MISCTactics MISCTOCACtivity MOPPLevel StaffHud PERSCMDR PERSXO PERSBC PERSS3 PERSS3NCO PERSS3RTO PERSS2 PERSFSO PERSENGR PERSALO PERSS3SGM SKYCLR SKYOC SKYRAIN WINDNONE WINDLOW WINDMOD WINDHIGH LightBG LightFG Noise TEMPDB TEMPWB Humidity TP1 TP2 TP3 TP4 TP5 TP6 TP7 TP8 TP9 TP10 TP11 TP12 TP13 TP14 TP15 TP16 TP17 TP18 TP19 TP20 TP21 TP22 TP23 TS1 TS2 TS3 TS4 TS5 TS6 TS7 TS8 TS9 TS10 TS11 TS12 TS13 TS14 TS15 TS16 TS17 TS18 TS19 TS20 TS21 TS22 TS23 TA1 TA2 TA3 TA4 TA5 TA6 TA7 TA8 TA9 TA10 TA11 TA12 TA13 TA14 TA15 TA16 TA17 TA18 TA19 TA20 TA21 TA22 TA23 Mission1 Mission2 Mission3 Mission4 Mission5 Mission6 ; CARDS ;

$\begin{array}{lllllllllllllllllllllllllllllllllllllllllllllllllll}0 & 0 & 0 & 1 & 0 & 0 & 0 & 0 & 0 & 0 & 0 & 0 & 0 & 0 & 0 & 0 & 0 & 0 & 0 & 0 & 0 & 0 & 0 & 0 & 0 & 0 & 0 & 0 & 0 & 0 & 0 & 0 & 1 & 0 & 0 & 0 & 0 & 0 & 0 & 0 & 0 & 0 & 0 & 0 & 0 & 1 & 0 & 1 & 0\end{array}$ $\begin{array}{lllllllllllllllllllllllllllllllllllllllllllllllllllllllll}1 & 0 & 0 & 0 & 0 & 1 & 0 & 0 & 0 & 0 & 0 & 0 & 0 & 0 & 0 & 0 & 0 & 0 & 0 & 0 & 0 & 0 & 0 & 0 & 0 & 0 & 1 & 0 & 0 & 0 & 1 & 1 & 1 & 1 & 1 & 0 & 1 & 0 & 0 & 0 & 0 & 1 & 0 & 1 & 1 & 1 & 0 & 0 & 0\end{array}$ $\begin{array}{llllllllllllllllllllllllllllllllllllllllllll}0 & 1 & 1 & 0 & 0 & 0 & 1 & 1 & 1 & 1 & 1 & 1 & 1 & 1 & 0.7 & 0.0 & 0.6 & 0.0 & 0.0 & 0.0 & 0.0 & 0.0 & 0.6 & 0.4 & 0.1 & 0.0 & 0.1 & 0.1 & 0.0 & 0.5 & 0.5\end{array}$

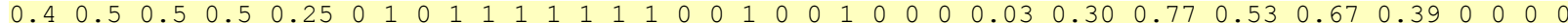

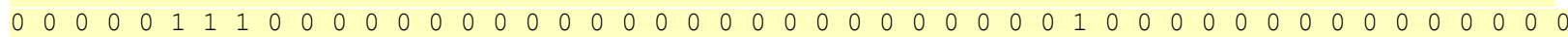
$\begin{array}{lllllllllllllllllllllllllllllllll}0 & 0 & 0 & 0 & 0 & 0 & 0 & 0 & 0 & 0 & 0 & 0 & 0 & 0 & 0 & 1 & 0.0 & 0.5 & 0.5 & 0.0 & 0.0 & 0.0\end{array}$

. .

$\begin{array}{lllllllllllllllllllllllllllllllllllllllllllllllllllllllllllllllllllllll}0 & 0 & 0 & 0 & 0 & 0 & 1 & 0 & 0 & 0 & 0 & 0 & 0 & 0 & 0 & 0 & 0 & 0 & 0 & 0 & 0 & 0 & 0 & 0 & 0 & 0 & 0 & 0 & 0 & 0 & 0 & 1 & 0 & 0 & 0 & 0 & 0 & 0 & 0 & 0 & 0 & 0 & 0 & 0 & 0 & 1 & 0 & 1 & 1\end{array}$ $\begin{array}{llllllllllllllllllllllllllllllllllllllllllllllllll}0 & 0 & 0 & 1 & 0 & 0 & 0 & 0 & 0 & 0 & 0 & 0 & 0 & 0 & 0 & 0 & 0 & 0 & 0 & 0 & 0 & 0 & 0 & 0 & 1 & 0 & 0 & 0 & 0 & 0 & 1 & 0 & 0 & 0 & 0 & 0 & 0 & 0 & 0 & 0 & 0 & 0 & 0 & 1 & 0 & 0 & 0 & 0 & 1\end{array}$

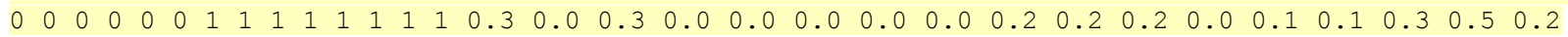

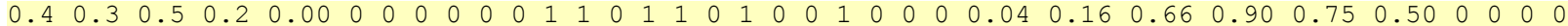

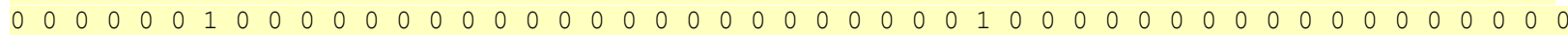
$\begin{array}{llllllllllllllllllllllllllllllllllll}0 & 0 & 0 & 0 & 0 & 0 & 0 & 0 & 0 & 0 & 0 & 0 & 0 & 1 & 0 & 0 & 0.0 & 0.4 & 0.0 & 0.0 & 0.0 & 0.0\end{array}$

;

* Io Correlation Analysis:

options pageno=1 formdlim="-"

proc corr data=Tngdata123.

TITLE "Misc Correlation for Exercises 1,2,3";

* Run corr on only the output variables to get simple statistics table showing the sum of each variable;

* which will indicate the number of observations for that variable;

*;

var TP1 TP2 TP3 TP4

TP5 TP6 TP7 TP8 TP9 TP10 TP11 TP12 TP13 TP14 TP15 TP16 TP17 TP18 TP19 TP20 TP21 TP22 TP23 TS1

TS2 TS3 TS4 TS5 TS6 TS7 TS8 TS9 TS10 TS11 TS12 TS13 TS14 TS15 TS16 TS17 TS18 TS19 TS20 TS21

TS22 TS23 TA1 TA2 TA3 TA4 TA5 TA6 TA7 TA8 TA9 TA10 TA11 TA12 TA13 TA14 TA15 TA16 TA17 TA18

TA19 TA20 TA21 TA22 TA23 Mission1 Mission2 Mission3 Mission4 Mission5 Mission6;

run; 


\section{SAS Output:}

(The sum of the data is shown in bold; those DV selected are underlined.)

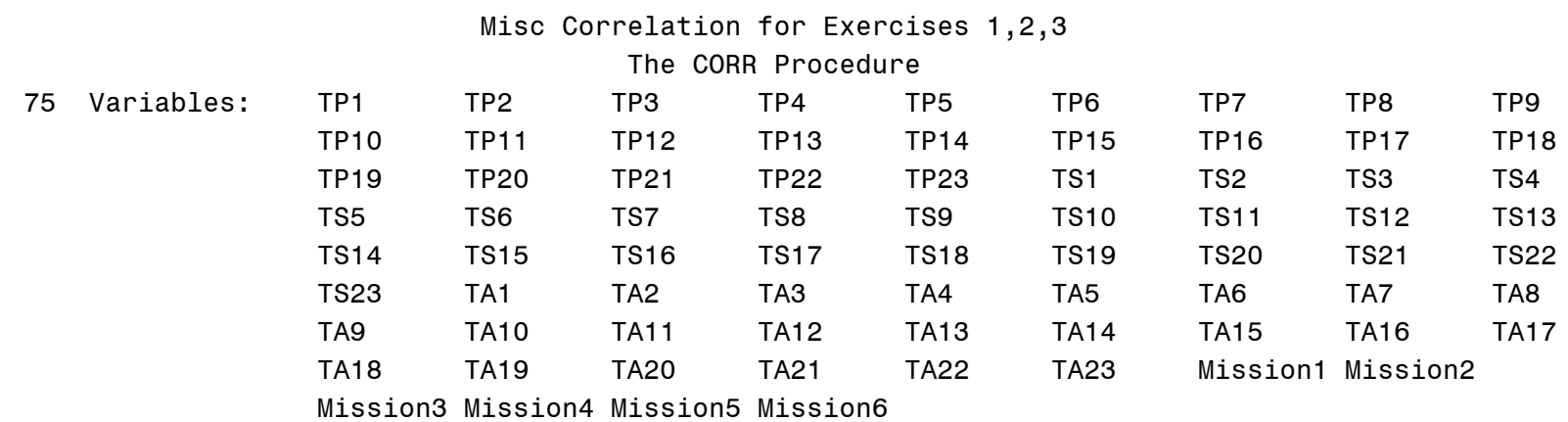

\begin{tabular}{|c|c|c|c|c|c|c|}
\hline \multicolumn{7}{|c|}{ Simple Statistics } \\
\hline Variable & $\mathrm{N}$ & Mean & Std Dev & Sum & Minimum & Maximum \\
\hline TP1 & 139 & 0 & 0 & 0 & 0 & 0 \\
\hline TP2 & 139 & 0.00719 & 0.08482 & 1.00000 & 0 & 1.00000 \\
\hline TP3 & 139 & 0.04317 & 0.20396 & 6.00000 & 0 & 1.00000 \\
\hline TP4 & 139 & 0.00719 & 0.08482 & 1.00000 & 0 & 1.00000 \\
\hline TP5 & 139 & 0.04317 & 0.20396 & 6.00000 & 0 & 1.00000 \\
\hline TP6 & 139 & 0 & 0 & 0 & 0 & 0 \\
\hline TP7 & 139 & 0.05755 & 0.23374 & 8.00000 & 0 & 1.00000 \\
\hline TP8 & 139 & 0.01439 & 0.11952 & 2.00000 & 0 & 1.00000 \\
\hline TP9 & 139 & 0 & 0 & 0 & 0 & 0 \\
\hline TP10 & 139 & 0.10791 & 0.31139 & 15.00000 & 0 & 1.00000 \\
\hline TP11 & 139 & 0.43165 & 0.49710 & 60.00000 & 0 & 1.00000 \\
\hline TP12 & 139 & 0.08633 & 0.28187 & 12.00000 & 0 & 1.00000 \\
\hline TP13 & 139 & 0 & 0 & 0 & 0 & 0 \\
\hline TP14 & 139 & 0 & 0 & 0 & 0 & 0 \\
\hline TP15 & 139 & 0.09353 & 0.29222 & 13.00000 & 0 & 1.00000 \\
\hline TP16 & 139 & 0 & 0 & 0 & 0 & 0 \\
\hline TP17 & 139 & 0 & 0 & 0 & 0 & 0 \\
\hline TP18 & 139 & 0 & 0 & 0 & 0 & 0 \\
\hline TP19 & 139 & 0 & 0 & 0 & 0 & 0 \\
\hline TP20 & 139 & 0.04317 & 0.20396 & 6.00000 & 0 & 1.00000 \\
\hline TP21 & 139 & 0.24460 & 0.43141 & 34.00000 & 0 & 1.00000 \\
\hline TP22 & 139 & 0.05036 & 0.21948 & 7.00000 & 0 & 1.00000 \\
\hline TP23 & 139 & 0.10791 & 0.31139 & 15.00000 & 0 & 1.00000 \\
\hline TS1 & 139 & 0.00719 & 0.08482 & 1.00000 & 0 & 1.00000 \\
\hline TS2 & 139 & 0.04317 & 0.20396 & 6.00000 & 0 & 1.00000 \\
\hline TS3 & 139 & 0 & 0 & 0 & 0 & 0 \\
\hline TS4 & 139 & 0 & 0 & 0 & 0 & 0 \\
\hline TS5 & 139 & 0.04317 & 0.20396 & 6.00000 & 0 & 1.00000 \\
\hline TS6 & 139 & 0 & 0 & 0 & 0 & 0 \\
\hline TS7 & 139 & 0 & 0 & 0 & 0 & 0 \\
\hline TS8 & 139 & 0.00719 & 0.08482 & 1.00000 & 0 & 1.00000 \\
\hline TS9 & 139 & 0 & 0 & 0 & 0 & 0 \\
\hline TS10 & 139 & 0 & 0 & 0 & 0 & 0 \\
\hline TS11 & 139 & 0.03597 & 0.18689 & 5.00000 & 0 & 1.00000 \\
\hline TS12 & 139 & 0.20144 & 0.40253 & 28.00000 & 0 & 1.00000 \\
\hline TS13 & 139 & 0 & 0 & 0 & 0 & 0 \\
\hline TS14 & 139 & 0 & 0 & 0 & 0 & 0 \\
\hline TS15 & 139 & 0.02878 & 0.16778 & 4.00000 & 0 & 1.00000 \\
\hline TS16 & 139 & 0 & 0 & 0 & 0 & 0 \\
\hline
\end{tabular}




\begin{tabular}{|c|c|c|c|c|c|c|}
\hline \multicolumn{7}{|c|}{ Simple Statistics } \\
\hline Variable & $\mathrm{N}$ & Mean & Std Dev & Sum & Minimum & Maximum \\
\hline TS17 & 139 & 0 & 0 & 0 & 0 & 0 \\
\hline TS18 & 139 & 0.10072 & 0.30205 & 14.00000 & 0 & 1.00000 \\
\hline TS19 & 139 & 0.08633 & 0.28187 & 12.00000 & 0 & 1.00000 \\
\hline TS20 & 139 & 0.01439 & 0.11952 & 2.00000 & 0 & 1.00000 \\
\hline TS21 & 139 & 0 & 0 & 0 & 0 & 0 \\
\hline TS22 & 139 & 0.01439 & 0.11952 & 2.00000 & 0 & 1.00000 \\
\hline TS23 & 139 & 0.12950 & 0.33696 & 18.00000 & 0 & 1.00000 \\
\hline TA1 & 139 & 0 & 0 & 0 & 0 & 0 \\
\hline TA2 & 139 & 0.00719 & 0.08482 & 1.00000 & 0 & 1.00000 \\
\hline TA3 & 139 & 0.08633 & 0.28187 & 12.00000 & 0 & 1.00000 \\
\hline TA4 & 139 & 0 & 0 & 0 & 0 & $\overline{0}$ \\
\hline TA5 & 139 & 0 & 0 & 0 & 0 & 0 \\
\hline TA6 & 139 & 0 & 0 & 0 & 0 & 0 \\
\hline TA7 & 139 & 0.04317 & 0.20396 & 6.00000 & 0 & 1.00000 \\
\hline TA8 & 139 & 0 & 0 & 0 & 0 & 0 \\
\hline TA9 & 139 & 0 & 0 & 0 & 0 & 0 \\
\hline TA10 & 139 & 0.01439 & 0.11952 & 2.00000 & 0 & 1.00000 \\
\hline TA11 & 139 & 0.05036 & 0.21948 & 7.00000 & 0 & 1.00000 \\
\hline TA12 & 139 & 0.02158 & 0.14584 & 3.00000 & 0 & 1.00000 \\
\hline TA13 & 139 & 0 & 0 & 0 & 0 & 0 \\
\hline TA14 & 139 & 0 & 0 & 0 & 0 & 0 \\
\hline TA15 & 139 & 0 & 0 & 0 & 0 & 0 \\
\hline TA16 & 139 & 0 & 0 & 0 & 0 & 0 \\
\hline TA17 & 139 & 0 & 0 & 0 & 0 & 0 \\
\hline TA18 & 139 & 0.05036 & 0.21948 & 7.00000 & 0 & 1.00000 \\
\hline TA19 & 139 & 0.02158 & 0.14584 & 3.00000 & 0 & 1.00000 \\
\hline TA20 & 139 & 0 & 0 & 0 & 0 & 0 \\
\hline TA21 & 139 & 0.15827 & 0.36632 & 22.00000 & 0 & 1.00000 \\
\hline TA22 & 139 & 0 & 0 & 0 & 0 & $\overline{0}$ \\
\hline TA23 & 139 & 0.19424 & 0.39705 & 27.00000 & 0 & 1.00000 \\
\hline Mission1 & 139 & 0.16835 & 0.33211 & 23.40000 & 0 & 1.00000 \\
\hline Mission2 & 139 & 0.22158 & 0.20913 & 30.80000 & 0 & 0.60000 \\
\hline Mission3 & 139 & 0.08417 & 0.22320 & 11.70000 & 0 & 0.85000 \\
\hline Mission4 & 139 & 0.00576 & 0.04781 & 0.80000 & 0 & 0.40000 \\
\hline Mission5 & 139 & 0.07050 & 0.21551 & 9.80000 & 0 & 0.90000 \\
\hline Mission6 & 139 & 0 & 0 & 0 & 0 & 0 \\
\hline
\end{tabular}




\title{
Appendix J - First Linear Regression Run To Select IV For Inclusion In DV Models
}

\section{SAS Code:}

\author{
options pageno=1 formdlim="-"; \\ TITLE "Training Data 123 Regression Analysis'; \\ DATA Tngdata123;
}

INPUT Day1 Day2 Day3 Day4 Day5 Day6 Day7 Day8 Day9 Day10 Day11 Day12 Day13 Day14 Day15 Day16 Day17 Day18 Day19 Day20 Hour1 Hour2 Hour3 Hour4 Hour5 Hour6 Hour7 Hour8 Hour9 Hour10 Hour11 Hour12 Hour13 Hour14 Hour15 Hour16 Hour17 Hour18 Hour19 Hour20 Hour21 Hour22 Hour23 Hour24 TOCAn TOCDg TOCBn TOCBde ExTyp1 ExTyp2 ExTyp3 ExTyp4 ExNo1 ExNo2 ExNo3 ExNo4 ExNo5 ExNo6 ExNo7 ExNo8 ExNo9 ExNo10 ExNo11 ExNo12 ExNo13 ExNo14 ExNo15 ExNo16 ExNo17 ExNo18 ExNo19 ExNo20 SimNo SimJANS SimJAST SimCBS SimCCTT SimSTRM BOSIntel1 BOSIntel2 BOSIntel3 BOSIntel4 BOSMan1 BOSMan2 BOSFS1 BOSFS2 BOSFS3 BOSFS4 BOSFS5 BOSFS 6 BOSFS7 BOSFS 8 BOSFS9 BOSMAS1 BOSMAS2 BOSMAS3 BOSMAS4 BOSMAS5 BOSMAS 6 BOSMAS7 BOSADA1 BOSADA2 BOSCC1 BOSCC2 BOSCC3 BRIntel BRMan BRFS BRMAS BRADA BRCSS BRC2 COMAFATDS COMAMPS COMASAS COMCTIS COMCSSCS COMFAADC2 COMFBCB2 COMIMETS COMMCS COMAMDWS COMJSTARS COMEPLARS INTELTUAV INTELJSTARS INTELGSR MISCBattleTiming MISCBattleTempo MISCReconops MISCInfoOps MISCTactics MISCTOCACtivity MOPPLevel StaffHud PERSCMDR PERSXO PERSBC PERSS3 PERSS3NCO PERSS3RTO PERSS2 PERSFSO PERSENGR PERSALO PERSS3SGM SKYCLR SKYOC SKYRAIN WINDNONE WINDLOW WINDMOD WINDHIGH LightBG LightFG Noise TEMPDB TEMPWB Humidity TP1 TP2 TP3 TP4 TP5 TP6 TP7 TP8 TP9 TP10 TP11 TP12 TP13 TP14 TP15 TP16 TP17 TP18 TP19 TP20 TP21 TP22 TP23 TS1 TS2 TS3 TS4 TS5 TS6 TS7 TS 8 TS9 TS10 TS11 TS12 TS13 TS14 TS15 TS16 TS17 TS18 TS19 TS20 TS21 TS22 TS23 TA1 TA2 TA3 TA4 TA5 TA6 TA7 TA8 TA9 TA10 TA11 TA12 TA13 TA14 TA15 TA16 TA17 TA18 TA19 TA20 TA21 TA22 TA23 Mission1 Mission2 Mission3 Mission4 Mission5 Mission6 ; CARDS;

$\begin{array}{lllllllllllllllllllllllllllllllllllllllllllllllllllll}0 & 0 & 0 & 1 & 0 & 0 & 0 & 0 & 0 & 0 & 0 & 0 & 0 & 0 & 0 & 0 & 0 & 0 & 0 & 0 & 0 & 0 & 0 & 0 & 0 & 0 & 0 & 0 & 0 & 0 & 0 & 0 & 1 & 0 & 0 & 0 & 0 & 0 & 0 & 0 & 0 & 0 & 0 & 0 & 0 & 1 & 0 & 1 & 0\end{array}$ $\begin{array}{llllllllllllllllllllllllllllllllllllllllllllllllllllllll}1 & 0 & 0 & 0 & 0 & 1 & 0 & 0 & 0 & 0 & 0 & 0 & 0 & 0 & 0 & 0 & 0 & 0 & 0 & 0 & 0 & 0 & 0 & 0 & 0 & 0 & 1 & 0 & 0 & 0 & 1 & 1 & 1 & 1 & 1 & 0 & 1 & 0 & 0 & 0 & 0 & 1 & 0 & 1 & 1 & 1 & 0 & 0 & 0\end{array}$ $\begin{array}{llllllllllllllllllllllllllllllllllllllllllll}0 & 1 & 1 & 0 & 0 & 0 & 1 & 1 & 1 & 1 & 1 & 1 & 1 & 1 & 0.7 & 0.0 & 0.6 & 0.0 & 0.0 & 0.0 & 0.0 & 0.0 & 0.6 & 0.4 & 0.1 & 0.0 & 0.1 & 0.1 & 0.0 & 0.5 & 0.5\end{array}$ $\begin{array}{lllllllllllllllllllllllllllllllllllllllllll}0.4 & 0.5 & 0.5 & 0.5 & 0.25 & 0 & 1 & 0 & 1 & 1 & 1 & 1 & 1 & 1 & 1 & 0 & 0 & 1 & 0 & 0 & 1 & 0 & 0 & 0 & 0 & 0 & 3 & 0.30 & 0.77 & 0.53 & 0.67 & 0.39 & 0 & 0 & 0 & 0\end{array}$ $\begin{array}{lllllllllllllllllllllllllllllllllllllllllllllllllllllll}0 & 0 & 0 & 0 & 0 & 1 & 1 & 1 & 0 & 0 & 0 & 0 & 0 & 0 & 0 & 0 & 0 & 0 & 0 & 0 & 0 & 0 & 0 & 0 & 0 & 0 & 0 & 0 & 0 & 0 & 0 & 0 & 0 & 1 & 0 & 0 & 0 & 0 & 0 & 0 & 0 & 0 & 0 & 0 & 0 & 0 & 0 & 0 & 0\end{array}$ $\begin{array}{llllllllllllllllllllllllllllllllll}0 & 0 & 0 & 0 & 0 & 0 & 0 & 0 & 0 & 0 & 0 & 0 & 0 & 0 & 0 & 1 & 0.0 & 0.5 & 0.5 & 0.0 & 0.0 & 0.0\end{array}$

$\cdots$

$\begin{array}{llllllllllllllllllllllllllllllllllllllllllllllllll}0 & 0 & 0 & 0 & 0 & 0 & 1 & 0 & 0 & 0 & 0 & 0 & 0 & 0 & 0 & 0 & 0 & 0 & 0 & 0 & 0 & 0 & 0 & 0 & 0 & 0 & 0 & 0 & 0 & 0 & 0 & 1 & 0 & 0 & 0 & 0 & 0 & 0 & 0 & 0 & 0 & 0 & 0 & 0 & 0 & 1 & 0 & 1 & 1\end{array}$ $\begin{array}{lllllllllllllllllllllllllllllllllllllllllllllllllllllllllll}0 & 0 & 0 & 1 & 0 & 0 & 0 & 0 & 0 & 0 & 0 & 0 & 0 & 0 & 0 & 0 & 0 & 0 & 0 & 0 & 0 & 0 & 0 & 0 & 1 & 0 & 0 & 0 & 0 & 0 & 1 & 0 & 0 & 0 & 0 & 0 & 0 & 0 & 0 & 0 & 0 & 0 & 0 & 1 & 0 & 0 & 0 & 0 & 1 & 0\end{array}$

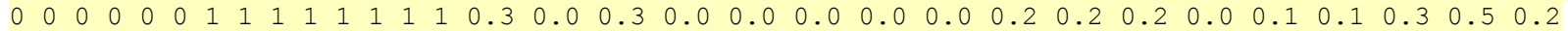

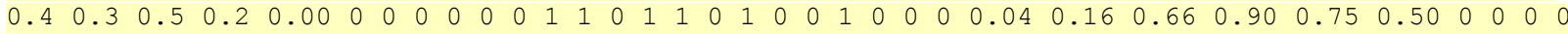

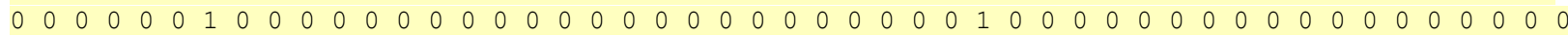
$\begin{array}{lllllllllllllllllllllllllllllllllllll}0 & 0 & 0 & 0 & 0 & 0 & 0 & 0 & 0 & 0 & 0 & 0 & 0 & 1 & 0 & 0 & 0.0 & 0.4 & 0.0 & 0.0 & 0.0 & 0.0\end{array}$

* Full Tng Regression Analysis;

* Make regression run with input/independent variables remaining after pruning with JNNS; * and output/dependent variables culled with less than 10 observations as shown in simple ; * statistics table from SAS PROC CORR run above;

options pageno=1 formdlim="-";

proc reg data=Tngdata123;

TITLE "Regression Analysis for Exercises 1,2,3";

* model TP2=BOSFS1 BOSFS2 BOSFS3 BOSFS7 BOSFS9;

model TP10=BOSIntel1 BOSIntel2 BOSIntel3 BOSIntel4 BOSMan1 BOSMan2 BOSFS2 BOSFS7 BOSFS9 BOSMAS1 BOSMAS2 BOSMAS4 BOSMAS5 BOSMAS6 BOSMAS7 BOSADA1 BOSADA2 BOSCC1 BOSCC2 BOSCC3 BRIntel BRMAS COMAFATDS

COMASAS COMMCS COMAMDWS INTELTUAV MISCBattleTiming MISCBattleTempo MISCReconOps MISCInfoOps MISCTOCACtivity MOPPLevel staffHud PERSCMDR PERSXO PERSBC PERSS3 PERSS3NCO PERSS3RTO PERSS2 PERSFSO

PERSENGR PERSALO PERSS3SGM Humidity;

model TP11=BOSIntel1 BOSIntel2 BOSIntel3 BOSIntel4 BOSMan1 BOSMan2 BOSFS2 BOSFS7 BOSFS9 BOSMAS1 BOSMAS2 BOSMAS 4 BOSMAS5 BOSMAS6 BOSMAS7 BOSADA1 BOSADA2 BOSCC1 BOSCC2 BOSCC3 BRIntel BRMAS COMAFATDS

COMASAS COMMCS COMAMDWS INTELTUAV MISCBattleTiming MISCBattleTempo MISCReconOps MISCInfoOps MISCTOCACtivity MOPPLevel StaffHud PERSCMDR PERSXO PERSBC PERSS3 PERSS3NCO PERSS3RTO PERSS2 PERSFSO

PERSENGR PERSALO PERSS3SGM Humidity;

model TP12=BOSIntel1 BOSIntel2 BOSIntel3 BOSIntel4 BOSMan1 BOSMan2 BOSFS2 BOSFS7 BOSFS9 BOSMAS1 BOSMAS2 BOSMAS4 BOSMAS5 BOSMAS6 BOSMAS7 BOSADA1 BOSADA2 BOSCC1 BOSCC2 BOSCC3 BRIntel BRMAS COMAFATDS 
COMASAS COMMCS COMAMDWS INTELTUAV MISCBattleTiming MISCBattleTempo MISCReconops MISCInfoOps MISCTOCActivity MOPPLevel StaffHud PERSCMDR PERSXO PERSBC PERSS3 PERSS3NCO PERSS3RTO PERSS2 PERSFSO

PERSENGR PERSALO PERSS3SGM Humidity;

model TP15=BOSIntel1 BOSIntel2 BOSIntel3 BOSIntel4 BOSMan1 BOSMan2 BOSFS2 BOSFS7 BOSFS9 BOSMAS1 BOSMAS2 BOSMAS4 BOSMAS5 BOSMAS6 BOSMAS7 BOSADA1 BOSADA2 BOSCC1 BOSCC2 BOSCC3 BRIntel BRMAS COMAFATDS

COMASAS COMMCS COMAMDWS INTELTUAV MISCBattleTiming MISCBattleTempo MISCReconOps MISCInfoOps MISCTOCACtivity MOPPLevel StaffHud PERSCMDR PERSXO PERSBC PERSS3 PERSS3NCO PERSS3RTO PERSS2 PERSFSO

PERSENGR PERSALO PERSS3SGM Humidity;

model TP21=BOSIntel1 BOSIntel2 BOSIntel3 BOSIntel4 BOSMan1 BOSMan2 BOSFS2 BOSFS7 BOSFS 9 BOSMAS1 BOSMAS2 BOSMAS4 BOSMAS5 BOSMAS6 BOSMAS7 BOSADA1 BOSADA2 BOSCC1 BOSCC2 BOSCC3 BRIntel BRMAS COMAFATDS

COMASAS COMMCS COMAMDWS INTELTUAV MISCBattleTiming MISCBattleTempo MISCReconOps MISCInfoOps MISCTOCActivity MOPPLevel StaffHud PERSCMDR PERSXO PERSBC PERSS3 PERSS3NCO PERSS3RTO PERSS2 PERSESO

PERSENGR PERSALO PERSS3SGM Humidity;

model TP23=BOSIntel1 BOSIntel2 BOSIntel3 BOSIntel4 BOSMan1 BOSMan2 BOSFS2 BOSFS7 BOSFS 9 BOSMAS1 BOSMAS2 BOSMAS4 BOSMAS5 BOSMAS6 BOSMAS7 BOSADA1 BOSADA2 BOSCC1 BOSCC2 BOSCC3 BRIntel BRMAS COMAFATDS

COMASAS COMMCS COMAMDWS INTELTUAV MISCBattleTiming MISCBattleTempo MISCReconOps MISCInfoOps MISCTOCActivity MOPPLevel StaffHud PERSCMDR PERSXO PERSBC PERSS3 PERSS3NCO PERSS3RTO PERSS2 PERSESO

PERSENGR PERSALO PERSS3SGM Humidity;

model TS12=BOSIntel1 BOSIntel2 BOSIntel3 BOSIntel4 BOSMan1 BOSMan2 BOSFS2 BOSFS7 BOSFS 9 BOSMAS1 BOSMAS2 BOSMAS4 BOSMAS5 BOSMAS6 BOSMAS7 BOSADA1 BOSADA2 BOSCC1 BOSCC2 BOSCC3 BRIntel BRMAS COMAFATDS

COMASAS COMMCS COMAMDWS INTELTUAV MISCBattleTiming MISCBattleTempo MISCReconOps MISCInfoOps MISCTOCActivity MOPPLevel StaffHud PERSCMDR PERSXO PERSBC PERSS3 PERSS3NCO PERSS3RTO PERSS2 PERSFSO

PERSENGR PERSALO PERSS3SGM Humidity;

model TS18=BOSIntel1 BOSIntel2 BOSIntel3 BOSIntel4 BOSMan1 BOSMan2 BOSFS2 BOSFS7 BOSFS9 BOSMAS1 BOSMAS2 BOSMAS4 BOSMAS5 BOSMAS6 BOSMAS7 BOSADA1 BOSADA2 BOSCC1 BOSCC2 BOSCC3 BRIntel BRMAS COMAFATDS

COMASAS COMMCS COMAMDWS INTELTUAV MISCBattleTiming MISCBattleTempo MISCReconOps MISCInfoOps MISCTOCActivity MOPPLevel StaffHud PERSCMDR PERSXO PERSBC PERSS3 PERSS3NCO PERSS3RTO PERSS2 PERSFSO

PERSENGR PERSALO PERSS3SGM Humidity;

model TS19=BOSIntel1 BOSIntel2 BOSIntel3 BOSIntel4 BOSMan1 BOSMan2 BOSFS2 BOSFS7 BOSFS 9 BOSMAS1 BOSMAS2 BOSMAS4 BOSMAS5 BOSMAS6 BOSMAS7 BOSADA1 BOSADA2 BOSCC1 BOSCC2 BOSCC3 BRIntel BRMAS COMAFATDS

COMASAS COMMCS COMAMDWS INTELTUAV MISCBattleTiming MISCBattleTempo MISCReconOps MISCInfoOps MISCTOCActivity MOPPLevel StaffHud PERSCMDR PERSXO PERSBC PERSS3 PERSS3NCO PERSS3RTO PERSS2 PERSFSO

PERSENGR PERSALO PERSS3SGM Humidity;

model TS23=BOSIntel1 BOSIntel2 BOSIntel3 BOSIntel4 BOSMan1 BOSMan2 BOSFS2 BOSFS7 BOSFS9 BOSMAS1 BOSMAS2 BOSMAS4 BOSMAS5 BOSMAS6 BOSMAS7 BOSADA1 BOSADA2 BOSCC1 BOSCC2 BOSCC3 BRIntel BRMAS COMAFATDS

COMASAS COMMCS COMAMDWS INTELTUAV MISCBattleTiming MISCBattleTempo MISCReconOps MISCInfoOps MISCTOCActivity MOPPLevel StaffHud PERSCMDR PERSXO PERSBC PERSS3 PERSS3NCO PERSS3RTO PERSS2 PERSFSO

PERSENGR PERSALO PERSS3SGM Humidity;

model TA3=BOSIntel1 BOSIntel2 BOSIntel3 BOSIntel4 BOSMan1 BOSMan2 BOSFS2 BOSFS7 BOSFS9 BOSMAS1 BOSMAS2 BOSMAS4 BOSMAS5 BOSMAS6 BOSMAS7 BOSADA1 BOSADA2 BOSCC1 BOSCC2 BOSCC3 BRIntel BRMAS COMAFATDS

COMASAS COMMCS COMAMDWS INTELTUAV MISCBattleTiming MISCBattleTempo MISCReconOps MISCInfoOps MISCTOCActivity MOPPLevel StaffHud PERSCMDR PERSXO PERSBC PERSS3 PERSS3NCO PERSS3RTO PERSS2 PERSFSO

PERSENGR PERSALO PERSS3SGM Humidity;

model TA21=BOSIntel1 BOSIntel2 BOSIntel3 BOSIntel4 BOSMan1 BOSMan2 BOSFS2 BOSFS7 BOSFS 9 BOSMAS1 BOSMAS2 BOSMAS4 BOSMAS5 BOSMAS6 BOSMAS7 BOSADA1 BOSADA2 BOSCC1 BOSCC2 BOSCC3 BRIntel BRMAS COMAFATDS

COMASAS COMMCS COMAMDWS INTELTUAV MISCBattleTiming MISCBattleTempo MISCReconOps MISCInfoOps MISCTOCActivity MOPPLevel StaffHud PERSCMDR PERSXO PERSBC PERSS3 PERSS3NCO PERSS3RTO PERSS2 PERSFSO

PERSENGR PERSALO PERSS3SGM Humidity;

model TA23=BOSIntel1 BOSIntel2 BOSIntel3 BOSIntel4 BOSMan1 BOSMan2 BOSFS2 BOSFS7 BOSFS 9 BOSMAS1 BOSMAS2 BOSMAS4 BOSMAS5 BOSMAS6 BOSMAS7 BOSADA1 BOSADA2 BOSCC1 BOSCC2 BOSCC3 BRIntel BRMAS COMAFATDS

COMASAS COMMCS COMAMDWS INTELTUAV MISCBattleTiming MISCBattleTempo MISCReconOps MISCInfoOps 
MISCTOCACtivity MOPPLevel StaffHud PERSCMDR PERSXO PERSBC PERSS3 PERSS3NCO PERSS3RTO PERSS2 PERSFSO

PERSENGR PERSALO PERSS3SGM Humidity;

model Mission1=BOSIntel1 BOSIntel2 BOSIntel3 BOSIntel4 BOSMan1 BOSMan2 BOSFS2 BOSFS7 BOSFS9 BOSMAS 1

BOSMAS2 BOSMAS 4 BOSMAS5 BOSMAS6 BOSMAS7 BOSADA1 BOSADA2 BOSCC1 BOSCC2 BOSCC3 BRIntel BRMAS COMAFATDS

COMASAS COMMCS COMAMDWS INTELTUAV MISCBattleTiming MISCBattleTempo MISCReconOps MISCInfoOps MISCTOCACtivity MOPPLevel staffHud PERSCMDR PERSXO PERSBC PERSS3 PERSS3NCO PERSS3RTO PERSS2 PERSESO

PERSENGR PERSALO PERSS3SGM Humidity;

model Mission2=BOSIntel1 BOSIntel2 BOSIntel3 BOSIntel4 BOSMan1 BOSMan2 BOSFS2 BOSFS7 BOSFS9 BOSMAS 1

BOSMAS2 BOSMAS4 BOSMAS5 BOSMAS6 BOSMAS7 BOSADA1 BOSADA2 BOSCC1 BOSCC2 BOSCC3 BRIntel BRMAS

COMAFATDS

COMASAS COMMCS COMAMDWS INTELTUAV MISCBattleTiming MISCBattleTempo MISCReconOps MISCInfoOps MISCTOCActivity MOPPLevel staffHud PERSCMDR PERSXO PERSBC PERSS3 PERSS3NCO PERSS3RTO PERSS2

PERSFSO

PERSENGR PERSALO PERSS3SGM Humidity;

model Mission3=BOSIntel1 BOSIntel2 BOSIntel3 BOSIntel4 BOSMan1 BOSMan2 BOSFS2 BOSFS7 BOSFS9 BOSMAS 1

BOSMAS2 BOSMAS 4 BOSMAS5 BOSMAS6 BOSMAS7 BOSADA1 BOSADA2 BOSCC1 BOSCC2 BOSCC3 BRIntEl BRMAS

COMAFATDS

COMASAS COMMCS COMAMDWS INTELTUAV MISCBattleTiming MISCBattleTempo MISCReconOps MISCInfoOps MISCTOCACtivity MOPPLevel staffHud PERSCMDR PERSXO PERSBC PERSS3 PERSS3NCO PERSS3RTO PERSS2 PERSFSO

PERSENGR PERSALO PERSS3SGM Humidity;

model Mission5=BOSIntel1 BOSIntel2 BOSIntel3 BOSIntel4 BOSMan1 BOSMan2 BOSFS2 BOSFS7 BOSFS9 BOSMAS1

BOSMAS2 BOSMAS 4 BOSMAS5 BOSMAS6 BOSMAS7 BOSADA1 BOSADA2 BOSCC1 BOSCC2 BOSCC3 BRIntel BRMAS COMAFATDS

COMASAS COMMCS COMAMDWS INTELTUAV MISCBattleTiming MISCBattleTempo MISCReconOps MISCInfoOps MISCTOCActivity MOPPLevel staffHud PERSCMDR PERSXO PERSBC PERSS3 PERSS3NCO PERSS3RTO PERSS2 PERSESO

PERSENGR PERSALO PERSS3SGM Humidity;

run; 
SAS Output:

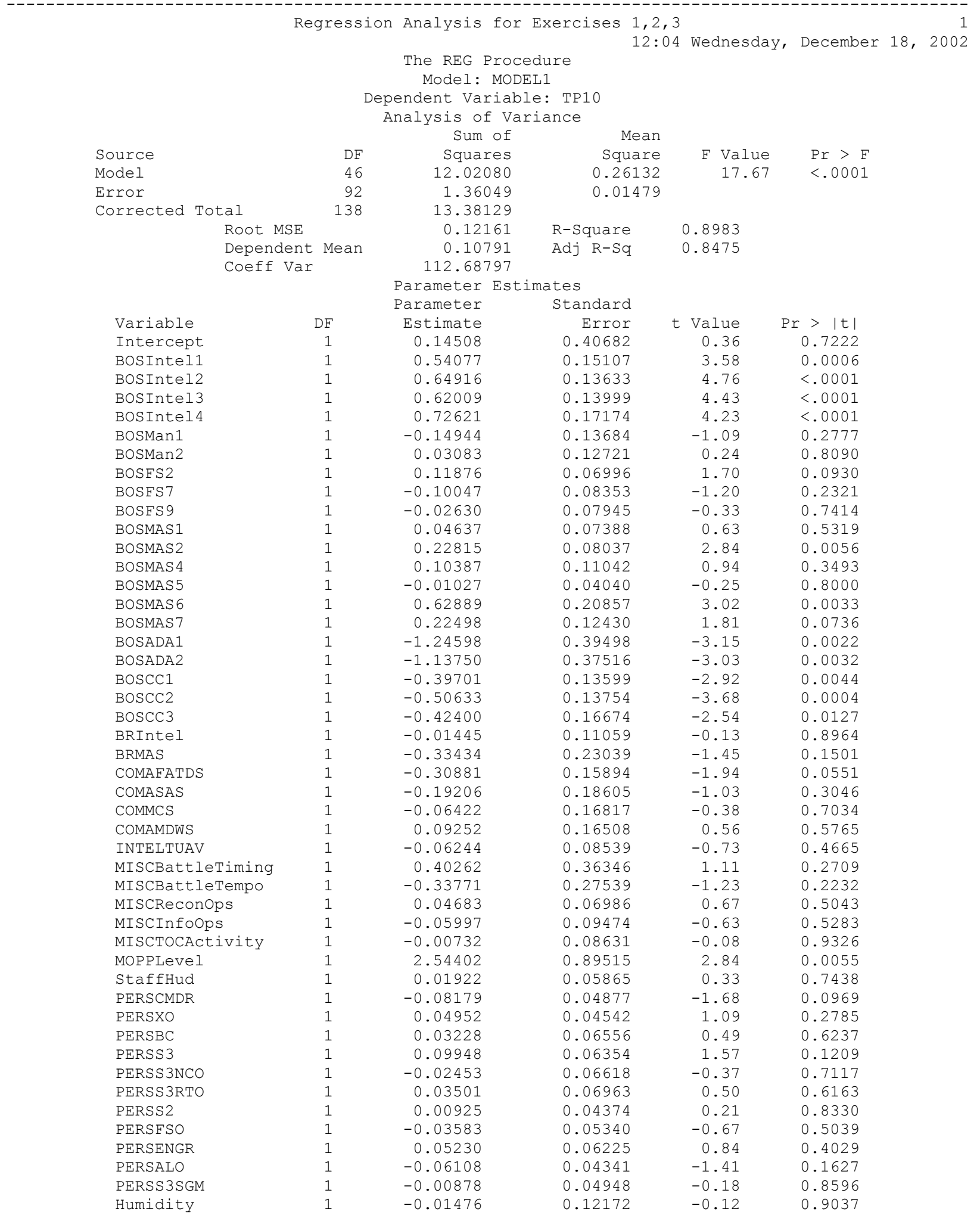




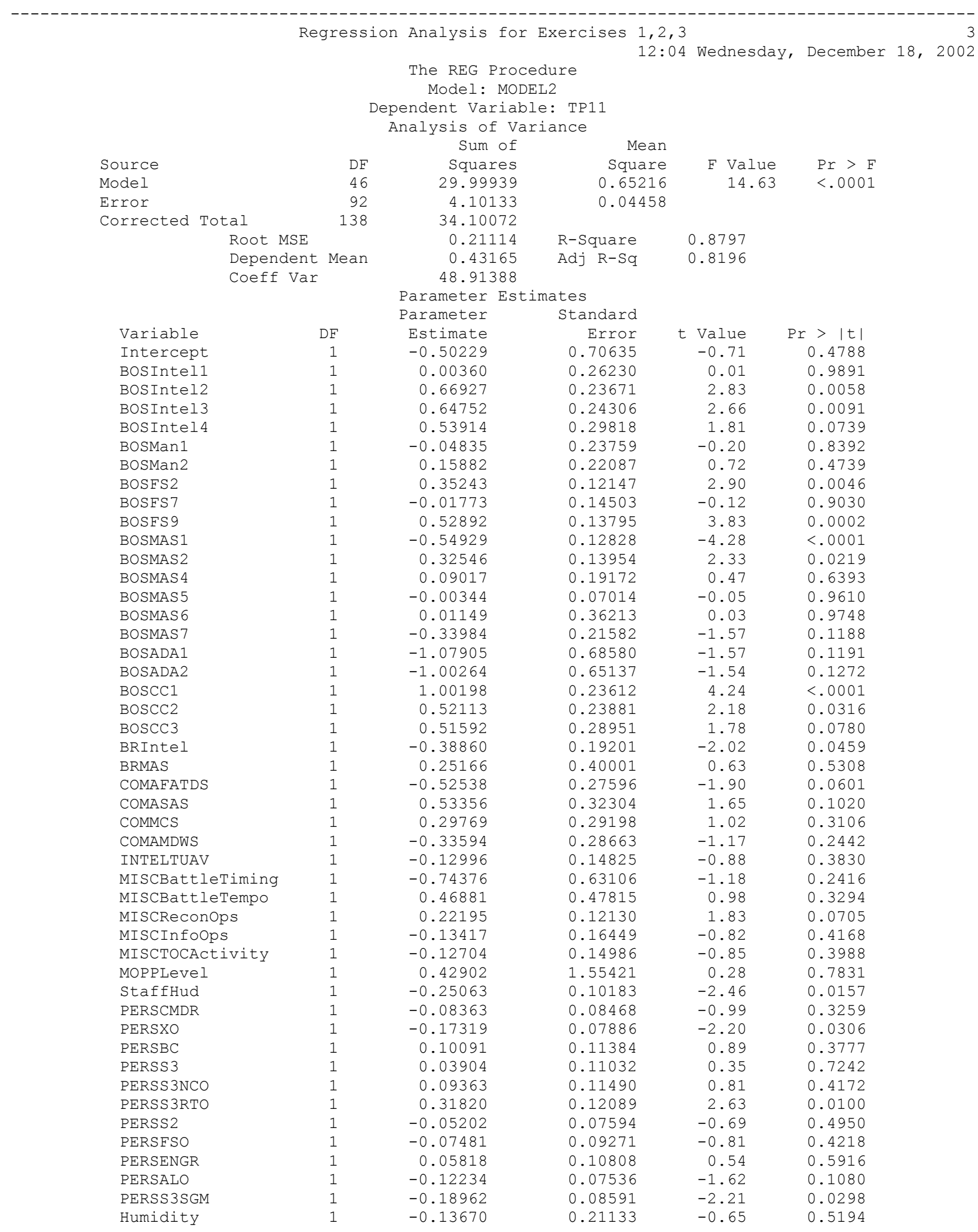




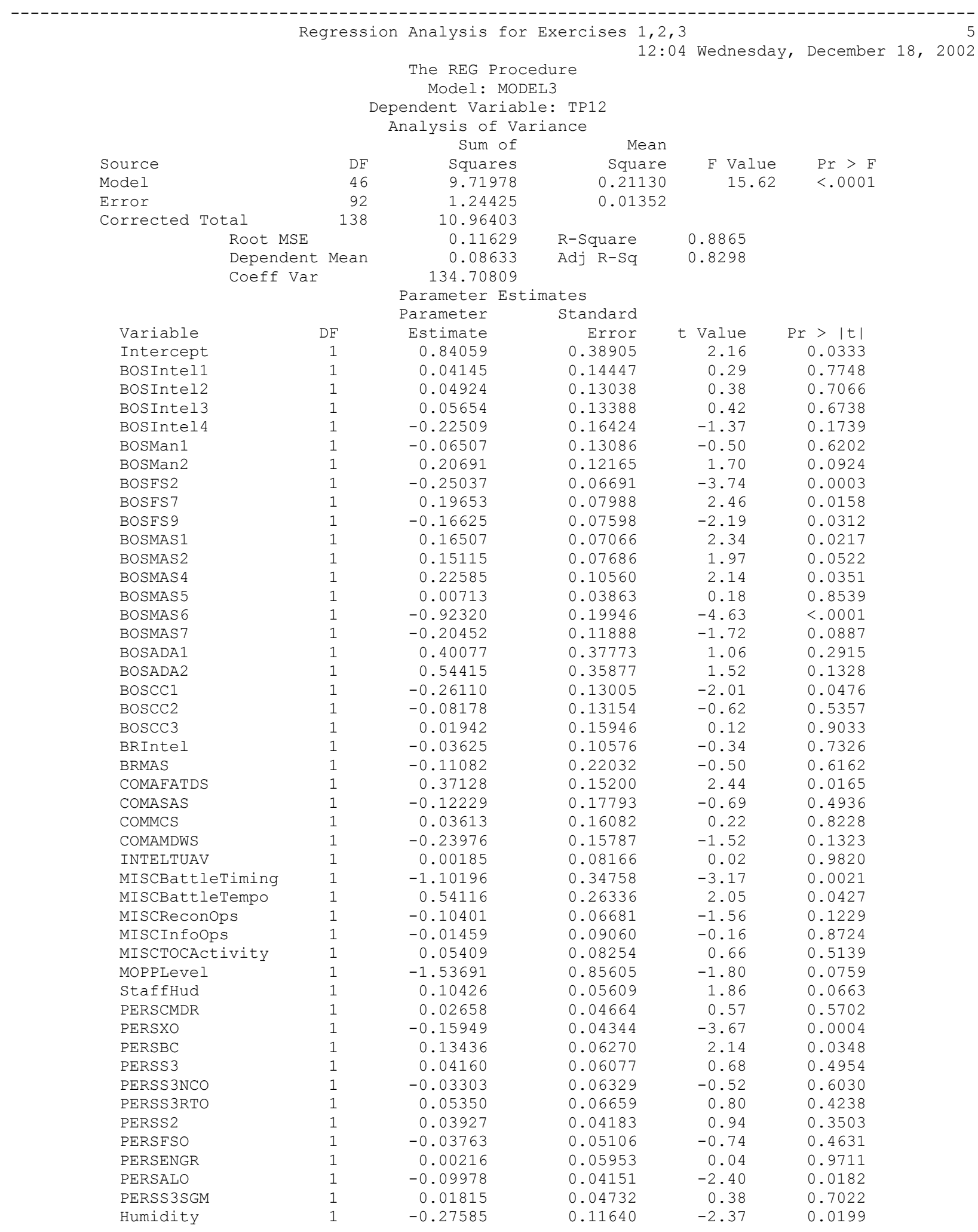




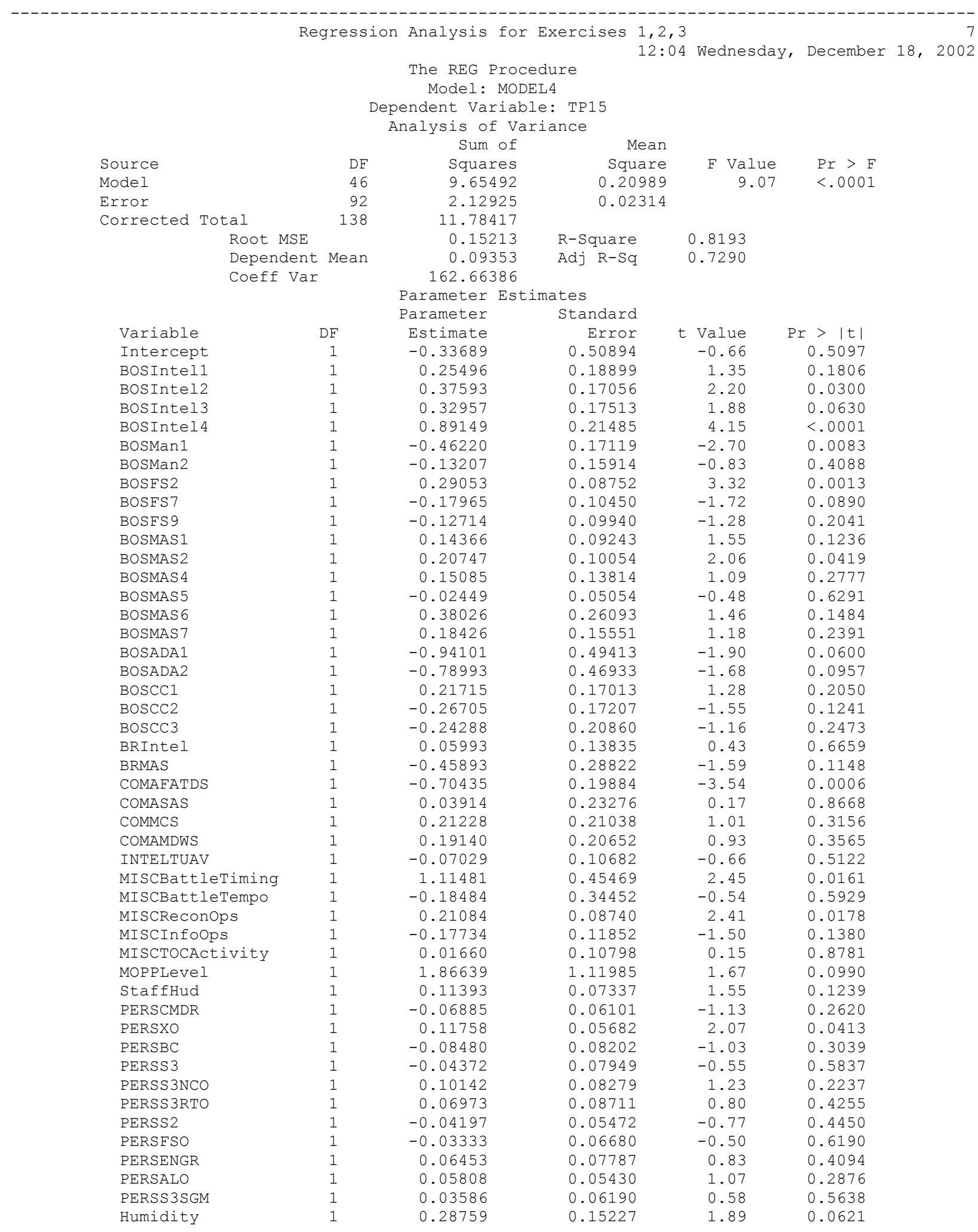




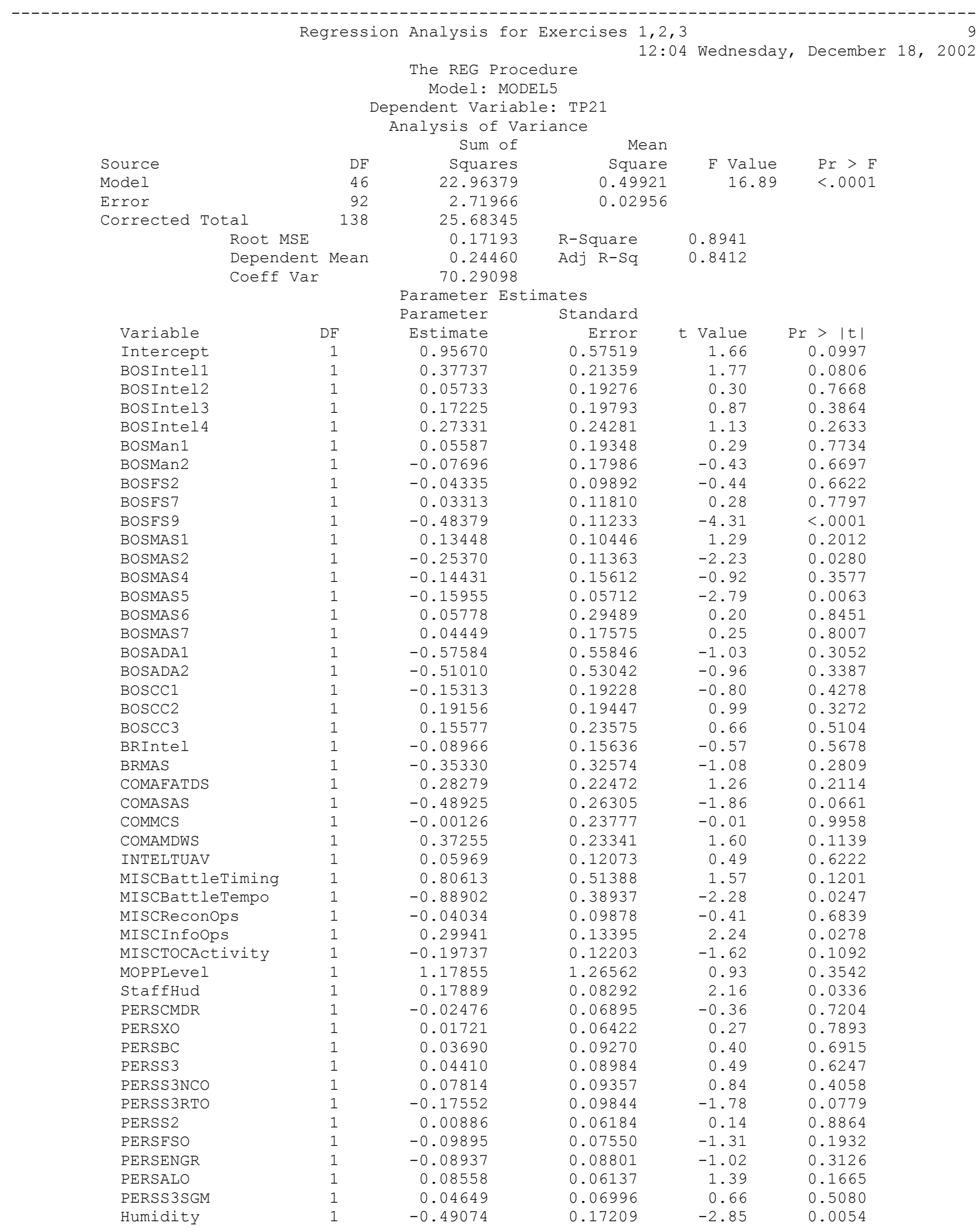




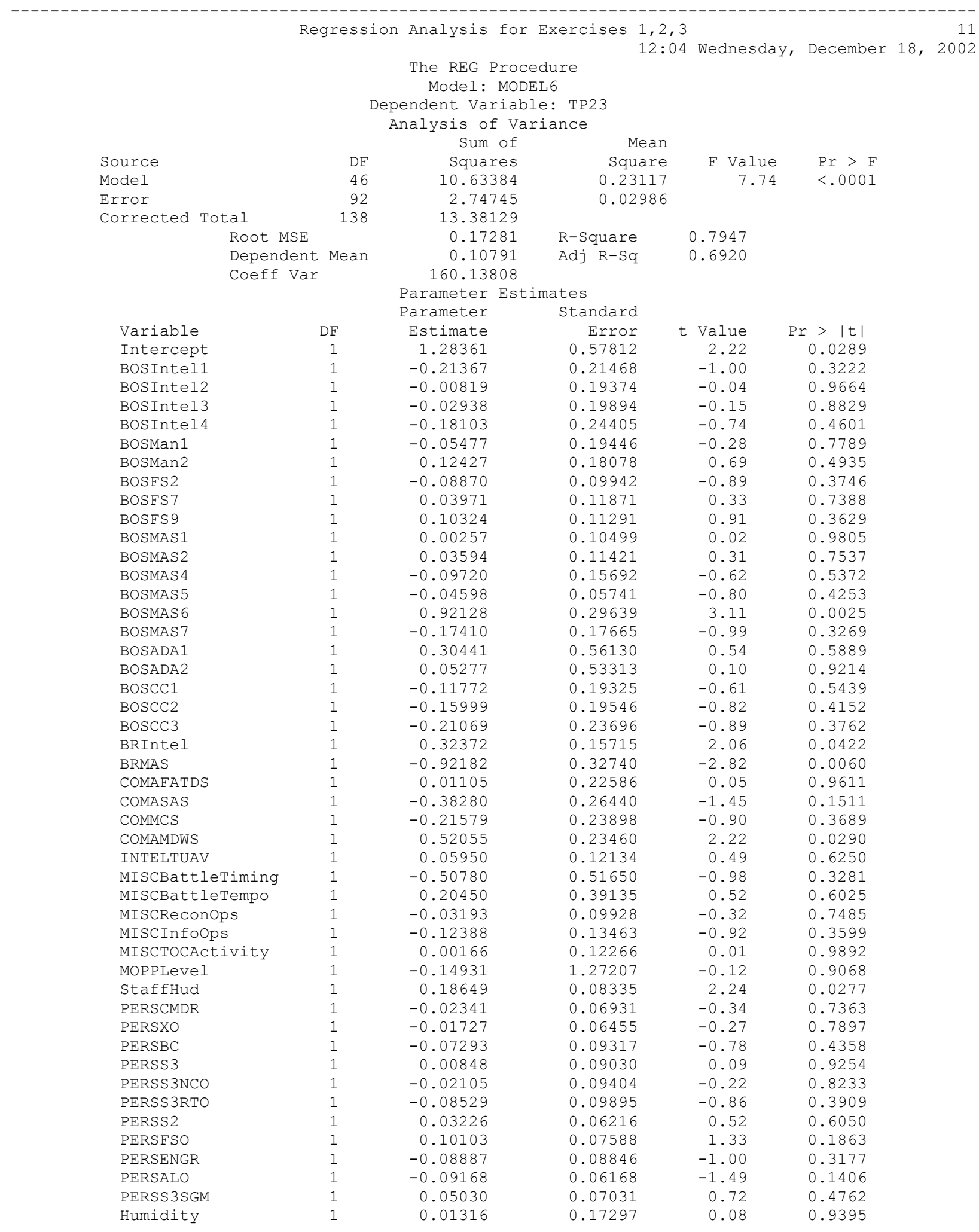




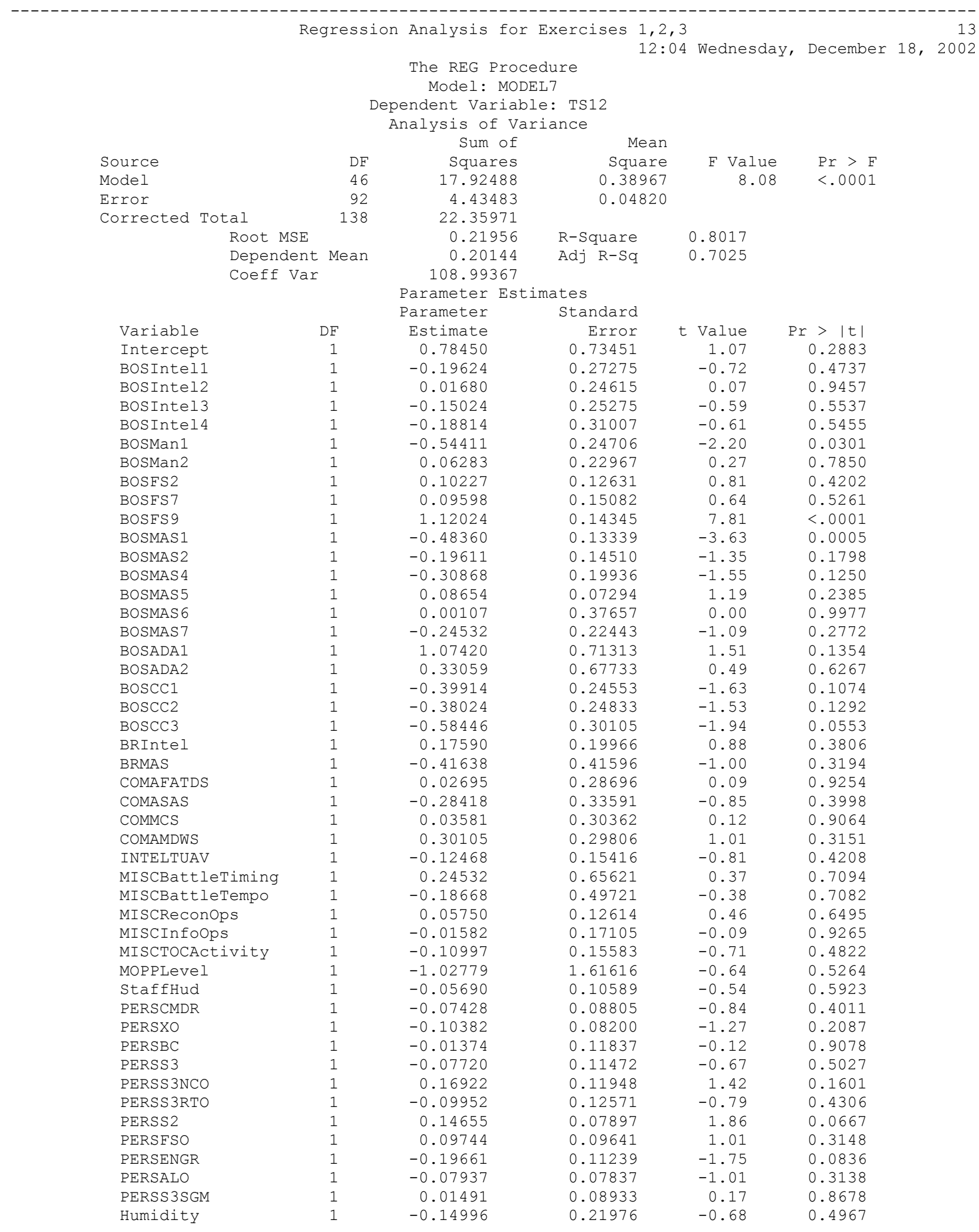




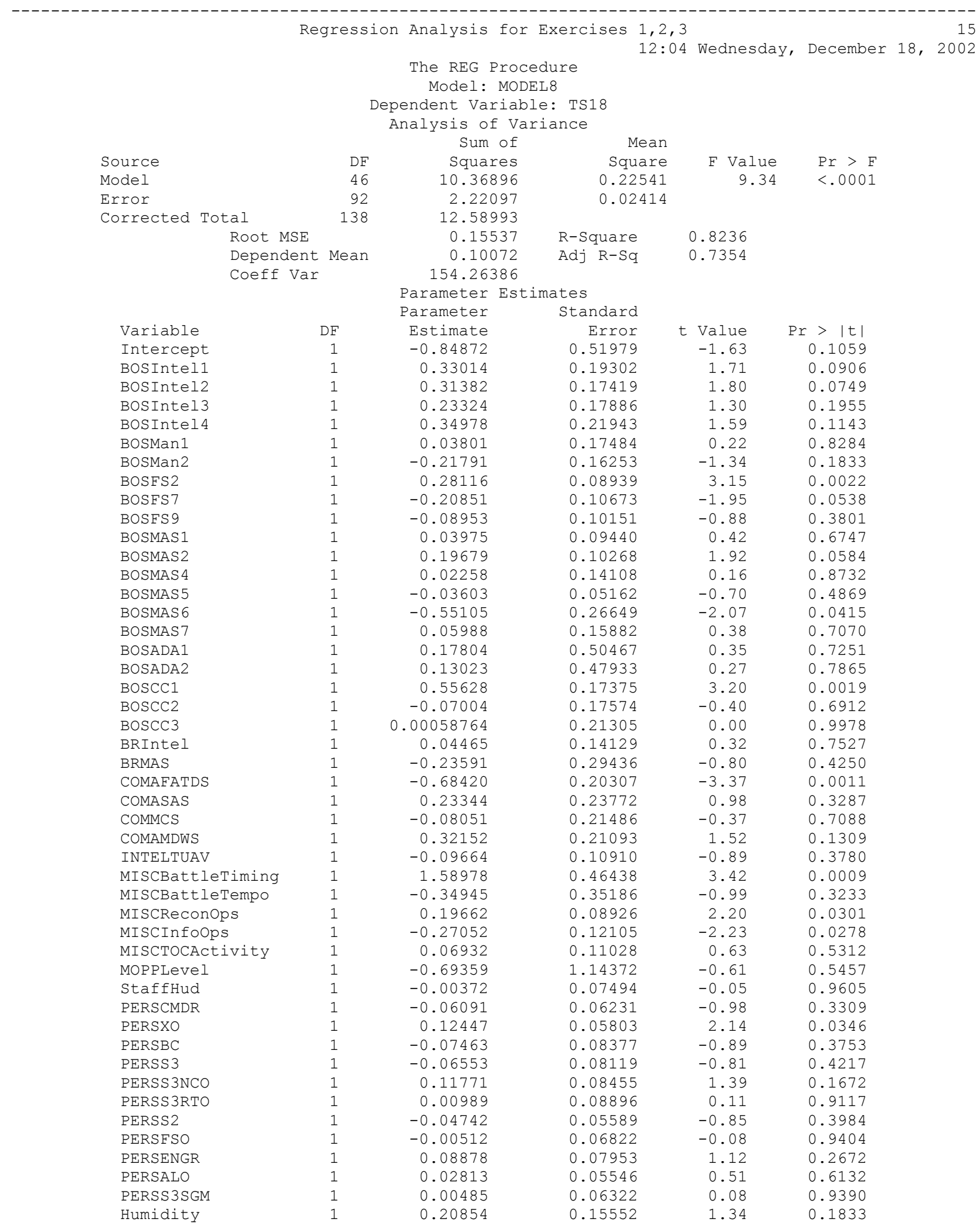




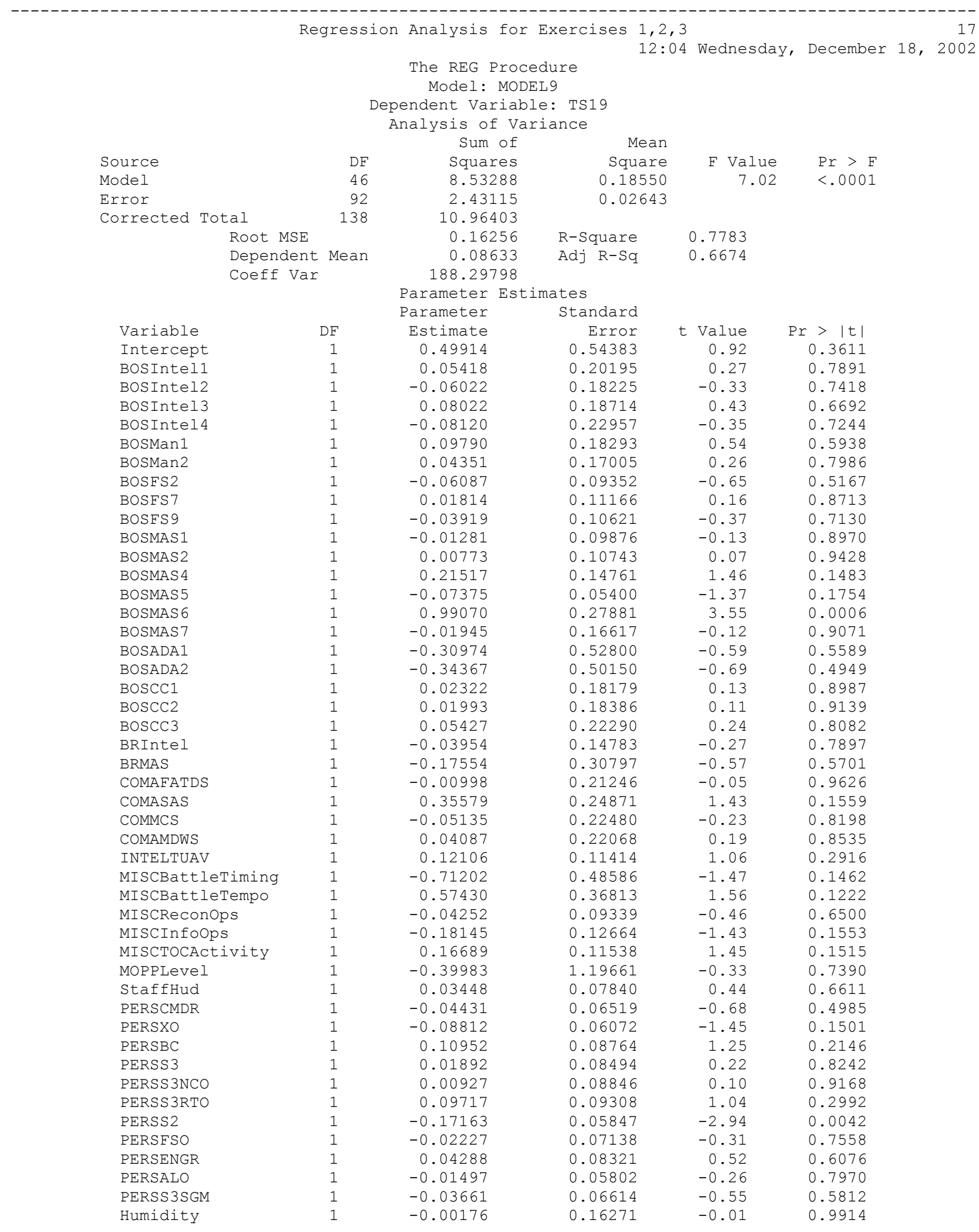




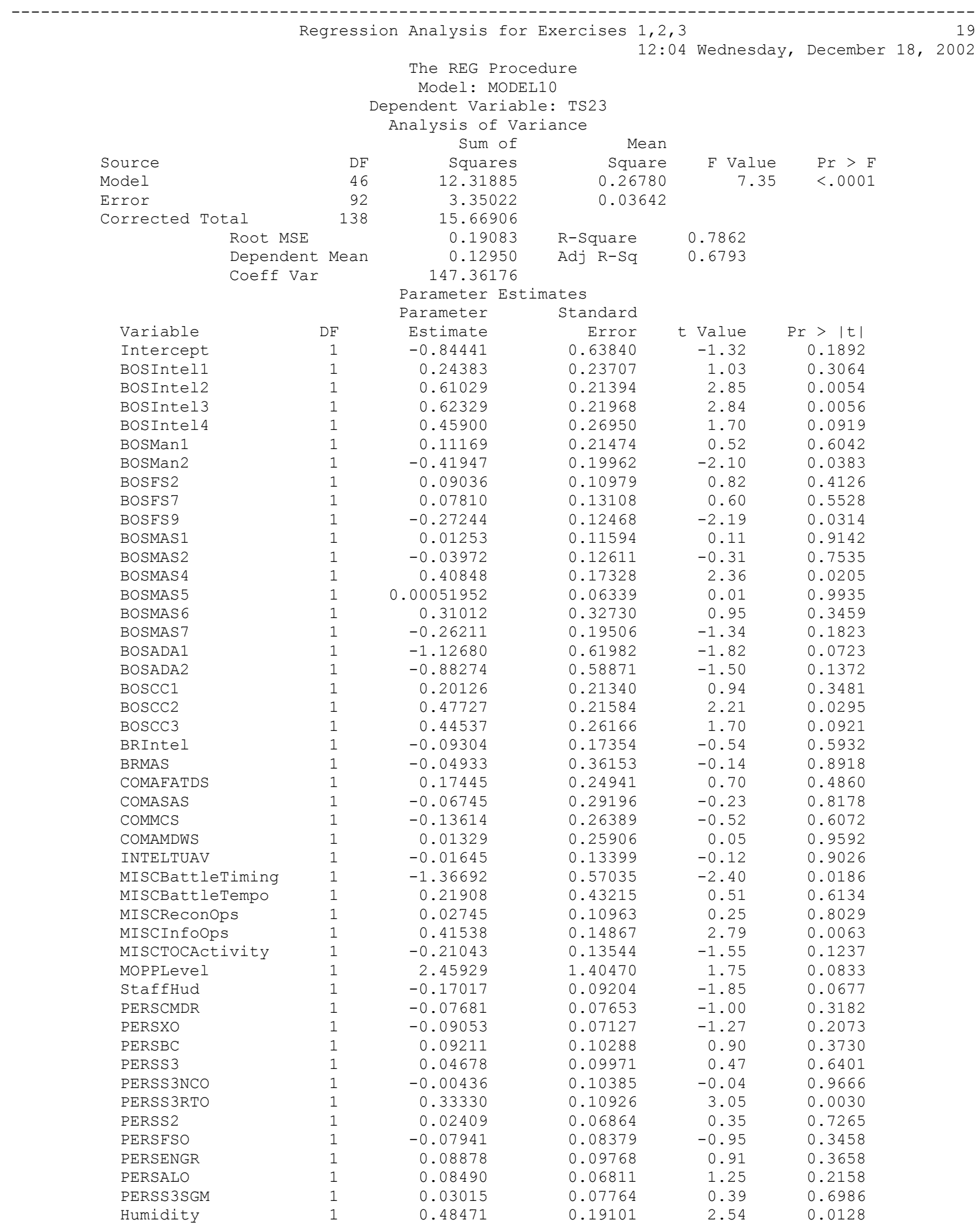




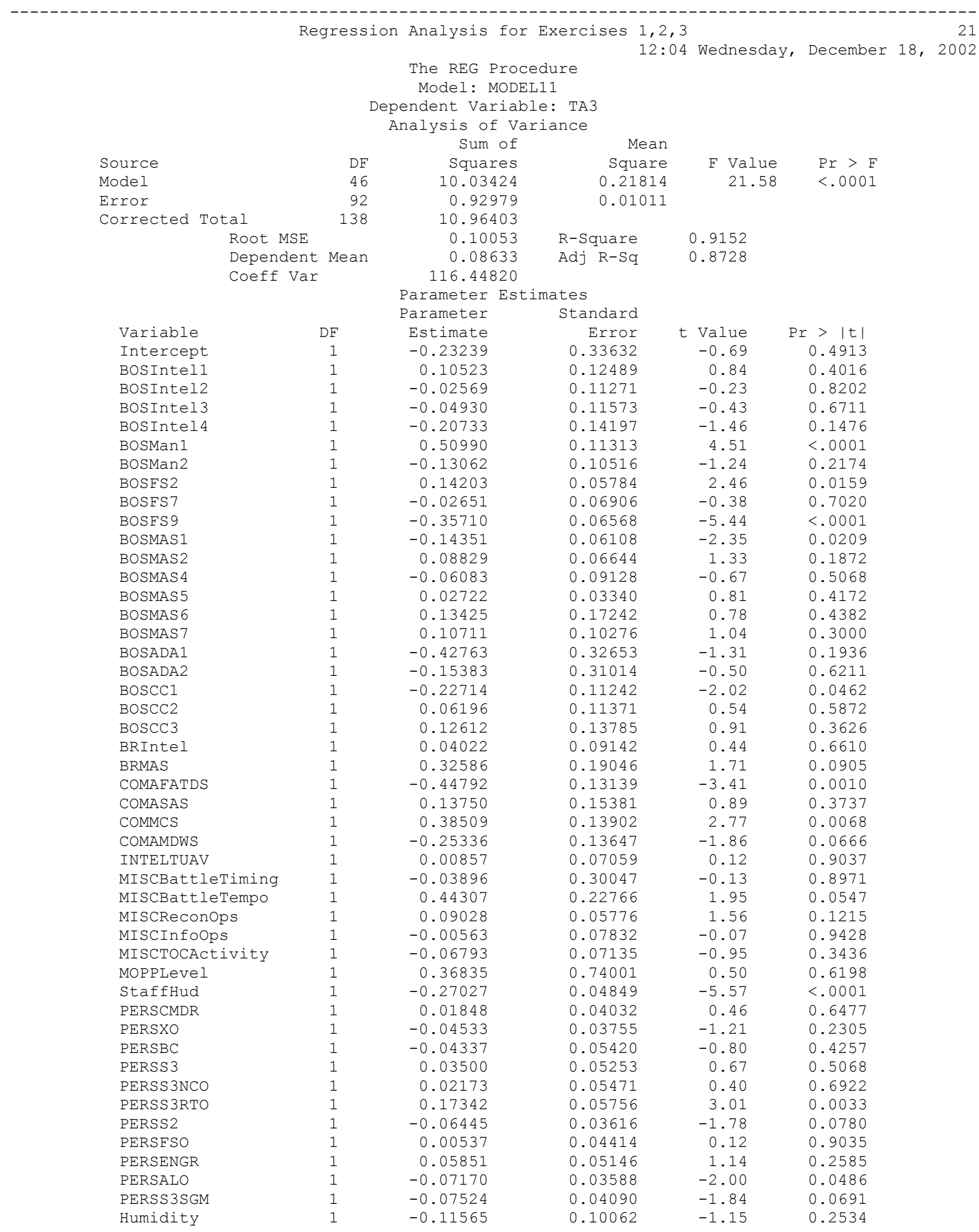




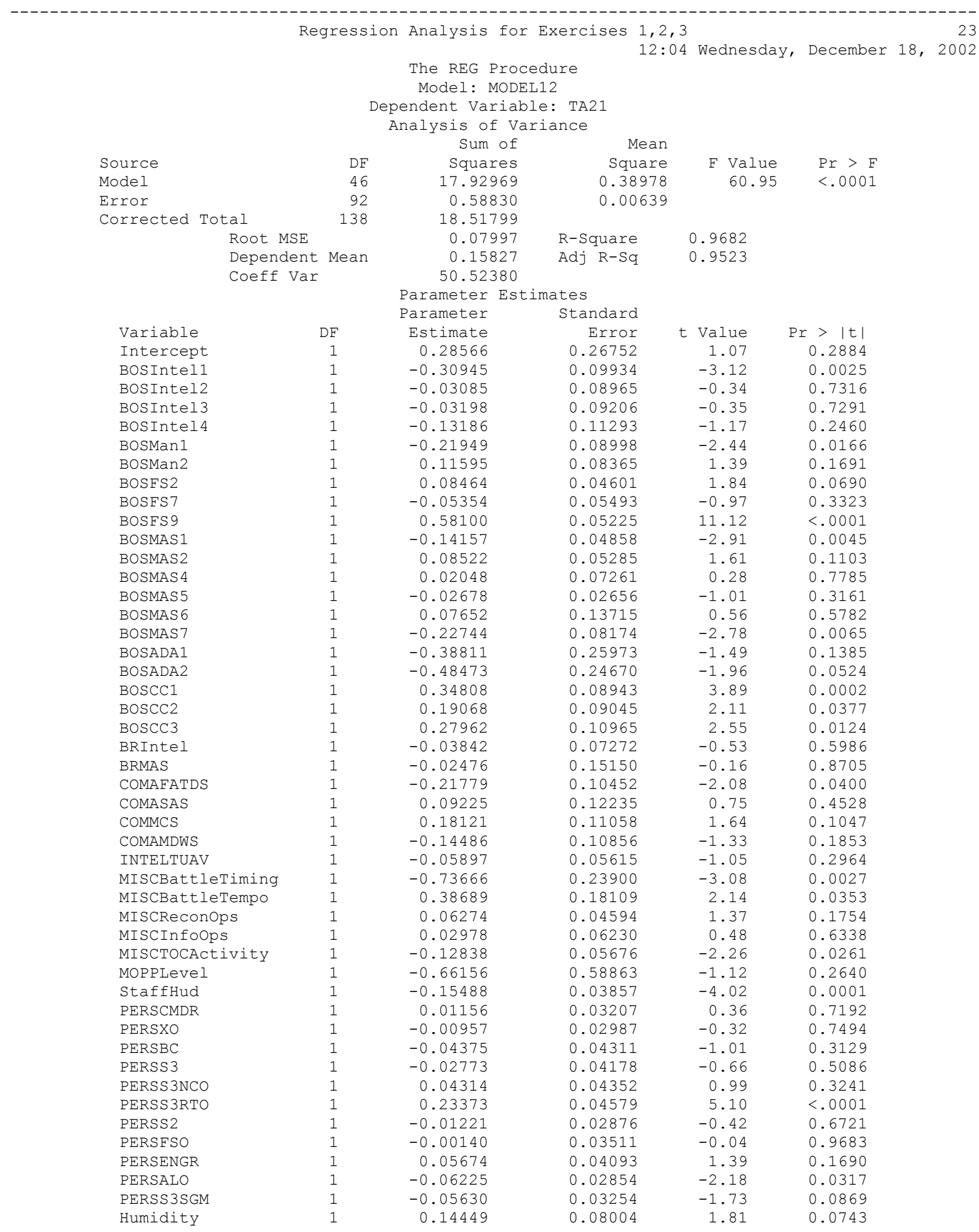




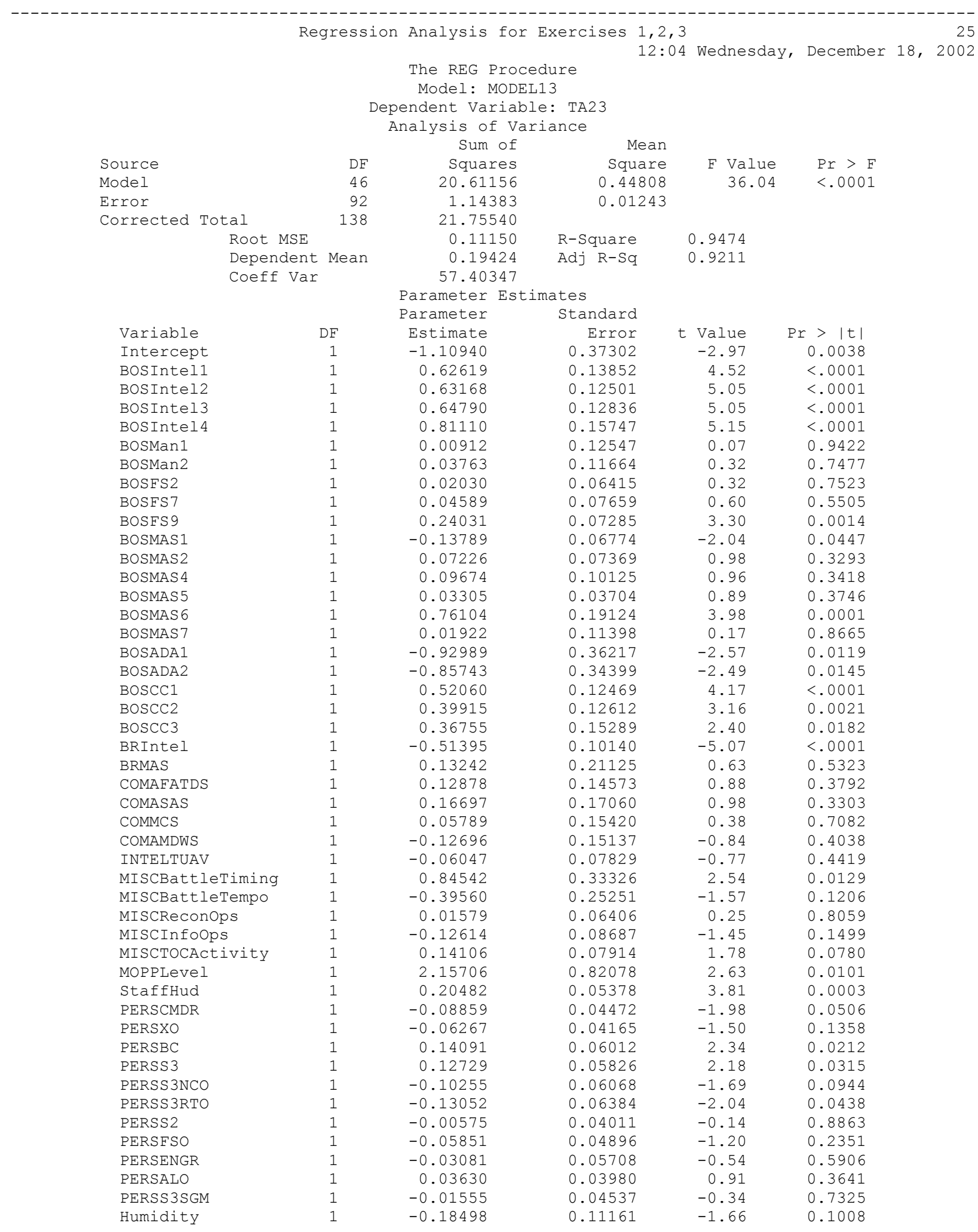




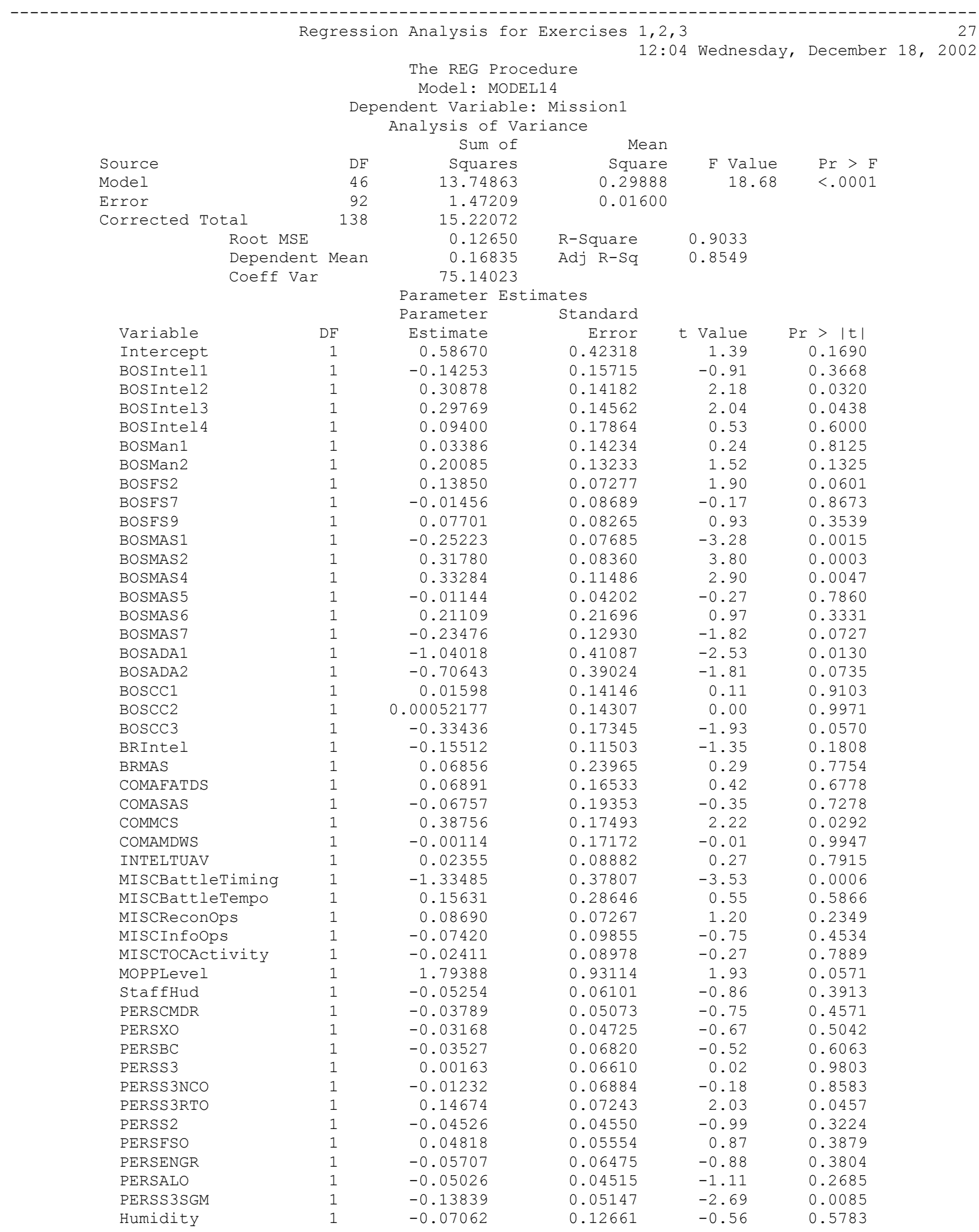




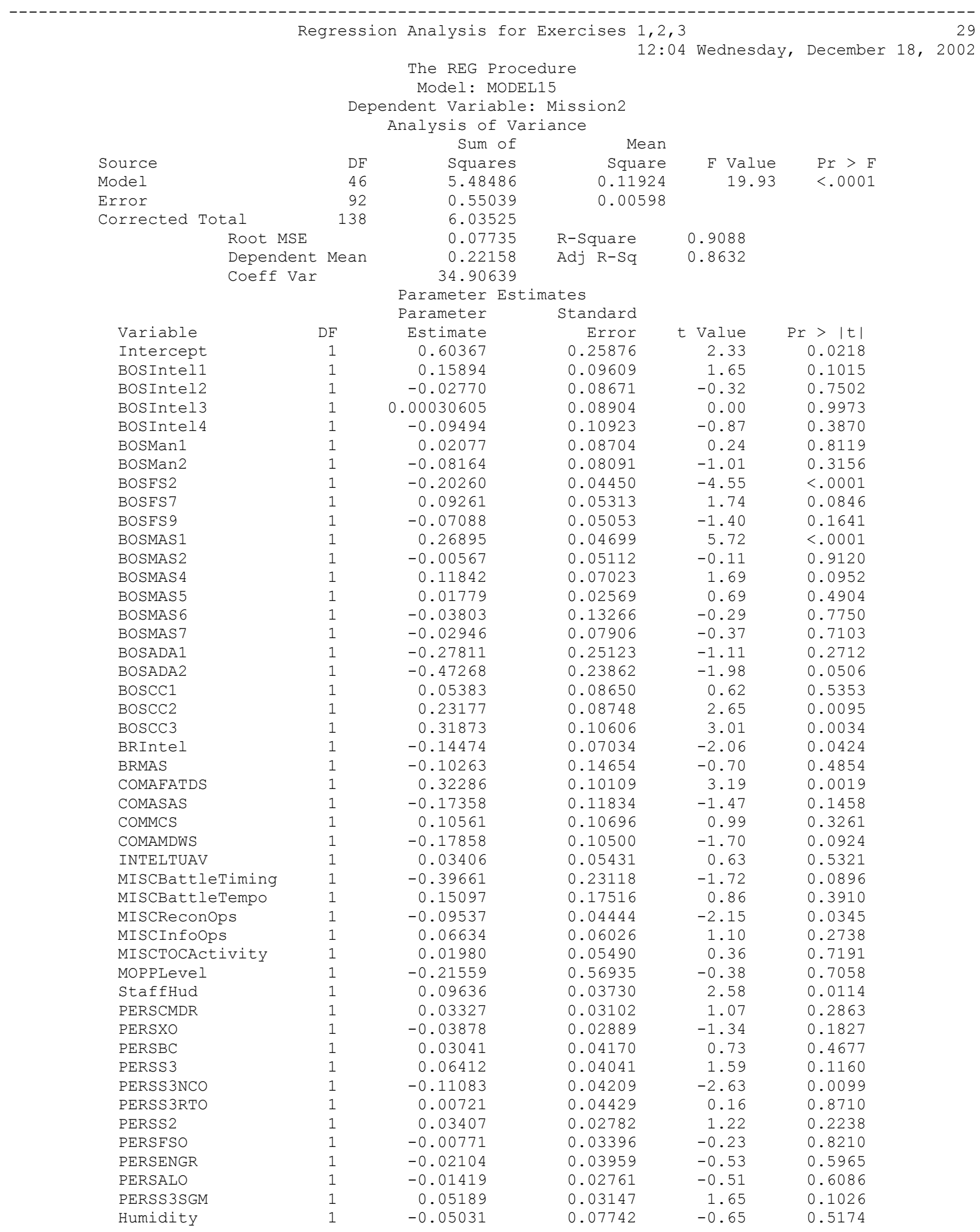




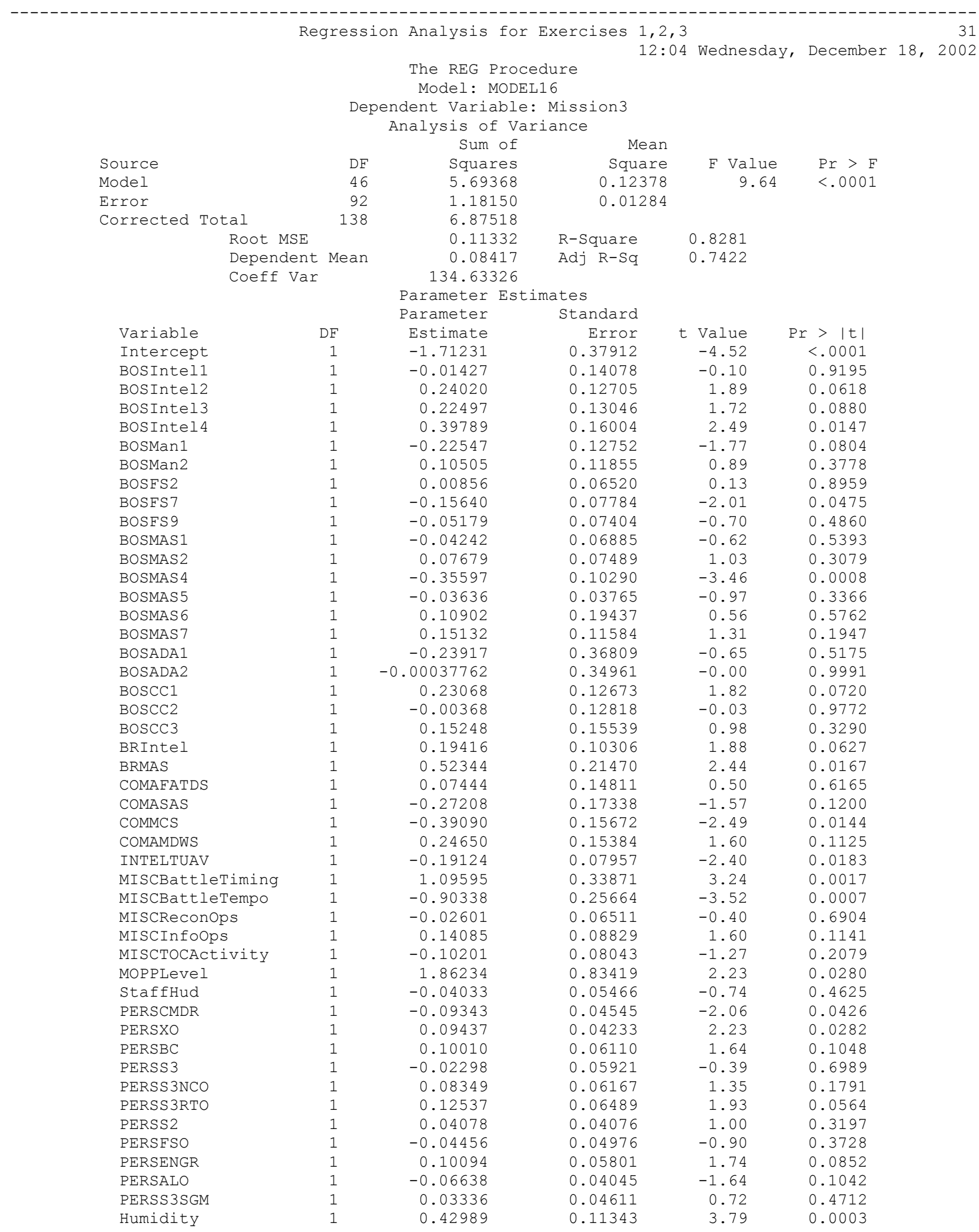




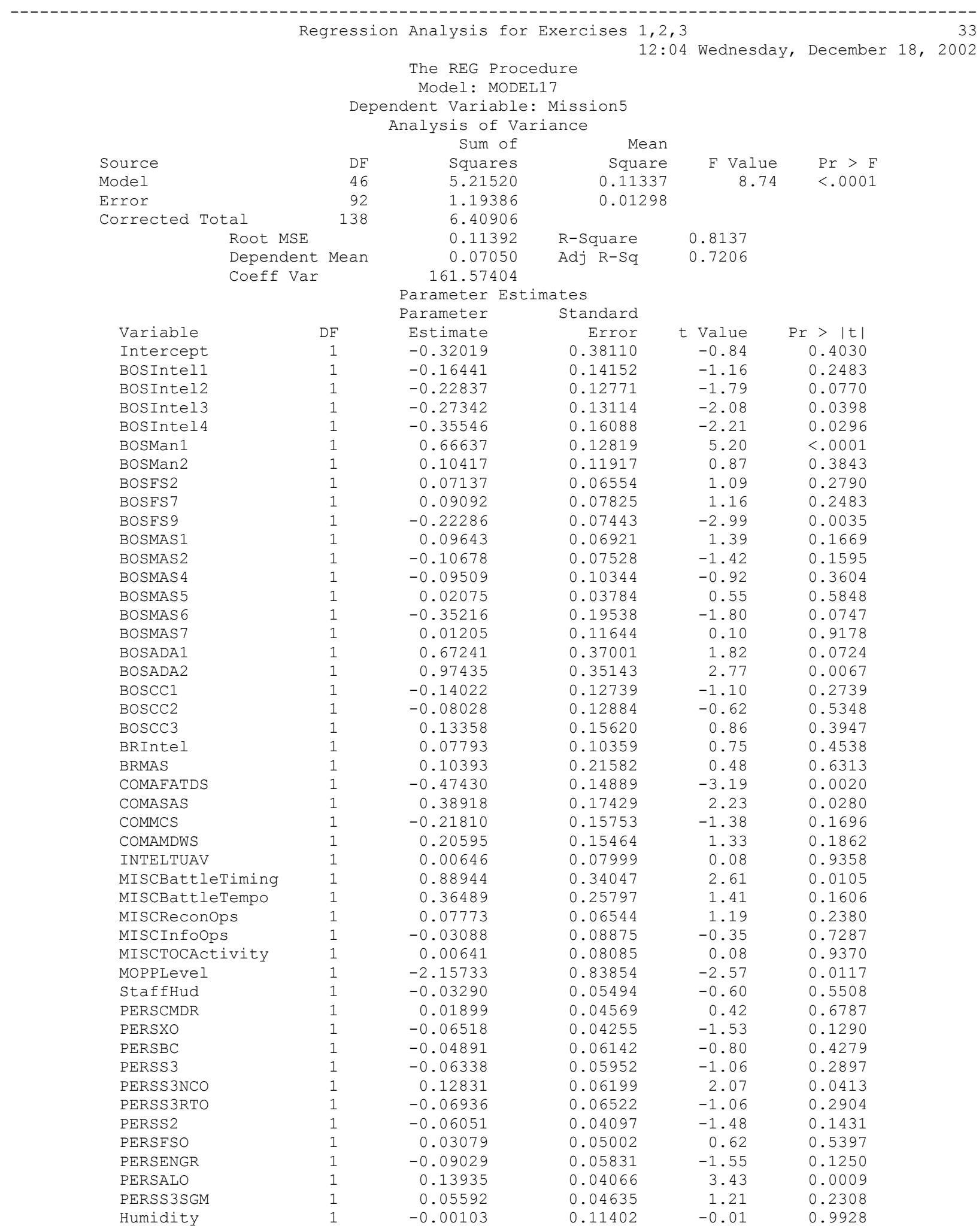




\title{
Appendix K - Second Linear Regression Run To Select IV For Inclusion In DV Models
}

\section{SAS Code (Pick those IV that had a significant $p$ value $(\alpha=.05)$ from the first run):}

\author{
options pageno=1 formdlim="-"; \\ TITLE "Training Data 123 Regression Analysis"; \\ DATA Tngdata123;
}

InPut Day1 Day2 Day3 Day4 Day5 Day6 Day7 Day8 Day9 Day10 Day11 Day12 Day13 Day14 Day15 Day16 Day17 Day18 Day19 Day20 Hour1 Hour2 Hour3 Hour4 Hour5 Hour6 Hour7 Hour8 Hour9 Hour10 Hour11 Hour12 Hour13 Hour14 Hour15 Hour16 Hour17 Hour18 Hour19 Hour20 Hour21 Hour22 Hour23 Hour24 TOCAn TOCDg TOCBn TOCBde ExTyp1 ExTyp2 ExTyp3 ExTyp4 ExNo1 ExNo2 ExNo3 ExNo4 ExNo5 ExNo6 ExNo7 ExNo8 ExNo9 ExNo10 ExNo11 ExNo12 ExNo13 ExNo14 ExNo15 ExNo16 ExNo17 ExNo18 ExNo19 ExNo20 SimNo SimJANS SimJAST SimCBS SimCCTT SimSTRM BOSIntel1 BOSIntel2 BOSIntel3 BOSIntel4 BOSMan1 BOSMan2 BOSFS1 BOSFS2 BOSFS 3 BOSFS 4 BOSFS 5 BOSFS 6 BOSFS 7 BOSFS 8 BOSFS 9 BOSMAS1 BOSMAS2 BOSMAS 3 BOSMAS4 BOSMAS5 BOSMAS 6 BOSMAS7 BOSADA1 BOSADA2 BOSCC1 BOSCC2 BOSCC3 BRIntel BRMan BRFS BRMAS BRADA BRCSS BRC2 COMAFATDS COMAMPS COMASAS COMCTIS COMCSSCS COMFAADC2 COMFBCB2 COMIMETS COMMCS COMAMDWS COMJSTARS COMEPLARS INTELTUAV INTELJSTARS INTELGSR MISCBattleTiming MISCBattleTempo MISCReconOps MISCInfoOps MISCTactics MISCTOCActivity MOPPLevel StaffHud PERSCMDR PERSXO PERSBC PERSS3 PERSS3NCO PERSS3RTO PERSS2 PERSFSO PERSENGR PERSALO PERSS3SGM SKYCLR SKYOC SKYRAIN WINDNONE WINDLOW WINDMOD WINDHIGH LightBG LightFG Noise TEMPDB TEMPWB Humidity TP1 TP2 TP3 TP4 TP5 TP6 TP7 TP8 TP9 TP10 TP11 TP12 TP13 TP14 TP15 TP16 TP17 TP18 TP19 TP20 TP21 TP22 TP23 TS1 TS2 TS3 TS4 TS5 TS6 TS7 TS8 TS9 TS10 TS11 TS12 TS13 TS14 TS15 TS16 TS17 TS18 TS19 TS20 TS21 TS22 TS23 TA1 TA2 TA3 TA4 TA5 TA6 TA7 TA8 TA9 TA10 TA11 TA12 TA13 TA14 TA15 TA16 TA17 TA18 TA19 TA20 TA21 TA22 TA23 Mission1 Mission2 Mission3 Mission4 Mission5 Mission6 ; CARDS;

$\begin{array}{lllllllllllllllllllllllllllllllllllllllllllllllll}0 & 0 & 0 & 1 & 0 & 0 & 0 & 0 & 0 & 0 & 0 & 0 & 0 & 0 & 0 & 0 & 0 & 0 & 0 & 0 & 0 & 0 & 0 & 0 & 0 & 0 & 0 & 0 & 0 & 0 & 0 & 0 & 1 & 0 & 0 & 0 & 0 & 0 & 0 & 0 & 0 & 0 & 0 & 0 & 0 & 1 & 0 & 1 & 0\end{array}$

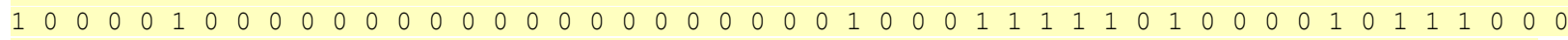
$\begin{array}{llllllllllllllllllllllllllllllllllllllllll}0 & 1 & 1 & 0 & 0 & 0 & 1 & 1 & 1 & 1 & 1 & 1 & 1 & 1 & 0.7 & 0.0 & 0.6 & 0.0 & 0.0 & 0.0 & 0.0 & 0.0 & 0.6 & 0.4 & 0.1 & 0.0 & 0.1 & 0.1 & 0.0 & 0.5 & 0.5\end{array}$ $\begin{array}{lllllllllllllllllllllllllllllllllllllllll}0.4 & 0.5 & 0.5 & 0.5 & 0.25 & 0 & 1 & 0 & 1 & 1 & 1 & 1 & 1 & 1 & 1 & 0 & 0 & 1 & 0 & 0 & 1 & 0 & 0 & 0 & 0.03 & 0.30 & 0.77 & 0.53 & 0.67 & 0.39 & 0 & 0 & 0 & 0\end{array}$ $\begin{array}{llllllllllllllllllllllllllllllllllllllllllllllllll}0 & 0 & 0 & 0 & 0 & 1 & 1 & 1 & 0 & 0 & 0 & 0 & 0 & 0 & 0 & 0 & 0 & 0 & 0 & 0 & 0 & 0 & 0 & 0 & 0 & 0 & 0 & 0 & 0 & 0 & 0 & 0 & 0 & 1 & 0 & 0 & 0 & 0 & 0 & 0 & 0 & 0 & 0 & 0 & 0 & 0 & 0 & 0 & 0\end{array}$ $\begin{array}{lllllllllllllllllllllllllllllll}0 & 0 & 0 & 0 & 0 & 0 & 0 & 0 & 0 & 0 & 0 & 0 & 0 & 0 & 0 & 1 & 0.0 & 0.5 & 0.5 & 0.0 & 0.0 & 0.0\end{array}$

...

$\begin{array}{llllllllllllllllllllllllllllllllllllllllllllllllll}0 & 0 & 0 & 0 & 0 & 0 & 1 & 0 & 0 & 0 & 0 & 0 & 0 & 0 & 0 & 0 & 0 & 0 & 0 & 0 & 0 & 0 & 0 & 0 & 0 & 0 & 0 & 0 & 0 & 0 & 0 & 1 & 0 & 0 & 0 & 0 & 0 & 0 & 0 & 0 & 0 & 0 & 0 & 0 & 0 & 1 & 0 & 1 & 1\end{array}$ $\begin{array}{lllllllllllllllllllllllllllllllllllllllllllllllll}0 & 0 & 0 & 1 & 0 & 0 & 0 & 0 & 0 & 0 & 0 & 0 & 0 & 0 & 0 & 0 & 0 & 0 & 0 & 0 & 0 & 0 & 0 & 0 & 1 & 0 & 0 & 0 & 0 & 0 & 1 & 0 & 0 & 0 & 0 & 0 & 0 & 0 & 0 & 0 & 0 & 0 & 0 & 1 & 0 & 0 & 0 & 0 & 1\end{array}$ $\begin{array}{llllllllllllllllllllllllllllllllllllllllll}0 & 0 & 0 & 0 & 0 & 0 & 1 & 1 & 1 & 1 & 1 & 1 & 1 & 1 & 0.3 & 0.0 & 0.3 & 0.0 & 0.0 & 0.0 & 0.0 & 0.0 & 0.2 & 0.2 & 0.2 & 0.0 & 0.1 & 0.1 & 0.3 & 0.5 & 0.2\end{array}$ $\begin{array}{llllllllllllllllllllllllllllllllllllllllll}0.4 & 0.3 & 0.5 & 0.2 & 0.00 & 0 & 0 & 0 & 0 & 0 & 0 & 1 & 1 & 0 & 1 & 1 & 0 & 1 & 0 & 0 & 1 & 0 & 0 & 0 & 0.04 & 0.16 & 0.66 & 0.90 & 0.75 & 0.50 & 0 & 0 & 0 & 0\end{array}$ $\begin{array}{llllllllllllllllllllllllllllllllllllllllllllllllll}0 & 0 & 0 & 0 & 0 & 0 & 1 & 0 & 0 & 0 & 0 & 0 & 0 & 0 & 0 & 0 & 0 & 0 & 0 & 0 & 0 & 0 & 0 & 0 & 0 & 0 & 0 & 0 & 0 & 0 & 1 & 0 & 0 & 0 & 0 & 0 & 0 & 0 & 0 & 0 & 0 & 0 & 0 & 0 & 0 & 0 & 0 & 0 & 0\end{array}$ $\begin{array}{llllllllllllllllllllllllllllll}0 & 0 & 0 & 0 & 0 & 0 & 0 & 0 & 0 & 0 & 0 & 0 & 0 & 1 & 0 & 0 & 0.0 & 0.4 & 0.0 & 0.0 & 0.0 & 0.0\end{array}$

* Make regression run with input/independent variables remaining that are significant after full regression run

* to try to maximize the adjusted $r * * 2$ showing the amount of the regression accounted for; options pageno=1 formdlim="-";

proc reg data=Tngdata123;

TITLE 'second level Regression Analysis for Exercises 1,2,3";

model TP10=BOSIntel3 BOSIntel 4 BOSMAS2 BOSMAS6 BOSADA1 BOSADA2 BOSCC1 BOSCC2 BOSCC3 MOPPLevel; model TP11=BOSIntel3 BOSFS2 BOSFS9 BOSMAS1 BOSMAS2 BOSCC1 BOSCC2 BOSCC3 BRIntel StaffHud PERSXO PERSS3RTO PERSS3SGM; model TP12=BOSFS2 BOSFS7 BOSFS9 BOSMAS1 BOSMAS4 BOSMAS6 BOSCC1 COMAFATDS COMASAS COMAMDWS MISCBattleTiming MISCBattleTempo MISCReconOps PERSXO PERSBC PERSALO Humidity;

model TP15=BOSIntel2 BOSIntel3 BOSIntel4 BOSMan1 BOSFS2 BOSMAS2 COMAFATDS MISCBattleTiming MISCReCONOPS PERSXO;

model TP21=BOSFS9 BOSMAS5 MISCBattleTempo MISCInfoOps StaffHud Humidity;

model TP23=BOSMAS6 BRIntel BRMAS COMAMDWS StaffHud;

model TS12=BOSMan1 BOSFS9 BOSMAS1;

model TS18=BOSFS2 BOSMAS6 BOSCC1 COMAFATDS MISCBattleTiming MISCReconOps MISCInfoOps PERSXO ; model TS19=BOSMAS 6 PERSS2;

model TS23=BOSIntel2 BOSIntel3 BOSMan2 BOSFS9 BOSMAS4 BOSCC2 MISCBattleTiming MISCInfoOps

PERSS3RTO Humidity;

model TA3=BOSMan1 BOSFS2 BOSFS9 BOSMAS1 BOSCC1 COMAFATDS COMMCS StaffHud PERSS3RTO PERSALO; model TA21=BOSIntel1 BOSMan1 BOSFS9 BOSMAS1 BOSMAS7 BOSCC1 BOSCC3 COMAFATDS MISCBattleTiming MISCBatt leTempo 
MISCTOCActivity staffHud PERSS3RTO PERSALO;

model TA23=BOSIntel1 BOSIntel2 BOSIntel3 BOSIntel4 BOSFS9 BOSMAS1 BOSMAS6 BOSADA1 BOSADA2 BOSCC1 BOSCC2 BOSCC3

BRIntel BRMAS MISCBattleTiming MOPPLevel StaffHud PERSBC PERSS3 PERSS3RTO Humidity; model Mission1=BOSIntel2 BOSIntel3 BOSMAS1 BOSMAS2 BOSMAS4 BOSADA1 COMMCS MISCBattleTiming PERSS3RTO PERSS3SGM ;

model Mission2=BOSFS2 BOSMAS1 BOSCC2 BOSCC3 BRIntel COMAFATDS MISCReconops StaffHud PERSS3NCO ; model Mission3=BOSIntel4 BOSFS7 BOSMAS4 BRMAS COMMCS INTELTUAV MISCBattleTiming MISCBattleTempo MOPPLevel PERSCMDR PERSXO Humidity;

model Mission5=BOSIntel3 BOSIntel4 BOSMan1 BOSFS9 BOSADA2 COMAFATDS COMASAS MISCBattleTiming $\mathrm{MOP}$ 
SAS Output:

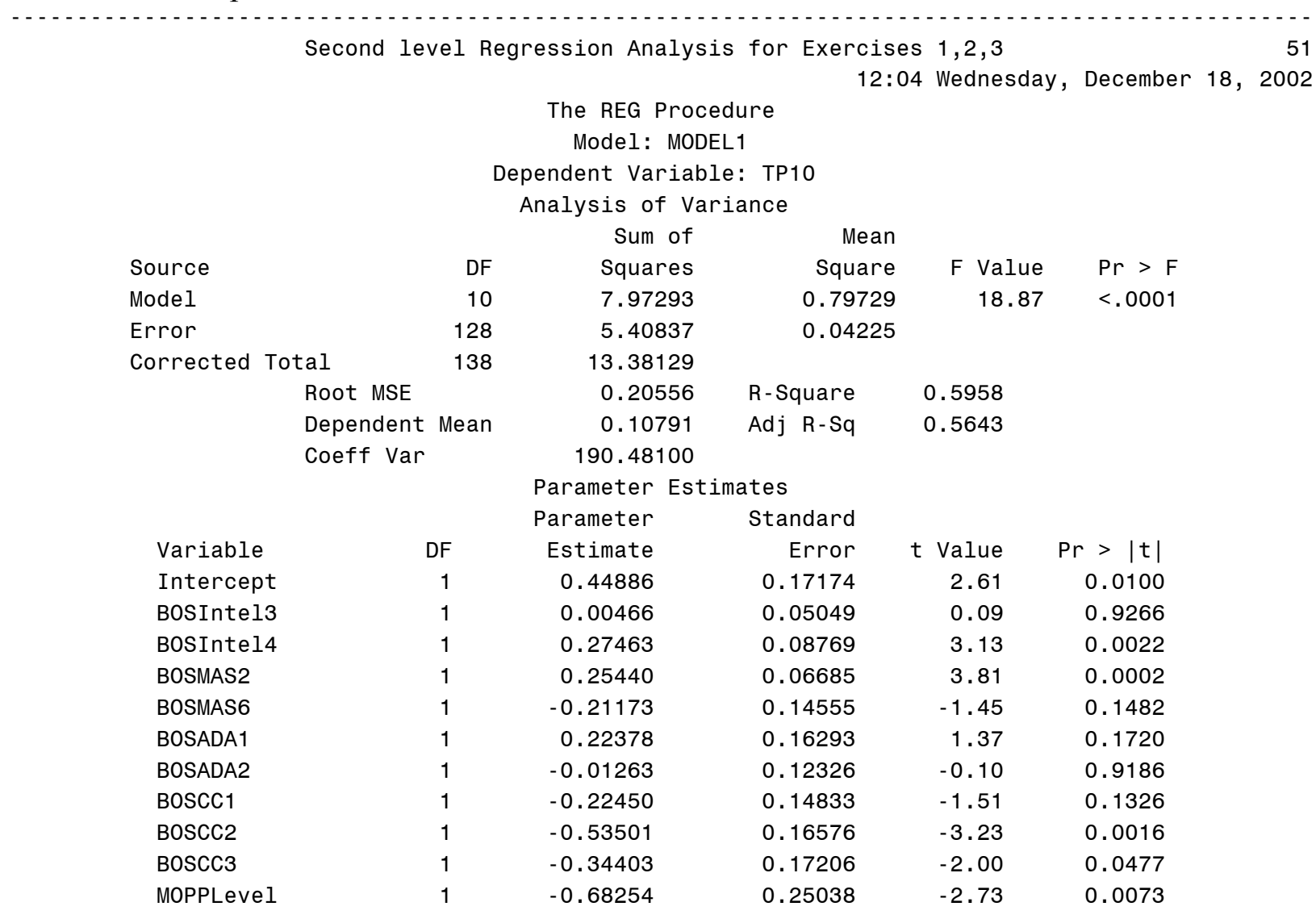


Second level Regression Analysis for Exercises 1,2,3

12:04 Wednesday, December 18, 2002

The REG Procedure

Model: MODEL2

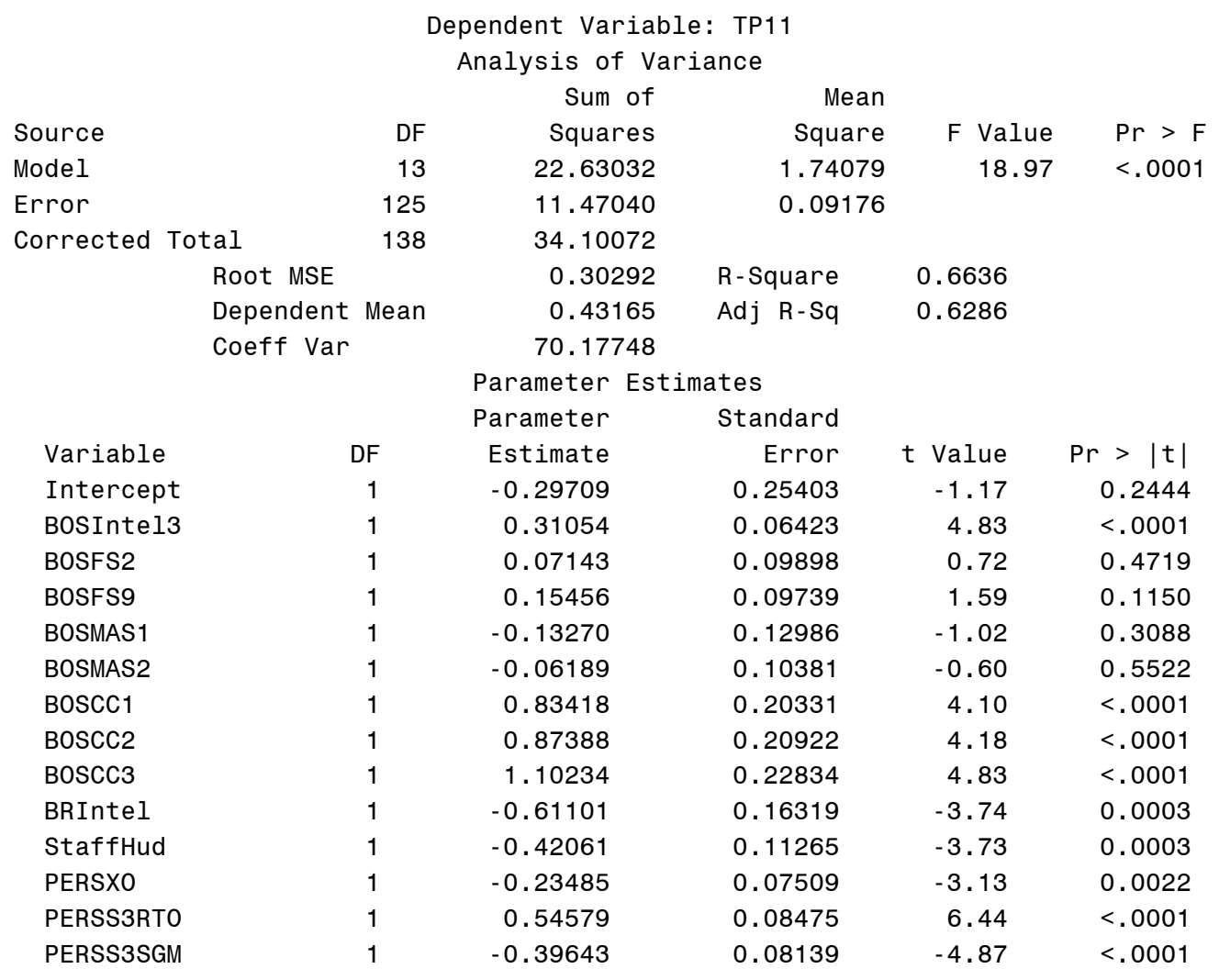




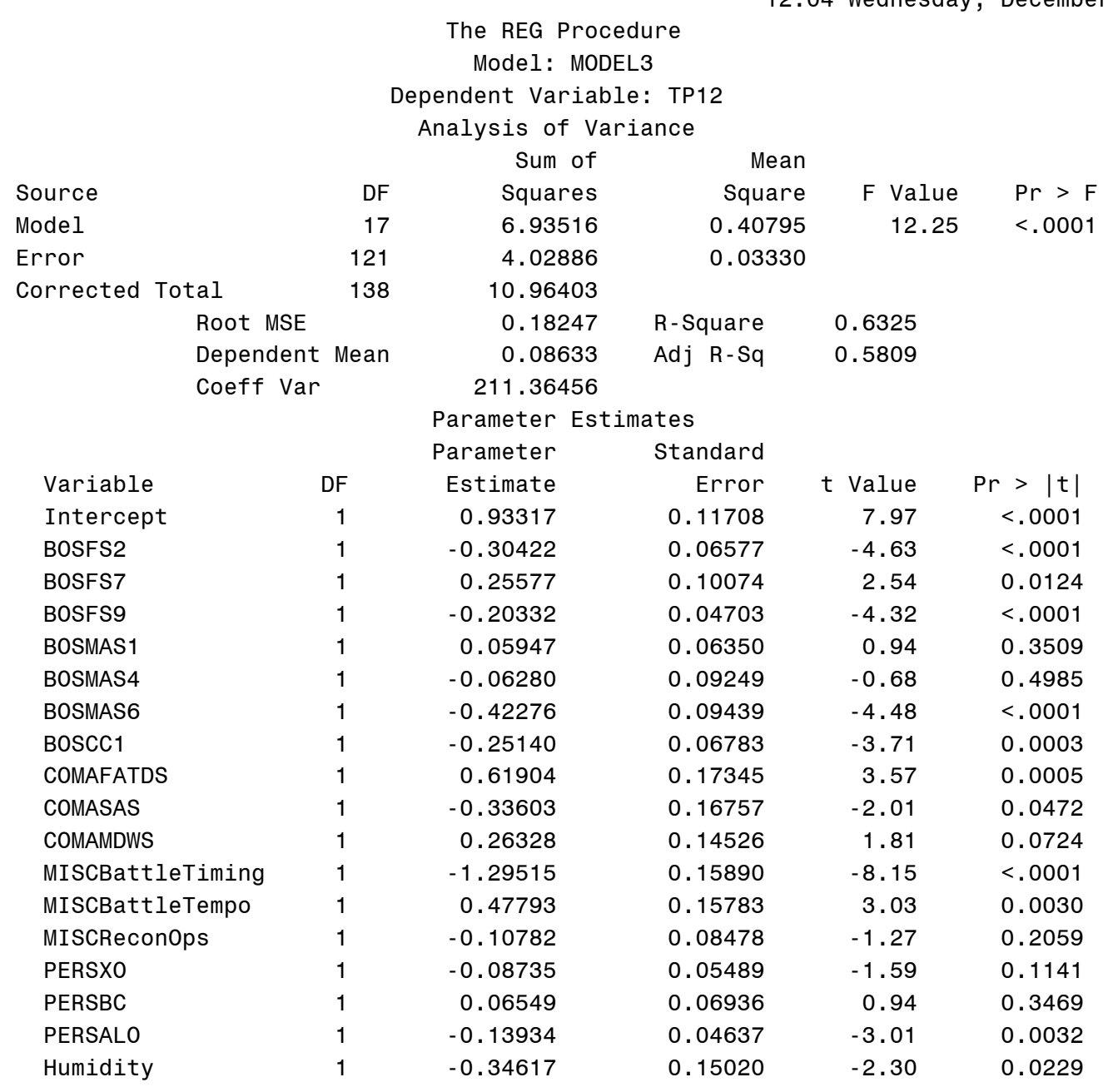


Second level Regression Analysis for Exercises 1,2,3

12:04 Wednesday, December 18, 2002

The REG Procedure

Model: MODEL4

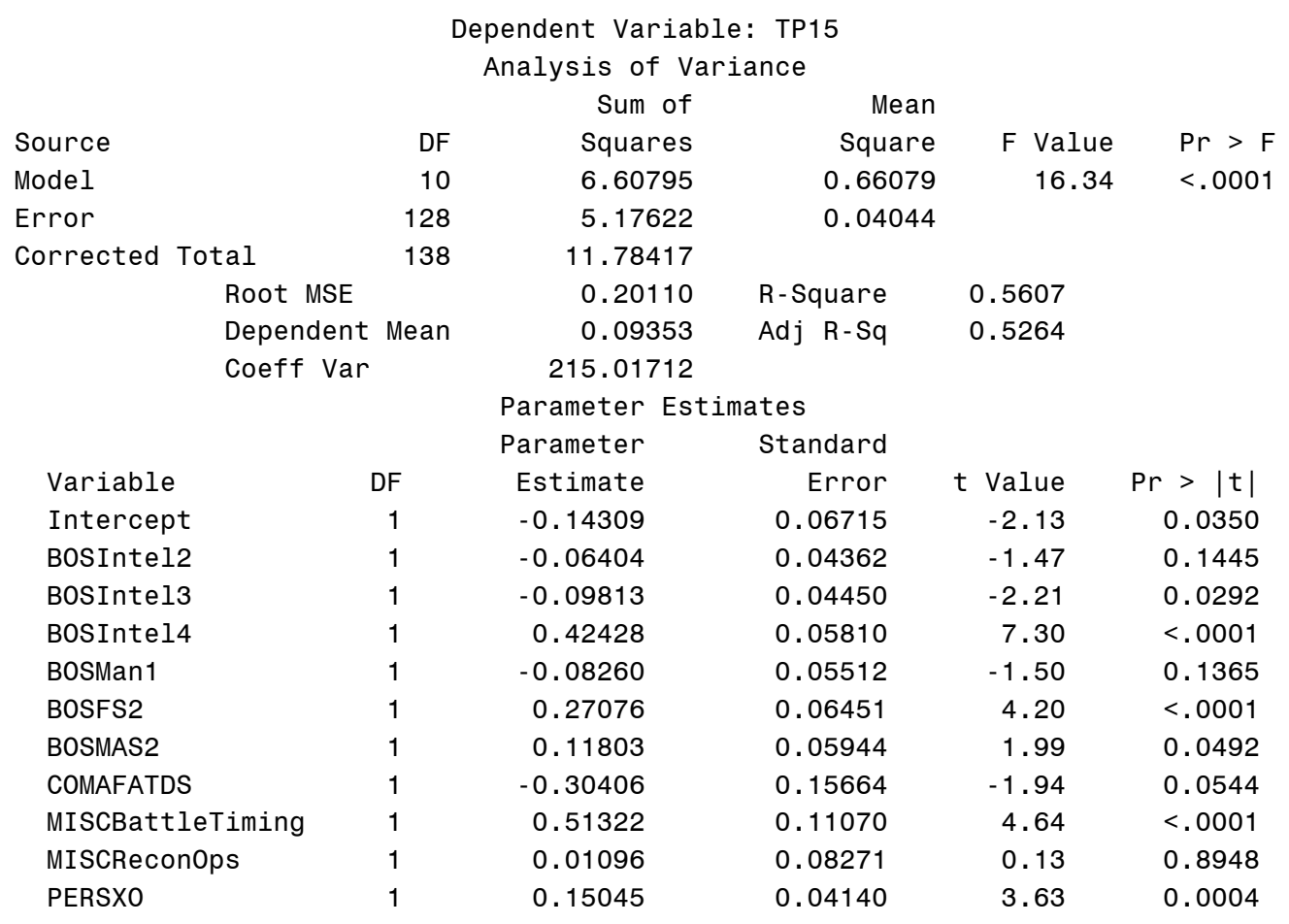




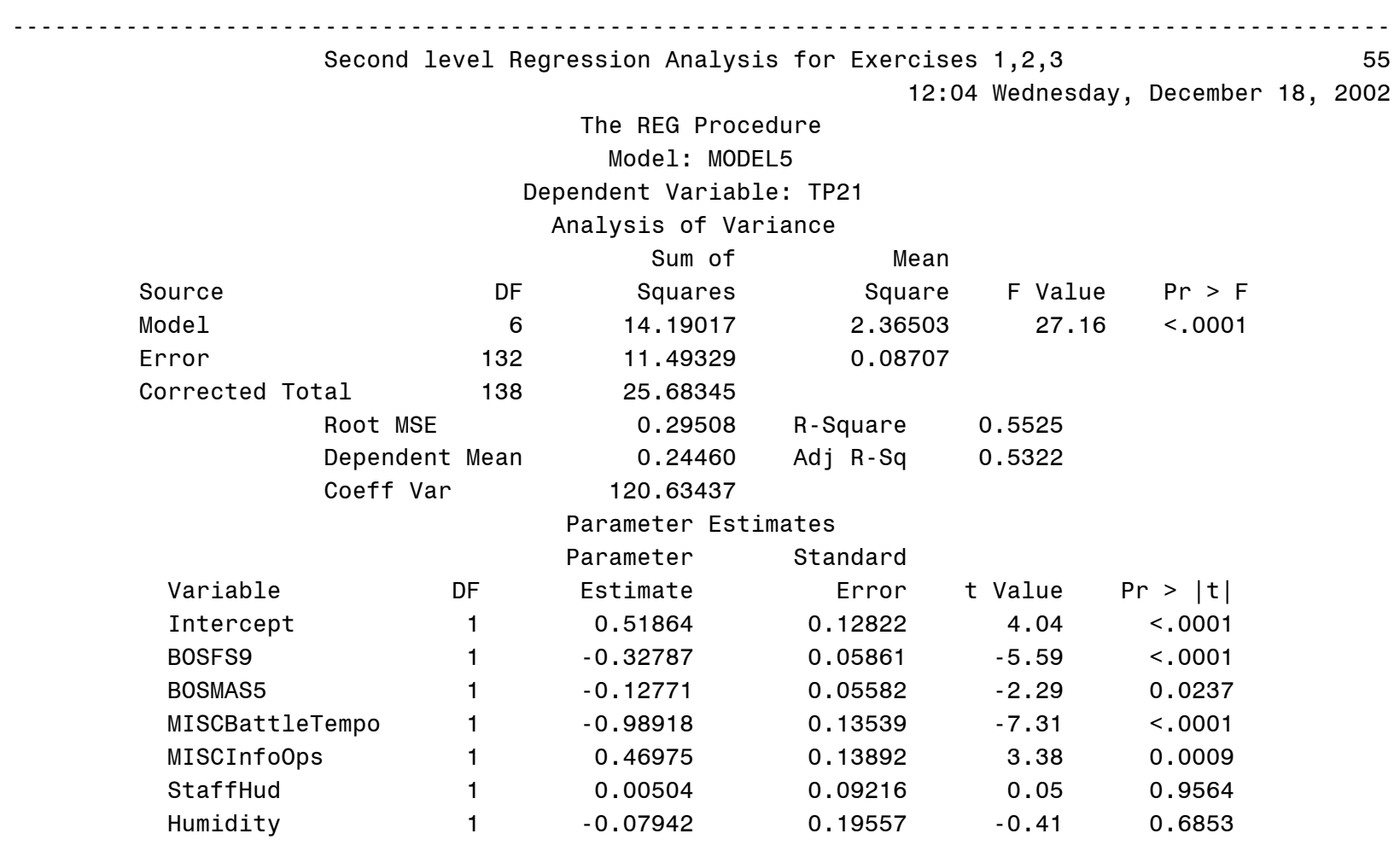




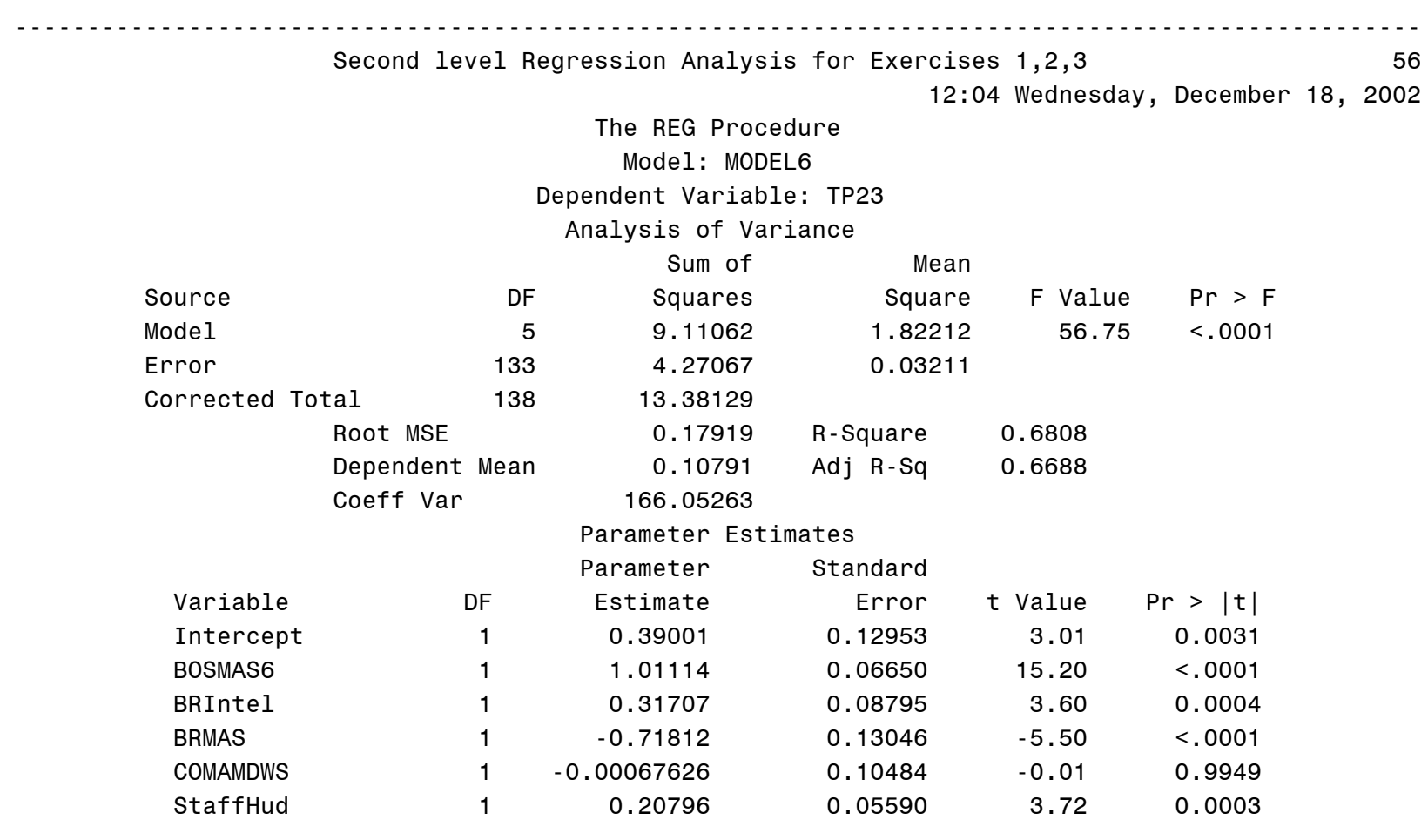




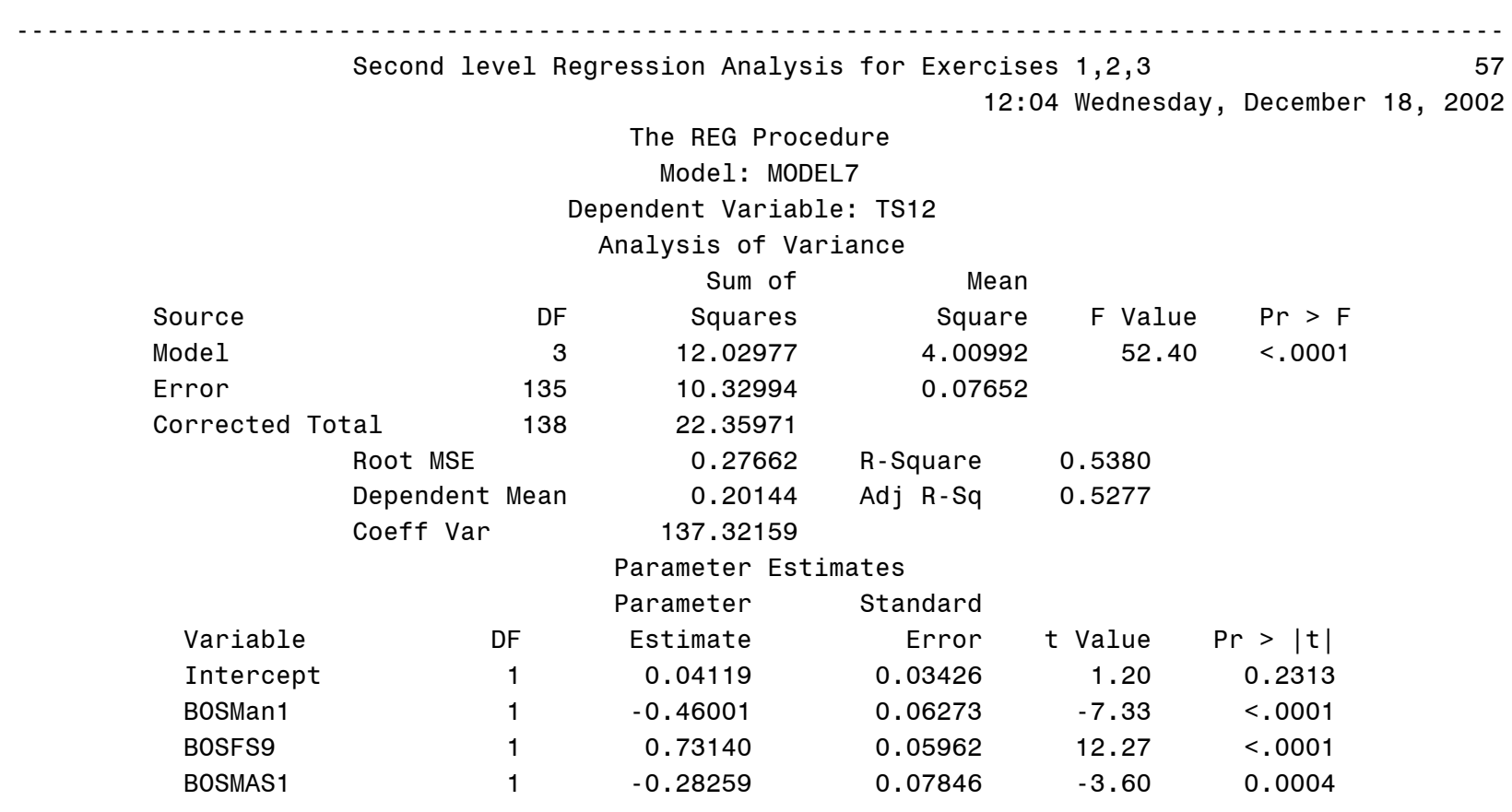




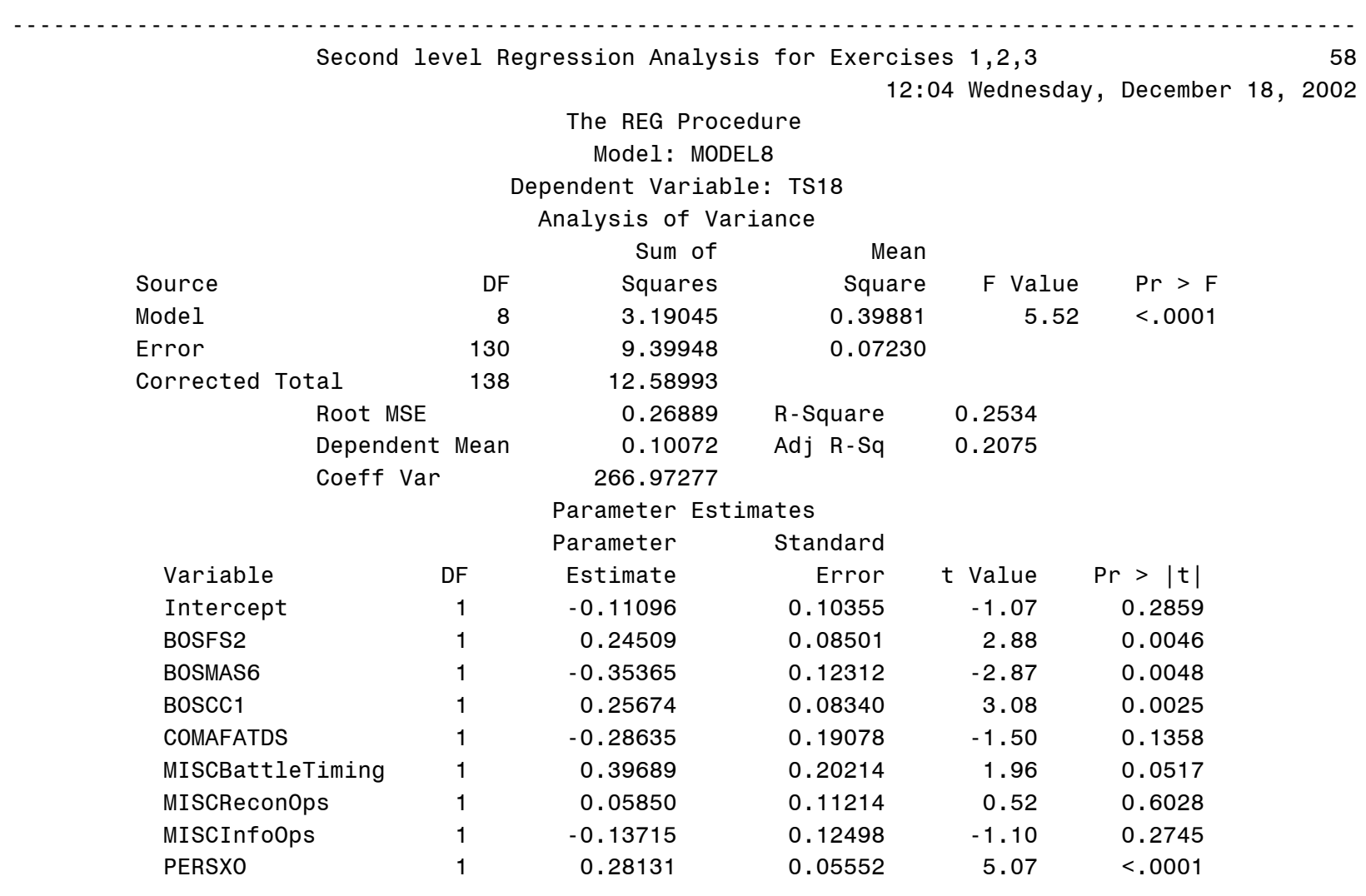




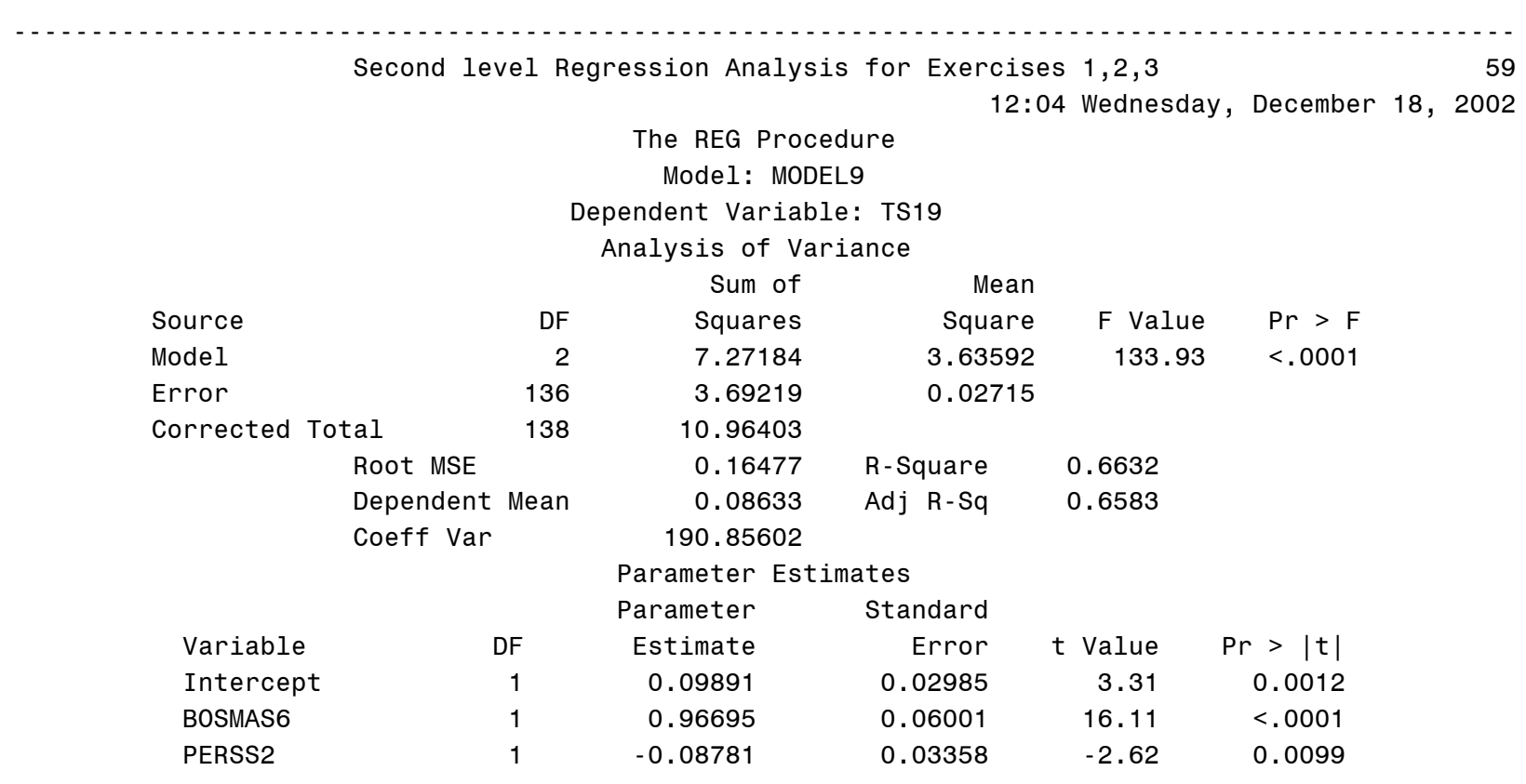




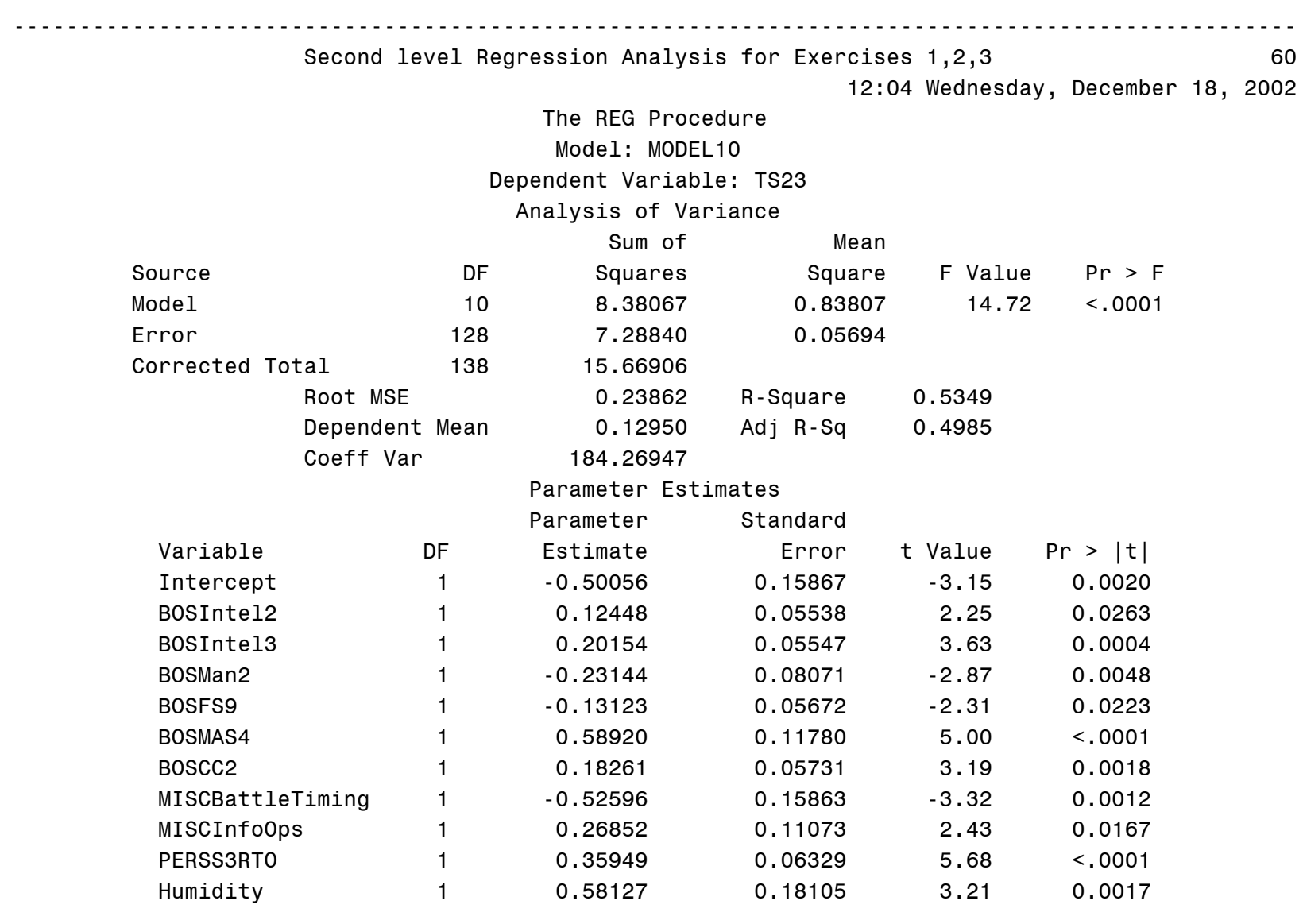


Second level Regression Analysis for Exercises 1,2,3

12:04 Wednesday, December 18, 2002

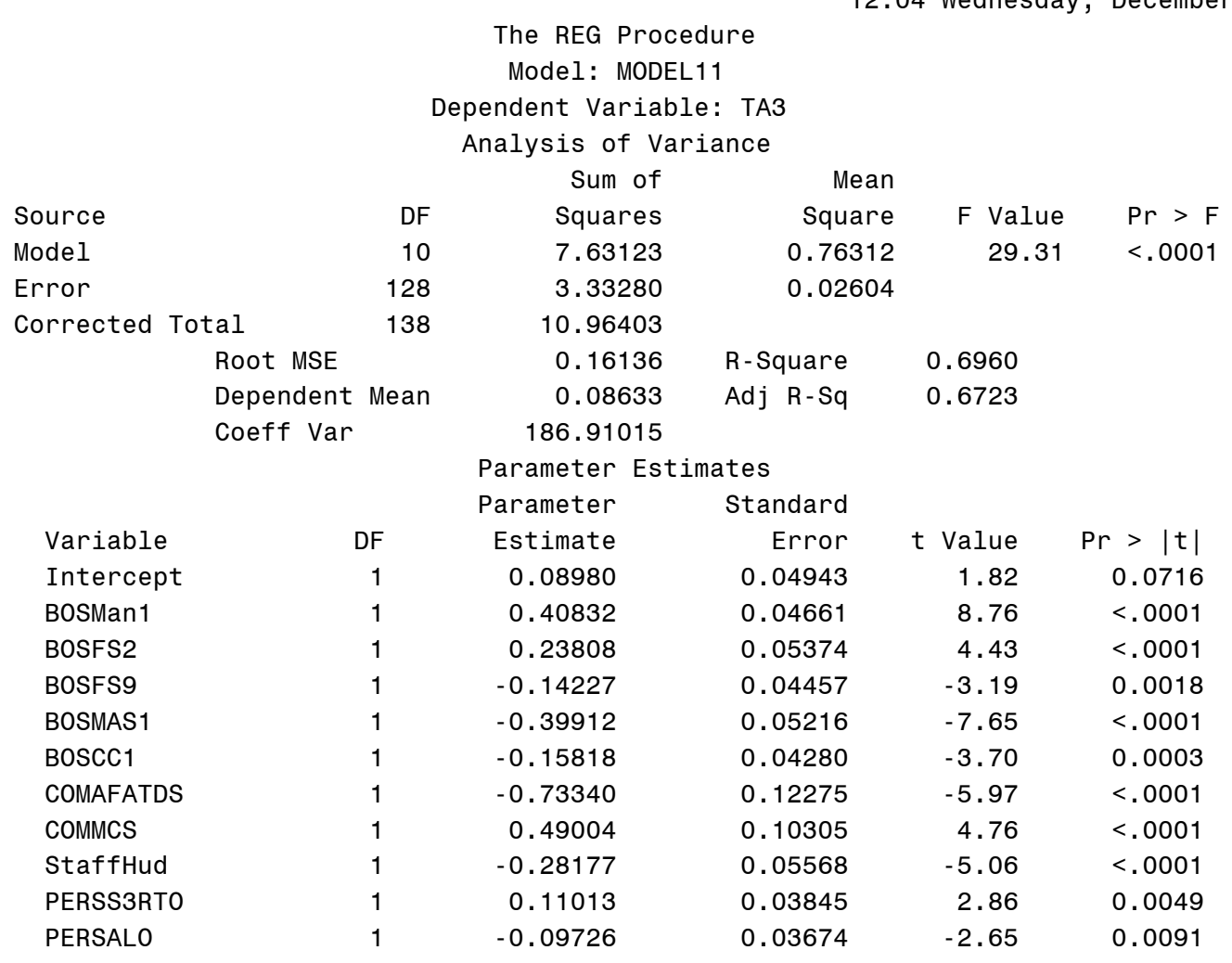


Second level Regression Analysis for Exercises 1,2,3

12:04 Wednesday, December 18, 2002

\begin{tabular}{|c|c|c|c|c|c|}
\hline \multicolumn{6}{|c|}{$\begin{array}{l}\text { The REG Procedure } \\
\text { Model: MODEL12 } \\
\text { pendent Variable: TA21 } \\
\text { Analysis of Variance }\end{array}$} \\
\hline & & Sum of & Mean & & \\
\hline Source & DF & Squares & Square & F Value & $\mathrm{Pr}>\mathrm{F}$ \\
\hline Model & 14 & 16.53220 & 1.18087 & 73.74 & $<.0001$ \\
\hline Error & 124 & 1.98579 & 0.01601 & & \\
\hline Corrected Total & 138 & 18.51799 & & & \\
\hline \multicolumn{2}{|l|}{ Root MSE } & 0.12655 & R-Square & \multicolumn{2}{|l|}{0.8928} \\
\hline \multicolumn{2}{|c|}{ Dependent Mean } & 0.15827 & Adj R-Sq & \multicolumn{2}{|l|}{0.8807} \\
\hline \multicolumn{2}{|c|}{ Coeff Var } & 79.95537 & & & \\
\hline \multicolumn{6}{|c|}{ Parameter Estimates } \\
\hline & & Parameter & Standard & & \\
\hline Variable & DF & Estimate & Error & t Value & $\operatorname{Pr}>|t|$ \\
\hline Intercept & 1 & -0.02767 & 0.07718 & -0.36 & 0.7206 \\
\hline BosIntel1 & 1 & -0.28954 & 0.04924 & -5.88 & $<.0001$ \\
\hline BoSMan1 & 1 & -0.45515 & 0.04625 & -9.84 & $<.0001$ \\
\hline BOSFS9 & 1 & 0.54835 & 0.04903 & 11.18 & $<.0001$ \\
\hline BOSMAS1 & 1 & -0.09739 & 0.05770 & -1.69 & 0.0939 \\
\hline BOSMAS7 & 1 & -0.14943 & 0.05374 & -2.78 & 0.0063 \\
\hline Boscc1 & 1 & 0.11514 & 0.05359 & 2.15 & 0.0336 \\
\hline Boscc3 & 1 & 0.09074 & 0.05007 & 1.81 & 0.0724 \\
\hline COMAFATDS & 1 & -0.15557 & 0.08974 & -1.73 & 0.0855 \\
\hline MISCBattleTiming & 1 & 0.44087 & 0.14014 & 3.15 & 0.0021 \\
\hline MISCBattleTempo & 1 & -0.46725 & 0.10193 & -4.58 & $<.0001$ \\
\hline MISCTOCActivity & 1 & -0.11781 & 0.05948 & -1.98 & 0.0498 \\
\hline StaffHud & 1 & -0.13192 & 0.04489 & -2.94 & 0.0039 \\
\hline PERSS3RTO & 1 & 0.33150 & 0.04868 & 6.81 & $<.0001$ \\
\hline PERSALO & 1 & -0.08447 & 0.02929 & -2.88 & 0.0046 \\
\hline
\end{tabular}


Second level Regression Analysis for Exercises 1,2,3

12:04 Wednesday, December 18, 2002

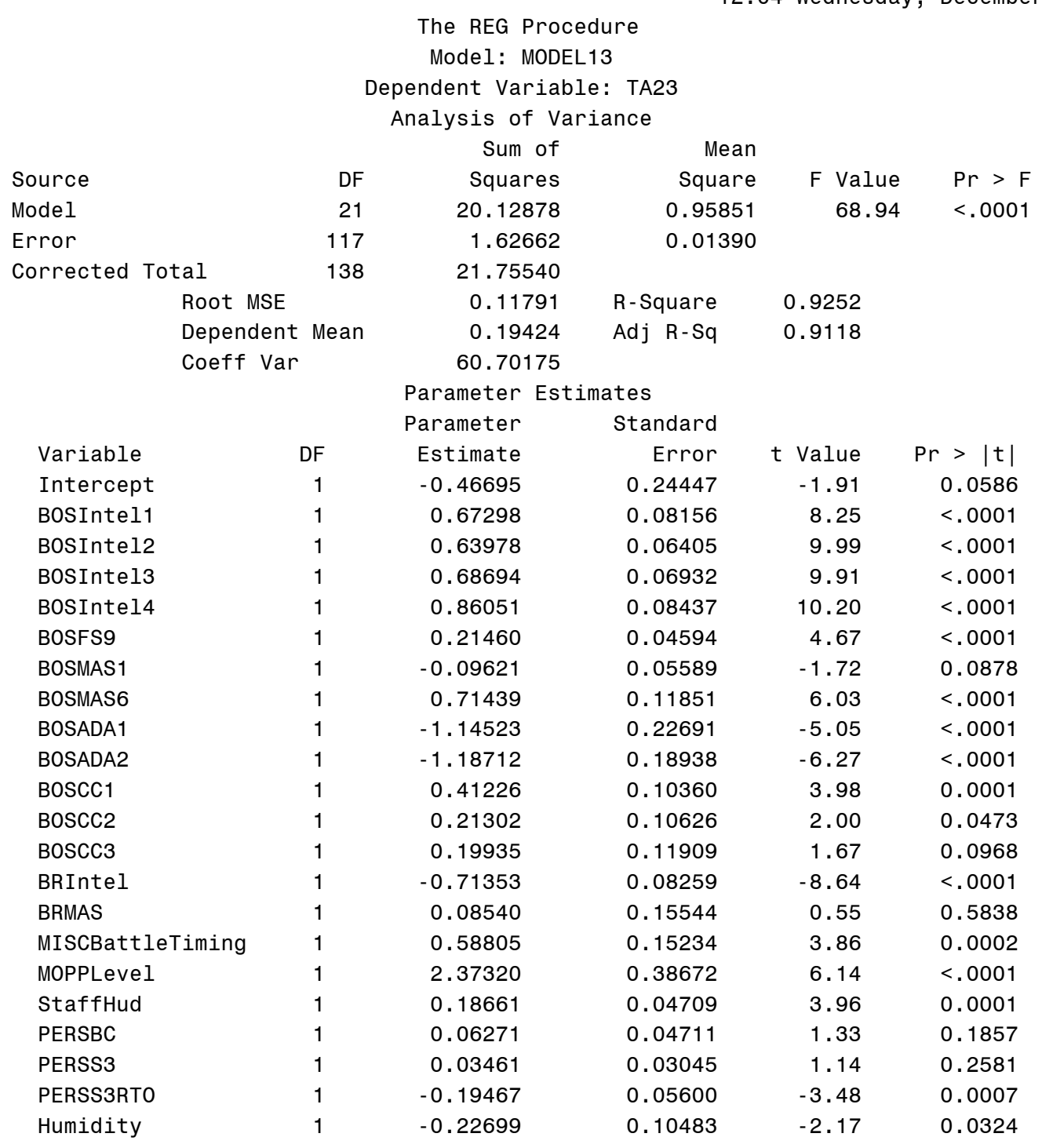


Second level Regression Analysis for Exercises 1,2,3

12:04 Wednesday, December 18, 2002

The REG Procedure

Model: MODEL14

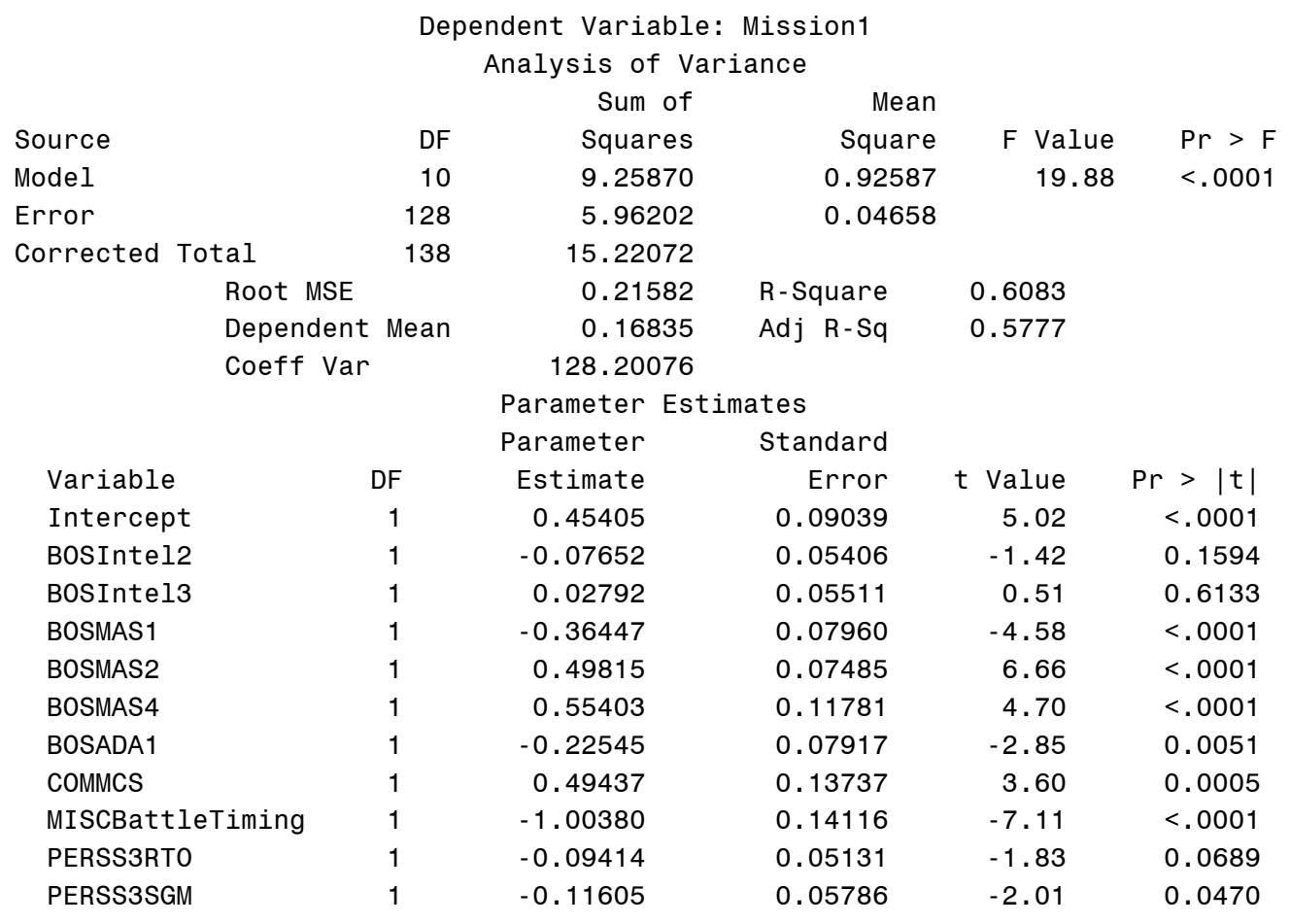




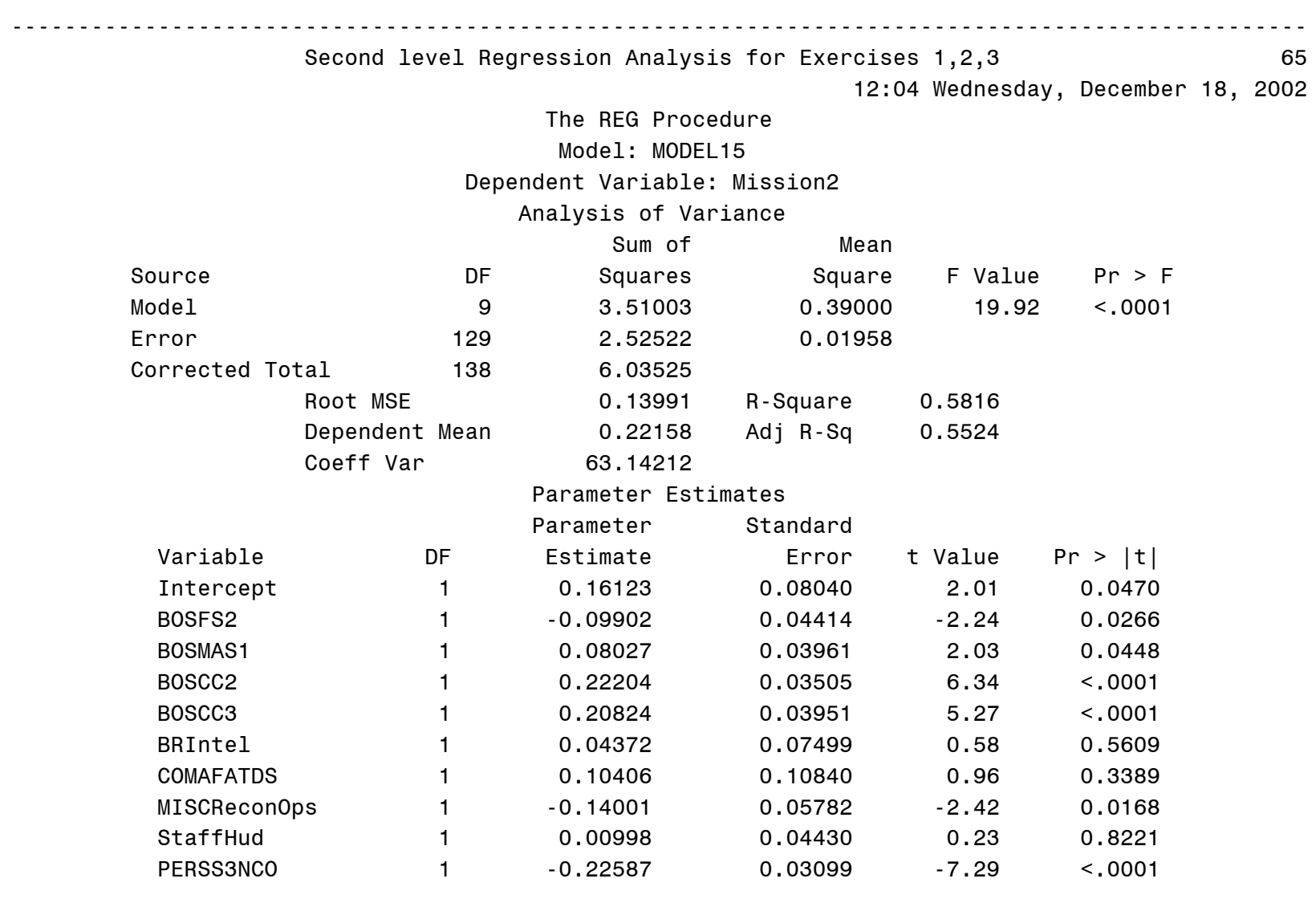


Second level Regression Analysis for Exercises 1,2,3

12:04 Wednesday, December 18, 2002

The REG Procedure

Model: MODEL16

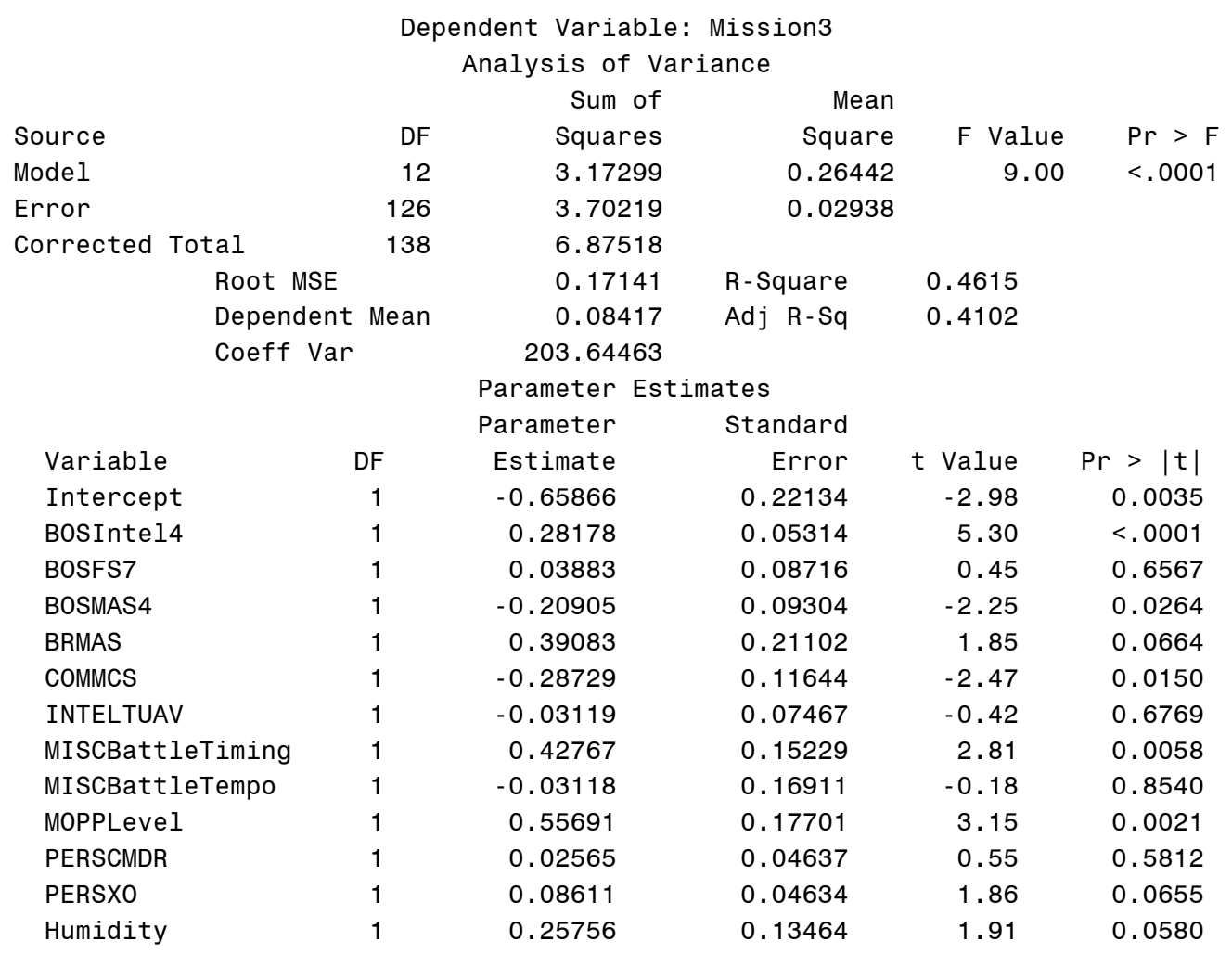


Second level Regression Analysis for Exercises 1,2,3

12:04 Wednesday, December 18, 2002

The REG Procedure

Model: MODEL17

\begin{tabular}{|c|c|c|c|c|c|}
\hline \multicolumn{6}{|c|}{$\begin{array}{c}\text { Dependent Variable: Mission5 } \\
\text { Analysis of Variance }\end{array}$} \\
\hline \multicolumn{6}{|c|}{ Mean } \\
\hline Source & DF & Squares & Square & F Value & $\mathrm{Pr}>\mathrm{F}$ \\
\hline Model & 11 & 4.04591 & 0.36781 & 19.77 & $<.0001$ \\
\hline Error & 127 & 2.36315 & 0.01861 & & \\
\hline Corrected Total & 138 & 6.40906 & & & \\
\hline \multicolumn{2}{|c|}{ Root MSE } & 0.13641 & R-Square & \multicolumn{2}{|l|}{0.6313} \\
\hline \multirow{2}{*}{\multicolumn{2}{|c|}{$\begin{array}{l}\text { Dependent Mean } \\
\text { Coeff Var }\end{array}$}} & 0.07050 & $\operatorname{Adj} R-S q$ & \multicolumn{2}{|l|}{0.5993} \\
\hline & & 193.47854 & & & \\
\hline \multicolumn{6}{|c|}{ Parameter Est } \\
\hline & Parameter & \multicolumn{3}{|l|}{ Standard } \\
\hline Variable & DF & Estimate & Error & $\mathrm{t}$ Value & $\operatorname{Pr}>|t|$ \\
\hline Intercept & 1 & -0.70717 & 0.06980 & -10.13 & $<.0001$ \\
\hline Bosintel3 & 1 & -0.03968 & 0.03086 & -1.29 & 0.2009 \\
\hline BosIntel4 & 1 & 0.10022 & 0.04249 & 2.36 & 0.0199 \\
\hline BosMan1 & 1 & 0.38117 & 0.06082 & 6.27 & $<.0001$ \\
\hline BOSFS9 & 1 & -0.03477 & 0.03193 & -1.09 & 0.2782 \\
\hline BOSADA2 & 1 & 0.40088 & 0.04714 & 8.50 & $<.0001$ \\
\hline COMAFATDS & 1 & -0.17253 & 0.10141 & -1.70 & 0.0913 \\
\hline COMASAS & 1 & 0.16611 & 0.11209 & 1.48 & 0.1408 \\
\hline MISCBattleTiming & 1 & 1.28222 & 0.11669 & 10.99 & $<.0001$ \\
\hline MOPPLevel & 1 & -0.73388 & 0.13344 & -5.50 & $<.0001$ \\
\hline PERSS3NCO & 1 & 0.12560 & 0.03324 & 3.78 & 0.0002 \\
\hline PERSALO & 1 & 0.06181 & 0.03226 & 1.92 & 0.0576 \\
\hline
\end{tabular}




\title{
Appendix L - Third Linear Regression Run To Select IV For Inclusion In DV Models
}

\section{SAS Code (Pick those IV that had a significant $p$ value $(\alpha=.05)$ from the Second run):}

\author{
options pageno=1 formdlim="-"; \\ TITLE "Training Data 123 Regression Analysis"; \\ DATA Tngdata123;
}

InPut Day1 Day2 Day3 Day4 Day5 Day6 Day7 Day8 Day9 Day10 Day11 Day12 Day13 Day14 Day15 Day16 Day17 Day18 Day19 Day20 Hour1 Hour2 Hour3 Hour4 Hour5 Hour6 Hour7 Hour8 Hour9 Hour10 Hour11 Hour12 Hour13 Hour14 Hour15 Hour16 Hour17 Hour18 Hour19 Hour20 Hour21 Hour22 Hour23 Hour24 TOCAn TOCDg TOCBn TOCBde ExTyp1 ExTyp2 ExTyp3 ExTyp4 ExNo1 ExNo2 ExNo3 ExNo4 ExNo5 ExNo6 ExNo7 ExNo8 ExNo9 ExNo10 ExNo11 ExNo12 ExNo13 ExNo14 ExNo15 ExNo16 ExNo17 ExNo18 ExNo19 ExNo20 SimNo SimJANS SimJAST SimCBS SimCCTT SimSTRM BOSIntel1 BOSIntel2 BOSIntel3 BOSIntel4 BOSMan1 BOSMan2 BOSFS1 BOSFS2 BOSFS 3 BOSFS 4 BOSFS5 BOSFS 6 BOSFS7 BOSFS 8 BOSFS 9 BOSMAS1 BOSMAS2 BOSMAS 3 BOSMAS 4 BOSMAS5 BOSMAS 6 BOSMAS7 BOSADA1 BOSADA2 BOSCC1 BOSCC2 BOSCC3 BRIntel BRMan BRFS BRMAS BRADA BRCSS BRC2 COMAFATDS COMAMPS COMASAS COMCTIS COMCSSCS COMFAADC2 COMFBCB2 COMIMETS COMMCS COMAMDWS COMJSTARS COMEPLARS INTELTUAV INTELJSTARS INTELGSR MISCBattleTiming MISCBattleTempo MISCReconOps MISCInfoOps MISCTactics MISCTOCACtivity MOPPLevel StaffHud PERSCMDR PERSXO PERSBC PERSS3 PERSS3NCO PERSS3RTO PERSS2 PERSFSO PERSENGR PERSALO PERSS3SGM SKYCLR SKYOC SKYRAIN WINDNONE WINDLOW WINDMOD WINDHIGH LightBG LightFG Noise TEMPDB TEMPWB Humidity TP1 TP2 TP3 TP4 TP5 TP6 TP7 TP8 TP9 TP10 TP11 TP12 TP13 TP14 TP15 TP16 TP17 TP18 TP19 TP20 TP21 TP22 TP23 TS1 TS2 TS3 TS4 TS5 TS6 TS7 TS8 TS9 TS10 TS11 TS12 TS13 TS14 TS15 TS16 TS17 TS18 TS19 TS20 TS21 TS22 TS23 TA1 TA2 TA3 TA4 TA5 TA6 TA7 TA8 TA9 TA10 TA11 TA12 TA13 TA14 TA15 TA16 TA17 TA18 TA19 TA20 TA21 TA22 TA23 Mission1 Mission2 Mission3 Mission4 Mission5 Mission6 ; *ARDS;

$\begin{array}{lllllllllllllllllllllllllllllllllllllllllllllllll}0 & 0 & 0 & 1 & 0 & 0 & 0 & 0 & 0 & 0 & 0 & 0 & 0 & 0 & 0 & 0 & 0 & 0 & 0 & 0 & 0 & 0 & 0 & 0 & 0 & 0 & 0 & 0 & 0 & 0 & 0 & 0 & 1 & 0 & 0 & 0 & 0 & 0 & 0 & 0 & 0 & 0 & 0 & 0 & 0 & 1 & 0 & 1 & 0\end{array}$ $100 \begin{array}{llllllllllllllllllllllllllllllllllllllllllllllll} & 0 & 0 & 0 & 1 & 0 & 0 & 0 & 0 & 0 & 0 & 0 & 0 & 0 & 0 & 0 & 0 & 0 & 0 & 0 & 0 & 0 & 0 & 0 & 0 & 1 & 0 & 0 & 0 & 1 & 1 & 1 & 1 & 1 & 0 & 1 & 0 & 0 & 0 & 0 & 1 & 0 & 1 & 1 & 1 & 0 & 0 & 0\end{array}$ $\begin{array}{llllllllllllllllllllllllllllllllllllllllll}0 & 1 & 1 & 0 & 0 & 0 & 1 & 1 & 1 & 1 & 1 & 1 & 1 & 1 & 0.7 & 0.0 & 0.6 & 0.0 & 0.0 & 0.0 & 0.0 & 0.0 & 0.6 & 0.4 & 0.1 & 0.0 & 0.1 & 0.1 & 0.0 & 0.5 & 0.5\end{array}$ $\begin{array}{llllllllllllllllllllllllllllllllllllllllll}0.4 & 0.5 & 0.5 & 0.5 & 0.25 & 0 & 1 & 0 & 1 & 1 & 1 & 1 & 1 & 1 & 1 & 0 & 0 & 1 & 0 & 0 & 1 & 0 & 0 & 0 & 0.03 & 0.30 & 0.77 & 0.53 & 0.67 & 0.39 & 0 & 0 & 0 & 0\end{array}$ $\begin{array}{llllllllllllllllllllllllllllllllllllllllllllllllll}0 & 0 & 0 & 0 & 0 & 1 & 1 & 1 & 0 & 0 & 0 & 0 & 0 & 0 & 0 & 0 & 0 & 0 & 0 & 0 & 0 & 0 & 0 & 0 & 0 & 0 & 0 & 0 & 0 & 0 & 0 & 0 & 0 & 1 & 0 & 0 & 0 & 0 & 0 & 0 & 0 & 0 & 0 & 0 & 0 & 0 & 0 & 0 & 0\end{array}$ $\begin{array}{lllllllllllllllllllllllllllllll}0 & 0 & 0 & 0 & 0 & 0 & 0 & 0 & 0 & 0 & 0 & 0 & 0 & 0 & 0 & 1 & 0.0 & 0.5 & 0.5 & 0.0 & 0.0 & 0.0\end{array}$

...

$\begin{array}{llllllllllllllllllllllllllllllllllllllllllllllllll}0 & 0 & 0 & 0 & 0 & 0 & 1 & 0 & 0 & 0 & 0 & 0 & 0 & 0 & 0 & 0 & 0 & 0 & 0 & 0 & 0 & 0 & 0 & 0 & 0 & 0 & 0 & 0 & 0 & 0 & 0 & 1 & 0 & 0 & 0 & 0 & 0 & 0 & 0 & 0 & 0 & 0 & 0 & 0 & 0 & 1 & 0 & 1 & 1\end{array}$ $\begin{array}{lllllllllllllllllllllllllllllllllllllllllllllllll}0 & 0 & 0 & 1 & 0 & 0 & 0 & 0 & 0 & 0 & 0 & 0 & 0 & 0 & 0 & 0 & 0 & 0 & 0 & 0 & 0 & 0 & 0 & 0 & 1 & 0 & 0 & 0 & 0 & 0 & 1 & 0 & 0 & 0 & 0 & 0 & 0 & 0 & 0 & 0 & 0 & 0 & 0 & 1 & 0 & 0 & 0 & 0 & 1\end{array}$ $\begin{array}{llllllllllllllllllllllllllllllllllllllllll}0 & 0 & 0 & 0 & 0 & 0 & 1 & 1 & 1 & 1 & 1 & 1 & 1 & 1 & 0.3 & 0.0 & 0.3 & 0.0 & 0.0 & 0.0 & 0.0 & 0.0 & 0.2 & 0.2 & 0.2 & 0.0 & 0.1 & 0.1 & 0.3 & 0.5 & 0.2\end{array}$

$\begin{array}{lllllllllllllllllllllllllllllllllllllllllll}0.4 & 0.3 & 0.5 & 0.2 & 0.00 & 0 & 0 & 0 & 0 & 0 & 0 & 1 & 1 & 0 & 1 & 1 & 0 & 1 & 0 & 0 & 1 & 0 & 0 & 0 & 0.04 & 0.16 & 0.66 & 0.90 & 0.75 & 0.50 & 0 & 0 & 0 & 0\end{array}$

$\begin{array}{llllllllllllllllllllllllllllllllllllllllllllllllll}0 & 0 & 0 & 0 & 0 & 0 & 1 & 0 & 0 & 0 & 0 & 0 & 0 & 0 & 0 & 0 & 0 & 0 & 0 & 0 & 0 & 0 & 0 & 0 & 0 & 0 & 0 & 0 & 0 & 0 & 1 & 0 & 0 & 0 & 0 & 0 & 0 & 0 & 0 & 0 & 0 & 0 & 0 & 0 & 0 & 0 & 0 & 0 & 0\end{array}$ $\begin{array}{llllllllllllllllllllllllllllll}0 & 0 & 0 & 0 & 0 & 0 & 0 & 0 & 0 & 0 & 0 & 0 & 0 & 1 & 0 & 0 & 0.0 & 0.4 & 0.0 & 0.0 & 0.0 & 0.0\end{array}$

;

* Regression third level run;

Partial Tng Regression Analysis;

* Make regression run with input/independent variables remaining that are significant after full regression run

* to try to maximize the adjusted $r * * 2$ showing the amount of the regression accounted for;

options pageno=1 formdlim="-";

proc reg data=Tngdata123;

TITLE "Third level Regression Analysis for Exercises 1,2,3";

* model TP2=BOSFS1 BOSFS2 BOSFS3 BOSFS7 BOSFS9;

model TP10=BOSIntel4 BOSMAS2 BOSCC2 BOSCC3 MOPPLevel;

model TP11=BOSIntel3 BOSCC1 BOSCC2 BOSCC3 BRIntel StaffHud PERSXO PERSS3RTO PERSS3SGM;

model TP12=BOSFS2 BOSFS7 BOSFS9 BOSMAS6 BOSCC1 COMAFATDS COMASAS MISCBattleTiming

MISCBattleTempo PERSALO Humidity;

model TP15=BOSIntel3 BOSIntel4 BOSFS2 BOSMAS2 COMAFATDS MISCBattleTiming PERSXO;

model TP21=BOSFS9 BOSMAS5 MISCBattleTempo MISCInfoOps;

model TP23=BOSMAS6 BRIntel BRMAS StaffHud;

model TS12=BOSMan1 BOSFS9 BOSMAS1;

model TS18=BOSFS2 BOSMAS6 BOSCC1 MISCBattleTiming PERSXO ;

model TS19=BOSMAS 6 PERSS2;

model TS23=BOSIntel2 BOSIntel3 BOSMan2 BOSFS9 BOSMAS4 BOSCC2 MISCBattleTiming MISCInfoOps

PERSS3RTO Humidity;

model TA3=BOSMAn1 BOSFS2 BOSFS9 BOSMAS1 BOSCC1 COMAFATDS COMMCS StaffHud PERSS3RTO PERSALO;

model TA21=BOSIntel1 BOSMan1 BOSFS9 BOSMAS7 BOSCC1 COMAFATDS MISCBattleTiming MISCBattleTempo MISCTOCActivity staffHud PERSS3RTO PERSALO;

model TA23=BOSIntel1 BOSIntel2 BOSIntel3 BOSIntel4 BOSFS9 BOSMAS6 BOSADA1 BOSADA2 BOSCC1 BOSCC2 
BRIntel MISCBattleTiming MOPPLevel StaffHud PERSS3RTO Humidity;

model Mission1=BOSMAS1 BOSMAS2 BOSMAS4 BOSADA1 COMMCS MISCBattleTiming PERSS3SGM ;

model Mission2=BOSFS2 BOSMAS1 BOSCC2 BOSCC3 MISCReConOps PERSS3NCO ;

model Mission3=BOSIntel4 BOSMAS4 COMMCS MISCBattleTiming MOPPLevel ;

model Mission5=BOSIntel4 BOSMan1 BOSADA2 MISCBattleTiming MOPPLevel PERSS3NCO;

run; 
SAS Output:

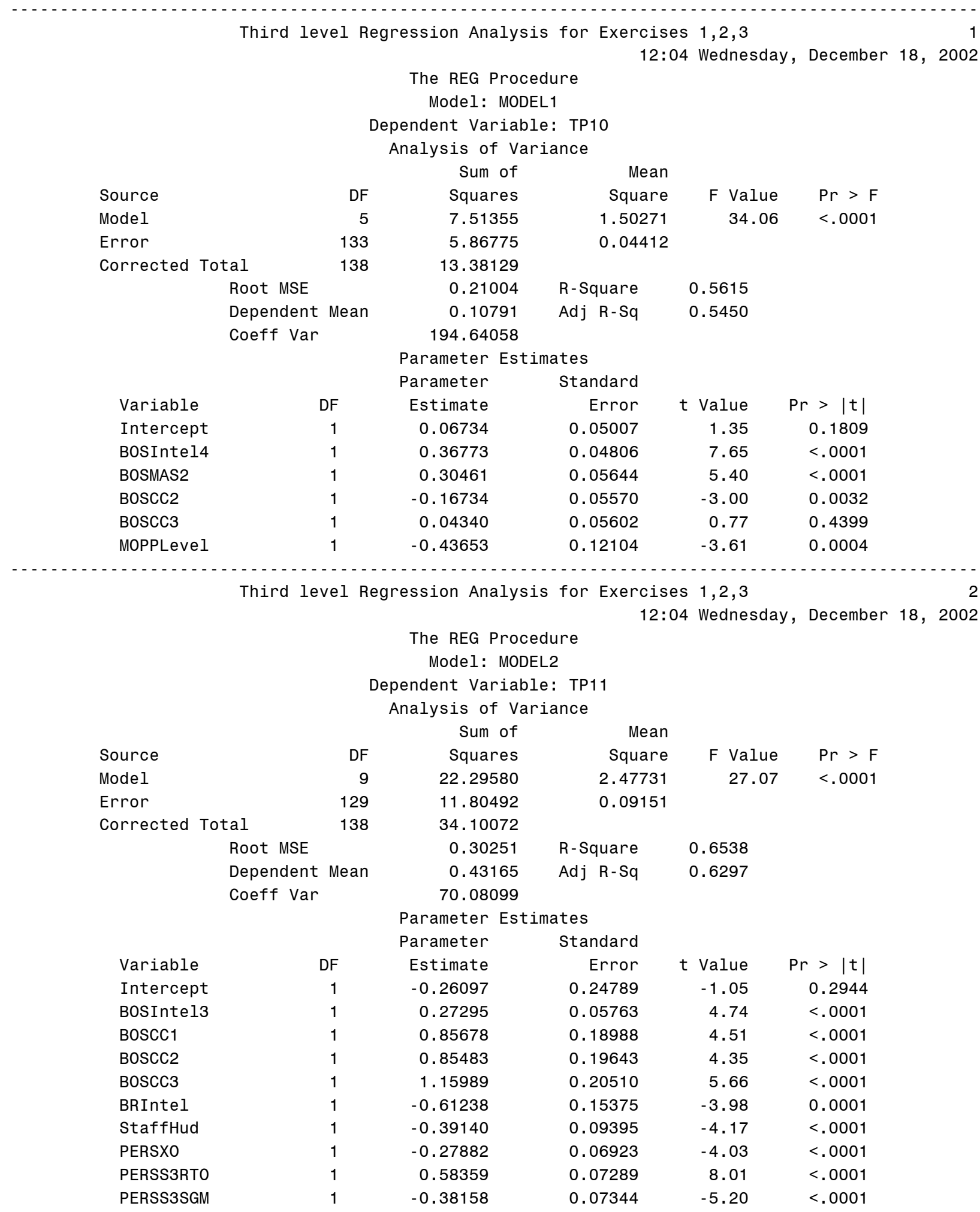


Third level Regression Analysis for Exercises 1,2,3

The REG Procedure

Model: MODEL3

Dependent Variable: TP12

Analysis of Variance

\begin{tabular}{|c|c|c|c|c|c|}
\hline \multicolumn{2}{|l|}{ Source } & $\begin{array}{l}\text { Sum of } \\
\text { Squares }\end{array}$ & $\begin{array}{r}\text { Mean } \\
\text { Square }\end{array}$ & F Value & $\mathrm{Pr}>\mathrm{F}$ \\
\hline Model & 11 & 6.59201 & 0.59927 & 17.41 & $<.0001$ \\
\hline Error & 127 & 4.37202 & 0.03443 & & \\
\hline orrected Total & 138 & 10.96403 & & & \\
\hline \multicolumn{2}{|l|}{ Root MSE } & 0.18554 & R-Square & 0.6012 & \\
\hline \multirow{2}{*}{\multicolumn{2}{|c|}{$\begin{array}{l}\text { Dependent Mean } \\
\text { Coeff Var }\end{array}$}} & 0.08633 & Adj $R-S q$ & 0.5667 & \\
\hline & & 214.91796 & & & \\
\hline \multicolumn{6}{|c|}{ Parameter Estimates } \\
\hline & & Parameter & Standard & & \\
\hline Variable & DF & Estimate & Error & t Value & $\operatorname{Pr}>|t|$ \\
\hline Intercept & 1 & 1.00078 & 0.09816 & 10.20 & $<.0001$ \\
\hline BOSFS2 & 1 & -0.25704 & 0.06338 & -4.06 & $<.0001$ \\
\hline BOSFS7 & 1 & 0.34609 & 0.09585 & 3.61 & 0.0004 \\
\hline BOSFS9 & 1 & -0.17123 & 0.04266 & -4.01 & 0.0001 \\
\hline BOSMAS6 & 1 & -0.39444 & 0.09020 & -4.37 & $<.0001$ \\
\hline Boscc1 & 1 & -0.28068 & 0.06474 & -4.34 & $<.0001$ \\
\hline COMAFATDS & 1 & 0.47345 & 0.16034 & 2.95 & 0.0038 \\
\hline COMASAS & 1 & -0.09658 & 0.13655 & -0.71 & 0.4807 \\
\hline MISCBattleTiming & 1 & -1.15387 & 0.14497 & -7.96 & $<.0001$ \\
\hline MISCBattleTempo & 1 & 0.18928 & 0.10964 & 1.73 & 0.0867 \\
\hline PERSALO & 1 & -0.15613 & 0.04215 & -3.70 & 0.0003 \\
\hline Humidity & 1 & -0.46752 & 0.13826 & -3.38 & 0.0010 \\
\hline
\end{tabular}

Third level Regression Analysis for Exercises 1,2,3

The REG Procedure

Model: MODEL4

Dependent Variable: TP15

Analysis of Variance

$\begin{array}{lrr}\text { Source } & \text { DF } \\ \text { Model } & 7 \\ \text { Error } & 131 \\ \text { Corrected Total } & 138 \\ & & \text { Root MSE } \\ & & \text { Dependent Mean } \\ & \text { Coeff Var }\end{array}$

Sum of

7

38

Variable
Intercept
BOSIntel3
BOSIntel4
BOSFS2
BOSMAS2
COMAFATDS
MISCBattleTiming
PERSXO

DF
1
1
1
1
1
1
1
1

$\begin{array}{rrrr}\begin{array}{r}\text { Sum of } \\ \text { Squares }\end{array} & \begin{array}{r}\text { Mean } \\ \text { Square }\end{array} & \mathrm{F} \text { Value } & \mathrm{Pr}>\mathrm{F} \\ 6.46417 & 0.92345 & 22.74 & <.0001 \\ 5.32001 & 0.04061 & & \\ 11.78417 & & & \\ 0.20152 & \text { R-Square } & 0.5485 & \\ 0.09353 & \text { Adj R-Sq } & 0.5244 & \\ 215.47250 & & & \\ \text { Parameter Estimates } & & \\ \text { Parameter } & \text { Standard } & & \\ \text { Estimate } & \text { Error } & \mathrm{t} \text { Value } & \mathrm{Pr}|\mathrm{t}| \\ -0.21474 & 0.05254 & -4.09 & <.0001 \\ -0.07527 & 0.04227 & -1.78 & 0.0773 \\ 0.38302 & 0.05236 & 7.31 & <.0001 \\ 0.26797 & 0.06303 & 4.25 & <.0001 \\ 0.07394 & 0.04446 & 1.66 & 0.0987 \\ -0.26475 & 0.14546 & -1.82 & 0.0710 \\ 0.52821 & 0.10705 & 4.93 & <.0001 \\ 0.14446 & 0.04134 & 3.49 & 0.0006\end{array}$


Third level Regression Analysis for Exercises 1,2,3

The REG Procedure

12:04 Wednesday, December 18, 2002

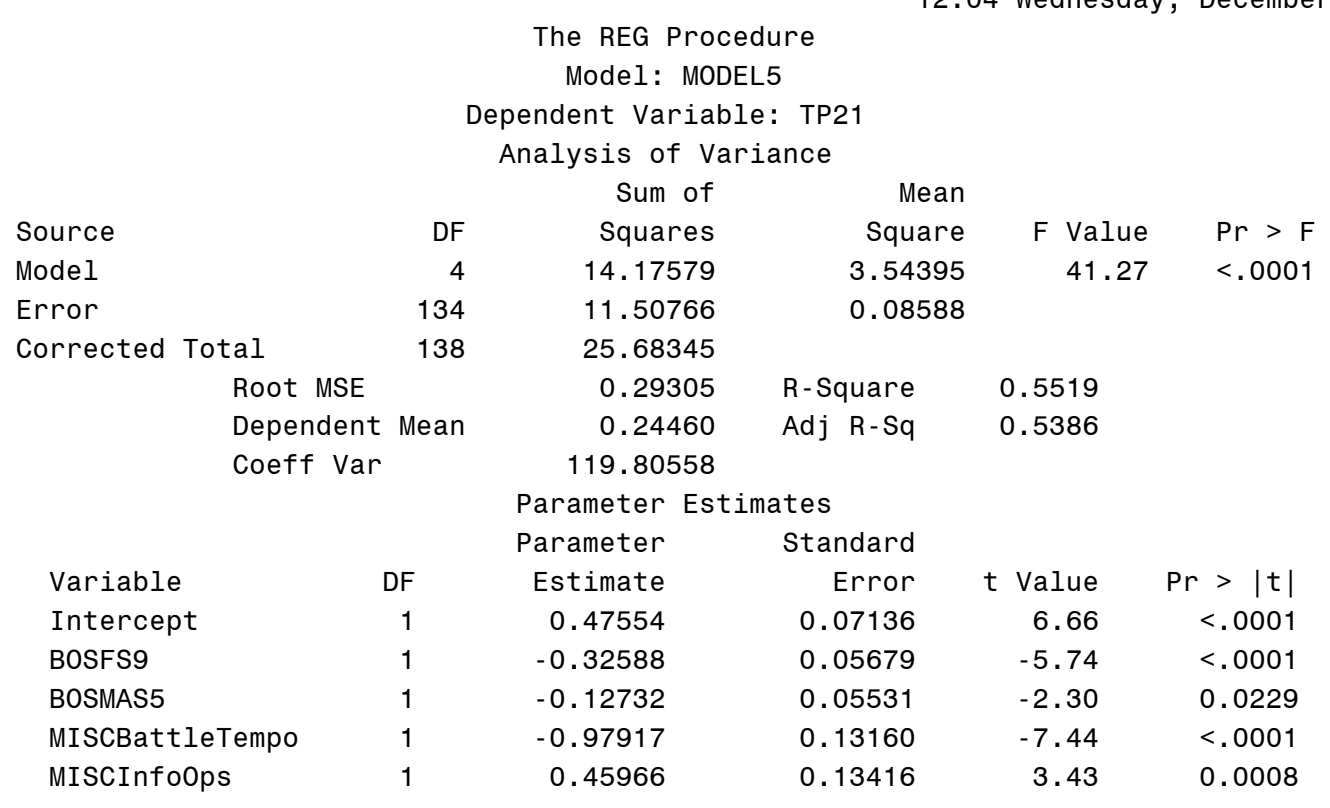

Third level Regression Analysis for Exercises 1,2,3

12:04 Wednesday, December 18, 2002

The REG Procedure

Model: MODEL6

Dependent Variable: TP23

Analysis of Variance

$\begin{array}{lr}\text { Source } & \text { DF } \\ \text { Model } & 4 \\ \text { Error } & 134 \\ \text { Corrected Total } & 138 \\ & \\ & \text { Root MSE } \\ & \text { Dependent Mean } \\ & \text { Coeff Var }\end{array}$

Sum of Mean

DF Squares Square

$4 \quad 9.11062 \quad 2.27766$

F Value $\mathrm{Pr}>\mathrm{F}$

9.11062

2.27766
0.03187

13.38129

0.17852

R-Square

.6808

0.10791

Adj R-Sq

0.6713

165.43190

Parameter Estimates

Variable

Intercept

BOSMAS6

BRIntel

BRMAS

StaffHud

\section{Parameter}

Estimate

0.39034

1.01120

0.31699

$-0.71853$

0.20794
Standard

Error

0.11841

0.06566

0.08691

0.11348

0.05561 t Value

3.30

15.40

3.65

$-6.33$

3.74
$\operatorname{Pr}>|\mathrm{t}|$

0.0013

$<.0001$

0.0004

$<.0001$

0.0003 
Third level Regression Analysis for Exercises 1,2,3

The REG Procedure

Model: MODEL7

Dependent Variable: TS12

Analysis of Variance

Sum of

Source

Model

Error

Corrected Total

Root MSE

Dependent Mean

Coeff Var
DF

3

135

138

an

12.02977

10.32994

22.35971

0.27662

0.20144

137.32159

12:04 Wednesday, December 18, 2002

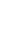

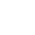

Sum of Mean

Squares Square

4.00992

F Value $\mathrm{Pr}>\mathrm{F}$

$52.40<.0001$

R-Square

0.5380

Adj R-Sq

0.5277

Parameter Estimates

Parameter Standard

Estimate Error

$0.04119=0.03426$

$t$ Value $\operatorname{Pr}>|t|$

0.04119

0.03426

1.20

0.2313

$-0.46001$

0.05962

$-7.33$

$<.0001$

0.73140

0.07846

12.27

$<.0001$

$-0.28259$

$-3.60$

0.0004

Third level Regression Analysis for Exercises 1,2,3

12:04 Wednesday, December 18, 2002

The REG Procedure

Model: MODEL8

Dependent Variable: TS18

Analysis of Variance

$\begin{array}{lr}\text { Source } & \text { DF } \\ \text { Model } & 5 \\ \text { Error } & 133 \\ \text { Corrected Total } & 138 \\ & \text { Root MSE } \\ & \\ & \text { Dependent Mean } \\ & \text { Coeff Var }\end{array}$

$\begin{array}{lr}\text { Variable } & \text { DF } \\ \text { Intercept } & 1 \\ \text { BOSFS2 } & 1 \\ \text { BOSMAS6 } & 1 \\ \text { BOSCC1 } & 1 \\ \text { MISCBattleTiming } & 1 \\ \text { PERSX0 } & 1\end{array}$

$\begin{array}{rrrl}\text { Sum of } & \text { Mean } & & \\ \text { Squares } & \text { Square } & \mathrm{F} \text { Value } & \mathrm{Pr}>\mathrm{F} \\ 2.93386 & 0.58677 & 8.08 & <.0001 \\ 9.65607 & 0.07260 & & \end{array}$

$\begin{array}{rll}12.58993 & & \\ 0.26945 & \text { R-Square } & 0.2330 \\ 0.10072 & \text { Adj R-Sq } & 0.2042\end{array}$

267.52295

Parameter Estimates

Parameter Standard

Estimate Error

$-0.14761 \quad 0.09511$

t Value $\mathrm{Pr}>|\mathrm{t}|$

0.15261

0.06459

$-1.55$

0.1230

$-0.31555$

0.11629

2.36

0.0196

0.22247

0.08148

$-2.71$

0.0075

0.26665

0.18858

0.24460

0.05043

2.73

0.0072

$1.41 \quad 0.1597$

$4.85<.0001$ 
Third level Regression Analysis for Exercises 1,2,3

The REG Procedure

12:04 Wednesday, December 18, 2002

Model: MODEL9

Dependent Variable: TS19

Analysis of Variance

\begin{tabular}{|c|c|c|c|c|c|c|}
\hline \multicolumn{2}{|l|}{ Source } & DF & $\begin{array}{l}\text { Sum of } \\
\text { Squares }\end{array}$ & $\begin{array}{r}\text { Mean } \\
\text { Square }\end{array}$ & F Value & $\mathrm{Pr}>\mathrm{F}$ \\
\hline \multicolumn{2}{|l|}{ Model } & 2 & 7.27184 & 3.63592 & 133.93 & $<.0001$ \\
\hline \multicolumn{2}{|l|}{ Error } & 136 & 3.69219 & 0.02715 & & \\
\hline \multicolumn{2}{|c|}{ Corrected Total } & 138 & 10.96403 & & & \\
\hline \multicolumn{3}{|c|}{ Root MSE } & 0.16477 & R-Square & 0.6632 & \\
\hline \multirow{2}{*}{\multicolumn{3}{|c|}{$\begin{array}{l}\text { Dependent Mean } \\
\text { Coeff Var }\end{array}$}} & 0.08633 & Adj R-Sq & 0.6583 & \\
\hline & & & 190.85602 & & & \\
\hline \multicolumn{7}{|c|}{ Parameter Estimates } \\
\hline & & & Parameter & Standard & & \\
\hline Variable & & DF & Estimate & Error & t Value & $\operatorname{Pr}>|t|$ \\
\hline Intercept & & 1 & 0.09891 & 0.02985 & 3.31 & 0.0012 \\
\hline BOSMAS6 & & 1 & 0.96695 & 0.06001 & 16.11 & $<.0001$ \\
\hline PERSS2 & & 1 & -0.08781 & 0.03358 & -2.62 & 0.0099 \\
\hline
\end{tabular}

Third level Regression Analysis for Exercises 1,2,3 10

12:04 Wednesday, December 18, 2002

The REG Procedure

Model: MODEL10

Dependent Variable: TS23

Analysis of Variance

$\begin{array}{lr}\text { Source } & \text { DF } \\ \text { Model } & 10 \\ \text { Error } & 128 \\ \text { Corrected Total } & 138 \\ & \\ & \text { Root MSE } \\ & \text { Dependent Mean } \\ & \text { Coeff Var }\end{array}$

Variable

Intercept

BoSIntel2

BosIntel3

BoSMan2

BOSFS9

BOSMAS4

BOSCC2

MISCBattleTiming

MISCInfo0ps

PERSS3RTO

Humidity

\section{Sum of}

Squares

8.38067

7.28840

15.66906

0.23862

0.12950

184.26947

Parameter Estimates

Parameter

Estimate

$-0.50056$

0.12448

0.20154

$-0.23144$

$-0.13123$

0.58920

0.18261

$-0.52596$

0.26852

0.35949

0.58127
Standard

\section{Error}

0.15867

0.05538

0.05547

0.08071

0.05672

0.11780

0.05731

0.15863

0.11073

0.06329

0.18105
F Value $\quad \mathrm{Pr}>\mathrm{F}$

$14.72<.0001$

0.5349

0.4985

$\begin{array}{rr}t \text { Value } & \mathrm{Pr}>|\mathrm{t}| \\ -3.15 & 0.0020 \\ 2.25 & 0.0263 \\ 3.63 & 0.0004 \\ -2.87 & 0.0048 \\ -2.31 & 0.0223 \\ 5.00 & <.0001 \\ 3.19 & 0.0018 \\ -3.32 & 0.0012 \\ 2.43 & 0.0167 \\ 5.68 & <.0001 \\ 3.21 & 0.0017\end{array}$


Third level Regression Analysis for Exercises 1,2,3

12:04 Wednesday, December 18, 2002

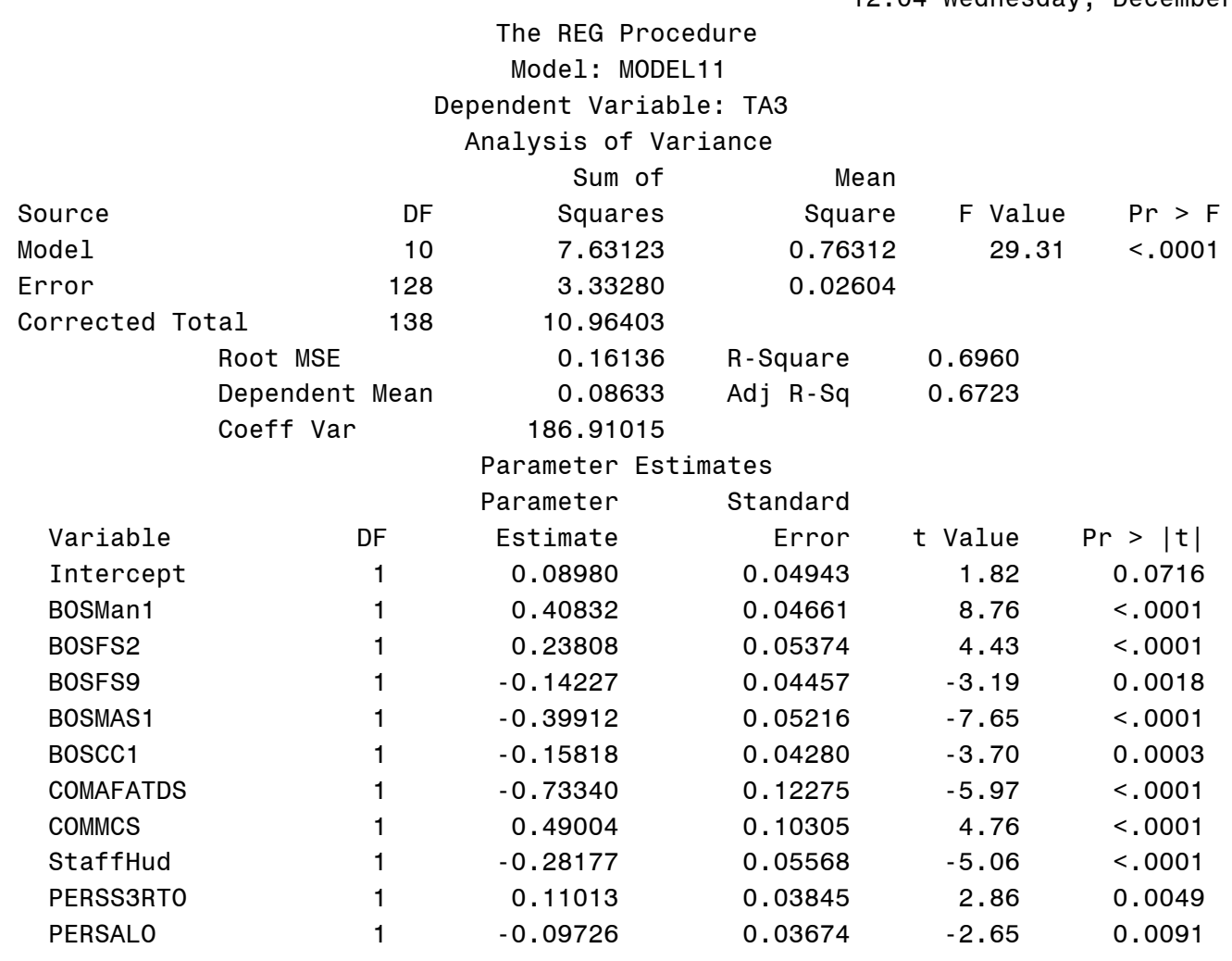


Third level Regression Analysis for Exercises 1,2,3

12:04 Wednesday, December 18, 2002

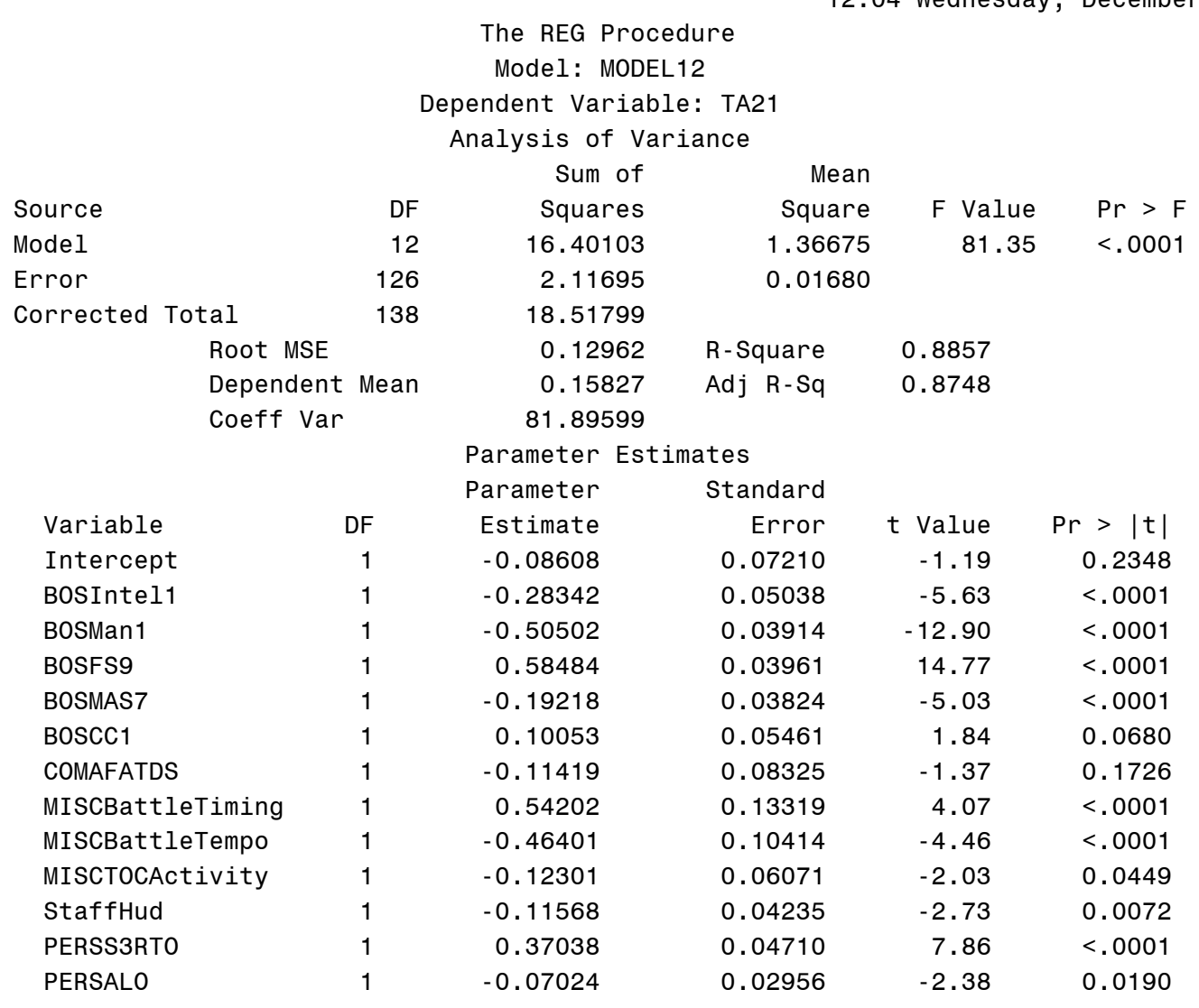


Third level Regression Analysis for Exercises 1,2,3

12:04 Wednesday, December 18, 2002

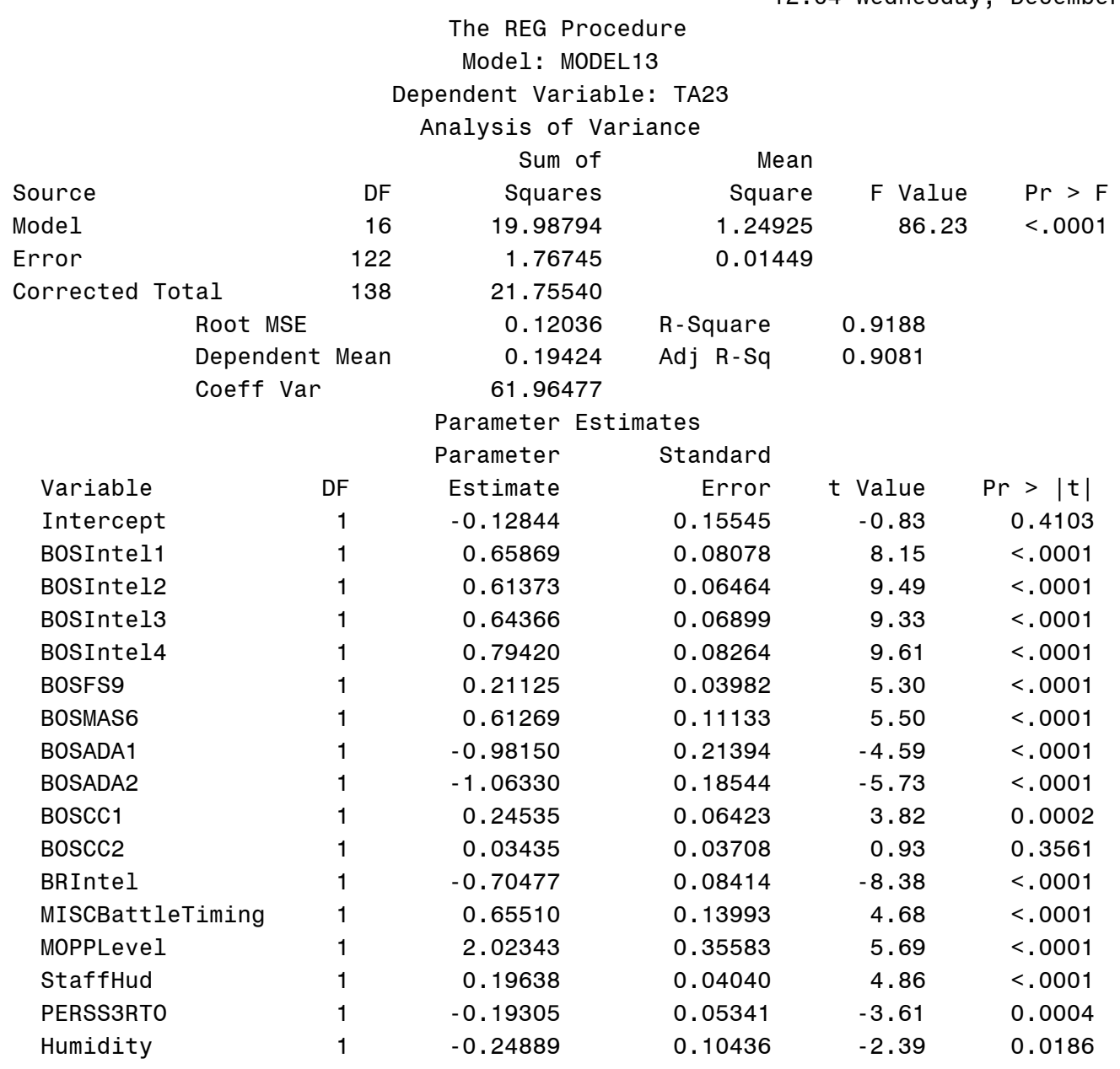


Third level Regression Analysis for Exercises 1,2,3

The REG Procedure

Model: MODEL14

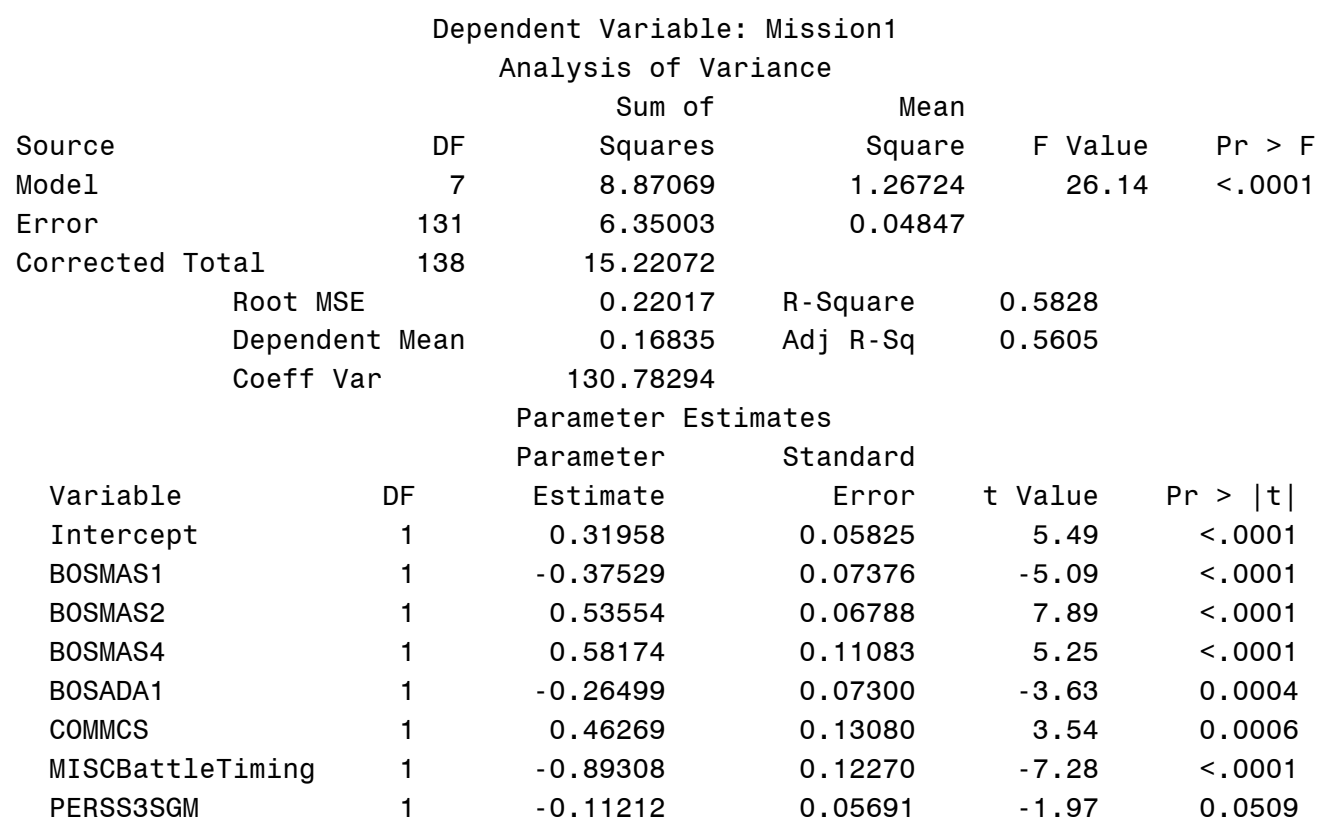

Third level Regression Analysis for Exercises 1,2,3

The REG Procedure

Model: MODEL15

Dependent Variable: Mission2

Analysis of Variance

$\begin{array}{lr}\text { Source } & \text { DF } \\ \text { Model } & 6 \\ \text { Error } & 132 \\ \text { Corrected Total } & 138 \\ & \\ & \text { Root MSE } \\ & \text { Dependent Mean } \\ & \text { Coeff Var }\end{array}$

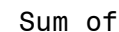

Sum of Mean

Squares

3.48794

Square

2.54731

0.58132

F Value $\mathrm{Pr}>\mathrm{F}$

6.03525

\begin{abstract}
0.13892
\end{abstract}
0.01930

30.12

$<.0001$

62.69290

R-Square

0.5779

Parameter Estimates

Variable
Intercept
BOSFS2
BOSMAS1
BOSCC2
BOSCC3
MISCReconOps
PERSS3NCO

Parameter

Estimate

0.21417

$-0.07909$

Standard

0.08428

0.03487

$\mathrm{t}$ Value

6.14

$\operatorname{Pr}>|\mathrm{t}|$

0.03552

$-2.23$

.0001

0.22173

0.03821

2.21

0.21830

0.03411

6.50

0.0291

$-0.12503$

0.03727

5.86

$<.0001$

$-0.21450$

0.05306

$-2.36$

$<.0001$

0.02813

$-7.62$

0.0199

$<.0001$ 
Third level Regression Analysis for Exercises 1,2,3

The REG Procedure

Model: MODEL16

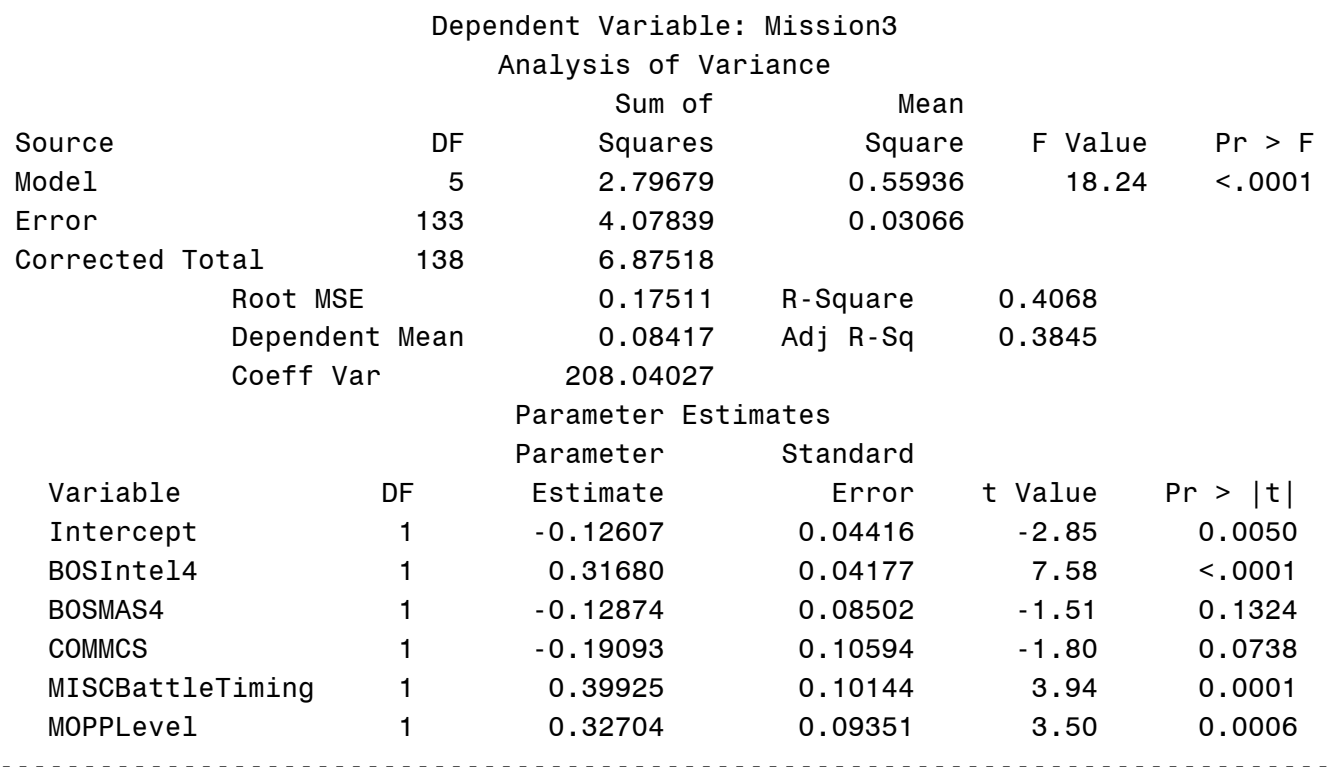

Third level Regression Analysis for Exercises 1,2,3

12:04 Wednesday, December 18, 2002

The REG Procedure

Model: MODEL17

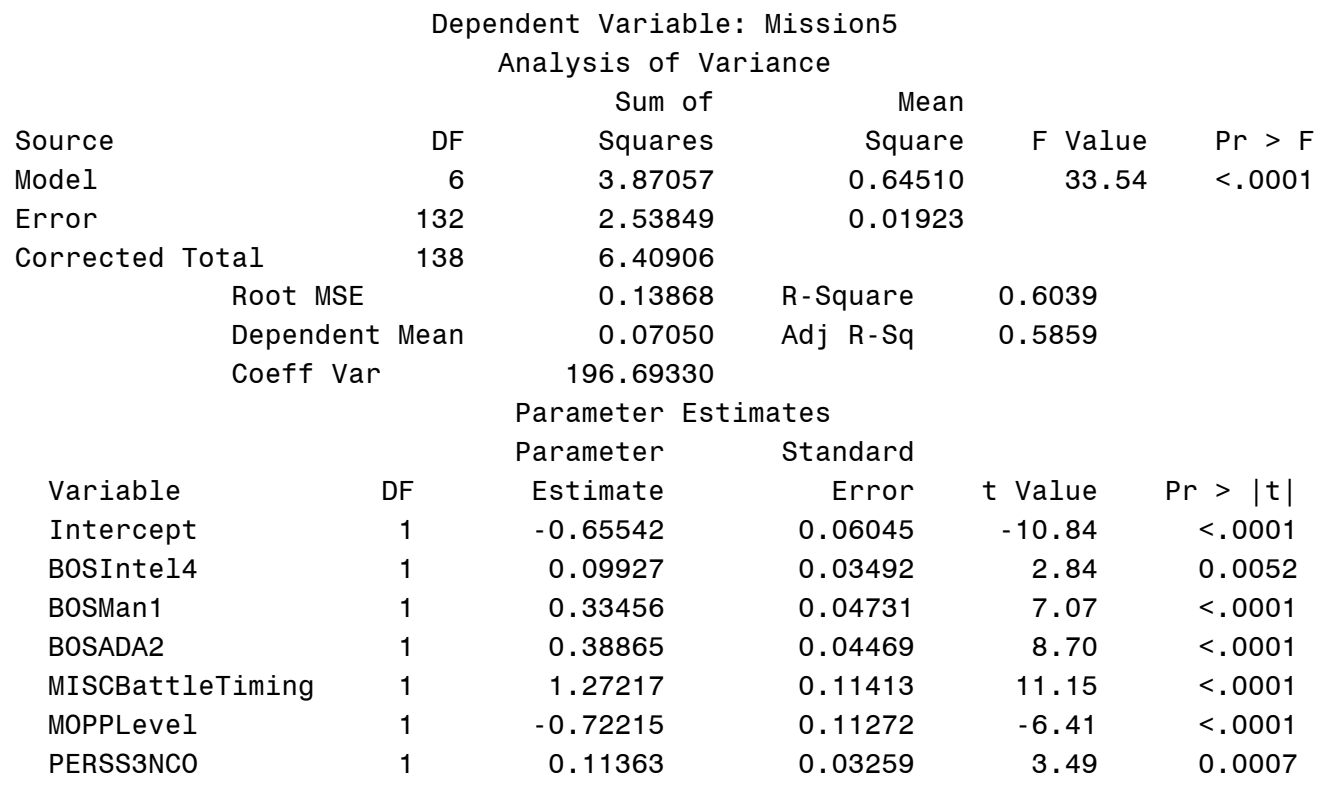




\title{
Appendix M - Fourth Linear Regression Run To Select IV For Inclusion In DV Models
}

\section{SAS Code (Pick those IV that had a significant $p$ value $(\alpha=.05)$ from the third run):}

\author{
options pageno=1 formdlim="-"; \\ TITLE "Training Data 123 Regression Analysis"; \\ DATA Tngdata123;
}

InPut Day1 Day2 Day3 Day4 Day5 Day6 Day7 Day8 Day9 Day10 Day11 Day12 Day13 Day14 Day15 Day16 Day17 Day18 Day19 Day20 Hour1 Hour2 Hour3 Hour4 Hour5 Hour6 Hour7 Hour8 Hour9 Hour10 Hour11 Hour12 Hour13 Hour14 Hour15 Hour16 Hour17 Hour18 Hour19 Hour20 Hour21 Hour22 Hour23 Hour24 TOCAn TOCDg TOCBn TOCBde ExTyp1 ExTyp2 ExTyp3 ExTyp4 ExNo1 ExNo2 ExNo3 ExNo4 ExNo5 ExNo6 ExNo7 ExNo8 ExNo9 ExNo10 ExNo11 ExNo12 ExNo13 ExNo14 ExNo15 ExNo16 ExNo17 ExNo18 ExNo19 ExNo20 SimNo SimJANS SimJAST SimCBS SimCCTT SimSTRM BOSIntel1 BOSIntel2 BOSIntel3 BOSIntel4 BOSMan1 BOSMan2 BOSFS1 BOSFS2 BOSFS 3 BOSFS 4 BOSFS 5 BOSFS 6 BOSFS 7 BOSFS 8 BOSFS 9 BOSMAS1 BOSMAS2 BOSMAS 3 BOSMAS4 BOSMAS5 BOSMAS 6 BOSMAS7 BOSADA1 BOSADA2 BOSCC1 BOSCC2 BOSCC3 BRIntel BRMan BRFS BRMAS BRADA BRCSS BRC2 COMAFATDS COMAMPS COMASAS COMCTIS COMCSSCS COMFAADC2 COMFBCB2 COMIMETS COMMCS COMAMDWS COMJSTARS COMEPLARS INTELTUAV INTELJSTARS INTELGSR MISCBattleTiming MISCBattleTempo MISCReconOps MISCInfoOps MISCTactics MISCTOCActivity MOPPLevel StaffHud PERSCMDR PERSXO PERSBC PERSS3 PERSS3NCO PERSS3RTO PERSS2 PERSFSO PERSENGR PERSALO PERSS3SGM SKYCLR SKYOC SKYRAIN WINDNONE WINDLOW WINDMOD WINDHIGH LightBG LightFG Noise TEMPDB TEMPWB Humidity TP1 TP2 TP3 TP4 TP5 TP6 TP7 TP8 TP9 TP10 TP11 TP12 TP13 TP14 TP15 TP16 TP17 TP18 TP19 TP20 TP21 TP22 TP23 TS1 TS2 TS3 TS4 TS5 TS6 TS7 TS8 TS9 TS10 TS11 TS12 TS13 TS14 TS15 TS16 TS17 TS18 TS19 TS20 TS21 TS22 TS23 TA1 TA2 TA3 TA4 TA5 TA6 TA7 TA8 TA9 TA10 TA11 TA12 TA13 TA14 TA15 TA16 TA17 TA18 TA19 TA20 TA21 TA22 TA23 Mission1 Mission2 Mission3 Mission4 Mission5 Mission6 ; CARDS;

$\begin{array}{lllllllllllllllllllllllllllllllllllllllllllllllll}0 & 0 & 0 & 1 & 0 & 0 & 0 & 0 & 0 & 0 & 0 & 0 & 0 & 0 & 0 & 0 & 0 & 0 & 0 & 0 & 0 & 0 & 0 & 0 & 0 & 0 & 0 & 0 & 0 & 0 & 0 & 0 & 1 & 0 & 0 & 0 & 0 & 0 & 0 & 0 & 0 & 0 & 0 & 0 & 0 & 1 & 0 & 1 & 0\end{array}$ $100 \begin{array}{llllllllllllllllllllllllllllllllllllllllllllllll} & 0 & 0 & 0 & 1 & 0 & 0 & 0 & 0 & 0 & 0 & 0 & 0 & 0 & 0 & 0 & 0 & 0 & 0 & 0 & 0 & 0 & 0 & 0 & 0 & 1 & 0 & 0 & 0 & 1 & 1 & 1 & 1 & 1 & 0 & 1 & 0 & 0 & 0 & 0 & 1 & 0 & 1 & 1 & 1 & 0 & 0 & 0\end{array}$ $\begin{array}{llllllllllllllllllllllllllllllllllllllllll}0 & 1 & 1 & 0 & 0 & 0 & 1 & 1 & 1 & 1 & 1 & 1 & 1 & 1 & 0.7 & 0.0 & 0.6 & 0.0 & 0.0 & 0.0 & 0.0 & 0.0 & 0.6 & 0.4 & 0.1 & 0.0 & 0.1 & 0.1 & 0.0 & 0.5 & 0.5\end{array}$ $\begin{array}{lllllllllllllllllllllllllllllllllllllll}0.4 & 0.5 & 0.5 & 0.5 & 0.25 & 0 & 1 & 0 & 1 & 1 & 1 & 1 & 1 & 1 & 1 & 0 & 0 & 1 & 0 & 0 & 1 & 0 & 0 & 0 & 0.03 & 0.30 & 0.77 & 0.53 & 0.67 & 0.39 & 0 & 0 & 0 & 0\end{array}$ $\begin{array}{llllllllllllllllllllllllllllllllllllllllllllllllll}0 & 0 & 0 & 0 & 0 & 1 & 1 & 1 & 0 & 0 & 0 & 0 & 0 & 0 & 0 & 0 & 0 & 0 & 0 & 0 & 0 & 0 & 0 & 0 & 0 & 0 & 0 & 0 & 0 & 0 & 0 & 0 & 0 & 1 & 0 & 0 & 0 & 0 & 0 & 0 & 0 & 0 & 0 & 0 & 0 & 0 & 0 & 0 & 0\end{array}$ $\begin{array}{lllllllllllllllllllllllllllllll}0 & 0 & 0 & 0 & 0 & 0 & 0 & 0 & 0 & 0 & 0 & 0 & 0 & 0 & 0 & 1 & 0.0 & 0.5 & 0.5 & 0.0 & 0.0 & 0.0\end{array}$

...

$\begin{array}{llllllllllllllllllllllllllllllllllllllllllllllllll}0 & 0 & 0 & 0 & 0 & 0 & 1 & 0 & 0 & 0 & 0 & 0 & 0 & 0 & 0 & 0 & 0 & 0 & 0 & 0 & 0 & 0 & 0 & 0 & 0 & 0 & 0 & 0 & 0 & 0 & 0 & 1 & 0 & 0 & 0 & 0 & 0 & 0 & 0 & 0 & 0 & 0 & 0 & 0 & 0 & 1 & 0 & 1 & 1\end{array}$

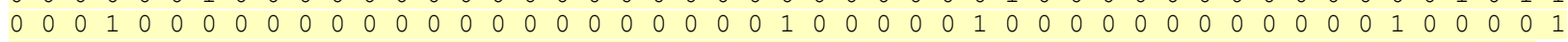
$\begin{array}{lllllllllllllllllllllllllllllllllllll}0 & 0 & 0 & 0 & 0 & 0 & 1 & 1 & 1 & 1 & 1 & 1 & 1 & 1 & 0.3 & 0.0 & 0.3 & 0.0 & 0.0 & 0.0 & 0.0 & 0.0 & 0.2 & 0.2 & 0.2 & 0.0 & 0.1 & 0.1 & 0.3 & 0.5 & 0.2\end{array}$ $\begin{array}{llllllllllllllllllllllllllllllllllllllllll}0.4 & 0.3 & 0.5 & 0.2 & 0.00 & 0 & 0 & 0 & 0 & 0 & 0 & 1 & 1 & 0 & 1 & 1 & 0 & 1 & 0 & 0 & 1 & 0 & 0 & 0 & 0.04 & 0.16 & 0.66 & 0.90 & 0.75 & 0.50 & 0 & 0 & 0 & 0\end{array}$ $\begin{array}{llllllllllllllllllllllllllllllllllllllllllllllllll}0 & 0 & 0 & 0 & 0 & 0 & 1 & 0 & 0 & 0 & 0 & 0 & 0 & 0 & 0 & 0 & 0 & 0 & 0 & 0 & 0 & 0 & 0 & 0 & 0 & 0 & 0 & 0 & 0 & 0 & 1 & 0 & 0 & 0 & 0 & 0 & 0 & 0 & 0 & 0 & 0 & 0 & 0 & 0 & 0 & 0 & 0 & 0 & 0\end{array}$ $\begin{array}{llllllllllllllllllllllllllllllll}0 & 0 & 0 & 0 & 0 & 0 & 0 & 0 & 0 & 0 & 0 & 0 & 0 & 1 & 0 & 0 & 0.0 & 0.4 & 0.0 & 0.0 & 0.0 & 0.0\end{array}$

;

* Partial Tng Regression Analysis;

* Make regression run with input/independent variables remaining that are significant after full regression run

* to try to maximize the adjusted $r * * 2$ showing the amount of the regression accounted for; options pageno=1 formdlim="-";

proc reg data=Tngdata123;

TITLE "Fourth level Regression Analysis for Exercises 1,2,3";

model TP10=BOSIntel4 BOSMAS2 BOSCC2 MOPPLevel;

model TP11=BOSIntel3 BOSCC1 BOSCC2 BOSCC3 BRIntel StaffHud PERSXO PERSS3RTO PERSS3SGM;

model TP12=BOSFS2 BOSFS7 BOSFS9 BOSMAS6 BOSCC1 COMAFATDS MISCBattleTiming PERSALO Humidity;

model TP15=BOSIntel4 BOSFS2 MISCBattleTiming PERSXO;

model TP21=BOSFS9 BOSMAS5 MISCBattleTempo MISCInfoops ;

model TP23=BOSMAS6 BRIntel BRMAS StaffHud;

model TS12=BOSMan1 BOSFS 9 BOSMAS1;

model TS18=BOSFS2 BOSMAS6 BOSCC1 PERSXO ;

model TS19=BOSMAS6 PERSS2;

model TS23=BOSIntel2 BOSIntel3 BOSMan2 BOSFS9 BOSMAS4 BOSCC2 MISCBattleTiming MISCInfoOps

PERSS3RTO Humidity;

model TA3=BOSMan1 BOSFS2 BOSFS9 BOSMAS1 BOSCC1 COMAFATDS COMMCS StaffHud PERSS3RTO PERSALO;

model TA21=BOSIntel1 BOSMan1 BOSFS9 BOSMAS7 BOSCC1 MISCBattleTiming MISCBattleTempo MISCTOCActivity StaffHud PERSS3RTO PERSALO;

model TA23=BOSIntel1 BOSIntel2 BOSIntel3 BOSIntel4 BOSFS9 BOSMAS6 BOSADA1 BOSADA2 BOSCC1 BRIntel MISCBattleTiming MOPPLevel StaffHud PERSS3RTO Humidity;

model Mission1=BOSMAS1 BOSMAS2 BOSMAS4 BOSADA1 COMMCS MISCBattleTiming ; 
model Mission2=BOSFS2 BOSMAS1 BOSCC2 BOSCC3 MISCReconOps PERSS3NCO ; model Mission3=BOSIntel 4 MISCBattleTiming MOPPLevel ;

model Mission5=BOSIntel4 BOSMan1 BOSADA2 MISCBattleTiming MOPPLevel PERSS3NCO; run; 


\section{SAS Output:}

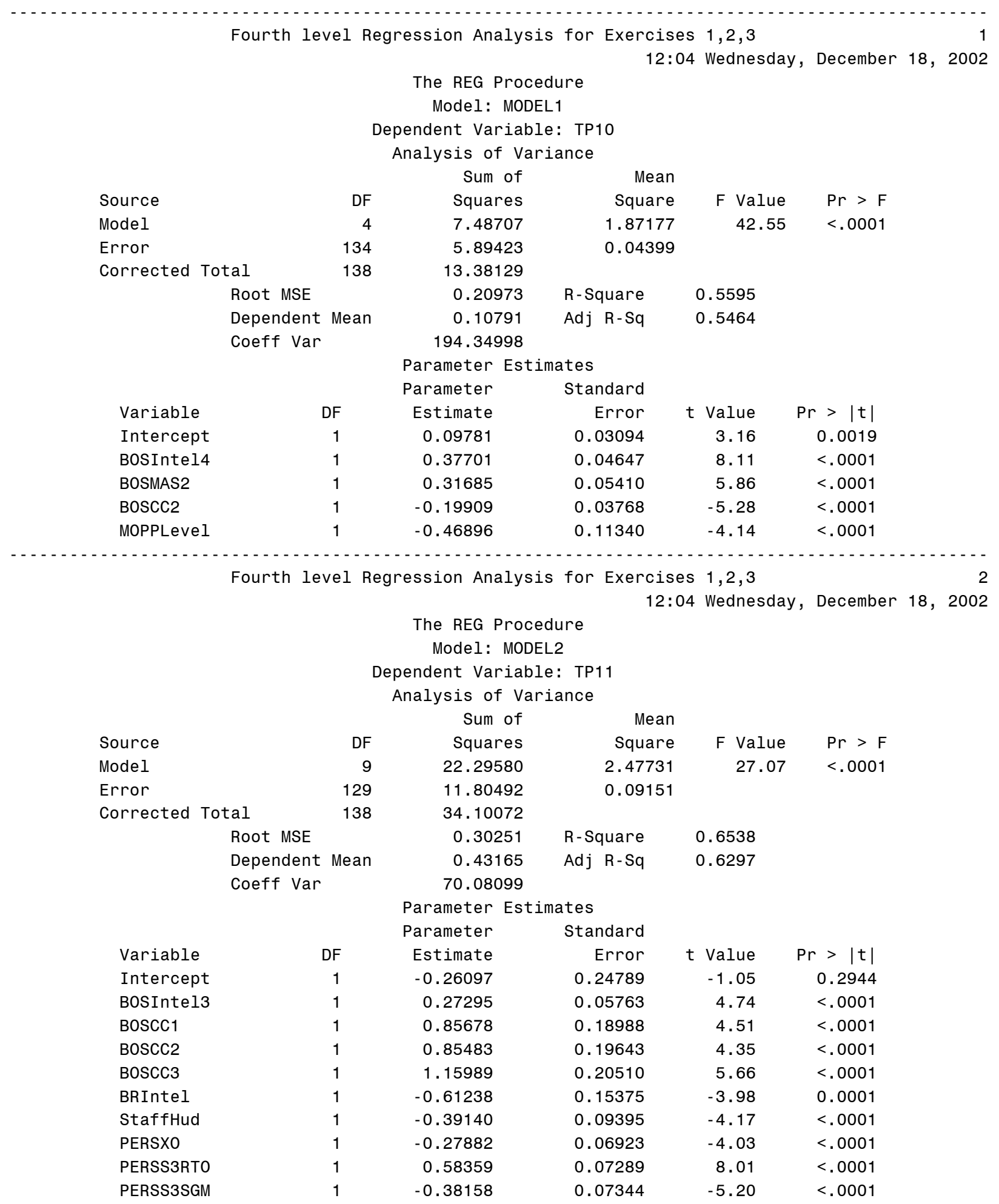


Fourth level Regression Analysis for Exercises 1,2,3

The REG Procedure

12:04 Wednesday, December 18, 2002

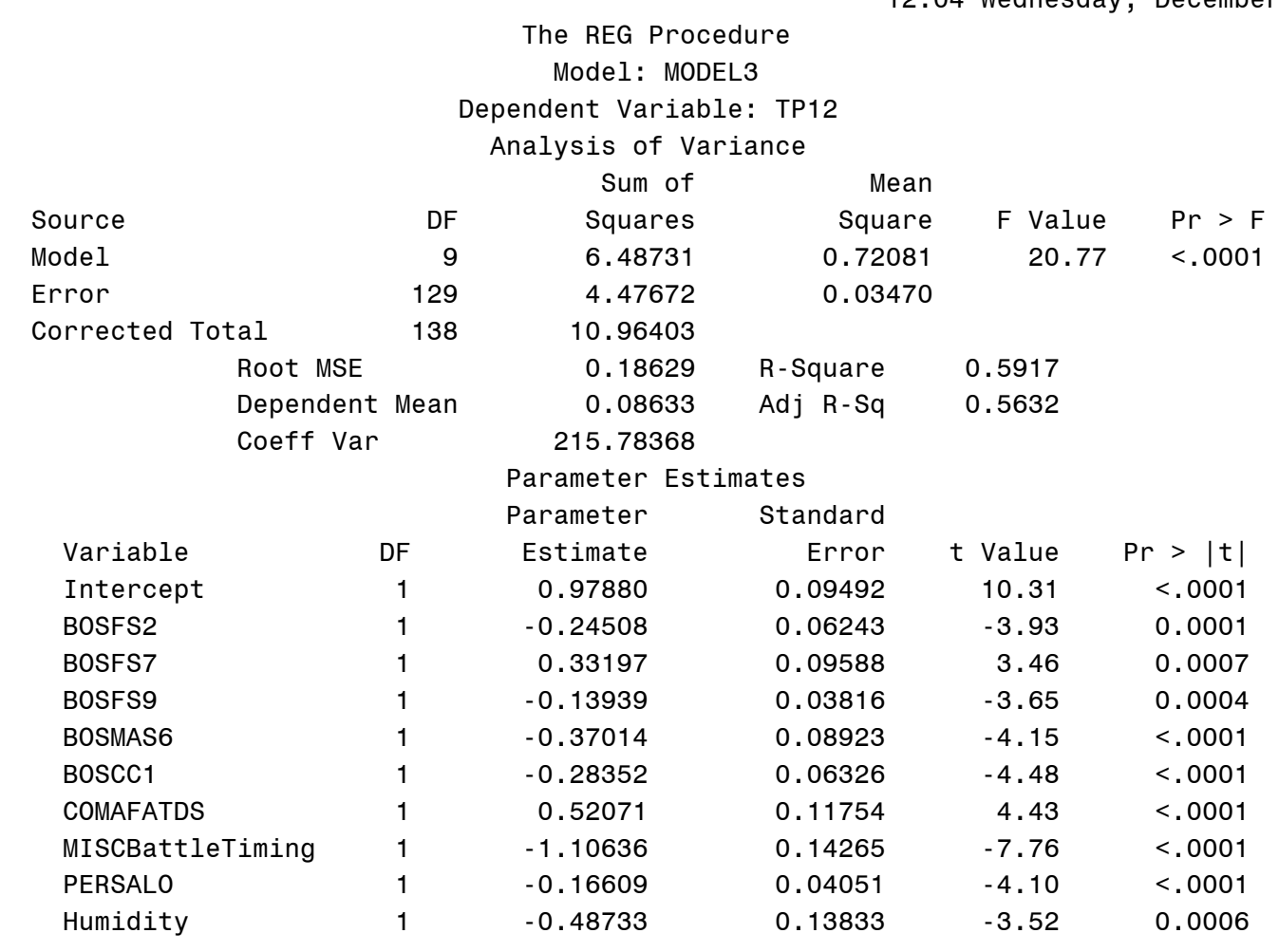

Fourth level Regression Analysis for Exercises 1,2,3

12:04 Wednesday, December 18, 2002

The REG Procedure

Model: MODEL4

Dependent Variable: TP15

Analysis of Variance

Sum of Mean

$\begin{array}{lr}\text { Source } & \text { DF } \\ \text { Model } & 4 \\ \text { Error } & 134 \\ \text { Corrected Total } & 138 \\ & \\ & \text { Root MSE } \\ & \text { Dependent Mean } \\ & \text { Coeff Var }\end{array}$

\section{DF}

4

38

Squares

6.10327

5.68090

11.78417

0.20590

0.09353

220.15451

Parameter Estimates

Variable
Intercept
BOSIntel4
BOSFS2
MISCBattleTiming
PERSXO

DF

Parameter

Estimate

$-0.25933$

0.32348

0.21054

0.49106

0.13550
F Value $\quad \mathrm{Pr}>\mathrm{F}$

$35.99<.0001$

1.52582

0.04239

R-Square

0.5179

0.5035

Adj R-Sq

Standard

Error

0.05076

0.04700

t Value

$\mathrm{Pr}>|\mathrm{t}|$

$-5.11<.0001$

$6.88<.0001$

$4.24<.0001$

$4.63<.0001$

$3.36 \quad 0.0010$ 
Fourth level Regression Analysis for Exercises 1,2,3

The REG Procedure

12:04 Wednesday, December 18, 2002

\begin{tabular}{|c|c|c|c|c|c|c|}
\hline \multicolumn{7}{|c|}{$\begin{array}{c}\text { The REG Procedure } \\
\text { Model: MODEL5 } \\
\text { ependent Variable: TP21 } \\
\text { Analysis of Variance }\end{array}$} \\
\hline \multirow{2}{*}{\multicolumn{2}{|c|}{ Source }} & & Sum of & Mean & & \\
\hline & & DF & Squares & Square & F Value & $\mathrm{Pr}>\mathrm{F}$ \\
\hline \multicolumn{2}{|l|}{ Model } & 4 & 14.17579 & 3.54395 & 41.27 & $<.0001$ \\
\hline \multicolumn{2}{|l|}{ Error } & 134 & 11.50766 & 0.08588 & & \\
\hline \multirow[t]{6}{*}{ Corrected $\mathrm{T}$} & Total & 138 & 25.68345 & & & \\
\hline & Root MSE & & 0.29305 & R-Square & 0.5519 & \\
\hline & Dependent & Mean & 0.24460 & $\operatorname{Adj~R-Sq}$ & 0.5386 & \\
\hline & Coeff Var & & 119.80558 & & & \\
\hline & & & Parameter Est & ates & & \\
\hline & & & Parameter & Standard & & \\
\hline \multicolumn{2}{|l|}{ Variable } & DF & Estimate & Error & t Value & $\operatorname{Pr}>|t|$ \\
\hline \multicolumn{2}{|l|}{ Intercept } & 1 & 0.47554 & 0.07136 & 6.66 & $<.0001$ \\
\hline \multicolumn{2}{|l|}{ BOSFS9 } & 1 & -0.32588 & 0.05679 & -5.74 & $<.0001$ \\
\hline \multicolumn{2}{|l|}{ BOSMAS5 } & 1 & -0.12732 & 0.05531 & -2.30 & 0.0229 \\
\hline \multicolumn{2}{|c|}{ MISCBattleTempo } & 1 & -0.97917 & 0.13160 & -7.44 & $<.0001$ \\
\hline \multicolumn{2}{|c|}{ MISCInfoops } & 1 & 0.45966 & 0.13416 & 3.43 & 0.0008 \\
\hline
\end{tabular}

Fourth level Regression Analysis for Exercises 1,2,3

12:04 Wednesday, December 18, 2002

The REG Procedure

Model: MODEL6

Dependent Variable: TP23

Analysis of Variance

Sum of Mean

$\begin{array}{lr}\text { Source } & \text { DF } \\ \text { Model } & 4 \\ \text { Error } & 134 \\ \text { Corrected Total } & 138 \\ & \\ & \text { Root MSE } \\ & \text { Dependent Mean } \\ & \text { Coeff Var }\end{array}$

\section{DF}

4
Squares

9.11062

\begin{abstract}
4.27067
\end{abstract}
13.38129

0.17852

0.10791

165.43190

Parameter Estimates

Variable

Intercept

BOSMAS6

BRIntel

BRMAS

StaffHud

DF
1
1
1
1
1

$\begin{array}{rr}\text { Parameter } & \text { Standard } \\ \text { Estimate } & \text { Error } \\ 0.39034 & 0.11841 \\ 1.01120 & 0.06566 \\ 0.31699 & 0.08691 \\ -0.71853 & 0.11348 \\ 0.20794 & 0.05561\end{array}$

Square

2.27766

0.03187

R-Square

0.6808

0.6713

F Value $\quad \mathrm{Pr}>\mathrm{F}$

$71.47<.0001$

Adj R-Sq

$\begin{array}{rr}\mathrm{t} \text { Value } & \mathrm{Pr}>|\mathrm{t}| \\ 3.30 & 0.0013 \\ 15.40 & <.0001 \\ 3.65 & 0.0004 \\ -6.33 & <.0001 \\ 3.74 & 0.0003\end{array}$


Fourth level Regression Analysis for Exercises 1,2,3

The REG Procedure

Model: MODEL7

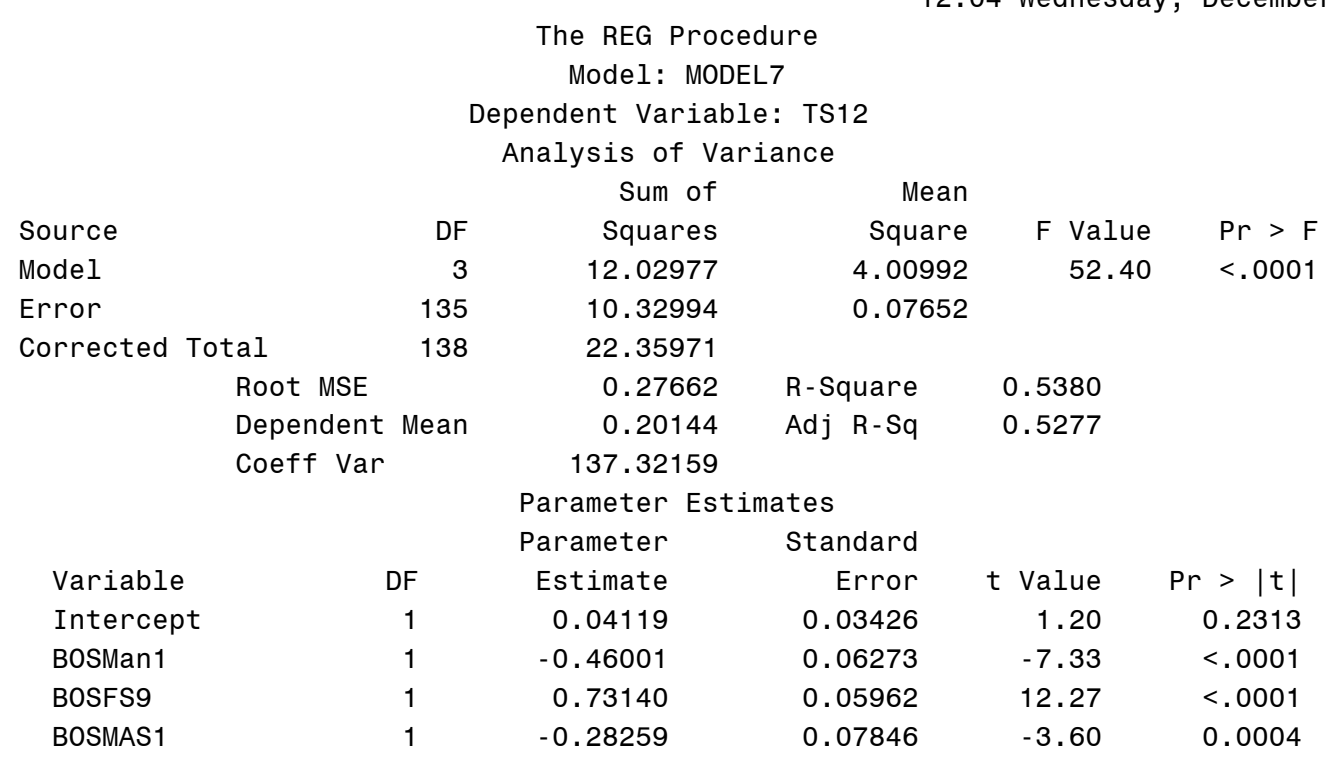

Fourth level Regression Analysis for Exercises 1,2,3

The REG Procedure

12:04 Wednesday, December 18, 2002

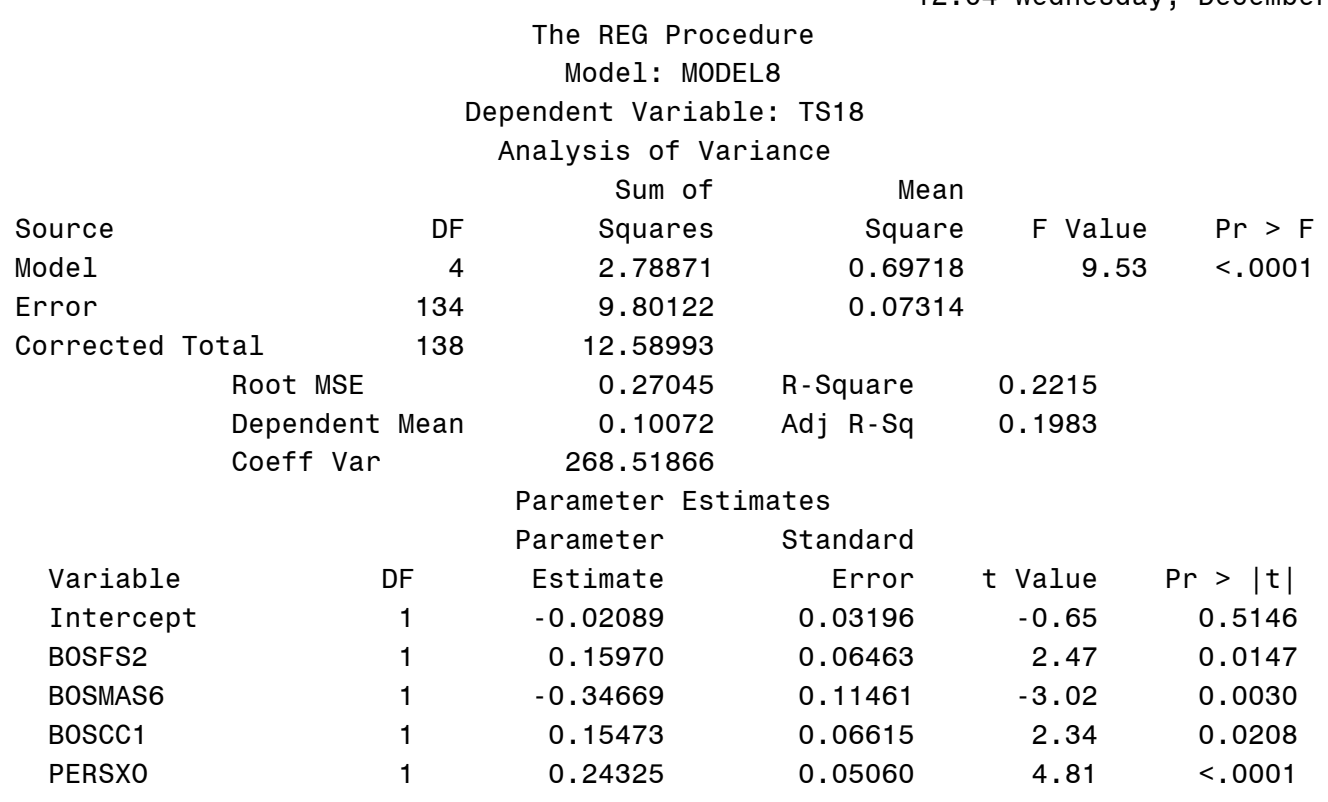


Fourth level Regression Analysis for Exercises 1,2,3

The REG Procedure

12:04 Wednesday, December 18, 2002 Model: MODEL9

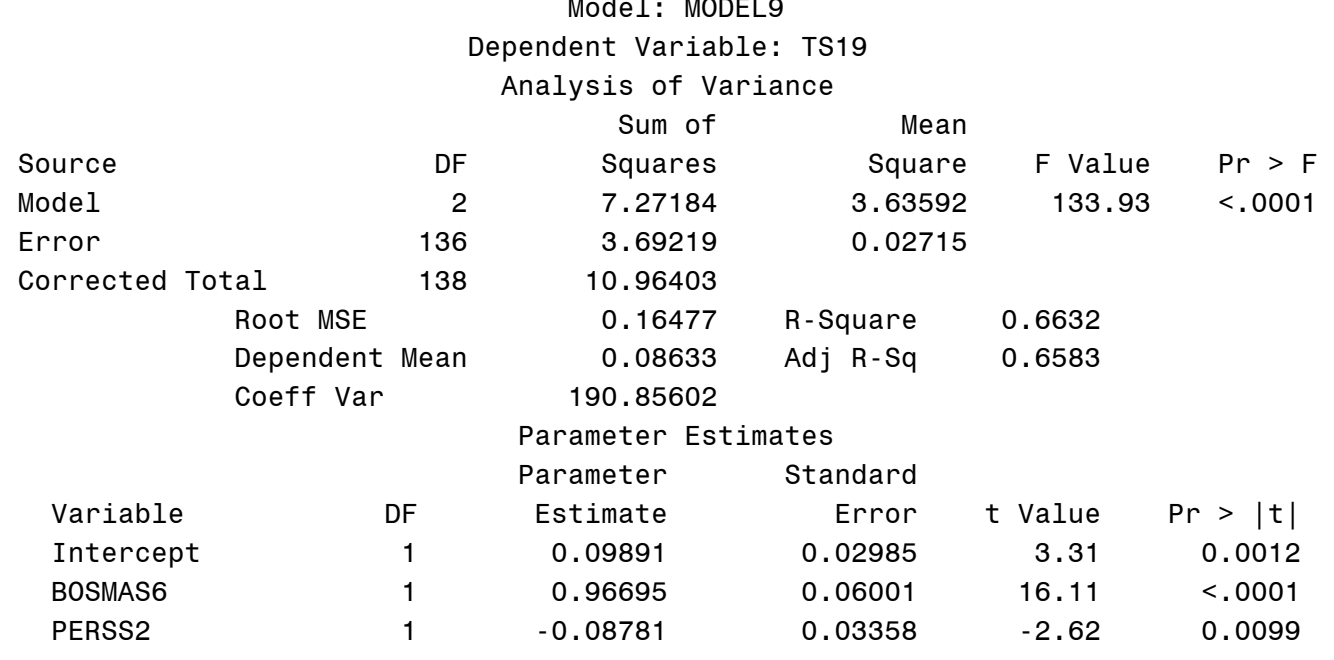

Fourth level Regression Analysis for Exercises 1,2,3

The REG Procedure

Model: MODEL10

Dependent Variable: TS23

Analysis of Variance

\begin{tabular}{lr} 
Source & DF \\
Model & 10 \\
Error & 128 \\
Corrected Total & 138 \\
\multicolumn{1}{l}{} & \\
& Root MSE \\
& Dependent Mean \\
& Coeff Var
\end{tabular}

Variable

Intercept

BoSIntel2

BosIntel3

BosMan2

BOSFS9

BOSMAS4

BOSCC2

MISCBattleTiming

MISCInfoOps

PERSS3RTO

Humidity

\section{Sum of}

Squares

8.38067

7.28840

15.66906

$$
0.23862
$$

0.12950

184.26947

Parameter Estimates

Parameter

Estimate

$-0.50056$

0.12448

0.20154

$-0.23144$

$-0.13123$

0.58920

0.18261

$-0.52596$

0.26852

0.35949

0.58127
Standard

\section{Error}

0.15867

0.05538

0.05547

0.08071

0.05672

0.11780

0.05731

0.15863

0.11073

0.06329

0.18105
F Value $\quad \mathrm{Pr}>\mathrm{F}$

$14.72<.0001$

0.5349

0.4985

$\begin{array}{rr}t \text { Value } & \mathrm{Pr}>|\mathrm{t}| \\ -3.15 & 0.0020 \\ 2.25 & 0.0263 \\ 3.63 & 0.0004 \\ -2.87 & 0.0048 \\ -2.31 & 0.0223 \\ 5.00 & <.0001 \\ 3.19 & 0.0018 \\ -3.32 & 0.0012 \\ 2.43 & 0.0167 \\ 5.68 & <.0001 \\ 3.21 & 0.0017\end{array}$


Fourth level Regression Analysis for Exercises 1,2,3

12:04 Wednesday, December 18, 2002

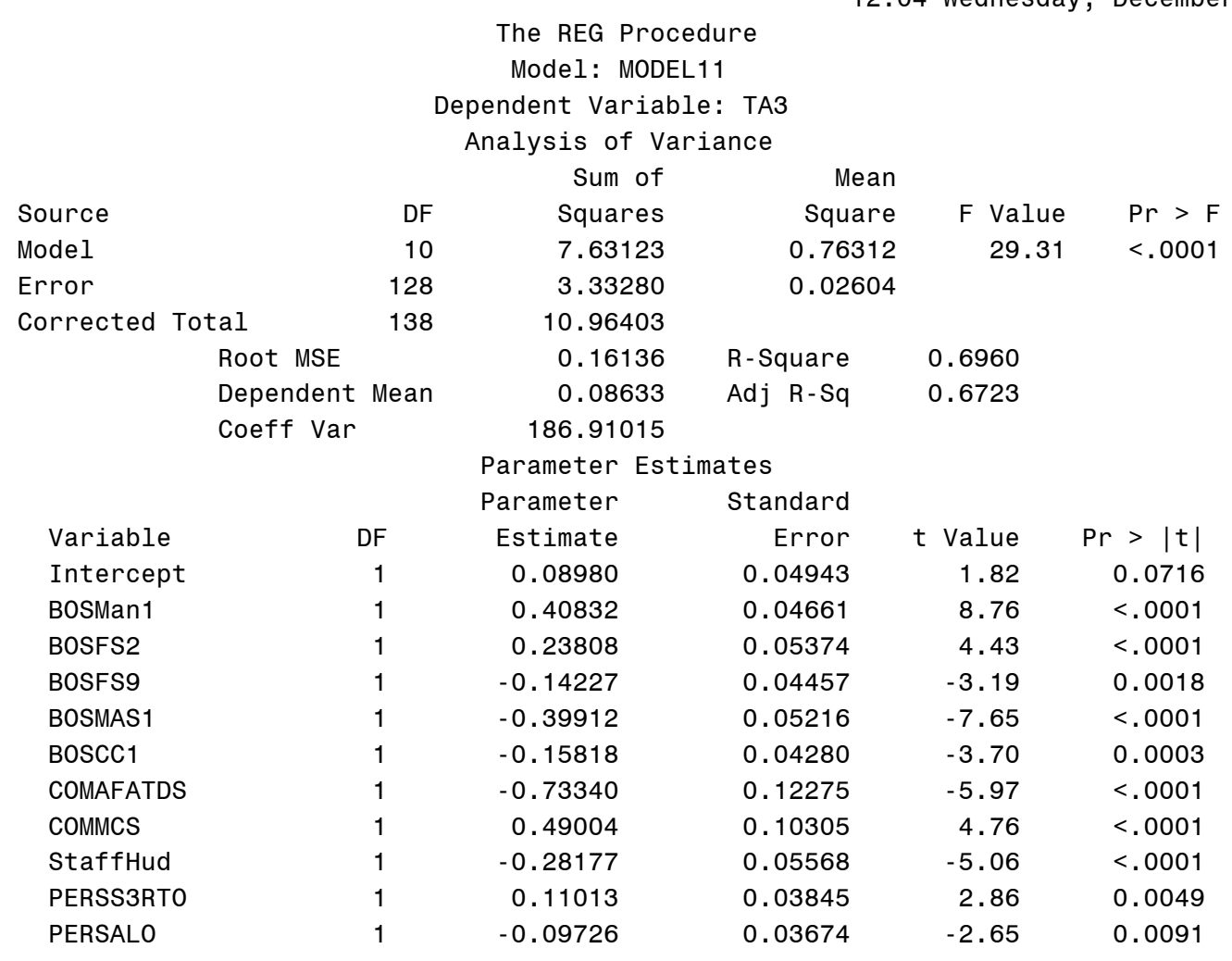


Fourth level Regression Analysis for Exercises 1,2,3

12:04 Wednesday, December 18, 2002

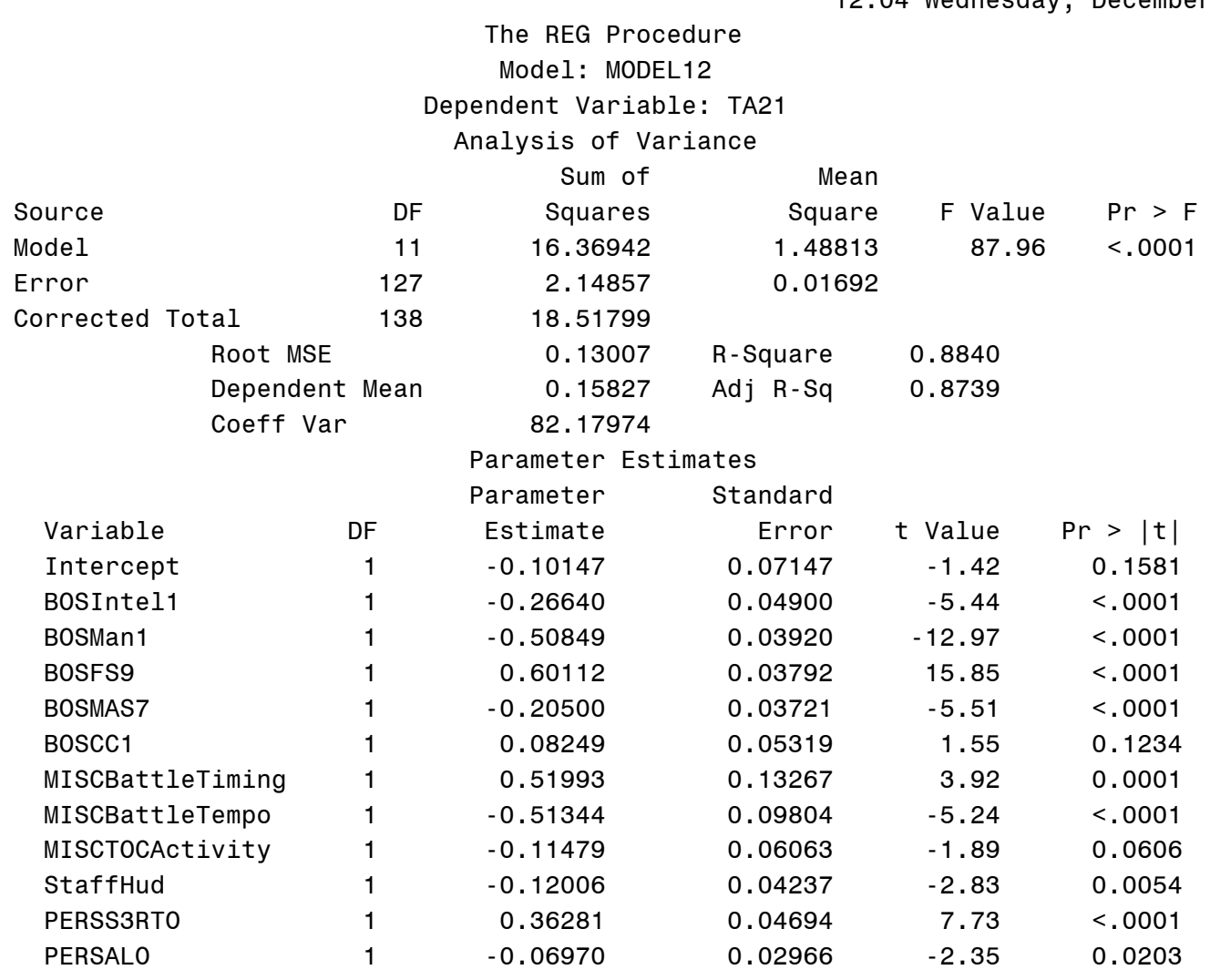


Fourth level Regression Analysis for Exercises 1,2,3

12:04 Wednesday, December 18, 2002

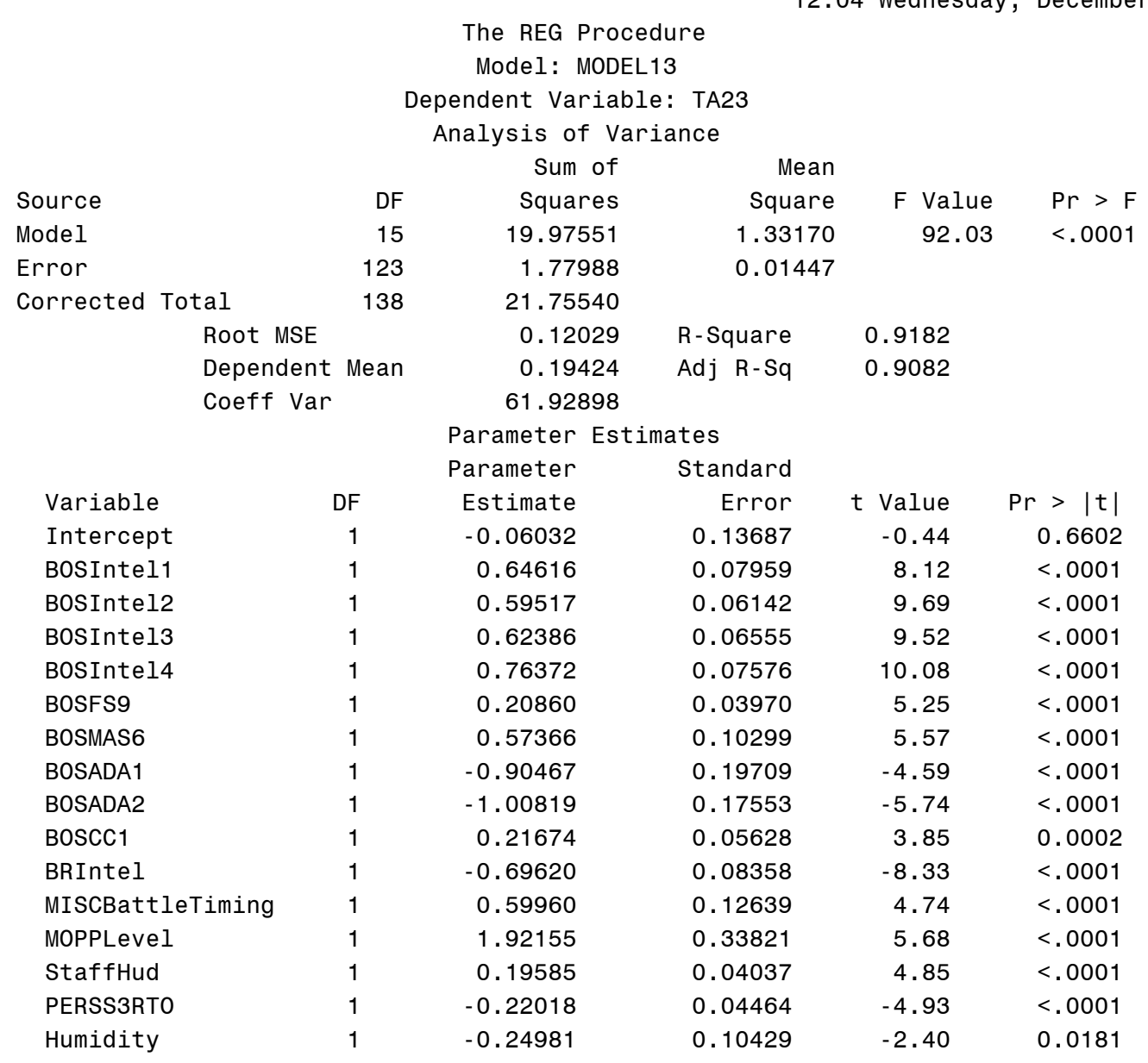


Fourth level Regression Analysis for Exercises 1,2,3

The REG Procedure

Model: MODEL14

Dependent Variable: Mission1

Analysis of Variance

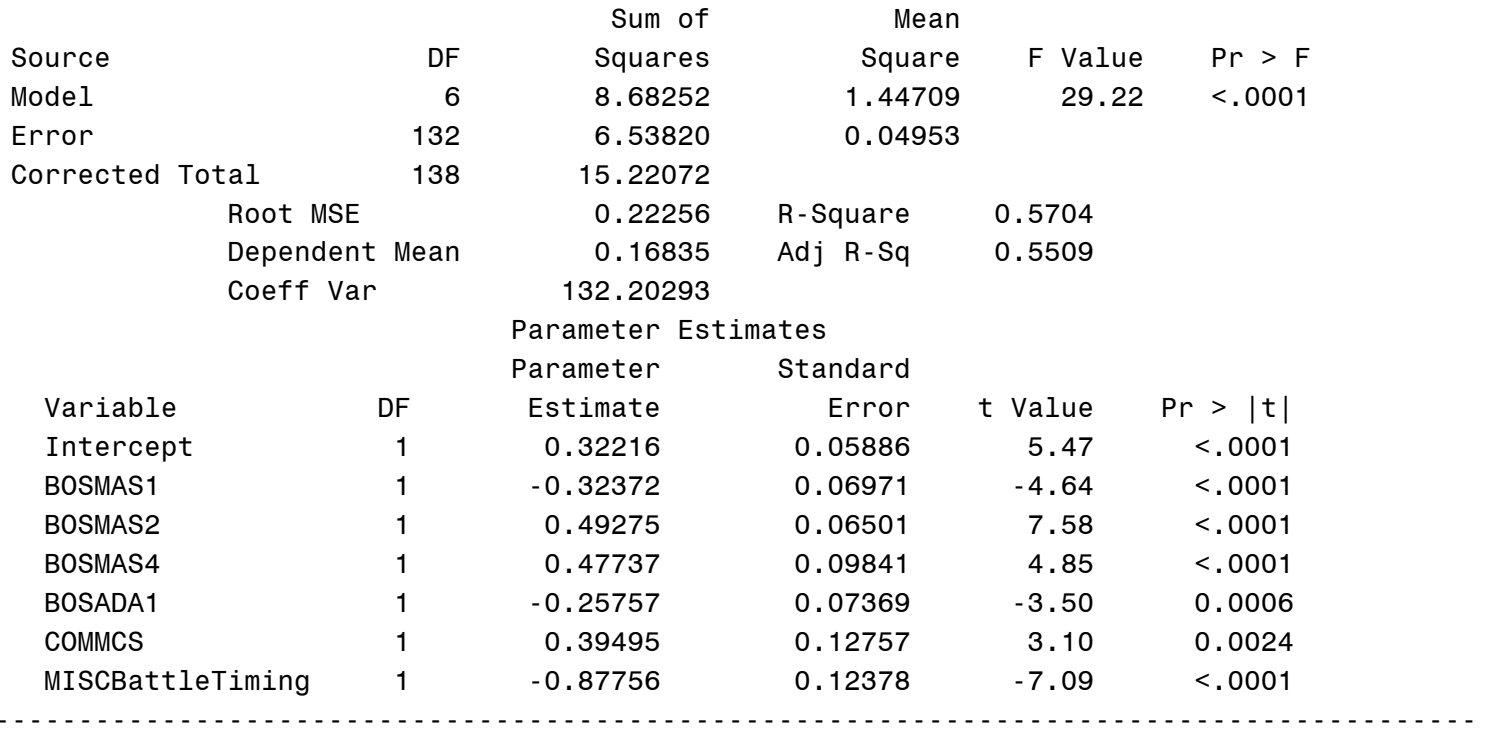

Fourth level Regression Analysis for Exercises 1,2,3

The REG Procedure

Model: MODEL15

Dependent Variable: Mission2

Analysis of Variance

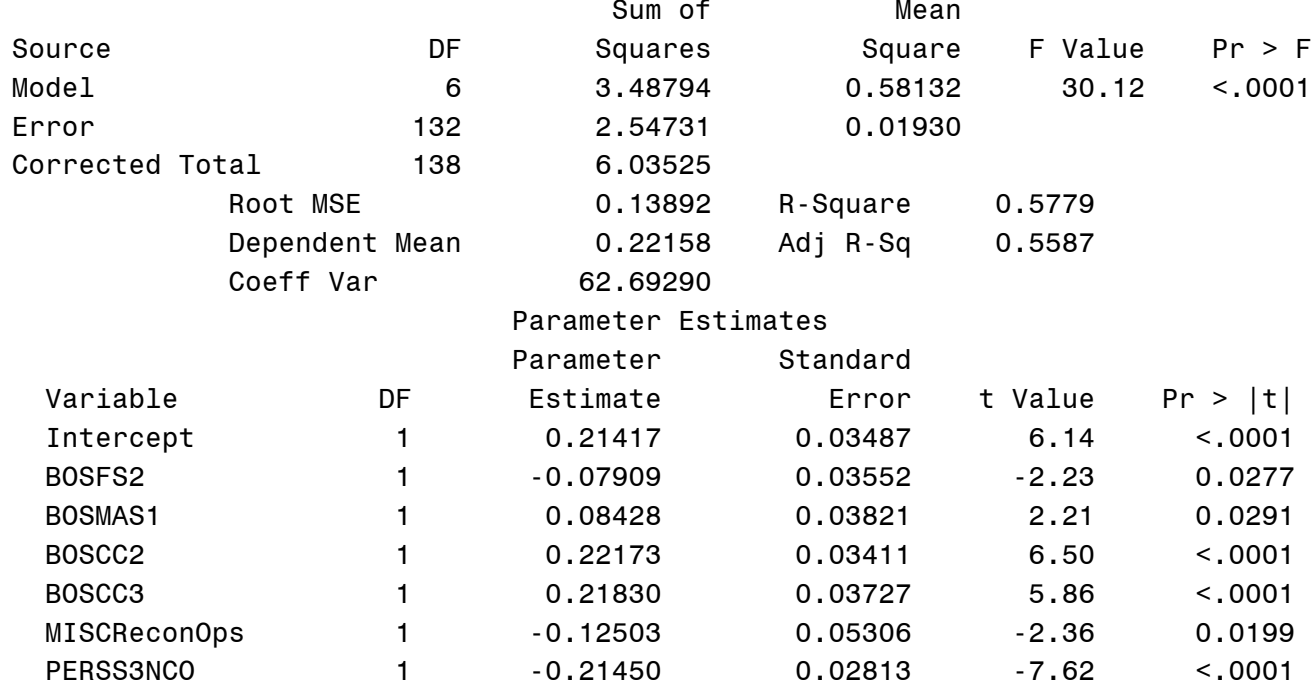


Fourth level Regression Analysis for Exercises 1,2,3

The REG Procedure

Model: MODEL16

Dependent Variable: Mission3

Analysis of Variance

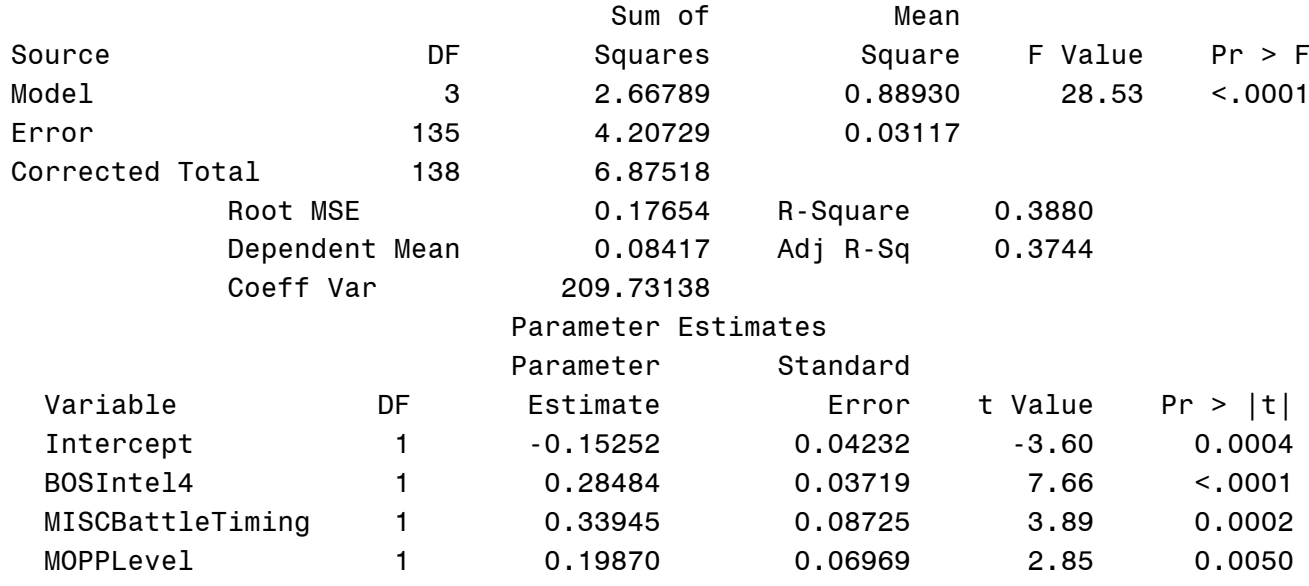

Fourth level Regression Analysis for Exercises 1,2,3

The REG Procedure

Model: MODEL17

Dependent Variable: Mission5

Analysis of Variance

$\begin{array}{lr}\text { Source } & \text { DF } \\ \text { Model } & 6 \\ \text { Error } & 132 \\ \text { Corrected Total } & 138 \\ & \\ & \text { Root MSE } \\ & \text { Dependent Mean } \\ & \text { Coeff Var }\end{array}$

$\begin{array}{rrrl}\text { Sum of } & \text { Mean } & & \\ \text { Squares } & \text { Square } & \mathrm{F} \text { Value } & \mathrm{Pr}>\mathrm{F} \\ 3.87057 & 0.64510 & 33.54 & <.0001 \\ 2.53849 & 0.01923 & & \end{array}$

$\begin{array}{lr}\text { Variable } & \text { DF } \\ \text { Intercept } & 1 \\ \text { BOSIntel4 } & 1 \\ \text { BOSMan1 } & 1 \\ \text { BOSADA2 } & 1 \\ \text { MISCBattleTiming } & 1 \\ \text { MOPPLevel } & 1 \\ \text { PERSS3NCO } & 1\end{array}$

6.40906
0.13868

$\begin{array}{lll}0.13868 & \text { R-Square } & 0.6039 \\ 0.07050 & \text { Adj R-Sq } & 0.5859\end{array}$

196.69330

Parameter Estimates

$\begin{array}{rrrr}\text { Parameter } & \text { Standard } & & \\ \text { Estimate } & \text { Error } & \mathrm{t} \text { Value } & \mathrm{Pr}>|\mathrm{t}| \\ -0.65542 & 0.06045 & -10.84 & <.0001 \\ 0.09927 & 0.03492 & 2.84 & 0.0052 \\ 0.33456 & 0.04731 & 7.07 & <.0001 \\ 0.38865 & 0.04469 & 8.70 & <.0001 \\ 1.27217 & 0.11413 & 11.15 & <.0001 \\ -0.72215 & 0.11272 & -6.41 & <.0001 \\ 0.11363 & 0.03259 & 3.49 & 0.0007\end{array}$


Appendix N - Linear Regression Expressions for the 17 DV Models

\# 1 - Primary Task10. Destruction, capture, or bypass of enemy force.

\begin{tabular}{|c|c|c|c|c|c|c|}
\hline & Variable & Intercept & BOSIntel4 & BOSMAS2 & BOSCC2 & MOPPLevel \\
\hline TP10 & Beta Weight & 0.09781 & 0.37701 & 0.31685 & -0.19909 & -0.46896 \\
\hline
\end{tabular}

\# 2 - Primary Task11. Fixing enemy in position.

\begin{tabular}{|c|c|c|c|c|c|c|}
\hline & Variable & Intercept & BOSIntel3 & BOSCC1 & BOSCC2 & BOSCC3 \\
\hline TP11 & Beta Weight & -0.26097 & 0.27295 & 0.85678 & 0.85483 & 1.15989 \\
\hline & Variable & & BRIntel & StaffHud & PERSXO & PERSS3RTO \\
\hline & Beta Weight & & -0.61668 & -0.3914 & -0.27882 & 0.58359 \\
\hline & Variable & & PERSS3SGM & & & \\
\hline & Beta Weight & & -0.46158 & & & \\
\hline
\end{tabular}

\# 4 - Primary Task15. Destruction of first echelon forces.

\begin{tabular}{|c|c|c|c|c|c|c|}
\hline & Variable & Intercept & BOS Intel4 & BOSFS2 & $\begin{array}{c}\text { MISCBattle } \\
\text { Timing }\end{array}$ & PERS XO \\
\hline TP15 & Beta Weight & -0.61941 & 0.41048 & 0.21054 & 0.49106 & 0.1355 \\
\hline
\end{tabular}

\# 5 - Primary Task 21. Peacekeeping operations.

\begin{tabular}{|c|c|c|c|c|c|c|}
\hline & Variable & Intercept & BOSFS9 & BOS MAS5 & $\begin{array}{c}\text { MISC Battle } \\
\text { Tempo }\end{array}$ & $\begin{array}{c}\text { MISC Info } \\
\text { Ops }\end{array}$ \\
\hline TP21 & Beta Weight & 0.47554 & -0.36188 & -0.12732 & -0.97917 & 0.45966 \\
\hline
\end{tabular}

\# 6 - Primary Task23. Planning

\begin{tabular}{|c|c|c|c|c|c|c|}
\hline & Variable & Intercept & BOS MAS6 & BR Intel & BR MAS & Staff Hud \\
\hline TP23 & Beta Weight & 0.39034 & 1.0112 & 0.31699 & 0.71853 & 0.20794 \\
\hline
\end{tabular}

\# 7 - Secondary Task12. Synchronization with supporting forces.

\begin{tabular}{|c|c|c|c|c|c|}
\hline & Variable & Intercept & BOS Man1 & BOS FS9 & BOS MAS1 \\
\hline TS12 & Beta Weight & 0.04119 & -0.46001 & 0.7314 & -0.35619 \\
\hline
\end{tabular}

\# 8 - Secondary Task18. Deception activities.

\begin{tabular}{|c|c|c|c|c|c|c|}
\hline & Variable & Intercept & BOS FS2 & BOS MAS6 & BOS CC1 & PERS XO \\
\hline TS18 & Beta Weight & -0.02089 & 0.1597 & -0.34669 & 0.15473 & 0.61261 \\
\hline
\end{tabular}

\# 9 - Secondary Task19. Rear operations.

\begin{tabular}{|c|c|c|c|c|}
\hline & Variable & Intercept & BOS MAS6 & PERS S2 \\
\hline TS19 & Beta Weight & 0.09891 & 0.96695 & -0.08781 \\
\hline
\end{tabular}

\# 10 - Secondary Task23. Planning

\begin{tabular}{|c|c|c|c|c|c|c|}
\hline & Variable & Intercept & BOS Intel2 & BOS Intel3 & BOS Man2 & BOSFS9 \\
\hline TS23 & Beta Weight & -0.50056 & 0.12448 & 0.20154 & -0.66144 & -0.1313 \\
\hline & Variable & & BOS MAS4 & BOS CC2 & MISC Battle Timing & MISC Info Ops \\
\hline & Beta Weight & & 0.5892 & 0.18261 & -0.56196 & 0.26861 \\
\hline & Variable & & PERS S3RTO & Humidity & & \\
\hline & Beta Weight & & 0.35949 & 0.58127 & & \\
\hline
\end{tabular}


\# 11 - Tertiary Task3. Movement to the line of departure.

\begin{tabular}{|c|c|c|c|c|c|c|}
\hline & Variable & Intercept & BOS Man1 & BOS FS2 & BOS FS9 & BOS MAS1 \\
\hline TA3 & Beta Weight & 0.0898 & 0.40832 & 0.66808 & -0.14227 & -0.39912 \\
\hline & Variable & & BOS CC1 & COM AFATDS & COM MCS & Staff Hud \\
\hline & Beta Weight & & -0.15818 & -0.7414 & 0.49004 & -0.35177 \\
\hline & Variable & & PERS S3RTO & PERS ALO & & \\
\hline & Beta Weight & & 0.11013 & -0.09726 & & \\
\hline
\end{tabular}

\# 12 - Tertiary Task21. Peacekeeping operations.

\begin{tabular}{|c|c|c|c|c|c|c|}
\hline & Variable & Intercept & BOS Intel1 & BOS Man1 & BOS FS9 & BOS MAS7 \\
\hline TA21 & Beta Weight & -0.10147 & -0.2664 & -0.50849 & 0.60112 & -0.205 \\
\hline & Variable & & BOS CC1 & MISC Battle Timing & $\begin{array}{c}\text { MISC Battle } \\
\text { Tempo }\end{array}$ & $\begin{array}{c}\text { MISC TOC } \\
\text { Activity }\end{array}$ \\
\hline & Beta Weight & & 0.08249 & 0.51993 & -0.51344 & -0.11479 \\
\hline & Variable & & Staff Hud & PERS S3RTO & & \\
\hline & Beta Weight & & -0.12006 & 0.36351 & & \\
\hline
\end{tabular}

\# 13 - Tertiary Task23. Planning

\begin{tabular}{|c|c|c|c|c|c|c|}
\hline & Variable & Intercept & BOS Intel1 & BOS Intel2 & BOS Intel3 & BOS Intel4 \\
\hline TA23 & Beta Weight & -0.06032 & 0.64616 & 0.59517 & 0.66686 & 0.76372 \\
\hline & Variable & & BOS FS9 & BOS MAS6 & BOS ADA1 & BOS ADA2 \\
\hline & Beta Weight & & 0.2086 & 0.66366 & -0.90467 & -1.00819 \\
\hline & Variable & & BOS CC1 & BR Intel & MISC Battle Timing & MOPP Level \\
\hline & Beta Weight & & 0.21674 & -0.6962 & 0.5996 & 1.92155 \\
\hline & Variable & & Staff Hud & PERS S3RTO & Humidity & \\
\hline & Beta Weight & & 0.19585 & -0.22018 & -0.24981 & \\
\hline
\end{tabular}

\# 14 - Mission1. Pre Operations Planning

\begin{tabular}{|l|c|c|c|c|c|c|}
\hline & Variable & Intercept & BOS MAS1 & BOS MAS2 & BOS MAS4 & BOS ADA1 \\
\hline MISSION1 & Beta Weight & 0.32216 & -0.41072 & 0.49275 & 0.47737 & -0.61766 \\
\hline & Variable & & COM MCS & MISC Battle Timing & & \\
\hline & Beta Weight & & 0.39495 & -0.87756 & & \\
\hline
\end{tabular}

\# 15 - Mission2. Movement to Contact

\begin{tabular}{|c|c|c|c|c|c|c|}
\hline & Variable & Intercept & BOSFS2 & BOSMAS1 & BOSCC2 & BOSCC3 \\
\hline MISSION2 & Beta Weight & 0.21417 & -0.07909 & 0.08561 & 0.22173 & 0.2183 \\
\hline & Variable & & MISCReconOps & PERSS3NCO & & \\
\hline & Beta Weight & & -0.13503 & -0.2145 & & \\
\hline
\end{tabular}

\# 16 - Mission3. Attack

\begin{tabular}{|c|c|c|c|c|c|}
\hline & Variable & Intercept & BOSIntel4 & MISCBattleTiming & MOPPLevel \\
\hline MISSION3 & Beta Weight & -0.15612 & 0.35484 & 0.41945 & 0.1987 \\
\hline
\end{tabular}

\# 17 - Mission5. River Crossing

\begin{tabular}{|l|c|c|c|c|c|c|}
\hline & Variable & Intercept & BOSIntel4 & BOSMan1 & BOSADA2 & MISCBattleTiming \\
\hline MISSION5 & Beta Weight & -0.65542 & 0.09927 & 0.41456 & 0.46865 & 1.27217 \\
\hline & Variable & & MOPPLevel & PERSS3NCO & & \\
\hline & Beta Weight & & -0.72215 & 0.11363 & & \\
\hline
\end{tabular}


Appendix O - Description of the CoHOST Simulation.

The CoHOST computer simulation models used in this dissertation were the result of a previous research study conducted by the Army. Their use in this dissertation represents how continued utilization can be made of computer simulations developed for other purposes. Considering the cost and time required to develop simulations such as CoHOST, their use in subsequent efforts like COMPASS represent a continued payback to the model developers in terms of utilization and effectiveness of the models.

\section{Background.}

In the late 1990's the U. S. Army was engaged in a number of design initiatives for improving the operation of command and control centers. The Human Research and Engineering Directorate of the U.S. Army Research Laboratory (HRED-ARL) was commissioned to develop a series of computer simulations to replicate existing configurations and to investigate potential configurations of battalion and brigade TOCs. A series of computer models called Computer modeling Of Human Operator System Tasks (CoHOST) (Middlebrooks et al., 1999b) was written and results were developed that addressed some of the questions being posed in regard to the modernization of TOCs especially in regard to the development of new digital communications systems for use in it. These new digital communications systems are at the heart of a change of the operational paradigm in command and control TOCs that the Army is now undergoing. Figure 55 illustrates the components in a typical Army battalion level TOC. The rectangles represent the different vehicles in the unit. Personnel are listed inside the vehicle box and the communication systems for each vehicle are listed beside it. This select group of 24 people, along with the vehicles and communications systems they use, represent those people directly concerned with battlespace management and it is this working group that is modeled in CoHOST. 


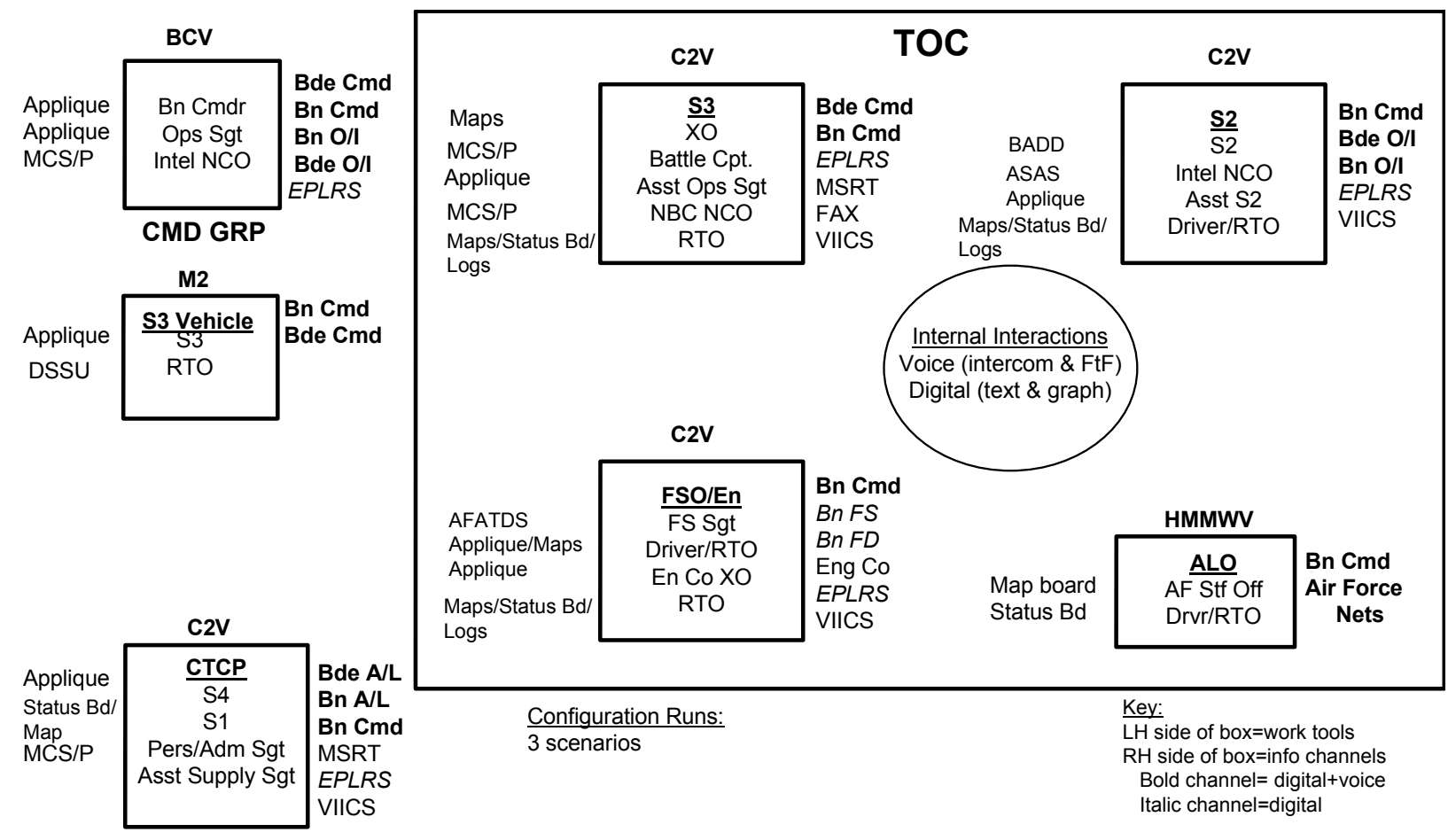

Figure 55 - TOC Diagram

(Middlebrooks et al., 1999b)

This project addressed the ability of the human component of the TOC system to perform under a new operational paradigm. As communications systems are passing greater and more accurate volumes of information in real time the question to be asked is "can the soldier absorb this information and be able to react to the stream of data being presented to him/her also in real time?" Can these activities be performed while the vehicle is moving over extended distances and during extended time periods? Do the combined effects of fatigue, noise, and vibration that are sustained by an operator cause that person to become what is described as a "cognitive causality"? The CoHOST computer models and project looked at some of these issues and provided findings that addressed some of these questions.

\section{Application of a Taxonomy of Human Performance.}

A With work first published in 1954, Edwin Fleishman (Fleishman, 1975) began what would turn into a lifetime effort focused on the development of taxonomic descriptors of work performance. The resulting taxonomy (Fleishman and Quaintance, 1984) presents a set of skills 
and abilities that can be used to describe human performance characteristics in any general work situation. Fleishman stated (Fleishman, 1975; Fleishman, 1978) that some kind of taxonomy of human performance is required which provides an integrative framework and common language applicable to a variety of basic and applied areas. He goes on further to state that it does appear that predictions and generalizations about human performance may be enhanced by some linkage of task classification systems based on human abilities and task characteristics. In 1988 Fleishman (Fleishman, 1988) quoted earlier 1947 work by others with the observation that apparatus tests of perceptual motor abilities had been found to have considerable validity for predicting the success of pilots and bombardiers in getting through training during World War II.

Comments by others point out that Fleishman's work tends to be neglected in the mainstream of human information processing research, perhaps due to the fact that the skills and abilities in the taxonomy are only based on factor analyses and are void of any process description. However, the tests used by Fleishman to develop the taxonomy belong to the same type of performance tests that are studied in Wickens' more accepted dual task experiments and therefore deserve closer scrutiny (Sanders, 1997). There have been many attempts in the human factors community to develop similar descriptions of human performance and while this taxonomy may not be generally accepted by all for every attempt at evaluations of human performance, it does provide a set of skill and ability descriptors that are heavily weighted to cognitive performance.

Previous work at the U.S. Army Research Laboratory (ARL) (Knapp, 1996a; Knapp, 1996b; Knapp, Johnson, Barnette, Wojciechowski, Kilduff, Bird, and Plott, 1997c; Schipani et al., 1998), and the U.S. Army Research Institute (ARI) (Seven, Akman, Muckler, Knapp, and Burnstein, 1991) identified this job skill and ability taxonomy (Fleishman, 1984; Fleishman and Quaintance, 1984) and stated that it showed promise to provide the basis for workload scaling in Army battalion level command and control modeling efforts. This taxonomy consists of 52 skills and abilities that include mental processing, sensory perception and fine and gross motor skills. The selection of this taxonomy was influenced by its detailed decomposition of mental abilities and the existence of behaviorally anchored rating scales (Knapp et al., 1997c). Subsequently, 50 of the 52 skills and abilities from the taxonomy were adopted to support work that was performed for the U.S. Army Intelligence Center at Fort Huachuca, Arizona. This work sought to determine basic soldier training requirements needed to provide requisite skills and 
abilities for various Military Occupational Specialty (MOS) at the Intelligence Center's basic soldier training units. As shown in Figure 56, the taxonomy was grouped into eight demand categories of reasoning, speed-loaded, conceptual, communications, visual, auditory, psychomotor, and gross motor. Knapp stated that (Knapp et al., 1997c) “Each skill and ability has an associated behaviorally anchored rating scale that ranges from " 1 " for a very low level demand, to "7" for the highest demand. Definitions for all 50 skills and abilities, along with their behaviorally anchored scales, is documented in a separate review of this taxonomy (Seven et al., 1991)."

Cognitive Skill and Experience Clusters

Communication
1. Oral Comprehension
2. Written Comprehension
3. Oral Expression
4. Written Expression

Conceptual (1.20)
6. Memorization
7. Oroblem Sensitivity
8. Fluency of Ideas
9. Flexibility of Closure
10. Selective Attention
11. Spatial Orientation
12. Visualization

Reasoning (1.25)

13. Inductive Reasoning

14. Category Flexibility

15. Deductive Reasoning

16. Information Ordering

17. Mathematical Reasoning

18. Number Facility

Cognitive skills weighted in the model
Perceptual-Motor Ability Clusters

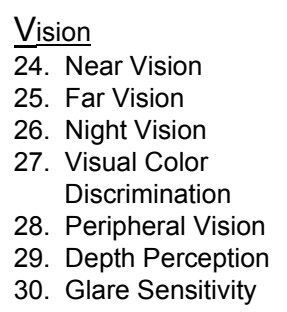

Psychomotor
34. Control Precision
35. Rate Control
36. Wrist-Finger Speed
37. Finger Dexterity
38. Manual Dexterity
39. Arm-hand Steadiness
40. Multi-Limb Coordination

\section{Gross Motor}

41. Extent Flexibility

42. Dynamic Flexibility

43. Speed of Limb Movement

44. Gross Body Equilibrium

45. Gross Body Coordination

46. Static Strength

47. Explosive Strength

48. Dynamic Strength

49. Trunk Strength

50. Stamina

Fleishman, E. A. and Quaintance, M. K. (1984) Taxonomies of Human Performance: The Description of Human Tasks., Orlando: Academic Press.

Figure 56 - Fleishman's Knowledge, Skills, and Abilities Taxonomy

(Middlebrooks et al., 1999b)

Using Fleishman's taxonomy, a database was developed using questionnaires using Likert - like 7 point behaviorally anchored questions and was administered to U.S. Army SMEs. This questionnaire associated physical and mental skills and abilities from the taxonomy to performance tasks such as "receive and record a radio message" that operators would be expected to execute in the performance of their duties in a TOC during the conduct of battlefield operations. This database then provided a numerical basis for a computer simulation model to 
calculate a workload estimate for each individual based on the tasks being performed at the instant of the calculation. The time interval selected for workload calculation updates was 100 seconds. Resulting from this, over the course of a simulation run, a profile of individual workload and utilization rates was established for each member of the workgroup at a 100 second resolution. The data was captured so that the workload rates could be decomposed into the individual elements of the taxonomy so that the amount of time spent by the individual in the different cognitive and physical performance categories could be determined. These workload and utilization profiles were then analyzed following the simulation run using multivariate statistical techniques to predict whether individuals became cognitively saturated and therefore unable to effectively perform their assigned tasks.

\section{Tactical Scenario.}

The tactical mission was modeled as a force-on-force operation occurring over several hours. Different scenarios that were developed include the phases of pre-operations planning, movement-to-contact, deliberate defense, and hasty attack. Some scenarios reflect heavy combat actions and others reflect extended movement and reconnaissance type operations as shown in Figure 57. A model input file consisting of scenario voice and digital messages expected to be sent to and from the TOC during the course of the tactical mission was generated using training scenarios for Southwest Asia operations and OMS/MP (Operations Mission Summary / Mission Profile) movement rates as provided by the U.S. Army Armor Center at Fort Knox, Kentucky. The input file indicates the time each message occurs, where it is received and who or what equipment receives it, and the subsequent routing and task flow initiated by this message. Tasks performed in response to these messages come from an external source (usually a radio, digital link, or coworker), and are labeled "reactive", and either "voice" or 'digital". In addition to external messages, the scenario file also contains "internal information messages' that are mental “triggers' for personnel to periodically perform "proactive" (self-initiated) tasks that are an essential part of $\mathrm{C} 2$ operations and workstation database manipulation (Knapp, Johnson, Barnette, Wojciechowski, Kilduff, and Swoboda, 1997a). Examples of these proactive tasks are situation assessment checks, updating documentation (plans, orders, etc.), preparing status reports, and calling up windows of information for review. 

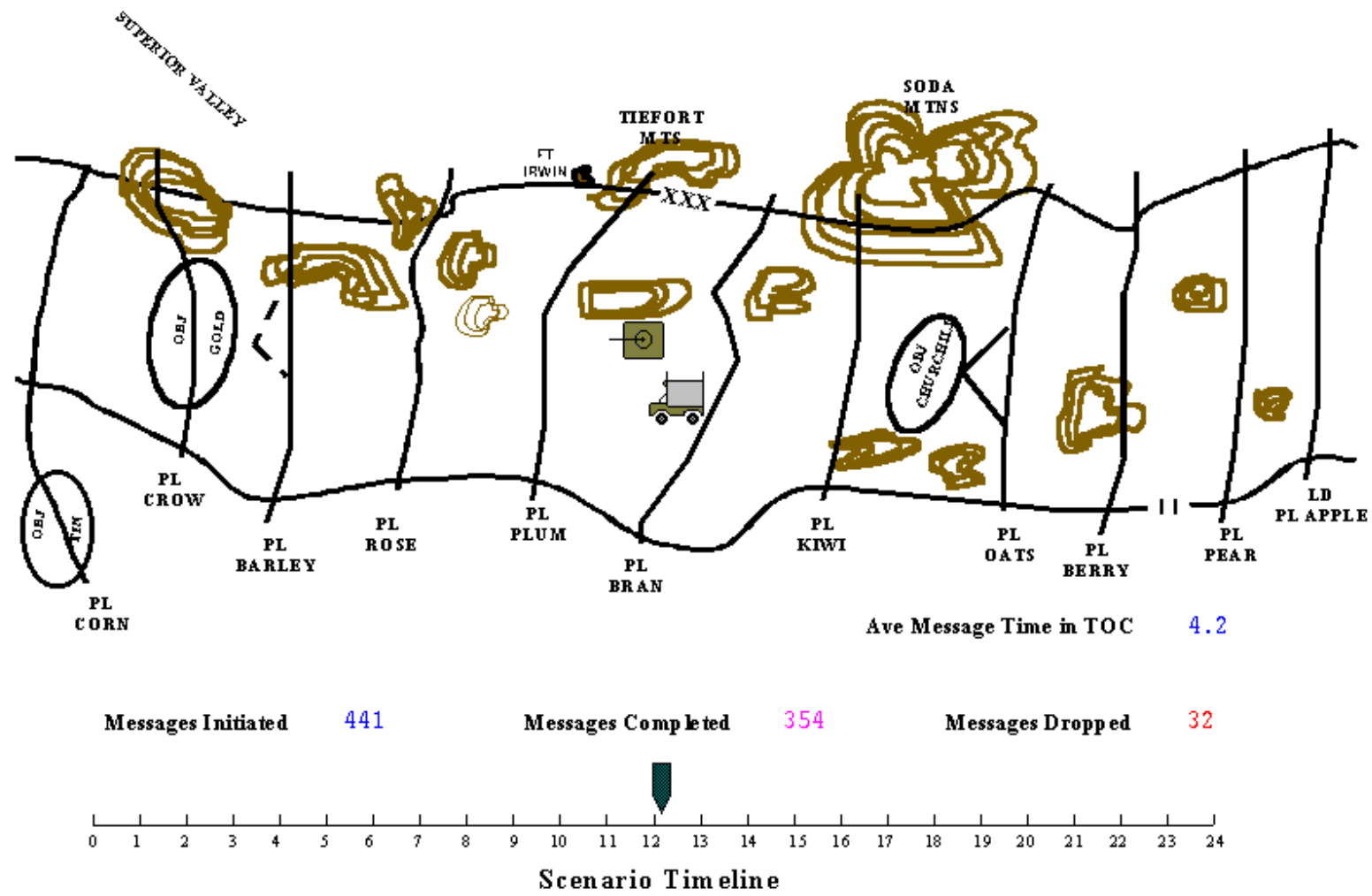

Figure 57 - Tactical Scenario Overlay

(Middlebrooks et al., 1999b)

\section{The CoHOST Computer Simulation Model.}

The discrete event programming language Micro Saint ${ }^{\mathrm{TM}}$ provided the CoHOST programming environment to develop the protocols and conventions to input the tasks, task sequences, flow logic, and task timing and workload data from the network diagrams into an executable model. The computer model works according to a basic "input-throughput-output" scheme as shown in Figure 58. That is, the inputs to the model are message events from the scenario input file, which present an information event stream in a time sequence synchronized to mission activity phases. As these information events enter the model, tasks are triggered and performed in a pattern that reflects the logic for task branching, interrupt priorities, time outs, and collaborative (interactive) tasks. Any information event that triggers a staff huddle always has the highest priority (Knapp et al., 1997a). 


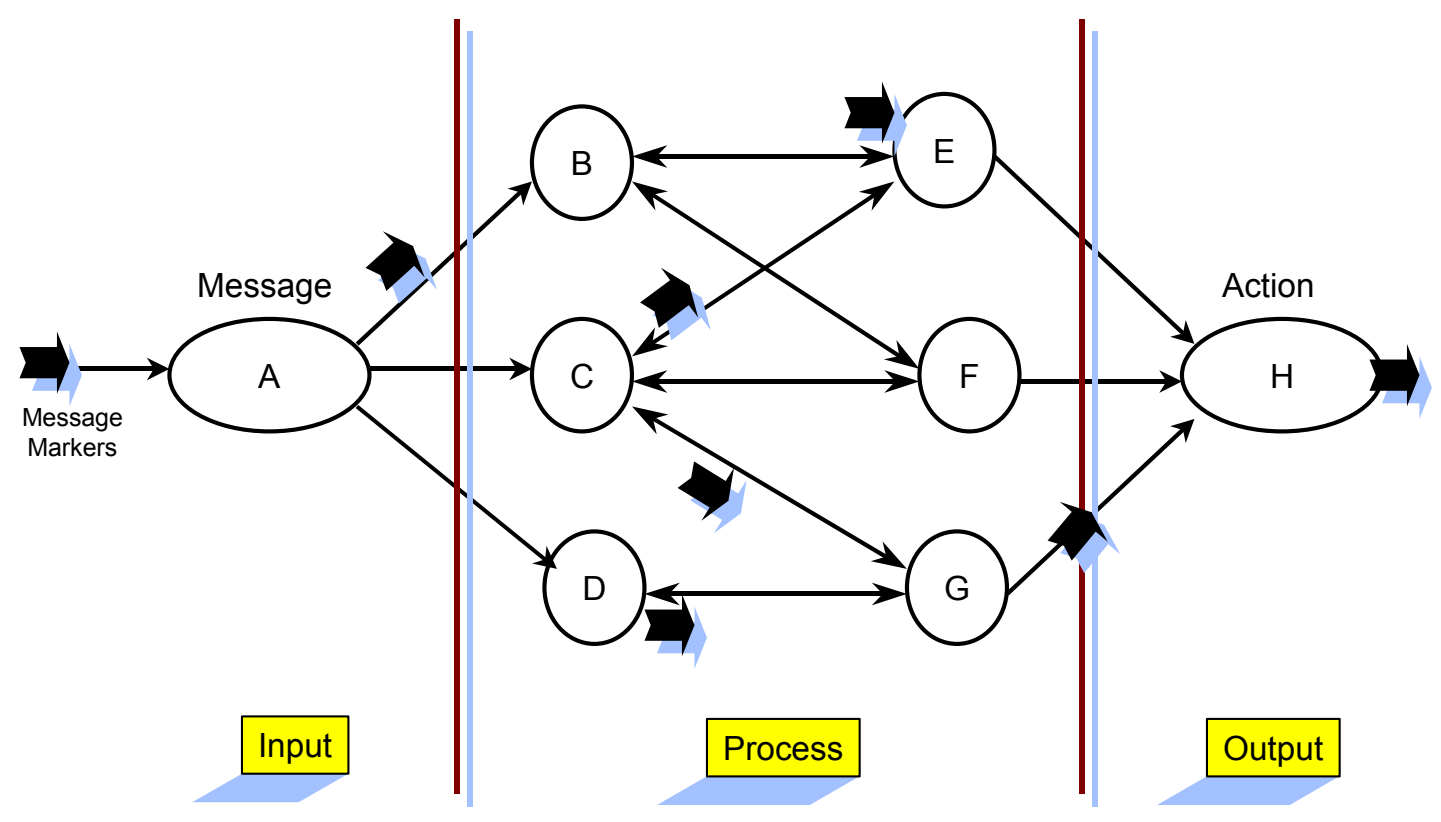

Figure 58 - Conceptual Model of TOC Operations

(Middlebrooks et al., 1999b)

CoHOST runs on an IBM-compatible PC running Windows 2000 (or higher). During model execution, a graphical user interface (GUI) screen displays the progress of tasks being performed by each $\mathrm{C} 2$ section and individual soldier position, as information messages enter the system. Bar and pie charts on the GUI display allow an observer to get an initial look at whether staff sections and individuals are keeping pace or falling behind in their information processing, as well as how busy or idle they are as scenario time goes on. A screen print from this real-time display is shown in Figure 59. 


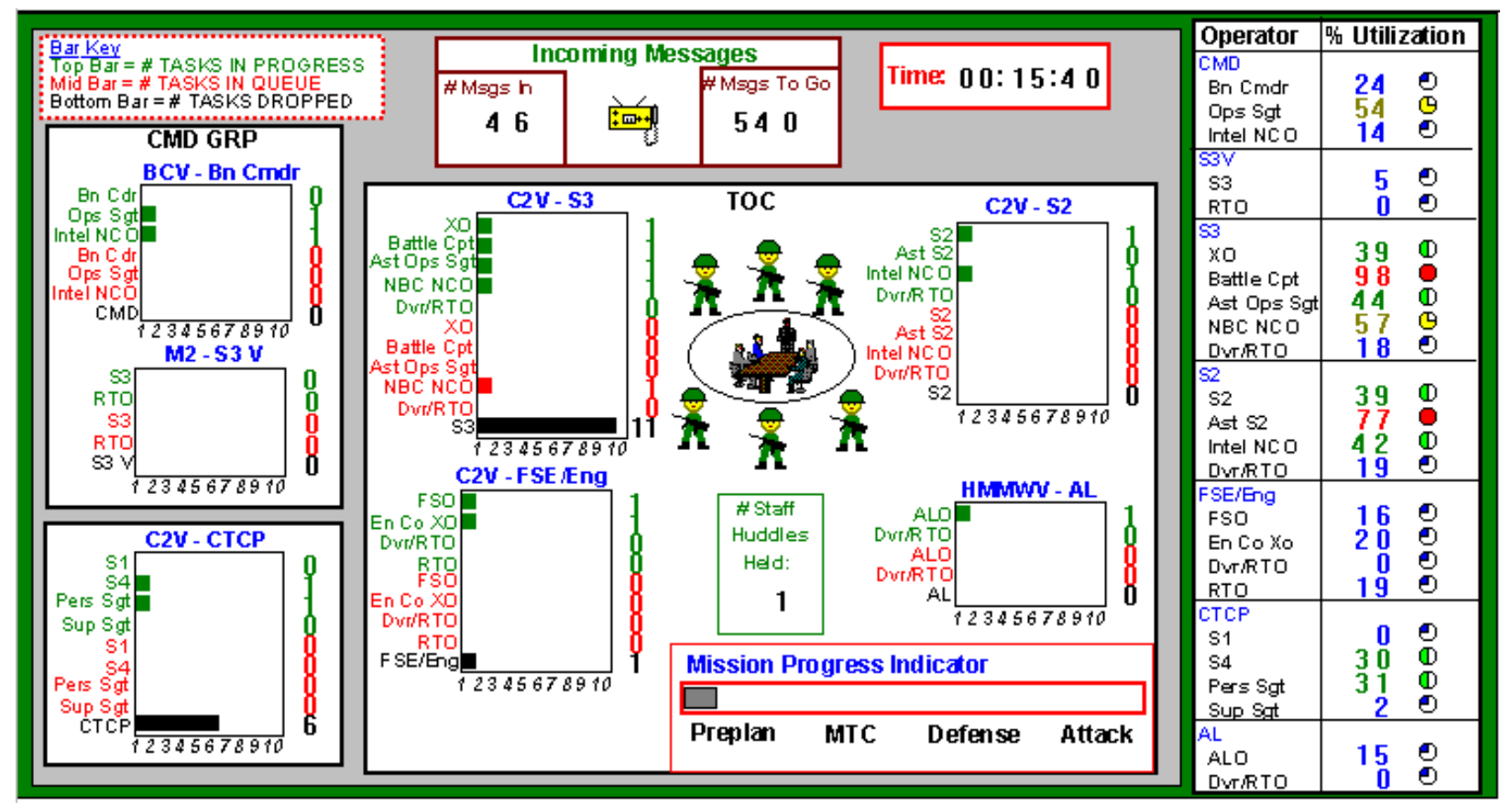

Figure 59 - CoHOST Model Action View Display

(Middlebrooks et al., 1999b)

The model was developed in three steps that occurred iteratively and in parallel:

(1) Cognitive task analysis and workload measurement for battalion command and control tasks, using techniques from the most recent human performance and related literature;

(2) Obtaining and translating scenarios and task flow data from pertinent documentation and battalion C2 SMEs;

(3) Exercising the Micro Saint ${ }^{\mathrm{TM}}$ discrete event simulation programming language to simulate the task and flow data from steps one and two. Following data input, the C2 computer model was debugged and executed, and the resulting output data were analyzed using descriptive and comparative statistics. An example of the task flow logic contained in one of the CoHOST models is shown in Figure 60. 


\section{Heavy Maneuver Battalion Command \& Control Work Group}

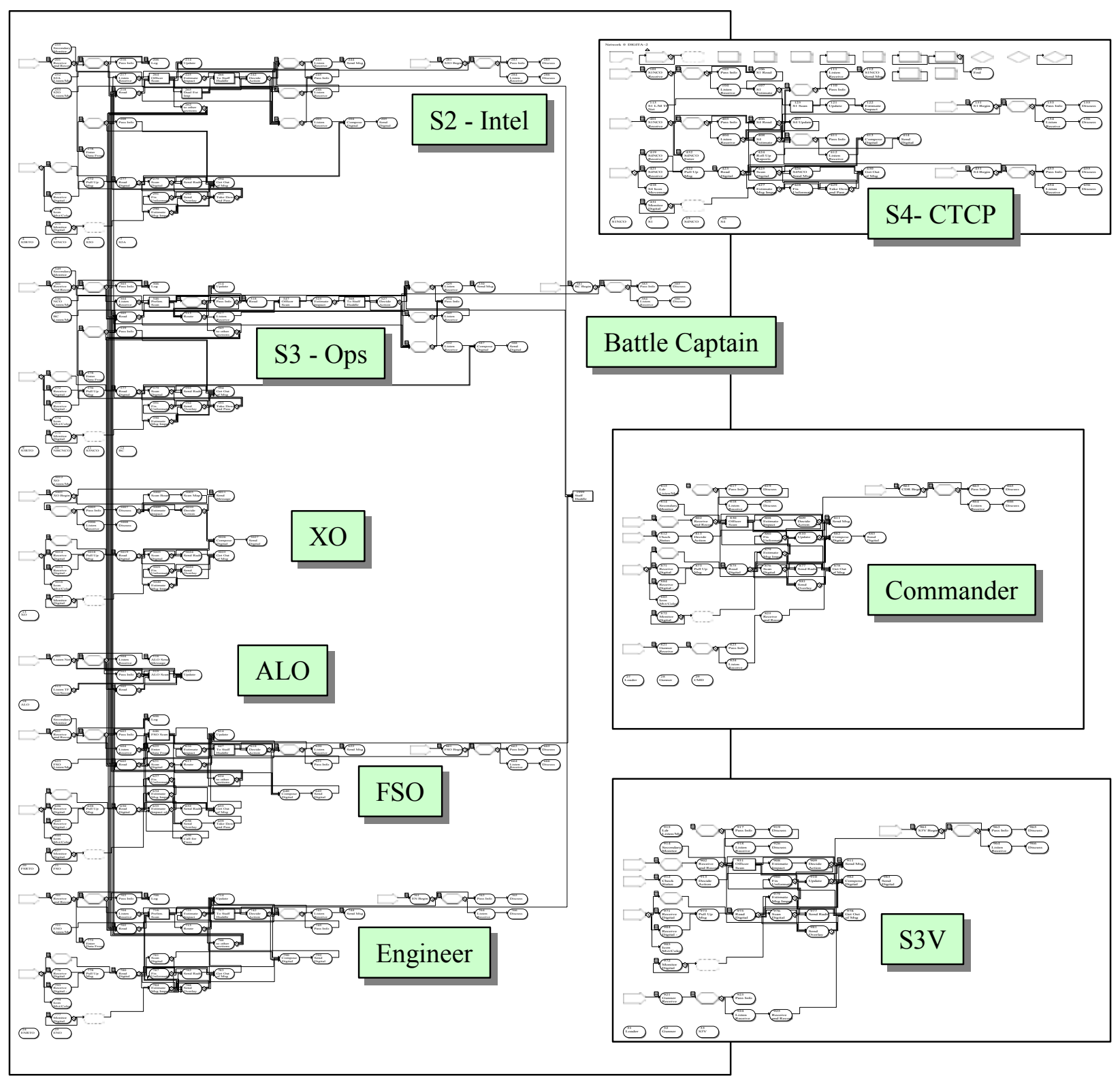

Figure 60 - CoHOST Model Network Flow Diagram

\section{Results From Original CoHOST Project.}

Each CoHOST model was executed using communication messages from the tactical scenario as driver events for the simulation. The dependent measures that were evaluated were: 
1) Tasks dropped - those tasks that an operator did not complete for any reason.

2) Tasks interrupted - those tasks whose performance was interrupted by another task or event of higher priority.

3) Number of task queues generated - the number of times an incoming task was assigned to a queue wait state because the operator identified to perform the task was busy performing another task of equal or higher priority.

4) Task backlog work - off time - the amount of time it took for an operator to eliminate the tasks that were queued up for execution.

Additional analyses were performed to assess the reasons for and types of information flow bottlenecks. The purpose of this review was to identify why tasks got dropped, queued, and/ or interrupted.

Initially, three CoHOST models were executed with varying configurations of organizational configuration and implementation of digital communications equipment. The results are summarized in Figure 61.

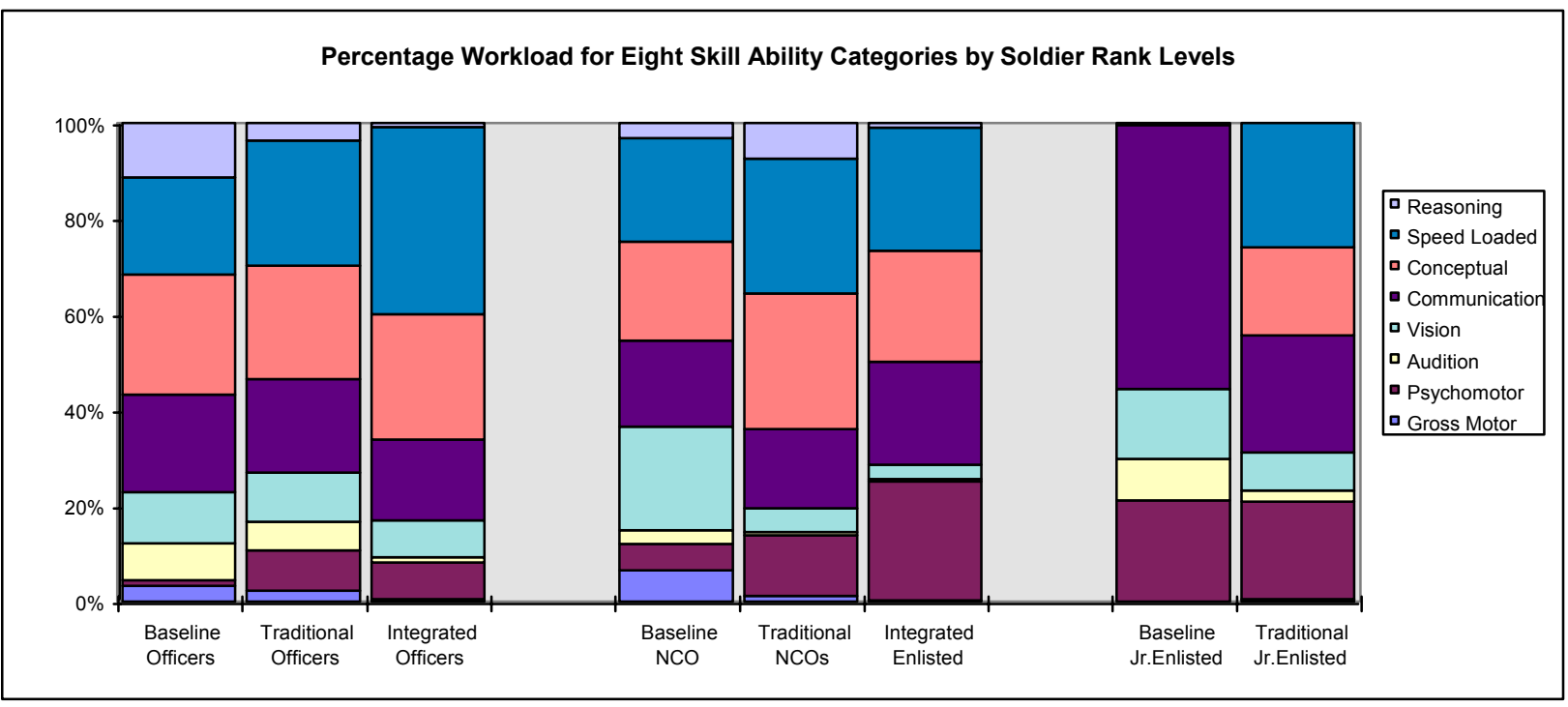

Figure 61 - Percent of Time Spent In Each Performance Category of the Taxonomy (Knapp et al., 1997c)

Figure 61 presents results from the three runs with information organized according to the taxonomy. The 3 groups of bars represent information from officers, NCO's and junior enlisted 
personnel. The 3 bars within each group represent the data from each run. The three runs were identified as:

1) Baseline Model - Personnel and equipment configuration according to the then (1996) mode of TOC operation with analog communications equipment (Knapp et al., 1997a).

2) Traditional Model - Same as the baseline model but with first generation digital communications equipment (Knapp, Johnson, Barnette, Wojciechowski, Kilduff, and Swoboda, 1997b).

3) Integrated Model - Reorganized personnel organizational structure to capitalize on enhanced communications equipment capabilities and objective digital communications equipment (Knapp et al., 1997c).

There was no data for the junior enlisted for the Integrated model run because all the junior enlisted personnel were eliminated by the personnel reorganization for that model run.

The sections of each bar graph are color coded to correspond to the eight categories of the taxonomy as indicated in the legend. The top category represented the amount of time spent performing the proactive think - ahead reasoning cognitive tasks that are critically important for situation analysis and decision making abilities. The next category is the cognitive speed loaded category that is indicative of activity that requires immediate attention for quick servicing of the activity before the content of the activity becomes obsolete. Looking at the three bar graphs for the 3 model runs for the officers at the left side of Figure 61, it can be seen that in the baseline model the officers were modeled as being able to spend about $10 \%$ of their time performing the proactive think - ahead tasks necessary to maintain cognitive awareness of the battlefield and develop decisions on what actions to take next. Subject Matter Expert opinion validated that this estimate roughly corresponded to the circumstances of actual battle. The middle bar from the traditional model run that simulated first generation digital communications equipment for the officers show that this activity was greatly reduced being almost totally supplanted by the speed loaded activity of the next taxonomic category. The third run from the integrated model that simulated the full capabilities of digital communications equipment being developed and a reorganization of personnel to take full advantage of it shows an even worse situation with almost all reasoning activity disappeared.

The explanation for this phenomenon comes from a realization that while each element of the communications equipment was performing exactly as it was designed, the design was based 
on maximizing hardware system performance that did not include the human as an integral component of the system. The result was that increased message arrival rates coming from enhanced communications systems were forcing the decision makers to focus their attention to just trying to keep up with and react to the messages with the result that there was no time left to analyze and interpret the information they were receiving. Thus, in this series of simulation runs the officer decision makers went from a pseudo proactive think - ahead reasoning mode to an almost total reactive speed loaded mode while trying to keep up with the increased message traffic. A contributing element to this situation was the elimination of the junior enlisted personnel whose primary duties were to function as equipment operators. With the organizational paradigm associated with the new digital communications equipment, the officer decision makers were required to sit at and operate their own communications consoles and had to personally interact with the incoming message traffic.

\section{Summary of Original CoHOST Project.}

By looking at which individuals were predicted to be workload saturated for each model run condition, a project conclusion was reached that increasing automation does not necessarily improve human decision making performance and may, in fact, degrade it. 
Appendix P - DV / IV Correlation Matrices for all Tasks

Table 41 - DV / IV Correlation Matrix For Each Performance Task

\begin{tabular}{|r|c|c|c|c|c|l|}
\hline \multicolumn{10}{|c|}{ \# 1 - Primary Task10. Destruction, capture, or bypass of enemy force. } \\
\cline { 2 - 7 } Variable & Intercept & BosInte14 & B0SMAS2 & B0ScC2 & MOPPLeve1 & \\
\hline All IVs 1.0 & & 1 & 1 & 1 & 1 & \\
\hline All IVs 0.0 & & 0 & 0 & 0 & 0 & \\
\hline DV .25 & & 0 & 0 & 0 & 0 & No IVs \\
\hline DV 0.5 & & 1 & 1 & 1 & 1 & BOSIntel4, BOSMAS2, BOSCC2, MOPPLevel \\
\hline DV .75 & & 1 & 1 & 1 & 1 & BOSIntel4, BOSMAS2, BOSCC2, MOPPLevel \\
\hline Beta Weight & 0.09781 & 0.37701 & 0.31685 & -0.19909 & -0.46896 & \\
\hline
\end{tabular}

\begin{tabular}{|r|c|c|c|c|c|c|c|c|c|c|}
\hline & $\#$ 2 - Primary Task11. Fixing enemy in position. & & & & \\
\hline Variable & Intercept & BOSInte13 & BOSCC1 & BOSCC2 & BOSCC3 & BRIntel & StaffHud & PERSX0 & PERSS3RT0 & PERSS3SGM \\
\hline All IVs 1.0 & & 1 & 1 & 1 & 1 & 1 & 1 & 1 & 1 & 1 \\
\hline All IVs 0.0 & & 0 & 0 & 0 & 0 & 0 & 0 & 0 & 0 & 0 \\
\hline DV .25 & & 1 & 0 & 0 & 0 & 0 & 1 & 0 & 1 & 0 \\
\hline DV 0.5 & & 0 & 1 & 1 & 0 & 0 & 1 & 1 & 0 & 0 \\
\hline DV .75 & & 0 & 0 & 0 & 1 & 0 & 0 & 1 & 1 & 0 \\
\hline Beta Weight & -0.26097 & 0.27295 & 0.85678 & 0.85483 & 1.15989 & -0.61668 & -0.3914 & -0.27882 & 0.58359 & -0.46158 \\
\hline
\end{tabular}

BOSIntel3, StaffHud, PERSS3RTO BOSCC1, BOSCC2, StaffHud, PERSXO BOSCC3, PERSXO, PERSS3RTO

\begin{tabular}{|r|c|c|c|c|c|c|c|c|c|c|}
\hline \multicolumn{2}{|c|}{ \# 3 - Primary Task12. Synchronization with supporting forces. } & & & \\
\hline Variable & Intercept & BOSFS2 & BOSFS7 & BOSFS9 & BOSMAS6 & BOSCC1 & COMAFATDS & MISCBattle PERSAL0 & Humidity \\
\hline All IVs 1.0 & & 1 & 1 & 1 & 1 & 1 & 1 & 1 & 1 & 1 \\
\hline All IVs 0.0 & & 0 & 0 & 0 & 0 & 0 & 0 & 0 & 0 & 0 \\
\hline DV .25 & & 1 & 0 & 0 & 1 & 1 & 0 & 0 & 0 & 1 \\
\hline DV 0.5 & & 0 & 1 & 0 & 1 & 0 & 0 & 1 & 0 & 0 \\
\hline DV .75 & & 1 & 0 & 1 & 0 & 0 & 1 & 0 & 1 & 1 \\
\hline Beta Weight & 0.9788 & -0.24508 & 0.41197 & -0.13939 & -0.37014 & -0.35361 & 0.61071 & -1.10636 & -0.16609 & -0.48741 \\
\hline
\end{tabular}

BOSFS2, BOSMAS6, BOSCC1, Humidity BOSFS7, BOSMAS6, MISCBattleTiming BOSFS2, BOSFS9, COMAFATDS, PERSALO, Humidity 


\begin{tabular}{|c|c|c|c|c|c|c|}
\hline & \# 4 - Prima & y Task15. & estruction & of first echelon forc & & \\
\hline Variable & Intercept & BoSIntel4 & BOSFS2 & MISCBattleTiming & PERSXO & \\
\hline All IVs 1.0 & & 1 & 1 & 1 & 1 & \\
\hline All IVs 0.0 & & 0 & 0 & 0 & 0 & \\
\hline DV .25 & & 0 & 1 & -0.0600 & 1 & BOSFS2, MISCBattleTiming, PERSXO \\
\hline DV 0.5 & & 1 & 0 & 0.43 & 0 & BOSIntel4, MISCBattleTiming, PERSXO \\
\hline DV .75 & & 1 & 1 & 0.36 & 1 & BOSIntel4, BOSFS2, MISCBattleTiming, PERSXO \\
\hline Beta Weight & -0.61941 & 0.41048 & 0.21054 & 0.49106 & 0.1355 & \\
\hline
\end{tabular}

\begin{tabular}{|c|c|c|c|c|c|c|}
\hline & \# 5 - Prime & y Task21. & Peacekeep & ing operations. & & \\
\hline Variable & Intercept & BOSFS9 & BOSMAS5 & MISCBattleTempo & MISCInfoops & \\
\hline All IVs 1.0 & & 1 & 1 & 1 & 1 & \\
\hline All IVs 0.0 & & 0 & 0 & 0 & 0 & \\
\hline DV .25 & & 0 & 0 & 1 & 0 & MISCBattleTempo \\
\hline DV 0.5 & & 0 & 0 & 1 & 1 & MISCBattleTempo, MISCInfoOps \\
\hline DV .75 & & 1 & 1 & 0 & 1 & BOSFS9, BOSMAS5, MISCInfoOps \\
\hline Beta Weight & 0.47554 & -0.36188 & -0.12732 & -0.97917 & 0.45966 & \\
\hline
\end{tabular}

\begin{tabular}{|r|c|c|c|c|c|l|}
\hline & \# 6 - Primary Task23. Planning & & \\
\hline Variable & Intercept & BOSMAS6 & BRIntel & BRMAS & StaffHud & \\
\hline All IVs 1.0 & & 1 & 1 & 1 & 1 & \\
\hline All IVs 0.0 & & 0 & 0 & 0 & 0 & \\
\hline DV .25 & & 0 & 1 & 0 & 1 & BRIntel, StaffHud \\
\hline DV 0.5 & & 1 & 0 & 0 & 1 & BOSMAS6, StaffHud \\
\hline DV .75 & & 1 & 1 & 0 & 1 & BOSMAS6, BRIntel, StaffHud \\
\hline Beta Weight & 0.39034 & 1.0112 & 0.31699 & 0.71853 & 0.20794 & \\
\hline
\end{tabular}

\begin{tabular}{|r|c|c|c|c|l|}
\hline \multicolumn{7}{|c|}{$\#$ 7 - Secondary Task12. Synchronization with supporting forces. } \\
\hline Variable & Intercept & BOSMan1 & BOSFS9 & BOSMAS1 & \\
\hline All IVs 1.0 & & 1 & 1 & 1 & \\
\hline All IVs 0.0 & & 0 & 0 & 0 & \\
\hline DV .25 & & 1 & 1 & 1 & BOSMan1, BOSFS9, BOSMAS1 \\
\hline DV 0.5 & & 1 & 1 & 1 & BOSMan1, BOSFS9, BOSMAS1 \\
\hline DV .75 & & 1 & 1 & 1 & BOSMan1, BOSFS9, BOSMAS1 \\
\hline Beta Weight & 0.04119 & -0.46001 & 0.7314 & -0.35619 & \\
\hline
\end{tabular}

\begin{tabular}{|r|c|c|c|c|c|l|}
\hline & $\# 8$ - Secondary Task18. Deception activities. & \\
\hline Variable & Intercept & BOSFS2 & BOSMAS6 & BOSCC1 & PERSX0 & \\
\hline All IVs 1.0 & & 1 & 1 & 1 & 1 & \\
\hline All IVs 0.0 & & 0 & 0 & 0 & 0 & \\
\hline DV .25 & 0 & 0 & 1 & 0 & BOSCC1 \\
\hline DV 0.5 & & 1 & 0 & 1 & 0 & BOSFS2, BOSCC1 \\
\hline DV .75 & & 1 & 1 & 0 & 1 & BOSFS2, BOSMAS6, PERSXO \\
\hline Beta Weight & -0.02089 & 0.1597 & -0.34669 & 0.15473 & 0.61261 & \\
\hline
\end{tabular}

\begin{tabular}{|r|c|c|c|l|}
\hline & $\# 9$ - Secondary Task19. Rear operations. \\
\hline Variable & Intercept & BOSMAS6 & PERSS2 & \\
\hline All IVs 1.0 & & 1 & 1 & \\
\hline All IVs 0.0 & & 0 & 0 & \\
\hline DV .25 & & 1 & 1 & BOSMAS6, PERSS2 \\
\hline DV 0.5 & & 1 & 1 & BOSMAS6, PERSS2 \\
\hline DV .75 & & 1 & 1 & BOSMAS6, PERSS2 \\
\hline Beta Weight & 0.09891 & 0.96695 & -0.08781 & \\
\hline
\end{tabular}




\begin{tabular}{|c|c|c|c|c|c|c|c|c|c|c|c|}
\hline & \multicolumn{6}{|c|}{ \# 10 - Secondary Task23. Planning } & \multirow{2}{*}{\multicolumn{2}{|c|}{ BOSMan2 }} & \multirow[b]{2}{*}{ BOSFS9 } & \multirow[b]{2}{*}{ BOSMAS4 } & \multirow[b]{2}{*}{ B0scC2 } \\
\hline Variable & \multicolumn{2}{|c|}{ Intercept } & \multicolumn{2}{|c|}{ BosIntel2 } & \multicolumn{2}{|c|}{ BosIntel3 } & & & & & \\
\hline All IVs 1.0 & & & \multicolumn{2}{|l|}{1} & \multicolumn{2}{|l|}{1} & \multicolumn{2}{|c|}{1} & 1 & 1 & 1 \\
\hline All IVs 0.0 & & & \multicolumn{2}{|l|}{0} & \multicolumn{2}{|l|}{0} & \multicolumn{2}{|c|}{0} & 0 & 0 & 0 \\
\hline DV .25 & & & \multicolumn{2}{|l|}{1} & \multicolumn{2}{|l|}{1} & \multicolumn{2}{|l|}{0} & 1 & 0 & 0 \\
\hline DV 0.5 & & & 0 & & \multirow{2}{*}{\multicolumn{2}{|c|}{$\frac{0}{0}$}} & \multicolumn{2}{|c|}{0} & 0 & 1 & 0 \\
\hline DV .75 & & & \multicolumn{2}{|l|}{0} & & & \multicolumn{2}{|c|}{0} & 1 & 1 & 0 \\
\hline Beta Weight & \multicolumn{2}{|c|}{-0.50056} & \multicolumn{2}{|c|}{0.12448} & \multicolumn{2}{|c|}{0.20154} & \multicolumn{2}{|c|}{-0.66144} & -0.1313 & 0.5892 & 0.18261 \\
\hline \multicolumn{2}{|c|}{ MISCBattleTiming } & \multicolumn{2}{|c|}{ MISCInfoOps } & & RSS3RTO & \multicolumn{3}{|c|}{ Humidity } & & & \\
\hline \multicolumn{2}{|l|}{1} & \multicolumn{2}{|r|}{1} & & 1 & \multicolumn{3}{|c|}{1} & & & \\
\hline \multicolumn{2}{|l|}{0} & \multicolumn{2}{|r|}{0} & & 0 & \multirow{2}{*}{\multicolumn{3}{|c|}{$\begin{array}{l}0 \\
0\end{array}$}} & & & \\
\hline \multicolumn{2}{|l|}{0} & \multicolumn{2}{|r|}{0} & & 0 & & & & SIntel2, B & SIntel3, & OSFS9 \\
\hline 0 & & & 0 & & 0 & & 0 & & SMAS4 & & \\
\hline 0 & & & 1 & & 0 & & 0 & $\mathrm{BO}$ & SFS9, BO & SMAS4, $\mathrm{N}$ & ISCInfoOps \\
\hline-0.56196 & & & 26861 & & .35949 & 0.5 & 8127 & & & & \\
\hline
\end{tabular}

\begin{tabular}{|c|c|c|c|c|c|c|c|c|c|c|c|}
\hline \multicolumn{10}{|c|}{ \# 11 - Tertiary Task3. Movement to the line of departure. } & \multirow[b]{2}{*}{ COMAFATDS } & \multirow[b]{2}{*}{ COMMCS } \\
\hline \multicolumn{2}{|c|}{ Variable } & \multicolumn{2}{|c|}{ Intercept } & \multicolumn{2}{|c|}{ BoSMan1 } & BOSFS2 & BOSFS9 & BOSMAS1 & Boscc1 & & \\
\hline \multicolumn{2}{|c|}{ All IVs 1.0} & & & \multicolumn{2}{|c|}{1} & 1 & 1 & 1 & 1 & 1 & 1 \\
\hline \multicolumn{2}{|c|}{ All IVs 0.0} & & & \multicolumn{2}{|c|}{0} & 0 & 0 & 0 & 0 & 0 & 0 \\
\hline \multicolumn{2}{|c|}{ DV .25 } & & & \multicolumn{2}{|c|}{1} & 0 & 1 & 0 & 1 & 0 & 0 \\
\hline \multicolumn{2}{|c|}{ DV 0.5} & & & \multicolumn{2}{|c|}{1} & 0 & 0 & 0 & 0 & 0 & 0 \\
\hline \multicolumn{2}{|c|}{ DV .75 } & & & \multicolumn{2}{|c|}{1} & 1 & 1 & 1 & 1 & 0 & 0 \\
\hline \multicolumn{2}{|c|}{ Beta Weight } & 0.089 & & \multicolumn{2}{|c|}{0.40832} & 0.66808 & -0.14227 & -0.39912 & -0.15818 & -0.7414 & 0.49004 \\
\hline StaffHud & & RSS3RTO & PERSA & & & & & & & & \\
\hline 1 & & 1 & & 1 & & & & & & & \\
\hline 0 & & 0 & & 0 & & & & & & & \\
\hline 0 & & 1 & & 0 & $\mathrm{BOs}$ & Man1, BO & SFS9, BOS & C1, PERS & S3RTO & & \\
\hline 0 & & 0 & & 0 & $\mathrm{BOs}$ & Man1 & & & & & \\
\hline 0 & & 1 & & 0 & $\mathrm{BOs}$ & Man1, BO & SFS2, BOS & S9, BOSN & AS1, BOSC & 1, PERSS3 & RTO \\
\hline-0.35177 & & .11013 & -0.09 & 9726 & & & & & & & \\
\hline
\end{tabular}

\begin{tabular}{|c|c|c|c|c|c|c|c|c|c|}
\hline \multicolumn{10}{|c|}{ \# 12 - Tertiary Task21. Peacekeeping operations. } \\
\hline Variable & Intercept & BoSIntel1 & BoSMan1 & \begin{tabular}{l|l} 
BOSFS9 & E \\
\end{tabular} & BOSMAS7 & BOScC1 & \multicolumn{2}{|c|}{ MISCBattleTiming } & MISCBattleTempo \\
\hline All IVs 1.0 & & 1 & 1 & 1 & 1 & 1 & \multicolumn{2}{|r|}{1} & 1 \\
\hline All IVs 0.0 & & 0 & 0 & 0 & 0 & 0 & \multicolumn{2}{|r|}{0} & 0 \\
\hline DV .25 & & 0 & 0 & 0 & \multirow{2}{*}{$\frac{0}{0}$} & 0 & \multicolumn{2}{|r|}{0} & 0 \\
\hline DV 0.5 & & 0 & 0 & 0 & & 0 & \multicolumn{2}{|r|}{0} & 0 \\
\hline DV .75 & & 0 & 0 & 0 & 0 & 0 & \multirow{2}{*}{\multicolumn{2}{|c|}{$\begin{array}{c}0 \\
0.51993\end{array}$}} & 0 \\
\hline Beta Weight & -0.10147 & -0.2664 & -0.50849 & 0.60112 & -0.205 & 0.0824 & & & -0.51344 \\
\hline & & \multicolumn{2}{|c|}{ MISCTOCActivity } & StaffHuc & \multicolumn{2}{|c|}{\begin{tabular}{l|l} 
ud & PERSS3RTO \\
\end{tabular}} & \multicolumn{3}{|l|}{ PERSALO } \\
\hline & & \multicolumn{2}{|r|}{1} & 1 & \multicolumn{2}{|c|}{1} & 1 & & \\
\hline & & \multicolumn{2}{|r|}{0} & 0 & \multicolumn{2}{|r|}{0} & 0 & \multicolumn{2}{|c|}{ 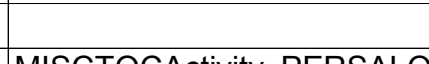 } \\
\hline & & & 1 & 0 & \multicolumn{2}{|r|}{0} & 1 & \multicolumn{2}{|c|}{ MISCTOCActivity, PERSALO } \\
\hline & & \multicolumn{2}{|r|}{1} & 0 & \multicolumn{2}{|c|}{0} & 0 & \multicolumn{2}{|c|}{ MISCTOCActivity } \\
\hline & & \multicolumn{2}{|c|}{0} & 0 & \multirow{2}{*}{\multicolumn{2}{|c|}{$\begin{array}{c}0 \\
026351\end{array}$}} & 1 & \multicolumn{2}{|l|}{ PERSALO } \\
\hline & & \multicolumn{2}{|c|}{-0.11479} & -0.12006 & \begin{tabular}{|l|l|}
36351 \\
\end{tabular} & & -0.0697 & & \\
\hline
\end{tabular}




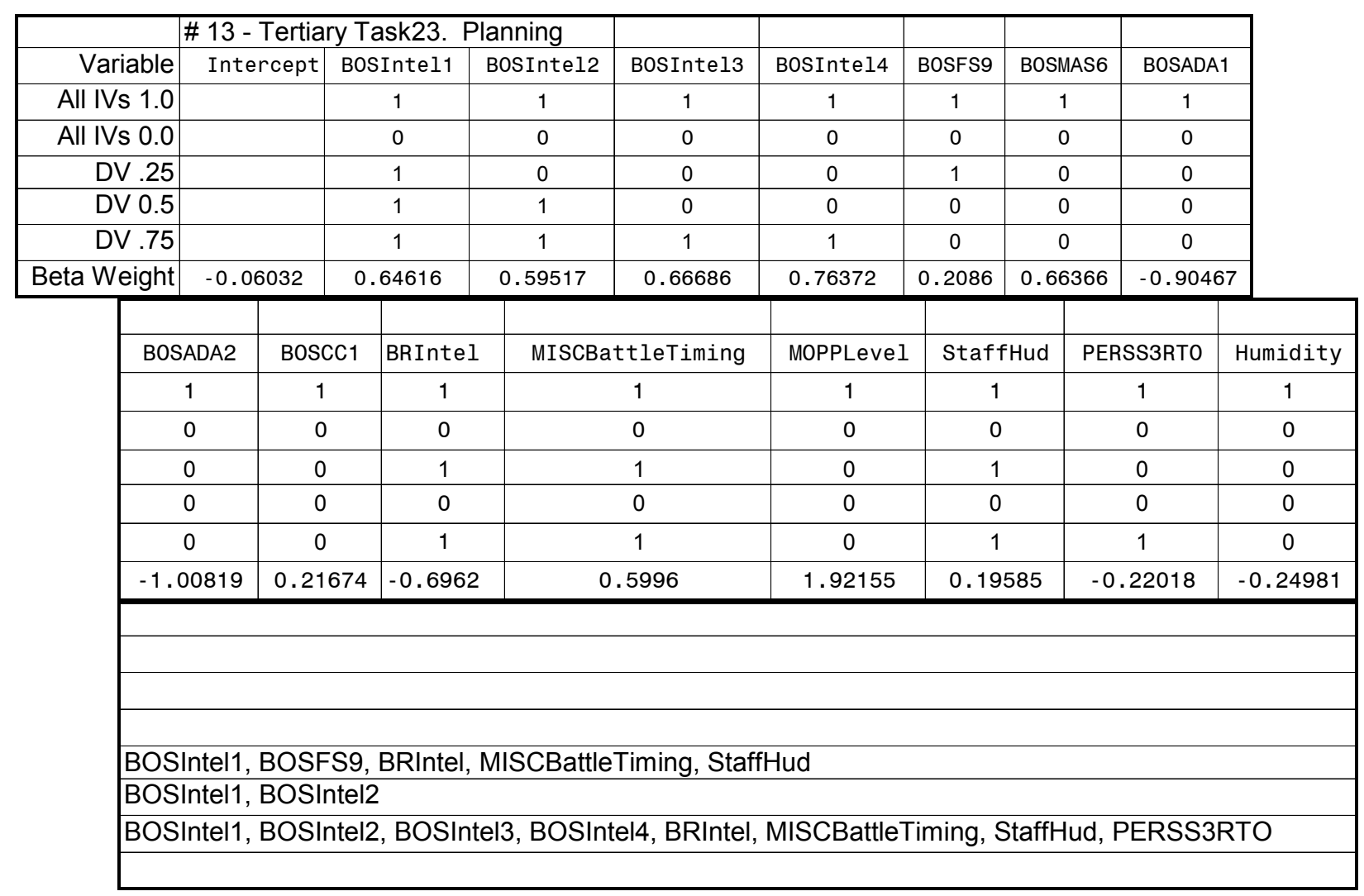

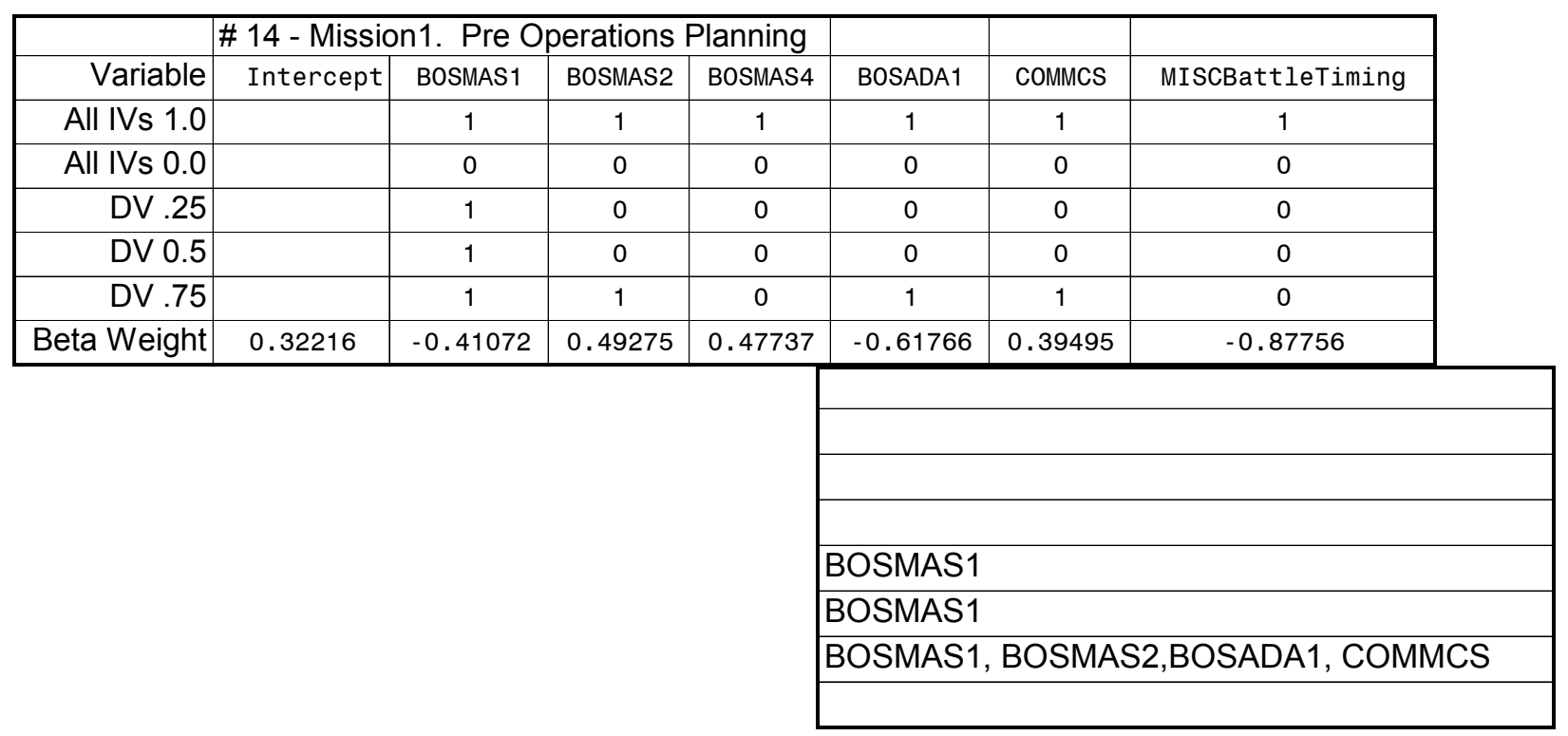




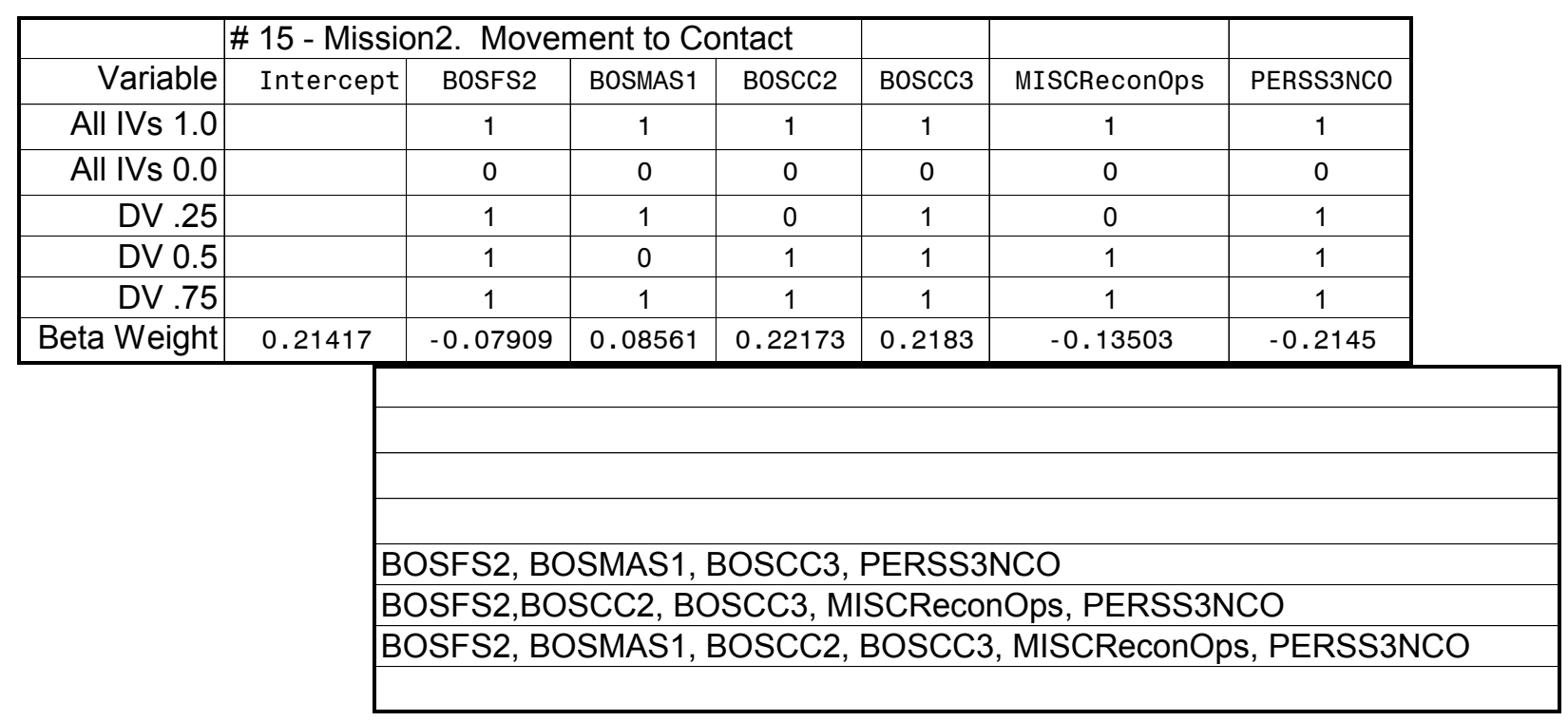

\begin{tabular}{|r|c|c|c|c|l|}
\hline & $\#$ 16 - Mission3. Attack & & & \\
\hline Variable & Intercept & BOSInte14 & MISCBattleTiming & MOPPLevel & \\
\hline All IVs 1.0 & & 1 & 1 & 1 & \\
\hline All IVs 0.0 & & 0 & 0 & 0 & \\
\hline DV .25 & & 0 & 0 & 1 & MOPPLevel \\
\hline DV 0.5 & & 1 & 0 & 1 & BOSIntel4, MOPPLevel \\
\hline DV .75 & 0 & 1 & 1 & MISCBattleTiming, MOPPLevel \\
\hline Beta Weight & -0.15612 & 0.35484 & 0.41945 & 0.1987 & \\
\hline
\end{tabular}

\begin{tabular}{|r|c|c|c|c|c|c|c|}
\hline & $\# 17$ - Mission5. River Crossing & & & & \\
\hline Variable & Intercept & BOSInte14 & BOSMan1 & BOSADA2 & MISCBattleTiming & MOPPLevel & PERSS3NC0 \\
\hline All IVs 1.0 & & 1 & 1 & 1 & 1 & 1 & 1 \\
\hline All IVs 0.0 & & 0 & 0 & 0 & 0 & 0 & 0 \\
\hline DV .25 & & 0 & 0 & 1 & 0 & 0 & 0 \\
\hline DV 0.5 & & 1 & 0 & 0 & 1 & 1 & 1 \\
\hline DV .75 & & 1 & 1 & 1 & 0 & 0 & 1 \\
\hline Beta Weight & -0.65542 & 0.09927 & 0.41456 & 0.46865 & 1.27217 & -0.72215 & 0.11363 \\
\hline
\end{tabular}

\section{BOSADA2}

BOSIntel4, MISCBattleTiming, MOPPLevel, PERSS3NCO BOSIntel4, BOSMan1, BOSADA2, PERSS3NCO 
Appendix Q - Determination Of CoHOST Simulation Replication Count.

With stochastic computer simulations, multiple runs must be conducted for each combination of the IVs in order to account for the variability induced by the random number generation (Whicker and Sigelman, 1991). When analysis is conducted on data generated by a simulation due to random effects then the variance of the output data must be controlled so that it falls within a desired precision limit (Banks et al., 1996). Stated succinctly, a computer simulation model involving Monte Carlo determinations needs to be repeated or "replicated" as many times as necessary to get the required precision (Kelton, 1995). This can be achieved by making multiple replications of the simulation runs by holding the IV levels constant and changing the random number seed at the start of each replication run. When a sufficient number of replications have been executed then the mean of the output data from the replications can be expected to fall within the desired confidence limit. For this study it is desired to have the output data from the simulation exhibit a $95 \%$ probability of falling within the confidence limit which gives a specified error level, e, equal to \pm .05 of the mean. Following Banks' procedures, (pages 429-449), the required number of replications can be determined that needs to be conducted to support the intended analysis.

An initial simulation run of 5 replications was made with a starting random number seed of 1 . The model automatically used the next random number at the end of each replication as the starting seed for the next replication. The resulting data for workload, utilization, tasks queued, tasks dropped, and tasks interrupted for the Battalion Commander is shown in Table 42. A replication analysis was performed for each of these DVs to determine the number of replications required to satisfy each of these measures. 
Table 42 - Data From Initial 5 Replication Simulation Run

\begin{tabular}{|l|c|c|c|c|c|c|}
\hline Battalion Commander & $\begin{array}{c}\text { Workload } \\
(* \text { Util })\end{array}$ & $\begin{array}{c}\text { Utilizatio } \\
\mathrm{n} \\
(* \text { Util })\end{array}$ & $\begin{array}{c}\text { Number of } \\
\text { Queues } \\
(* \text { Opdata })\end{array}$ & $\begin{array}{c}\text { Tasks } \\
\text { Interrupted } \\
(* \text { Opdata })\end{array}$ & $\begin{array}{c}\text { Tasks } \\
\text { Suspended } \\
(* \text { Opdata })\end{array}$ & $\begin{array}{c}\text { Tasks } \\
\text { Dropped } \\
(* \text { Opdata })\end{array}$ \\
\hline Replication 1 & 3561179.34 & $92.30 \%$ & 50 & 90 & 11 & 11 \\
\hline Replication 2 & 3549435.31 & $92.07 \%$ & 48 & 90 & 11 & 13 \\
\hline Replication 3 & 3556732.44 & $92.39 \%$ & 54 & 90 & 10 & 11 \\
\hline Replication 4 & 3548795.69 & $93.21 \%$ & 52 & 88 & 11 & 9 \\
\hline Replication 5 & 3526501.95 & $91.95 \%$ & 51 & 91 & 11 & 13 \\
\hline Mean & 3548528.95 & $92.38 \%$ & 51.00 & 89.80 & 10.80 & 11.40 \\
\hline Standard Deviation & 13354.88 & 0.0049 & 2.24 & 1.10 & 0.45 & 1.67 \\
\hline $\begin{array}{l}5 \% \text { Error Limit }= \\
.05, \text { relative to the mean }\end{array}$ & 177426.45 & 0.0462 & 2.55 & 4.49 & 0.54 & 0.57 \\
\hline \multicolumn{7}{|l|}{$*$ (filename) $)=$ name of model output data file } \\
\hline
\end{tabular}

The desire is to determine the number of replications required so that the relative error (relative to the mean) for any of the DVs does not exceed 5 percent. The iterative formula to determine the number of replications is (Banks et al., 1996) (eq. 12.29, p. 449) is shown as Equation 2.

$$
R \geq\left(\frac{\frac{Z_{\alpha}}{2} S_{0}}{\varepsilon}\right)^{2}
$$

Equation 1 - Initial Estimate for Number of Required Replications, R

where,

$$
\begin{array}{lll}
\mathrm{R} & \equiv \quad \text { number of replications required to achieve the desired error level } \\
\mathrm{Z} \quad \equiv \quad \mathrm{Z} \text { statistic } & \begin{array}{l}
\text { Standard Deviation of the computed parameter across the } \\
\text { simulation replications }
\end{array} \\
\mathrm{S}_{0} \quad \equiv \quad \text { Error Level Threshold } \\
\varepsilon \quad \equiv \quad \begin{array}{l}
\text { Percent Error Level of the mean value of the computed parameter } \\
\text { across the simulation replications. }
\end{array}
\end{array}
$$


This expression is iteratively computed with the value of the computed replications being substituted for $\mathrm{R}$ until the value for $\mathrm{R}$ satisfies the greater than or equal to condition at which time the value for $\mathrm{R}$ becomes the required number of replications necessary to compensate for the random effects of the simulation.

\section{Example Calculation For Replication Analysis For Workload Parameter:}

From Table 42:

$$
\begin{aligned}
& \mathrm{S}_{0}=1354.88 \\
& \varepsilon=117426.45 \\
& \alpha=.05 ; \quad \alpha / 2=.025 ; \quad 1-\alpha / 2=.975 \\
& \therefore Z_{.975}=1.96, \text { from } Z \text { table, page } 966 \text { (Winer et al., 1991) }
\end{aligned}
$$

Thus,

$$
\begin{aligned}
& \mathrm{R} \geq\left\{\left(\mathrm{Z}_{\alpha / 2} \times \mathrm{S}_{0}\right) / \varepsilon\right\}^{2} \\
& \mathrm{R} \geq\{(1.96 \times 13354.88) / 177426.45\}^{2}=.02176 \approx 1
\end{aligned}
$$

So, use $\mathrm{R}=1$. Since this is less than 50 , use the $t$ distribution, plug back into the formula and evaluate. From the $t$ table, (Winer et al., 1991), page 967, $t_{\alpha / 2,1}=$ 12.71

$$
\begin{aligned}
& \mathrm{R} \geq\left\{\left(t_{97.5,2} \times \mathrm{S}_{0}\right) / \varepsilon\right\}^{2} \\
& \mathrm{R} \geq\{(12.71 \times 13354.88) / 177426.45\}^{2}=.91524 \approx 1
\end{aligned}
$$

$\therefore 5 \geq 1$ relationship is verified. As one run is required and 5 have been made, no additional runs are required to satisfy this parameter.

Table 43 shows the replication analysis for all the DVs. From this analysis it is determined that the parameter 'Number of Queues' is the defining variable and will require 15 replication runs to satisfy the criteria. 
Table 43 - Replication Analysis For Initial 5 Replication Run

\begin{tabular}{|c|c|c|c|c|c|c|c|}
\hline \multicolumn{8}{|c|}{$\mathrm{R} \geq\left[\left(\mathrm{Z}_{\alpha / 2} \times \mathrm{S}_{0}\right) / \varepsilon\right]^{2}$} \\
\hline $\begin{array}{l}\text { Battalion } \\
\text { Commander }\end{array}$ & $Z_{\alpha / 2}$ & $t_{\alpha / 2, \mathrm{df}}$ & $\mathrm{S}_{0}$ & $\varepsilon$ & $\mathrm{R}$ & R Adjusted & Conclusion \\
\hline \multirow[t]{4}{*}{ Workload: } & & & & & & & \multirow{4}{*}{$\begin{array}{l}\text { Since } \mathrm{R}<50 \text {, use } \mathrm{t} \text { disbribution and recalculate } \\
\therefore 5 \geq 1 \text { relationship is satisfied. No more runs required }\end{array}$} \\
\hline & $\mathrm{Z}_{.975}$ & $t_{97.5,1}$ & $\mathrm{~S}_{0}$ & $\varepsilon$ & $\mathrm{R}$ & R Adjusted & \\
\hline & 1.96 & & 13354.88 & 177426.45 & 0.02176 & 1 & \\
\hline & & 12.71 & 13354.88 & 177426.45 & 0.91524 & 1 & \\
\hline \multirow[t]{4}{*}{ Utilization: } & & & & & & & \multirow{4}{*}{$\begin{array}{l}\text { Since } \mathrm{R}<50 \text {, use } \mathrm{t} \text { disbribution and recalculate } \\
\therefore 5 \geq 2 \text { relationship is satisfied. No more runs required }\end{array}$} \\
\hline & $\mathrm{Z}_{.975}$ & $t_{97.5,1}$ & $\mathrm{~S}_{0}$ & $\varepsilon$ & $\mathrm{R}$ & R Adjusted & \\
\hline & 1.96 & & 0.0049 & 0.0462 & 0.04395 & 1 & \\
\hline & & 12.71 & 0.0049 & 0.0462 & 1.84795 & 2 & \\
\hline \multirow[t]{6}{*}{ Number of Queues } & & & & & & & \multirow{4}{*}{$\begin{array}{l}\text { Since } \mathrm{R}<50 \text {, use } \mathrm{t} \text { disbribution and recalculate } \\
\therefore 5 \geq 15->\mathrm{No} \text {; Set } \mathrm{R}=15 \text { and reevaluate } \\
\end{array}$} \\
\hline & $Z_{.975}$ & & $\mathrm{~S}_{0}$ & $\varepsilon$ & $\mathrm{R}$ & R Adjusted & \\
\hline & 1.96 & $t_{97.5,2}$ & 2.24 & 2.55 & 2.95394 & 3 & \\
\hline & & 4.3 & 2.24 & 2.55 & 14.21761 & 15 & \\
\hline & & $t_{97.5,14}$ & $\mathrm{~S}_{0}$ & $\varepsilon$ & $\mathrm{R}$ & & \multirow[b]{2}{*}{$\therefore 15 \geq 4->$ Yes, therefore Use $R=15$} \\
\hline & & 2.14 & 2.24 & 2.55 & 3.52141 & 4 & \\
\hline \multirow[t]{6}{*}{ Tasks Interrupted } & & & & & & & \multirow{4}{*}{$\begin{array}{l}\text { Since } \mathrm{R}<50 \text {, use } \mathrm{t} \text { disbribution and recalculate } \\
\therefore 5 \geq 10->\text { No; Set } \mathrm{R}=10 \text { and reevaluate }\end{array}$} \\
\hline & $Z_{.975}$ & & $\mathrm{~S}_{0}$ & $\varepsilon$ & $\mathrm{R}$ & R Adjusted & \\
\hline & 1.96 & $t_{97.5,1}$ & 1.10 & 4.49 & 0.22867 & 1 & \\
\hline & & 12.71 & 1.10 & 4.49 & 9.61567 & 10 & \\
\hline & & $t_{97.5,9}$ & $\mathrm{~S}_{0}$ & $\varepsilon$ & $\mathrm{R}$ & & \multirow[b]{2}{*}{$\therefore 10 \geq \mathbf{1}->$ Yes, therefore Use $\mathbf{R}=\mathbf{1 0}$} \\
\hline & & 2.26 & 1.10 & 4.49 & 0.30402 & 1 & \\
\hline \multirow[t]{6}{*}{ Tasks Suspended } & & & & & & & \multirow{4}{*}{$\begin{array}{l}\text { Since } \mathrm{R}<50 \text {, use } \mathrm{t} \text { disbribution and recalculate } \\
\therefore 5 \geq 13->\text { No; Set } \mathrm{R}=13 \text { and reevaluate }\end{array}$} \\
\hline & $Z_{.975}$ & & $\mathrm{~S}_{0}$ & $\varepsilon$ & $\mathrm{R}$ & R Adjusted & \\
\hline & 1.96 & $t_{97.5,2}$ & 0.45 & 0.54 & 2.63484 & 3 & \\
\hline & & 4.3 & 0.45 & 0.54 & 12.68176 & 13 & \\
\hline & & $t_{97.5,12}$ & $\mathrm{~S}_{0}$ & $\varepsilon$ & $\mathrm{R}$ & & \multirow[b]{2}{*}{$\therefore 13 \geq 4->$ Yes, therefore Use $R=13$} \\
\hline & & 2.18 & 0.45 & 0.54 & 3.25953 & 4 & \\
\hline
\end{tabular}


This page left intentionally blank. 
Vita

Sam E. Middlebrooks, a career Federal Civil Service employee for over 20 years, is a member of the Human Research and Engineering Directorate (HRED) of the U.S. Army Research Laboratory (ARL) where he is the Chief of the ARL-HRED Field Element at Fort Hood, Texas. Previously, through 17 years of Federal service in progressive assignments in the Federal Republic of Germany, he was chief of the programming team at a joint U.S. Army and U.S. Air Force computer wargaming center in Germany and later became the director of the largest corps level battle simulation center in Europe. Sam holds a Bachelor of Science degree in Mechanical Engineering from the University of Texas at Arlington and a Master of Science degree in Industrial and Systems Engineering (Human Factors option) from the Virginia Polytechnic Institute and State University in Blacksburg, Virginia. He is a retired U.S. Army Armor officer with operational experience in armored and cavalry units from platoon through corps levels. After two years of full time study on campus at Virginia Tech, beginning in the Fall of 1999, Sam is completing work on his Ph.D. with a dissertation project that has the long term goal of developing a new research program at Fort Hood conducting modeling and simulation of human cognitive performance in military command and control teams. Membership in professional societies include the Human Factors and Ergonomics Society (HFES), the Military Operations Research Society (MORS), the Association for Computing Machinery (ACM), and the Alpha Pi Mu Industrial Engineering Honor Society. 